\title{
ANÁLISE MULTIVARIADA
} EM

\section{INVENTÁRIO FLORESTAL CONTÍNUO}

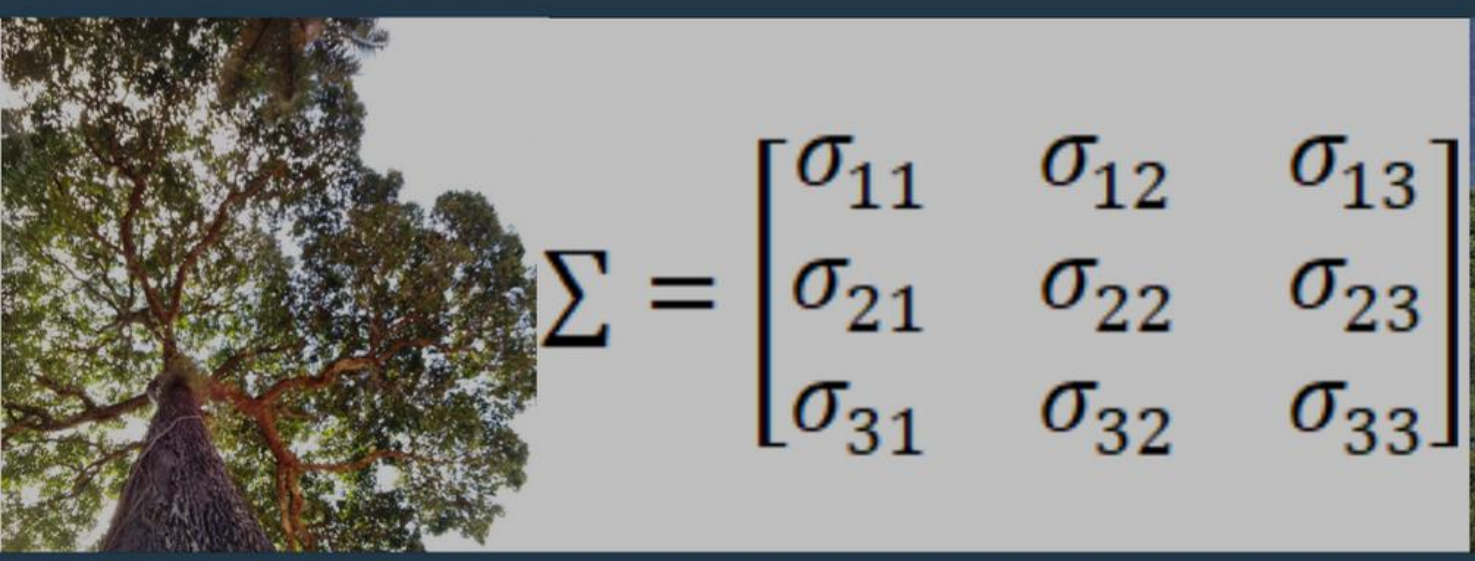

WALDENEI TRAVASSOS DE QUEIROZ

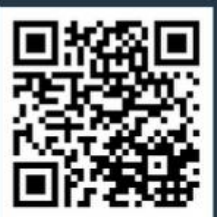


WALDENEI TRAVASSOS DE QUEIROZ

\section{Análise Multivariada em Inventário Florestal Contínuo}

1a Edição

Belo Horizonte

Poisson

2021 
Editor Chefe: Dr. Darly Fernando Andrade

\section{Conselho Editorial}

Dr. Antônio Artur de Souza - Universidade Federal de Minas Gerais

Ms. Davilson Eduardo Andrade

Dra. Elizângela de Jesus Oliveira - Universidade Federal do Amazonas

Msc. Fabiane dos Santos

Dr. José Eduardo Ferreira Lopes - Universidade Federal de Uberlândia

Dr. Otaviano Francisco Neves - Pontifícia Universidade Católica de Minas Gerais

Dr. Luiz Cláudio de Lima - Universidade FUMEC

Dr. Nelson Ferreira Filho - Faculdades Kennedy

Ms. Valdiney Alves de Oliveira - Universidade Federal de Uberlândia

Imagem da Capa: Bertholetia excelsa (quantumambiental@gmail.com) e Araucária angustifólia (www.stcp.com.br).

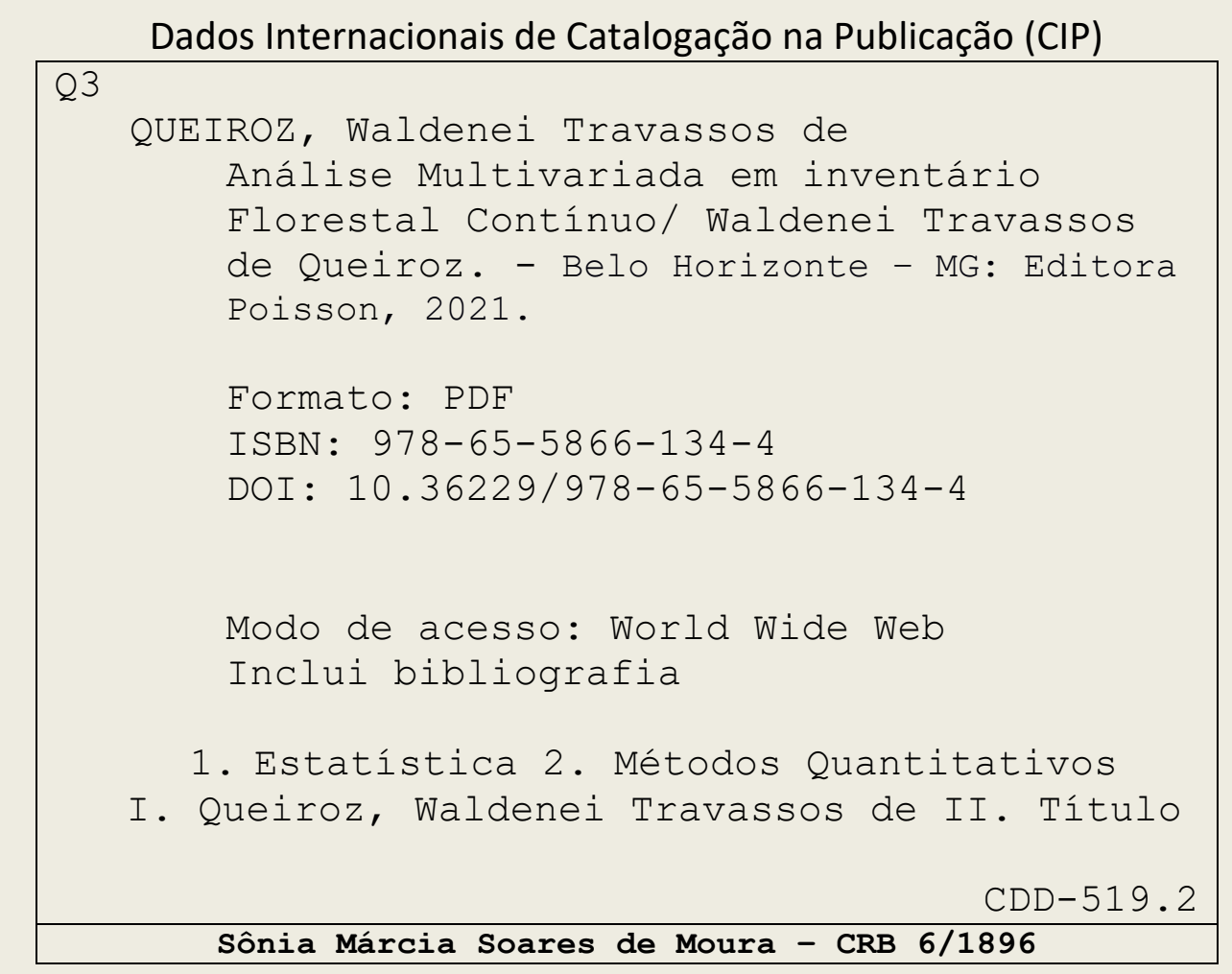

O conteúdo deste livro está licenciado sob a Licença de Atribuição Creative Commons 4.0.

Com ela é permitido compartilhar o livro, devendo ser dado o devido crédito, não podendo ser utilizado para fins comerciais e nem ser alterada.

O conteúdo dos artigos e seus dados em sua forma, correção e confiabilidade são de responsabilidade exclusiva dos seus respectivos autores.

www.poisson.com.br

contato@poisson.com.br 
À minha amada esposa Izabel. Aos meus filhos Anderson e Alex.

Às noras Alessandra e Lorena. Aos netos Lucas, Gabriel e Alícia. Aos meus pais Wagner e Erecina in memoriam.

Viva a vida, como se estivesse construindo com alegria, ternura e amor, a cada tempo de Planck, uma grande obra de arte matemática que é você mesmo.

Adaptado do ensinamento do Deus Tolteca Quetzalcóatl (Serpente Emplumada) 


\section{AGRADECIMENTOS}

À Editora Poisson, pelo empenho e dedicação na publicação deste livro. À Inácia Faro Libonati pela cuidadosa revisão do texto final.

À Universidade Federal Rural da Amazônia (UFRA), pela oportunidade que tive de exercer o magistério por 41 anos, e assim poder ensinar e aprender, não somente os conhecimentos técnicos sobre inventário florestal, mas, em especial, com os estudantes, colegas professores e funcionários, compreender a arte real do amor incondicional, atributo espiritual que estabelece as bases das relações humanas, condição importante para deslindar, em nosso planeta azul, os segredos da nossa missão de vida.

Ao Serviço Florestal Brasileiro (MMA), pelo convite para participar de todos os eventos que culminaram na construção e na implantação do Projeto do Inventário Florestal Nacional do Brasil (IFN), o que possibilitou produzir grandes discussões e aprendizados na área de inventário florestal.

Ao Professor Sylvio Péllico Netto (UFPR) pelos conhecimentos na área de inventário florestal e aos professores da ESALQ-USP: Cassio Roberto de Melo Godoi, Humberto de Campos, Frederico Pimentel Gomes (in memoriam), pelos ensinamentos que recebi na área de análise estatística uni e multivariada, em nossa formação pós-graduada, e ao Professor Carlos Tadeu dos Santos Dias pelos conhecimentos na elaboração de programas na linguagem do Sistema de Análise Estatística SAS.

Aos revisores deste trabalho: à Engenheira Florestal Anabel Aparecida de Mello, Professora da UFS, Mestrado pela UFLA e Doutorado pela UFPR; ao Engenheiro Florestal Joberto Veloso de Freitas, Professor da UFAM, Mestrado no INPA e Doutor pela Universidade de Aberdeen, Escócia; ao Engenheiro Florestal Joésio Deoclécio Pierin Siqueira, Vice-Presidente da STCP, Professor Aposentado da UFPR, Mestrado e Doutorado pela UFPR; ao Engenheiro Florestal José Natalino Macedo Silva, Professor Visitante da UFRA, Mestrado na UFPR e Doutor pela Universidade de Oxford; ao Engenheiro Florestal Lenilson Ferreira Palheta, Mestrado e Doutorado pela UFRA; à Engenheira Florestal Maria de Nazaré Maciel, Professora da UFRA, Mestrado pela UFRA e Doutorado pela UFPR; ao Engenheiro Florestal Paulo Luiz Contente de Barros, Professor Aposentado da UFRA, Mestrado e Doutorado pela UFPR; ao Bacharel em Estatística e Licenciado Pleno em Matemática Pedro Silvestre da Silva Campos, Professor da UFRA, Mestrado em Estatística pela UFPA e Doutorado em Ciências Agrárias pela UFRA.

A todos os colegas professores de Inventário Florestal com os quais tive o prazer de conviver e aprender, os quais homenageio em nome dos que participaram do lançamento do meu livro de "Amostragem em inventário florestal", quando da realização do I Simpósio Nacional de Inventario Florestal, Natal-RN, em 26-28/11/2012, promovido pelo Serviço Florestal Brasileiro (SFB/MMA). Fotografia do evento de lançamento: Paulo Luiz Contente de Barros, Daniel Piotto, Alexander Christian Vibrans, Doádi Antonio Brena, Waldenei Travassos de Queiroz, Joberto Veloso de Freitas, Anabel Aparecida de Mello, Sylvio Péllico Netto, Fabio Venturoli, José Enílcio Rocha Collares, 
Versides Sebastião Moraes e Silva, José Roberto Soares Scolforo, Afonso Figueiredo Filho e Paulo Rogério Soares de Oliveira.

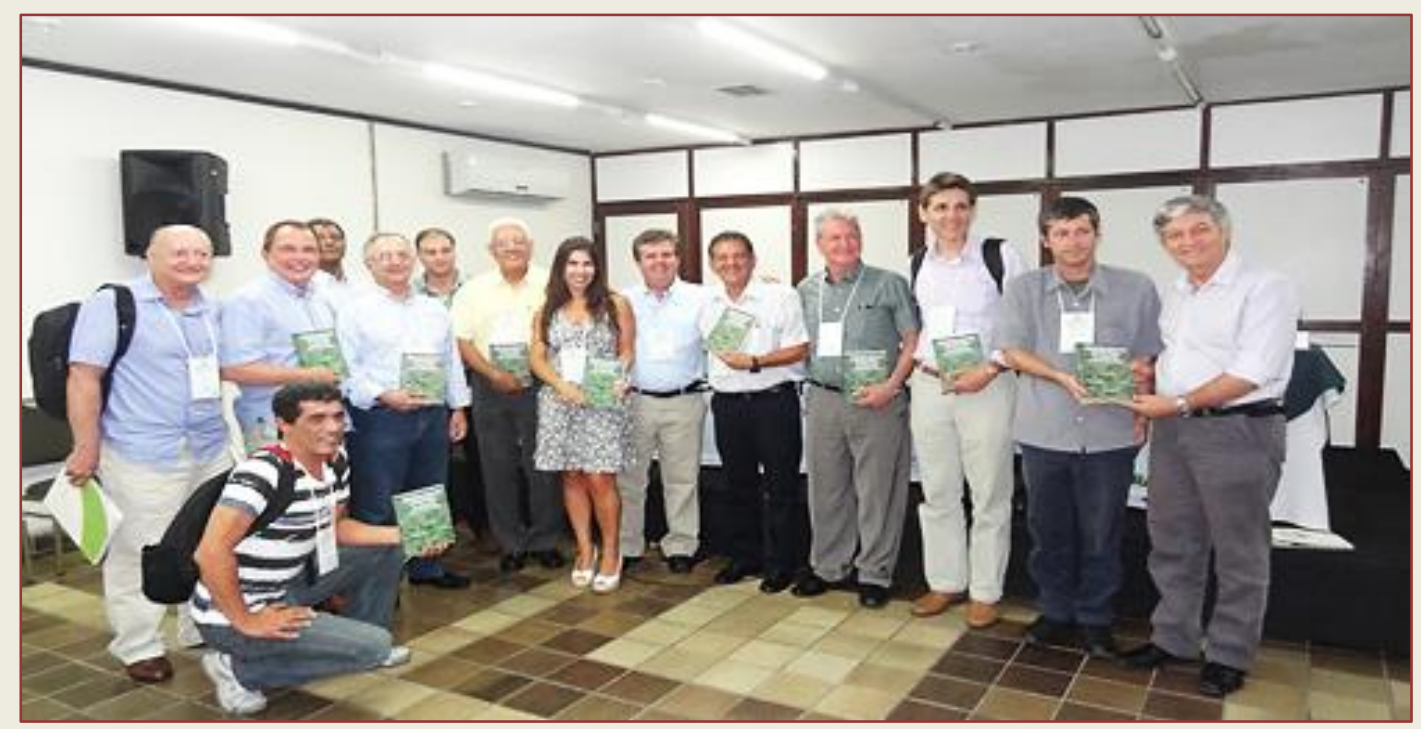

Aos colegas professores e aos profissionais, que com amor e dedicação, adotaram o inventário florestal como ideal profissional, a amizade, o convívio e os ensinamentos recebidos: Agostinho Lopes de Souza, Alba Valeria Rezende, Armando Pinheiro de Carvalho Filho (in memoriam), Arne Nyyssönen, Bem-Hur Borges, Carlos Roberto Sanquetta, Bernard Rollet, Fernando Cristóvam da Silva Jardim (in memoriam), Floralin de Jesus Fonseca Coelho, Evaristo Francisco de Moura Terezo, Fernando Antônio Souza Bemerguy, Francisco de Paula Neto (in memoriam), Francisco José de Barros Cavalcante, Henrique de Castro Ferreira (in memoriam), Hilton Tadeu Zarate do Couto, Niro Higuchi, Hugo Barbosa Amorim, Jean Clement Laurent Dubois (in memoriam), Jeanine Maria Felfili (in memoriam), João Olegário Pereira de Carvalho, João Ricardo Vasconcellos Gama, John Palmer, John Welker, Jorge Alberto Gazel Yared, José de Arimatéia Silva, Juris Jankauskis, Lia de Oliveira Melo, Marco Antonio Amaro, Paulo Renato Schneider, Permínio Pascoal Costa Filho (in memoriam), Rafael de Paiva Salomão, Rosângela de Jesus Souza, Rui Lopes Loureiro (in memoriam), Sebastião do Amaral Machado, Ulisses Silva da Cunha, Yeda Maria Malheiros Oliveira e ao Estatístico Professor Carlos Roberto Padovani (Campus de Botucatu/UNESP), colega no curso de doutorado na ESALQ/USP, pela amizade e aprendizado quando das nossas discussões sobre análise multivariada, e a todos que me incentivaram na tarefa de oferecer aos usuários da teoria de análise multivariada aplicada em Inventário Florestal Contínuo, uma obra que reúne algumas experiências condizentes, principalmente, com as condições impostas pelos tipos florestais ocorrentes no Brasil. 


\section{BIOGRAFIA DO AUTOR}

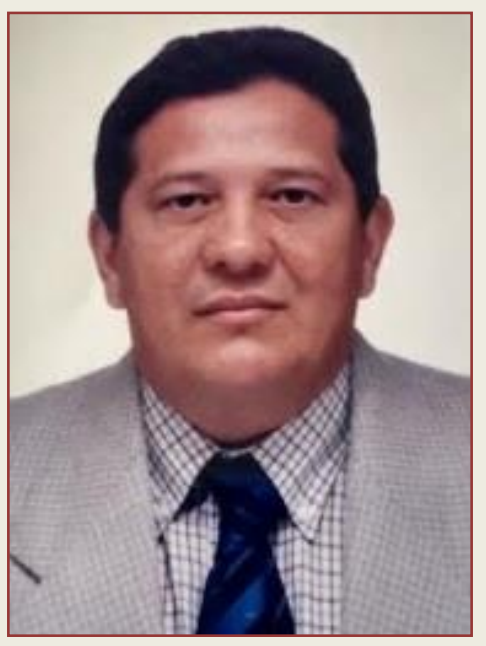

Waldenei Travassos de Queiroz nasceu, em Belém do Pará, no ano de 1950. É graduado em Engenharia Florestal e Mestre em Manejo Florestal pela Universidade Federal do Paraná (UFPR) e doutor em Estatística e Experimentação Agronômica pela Escola Superior de Agricultura "Luiz de Queiroz" (USP). Em 1974, iniciou suas atividades profissionais no Projeto RadamBrasil. Em 1975/1976, Contraparte Nacional do Setor de Inventário Florestal do Projeto BRA-45 FAO/IBDF. É Professor Titular aposentado da Universidade Federal Rural da Amazônia (UFRA). Em 1979, membro da comissão do IBDF para traçar diretrizes básicas para o estabelecimento de um Sistema Nacional de Avaliação de Recursos Naturais do Brasil. Em 1997, consultor do Programa de Desenvolvimento das Nações Unidas (PNUD) em Estatística e Planejamento de Inventários Florestais no Programa de Cooperação Técnica ao Zoneamento Ecológico Econômico do Estado de Rondônia (Projeto BRA/94/007). Foi Pró-reitor de Pesquisa e de Pós-Graduação e Vice Reitor da UFRA. De 1988-2002, professor de Inventário Florestal e Análise Multivariada do Curso de Pós-Graduação em Ciências Florestais Tropicais do INPA. Em 2009/2010, consultor da Organização das Nações Unidas para a Agricultura e Alimentação (FAO), junto ao Serviço Florestal Brasileiro (SFB) do MMA, para o planejamento do Sistema de Amostragem do Inventário Florestal Nacional do Brasil. Publicou os livros "Técnicas de amostragem em Inventário Florestal nos Trópicos”, em 1998, e “Amostragem em Inventário Florestal”, em 2012. É membro do Conselho Editorial e Científico da Revista Ciência Florestal da Universidade Federal de Santa Maria (UFSM) e também membro da Comissão Editorial da Revista Árvore da Universidade Federal de Viçosa (UFV). 


\section{PREFÁCIO}

O livro que o Prof. Waldenei Travassos de Queiroz nos entrega foi elaborado para ampliar as alternativas de estudantes e profissionais para lidar com a complexidade das florestas, por meio da aplicação de métodos estatísticos multivariados.

As florestas desempenham importantes e múltiplas funções que dão suporte à vida humana na terra. Elas nos entregam produtos, serviços ambientais e valores culturais, continuamente. 0 desafio de conservar e usar sustentavelmente essas florestas deve ser enfrentado com base na ciência e no conhecimento, como forma de garantir, também às gerações futuras, os benefícios que temos recebido delas.

O Brasil detém cerca de 500 milhões de hectares de florestas, posicionando-se como a segunda maior extensão florestal do mundo, segundo estimativas da FAO em 2020. Não há como conhecer e manejar as nossas florestas sustentavelmente para atender as necessidades da sociedade sem dispor de dados e métodos objetivos para subsidiar as melhores decisões, em todas as escalas. Conhecer as florestas é do que tratam os inventários florestais, seja em levantamentos pontuais ou em múltiplas ocasiões no tempo.

O inventário florestal contínuo é a principal ferramenta de monitoramento florestal a partir de dados de campo, sendo que quando este é baseado em medidas repetidas obtidas de parcelas permanentes, torna-se uma preciosa fonte de dados sobre a dinâmica, o crescimento e a produção florestal.

O manejo de florestas naturais da Amazônia é uma alternativa legítima de uso com a garantia da permanência da floresta, seus serviços e funções, em longo prazo. Mas ainda há muito que descobrir para o seu refinamento. No caso da produção de madeira, o manejo das florestas amazônicas baseia-se na colheita de algumas árvores por unidade de área e de um período de tempo de espera, ou de pousio, para que a floresta se recupere e possa ser explorada novamente.

Durante o período de pousio, que leva anos, as parcelas permanentes são medidas periodicamente, gerando dados sobre o crescimento das espécies e a evolução da recuperação da capacidade produtiva da floresta. Como o manejo florestal é uma atividade de longo prazo, dispor de métodos que estimem o tempo exato em que as árvores de espécies comerciais atingirão a maturidade para nova colheita é um importante passo para um bom planejamento.

E ao observarmos as variáveis e complexidades das florestas tropicais, que são múltiplas, vemos que o emprego da análise multivariada pode alavancar o potencial de resposta dos inventários florestais substancialmente, ampliando as possibilidades para um melhor uso e conservação das florestas. Este é o caso, por exemplo, do Inventário Florestal Nacional, que além de ser um inventário florestal contínuo, em escala nacional, também abrange variáveis biofísicas, ambientais e sociais relacionadas às florestas, compondo múltiplas dimensões e variáveis. 
O livro Análise Multivariada em Inventário Florestal Contínuo cobre uma lacuna na ciência florestal, particularmente de métodos para análise de medidas repetidas no tempo, mas também com aplicação desses métodos na solução de vários problemas clássicos desta área da ciência. De forma detalhada e didática, ele oferece os fundamentos necessários para o entendimento teórico, como a álgebra de matrizes e vetores, as pressuposições para a análise de inventários florestais contínuos, a análise de variância multivariada e noções sobre a regressão não linear no contexto do tema central do livro. Objetivamente, o livro oferece exemplos de situações típicas de inventário florestal contínuo em que os métodos de análise multivariada podem ser aplicados pelo manejador, envolvendo perfis de espécies e métodos de manejo, como bases para subsidiar importantes decisões econômicas e silviculturais. Exemplos numéricos, bancos de dados, rotinas computacionais e os procedimentos necessários para a aplicação dos métodos de análise também são apresentados e tornam o livro didático e ainda mais valioso.

As florestas da Amazônia são complexas e ainda há muito a conhecer, e assim é também o seu manejo e, por isso, precisamos dispor de dados e métodos que nos permitam maior aproximação de seu entendimento e compreensão, de modo que possamos encontrar melhores caminhos para a sustentabilidade, e a estatística e os inventários florestais, sem dúvida são ferramentas de que dispomos para isso.

Finalmente, é muito importante destacar a grande contribuição que o autor trouxe, ao longo de sua brilhante carreira como Engenheiro Florestal com Pós-graduação em Métodos Estatísticos, para o ensino e compreensão das teorias que suportam e permitem, cada vez mais, uma visão ampliada dos inventários florestais.

Joberto Veloso de Freitas-Professor da UFAM Doutor pela Universidade de Aberdeen, Escócia. 


\section{APRESENTAÇÃO}

São muitas as dificuldades enfrentadas pelos profissionais e estudantes que se dedicam aos estudos das ciências florestais e que necessitam fazer uso de métodos estatísticos multivariados na análise de inventários florestais multidimensionais, decorrentes da falta de livros didáticos com aplicações práticas dentro desse contexto.

Objetivando ajudar a minimizar esse problema, o presente livro foi elaborado com a finalidade de contribuir para a diminuição dessa lacuna existente na literatura florestal e, de colaborar com o aprendizado de disciplinas que tratam da análise de levantamentos florestais.

O conteúdo apresentado nesta obra constitui a base dos cursos de análise multivariada que foram ministrados aos alunos de graduação do curso de Engenharia Florestal e no programa de pós-graduação em Ciências Florestais da Universidade Federal Rural da Amazônia (UFRA), quando do exercício ativo da docência deste professor aposentado.

Este livro nada mais seria que "um pingo d'água no oceano", dentro do mar diversificado de alternativas que caracterizam o ramo da análise estatística multivariada, caso o objetivo fosse apresentar com profundidade todos os métodos estatísticos, visando a subsidiar a análise de levantamentos e de experimentos longitudinais, considerando a complexidade requerida e a grande quantidade de problemas a serem equacionados dentro da engenharia florestal. A seguir serão abordados alguns pontos para aguçar a discussão sobre o panorama da análise multidimensional nos inventários florestais.

No caso do estudo dos recursos naturais de uma floresta, o planejamento dos inventários para subsidiar a operacionalização de um plano de manejo florestal sustentado, obrigatoriamente, deve, em seu levantamento, definir, distinguir e delimitar os ecossistemas com características semelhantes, e obviamente, os métodos estatísticos univariados não oferecem o suporte necessário e suficiente de análise para atender referido objetivo.

A experiência na Amazônia tem demonstrado que as estratificações dos ecossistemas estabelecidas mediante a interpretação de imagens de satélite e de fotografias aéreas, apesar de importantes e informativas, isoladamente não são capazes de explicar com o detalhamento requerido as inter-relações existentes entre as variáveis envolvidas para a definição dos diferentes estratos, principalmente na composição da vegetação localizada no sub-bosque da floresta. Desse modo, seria indicado compatibilizar os recursos de sensoriamento remoto e de planejamento de amostragem com a análise estatística multivariada. Entretanto, durante a execução de uma análise multivariada ocorrem algumas dificuldades, sejam em relação à compreensão de sua teoria estatística ou com relação à computação dessas análises.

É inegável, nas análises fitossociológicas de uma floresta natural, ser o uso da análise multidimensional um instrumento poderoso para interpretar as correlações inter e intra, existentes nos seus ecossistemas. Este fato fica ainda mais evidente atualmente, quando os métodos mais comumente utilizados nos estudos da fitossociologia e, amplamente disponíveis na literatura, através dos diversos "índices fitossociológicos" unidimensionais que, de acordo com Queiroz et al. (2017), tornam difícil a interpretação 
ecológica dos tipos florestais, principalmente por não apresentarem estruturas de lógica matemática e de método estatístico capazes de explicarem a complexidade dos problemas envolvidos.

Atualmente, um dos problemas mais complexos enfrentados pelos profissionais que atuam no campo do manejo das florestas de terra firme da Amazônia, é a análise dos inventários florestais contínuos, com parcelas permanentes, cujo objetivo principal é o de monitorar e avaliar a dinâmica de crescimento e produção da floresta manejada. Por outro lado, para subsidiar o planejamento, definição e aplicação dos tratos silviculturais com vista à aceleração da recomposição da floresta manejada, torna-se imprescindível o uso de métodos estatísticos, que levem em consideração a detectação e a classificação de agrupamentos de espécies que possuam perfis de crescimento semelhantes.

Em se tratando do manejo de florestas naturais, a análise estatística do inventário contínuo com parcelas permanentes, ajuda a explicar a tendência de crescimento de diferentes grupos de espécies com características de desenvolvimento semelhantes e, portanto, facilita o planejamento dos tratamentos silviculturais a serem aplicados para estimular a regeneração natural e melhorar o crescimento da floresta. A análise multivariada, denominada de medidas repetidas, com o uso das técnicas de análises de levantamentos longitudinais, pode ajudar na determinação desses grupos. Em alguns casos, é até recomendável a aplicação conjunta de vários métodos multidimensionais.

As técnicas multivariadas de componentes principais e análise de fatores podem ser utilizadas na diminuição da dimensionalidade dos dados, o que possibilitaria a partir de um número menor de variáveis, mais facilmente explicar suas inter-relações. Essas técnicas são importantes para construir índices fitossociológicos multivariados, e, a partir deles, produzir mapas delimitando as comunidades florestais.

As técnicas de análises de agrupamento, de discriminante e de variância multivariada podem ser usadas para delimitar, classificar e comparar os diferentes tipos de ecossistemas, e assim obter mapas tipológicos baseados nas variáveis mais importantes na explicação das estruturas florísticas e ecológicas da vegetação.

Pode ser interesse de o pesquisador estudar as inter-relações entre grupos de variáveis. Por exemplo, um grupo pode ser formado por variáveis da flora e o outro pelas variáveis que refletem a fertilidade de solo. A análise de correlação canônica ajuda a explicar essas correlações gerando, inclusive, mapas com a estratificação das diversas relações solovegetação.

Nesta obra, os programas para efetuar as análises foram elaborados utilizando a linguagem de programação do Sistema de Análise Estatística (SAS), haja vista o seu grande potencial e amplitude, assim como a sua facilidade operacional e possibilidade de adaptação a outros aplicativos disponíveis no mercado. 


\section{SUMÁRIO}

CAPÍTULO 2 - ÁLGEBRA DE VETORES E DE MATRIZES ............................................... 21

2.1. - Produto interno ou produto escalar de dois vetores ....................................... 22

2.2. - Ortogonalidade e base ortonormal. .............................................................. 23

2.3. - Vetores linearmente dependentes e independentes ...................................... 23

2.4. - Ortonormalização de Gram-Schmidt ................................................................ 24

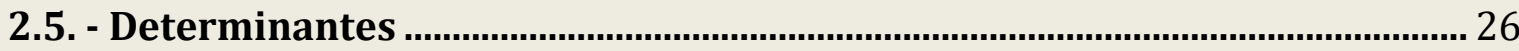

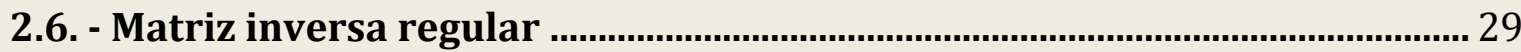

2.7. - Matrizes inversas generalizadas (Inversa-g) ................................................... 31

2.7.1. - Matriz generalizada de Moore-Penrose ...................................................................... 32

2.7.2. - Matriz inversa generalizada condicional. ....................................................................... 32

2.7.3. - Matriz inversa generalizada de quadrados mínimos................................................ 32

2.7.4. - Matriz inversa reflexiva ou inversa-g2. …………………………………………….... 33

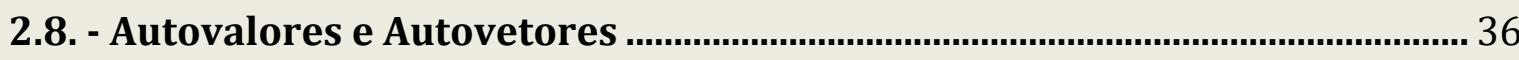

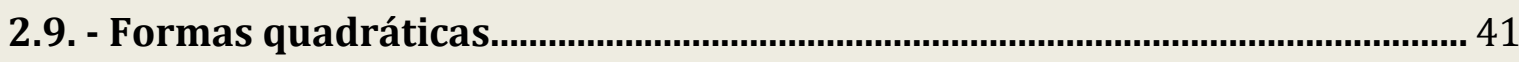

2.10. - Matriz Helmert ............................................................................................... 44

CAPÍTULO 3 - MULTINORMALIDADE E ESTRUTURAS DE MATRIZES DE

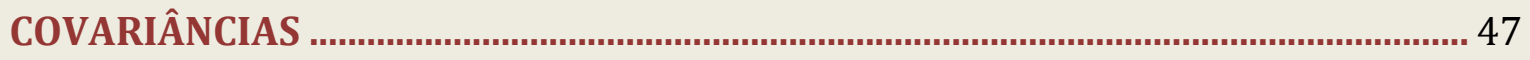

3.1. - Teste de multinormalidade de Mardia. .............................................................. 49

3.2. - Teste de multinormalidade de Henze-Zirkler .................................................. 51

3.3. - Teste de homogeneidade de matrizes de covariâncias considerando $p$

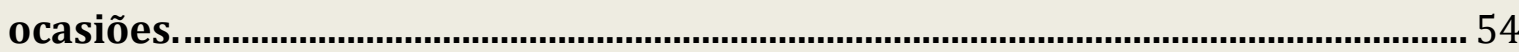

3.4. - Teste de uniformidade da matriz de covariância considerando p ocasiões.

3.4.1. - Teste de uniformidade da matriz de covariância de Wilks..................................... 57

3.4.2. - Circularidade ou esfericidade de uma matriz............................................................. 59

3.4.3. - O critério de Mauchly para testar a esfericidade de uma matriz ............................ 62

3.4.4. - Correção de Greenhouse-Geisser (g-g).................................................................... 64

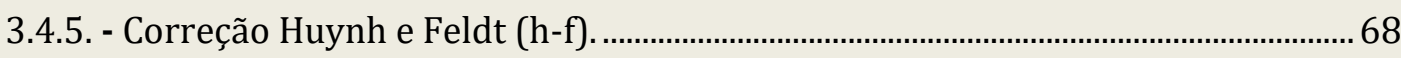


4.1. - Análise multivariada para testar um vetor de médias de tratamento versus um vetor padrão opulacional. 70

4.2. - Análise multivariada para testar vetores de médias para dois tratamentos independentes. 74

4.3. - Modelo linear univariado para mais de dois tratamentos............................ 76

4.4. - Análise de variância multivariada do ensaio inteiramente ao acaso......... 79

4.5. - Testes de hipóteses multivariados e aproximações para a distribuição F. 82

4.6. - A função discriminante ou variável canônica Z1................................................ 84

4.7. - Análise de um experimento inteiramente ao acaso bivariado ..................... 85

CAPÍTULO 5 - NOÇÕES SOBRE REGRESSÃO NÃO LINEAR .................................. 97

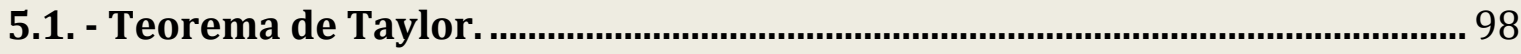

5.2. - Estimadores de máxima verossimilhança. ...................................................... 99

5.3. - Teste da razão de verossimilhança. ................................................................... 101

5.4. - Teste para verificar a igualdade de parâmetros e a identidade de modelos de

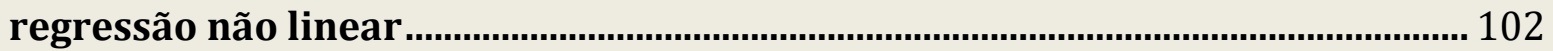

5.5. - Análise de resíduos ........................................................................................... 104

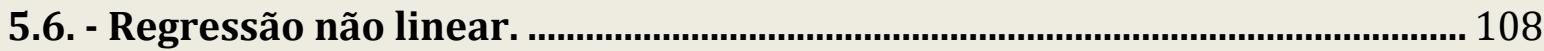

5.6.1. - Determinação das estimativas dos parâmetros, para o modelo de Mitscherlich, pelo método dos mínimos quadrados ordinários.

5.6.2. - Determinação das estimativas dos parâmetros, pelo método dos mínimos quadrados ordinários, usando a forma matricial para o modelo de Mitscherlich

5.7. - Método de Gauss-Newton

5.8. - Avaliação da adequacidade dos modelos testados.

5.9. - Validação da predição do modelo de regressão pelo teste de Graybill .... 118

CAPÍTULO 6 - CICLO DE CORTE ECONOMICAMENTE ACONSELHÁVEL

6.1. - Derivadas

6.2. - Máximo e mínimo de uma função com uma variável 
6.4. - Exemplo de obtenção de máximo e mínimo de uma função com três variáveis

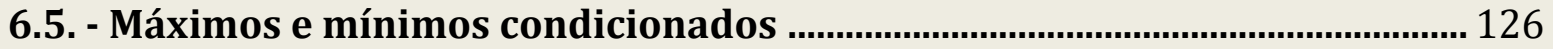

6.6. - Método dos Multiplicadores de Lagrange (Joseph Louis Lagrange 17361813)

6.7. - Equação de Mitscherlich para estimar o crescimento de uma floresta ..... 131

6.8. - Ciclo de corte economicamente aconselhável com a equação de Mitscherlich 134

6.9. - Ciclo de corte economicamente aconselhável pela equação de VonBertalanffy. 136

CAPÍTULO 7 - ANÁLISE DE INVENTÁRIOS FLORESTAIS CONTÍNUOS. 138

7.1. - Análise univariada de inventários florestais com parcelas permanentes pelo método de parcelas subdivididas 140

7.2. - Inventários florestais com parcelas permanentes com um tratamento... 142

7.2.1. - Análise de um inventário florestal contínuo com um método de manejo

7.3. - Inventários florestais com parcelas permanentes com dois tratamentos 148

7.3.1. - Análise de um inventário florestal contínuo com dois métodos de manejo

7.4. - Inventários florestais com parcelas permanentes com mais de dois tratamentos 154

7.5. - Testes de hipóteses multivariados e as aproximações para a distribuição F. 158

7.6. - Análise de agrupamento pelo método de Ward 159

7.7. - Análise de um inventário florestal contínuo com quatro métodos de manejo

7.7.1. - Análise da interação Ocasião×Método de Manejo. 161

7.7.2. - Estimativa do ciclo de corte economicamente aconselhável para M1, M2, M3 e M4.

7.7.3. - Teste da razão da máxima verossimilhança, de verificação de igualdade de parâmetros e a identidade de modelos de regressão não linear, para M3 e M4 
7.7.4. - Teste da razão da máxima verossimilhança, considerando os métodos de manejos M1, M2, M3 e M4, para verificar a igualdade de parâmetros e a identidade de modelos de regressão não linear. 175

7.7.5. - Análise dos resultados e conclusões do exemplo do inventario florestal contínuo.

\section{CAPÍTULO 8 - ANÁLISE DE PERFIL DE ESPÉCIES EM FLORESTAS NATURAIS...... 179}

8.1. - Análise de perfil de crescimento de quatro espécies florestais. .................. 180

8.1.1. - Análise da interação Ocasião×Espécie ...................................................................... 180

8.1.2. - Ciclo de corte economicamente aconselhável para E1, E2, E3 e E4 ..................... 188

8.1.3. - Teste da razão da máxima verossimilhança para verificar a igualdade de parâmetros e a identidade de modelos de regressão não linear .......................................... 193

8.1.4 - Considerações finais da análise do IFC para as espécies E1, E2, E3 e E4............. 198

\section{CAPÍTULO 9 - TÉCNICAS MULTIVARIADAS EM INVENTÁRIO FLORESTAL ........... 201}

\section{1. - Análise de componentes principais..................................................................... 203}

9.1.1. - Significado geométrico dos componentes principais .................................. 206

9.1.2. - Metodologia dos índices normalizados ............................................................. 207

9.1.3. - Análise de componentes principais para as espécies E1, E2, E3, E4, considerando como variáveis as ocasiões OC1, OC2, OC3, OC4 e 0C5................. 208

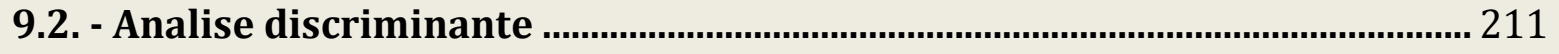

9.2.1. - Função linear discriminante de Fisher..................................................................... 211

9.2.2. - Caso de duas populações minimizando a probabilidade total de classificação.

9.2.3. - Caso de duas populações multinormais com dispersões conhecidas e iguais. 213

9.2.4. - Caso de três populações multinormais com dispersões conhecidas e iguais, assim como (p1 = p2)

9.2.5. - Método não paramétrico para calcular a probabilidade de má classificação... 216

9.2.6. - Análise de discriminante, considerando as variáveis (OC1, OC2, OC3, OC4, OC5) para as quatro populações (E1, E2, E3 e E4)......................................................................... 216

9.3. - Análise de correlação canônica. ..................................................................... 220

9.3.1. - Análise de correlação canônica considerando os métodos de manejo (M1, M2, M3, M4) e as quatro espécies (E1, E2, E3, E4) 


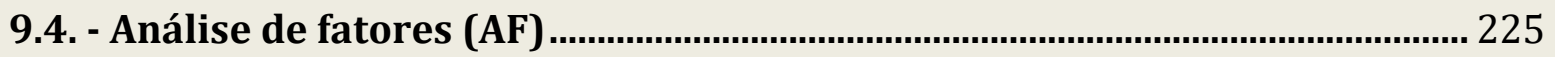

9.4.1. - Análise de fatores pelo método da máxima verossimilhança................................. 228

9.4.2. - Teste de hipótese para determinar o número de fatores (m) adequado para reproduzir o modelo (método de máxima verossimilhança) ............................................... 232

9.4.3. - Estimação dos escores dos fatores. .................................................................................. 232

9.4.4. - Metodologia dos índices normalizados ................................................................. 232

9.4.5. - Análise de fatores por componentes principais........................................................ 233

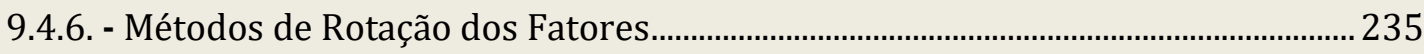

9.4.7. - Análise de fatores, pelo método dos componentes principais, para quatro espécies (E1, E2, E3, E4) em relação às variáveis (OC1, OC2, OC3, OC4, OC5)................................. 236

CONSIDERAÇÕES FINAIS.................................................................................. 240

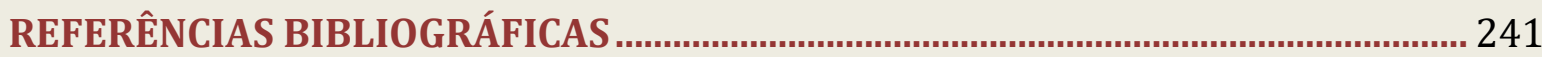

APÊNDICE A: Distribuições estatísticas .......................................................... 247

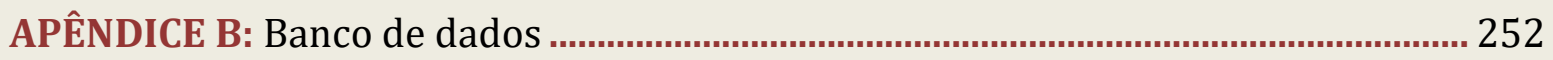

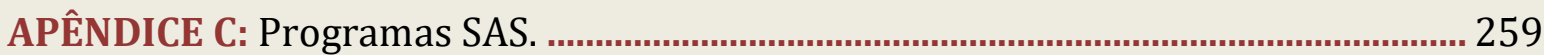




\section{Capítulo 1}

\section{Introdução ao inventário florestal contínuo}

Neste capítulo serão abordados, de um modo geral, os procedimentos denominados de medidas repetidas, por meio das técnicas de análise de levantamentos longitudinais, que são importantes quando se estuda perfil de crescimento, em particular, em inventário florestal contínuo com uso de parcelas permanentes, destacando temas fundamentais da estatística e da matemática, para que os estudantes da disciplina de inventário florestal percebam a importância do uso da estatística multidimensional na análise e na solução dos problemas florestais. 


\section{INTRODUÇÃO}

Para avaliar e analisar a dinâmica de recomposição de uma floresta explorada, como parte integrante de um plano de manejo florestal sustentável, é necessário utilizar um processo de monitoramento da dinâmica da floresta para determinação do crescimento e produção dos seus recursos. Na ciência florestal, este procedimento é conhecido como Inventário Florestal Contínuo (IFC), onde são efetuadas mensurações repetidas no tempo, ou seja, medições em várias ocasiões. 0 tipo de alocação de parcelas mais comum no IFC são as permanentes, constituindo-se em um caso de população multivariada, onde variáveis aleatórias, como por exemplo, o volume de madeira avaliado em $p$ ocasiões segue uma distribuição de probabilidade com um vetor de médias e uma matriz de covariância. Os sucessivos inventários florestais realizados em ocasiões diferentes permitirão ajustar a respectiva função de crescimento, e assim, irão possibilitar a definição da idade ou ciclo de corte economicamente aconselhável para promover a colheita dos produtos da floresta.

A análise de levantamentos com parcelas permanentes enquadra-se no grupo de experimentos com medidas repetidas, mais precisamente na classe dos levantamentos longitudinais, por envolver observações, em diversas ocasiões, em uma mesma unidade experimental. As medidas são repetidas no tempo de forma sistemática e, assim sendo, espera-se existir uma correlação não nula entre as medidas obtidas nos vários períodos de tempo analisados, bem como uma heterocedasticidade ou heteroscedasticidade das variâncias nas diversas ocasiões.

Experimentos planejados com medidas repetidas, normalmente têm como objetivo estudar o efeito global dos tratamentos ao fim do experimento, a variação do efeito dos tratamentos ao longo do tempo, ou mesmo estudar o efeito em alguma ocasião em particular.

Existem dois métodos para testar as hipóteses sobre o efeito dos tratamentos e a correspondente variação ao longo do tempo. 0 primeiro adota um procedimento univariado que trata as observações medidas no tempo como se fossem originadas de subdivisões das unidades experimentais (split-plot), sendo estudado por meio do delineamento em parcelas subdivididas, mas com a exigência de que as medidas tomadas em ocasiões distintas tenham variâncias homogêneas e sejam igualmente correlacionadas. O segundo método, denominado de análise de perfil ("profile analysis"), adota um procedimento multivariado, ou seja, considera as observações repetidas sobre cada unidade experimental como um vetor de respostas.

A análise de perfil deve ser usada quando as ocasiões distintas não possuem variâncias homogêneas e/ou também não são igualmente correlacionadas. Este procedimento adota hipótese completamente geral sobre a estrutura de covariâncias entre medidas repetidas tomadas sobre uma mesma unidade experimental.

A escolha do procedimento de análise de perfil de crescimento no IFC, se univariado ou multivariado, depende de suposições para o conjunto de dados em estudo. 0 modelo de análise univariada de perfil em parcelas subdivididas ("split-plot") não é recomendado para a análise de dados com medidas repetidas, pois considerando o modo sistemático 
como são feitas as observações nas ocasiões, geralmente não é esperado uma matriz de covariância do tipo uniforme.

De acordo com Huynh e Feldt (1970), uma condição necessária e suficiente para que possa ser empregada a análise de variância univariada, no esquema de parcelas subdivididas, para um experimento com medidas repetidas, é que a matriz de covariância entre os tempos satisfaça a condição de esfericidade ou circularidade, o que é equivalente a especificar que as covariâncias entre pares de erros sejam todas iguais. Para testar tal condição, Mauchly (1940) propôs um teste de esfericidade que verifica se uma população normal multivariada apresenta variâncias iguais e correlações nulas. Caso o teste de esfericidade de Mauchly resulte ser não significativo, conclui-se que a matriz de covariância é classificada como esférica. Logo o experimento pode ser analisado na forma de parcela subdividida, caso contrário, recomenda-se o uso da análise multivariada de perfil.

Uma aplicação da análise univariada, considerando o delineamento em parcelas subdivididas, com o objetivo de estudar curvas de crescimento e análise de perfil de um ensaio com parcelas permanentes, pode ser vista em Queiroz (2012).

Queiroz (2014) utilizou a análise multivariada de perfil, a partir de dados de um inventário florestal contínuo com parcelas permanentes, em um experimento desenvolvido por Yared (1983), considerando nove procedências da espécie Cordia alliodora (Ruiz \& Pav) Oken e as ocasiões de 6,12, 18, 24 e 30 meses. 0 objetivo foi estudar curvas de crescimento e análise de perfil para a variável altura da espécie.

Morrison (1976) descreveu o teste de Mauchly (1940) para a validade da condição de esfericidade da matriz de covariância. 0 referido teste está disponível no PROC GLM do SAS (2002), com o comando repeated e a opção printe.

Para evitar problemas de singularidade das matrizes de covariâncias, o tamanho da amostra, no mínimo, deve ser maior do que o número de variáveis dependentes. A maioria dos experimentos biológicos apresenta tamanho amostral insuficiente para proceder ao teste de multinormalidade e de homogeneidade de matrizes de covariâncias. Desse modo, Hair et al. (2009) recomendam que, na MANAVA, o tamanho da amostra seja maior do que na ANAVA.

Segundo Anderson (1958) a pressuposição de homogeneidade de matrizes de covariâncias, supondo populações normais multidimensionais, pode ser feita pelo critério da razão de verossimilhança generalizada.

O modelo de análise multivariada de perfil, geralmente é parametrizado por meio das médias e tem a vantagem de ser de fácil interpretação. 0 modelo pode ser representado matricialmente na forma usual da análise multivariada de variância: $Y=X \beta+\varepsilon$, tal que $Y$ é uma matriz de dados, $X$ é a matriz de especificação do modelo, $\beta$ é a matriz de parâmetros e $\varepsilon$ é a matriz dos resíduos. Qualquer hipótese sobre os parâmetros pode ser expressa na forma linear geral $H: C \beta U=\phi$, onde $C(c \times g)$ e $U(t \times u)$ são matrizes com postos $c$ e $u$ de constantes conhecidas. A matriz $C$ é responsável por comparações entre os tratamentos (linhas da matriz $\beta$ ), enquanto que a matriz $U$ efetua as comparações entre as ocasiões (colunas da matriz $\beta$ ). 
0 teste da hipótese linear geral $H: C \beta U=\phi$ pode ser feito através das estatísticas: Lambda de Wilks, Traço de Pillai, Traço de Hotelling-Lawley e a Maior Raiz Característica de Roy. Estas estatísticas são funções das raízes características da matriz $H E^{-1}$, donde $H$ é a matriz de somas de quadrados e produtos cruzados devido à hipótese nula e $E$ é a matriz de somas de quadrados e produtos cruzados devido ao erro. Detalhes sobre essas estatísticas podem ser encontradas em Ferreira (2011), Fávero et al. (2009) e Hair et al. (2009).

Com o intuito de melhor embasamento teórico aos estudantes e demais leitores deste livro, serão apresentados, no contexto estatístico e matemático, os seguintes temas: noções sobre álgebra de vetores e de matrizes; estudo de máximos e mínimos condicionados de uma função; noções sobre regressão não linear pelo método de GaussNewton; teste da razão de verossimilhança para verificar a igualdade de parâmetros e identidade de modelos de regressão não linear; teste de validade de regressão de Graybill; equação de Mitscherlich na obtenção do ciclo de corte economicamente aconselhável; o critério de Mauchly para testar se uma matriz de covariância é esférica; as correções de Geisser-Greenhouse $(g-g)$ e de Huynh e Feldt (h-f) no estudo da esfericidade de matrizes; teste de uniformidade de matriz de covariância; teste de homoscedasticidade de matrizes de covariâncias; testes de multinormalidade de assimetria e de curtose de Mardia; teste de normalidade multivariada de Henze-Zirkler; noções sobre análise de variância multivariada (variável discriminante ou canônica); testes de paralelismo e de coincidência de perfis de crescimento; análise de agrupamento pelo método de Ward; análise de componentes principais, análise de fatores, análise discriminante; análise de correlação canônica.

Os dados usados nas diferentes aplicações apresentadas, neste livro, foram simulados, mas procurando ser o mais próximo possível da realidade Amazônica, haja vista a indisponibilidade de observações no formato completo requerido pelo método de análise de perfil. A partir da definição de vetores de médias e de matrizes de covariâncias foi gerado um banco de dados, considerando quatro tratamentos, denominados de métodos de manejo M1, M2, M3 e M4, aplicados em 30 parcelas permanentes de 0,25 ha por tratamento, para as ocasiões $0,5,10,15$ e 20 anos. 0 tempo 0 (zero) correspondeu à data logo após a exploração florestal.

Definido o melhor método de manejo, então foram simulados dados para quatro espécies, denominadas de E1, E2, E3, E4, com base nas suas importâncias econômicas, abundâncias de ocorrência e rápido crescimento, para ajustar as respectivas funções de crescimentos e a partir dessas definir os seus respectivos ciclos de corte economicamente aconselháveis.

O objetivo principal deste livro é apresentar o método multivariado de análise de perfil e suas relações com outros métodos multidimensionais. Em especial, servir de guia prático aos estudantes do curso de Engenharia Florestal, em particular da disciplina de Inventário Florestal. Por outro lado, visa também contribuir com a análise dos inventários florestais contínuos realizados dentro da programação do Inventario Florestal Nacional do Brasil (IFN), assim como, apresentar uma metodologia que possa servir de base para predizer o ciclo de corte viável economicamente em uma floresta manejada. 


\section{Capítulo 2}

\section{Álgebra de vetores e de matrizes}

Este capítulo trata de definições, conceitos e aplicações de álgebra de vetores e de matrizes que são importantes no estudo da análise multivariada. Aborda os seguintes temas: produto interno ou produto escalar de dois vetores; ortogonalidade e base ortonormal; vetores linearmente dependentes e independentes; procedimento de ortonormalização de Gram-Schmidt; determinantes; matriz inversa regular e matrizes inversas generalizadas (inversa-g); autovalores e autovetores; formas quadráticas; matriz Helmert. 


\section{INTRODUÇÃO}

Matriz é uma tabela de dados organizada no formato $n \times p$, onde $n$ representa o número de linhas (horizontal) e $p$ o número de colunas (vertical). No caso de uma amostra $p$ dimensional, donde a matriz $Y(n \times p)$ representa a tabela dos dados, então as linhas (vetores) representarão as $n$ observações $p$-dimensionais.

Sejam os vetores: $a_{v}^{\prime}=\left[\begin{array}{llll}a_{1} & a_{2} & \cdots & a_{p}\end{array}\right]$ e $y_{v}^{\prime}=\left[\begin{array}{llll}y_{1} & y_{2} & \cdots & y_{p}\end{array}\right]$. O produto $a_{v}^{\prime} y_{v}$ é dado por:

$$
a_{v}^{\prime} y_{v}=a_{1} y_{1}+a_{2} y_{2}+\cdots+a_{p} y_{p}=\sum_{i=1}^{p} a_{i} y_{i}
$$

Se $f\left(y_{1}, \cdots, y_{p}\right)=a_{1} y_{1}+a_{2} y_{2}+\cdots+a_{p} y_{p}$, então, pode-se escrever que $f\left(y_{1}, \cdots, y_{p}\right)=a_{v}^{\prime} y_{v}$.

\subsection{PRODUTO INTERNO OU PRODUTO ESCALAR DE DOIS VETORES}

Sejam os vetores: $y_{v 1}^{\prime}=\left[\begin{array}{llll}y_{11} & y_{12} & \cdots & y_{1 p}\end{array}\right]$ e $y_{v 2}^{\prime}=\left[\begin{array}{llll}y_{21} & y_{22} & \cdots & y_{2 p}\end{array}\right]$. O produto interno ou produto escalar em $R^{p}$ entre dois vetores $y_{v 1}$ e $y_{v 2}$, com o mesmo número de elementos, é definido como a soma dos produtos dos seus elementos correspondentes. 0 produto interno de dois vetores $y_{v 1}^{\prime} y_{v 2}$ é um escalar (constante).

$$
y_{v 1}^{\prime} y_{v 2}=y_{11} y_{21}+y_{12} y_{22}+\cdots+y_{1 p} y_{2 p}=\sum_{i=1}^{p} y_{1 i} y_{2 i}
$$

0 produto interno, de um vetor $y_{v}^{\prime}=\left[\begin{array}{llll}y_{1} & y_{2} & \cdots & y_{n}\end{array}\right]$ com ele mesmo $\left(y_{v}^{\prime} y_{v}\right)$, é a soma de quadrados dos elementos de $y_{v}$, ou seja, $y_{v}^{\prime} y_{v}=\sum_{i=1}^{n} y_{i}^{2}$. 0 valor $y_{v}^{\prime} y_{v}$ é definido como o quadrado do comprimento do vetor $y_{v}$ e sendo representado por $d^{2}=y_{v}^{\prime} y_{v}$.

A interpretação geométrica de $d=\sqrt{y_{v}^{\prime} y_{v}}$ corresponde à distância entre o ponto referente aos elementos de $y_{v}$ e a origem no sistema $p$-dimensional. 0 comprimento de um vetor também é chamado de norma. Se ocorrer $y_{v}^{\prime} y_{v}=1$, implica que $d=1$, então $y_{v}$ é definido como um vetor normalizado, isto é, possui o comprimento ou norma igual à unidade.

Seja a matriz $Y=\left[\begin{array}{ll}y_{v 1} & y_{v 2}\end{array}\right]$ formada pelos vetores:

$$
y_{v 1}^{\prime}=\left[\begin{array}{llll}
y_{11} & y_{12} & \cdots & y_{1 p}
\end{array}\right] \quad y_{v 2}^{\prime}=\left[\begin{array}{llll}
y_{21} & y_{22} & \cdots & y_{2 p}
\end{array}\right]
$$

Pode-se definir a distância entre os pontos que correspondem às coordenadas dos vetores $y_{v 1}$ e $y_{v 2}$, como: $d=\sqrt{\sum_{i=1}^{p}\left(y_{1 i}-y_{2 i}\right)^{2}}$, denominada distância Euclidiana. 
É importante em algumas técnicas multivariadas que a representação vetorial das variáveis tenha norma igual à unidade. Para obter a normalização igual à unidade de um vetor basta dividir os seus elementos pela sua norma.

Se $y_{v 1}$ e $y_{v 2}$ são vetores normalizados, isto é, $y_{v 1}^{\prime} y_{v 1}=1$ e $y_{v 2}^{\prime} y_{v 2}=1$, implica que $y_{v 1}^{\prime} y_{v 2}=1$, ou seja, o produto interno de vetores normalizados é igual à unidade.

\subsection{ORTOGONALIDADE E BASE ORTONORMAL}

Sejam os vetores: $y_{v 1}^{\prime}=\left[\begin{array}{llll}y_{11} & y_{12} & \cdots & y_{1 p}\end{array}\right]$ e $y_{v 2}^{\prime}=\left[\begin{array}{llll}y_{21} & y_{22} & \cdots & y_{2 p}\end{array}\right]$.

0 cosseno do ângulo $\theta$ entre dois vetores é igual à razão entre o produto interno deles e a raiz quadrada do produto de suas normas.

$$
\cos \theta=\frac{y_{v 1}^{\prime} y_{v 2}}{\sqrt{\left(y_{v 1}^{\prime} y_{v 1}\right)\left(y_{v 2}^{\prime} y_{v 2}\right)}}
$$

Dois vetores são ortogonais entre si se o angulo $\theta$ entre eles for igual a $90^{\circ}$, ou seja, $\cos \theta=0$, então para que dois vetores sejam ortogonais é necessário que o produto interno entre eles seja igual a zero. Destarte, os vetores $y_{v 1}^{\prime}$ e $y_{v 2}^{\prime}$ são ortogonais se $y_{v 1}^{\prime} y_{v 2}=0$.

Existem situações, na análise multivariada, em que há a necessidade de estabelecer uma base ortonormal de vetores. Uma base é definida como ortonormal quando é constituída por um conjunto de vetores que são mutuamente ortogonais e com normas iguais a unidade.

Caso nesse conjunto os vetores forem 2 a 2 ortogonais, mas não necessariamente de norma unitária, a base vetorial é dita ortogonal. Por outro lado, pode-se obter uma base ortonormal, normalizando os vetores da base ortogonal. Para obter a normalização de um vetor basta dividir cada elemento do vetor pela sua norma.

\subsection{VETORES LINEARMENTE DEPENDENTES E INDEPENDENTES}

Dois vetores $y_{v 1}$ e $y_{v 2}$ com $p$ componentes são definidos como sendo linearmente dependentes, se os elementos de um dos vetores forem proporcionais aos elementos do outro vetor. Os vetores $y_{v 1}^{\prime}=\left[\begin{array}{lll}2 & 1 & 3\end{array}\right]$ e $y_{v 2}^{\prime}=\left[\begin{array}{lll}4 & 2 & 6\end{array}\right]$ são linearmente dependentes, pois $y_{v 2}=2 y_{v 1}$.

Seja um conjunto formado por $p$ vetores $y_{v 1}, y_{v 2}, \cdots y_{v p}$. Estes vetores são denominados linearmente independentes, caso seja impossível escrever qualquer vetor desse conjunto como função linear dos vetores remanescentes. Um conjunto formado por $p$ vetores $y_{v 1}, y_{v 2}, \cdots y_{v p}$ é denominado linearmente independente, se não existirem escalares 
$c_{1}, c_{2}, \cdots, c_{p}$ que possam gerar algum vetor $y_{v i}$ do conjunto, ou seja, não é possível ocorrer:

$$
c_{i} y_{v i}=c_{1} y_{v 1}+\cdots+c_{i-1} y_{v(i-1)}+c_{i+1} y_{v(i+1)}+\cdots+c_{p} y_{v p}
$$

Por exemplo, sejam os vetores $y_{v 1}^{\prime}=\left[\begin{array}{lll}1 & 2 & -1\end{array}\right], y_{v 2}^{\prime}=\left[\begin{array}{lll}1 & 3 & 2\end{array}\right]$ e $y_{v 3}^{\prime}=\left[\begin{array}{lll}4 & 11 & 5\end{array}\right]$. Estes constituem um conjunto linearmente dependente, dado que: $y_{v 3}=y_{v 1}+3 y_{v 2}$.

Dado um conjunto de vetores dependentes, então se pode sempre obter pelo menos um vetor como função linear dos demais.

Define-se como posto ou rank de uma matriz o número de vetores linhas (colunas) linearmente independentes. Abaixo algumas propriedades:

[1] Uma matriz é definida como de posto completo quando todos os vetores linhas (colunas) forem linearmente independentes;

[2] 0 posto de $A^{\prime}$ (transposta) é igual ao posto de $A$. Este fato mostra que existe equivalência das linhas e das colunas na definição do posto;

[3] 0 posto de $A^{\prime} A$ ou $A A^{\prime}$ é igual ao posto de $A$;

[4] 0 posto da matriz $A$ não muda ao pré-multiplicá-la ou pós-multiplicá-la por outra não singular. Matriz não singular é aquela que admite inversa normal (possui posto completo);

[5] 0 posto de uma matriz é invariante a operações elementares de linhas e de colunas.

\subsection{ORTONORMALIZAÇÃO DE GRAM-SCHMIDT}

Seja a base vetorial composta pelos vetores $v_{1}, v_{2}, \cdots, v_{p}$. Ferreira (2011) apresenta os passos para, a partir dessa base, pelo processo de Gram-Schmitd, obter os vetores ortonormais $y_{1}, y_{2}, \cdots, y_{p}$. No caso de ocorrer alguns vetores linearmente dependentes no conjunto, então será originada uma base ortonormal com um número $(r)$ menor de vetores $(r<p)$.

a) 0 primeiro passo consiste em ortogonalizar os vetores da base:

$$
\begin{gathered}
e_{1}=v_{1} \\
e_{2}=v_{2}-\frac{v_{2}^{\prime} e_{1}}{\left\|e_{1}\right\|^{2}} e_{1} \\
e_{3}=v_{3}-\frac{v_{3}^{\prime} e_{2}}{\left\|e_{2}\right\|^{2}} e_{2}-\frac{v_{3}^{\prime} e_{1}}{\left\|e_{1}\right\|^{2}} e_{1}
\end{gathered}
$$




$$
e_{p}=v_{p}-\frac{v_{p}^{\prime} e_{p-1}}{\left\|e_{p-1}\right\|^{2}} e_{p-1}-\cdots-\frac{v_{p}^{\prime} e_{1}}{\left\|e_{1}\right\|^{2}} e_{1}
$$

b) O segundo passo consiste em normalizar todos os vetores ortogonalizados:

$$
y_{i}=\frac{e_{i}}{\sqrt{e_{i}^{\prime} e_{i}}}, \text { para } e_{i}^{\prime} e_{i} \neq 0,(i=1,2, \cdots, p)
$$

Exemplo 2.4.1. Dados os vetores $v_{1}^{\prime}=\left[\begin{array}{ll}3 & 1\end{array}\right]$ e $v_{2}^{\prime}=\left[\begin{array}{ll}2 & 2\end{array}\right]$. Obter a base ortonormal.

Primeiro passo: $e_{1}=v_{1}=\left[\begin{array}{l}3 \\ 1\end{array}\right]$

$$
\begin{gathered}
e_{2}=v_{2}-\frac{v_{2}^{\prime} e_{1}}{\left\|e_{1}\right\|^{2}} e_{1}=\left[\begin{array}{l}
2 \\
2
\end{array}\right]-\frac{8}{10}\left[\begin{array}{l}
3 \\
1
\end{array}\right]=\left[\begin{array}{c}
-2 / 5 \\
6 / 5
\end{array}\right] \\
\left\|e_{1}\right\|^{2}=3^{2}+1^{2}=10 \text { e } v_{2}^{\prime} e_{1}=\left[\begin{array}{ll}
2 & 2
\end{array}\right]\left[\begin{array}{l}
3 \\
1
\end{array}\right]=8
\end{gathered}
$$

Segundo passo: $y_{i}=\frac{e_{i}}{\sqrt{e_{i}^{\prime} e_{i}}}$

$$
\begin{gathered}
\sqrt{e_{1}^{\prime} e_{1}}=\sqrt{3^{2}+1^{2}}=\sqrt{10} \\
\sqrt{e_{2}^{\prime} e_{2}}=\sqrt{\left(-\frac{2}{5}\right)^{2}+\left(\frac{6}{5}\right)^{2}}=\sqrt{\frac{40}{25}}=\frac{2}{5} \sqrt{10} \\
y_{1}=\left[\begin{array}{c}
3 / \sqrt{10} \\
1 / \sqrt{10}
\end{array}\right] \quad y_{2}=\left[\begin{array}{c}
-1 / \sqrt{10} \\
3 / \sqrt{10}
\end{array}\right] \\
y_{v 1}^{\prime} y_{v 1}^{\prime}=1 ; \quad y_{v 2}^{\prime} y_{v 2}^{\prime}=1 ; \quad y_{v 1}^{\prime} y_{v 2}^{\prime}=y_{v 2}^{\prime} y_{v 1}=0
\end{gathered}
$$

Ferreira (2011) cita que o processo de Gram-Schmidt é importante para a obtenção da matriz inversa generalizada de Moore-Penrose, a qual é utilizada em processos iterativos de estimativas de parâmetros em modelos não lineares. Dada uma matriz $X$ $(p \times k)$ formada por um conjunto de $k$ vetores (colunas). Seja a matriz $U$, de dimensão coluna $r$ e dimensão linha igual a $p$, a base resultante da ortonormalização de $X(p \times k)$. A matriz inversa generalizada de Moore-Penrose de $X(p \times k)$ será dada por:

$$
X^{+}=\Gamma^{\prime}\left(\Gamma \Gamma^{\prime}\right)^{-1} U^{\prime}, \text { tal que: } \Gamma=U^{\prime} X
$$




\subsection{DETERMINANTES}

O conceito de um número associado a uma matriz quadrada é importante na solução de muitos problemas, como por exemplo, verificar se uma matriz possui inversa. Para cada matriz quadrada existe um número real único, denominado determinante. As notações mais usadas para representar o determinante de uma matriz quadrada $A$ são $\operatorname{det} A$ e $|\mathrm{A}|$. Seja o sistema de equação mais simples $a x_{1}=c, a \neq 0$, cuja solução é $x_{1}=c / a$. Observe que o denominador $(a)$, de $x_{1}=c / a$, está associado à matriz dos coeficientes. Escrevendo na forma matricial $A x_{v}=b_{v}$, ou seja, $A=[a], x_{v}=\left[x_{1}\right]$ e $b_{v}=[c]$, então o denominador (a) é definido como o determinante de $A=[a]$, isto é, $\operatorname{det} A=a$.

Seja um sistema com duas equações cujas soluções $x_{1}$ e $x_{2}$ sejam possíveis:

$$
\left\{\begin{array}{l}
a_{11} x_{1}+a_{12} x_{2}=c_{1} \\
a_{21} x_{1}+a_{22} x_{2}=c_{2}
\end{array}\right.
$$

As soluções $x_{1}$ e $x_{2}$ são: $x_{1}=\frac{c_{1} a_{22}-c_{2} a_{12}}{a_{11} a_{22}-a_{12} a_{21}}$ e $x_{2}=\frac{c_{2} a_{11}-c_{1} a_{21}}{a_{11} a_{22}-a_{12} a_{21}}$.

Verifica-se que, os denominadores $a_{11} a_{22}-a_{12} a_{21}$ das soluções $x_{1}$ e $x_{2}$ são iguais e associados à matriz dos coeficientes do sistema de equação escrito na forma matricial $A x_{v}=b_{v}$.

$$
A=\left[\begin{array}{ll}
a_{11} & a_{12} \\
a_{21} & a_{22}
\end{array}\right] \quad x_{v}=\left[\begin{array}{l}
x_{1} \\
x_{2}
\end{array}\right] \quad b_{v}=\left[\begin{array}{l}
c_{1} \\
c_{2}
\end{array}\right]
$$

0 denominador de $x_{1}$ e $x_{2}$ é definido como o determinante $\operatorname{de} A$, ou seja, $\operatorname{det} A=$ $a_{11} a_{22}-a_{12} a_{21}$.

Para uma matriz de primeira ordem: $\operatorname{det}\left[a_{11}\right]=a_{11}$;

Para uma matriz de segunda ordem: $\operatorname{det}\left[\begin{array}{ll}a_{11} & a_{12} \\ a_{21} & a_{22}\end{array}\right]=a_{11} a_{22}-a_{12} a_{21}$;

Para uma matriz de terceira ordem: $\operatorname{det}\left[\begin{array}{lll}a_{11} & a_{12} & a_{13} \\ a_{21} & a_{22} & a_{23} \\ a_{31} & a_{32} & a_{33}\end{array}\right]=a_{11} a_{22} a_{33}+a_{12} a_{23} a_{31}+$ $a_{21} a_{32} a_{13}-a_{13} a_{22} a_{31}-a_{12} a_{21} a_{33}-a_{32} a_{23} a_{11}$

Para uma matriz $A$ de qualquer ordem, neste caso, o determinante pode ser calculado pela regra de Laplace, onde o determinante de qualquer matriz quadrada de ordem $p$ é igual à soma dos produtos dos elementos de qualquer linha $i$ (ou coluna $j$ ) pelos seus respectivos cofatores. 
$\operatorname{det} A=\sum_{i=1}^{p} a_{i j} A_{i j}$, quando se considera a coluna $j$;

$\operatorname{det} A=\sum_{j=1}^{p} a_{i j} A_{i j}$, quando se considera a linha $i$.

A regra de Laplace obedece às seguintes etapas:

a. Obter os menores complementares $A_{i j}$ de qualquer linha $i$ ou de qualquer coluna $j$ de $A$. Menor complementar $A_{i j}$, de um elemento $a_{i j}$ de uma matriz $A$, é o determinante da submatriz resultante quando da eliminação da linha $i$ e da coluna $j$;

b. Obter a matriz dos cofatores $\bar{A}=\left[\Delta_{i j}\right]$ dos elementos $a_{i j}$ de $A$. 0 cofator $\Delta_{i j}$ do elemento $a_{i j}$ de $A$ é o menor complementar de $a_{i j}$ multiplicado pelo termo $(-1)^{i+j}$, ou seja, $\Delta_{i j}=(-1)^{i+j} A_{i j}$.

Exercício 2.5.1. Dada a matriz A.

$$
A=\left[\begin{array}{ccc}
1 & 3 & 1 \\
2 & -1 & 2 \\
1 & 2 & -1
\end{array}\right]
$$

a) Obter o menor complementar do elemento $a_{22}=-1$ :

Eliminando a segunda linha e a segunda coluna, a submatriz resultante será:

$$
\left[\begin{array}{cc}
1 & 1 \\
1 & -1
\end{array}\right]
$$

O menor complementar do elemento $a_{22}=-1$, é:

$$
A_{22}=\operatorname{det}\left[\begin{array}{cc}
1 & 1 \\
1 & -1
\end{array}\right]=-2
$$

b) Obter o cofator do elemento $a_{22}=-1$, ou seja, $\Delta_{22}$ :

Dado $\Delta_{i j}=(-1)^{i+j} A_{i j}$, então: $\Delta_{22}=(-1)^{2+2} A_{22}=(-1)^{4} \times-2=-2$.

c) Obter o determinante da matriz $A$ pela regra de Laplace: 
Pode-se trabalhar com qualquer linha ou qualquer coluna. Considerando a primeira linha, têm-se:

$$
\begin{gathered}
\operatorname{det} A=a_{11} \times(-1)^{1+1} \operatorname{det}\left[\begin{array}{cc}
-1 & 2 \\
2 & -1
\end{array}\right]+a_{12} \times(-1)^{1+2} \operatorname{det}\left[\begin{array}{cc}
2 & 2 \\
1 & -1
\end{array}\right]+a_{13} \times(-1)^{1+3} \operatorname{det}\left[\begin{array}{cc}
2 & -1 \\
1 & 2
\end{array}\right] \\
\operatorname{det} A=1 \times(1-4)-3 \times(-2-2)+1 \times(4+1)=-3+12+5=14
\end{gathered}
$$

Algumas propriedades dos determinantes:

a. O determinante de uma matriz triangular ou de uma matriz diagonal é igual ao produto dos elementos da diagonal principal;

b. O determinante é igual a zero quando todos os elementos de uma linha ou de uma coluna são iguais a zero;

c. O determinante é igual a zero quando duas linhas ou duas colunas são idênticas, ou seja, os seus elementos são iguais;

d. O determinante é igual a zero quando uma linha é uma relação linear de outra linha, similarmente quando uma coluna é uma relação linear de outra coluna, ou seja, uma é múltipla da outra;

e. Se duas linhas ou duas colunas são permutadas entre si, então o determinante troca de sinal.

A matriz adjunta de uma matriz $A$ é a matriz transposta da matriz dos seus cofatores, ou seja, $\operatorname{adj} A=\bar{A}^{\prime}$.

Exercício 2.5.2. Obter a matriz adjunta de $A$.

$$
A=\left[\begin{array}{ccc}
1 & 3 & 1 \\
2 & -1 & 2 \\
1 & 2 & -1
\end{array}\right]
$$

Inicialmente, calcula-se a matriz de cofatores de $A$, isto é, a matriz $\bar{A}$.

$$
\begin{aligned}
\Delta_{11}=(-1)^{1+1} \operatorname{det}\left[\begin{array}{cc}
-1 & 2 \\
2 & -1
\end{array}\right]=-3 & \Delta_{12}=(-1)^{1+2} \operatorname{det}\left[\begin{array}{cc}
2 & 2 \\
1 & -1
\end{array}\right]=4 \\
\Delta_{13}=(-1)^{1+3} \operatorname{det}\left[\begin{array}{cc}
2 & -1 \\
1 & 2
\end{array}\right]=5 & \Delta_{21}=(-1)^{2+1} \operatorname{det}\left[\begin{array}{cc}
3 & 1 \\
2 & -1
\end{array}\right]=5 \\
\Delta_{22}=(-1)^{2+2} \operatorname{det}\left[\begin{array}{cc}
1 & 1 \\
1 & -1
\end{array}\right]=-2 & \Delta_{23}=(-1)^{2+3} \operatorname{det}\left[\begin{array}{ll}
1 & 3 \\
1 & 2
\end{array}\right]=1
\end{aligned}
$$




$$
\begin{gathered}
\Delta_{31}=(-1)^{3+1} \operatorname{det}\left[\begin{array}{cc}
3 & 1 \\
-1 & 2
\end{array}\right]=7 \quad \Delta_{32}=(-1)^{3+2} \operatorname{det}\left[\begin{array}{ll}
1 & 1 \\
2 & 2
\end{array}\right]=0 \\
\Delta_{33}=(-1)^{3+3} \operatorname{det}\left[\begin{array}{cc}
1 & 3 \\
2 & -1
\end{array}\right]=-7
\end{gathered}
$$

Resultando $\bar{A}$ que é a matriz dos cofatores de $A$ :

$$
\bar{A}=\left[\begin{array}{ccc}
-3 & 4 & 5 \\
5 & -2 & 1 \\
7 & 0 & -7
\end{array}\right]
$$

Para calcular a matriz adjunta de $A$, basta obter a transposta da matriz dos cofatores, ou seja, $\operatorname{adj} A=\bar{A}^{\prime}$ :

$$
\operatorname{adj} A=\left[\begin{array}{ccc}
-3 & 5 & 7 \\
4 & -2 & 0 \\
5 & 1 & -7
\end{array}\right]
$$

\subsection{MATRIZ INVERSA REGULAR}

Sejam as matrizes quadradas $A$ e $A^{-1}$, se $A A^{-1}=A^{-1} A=I$, então $A^{-1}$ é a matriz inversa regular de $A$. Uma matriz é dita não singular se a mesma admite inversa regular, e se não admite inversa é denominada singular. A matriz inversa regular é única e será representada por $A^{-1}$.

Da teoria de determinantes tem-se que, em uma matriz quadrada não singular $A$, os elementos correspondentes à sua inversa $A^{-1}$ são os elementos da matriz adjunta de $A$ ( $\operatorname{adj} A$ ) divididos pelo determinante de $A$.

$$
A^{-1}=\frac{(\operatorname{adj} A)}{\operatorname{det} A} \quad(\operatorname{det} A \neq 0)
$$

Exercício 2.6.1. Obter a solução do sistema de equações.

$$
\left\{\begin{array}{c}
x_{1}+3 x_{2}+x_{3}=10 \\
2 x_{1}-x_{2}+2 x_{3}=6 \\
x_{1}+2 x_{2}-x_{3}=2
\end{array}\right.
$$

Da solução matricial $x_{v}=A^{-1} b_{v}$, tem-se:

$$
\left[\begin{array}{l}
x_{1} \\
x_{2} \\
x_{3}
\end{array}\right]=\left[\begin{array}{ccc}
1 & 3 & 1 \\
2 & -1 & 2 \\
1 & 2 & -1
\end{array}\right]^{-1} \times\left[\begin{array}{c}
10 \\
6 \\
2
\end{array}\right]
$$


Da teoria de determinantes:

$A^{-1}=\frac{(\operatorname{adj} A)}{\operatorname{det} A}$, onde $A \neq 0$ e $\operatorname{adj} A$ é a matriz adjunta de $A$.

Como visto anteriormente, têm-se $\operatorname{det} A=14$ e $\operatorname{adj} A$ :

$$
\begin{gathered}
\operatorname{adj} A=\left[\begin{array}{ccc}
-3 & 5 & 7 \\
4 & -2 & 0 \\
5 & 1 & -7
\end{array}\right] \rightarrow A^{-1}=\frac{1}{14}\left[\begin{array}{ccc}
-3 & 5 & 7 \\
4 & -2 & 0 \\
5 & 1 & -7
\end{array}\right] \\
{\left[\begin{array}{l}
x_{1} \\
x_{2} \\
x_{3}
\end{array}\right]=\frac{1}{14}\left[\begin{array}{ccc}
-3 & 5 & 7 \\
4 & -2 & 0 \\
5 & 1 & -7
\end{array}\right] \times\left[\begin{array}{c}
10 \\
6 \\
2
\end{array}\right]=\frac{1}{14}\left[\begin{array}{c}
-30+30+14 \\
40-12+0 \\
50+6-14
\end{array}\right]=\frac{1}{14}\left[\begin{array}{l}
14 \\
28 \\
42
\end{array}\right]=\left[\begin{array}{l}
1 \\
2 \\
3
\end{array}\right]}
\end{gathered}
$$

Conclusão: $x_{1}=1, x_{2}=2, x_{3}=3$.

Efetuando a verificação da definição, $A^{-1} A=A A^{-1}=I$, têm-se:

$$
\begin{aligned}
A A^{-1} & =\left[\begin{array}{ccc}
1 & 3 & 1 \\
2 & -1 & 2 \\
1 & 2 & -1
\end{array}\right] \times \frac{1}{14}\left[\begin{array}{ccc}
-3 & 5 & 7 \\
4 & -2 & 0 \\
5 & 1 & -7
\end{array}\right]=\frac{1}{14}\left[\begin{array}{ccc}
14 & 0 & 0 \\
0 & 14 & 0 \\
0 & 0 & 14
\end{array}\right]=\left[\begin{array}{lll}
1 & 0 & 0 \\
0 & 1 & 0 \\
0 & 0 & 1
\end{array}\right] \\
A^{-1} A & =\frac{1}{14}\left[\begin{array}{ccc}
-3 & 5 & 7 \\
4 & -2 & 0 \\
5 & 1 & -7
\end{array}\right] \times\left[\begin{array}{ccc}
1 & 3 & 1 \\
2 & -1 & 2 \\
1 & 2 & -1
\end{array}\right]=\frac{1}{14}\left[\begin{array}{ccc}
14 & 0 & 0 \\
0 & 14 & 0 \\
0 & 0 & 14
\end{array}\right]=\left[\begin{array}{lll}
1 & 0 & 0 \\
0 & 1 & 0 \\
0 & 0 & 1
\end{array}\right]
\end{aligned}
$$

Como $A A^{-1}=A^{-1} A=I$, conclui-se que $A^{-1}$ é a inversa regular de $A$.

Propriedades de uma matriz não singular, ou seja, matriz que possui inversa regular:
a. $A^{-1} A=A A^{-1}=I$;
b. $A^{-1}$ é única;
c.$$
\left|A^{-1}\right|=\frac{1}{|A|}
$$
d. $\left(A^{-1}\right)^{-1}=A$;
e. $\left(A^{\prime}\right)^{-1}=\left(A^{-1}\right)^{\prime}$;
f. Se $A$ é simétrica, então $A^{-1}$ também é simétrica;
g. $(A B)^{-1}=B^{-1} A^{-1}$ (se $A$ e $B$ são matrizes não singulares);
h. Se a inversa da matriz $A$ é igual a sua transposta $\left(A^{-1}=A^{\prime}\right)$, então, diz-se que $A$ é uma matriz ortogonal $\left(A^{\prime} A=I\right)$. 
As matrizes ortogonais $\left(A^{\prime} A=I\right)$, e consequentemente, $A^{-1}=A^{\prime}$, são importantíssimas na estatística multivariada.

Uma matriz $A$ é definida como ortogonal se suas linhas (colunas) são formadas por vetores ortonormais. Vetores ortonormais são vetores independentes (o produto interno entre eles é igual a zero) e possuem normas iguais à unidade.

Se $A$ é ortogonal $A^{\prime} A=A A^{\prime}=I$, a inversa de $A$, simplesmente, é a transposta $A^{\prime}$. A matriz $A=\left[\begin{array}{ll}0 & 1 \\ 1 & 0\end{array}\right]$ é um exemplo de uma matriz ortogonal, pois:

$$
A^{\prime} A=\left[\begin{array}{ll}
0 & 1 \\
1 & 0
\end{array}\right] \times\left[\begin{array}{ll}
0 & 1 \\
1 & 0
\end{array}\right]=\left[\begin{array}{ll}
1 & 0 \\
0 & 1
\end{array}\right] \quad A A^{\prime}=\left[\begin{array}{ll}
0 & 1 \\
1 & 0
\end{array}\right] \times\left[\begin{array}{ll}
0 & 1 \\
1 & 0
\end{array}\right]=\left[\begin{array}{ll}
1 & 0 \\
0 & 1
\end{array}\right]
$$

$A=\left[\begin{array}{cc}\cos \theta & \operatorname{sen} \theta \\ -\operatorname{sen} \theta & \cos \theta\end{array}\right]$ é a mais geral matriz ortogonal de ordem $2 \times 2$, pois $A A^{\prime}=A^{\prime} A=I$.

$$
\begin{gathered}
A A^{\prime}=\left[\begin{array}{cc}
\cos \theta & \operatorname{sen} \theta \\
-\operatorname{sen} \theta & \cos \theta
\end{array}\right] \times\left[\begin{array}{cc}
\cos \theta & -\operatorname{sen} \theta \\
\operatorname{sen} \theta & \cos \theta
\end{array}\right] \\
A A^{\prime}=\left[\begin{array}{cc}
\cos ^{2} \theta+\operatorname{sen}^{2} \theta & -\cos \theta \times \operatorname{sen} \theta+\operatorname{sen} \theta \times \cos \theta \\
-\operatorname{sen} \theta \times \cos \theta+\cos \theta \times \operatorname{sen} \theta & \operatorname{sen}^{2} \theta+\cos ^{2} \theta
\end{array}\right]=\left[\begin{array}{ll}
1 & 0 \\
0 & 1
\end{array}\right]
\end{gathered}
$$

\subsection{MATRIZES INVERSAS GENERALIZADAS (INVERSA-G)}

0 conceito sobre inversa normal de uma matriz é "se uma matriz $A$ tem inversa $A^{-1}$, essa matriz necessariamente é quadrada e deve ter determinante diferente de zero". Isto equivale a dizer que a matriz $A$ é não singular, ou ainda, que tem posto completo (linhas e colunas linearmente independentes). Uma matriz quadrada é dita singular se tiver determinante nulo.

Muitos problemas estatísticos, teóricos e aplicados, envolvem a solução de sistemas de equações lineares do tipo $A x_{v}=b_{v}$ ou $y_{v}=X \beta_{v}$. Se as matrizes $A$ e $X$ são de ordem $n \times n$, não singulares, então, as soluções dos sistemas existem (sistemas consistentes) e são únicas (sistemas determinados), sendo dadas por: $x_{v}=A^{-1} b_{v}$ e $\beta_{v}=X^{-1} y_{v}$. Existem casos em que $A$ e $X$ não são quadradas, ou mesmo sendo as matrizes $A$ e $X$ quadradas, pode ocorrer de serem singulares (matrizes com determinantes iguais a zero) ou, mesmo, apresentarem postos incompletos. Nestas situações, uma abordagem neste sentido envolve o uso das inversas generalizadas de matrizes.

Alguns tipos de matrizes inversas generalizadas que são importantes na análise estatística são: inversa generalizada de Moore-Penrose, inversa generalizada condicional, inversa generalizada de quadrados mínimos e a inversa generalizada reflexiva (ou inversa-g2). 


\subsubsection{MATRIZ GENERALIZADA DE MOORE-PENROSE}

Denomina-se matriz generalizada de Moore-Penrose (ou pseudo-inversa) de $A(m \times n)$, a matriz $A^{+}(n \times m)$ que satisfaz as condições:
a. $A A^{+} A=A$;
b. $A^{+} A A^{+}=A^{+}$;
c. $A A^{+}=\left(A A^{+}\right)^{\prime}$ (simétrica);
d. $A^{+} A=\left(A^{+} A\right)^{\prime}$ (simétrica).

Um procedimento para obter a matriz de Moore-Penrose está apresentado na seção 2.4.

\subsubsection{MATRIZ INVERSA GENERALIZADA CONDICIONAL}

Inversa generalizada condicional de uma matriz $A$, de ordem $m \times n$, é uma matriz denotada por $A^{-}$, de ordem $n \times m$, que satisfaz a condição: $A A^{-} A=A$.

Duarte (1996) cita que a inversa de Moore-Penrose é uma inversa condicional $\left(A^{-}\right) \mathrm{de}$ $A$, pois satisfaz a primeira propriedade da inversa de Moore-Penrose; a recíproca, no entanto, não é verdadeira, acrescentando, também, que $A^{-}$é "uma" inversa-g de $A$ e não "a" inversa- $g$ de $A$, pois podem existir muitas matrizes que satisfazem tal condição. A exceção ocorre quando $A$ é não singular, situação em que $A^{-}=A^{-1}$, sendo, portanto, única. A obtenção de uma matriz inversa generalizada condicional não apresenta uma expressão definida, já que pode haver várias inversas condicionais para uma mesma matriz. Há diversos procedimentos, entre os quais o algoritmo de Searle (1971), o qual consiste em observar as seguintes etapas:

a) Dada uma matriz $A(m \times n)$ de posto $(r)$, retirar de $A$ qualquer submatriz $P$, não singular e de posto $(r)$, e obter a sua inversa normal $P^{-1}$ e a sua transposta $\left(P^{-1}\right)^{\prime}$;

b) Substituir em $A$ os elementos que constituíram a matriz $P$ pelos correspondentes de $\left(P^{-1}\right)^{\prime}$, acrescentando zeros aos elementos restantes. A transposta da matriz resultante é uma inversa condicional.

\subsubsection{MATRIZ INVERSA GENERALIZADA DE QUADRADOS MÍNIMOS}

É uma matriz muito importante na aplicação da teoria do Método dos Quadrados Mínimos. Os sistemas de equações lineares dessa teoria podem ser resolvidos usando-se as inversas de quadrados mínimos.

Seja uma matriz $A(m \times n)$, denota-se $A^{*}$ de ordem $n \times m$ como uma inversa generalizada de quadrados mínimos de $A$, se e somente se, a matriz $A^{*}$ satisfazer as condições:
a. $A A^{*} A=A$;
b. $A A^{*}=\left(A A^{*}\right)^{\prime}$ (forma simétrica). 
Duarte (1996) menciona que toda matriz inversa de quadrados mínimos é uma inversa condicional $\left(A^{-}\right)$e que $A^{+}$(Moore-Penrose) é também uma inversa de quadrados mínimos. 0 mesmo autor cita que uma matriz $A^{*}$ pode ser obtida a partir de uma matriz inversa condicional por meio da seguinte expressão:

$$
A^{*}=\left(A^{\prime} A\right)^{-} A^{\prime} .
$$

\subsubsection{MATRIZ INVERSA REFLEXIVA OU INVERSA-G2}

É assim denominada por atender a duas condições recíprocas (inversa-g2). Este tipo de inversa é, particularmente, importante no uso do sistema estatístico SAS.

Dada uma matriz qualquer $A(m \times n)$, uma matriz $A^{R}$ ou $A^{g 2}$, de ordem $n \times m$, é definida inversa reflexiva de $A$, se e somente se, atender as condições: $A A^{R} A=A$ e $A^{R} A A^{R}=A^{R}$. De acordo com Duarte (1996), toda inversa reflexiva é uma inversa condicional ( $A^{-}$) e que $A^{+}$(Moore-Penrose) é também uma inversa reflexiva.

As inversas reflexivas também não são únicas e uma forma de obtê-las é por meio da expressão $A^{R}=A A^{-} A$, ou seja, é possível obter, a partir de uma inversa condicional de $A$, uma inversa reflexiva de $A$.

0 procedimento de Gauss-Jordan consiste em um algoritmo que utiliza operações pivotais para obtenção de matrizes inversas e inversas generalizadas de matrizes quadradas. 0 procedimento estabelece uma sequência de passos.

Seja a matriz $A=A^{(0)}$ ( $\left.p \times p\right)$ que será repetidamente alterada em $m \leq p$ passos, ou seja, $A^{(1)}, A^{(2)}, \cdots, A^{(m)}$. A matriz resultante $A^{(m)}$ corresponderá à matriz inversa $A^{-1}$ se o posto de $A^{(m)}$ for $m=p$, entretanto, no caso de $m \leq p$, então será uma matriz inversa generalizada.

Em cada fase do procedimento é escolhido um elemento pivô $a_{j k}^{(i)}$ da matriz $A^{(i)}$. As seguintes condições devem ser obedecidas:

a. O pivô deve ser diferente de zero;

b. As linhas e as colunas já usadas para definir o pivô nas $(i-1)$ fases anteriores não podem ser utilizadas novamente.

0 ideal é escolher os elementos pivôs na sequência $a_{11}^{(i)}, a_{22}^{(i)}, \cdots, a_{p p}^{(i)}$. Escolhe-se o próximo pivô na sequência quando da ocorrência de um pivô nulo, resultando que um número $m \leq p$ de iterações será realizado.

As linhas e colunas não utilizadas como pivô serão preenchidas por zeros. 0 processo é interrompido quando não existir mais pivô para ser utilizado. 0 posto da matriz $A$ é definido pelo número de pivôs não nulos utilizados. Ferreira (2011) cita que no caso do resultado ser uma matriz generalizada, a mesma corresponderá à matriz reflexiva (g2), 
assim como apresenta os passos do procedimento considerando a escolha do pivô $a_{j j}^{(i)}$, descritos a seguir:

a) Obter os elementos da $j$-ésima linha e $j$-ésima coluna de $A^{(i+1)}$ :

$$
a_{j j}^{(i+1)}=\frac{1}{a_{j j}^{(i)}}
$$

b) Obter os elementos da $j$-ésima coluna de $A^{(i+1)}$ :

$$
a_{k j}^{(i+1)}=-\frac{a_{k j}^{(i)}}{a_{j j}^{(i)}}(k \neq j)
$$

c) obter os elementos da $j$-ésima linha de $A^{(i+1)}$ :

$$
a_{j l}^{(i+1)}=\frac{a_{j l}^{(i)}}{a_{j j}^{(i)}} \quad(l \neq j)
$$

d) obter os elementos das linhas e colunas diferentes de $j \operatorname{de} A^{(i+1)}$ :

$$
a_{k l}^{(i+1)}=a_{k l}^{(i)}-\frac{a_{k j}^{(i)} a_{j l}^{(i)}}{a_{j j}^{(i)}} \forall k, l \neq j
$$

Exercício 2.7.1. Obter a inversa da matriz $A^{(0)}$ :

$$
A^{(0)}=\left[\begin{array}{ccc}
1 & 3 & 1 \\
2 & -1 & 2 \\
1 & 2 & -1
\end{array}\right]
$$

Definindo $a_{11}^{(0)}=1$ como primeiro pivô, tem-se $A^{(1)}$ :

$$
A^{(1)}=\left[\begin{array}{ccc}
1 & 3 & 1 \\
-2 & -1-\frac{2 \times 3}{1} & 2-\frac{2 \times 1}{1} \\
-1 & 2-\frac{1 \times 3}{1} & -1-\frac{1 \times 1}{1}
\end{array}\right] \rightarrow A^{(1)}=\left[\begin{array}{ccc}
1 & 3 & 1 \\
-2 & -7 & 0 \\
-1 & -1 & -2
\end{array}\right]
$$

Definindo $a_{22}^{(1)}=-7$ como segundo pivô, tem-se $A^{(2)}$ :

$$
A^{(2)}=\left[\begin{array}{ccc}
1-\left(\frac{3 \times-2}{-7}\right) & \frac{3}{7} & 1-\left(\frac{3 \times 0}{-7}\right) \\
\frac{2}{7} & -\frac{1}{7} & 0 \\
-1-\left(\frac{-1 \times-2}{-7}\right) & -\frac{1}{7} & -2-\left(\frac{-1 \times 0}{-7}\right)
\end{array}\right] \rightarrow A^{(2)}=\left[\begin{array}{ccc}
1 / 7 & 3 / 7 & 1 \\
2 / 7 & -1 / 7 & 0 \\
-5 / 7 & -1 / 7 & -2
\end{array}\right]
$$


Definindo $a_{33}^{(2)}=-2$ como terceiro e último pivô, tem-se $A^{(3)}$ :

$$
\begin{gathered}
A^{(3)}=\left[\begin{array}{ccc}
\frac{1}{7}-\left(\frac{1 \times-5 / 7}{-2}\right) & \frac{3}{7}-\left(\frac{1 \times-1 / 7}{-2}\right) & \frac{1}{2} \\
\frac{2}{7}-\left(\frac{0 \times-5 / 7}{-2}\right) & -\frac{1}{7}-\left(\frac{0 \times-1 / 7}{-2}\right) & 0 \\
\frac{5}{14} & \frac{1}{14} & -\frac{1}{2}
\end{array}\right] \\
A^{(3)}=\left[\begin{array}{ccc}
-3 / 14 & 5 / 14 & 1 / 2 \\
2 / 7 & -1 / 7 & 0 \\
5 / 14 & 1 / 14 & -1 / 2
\end{array}\right]=\frac{1}{14}\left[\begin{array}{ccc}
-3 & 5 & 7 \\
4 & -2 & 0 \\
5 & 1 & -7
\end{array}\right]
\end{gathered}
$$

A matriz $A^{(3)}$, como tem posto completo, é a inversa regular de $A^{(0)}$.

Exercício 2.7.2. Resolver o sistema de equações.

Obter $x_{v}=B^{R} b_{v}$ :

Dado: $\left\{\begin{array}{c}x_{1}+3 x_{2}+x_{3}=10 \\ 2 x_{1}-x_{2}+2 x_{3}=6 \\ 3 x_{1}+2 x_{2}+3 x_{3}=16\end{array} \quad B^{(0)}=\left[\begin{array}{ccc}1 & 3 & 1 \\ 2 & -1 & 2 \\ 3 & 2 & 3\end{array}\right]\right.$

Observar que $B^{(0)}$ é uma matriz singular, haja vista a terceira linha ser a soma da primeira e da segunda. Então, a matriz inversa generalizada a ser obtida será uma reflexiva g2. Verificar se: $B B^{R} B=B$ e $B^{R} B B^{R}=B^{R}$.

Definindo $b_{11}^{(0)}=1$ como primeiro pivô, tem-se $B^{(1)}$ :

$$
B^{(1)}=\left[\begin{array}{ccc}
1 & 3 & 1 \\
-2 & -1-\frac{2 \times 3}{1} & 2-\frac{2 \times 1}{1} \\
-3 & 2-\frac{3 \times 3}{1} & 3-\frac{3 \times 1}{1}
\end{array}\right] \rightarrow B^{(1)}=\left[\begin{array}{ccc}
1 & 3 & 1 \\
-2 & -7 & 0 \\
-3 & -7 & 0
\end{array}\right]
$$

Definindo $b_{22}^{(1)}=-7$ como segundo pivô, tem-se $B^{(2)}$ :

$$
B^{(2)}=\left[\begin{array}{ccc}
1-\left(\frac{3 \times-2}{-7}\right) & \frac{3}{7} & 1-\left(\frac{3 \times 0}{-7}\right) \\
\frac{2}{7} & -\frac{1}{7} & 0 \\
-3-\left(\frac{-7 \times-2}{-7}\right) & -1 & 0-\left(\frac{-7 \times 0}{-7}\right)
\end{array}\right] \rightarrow B^{(2)}=\left[\begin{array}{ccc}
1 / 7 & 3 / 7 & 1 \\
2 / 7 & -1 / 7 & 0 \\
-1 & -1 & 0
\end{array}\right]
$$


Definindo $b_{33}^{(2)}=0$ como terceiro e último pivô, tem-se que $B^{(3)}$ é a inversa reflexiva de $B^{(0)}$, haja vista o pivô ser nulo $\left(b_{33}^{(2)}=0\right)$. As linhas e colunas não utilizadas como pivô serão preenchidas por zeros.

$$
B^{R}=\left[\begin{array}{ccc}
1 / 7 & 3 / 7 & 0 \\
2 / 7 & -1 / 7 & 0 \\
0 & 0 & 0
\end{array}\right] \rightarrow x_{v}=B^{R} b_{v}=\left[\begin{array}{ccc}
1 / 7 & 3 / 7 & 0 \\
2 / 7 & -1 / 7 & 0 \\
0 & 0 & 0
\end{array}\right] \times\left[\begin{array}{c}
10 \\
6 \\
16
\end{array}\right]=\left[\begin{array}{l}
4 \\
2 \\
0
\end{array}\right]
$$

Verificação de:

$$
\begin{gathered}
B B^{R} B=B \text { e } B^{R} B B^{R}=B^{R} \\
B B^{R} B=\left[\begin{array}{ccc}
1 & 3 & 1 \\
2 & -1 & 2 \\
3 & 2 & 3
\end{array}\right] \times\left[\begin{array}{ccc}
\frac{1}{7} & \frac{3}{7} & 0 \\
\frac{2}{7} & -\frac{1}{7} & 0 \\
0 & 0 & 0
\end{array}\right] \times\left[\begin{array}{ccc}
1 & 3 & 1 \\
2 & -1 & 2 \\
3 & 2 & 3
\end{array}\right]=\left[\begin{array}{ccc}
1 & 3 & 1 \\
2 & -1 & 2 \\
3 & 2 & 3
\end{array}\right]=B \\
B^{R} B B^{R}=\left[\begin{array}{ccc}
1 / 7 & 3 / 7 & 0 \\
2 / 7 & -1 / 7 & 0 \\
0 & 0 & 0
\end{array}\right]\left[\begin{array}{ccc}
1 & 3 & 1 \\
2 & -1 & 2 \\
3 & 2 & 3
\end{array}\right] \times\left[\begin{array}{ccc}
1 / 7 & 3 / 7 & 0 \\
2 / 7 & -1 / 7 & 0 \\
0 & 0 & 0
\end{array}\right]=\left[\begin{array}{ccc}
1 / 7 & 3 / 7 & 0 \\
2 / 7 & -1 / 7 & 0 \\
0 & 0 & 0
\end{array}\right]=B^{R}
\end{gathered}
$$

O SAS usa o comando ginv, para obter a inversa generalizada de uma matriz $A$, ou seja, IGA=ginv (A).

\subsection{AUTOVALORES E AUTOVETORES}

Os autovalores e autovetores de uma matriz quadrada são importantes no desenvolvimento teórico das técnicas multivariadas. Os autovalores e autovetores possuem relevância nas estruturas metodológicas, principalmente, dos métodos de componentes principais, de análise de fatores, de análise de correlação canônica e nos testes de significância multivariados.

O pesquisador na área de recursos naturais renováveis, muitas vezes, tem como objetivo identificar fatores ambientais que influenciam a estrutura de um ecossistema natural, avaliando, geralmente, um grande número de variáveis respostas, o que dificulta a visualização das inter-relações entre elas.

Para facilitar essas interpretações, a análise multivariada por meio das análises de componentes principais e de fatores, cria novas variáveis independentes, respectivamente componentes principais e fatores, que são funções lineares das variáveis originais e que são extraídas da estrutura de dispersão das informações 
originais. Na análise de variância multivariada é importante obter as variáveis discriminantes ou canônicas para melhor interpretar os resultados.

Essas técnicas consistem em, a partir da matriz de covariância ou de correlação, usando transformação linear, obter uma matriz diagonal $\Lambda$ onde todos os coeficientes da diagonal principal são formados pelos autovalores $\lambda_{i}$. Estes estão associados a uma matriz formada pelos vetores chamados autovetores representados por eixos ortogonais (produto interno igual a zero).

Autovalores são conhecidos também como raízes características, valores característicos, valores próprios ou latentes. Os autovalores de uma matriz $A(p \times p)$ são as soluções da equação determinantal $|A-\lambda I|=0$ ou $\operatorname{det}(A-\lambda I)=0$. 0 determinante é um polinômio de grau $p$ em $\lambda$, assim a matriz $A(p \times p)$ possuirá $p$ raízes características ou autovalores.

Associado a cada autovalor $\lambda_{i}$ de uma matriz quadrada $A(\mathrm{p} \times \mathrm{p})$ existe um autovetor $a_{v i}$, também conhecido como vetor característico, vetor próprio ou vetor latente, cujos elementos satisfazem o sistema de equações homogêneas: $\left(A-\lambda_{i} I\right) a_{v i}=0_{v}$. Se $\lambda_{\mathrm{i}}$ e $\lambda_{\mathrm{j}}$ são raízes características distintas de uma matriz simétrica $A$, seus vetores associados $a_{v i} \mathrm{e}$ $a_{v j}$ são ortogonais. Da teoria dos autovalores e dos autovetores de matrizes:

a. 0 produto das $p$ raízes características de $A(p \times p)$ é igual ao $\operatorname{det} A$;

b. A soma das raízes características de $A(p \times p)$ será igual ao traço de $A(p \times p)$.

0 traço de uma matriz quadrada $A(p \times p)$ é definido como a soma dos elementos da diagonal principal.

Dado $\left(A-\lambda_{i} I\right) a_{v i}=0_{v}$, então $A a_{v i}=\lambda_{i} a_{v i}$, assim como se $\left(A-\lambda_{j} I\right) a_{v j}=0_{v}$, logo $A a_{v j}=\lambda_{j} a_{v j}$.

Pré-multiplicando por $a_{v j}^{\prime}$ a expressão $A a_{v i}=\lambda_{i} a_{v i}$, tem-se: $a_{v j}^{\prime} A a_{v i}=\lambda_{i} a_{v j}^{\prime} a_{v i}$.

Pré-multiplicando por $a_{v i}^{\prime}$ a expressão $A a_{v j}=\lambda_{j} a_{v j}$, tem-se: $a_{v i}^{\prime} A a_{v j}=\lambda_{j} a_{v i}^{\prime} a_{v j}$.

Dado que $a_{v j}^{\prime} A a_{v i}=a_{v i}^{\prime} A a_{v j}$, então $\lambda_{i} a_{v j}^{\prime} a_{v i}=\lambda_{j} a_{v i}^{\prime} a_{v j}$. Por outro lado, como $a_{v j}^{\prime} a_{v i}=a_{v i}^{\prime} a_{v j}$ e $\lambda_{i} \neq \lambda_{j}$, logo para atender $\lambda_{i} a_{v j}^{\prime} a_{v i}=\lambda_{j} a_{v i}^{\prime} a_{v j}$, resulta a solução única: $a_{v j}^{\prime} a_{v i}=0$ e $a_{v i}^{\prime} a_{v j}=0$, concluindo-se, então, que $a_{v i}$ e $a_{v j}$ são vetores ortogonais.

Os autovetores podem ser definidos satisfazendo a condição de ter comprimento igual à unidade: $a_{v 1}^{\prime} a_{v 1}=a_{v 2}^{\prime} a_{v 2}=\cdots=a_{v p}^{\prime} a_{v p}=1$, isto é, normalizados e também mutuamente perpendiculares (ortogonais). 
Para qualquer matriz simétrica real existe uma matriz ortogonal $P$, tal que $P^{\prime} D P=A$, onde $D$ é a matriz diagonal cujos elementos da diagonal principal são os autovalores de $A$, dispostos na condição $\lambda_{1}>\lambda_{2}>\cdots>\lambda_{r}$, onde $r$ é o número de autovetores não nulos (posto da matriz $A$ ). Os autovetores normalizados de $A$, associados aos autovalores, podem ser tomados como colunas de $P$. Como $A$ é uma matriz simétrica, então $A=P^{\prime} D P=P D P^{\prime}$.

$$
D=\left[\begin{array}{cccc}
\lambda_{1} & 0 & 0 & 0 \\
0 & \lambda_{2} & 0 & 0 \\
0 & 0 & \ddots & 0 \\
0 & 0 & 0 & \lambda_{p}
\end{array}\right]
$$

Tem-se da álgebra de matrizes que se $A$ é uma matriz simétrica real, então $A=\lambda_{1} a_{v 1} a_{v 1}^{\prime}+\lambda_{2} a_{v 2} a_{v 2}^{\prime}+\cdots+\lambda_{r} a_{v r} a_{v r}^{\prime}$, onde $r$ é o posto da matriz $A$. Fazendo-se $L=P D\left(\sqrt{\lambda_{i}}\right)$ e $L^{\prime}=D\left(\sqrt{\lambda_{i}}\right) P^{\prime}$, tem-se que $A=L L^{\prime}$.

Se a matriz $A(p \times p)$ é simétrica definida positiva (posto completo), implica que $A$ tem $p$ pares de autovalores e autovetores, então:

$$
A=\lambda_{1} a_{v 1} a_{v 1}^{\prime}+\lambda_{2} a_{v 2} a_{v 2}^{\prime}+\cdots+\lambda_{p} a_{v p} a_{v p}^{\prime}
$$

Da álgebra de matrizes, tem-se que os autovetores não são únicos se dois ou mais autovalores são iguais.

Exercício 2.8.1. Obter os autovalores e os autovetores correspondentes à matriz A.

$$
A=\left[\begin{array}{ll}
6 & 2 \\
2 & 3
\end{array}\right]
$$

$$
\begin{aligned}
& \operatorname{Det}(A-\lambda I)=\operatorname{det}\left(\left[\begin{array}{ll}
6 & 2 \\
2 & 3
\end{array}\right]-\lambda\left[\begin{array}{ll}
1 & 0 \\
0 & 1
\end{array}\right]\right)=0, \text { pois, } \operatorname{det}(A-\lambda I)=0 \\
& \operatorname{det}(A-\lambda I)=\operatorname{det}\left[\begin{array}{cc}
6-\lambda & 2 \\
2 & 3-\lambda
\end{array}\right]=0 \rightarrow \operatorname{det}(A-\lambda I)=\lambda^{2}-9 \lambda+14=0
\end{aligned}
$$

Os autovalores $\lambda_{1}=7$ e $\lambda_{2}=2$ são as soluções de $\lambda^{2}-9 \lambda+14=0$.

Os autovalores ou as raízes características da matriz $A$ são $\lambda_{1}=7$ e $\lambda_{2}=2$;

0 determinante da matriz $A$ é dado pelo produto $\lambda_{1} \times \lambda_{2}=7 \times 2=14$;

0 traço da matriz $A$ é dado pela soma $\lambda_{1}+\lambda_{2}=7+2=9$.

Procedimento para obter os autovetores $a_{v 1}$ e $a_{v 2}$ associados aos autovalores $\lambda_{1}=7$ e $\lambda_{2}=2$. Para cada autovalor $\lambda_{i}$ de uma matriz quadrada $A(p \times p)$ existe um autovetor $a_{v i}$, cujos seus elementos satisfazem o sistema de equações homogêneas $\left(A-\lambda_{i} I\right) a_{v i}=0_{v}$, 
então o primeiro passo é obter o autovetor associado ao autovalor $\lambda_{1}=7$, com a resolução do sistema de equações homogêneas $\left(A-\lambda_{1} I\right) a_{v 1}=0_{v}$.

$$
(A-7 I) a_{v 1}=\left(\left[\begin{array}{ll}
6 & 2 \\
2 & 3
\end{array}\right]-7 \times\left[\begin{array}{ll}
1 & 0 \\
0 & 1
\end{array}\right]\right) \times\left[\begin{array}{l}
a_{11} \\
a_{21}
\end{array}\right]=\left[\begin{array}{cc}
6-7 & 2 \\
2 & 3-7
\end{array}\right] \times\left[\begin{array}{l}
a_{11} \\
a_{21}
\end{array}\right]=\left[\begin{array}{l}
0 \\
0
\end{array}\right]
$$

Resultando: $\left\{\begin{array}{l}-a_{11}+2 a_{21}=0 \\ 2 a_{11}-4 a_{21}=0\end{array}\right.$

Este sistema de equações homogêneas admite infinitas soluções. Considerando a solução para $a_{11}=1$, então $a_{21}=1 / 2=0,5$. 0 próximo passo é transformar o vetor $a_{v 1}=\left[\begin{array}{c}a_{11} \\ a_{21}\end{array}\right]=\left[\begin{array}{c}1 \\ 0,5\end{array}\right]$, em um vetor normalizado $\dot{a}_{v 1}=\left[\begin{array}{c}\dot{a}_{11} \\ \dot{a}_{21}\end{array}\right]$.

Para que o vetor $\dot{a}_{v 1}$ seja considerado um vetor normalizado, o seu comprimento ou norma deve ser igual à unidade. Para transformar $a_{v 1}$ em um vetor normalizado $\dot{a}_{v 1}$, basta dividir os seus elementos pela sua norma, então:

$$
\begin{gathered}
\dot{a}_{11}=\frac{a_{11}}{\sqrt{a_{11}^{2}+a_{21}^{2}}}=\frac{1}{\sqrt{1^{2}+0,5^{2}}}=\frac{1}{\sqrt{1,25}} \\
\dot{a}_{21}=\frac{a_{21}}{\sqrt{a_{11}^{2}+a_{21}^{2}}}=\frac{0,5}{\sqrt{1^{2}+0,5^{2}}}=\frac{0,5}{\sqrt{1,25}} \\
\dot{a}_{v 1}=\left[\begin{array}{l}
\dot{a}_{11} \\
\dot{a}_{21}
\end{array}\right]=\frac{1}{\sqrt{1,25}}\left[\begin{array}{c}
1 \\
0,5
\end{array}\right]
\end{gathered}
$$

Com efeito, pode-se mostrar que $\dot{a}_{v 1}$ é um vetor normalizado, pois $\dot{a}_{v 1}^{\prime} \dot{a}_{v 1}=1$ :

$$
\dot{a}_{v 1}^{\prime} a_{v 1}=\left[\begin{array}{ll}
\dot{a}_{11} & \dot{a}_{21}
\end{array}\right] \times\left[\begin{array}{l}
\dot{a}_{11} \\
\dot{a}_{21}
\end{array}\right]=\dot{a}_{11}^{2}+\dot{a}_{21}^{2}=\left(\frac{1}{\sqrt{1,25}}\right)^{2}+\left(\frac{0,5}{\sqrt{1,25}}\right)^{2}=\frac{1+0,25}{1,25}=1
$$

Tem-se, então, o autovetor normalizado associado ao maior autovalor $\lambda_{1}=7$ :

$$
\dot{a}_{v 1}=\left[\begin{array}{l}
\dot{a}_{11} \\
\dot{a}_{21}
\end{array}\right]=\frac{1}{\sqrt{1,25}}\left[\begin{array}{c}
1 \\
0,5
\end{array}\right]
$$

Cálculo do autovetor $a_{v 2}$ associado ao menor autovalor $\lambda_{2}=2$ :

$$
\left.(A-2 I) a_{v 2}=\left(\begin{array}{ll}
6 & 2 \\
2 & 3
\end{array}\right]-2 \times\left[\begin{array}{ll}
1 & 0 \\
0 & 1
\end{array}\right]\right) \times\left[\begin{array}{l}
a_{12} \\
a_{22}
\end{array}\right]=\left[\begin{array}{cc}
6-2 & 2 \\
2 & 3-2
\end{array}\right] \times\left[\begin{array}{l}
a_{12} \\
a_{22}
\end{array}\right]=\left[\begin{array}{l}
0 \\
0
\end{array}\right]
$$




$$
\left\{\begin{array}{c}
4 a_{12}+2 a_{22}=0 \\
2 a_{12}+a_{22}=0
\end{array}\right.
$$

Este sistema de equações homogêneas admite infinitas soluções. Considerando a solução para $a_{12}=1$, então $a_{22}=-2$. 0 próximo passo é transformar o vetor $a_{v 2}=\left[\begin{array}{c}a_{12} \\ a_{22}\end{array}\right]=\left[\begin{array}{c}1 \\ -2\end{array}\right]$, em um vetor normalizado $\dot{a}_{v 2}=\left[\begin{array}{c}\dot{a}_{12} \\ \dot{a}_{22}\end{array}\right]$.

Seja a transformação do vetor $a_{v 2}$, no vetor normalizado $\dot{a}_{v 2}$. Então:

$$
\begin{aligned}
& \dot{a}_{12}=\frac{a_{12}}{\sqrt{a_{12}^{2}+a_{22}^{2}}}=\frac{1}{\sqrt{1^{2}+(-2)^{2}}}=\frac{1}{\sqrt{5}} \\
& \dot{a}_{22}=\frac{a_{22}}{\sqrt{a_{12}^{2}+a_{22}^{2}}}=\frac{-2}{\sqrt{1^{2}+(-2)^{2}}}=\frac{-2}{\sqrt{5}}
\end{aligned}
$$

Donde, resulta:

$$
\dot{a}_{v 2}=\left[\begin{array}{l}
\dot{a}_{12} \\
\dot{a}_{22}
\end{array}\right]=\frac{1}{\sqrt{5}}\left[\begin{array}{c}
1 \\
-2
\end{array}\right]
$$

Da álgebra de matrizes tem-se que, pela decomposição espectral, dada uma matriz simétrica $A$, existe uma matriz $P$, tal que, $A=P D P^{\prime}=\sum_{i=1}^{p} \lambda_{i} a_{v i} a_{v i}^{\prime}$, onde $D$ é a matriz diagonal cujos elementos correspondem aos autovalores de $A$. Os vetores característicos da matriz $A$ são tomados como colunas de $P$.

Verificação de $P D P^{\prime}=A$, considerando:

$$
A=\left[\begin{array}{ll}
6 & 2 \\
2 & 3
\end{array}\right]
$$

De onde resultaram os autovalores $\lambda_{1}=7$ e $\lambda_{2}=2$, assim como os autovetores associados normalizados correspondentes:

$$
\begin{gathered}
\dot{a}_{v 1}=\frac{1}{\sqrt{1,25}}\left[\begin{array}{c}
1 \\
0,5
\end{array}\right] \quad \dot{a}_{v 2}=\frac{1}{\sqrt{5}}\left[\begin{array}{c}
1 \\
-2
\end{array}\right] \\
A=\left[P D P^{\prime}\right]=\left[\begin{array}{cc}
\frac{1}{\sqrt{1,25}} & \frac{1}{\sqrt{5}} \\
\frac{0,5}{\sqrt{1,25}} & -\frac{2}{\sqrt{5}}
\end{array}\right] \times\left[\begin{array}{ll}
7 & 0 \\
0 & 2
\end{array}\right] \times\left[\begin{array}{cc}
\frac{1}{\sqrt{1,25}} & \frac{0,5}{\sqrt{1,25}} \\
\frac{1}{\sqrt{5}} & -\frac{2}{\sqrt{5}}
\end{array}\right]=\left[\begin{array}{ll}
6 & 2 \\
2 & 3
\end{array}\right]
\end{gathered}
$$


Dado $A=\lambda_{1} \dot{a}_{1} \dot{a}_{1}^{\prime}+\lambda_{2} \dot{a}_{2} \dot{a}_{2}^{\prime}$, tem-se:

$$
\begin{aligned}
& \lambda_{1} \dot{a}_{v 1} \dot{a}_{v 1}^{\prime}=7 \frac{1}{\sqrt{1,25}}\left[\begin{array}{c}
1 \\
05
\end{array}\right] \times \frac{1}{\sqrt{1,25}}[1 \quad 0,5]=\frac{7}{1,25}\left[\begin{array}{cc}
1 & 0,5 \\
0,5 & 0,25
\end{array}\right]=\left[\begin{array}{cc}
5,6 & 2,8 \\
2,8 & 1,4
\end{array}\right] \\
& \lambda_{2} \dot{a}_{v 2} \dot{a}_{v 2}^{\prime}=2 \frac{1}{\sqrt{5}}\left[\begin{array}{c}
1 \\
-2
\end{array}\right] \times \frac{1}{\sqrt{5}}\left[\begin{array}{ll}
1 & -2
\end{array}\right]=\frac{2}{5}\left[\begin{array}{cc}
1 & -2 \\
-2 & 4
\end{array}\right]=\left[\begin{array}{cc}
0,4 & -0,8 \\
-0,8 & 1,6
\end{array}\right] \\
& A=\lambda_{1} \dot{a}_{v 1} \dot{a}_{v 1}^{\prime}+\lambda_{2} \dot{a}_{v 2} \dot{a}_{v 2}^{\prime}=\left[\begin{array}{cc}
5,6 & 2,8 \\
2,8 & 1,4
\end{array}\right]+\left[\begin{array}{cc}
0,4 & -0,8 \\
-0,8 & 1,6
\end{array}\right]=\left[\begin{array}{ll}
6 & 2 \\
2 & 3
\end{array}\right]
\end{aligned}
$$

Como a matriz $P$ dos autovetores é ortogonal, sendo $A$ simétrica e $D$ diagonal, então:

$$
A=P D P^{\prime} \quad P^{\prime} A P=P^{\prime} P D P^{\prime} P \quad D=P^{\prime} A P
$$

\subsection{FORMAS QUADRÁTICAS}

Sejam os vetores $x_{v}^{\prime}=\left[\begin{array}{llll}x_{1} & x_{2} & \ldots & x_{p}\end{array}\right], y_{v}=\left[\begin{array}{llll}y_{1} & y_{2} & \ldots & y_{p}\end{array}\right]$ e a matriz $A(p \times p)$.

$$
{ }_{p} A_{p}=\left[\begin{array}{cccc}
a_{11} & a_{12} & \cdots & a_{1 p} \\
a_{21} & a_{22} & \cdots & a_{2 p} \\
\cdots & \cdots & \cdots & \cdots \\
a_{p 1} & a_{p 2} & \cdots & a_{p p}
\end{array}\right] .
$$

A expressão $Q\left(x_{v}, y_{v}\right)=x_{v}^{\prime} A y_{v}$ é denominada de "Forma Bilinear".

Se $x_{v}=\left[\begin{array}{l}x_{1} \\ x_{2}\end{array}\right], y_{v}=\left[\begin{array}{l}y_{1} \\ y_{2}\end{array}\right]$ e $A=\left[\begin{array}{ll}a_{11} & a_{12} \\ a_{21} & a_{22}\end{array}\right]$, tem-se:

$Q\left(x_{v}, y_{v}\right)=\left[\begin{array}{ll}x_{1} & x_{2}\end{array}\right] \times\left[\begin{array}{ll}a_{11} & a_{12} \\ a_{21} & a_{22}\end{array}\right] \times\left[\begin{array}{l}y_{1} \\ y_{2}\end{array}\right]$, desenvolvendo-se, tem-se:

$$
Q\left(x_{v}, y_{v}\right)=a_{11} x_{1} y_{1}+a_{21} x_{2} y_{1}+a_{12} x_{1} y_{2}+a_{22} x_{2} y_{2}
$$

No caso particular dos vetores serem iguais $\left(x_{v}=y_{v}\right)$ e $A$ uma matriz simétrica $\left(a_{i j}=a_{j i}\right)$ para $(i \neq j)$, resulta que: $Q\left(x_{v}\right)=x_{v}^{\prime} A x_{v}$ que é denominada de forma quadrática. Seja o exemplo:

$$
x_{v}=\left[\begin{array}{l}
x_{1} \\
x_{2}
\end{array}\right] \quad A=\left[\begin{array}{ll}
a_{11} & a_{12} \\
a_{21} & a_{22}
\end{array}\right] \quad\left(a_{12}=a_{21}\right)
$$




$$
Q\left(x_{v}\right)=a_{11} x_{1}^{2}+2 a_{12} x_{1} x_{2}+a_{22} x_{2}^{2}
$$

Generalizando para:

$$
x_{v}=\left[\begin{array}{c}
x_{1} \\
x_{2} \\
\vdots \\
x_{n}
\end{array}\right] \quad{ }_{n} A_{p}=\left[\begin{array}{cccc}
a_{11} & a_{12} & \cdots & a_{1 p} \\
a_{21} & a_{22} & \cdots & a_{2 p} \\
\cdots & \cdots & \cdots & \cdots \\
a_{p 1} & a_{p 2} & \cdots & a_{p p}
\end{array}\right]
$$

$Q\left(x_{v}\right)=x_{v}^{\prime} A x_{v}=f\left(x_{1}, \cdots, x_{p}\right)$ que é a expressão da forma quadrática para as variáveis $x_{1}, x_{2}, \cdots, x_{p}$, sendo ${ }_{n} A_{p}$ simétrica. Podendo ser escrita nas seguintes formas:

$$
\begin{aligned}
& f\left(x_{1}, \cdots, x_{p}\right)=\sum_{i=1}^{p} a_{i i} x_{i}^{2}+2 \sum_{i=1}^{p-1} \sum_{j=i+1}^{p} a_{i j} x_{i} x_{j} \\
& f\left(x_{1}, \cdots, x_{p}\right)=\sum_{i=1}^{p} \sum_{j=1}^{p} a_{i j} x_{i} x_{j}
\end{aligned}
$$

A matriz simétrica $A(p \times p)$ e suas formas quadráticas associadas são denominadas para todo $x_{v} \neq 0$, de:
a. Positiva definida se: $x_{v}^{\prime} A x_{v}>0$;
b. Positiva semi-definida se: $x_{v}^{\prime} A x_{v} \geq 0$;
c. Negativa definida se: $x_{v}^{\prime} A x_{v}<0$;
d. Negativa semi-definida se: $x_{v}^{\prime} A x_{v} \leq 0$.

0 posto $(r)$ ou característica de uma forma quadrática $Q\left(x_{v}\right)=x_{v}^{\prime} A x_{v}$ é o mesmo da matriz $A(p \times p)$. A forma quadrática é dita "singular" se o posto ou característica é $r<p$. Por outro lado, se $r=p$ é classificada como "não singular".

Em toda forma quadrática definida positiva a matriz $A(p \times p)$ dos coeficientes possui posto completo, ou seja, é uma matriz não singular, $A^{-1} A=A A^{-1}=I$, pois admite inversa regular, assim como os seus autovalores são todos positivos.

Seja o exemplo onde se deseja obter o valor máximo de uma forma quadrática $f\left(x_{1}, \cdots, x_{p}\right)=x_{v}^{\prime} A x_{v}$, donde $A$ é uma matriz $p \times p$ positiva definida e o vetor $x_{v}^{\prime}=\left[\begin{array}{llll}x_{1} & x_{2} & \cdots & x_{p}\end{array}\right]$ sujeito a restrição $x_{v}^{\prime} x_{v}=1$. A condição $x_{v}^{\prime} x_{v}=1$ significa que o vetor $x_{v}$ possui comprimento igual à unidade. 
Seja a equação $L\left(x_{1}, \cdots, x_{p}, \lambda\right)$ segundo o método dos multiplicadores de Lagrange (capítulo 6, seção 6.6):

$$
\begin{gathered}
L\left(x_{1}, \cdots, x_{p}, \lambda\right)=x_{v}^{\prime} A x_{v}-\lambda\left(x_{v}^{\prime} x_{v}-1\right) \\
\frac{\partial f\left(x_{1}, \cdots, x_{p}\right)}{\partial x_{v}}=2 A x_{v}-2 \lambda x_{v} \\
\frac{\partial f\left(x_{1}, \cdots, x_{p}\right)}{\partial x_{v}}=0_{v}
\end{gathered}
$$

De $2\left(A x_{v}-\lambda x_{v}\right)=0_{v}$, resulta: $(A-\lambda I) x_{v}=0_{v}$. Então:

$A x_{v}-\lambda x_{v}=0_{v}$, onde $A x_{v}=\lambda x_{v}$, tal que pré-multiplicando por $x_{v}^{\prime}$, tem-se:

$x_{v}^{\prime} A x_{v}=\lambda x_{v}^{\prime} x_{v}$, mas como $x_{v}^{\prime} x_{v}=1$, resulta: $x_{v}^{\prime} A x_{v}=\lambda$.

Conforme visto no estudo de autovalores e autovetores, $(A-\lambda I) x_{v}=0_{v}$ é o sistema homogêneo que define os autovalores e os vetores próprios da matriz $A$, onde os autovalores ou raízes características, ou valores próprios da matriz $A$ são obtidos da equação determinantal $|A-\lambda I|=0$.

Dada a matriz $A$ positiva definida e as suas raízes características colocadas na ordem decrescente, isto é, $\lambda_{1}>\lambda_{2}>\cdots>\lambda_{p}$, então o máximo de $f\left(x_{1}, \cdots, x_{p}\right)=x_{v}^{\prime} A x_{v}$ corresponderá ao maior autovalor $\lambda_{1}$ da matriz. 0 valor mínimo de $f\left(x_{1}, \cdots, x_{p}\right)=x_{v}^{\prime} A x_{v}$ corresponderá ao menor autovalor $\lambda_{p}$ da matriz, pois $x_{v}^{\prime} A x_{v}=\lambda$.

Seja $A$ uma matriz definida positiva $p \times p$ com autovalores $\lambda_{1} \geq \lambda_{2} \geq \cdots \geq \lambda_{p}>0$ e associados aos autovetores normalizados $a_{v 1}, a_{v 2}, \cdots, a_{v p}$. Tem-se, então:

$$
\begin{aligned}
& \max _{x_{v} \neq 0} \frac{x_{v}^{\prime} A x_{v}}{x_{v}^{\prime} x_{v}}=\lambda_{1} \text {, atendendo } x_{v}=a_{v 1} \\
& \min _{x_{v} \neq 0} \frac{x_{v}^{\prime} A x_{v}}{x_{v}^{\prime} x_{v}}=\lambda_{p}, \text { atendendo } x_{v}=a_{v p}
\end{aligned}
$$

A variância de uma função linear $y=a_{1} x_{1}+a_{2} x_{2}+\cdots+a_{p} x_{p}$, de um conjunto de variáveis aleatórias $x_{1}, x_{2}, \cdots, x_{p}$, é a forma quadrática $\sigma_{y y}=a_{v}^{\prime} \sum a_{v}$, tal que $\sum$ é a matriz de covariância. 
Sejam as variáveis aleatórias $x_{1}, x_{2}, \cdots, x_{p}$ observadas em um determinado experimento. Deseja-se obter a variância $\sigma_{y y}$ da função linear $y=a_{1} x_{1}+a_{2} x_{2}+\cdots+a_{p} x_{p}$. Então:

$$
\begin{gathered}
\operatorname{Var}(y)=\sigma_{y y}=\operatorname{Var}\left(a_{1} x_{1}+a_{2} x_{2}+\cdots+a_{p} x_{p}\right) \\
\sigma_{y y}=E\left\{\left[\left(a_{1} x_{1}+a_{2} x_{2}+\cdots+a_{p} x_{p}\right)-E\left(a_{1} x_{1}+a_{2} x_{2}+\cdots+a_{p} x_{p}\right)\right]^{2}\right\} \\
\sigma_{y y}=\sum_{i=1}^{p} a_{i i}^{2} \sigma_{i i}+2 a_{i} a_{j} \sum_{i<j}^{p} \operatorname{cov}\left(x_{i}, x_{j}\right)
\end{gathered}
$$

Se $\sum_{i<j}^{p} \operatorname{cov}\left(x_{i}, x_{j}\right)=0$, resulta que $x_{i}$ e $x_{j}$ são independentes, então $\sigma_{y y}$ é uma forma quadrática, escrita sob a forma matricial $\sigma_{y y}=a_{v}^{\prime} \sum a_{v}$, donde $a_{v}^{\prime}=\left[\begin{array}{llll}a_{1} & a_{2} & \cdots & a_{p}\end{array}\right]$ e $\sum$ é a matriz de covariância das variáveis $x_{1}, x_{2}, \cdots, x_{p}$.

Matriz de covariância é a matriz simétrica que contém na diagonal principal as variâncias de cada uma das variáveis, e fora da diagonal as covariâncias combinadas aos pares de variáveis. Na análise multivariada a matriz de covariância é o elemento de ponderação que permite estudar a estrutura de dependência das variáveis. Utilizar-se-á a notação $\sum$ para representar a matriz de covariância populacional e $S$ para representar a matriz amostral.

$$
\Sigma=\left[\begin{array}{cccc}
\sigma_{11} & \sigma_{12} & \ldots & \sigma_{1 \mathrm{p}} \\
\sigma_{21} & \sigma_{22} & \ldots & \sigma_{2 \mathrm{p}} \\
\ldots & \ldots & \ldots & \ldots \\
\sigma_{\mathrm{p} 1} & \sigma_{\mathrm{p} 2} & \ldots & \sigma_{\mathrm{pp}}
\end{array}\right] \quad S=\left[\begin{array}{cccc}
s_{11} & s_{12} & \ldots & s_{1 p} \\
s_{21} & s_{22} & \ldots & s_{2 p} \\
\ldots & \ldots & \ldots & \ldots \\
s_{p 1} & s_{p 2} & \ldots & s_{p p}
\end{array}\right]
$$

Deve-se observar que para $(i \neq j): \sigma_{i j}=\sigma_{j i}$ e $s_{i j}=s_{j i}$.

\subsection{MATRIZ HELMERT}

A matriz Helmert é uma matriz quadrada ortogonal, de ordem $p \times p$, de aplicação muito importante na álgebra linear e na teoria de matrizes. Ela foi introduzida por Lancaster (1965), sendo muito utilizada em cálculos matemáticos, em processos estocásticos, na análise de variância e, em especial, em ensaios com parcelas subdivididas. A matriz Helmert $\left(H_{p}\right)$ apresenta a seguinte formatação:

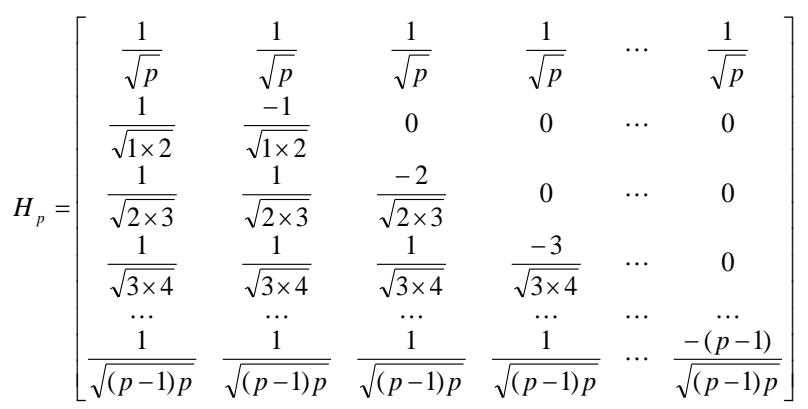


A primeira linha da matriz Helmert de ordem $p$ tem a seguinte composição:

$$
\left[\begin{array}{llll}
\frac{1}{\sqrt{p}} & \frac{1}{\sqrt{p}} & \cdots & \frac{1}{\sqrt{p}}
\end{array}\right]
$$

As demais linhas, ou seja, $2 \leq i \leq p$, são formadas por:

$$
\left[\begin{array}{lllll}
\frac{1}{\sqrt{i(i-1)}} & \frac{1}{\sqrt{i(i-1)}} & \cdots & \frac{1}{\sqrt{i(i-1)}} & \frac{-(i-1)}{\sqrt{i(i-1)}}
\end{array}\right]
$$

Verifica-se que $1 / \sqrt{i(i-1)}$ está alocado em $(i-1)$ itens da $i$-ésima linha e $0, \ldots, 0$ em $(p-i)$ itens da $i$-ésima linha.

Seja o exemplo da matriz Helmert de quinta ordem:

$$
H_{5}=\left[\begin{array}{ccccc}
\frac{1}{\sqrt{5}} & \frac{1}{\sqrt{5}} & \frac{1}{\sqrt{5}} & \frac{1}{\sqrt{5}} & \frac{1}{\sqrt{5}} \\
\frac{1}{\sqrt{2}} & \frac{-1}{\sqrt{2}} & 0 & 0 & 0 \\
\frac{1}{\sqrt{6}} & \frac{1}{\sqrt{6}} & \frac{-2}{\sqrt{6}} & 0 & 0 \\
\frac{1}{\sqrt{12}} & \frac{1}{\sqrt{12}} & \frac{1}{\sqrt{12}} & \frac{-3}{\sqrt{12}} & 0 \\
\frac{1}{\sqrt{20}} & \frac{1}{\sqrt{20}} & \frac{1}{\sqrt{20}} & \frac{1}{\sqrt{20}} & \frac{-4}{\sqrt{20}}
\end{array}\right]
$$

Seja, por exemplo, a composição da terceira linha. Ela terá $(i-1=2)$ itens iguais a $\frac{1}{\sqrt{i(i-1)}}=\frac{1}{\sqrt{3(3-1)}}=\frac{1}{\sqrt{6}}$, depois o item $\frac{-(i-1)}{\sqrt{i(i-1)}}=\frac{-(3-1)}{\sqrt{3(3-1)}}=\frac{-2}{\sqrt{6}}$ e finalizando a composição da terceira seria alocado $(p-i)=2$ (zeros).

As matrizes Helmert são ortogonais, então: $H^{\prime} H=H H^{\prime}=I, H^{-1}=H^{\prime}, \operatorname{det} H=1$. Iemma (1982) cita que as matrizes de Helmert são ortogonais e sustentam as propriedades necessárias para a decomposição ortogonal de matrizes definidas não negativas.

Farhadian \& Asadian (2017) mostram que é possível construir uma matriz estocástica a partir de uma matriz Helmert. Eles mostram que se $H=\left[h_{i j}\right]$ é uma matriz Helmert, então $P=\left[h_{i j}^{2}\right]$ é uma matriz estocástica, ou seja, $h_{i 1}^{2}+h_{i 2}^{2}+\cdots+h_{i p}^{2}=1 \quad \forall_{i, j} \in(1,2, . ., p)$.

Iemma (1982) considerando o estreito relacionamento entre as matrizes ortogonais $P_{p}$, $P_{p}^{\prime} V P_{p}=\operatorname{diag}\left(\lambda_{1}, \lambda_{2}, \cdots, \lambda_{p}\right)$, e as matrizes $H_{p}$ de Helmert, $H_{p} S H_{p}^{\prime}=\operatorname{diag}\left(\lambda_{1}, \lambda_{2}, \cdots, \lambda_{p}\right)$, $k=1,2, \cdots, p$, achou mais conveniente utilizar a matriz Helmert na decomposição da matriz uniforme de covariância $V_{p}$, e assim evitar o uso de algum processo que envolva o uso determinante. A uniformidade de $V_{p}$ garante sua simetria. Por outro lado, o estudo considerou apenas o caso em que $V_{p}$ é positiva definida, donde $\sigma^{2}$ é a variância e $\rho$ é o coeficiente de correlação constante. 
Na tabela $C 1$, do apêndice $C$, está apresentado o programa SAS com a aplicação das principais operações com vetores e matrizes.

$$
V_{p}=\left[\begin{array}{cccc}
\sigma^{2} & \rho \sigma^{2} & \cdots & \rho \sigma^{2} \\
\rho \sigma^{2} & \sigma^{2} & \cdots & \rho \sigma^{2} \\
\cdots & \cdots & \cdots & \cdots \\
\rho \sigma^{2} & \rho \sigma^{2} & \cdots & \sigma^{2}
\end{array}\right]
$$




\section{Capítulo 3}

\section{Multinormalidade e estruturas de matrizes de covariâncias}

Neste capítulo serão apresentados os testes para algumas pressuposições aplicadas na análise de inventário florestal contínuo, com parcelas permanentes, medidas em ocasiões equidistantes. Destarte, vários temas serão abordados tais como: testes de multinormalidade das extensões multivariadas de assimetria e de curtose de Mardia; teste de multinormalidade de Henze-Zirkler (HZ); teste de uniformidade da matriz de covariância de Wilks; teste de homogeneidade de matrizes de covariâncias; o critério de Mauchly sobre circularidade (esfericidade) de uma matriz; a correção de GreenhouseGeisser $(g-g)$ e a de Huynh e Feldt ( $h-f)$. 


\section{INTRODUÇÃo}

Diz-se que um vetor aleatório $y_{v}^{\prime}=\left[y_{1}, y_{2}, \ldots, y_{p}\right]$ possui distribuição multinormal de probabilidade, se a função de densidade de probabilidade (f.d.p.) de $y_{1}, y_{2}, \ldots, y_{p}$ for do tipo:

$$
f\left(y_{1}, y_{2}, \cdots, y_{p}\right)=\frac{1}{(2 \pi)^{p / 2}|\Sigma|^{1 / 2}} \exp \left[-\frac{1}{2}\left(y_{v}-\mu_{v}\right)^{\prime} \Sigma^{-1}\left(y_{v}-\mu_{v}\right)\right]
$$

$\sum(p \times p)$ : é denominada de matriz de covariância do vetor $y_{v}$.

Matriz de covariância é a matriz simétrica que contém na diagonal principal as variâncias de cada uma das variáveis, e fora da diagonal as covariâncias combinadas aos pares de variáveis. Na análise multivariada a matriz de covariâncias é o elemento de ponderação que permite considerar a estrutura de dependência das variáveis.

$$
\Sigma=\left[\begin{array}{cccc}
\sigma_{11} & \sigma_{12} & \ldots & \sigma_{1 p} \\
\sigma_{21} & \sigma_{22} & \ldots & \sigma_{2 p} \\
\ldots & \ldots & \ldots & \ldots \\
\sigma_{p 1} & \sigma_{p 2} & \ldots & \sigma_{p p}
\end{array}\right]
$$

No caso de duas variáveis $y_{1}$ e $y_{2}$, a função densidade normal bivariada, será:

$$
f\left(y_{1}, y_{2}\right)=\frac{1}{2 \pi \sqrt{\sigma_{11} \sigma_{22}\left(1-\rho_{12}^{2}\right)}} \exp \left\{\frac{-1}{2\left(1-\rho_{12}^{2}\right)}\left[\left(\frac{y_{1}-\mu_{1}}{\sqrt{\sigma_{11}}}\right)^{2}+\left(\frac{y_{2}-\mu_{2}}{\sqrt{\sigma_{22}}}\right)^{2}-2 \rho_{12}\left(\frac{y_{1}-\mu_{1}}{\sqrt{\sigma_{11}}}\right)\left(\frac{y_{2}-\mu_{2}}{\sqrt{\sigma_{22}}}\right)\right]\right\}
$$

Johnson \& Wichern (1992) apresentam os seguintes exemplos de gráficos de duas distribuições normais bivariadas:

Caso (a): Variâncias iguais $\left(\sigma_{11}=\sigma_{22}\right)$ e correlação igual a zero $\left(\rho_{12}=0\right)$ :

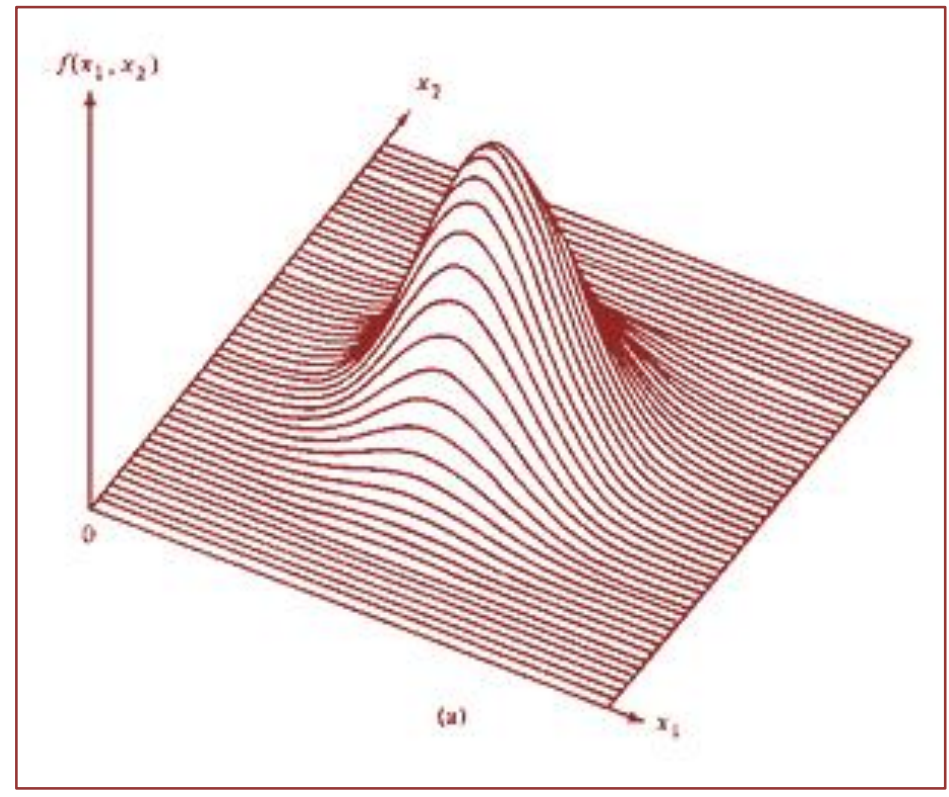


Caso (b): Variâncias iguais $\left(\sigma_{11}=\sigma_{22}\right)$ e correlação igual a $0,75\left(\rho_{12}=0,75\right)$.

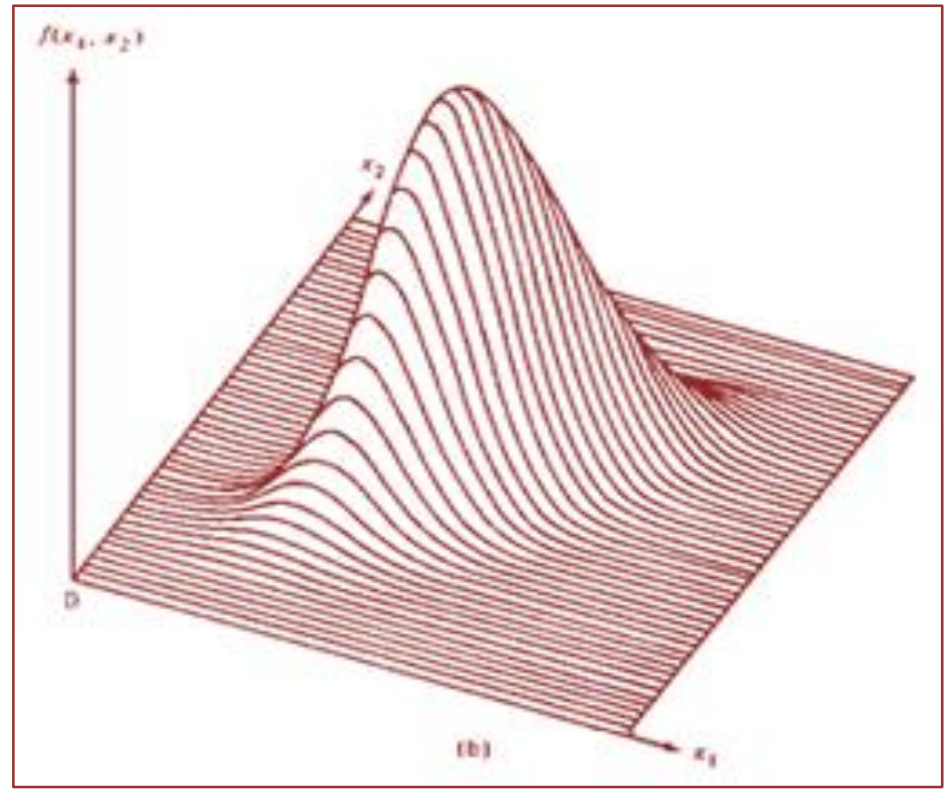

Existem vários testes para verificar a multinormalidade de um conjunto de dados. Neste livro, serão empregados os testes de Mardia (1970) que desenvolveu extensões multivariadas de assimetria e de curtose; o teste de Henze-Zirkler (HZ) que se baseia numa distância funcional não negativa e que mede a distância entre as duas funções de distribuição. Se os dados são distribuídos como uma normal multivariada, a estatística do teste HZ é aproximadamente distribuída como uma lognormal.

Johnson \& Wichern (1992) citam que algumas técnicas multivariadas assumem que o vetor das observações para grandes amostras possui distribuição multinormal, principalmente as que utilizam distâncias que envolvem vetores de médias. Contudo, a qualidade das inferências feitas por esses métodos depende da proximidade da aderência dos dados à multinormalidade.

A análise multivariada clássica baseia-se no pressuposto de que os dados vêm de uma distribuição normal multivariada. De acordo com Silva (2009), o desenvolvimento de testes multivariados começou com o trabalho de Wayle, em 1968, intitulado "Distribuição beta multivariada e teste para normalidade multivariada".

\subsection{TESTE DE MULTINORMALIDADE DE MARDIA}

Mardia (1970, 1974, 1980) introduziu as medidas multivariadas de assimetria e de curtose para testar a multinormalidade. Os testes de Mardia de assimetria e de curtose multivariados são baseados nos terceiro e quarto momentos padronizados. A construção da estatística do teste de Mardia é invariável, os vetores de dados são primeiro padronizados, sendo usado o vetor de médias e a matriz de covariância amostral. A assimetria e a curtose de Mardia são funções de distâncias quadráticas de Mahalanobis, o que torna essas medidas úteis na verificação de dados discrepantes. 
Considerando o caso univariado, onde $y$ é uma variável aleatória com média $\mu$ e variância $\sigma^{2}$, têm-se:
a. $\sqrt{\beta_{1}}=\frac{\mu_{3}}{\left(\mu_{2}\right)^{\frac{3}{2}}}$ : é o coeficiente de assimetria;
b. $\sqrt{\beta_{2}}=\frac{\mu_{4}}{\left(\mu_{2}\right)^{2}}$ : é o coeficiente de curtose;
c. $\mu_{k}=E(y-\mu)^{k}$ : são os momentos de ordem $k$ centrados na média $\mu$;
d. $\sqrt{\beta_{1}}=0$ e $\sqrt{\beta_{2}}=3$ : no caso da variável $y$ seguir a distribuição normal.

Sejam $y_{v 1}$ e $y_{v 2}$ dois vetores aleatórios independentes e originários de uma população p-dimensional, apresentando a mesma função densidade de probabilidade com vetor de médias $E\left(y_{v}\right)=\mu_{v}$ e matriz de covariâncias $E(S)=\Sigma$. Mardia (1970) definiu os coeficientes multivariados de assimetria $\beta_{1, p}$ e $\mathrm{o}$ de curtose $\beta_{2, p}$, como uma generalização dos coeficientes de assimetria e de curtose univariados. De acordo com Kankainen, A; Taskinen, S; Oja, H (1991), Kollo (2008), Koizumi, K; Sumikawa, T; Pavlenko, T. (2014), têm-se:

$$
\beta_{1, p}\left(y_{v}\right)=E\left[\left(y_{v 1}-\mu_{v}\right)^{\prime} \Sigma^{-1}\left(y_{v 2}-\mu_{v}\right)\right]^{3} \quad \beta_{2, p}\left(y_{v}\right)=E\left[\left(y_{v}-\mu_{v}\right)^{\prime} \Sigma^{-1}\left(y_{v}-\mu_{v}\right)\right]^{2}
$$

De acordo com Pontes (2005), na distribuição normal multivariada, a assimetria é nula, ou seja, $\beta_{1, p}=0$ e a curtose é dada por $\beta_{2, p}=p(p+2)$. As fórmulas para estimar a assimetria e a curtose de um conjunto de dados multivariados são dadas por:

$$
\hat{\beta}_{1, p}=\frac{1}{n^{2}} \sum_{i=1}^{n} \sum_{j=1}^{n} g_{i j}^{3} \quad \hat{\beta}_{2, p}=\frac{1}{n} \sum_{i=1}^{n} g_{i i}^{2}=\frac{1}{n} \sum_{i=1}^{n} d_{i}^{4}
$$

$\mathrm{Ou}$,

$$
\left.\hat{\beta}_{1, p}=\frac{1}{n^{2}} \sum_{i=1}^{n} \sum_{j=1}^{n}\left[\left(y_{v i}-\bar{y}\right)^{\prime} S_{n}^{-1}\left(y_{v j}-\bar{y}\right)\right]^{3} \quad \hat{\beta}_{2, p}=\frac{1}{n} \sum_{i=1}^{n}\left(y_{v i}-\bar{y}_{v}\right)^{\prime} S_{n}^{-1}\left(y_{v i}-\bar{y}_{v}\right)\right]^{2}
$$

$n$ : número de repetições $\left(y_{v 1}, y_{v 2}, \cdots, y_{v_{n}}\right)$;

$g_{i j}=\left(y_{v i}-\bar{y}_{v}\right)^{\prime} S_{n}^{-1}\left(y_{v j}-\bar{y}_{v}\right)$;

$S_{n}=\frac{1}{n} \sum_{i=1}^{n}\left(y_{v i}-\bar{y}_{v}\right)\left(y_{v i}-\bar{y}_{v}\right)^{\prime}$ : é o estimador da matriz de covariância populacional;

$d_{i}=\sqrt{g_{i i}}$ : é a distância amostral de Mahalanobis entre $y_{v i}$ e $\bar{y}$. 
Sendo:
a. $E\left(\hat{\beta}_{1, p}\right)=0 \quad$ e $\operatorname{Var}\left(\hat{\beta}_{1, p}\right)=6 / n$
b. $E\left(\hat{\beta}_{2, p}\right)=p(p+2)(n-1) /(n+1) \quad$ e $\operatorname{Var}\left(\hat{\beta}_{2, p}\right)=8 p(p+2) / n$

Denotando que o estimador do coeficiente de curtose populacional $\beta_{2, p}$ é viesado, o que não ocorre com o estimador do coeficiente de assimetria $\beta_{1, p}$.

Seja a hipótese $H_{0}$ para testar a assimetria: $H_{0}: \beta_{1, p}=0$.

A estatística $n \frac{\hat{\beta}_{1, p}}{6}$ é usada para testar $H_{0}: \beta_{1, p}=0$, a qual é assintoticamente distribuída como uma variável aleatória qui-quadrado $\operatorname{com} p(p+1)(p+2) / 6$ graus de liberdade.

$$
\frac{n \hat{\beta}_{1, p}}{6} \sim \chi_{[p(p+1)(p+2) / 6]}^{2}
$$

Seja a hipótese $H o$ para testar os desvios de curtose:

$$
H_{0}: \beta_{2, p}=p(p+2)
$$

A estatística $\frac{\hat{\beta}_{2, p}-p(p+2)(n-1) /(n+1)}{\sqrt{8 p(p+2) n}}$ é usada para testar $H_{0}: \beta_{2, p}=p(p+2)$, a qual é assintoticamente distribuída segundo uma distribuição $N(0,1)$. No caso de grandes amostras, tem-se:

$$
\frac{\hat{\beta}_{2, p}-p(p+2)}{\sqrt{8 p(p+2) n}} \sim N(0,1)
$$

De acordo com Kankainen, A; Taskinen, S; Oja, H (1991), sob multinormalidade $\beta_{1, p}$ e $\beta_{2, p}$ são invariantes afins, todos os terceiro e quarto momentos padronizados são normais assintoticamente independentes e, consequentemente, as distribuições são limitantes de $n$.

\subsection{TESTE DE MULTINORMALIDADE DE HENZE-ZIRKLER}

De acordo Korkmaz, Goksuluk e Zararsiz (2014), o teste de Henze-Zirkler (HZ) é baseado em uma distância funcional não negativa que mede a distância entre duas funções de distribuição. Se os dados são distribuídos como uma normal multivariada, a estatística do teste tem distribuição aproximadamente log-normal. Inicialmente, são calculados a média, a variância e o parâmetro de suavidade. Em seguida, a média e a variância são log-normalizadas e o $p$-valor é estimado. Seja HZ a estatística de teste descrita por esses autores: 


$$
H Z=\frac{1}{n} \sum_{i}^{n} \sum_{j}^{n} e^{-\frac{\beta^{2}}{2} D_{i j}}-2\left(1+\beta^{2}\right)^{-\frac{p}{2}} \sum_{i=1}^{n} e^{-\frac{\beta^{2}}{2\left(1+\beta^{2}\right)} D_{i}}+n\left(1+2 \beta^{2}\right)^{-\frac{p}{2}}
$$

Onde $p$ é o número de variáveis.

$$
\begin{gathered}
\beta=\frac{1}{\sqrt{2}}\left[\frac{n(2 p+1)}{4}\right]^{\frac{1}{p+4}} \\
D_{i j}=\left(y_{v i}-y_{v j}\right)^{\prime} S^{-1}\left(y_{v i}-y_{v j}\right) \\
D_{i}=\left(y_{v i}-\bar{y}_{v}\right)^{\prime} S^{-1}\left(y_{v i}-\bar{y}_{v}\right)
\end{gathered}
$$

Na equação 3.2.1, $D_{i}$ é a distância de Mahalanobis ao quadrado da $i$-ésima observação ao centroide e $D_{i j}$ expressa a distância ao quadrado de Mahalanobis entre a $i$-ésima e a $j$ ésima observações. Se os dados são multivariados normais, a estatística do teste (HZ) é aproximadamente log-normalmente distribuída com média $\mu$ e variância $\sigma^{2}$.

$$
\begin{gathered}
\mu=1-\frac{a^{-\frac{p}{2}}\left[1+p \beta^{\frac{2}{a}}+p(p+2) \beta^{4}\right]}{2 a^{2}} \\
\sigma^{2}=2(1+4 \beta)^{-\frac{p}{2}}+\frac{2 a^{-p}\left(1+2 p \beta^{4}\right)}{a^{2}}+\frac{3 p(p+2) \beta^{8}}{4 a^{4}}-4 \omega_{\beta}^{-\frac{p}{2}}\left[1+\frac{3 p \beta^{4}}{2 \omega_{\beta}}+\frac{p(p+2) \beta^{8}}{2 \omega_{\beta}^{2}}\right]
\end{gathered}
$$

Donde $a=1+2 \beta^{2}$ e $\omega_{\beta}=\left(1+\beta^{2}\right)\left(1+3 \beta^{2}\right)$. Então, a média e a variância da estatística HZ log-normalizada podem ser calculadas:

$$
\log (\mu)=\log \left(\sqrt{\frac{\mu^{4}}{\sigma^{2}+\mu^{2}}}\right) \quad \log \left(\sigma^{2}\right)=\log \left(\frac{\sigma^{2}+\mu^{2}}{\sigma^{2}}\right)
$$

Usando os parâmetros da distribuição log-normal, $\mu$ e $\sigma$, testa-se a normalidade multivariada dos dados. A estatística de teste de Wald, que é simplesmente um teste de escore (z) para normalidade multivariada, é dada por: $Z=\frac{\log (H Z)-\log (\mu)}{\log (\sigma)}$.

Existem na literatura outros procedimentos que objetivam verificar a normalidade multivariada, tal como o teste de Kolmogorov-Von-Mises, citados em Pontes (2005), que são baseados no teorema que diz: se um vetor aleatório $y_{v}$ ( $p$-dimensional) com distribuição normal multivariada, ou seja, $y_{v} \sim N_{p}\left(\mu_{v}, \Sigma\right)$, então $\left(y_{v}-\mu_{v}\right)^{\prime} \Sigma^{-1}\left(y_{v}-\mu_{v}\right)$ tem distribuição $\chi^{2} \operatorname{com} p$ graus de liberdade. 


$$
\left(y_{v}-\mu_{v}\right)^{\prime} \Sigma^{-1}\left(y_{v}-\mu_{v}\right) \sim \chi_{p}^{2}
$$

As técnicas gráficas univariadas, denominadas de Q-Q plots, podem ser ampliadas para o espaço multidimensional. A generalização é fundamentada no teorema acima descrito.

Melo (2016) cita que a verificação da normalidade de uma distribuição, tanto univariada quanto multivariada, pode ser feita por meio de gráfico, como o denominado de quantilquantil (Q-Q plot). 0 gráfico Q-Q plot é uma ferramenta importante para visualizar os valores discrepantes, embora esse procedimento contemple certa subjetividade na sua avaliação, não sendo suficiente para fazer inferência sobre a normalidade dos dados, sendo necessário a aplicação de testes estatísticos. Uma desvantagem do Q-Q plot clássico é que os quantis observados não são independentes, mas apenas identicamente distribuídos, tornando-se independentes apenas assintoticamente.

Nesses gráficos são plotados os percentis amostrais esperados pelo ajuste de uma distribuição normal. Se os pontos estiveram localizados próximos a uma reta de mínimos quadrados a suposição de normalidade deve ser aceita. No caso multivariado, as observações são transformadas em distâncias de Mahalanobis para o vetor de médias amostral, sendo os quantis amostrais e os esperados dessas distâncias plotados para a obtenção do gráfico de quantil-quantil.

Melo (2016) apresenta os passos para a construção do gráfico quantil-quantil:

a) Calcular cada distância de Mahalanobis:

$$
D_{j}^{2}=\left(y_{v j}-\bar{y}_{v}\right)^{\prime} S^{-1}\left(y_{v j}-\bar{y}_{v}\right)
$$

b) Efetuar a ordenação dos valores das distâncias (estatísticas de ordem):

$$
D_{(1)}^{2}, D_{(2)}^{2}, \cdots, D_{(j)}^{2}, \cdots, D_{n}^{2}
$$

c) Calcular $\chi_{j}^{2}$ para $(j=1,2, \ldots, n)$ :

$$
\chi_{j}^{2}=F_{\chi_{v}^{2}}^{-1}\left(\frac{j-\frac{1}{2}}{n}\right)
$$

$F_{\chi_{v}^{2}}^{-1}$ : é a função inversa da distribuição qui-quadrado $\operatorname{com} v=p$ graus de liberdade.

d) Plotar as coordenadas:

$$
\left(D_{(j)}^{2}, \chi_{(j)}^{2}\right)
$$

Deve ser observado que o gráfico só precisa ser usado para grandes amostras, haja vista a distribuição marginal de $D_{j}^{2}$, sob a hipótese nula, não ser precisamente um quiquadrado para pequenas amostras. 
Melo (2016) apresenta outro método para construção do gráfico de quantil-quantil para dados multivariados, não baseados em distâncias quadráticas, mas usando a transformação " $t$ " de Student.

As análises de multinormalidade, realizadas nas aplicações práticas apresentadas neste livro, foram feitas no programa SAS: \% Multinorm Macro: Mardia tests of multivariate normality, version 1.1, disponibilizado pelo SAS Institute Inc., no link:

http://homepages.wmich.edu/ applegab/EMR675/SASFiles/multnorm.sas

A linguagem de programação "Macro \%Multinorm" realiza os testes de normalidade multivariada de Mardia, com base nas estatísticas de assimetria e de curtose, e o de Henze-Zirkler (HZ), assim como, para cada uma das variáveis respostas, executa alguns testes de normalidade univariado. Apresenta o gráfico qui-quadrado quantil-quantil das observações ao quadrado das distâncias de Mahalanobis, o que permite obter uma avaliação visual da normalidade multivariada.

\subsection{TESTE DE HOMOGENEIDADE DE MATRIZES DE COVARIÂNCIAS CONSIDERANDO P OCASIÕES}

Dada a hipótese de nulidade $H_{0}: \Sigma_{1}=\Sigma_{2}=\cdots=\Sigma_{g}=\sum$, que representa a pressuposição de homogeneidade de matrizes de covariâncias, considerando $g$ populações normais $p$ dimensionais com vetores de médias $\mu_{v i}$ e matrizes de covariâncias $\sum_{i}$, assim como a hipótese alternativa $H_{A}: \sum_{i} \neq \sum_{i^{\prime}}$, donde $i \neq i^{\prime}$ e $n_{i}$ é o número de repetições por tratamento.

Seja o teste estatístico desenvolvido por Box (1950), a partir da razão de máxima verossimilhança generalizada:

$$
M=\sum_{i=1}^{g}\left(n_{i}-1\right) \operatorname{Ln}|S|-\sum_{i=1}^{g}\left(n_{i}-1\right) \operatorname{Ln}\left|S_{i}\right|
$$

$S_{i}$ e $n_{i}$-1 são respectivamente a matriz de covariância amostral e o número de graus de liberdade correspondente ao tratamento $i$. Então:

$$
S=\frac{1}{\sum_{i=1}^{g}\left(n_{i}-1\right)}\left[\sum_{i=1}^{g}\left(n_{i}-1\right) S_{i}\right]
$$

$S$ : é a matriz de covariância comum amostral e obtida como uma matriz de ponderação das matrizes $S_{i}$.

No teste os determinantes das matrizes de covariâncias assumem o conceito de variâncias generalizadas. 0 teste de Box é uma generalização do teste de Bartlett, citado 
em Steel \& Torrie (1960), que é utilizado, no caso univariado, para testar a homogeneidade de variâncias:

$$
\begin{gathered}
H_{0}: \sigma_{1}^{2}=\sigma_{2}^{2}=\cdots=\sigma_{g}^{2} \\
\chi^{2}=\sum_{i=1}^{g}\left(n_{i}-1\right) L n S^{2}-\sum_{i}^{g}\left(n_{i}-1\right) \operatorname{Ln} S_{i}^{2}
\end{gathered}
$$

De acordo com BOX (1949), citado por MORRISON (1976), a estatística $M C^{-1}$ é aproximadamente distribuída, quando $n_{i}$ tende para infinito, como uma variável quiquadrado $\left(\chi^{2}\right.$ assintótico) com $v=\frac{1}{2}(g-1) p(p+1)$ graus de liberdade. Onde:

$$
C^{-1}=1-\frac{2 p^{2}+3 p-1}{6(p+1)(g-1)}\left[\sum_{i=1}^{g} \frac{1}{n_{i}-1}-\frac{1}{\sum_{i=1}^{g}\left(n_{i}-1\right)}\right]
$$

Caso os tratamentos apresentem o mesmo número de repetições, $n_{1}=n_{2}=\cdots=n_{g}=n$, a fórmula de $C^{-1}$ será dada por:

$$
C^{-1}=1-\frac{\left(2 p^{2}+3 p-1\right)(g+1)}{6(p+1) g \times(n-1)}
$$

Exercício 3.3.1. Dado um experimento bidimensional com três tratamentos A, B e C e dez repetições. Testar se as matrizes de covariâncias $S_{A}, S_{B}$ e $S_{C}$ são homogeneas.

$$
\begin{gathered}
H_{0}: \sum_{A}=\sum_{B}=\sum_{C}=\sum \\
S_{A}=\left[\begin{array}{ll}
0,0520107626 & 0,0318025028 \\
0,0318025028 & 0,0316255055
\end{array}\right] \quad S_{B}=\left[\begin{array}{ll}
0,0751199608 & 0,0027858932 \\
0,0027858932 & 0,0249834745
\end{array}\right] \\
S_{C}=\left[\begin{array}{ll}
0,0212345752 & 0,0020700814 \\
0,0020700814 & 0,0152507080
\end{array}\right] \\
S=\frac{S_{A}+S_{B}+S_{C}}{3}=\left[\begin{array}{ll}
0,0494551 & 0,0122195 \\
0,0122195 & 0,0239532
\end{array}\right] \\
M=\sum_{i=1}^{g}\left(n_{i}-1\right) \operatorname{Ln}|S|-\sum_{i=1}^{g}\left(n_{i}-1\right) \operatorname{Ln}\left|S_{i}\right|
\end{gathered}
$$$$
\operatorname{Det} A=-7,36430 \operatorname{Det} B=-6,28235 \operatorname{Det} C=-8,04857 \operatorname{Det} S=-6,87307
$$$$
M=-185,57289+195,25698=9,68409
$$ 


$$
\begin{gathered}
C^{-1}=1-\frac{2 p^{2}+3 p-1}{6(p+1)(g-1)}\left[\sum_{i=1}^{g} \frac{1}{n_{i}-1}-\frac{1}{\sum_{i=1}^{g}\left(n_{i}-1\right)}\right] \\
C^{-1}=1-\frac{\left(2 p^{2}+3 p-1\right)(g+1)}{6(p+1) g(n-1)}=1-\frac{52}{486}=0,893004115 \quad\left(n_{1}=n_{2}=n_{3}=n=10\right) \\
M C^{-1}=8,647932219 \\
v=\frac{1}{2}(g-1) p(p+1)=6
\end{gathered}
$$

Dado que $\chi_{6 ; 0,01}^{2}=16,8$ e $M C^{-1}=8,647932219$, a conclusão é pela não rejeição de $H_{0}: \sum_{A}=\sum_{B}=\sum_{C}=\sum$, isto é, as matrizes de covariância dos tratamentos A, B e C podem ser consideradas homogêneas.

\subsection{TESTE DE UNIFORMIDADE DA MATRIZ DE COVARIÂNCIA CONSIDERANDO $P$ OCASIÕES}

É possível analisar um inventário florestal contínuo usando a técnica univariada de parcelas subdivididas, mas, neste caso, a matriz de covariância considerando as $p$ ocasiões deverá ser uniforme. Caso não ocorra a uniformidade, então deve ser aplicado o método multivariado.

A matriz uniforme, ou a que tem a forma simétrica composta, possui variâncias iguais e, também covariâncias iguais entre os pares de ocasiões. No caso de um inventário florestal contínuo, com parcelas permanentes, significa que existe homogeneidade de variâncias considerando as $p$ ocasiões, assim como as covariâncias ou correlações lineares entre as ocasiões são estatisticamente iguais. A hipótese $H_{0}$ a ser testada é:

$$
H_{0}: \sum=\sigma^{2}\left[\begin{array}{cccc}
1 & \rho & \cdots & \rho \\
\rho & 1 & \cdots & \rho \\
\cdots & \cdots & \cdots & \cdots \\
\rho & \rho & \cdots & 1
\end{array}\right]
$$

De acordo com Greenhouse \& Geisser (1959), na análise de dados de medidas repetidas (análise de perfil), as razões de quadrados médios calculados, usando o modelo misto, só terão uma distribuição exata de $F$, se as observações no tempo forem normalmente distribuídas com variâncias iguais e mutuamente independentes, ou seja, igualmente correlacionadas. Mas, consideram que essas pressuposições são muito restritivas, então recomendam que as medições no tempo sejam consideradas como um vetor de observações com uma distribuição normal multivariada e matriz de covariância arbitrária.

No caso do inventário contínuo com parcelas permanentes significa que a covariância ou correlação linear entre as ocasiões são estatisticamente iguais. Pode-se verificar a uniformidade da matriz de covariâncias pelo critério de Mauchly (estatística $W$ ) ou pelo critério de Wilks (1946). 


\subsubsection{TESTE DE UNIFORMIDADE DA MATRIZ DE COVARIÂNCIA DE WILKS}

A pressuposição de igualdade de variâncias e covariâncias, considerando as medidas nas $p$ ocasiões, pode ser testada pelo critério $(\Delta)$ de Wilks (1946). Seja a hipótese de nulidade:

$$
H_{0}: \sum=\sigma^{2}\left[\begin{array}{cccc}
1 & \rho & \cdots & \rho \\
\rho & 1 & \cdots & \rho \\
\cdots & \cdots & \cdots & \cdots \\
\rho & \rho & \cdots & 1
\end{array}\right]
$$

0 teste consiste em obter a estatística $M=-(N-g) \operatorname{Ln} \Delta$, onde $(N-g)$ são os graus de liberdade da matriz de covariância que está sendo testada, donde $N=n_{1}+n_{2}+\cdots+n_{g}$.

$$
\begin{aligned}
\Delta=\frac{|S|}{\left|S^{*}\right|} & =\frac{\left|\begin{array}{cccc}
s_{11} & s_{12} & \cdots & s_{1 p} \\
s_{12} & s_{22} & \cdots & s_{2 p} \\
\cdots & \cdots & \cdots & \cdots \\
s_{p 1} & s_{p 2} & \cdots & s_{p p}
\end{array}\right|}{\left|\begin{array}{cccc}
s^{2} & \hat{\rho} s^{2} & \cdots & \hat{\rho} s^{2} \\
\hat{\rho} s^{2} & s^{2} & \cdots & \hat{\rho} s^{2} \\
\cdots & \cdots & \cdots & \cdots \\
\hat{\rho} s^{2} & \hat{\rho} s^{2} & \cdots & s^{2}
\end{array}\right|} \\
s^{2}=\frac{1}{p} \sum_{i=1}^{p} s_{i j}(i=j) \quad \hat{\rho} s^{2} & =\frac{1}{p(p-1)} \sum_{i=1}^{p} \sum_{j=1}^{p} s_{i j}(i \neq j) \quad i, j=1,2, \cdots, p
\end{aligned}
$$

$s_{i j}$ : se $(i=j)$ são as variâncias dentro de cada ocasião;

$s_{i j}$ : se $(i \neq j)$ são as covariâncias entre as ocasiões;

S e $S^{*}$ : são matrizes simétricas, $s^{2}$ e $\hat{\rho} s^{2}$ são as estimativas não tendenciosas de $\sigma^{2}$ e $\rho \sigma^{2}$.

Calcula-se então a estatística $M=-(N-g) \operatorname{Ln} \Delta$, donde $(N-g)$ é o número de graus de liberdade da matriz de covariâncias testada.

A estatística $\left(1-A_{1}\right) M$, sob a hipótese de nulidade, é aproximadamente distribuída como $\chi^{2} \operatorname{com} v=\left(p^{2}+p-4\right) / 2$ graus de liberdade.

Então $H_{0}$ será aceita se $\left(1-A_{1}\right) M<\chi_{\left.\alpha ;\left(p^{2}+p-4\right) / 2\right)}^{2}$. Onde:

$$
A_{1}=\frac{p(p+1)^{2}(2 p-3)}{6(N-g)(p-1)\left(p^{2}+p-4\right)}
$$


Exercício 3.4.1.1. Verificar se a matriz $\mathrm{S}$ pode ser considerada uma matriz uniforme, ou seja, apresenta homogeneidade de variâncias e correlações iguais entre as ocasiões, donde $\mathrm{S}$ é a matriz de covariância comum dos quatro métodos de manejo florestal M1, M2, M3 e M4, referente ao experimento apresentado no capítulo 7, seção 7.7.1, na análise de um inventario florestal com trinta parcelas permanentes e cinco ocasiões. Programa SAS (Proc IML) desenvolvido e apresentado na tabela C2 do apêndice C.

$$
\begin{aligned}
& S=\left[\begin{array}{rrrrr}
2,1061385 & 2,2793064 & 2,2381011 & 2,1368150 & 1,9697176 \\
2,2793064 & 4,7718804 & 5,5400208 & 5,8451530 & 5,5819684 \\
2,2381011 & 5,5400208 & 7,2915454 & 7,9891048 & 7,9497102 \\
2,1368150 & 5,8451530 & 7,9891048 & 9,3602169 & 9,5140274 \\
1,9697176 & 5,5819684 & 7,9497102 & 9,5140274 & 10,1532850
\end{array}\right] \\
& S^{*}=\left[\begin{array}{lllll}
6,736613 & 5,104392 & 5,104392 & 5,104392 & 5,104392 \\
5,104392 & 6,736613 & 5,104392 & 5,104392 & 5,104392 \\
5,104392 & 5,104392 & 6,736613 & 5,104392 & 5,104392 \\
5,104392 & 5,104392 & 5,104392 & 6,736613 & 5,104392 \\
5,104392 & 5,104392 & 5,104392 & 5,104392 & 6,736613
\end{array}\right] \\
& \Delta=\frac{|S|}{\left|S^{*}\right|}=\frac{\operatorname{det}(S)}{\operatorname{det}\left(S^{*}\right)}=\frac{0,6097049}{192,73143}=0,00316349 \\
& M=-(N-g) \operatorname{Ln} \Delta=-(120-4) \operatorname{Ln} \Delta=667,7052 \\
& A_{1}=\frac{p(p+1)^{2}(2 p-3)}{6(N-g)(p-1)\left(p^{2}+p-4\right)} \\
& A_{1}=\frac{p(p+1)^{2}(2 p-3)}{6(N-g)(p-1)\left(p^{2}+p-4\right)}=\frac{5(5+1)^{2}(2 \times 5-3)}{6 \times(120-4)(5-1)\left(5^{2}+5-4\right)}=\frac{1260}{72384}=0,0174072 \\
& \left(1-A_{1}\right) M=(1-0,0174072) \times 667,7052=656,0823 \\
& \chi_{\text {Cal }}^{2}=656,0823 \quad \chi_{(13 ; 0,01)}^{2}=27,7 \\
& v=\left(p^{2}+p-4\right) / 2=\left(5^{2}+5-4\right) / 2=13
\end{aligned}
$$

Dado que $\chi_{\text {Cal }}^{2}>\chi_{(13 ; 0,01)}^{2}$, conclui-se que a matriz de covariância comum $(S)$ referente aos quatro métodos de manejo não é uniforme. 


\subsubsection{CIRCULARIDADE OU ESFERICIDADE DE UMA MATRIZ}

Huynh \& Feldt (1970) mostram as condições para que as razões de quadrados médios, nos delineamentos de medidas repetidas, possuam distribuições exatas de $F$. Considerando que os tratamentos sejam multinormais com matrizes de covariâncias $\Sigma_{1}, \Sigma_{2}, \cdots, \Sigma_{g}$, em vez de uma matriz comum $\Sigma$, estes autores concluem que os testes de significância sobre os efeitos de ocasião e de interação são exatos se, e somente se, os elementos das matrizes dos tratamentos $\Sigma_{i}(i=1,2 \cdots, g)$ atendam a seguinte condição, denominada de circularidade ou esfericidade, considerando as $k=1,2 \cdots, p$ ocasiões:

$$
\sigma_{k k^{\prime}} \begin{cases}a_{k}+a_{k^{\prime}}+\lambda & \left(k=k^{\prime}\right) \\ a_{k}+a_{k^{\prime}} & \left(k \neq k^{\prime}\right)\end{cases}
$$

A condição 3.4.2.1 é denominada de condição $H$-F. 0 padrão de uniformidade é um caso particular de circularidade quando $a_{k}=a_{k^{\prime}}=\sigma^{2} \rho / 2$ e $\lambda=\sigma^{2}(1-\rho)$, ou seja:

$$
\sum=\sigma^{2}\left[\begin{array}{cccc}
1 & \rho & \cdots & \rho \\
\rho & 1 & \cdots & \rho \\
\cdots & \cdots & \cdots & \cdots \\
\rho & \rho & \cdots & 1
\end{array}\right]
$$

O padrão de uniformidade ou de simetria composta consiste ter igualdade de variâncias no decorrer do tempo, assim como todas as covariâncias sejam iguais. Toda matriz uniforme é circular, mas a recíproca não é verdadeira. As matrizes de covariâncias que possuem variâncias iguais e correlações nulas são casos especiais da condição $H-F$.

Conforme citado em Leal (1979), no caso de uma matriz de covariância, a expressão 3.4.2.1 pode ser escrita também da seguinte forma:

$$
\sigma_{k k^{\prime}} \begin{cases}\bar{\sigma}_{k .}+\bar{\sigma}_{k^{\prime} .}-\bar{\sigma}_{. .}+\lambda\left(1-\frac{1}{p}\right) & \left(k=k^{\prime}\right) \\ \bar{\sigma}_{k .}+\bar{\sigma}_{k^{\prime} .}-\bar{\sigma}_{. .}-\frac{\lambda}{p} & \left(k \neq k^{\prime}\right)\end{cases}
$$

$\bar{\sigma}_{k}$ : é a média dos elementos da $k$-ésima linha (ou $k$-ésima coluna) de $\sum(p \times p)$;

$\bar{\sigma}_{k^{\prime}}$ : é a média dos elementos da $k^{\prime}$-ésima linha (ou $k^{\prime}$-ésima coluna) de $\sum(p \times p)$;

$\bar{\sigma}_{. .}$: é a média de todos os elementos de $\sum(p \times p)$; 
A constante $\lambda$ é a diferença entre as médias das variâncias e as médias das covariâncias. As expressões 3.4.2.1 e 3.4.2.2 também são equivalentes à equação $P^{\prime} \sum P=\lambda I_{(p-1)}$. Onde, ${ }_{p} \Sigma_{p}$ é a matriz de covariância considerando as $p$ ocasiões e $P$ é uma matriz contraste ortonormal de ordem $p \times(p-1)$.

Tem-se, também, da condição $H-F$, que:

$$
\sigma_{k k^{\prime}}^{2}=\frac{\sigma_{k}^{2}+\sigma_{k^{\prime}}^{2}}{2}-\lambda \quad\left(k \neq k^{\prime}\right)
$$

A condição $H-F$ ou condição de esfericidade (ou circularidade) da matriz $\sum$, também equivale a especificar que as variâncias das diferenças entre pares de ocasiões sejam todas iguais (constante), ou seja: $\sigma^{2}\left(y_{k}-y_{k^{\prime}}\right)=2 \lambda\left(\forall k \neq k^{\prime}\right)$.

Exercício 3.4.2.1. Para melhor entendimento da condição de H-F, seja o exemplo fictício de um experimento com $g=4$ tratamentos e $n=30$ parcelas permanentes medidas em $p=4$ ocasiões, onde $N=n g=120$, e apresentando a matriz não uniforme A de covariância comum. Usando o programa desenvolvido no SAS, tabela C2 do apêndice $C$, o resultado do teste de uniformidade de Wilks foi significativo para a matriz A, pois $\chi_{\text {Cal }}^{2}=143,15>\chi_{8 ; 0,01 l}^{2}=20,1$, tal que: $v=\left(p^{2}+p-4\right) / 2=\left(4^{2}+4-4\right) / 2=8$.

$$
A=\left[\begin{array}{cccc}
10 & 5 & 10 & 15 \\
5 & 20 & 15 & 20 \\
10 & 15 & 30 & 25 \\
15 & 20 & 25 & 40
\end{array}\right]=25\left[\begin{array}{cccc}
0,4 & 0,2 & 0,4 & 0,6 \\
0,2 & 0,8 & 0.6 & 0,8 \\
0,4 & 0,6 & 1,2 & 1 \\
0,6 & 0,8 & 1 & 1,6
\end{array}\right]
$$

a. Calcular o valor da constante $\lambda$ :

$$
\lambda=\frac{10+20+30+40}{4}-\frac{2(5+10+15+15+20+25)}{12}=25-15=10
$$

b. Calcular os valores:

$$
\begin{gathered}
\bar{\sigma}_{. .}=(100+2 \times 90) / 16=17,5 \\
\lambda(1-1 / p)=7,5 \quad \lambda / p=2,5 \\
\bar{\sigma}_{1 .}=10 \quad \bar{\sigma}_{2 .}=15 \quad \bar{\sigma}_{3 .}=20 \quad \bar{\sigma}_{4 .}=25
\end{gathered}
$$

c. Obter a matriz atendendo a condição:

$$
\sigma_{k k^{\prime}}= \begin{cases}\bar{\sigma}_{k .}+\bar{\sigma}_{k^{\prime} .}-\bar{\sigma}_{. .}+\lambda\left(1-\frac{1}{p}\right) & \left(k=k^{\prime}\right) \\ \bar{\sigma}_{k .}+\bar{\sigma}_{k^{\prime} \cdot}-\bar{\sigma}_{. .}-\frac{\lambda}{p} & \left(k \neq k^{\prime}\right)\end{cases}
$$




$$
\begin{array}{ll}
\sigma_{11}=10+10-17,5+7,5=10 & \sigma_{22}=15+15-17,5+7,5=20 \\
\sigma_{33}=20+20-17,5+7,5=30 & \sigma_{44}=25+25-17,5+7,5=40 \\
\sigma_{12}=10+15-17,5-2,5=5 & \sigma_{13}=10+20-17,5-2,5=10 \\
\sigma_{14}=10+25-17,5-2,5=15 & \sigma_{23}=15+20-17,5-2,5=15 \\
\sigma_{24}=15+25-17,5-2,5=20 & \sigma_{34}=20+25-17,5-2,5=25
\end{array}
$$

Resultando:

$$
A=\left[\begin{array}{cccc}
10 & 5 & 10 & 15 \\
5 & 20 & 15 & 20 \\
10 & 15 & 30 & 25 \\
15 & 20 & 25 & 40
\end{array}\right]
$$

Concluindo-se que a matriz $A$ atende a condição $H-F$.

Exercício 3.4.2.2. Verificar se matriz $A$ atende a condição: $\sigma_{k k^{\prime}}^{2}=\frac{\sigma_{k}^{2}+\sigma_{k^{\prime}}^{2}}{2}-\lambda$.

$$
\begin{gathered}
A=\left[\begin{array}{cccc}
10 & 5 & 10 & 15 \\
5 & 20 & 15 & 20 \\
10 & 15 & 30 & 25 \\
15 & 20 & 25 & 40
\end{array}\right] \\
\sigma_{12}=\frac{10+20}{2}-10=5 \quad \sigma_{14}=\frac{10+40}{2}-10=15 \\
\sigma_{23}=\frac{20+30}{2}-10=15 \quad \sigma_{24}=\frac{20+40}{2}-10=20 \quad \sigma_{13}=\frac{10+30}{2}-10=10 \\
\end{gathered}
$$

Conclui-se que a matriz A atende a condição $H-F$.

Exercício 3.4.2.3. Verificar se $A$ atende a condição: $\sigma^{2}\left(y_{k}-y_{k^{\prime}}\right)=2 \lambda\left(\forall k \neq k^{\prime}\right)$.

$$
\begin{gathered}
A=\left[\begin{array}{cccc}
10 & 5 & 10 & 15 \\
5 & 20 & 15 & 20 \\
10 & 15 & 30 & 25 \\
15 & 20 & 25 & 40
\end{array}\right] \\
V\left(y_{1}-y_{2}\right)=\sigma_{11}+\sigma_{22}-2 \sigma_{12}=10+20-2 \times 5=2 \lambda=20 \\
V\left(y_{1}-y_{3}\right)=\sigma_{11}+\sigma_{33}-2 \sigma_{13}=10+30-2 \times 10=20 \\
V\left(y_{1}-y_{4}\right)=\sigma_{11}+\sigma_{44}-2 \sigma_{14}=10+40-2 \times 15=20 \\
V\left(y_{2}-y_{3}\right)=\sigma_{22}+\sigma_{33}-2 \sigma_{23}=20+30-2 \times 15=20 \\
V\left(y_{2}-y_{4}\right)=\sigma_{22}+\sigma_{44}-2 \sigma_{24}=20+40-2 \times 20=20 \\
V\left(y_{3}-y_{4}\right)=\sigma_{33}+\sigma_{44}-2 \sigma_{34}=30+40-2 \times 25=20
\end{gathered}
$$

Conclui-se que a matriz A atende a condição $H-F$. 


\subsection{3. $O$ CRITÉRIO DE MAUCHLY PARA TESTAR A ESFERICIDADE DE UMA MATRIZ}

0 método de esfericidade de Mauchly (1940) objetiva verificar se uma população normal multivariada possui uma matriz de covariância esférica, ou seja, suas variâncias são iguais e suas correlações nulas.

0 método utiliza, para que a esfericidade seja medida pelo teste, uma matriz $C$ de contrastes ortonormais para transformar a matriz de covariância $\sum$ em uma forma ortonormalizada $\Sigma^{*}$. Caso aceita a hipótese de que $\sum^{*}$ seja esférica, então a matriz original $\sum$ atenderá a condição $H-F$ que seria a garantia de que, caso a opção seja de usar a analise pelo método univariado de parcelas subdividida, os testes $F$ sejam exatos.

Seja $H_{0}: C \sum C^{\prime}=\lambda I_{p-1}$, a hipótese de que a matriz de covariância satisfaz à condição de esfericidade. Seja a estatística $W$ de Mauchly (1940) para testar essa hipótese.

$$
W=\frac{(p-1)^{p-1}\left|C S C^{\prime}\right|}{\left(t r C S C^{\prime}\right)^{p-1}}
$$

Donde $S$ é a estimativa da matriz de covariância considerando as $p$ ocasiões e $C$ uma submatriz semiortoganal $(p-1) \times p$ de uma matriz ortogonal Helmert $(H)$ e $\lambda$ é um dos $p+1$ parâmetros definido na matriz padrão.

$$
H=\left[\begin{array}{c}
j^{\prime} \\
\sqrt{p} \\
C
\end{array}\right]
$$

Para grandes amostras, quando o número de parcelas permanentes é igual ou superior a 30 unidades, pode-se aplicar a seguinte estatística qui-quadrado:

$$
\chi_{f}^{2}=-\left[v-\frac{2 p^{2}-3 p+3}{6(p-1)}\right] \ln W
$$

que apresenta uma distribuição qui-quadrado com $f=\frac{1}{2} p(p-1)-1$ graus de liberdade. Onde $v$ é o número de graus de liberdade usado para obter a matriz $\mathrm{S}$.

Para o caso de não atingir a uniformidade desejada e a opção seja de aplicar o método de parcelas subdivididas, pode-se usar a correção de Greenhouse-Geisser $(g-g)$ e/ou a de Huynh e Feldt ( $h-f)$, objetivando corrigir os graus de liberdades correspondentes. Neste caso, corre-se o risco de perder uma boa precisão nas estimativas dos resultados. 
Exercício 3.4.3.1. Seja (S) a matriz de covariância comum originada de um inventário florestal com cinco ocasiões e trinta parcelas permanentes. Testar pelo critério de Mauchly se a matriz de covariância (S) pode ser considerada esférica, onde S é a matriz de covariância comum dos quatro métodos de manejo florestal M1, M2, M3 e M4, referente ao experimento apresentado no capítulo 7, seção 7.7.1. A tabela C3, do apêndice $C$, mostra o programa desenvolvido no SAS para calcular o teste de Mauchly, e obter as correções de Greenhouse-Geisser e de Huynh e Feldt.

$$
S=\left[\begin{array}{rrrrr}
2,1061385 & 2,2793064 & 2,2381011 & 2,1368150 & 1,9697176 \\
2,2793064 & 4,7718804 & 5,5400208 & 5,8451530 & 5,5819684 \\
2,2381011 & 5,5400208 & 7,2915454 & 7,9891048 & 7,9497102 \\
2,1368150 & 5,8451530 & 7,9891048 & 9,3602169 & 9,5140274 \\
1,9697176 & 5,5819684 & 7,9497102 & 9,5140274 & 10,1532850
\end{array}\right]
$$

De acordo com o capítulo 2, seção 2.10, seja a matriz Helmert H (5×5).

$$
H_{5}=\left[\begin{array}{ccccc}
\frac{1}{\sqrt{5}} & \frac{1}{\sqrt{5}} & \frac{1}{\sqrt{5}} & \frac{1}{\sqrt{5}} & \frac{1}{\sqrt{5}} \\
\frac{1}{\sqrt{2}} & \frac{-1}{\sqrt{2}} & 0 & 0 & 0 \\
\frac{1}{\sqrt{6}} & \frac{1}{\sqrt{6}} & \frac{-2}{\sqrt{6}} & 0 & 0 \\
\frac{1}{\sqrt{12}} & \frac{1}{\sqrt{12}} & \frac{1}{\sqrt{12}} & \frac{-3}{\sqrt{12}} & 0 \\
\frac{1}{\sqrt{20}} & \frac{1}{\sqrt{20}} & \frac{1}{\sqrt{20}} & \frac{1}{\sqrt{20}} & \frac{-4}{\sqrt{20}}
\end{array}\right]=\left[\begin{array}{c}
j^{\prime} \\
\sqrt{p} \\
C
\end{array}\right]
$$

C: é a submatriz ( $p-1) \times p$ semiortoganal originada da matriz ortogonal Helmert $H(p \times p)$.

$$
C=\left[\begin{array}{ccccc}
\frac{1}{\sqrt{2}} & \frac{-1}{\sqrt{2}} & 0 & 0 & 0 \\
\frac{1}{\sqrt{6}} & \frac{1}{\sqrt{6}} & \frac{-2}{\sqrt{6}} & 0 & 0 \\
\frac{1}{\sqrt{12}} & \frac{1}{\sqrt{12}} & \frac{1}{\sqrt{12}} & \frac{-3}{\sqrt{12}} & 0 \\
\frac{1}{\sqrt{20}} & \frac{1}{\sqrt{20}} & \frac{1}{\sqrt{20}} & \frac{1}{\sqrt{20}} & \frac{-4}{\sqrt{20}}
\end{array}\right]=\left[\begin{array}{cccccc}
0,7071068 & -0,7071068 & 0 & 0 & 0 \\
0,4082483 & 0,4082483 & -0,816497 & 0 & 0 \\
0,2886751 & 0,2886751 & 0,2886751 & -0,866025 & 0 \\
0,2236068 & 0,2236068 & 0,2236068 & 0,2236068 & -0,894427
\end{array}\right]
$$

$$
\begin{gathered}
C S C^{\prime}=\left[\begin{array}{ll}
1,597031 & 1,13683181,05273930,7546776 \\
1,13683181,58172321,53962401,3209349 \\
1,05273931,53962401,89165881,7056839 \\
0,75467761,32093491,70568391,8957967
\end{array}\right] \\
\left|C S C^{\prime}\right|=0,0599928 \quad \operatorname{tr}\left(C S C^{\prime}\right)=6,5288818
\end{gathered}
$$




$$
\begin{gathered}
W=\frac{(p-1)^{p-1}\left|C S C^{\prime}\right|}{\left(t r C S C^{\prime}\right)^{p-1}}=\frac{4^{4} \times 0,0599928}{6,5288818^{4}}=0,0084525 \\
\chi_{\text {Cal }}^{2}=-\left[v-\frac{2 p^{2}-3 p+3}{6(p-1)}\right] \ln W=-\left[116-\frac{2 \times 5^{2}-3 \times 5+3}{6(5-1)}\right] \times-3,459709=546,1448 \\
\chi_{9 ; 0,05}^{2}=21,7 \quad f=\frac{1}{2} p(p-1)-1=9
\end{gathered}
$$

Dado que $\chi_{\text {Cal }}^{2}>\chi_{9 ; 0,05}^{2}$, então há razão para considerar que a matriz comum, referente aos quatro métodos de manejo M1, M2, M3 e M4 não é esférica.

\subsubsection{CORREÇÃO DE GREENHOUSE-GEISSER ( $G-G)$}

Greenhouse e Geisser (1959) mostram que para o caso da matriz de covariância $\sum$ ter a forma geral, as estatísticas $F_{2}$ (teste de significância sobre o efeito de ocasião) e $F_{3}$ (teste de significância da interação tratamentos versus ocasiões) terem distribuições aproximadas de $F$ central, implica na necessidade de corrigir os correspondentes graus de liberdade, isto é, $F_{2}$ deverá ter $(p-1) \varepsilon_{g g}$ e $(N-g)(p-1) \varepsilon_{g g}$, enquanto que $F_{3}$ com $(g-1)(p-1) \varepsilon_{g g}$ e $(N-g)(p-1) \varepsilon_{g g}$. Tal que:

$$
\varepsilon_{g g}=\frac{\left[\operatorname{tr}\left(C S C^{\prime}\right)\right]^{2}}{(p-1) \operatorname{tr}\left[\left(C S C^{\prime}\right)^{2}\right]}
$$

Sendo a condição de circularidades dada por: $C \Sigma C^{\prime}=\lambda I_{(p-1)}$.

A matriz $C$ de ordem $(p-1) \times p$ define os $p$ - 1 contrastes ortonormais entre as $p$ medidas no tempo. $I_{(p-1)}$ é a matriz identidade de ordem $p$-1 e $\lambda$ é um valor maior que zero.

Substituindo $C \Sigma C^{\prime}=\lambda I_{(p-1)}$, em 3.4.4.1, tem-se que:

$$
\varepsilon_{g g}=\frac{\left[\operatorname{tr}\left(\lambda I_{(p-1)}\right]^{2}\right.}{(p-1) \operatorname{tr}[(\lambda I)]^{2}}=\frac{[\lambda(p-1)]^{2}}{(p-1) \lambda^{2}(p-1)}=1
$$

Como consequência, tem-se que não é necessário fazer correções nos graus de liberdade de $F_{2}$ e $F_{3}$, haja vista serem distribuições exatas.

Exercício 3.4.4.1. Verificar se matriz $A$ atende a condição: $C A C^{\prime}=\lambda I_{(p-1)}$.

$$
A=\left[\begin{array}{cccc}
10 & 5 & 10 & 15 \\
5 & 20 & 15 & 20 \\
10 & 15 & 30 & 25 \\
15 & 20 & 25 & 40
\end{array}\right]
$$


Seja a matriz Helmert $H(4 \times 4)$ e a submatriz semiortoganal $C$ de ordem $(p-1) \times p$.

$$
\begin{aligned}
H_{4} & =\left[\begin{array}{cccc}
\frac{1}{\sqrt{4}} & \frac{1}{\sqrt{4}} & \frac{1}{\sqrt{4}} & \frac{1}{\sqrt{4}} \\
\frac{1}{\sqrt{2}} & \frac{-1}{\sqrt{2}} & 0 & 0 \\
\frac{1}{\sqrt{6}} & \frac{1}{\sqrt{6}} & \frac{-2}{\sqrt{6}} & 0 \\
\frac{1}{\sqrt{12}} & \frac{1}{\sqrt{12}} & \frac{1}{\sqrt{12}} & \frac{-3}{\sqrt{12}}
\end{array}\right] \quad C=\left[\begin{array}{cccc}
\frac{1}{\sqrt{2}} & \frac{-1}{\sqrt{2}} & 0 & 0 \\
\frac{1}{\sqrt{6}} & \frac{1}{\sqrt{6}} & \frac{-2}{\sqrt{6}} & 0 \\
\frac{1}{\sqrt{12}} & \frac{1}{\sqrt{12}} & \frac{1}{\sqrt{12}} & \frac{-3}{\sqrt{12}}
\end{array}\right] \\
C A C^{\prime} & =\left[\begin{array}{cccc}
\frac{1}{\sqrt{2}} & \frac{-1}{\sqrt{2}} & 0 & 0 \\
\frac{1}{\sqrt{6}} & \frac{1}{\sqrt{6}} & \frac{-2}{\sqrt{6}} & 0 \\
\frac{1}{\sqrt{12}} & \frac{1}{\sqrt{12}} & \frac{1}{\sqrt{12}} & \frac{-3}{\sqrt{12}}
\end{array}\right] \times\left[\begin{array}{ccccc}
10 & 5 & 10 & 15 \\
5 & 20 & 15 & 20 \\
10 & 15 & 30 & 25 \\
15 & 20 & 25 & 40
\end{array}\right] \times\left[\begin{array}{ccc}
\frac{1}{\sqrt{2}} & \frac{1}{\sqrt{6}} & \frac{1}{\sqrt{12}} \\
\frac{-1}{\sqrt{2}} & \frac{1}{\sqrt{6}} & \frac{1}{\sqrt{12}} \\
0 & \frac{-2}{\sqrt{6}} & \frac{1}{\sqrt{12}} \\
0 & 0 & \frac{-3}{\sqrt{12}}
\end{array}\right] \\
& C A C^{\prime}=\left[\begin{array}{ccc}
10 & 0 & 0 \\
0 & 10 & 0 \\
0 & 0 & 10
\end{array}\right]=10 I_{3}
\end{aligned}
$$

Conclui-se que a matriz $A$, onde $\lambda=10$, atende a condição: $C A C^{\prime}=\lambda I_{(p-1)}$. Logo a matriz $A$ satisfaz a condição de esfericidade.

Verificação de: $\varepsilon_{g g}=\frac{\left[\operatorname{tr}\left(\lambda I_{(p-1)}\right)\right]^{2}}{(p-1) \operatorname{tr}\left[\lambda I_{(p-1)}\right]^{2}}=\frac{[\lambda(p-1)]^{2}}{(p-1) \lambda^{2}(p-1)}=1$

$$
\begin{gathered}
\varepsilon_{g g}=\frac{\left[\operatorname{tr}\left(\lambda I_{(p-1)}\right)\right]^{2}}{(p-1) \operatorname{tr}\left[\lambda I_{(p-1)}\right]^{2}}=\frac{(30)^{2}}{3 \times 300}=1 \\
\varepsilon_{g g}=\frac{[\lambda(p-1)]^{2}}{(p-1) \lambda^{2}(p-1)}=\frac{(10 \times 3)^{2}}{3 \times 10^{2} \times 3}=1 \\
\left(\operatorname{tr} \lambda I_{3}\right)^{2}=\lambda^{2} \operatorname{tr}\left[\begin{array}{lll}
1 & 0 & 0 \\
0 & 1 & 0 \\
0 & 0 & 1
\end{array}\right]=10^{2} \times \operatorname{tr}\left[\begin{array}{lll}
1 & 0 & 0 \\
0 & 1 & 0 \\
0 & 0 & 1
\end{array}\right]=300
\end{gathered}
$$

0 estimador de verossimilhança de $\varepsilon_{g g}$, citado em Leal (1979), também pode ser dado por:

$$
\hat{\varepsilon}_{g g}=\frac{p^{2}\left(\bar{s}_{d}-\bar{s}_{. .}\right)^{2}}{(p-1)\left[\sum_{k=1}^{p} \sum_{k^{\prime}=1}^{p}\left(s_{k k^{\prime}}\right)^{2}-2 p \sum_{k=1}^{p}\left(\bar{s}_{k .}\right)^{2}+p^{2}\left(\bar{s}_{. .}\right)^{2}\right]}
$$


Seja $S$ a matriz de covariância considerando as $p$ condições $(k=1,2, \cdots, p)$ :

$$
S=\left[\begin{array}{cccc}
s_{11} & s_{12} & \cdots & s_{1 p} \\
S_{21} & s_{22} & \cdots & s_{2 p} \\
\cdots & \cdots & \cdots & \cdots \\
S_{p 1} & s_{p 2} & \cdots & s_{p p}
\end{array}\right]
$$

$\bar{S}_{d}$ : média das variâncias da diagonal da matriz de covariância amostral S;

$\bar{S}_{\text {.. }}$ : média geral considerando todas as variâncias e covariâncias $(p \times p)$ elementos de $\mathrm{S}$;

$\sum_{k=1}^{p} \sum_{k^{\prime}}^{p}\left(s_{k k^{\prime}}\right)^{2}$ : soma de quadrados de todos os elementos (variâncias e covariâncias) da matriz $\mathrm{S}\left(k, k^{\prime}=1,2, \cdots, p\right)$;

$\sum_{k=1}^{p}\left(s_{k}\right)^{2}$ : soma de quadrados das médias dos elementos da $k$-ésima coluna da matriz de covariância amostral $(S)$. Box (1954) mostra que: $\frac{1}{(p-1)} \leq \varepsilon_{g g} \leq 1$.

Greenhouse \& Geisser (1959) sugerem utilizar o seguinte procedimento de três estágios descritos da seguinte forma:

a. Considerar $\varepsilon_{g g}=1$ e usar o teste exato $F$, ou seja, não usar as correções dos graus de liberdade. No caso de o teste não ser significativo, ou seja, não rejeitar a hipótese $H_{0}$, o procedimento é encerrado; caso contrário, isto é, se houver rejeição de $H_{0}$, aplicar o próximo estágio (b);

b. Como houve rejeição de $H_{0}$ no primeiro estágio, então usar a correção $\varepsilon_{g g}=1 /(p-1)^{-1}$, fazendo com que haja uma grande redução nos números de graus de liberdade. No caso de o teste ser significativo, isto é, se a hipótese for rejeitada, o procedimento é encerrado. Caso contrário, passar para o último estágio (c);

c. Usar o teste aproximado $F$ com $\hat{\varepsilon}_{g g}$ estimado a partir da matriz de covariância amostral.

Exercício 3.4.4.2. Seja a matriz comum de covariância $S$ originada de um inventário florestal contínuo com cinco ocasiões e trinta parcelas permanentes. Obter a correção de Greenhouse-Geisser, sendo $S$ a matriz de covariância comum dos quatro métodos de manejo florestal M1, M2, M3 e M4 referente ao experimento apresentado no capítulo 7, seção 7.7.1, exemplo de um inventario florestal contínuo para fins de manejo florestal. 


$S=\left[\begin{array}{rrrrr}2,1061385 & 2,2793064 & 2,2381011 & 2,1368150 & 1,9697176 \\ 2,2793064 & 4,7718804 & 5,5400208 & 5,8451530 & 5,5819684 \\ 2,2381011 & 5,5400208 & 7,2915454 & 7,9891048 & 7,9497102 \\ 2,1368150 & 5,8451530 & 7,9891048 & 9,3602169 & 9,5140274 \\ 1,9697176 & 5,5819684 & 7,9497102 & 9,5140274 & 10,1532850\end{array}\right]$

a) Usando a expressão:

$$
\begin{gathered}
\hat{\varepsilon}_{g g}=\frac{p^{2}\left(\bar{s}_{d}-\bar{s}_{. .}\right)^{2}}{(p-1)\left[\sum_{k=1}^{p} \sum_{k^{\prime}=1}^{p}\left(s_{k k^{\prime}}\right)^{2}-2 p \sum_{k=1}^{p}\left(\bar{s}_{k .}\right)^{2}+p^{2}\left(\bar{s}_{. .}\right)^{2}\right]} \\
\bar{S}_{d}=6,7366134 \bar{S}_{. .}=5,43083662 \sum_{k=1}^{p}\left(s_{k .}\right)^{2}=164,182995 \quad \sum_{k=1}^{p} \sum_{k^{\prime}}^{p}\left(s_{k k^{\prime}}\right)^{2}=935,489194 \\
\hat{\varepsilon}_{g g}=\frac{5^{2}(6,7366134-5,43083662)^{2}}{4\left(935,489194-2 \times 5 \times 164,182995+5^{2} \times 5,43083662^{2}\right)}=0,3437
\end{gathered}
$$

b) No caso da expressão:

$$
\begin{aligned}
& \hat{\varepsilon}_{g g}=\frac{\left[\operatorname{tr}\left(C S C^{\prime}\right)\right]^{2}}{(p-1) \operatorname{tr}\left[\left(C S C^{\prime}\right)^{2}\right]} \\
& C S C^{\prime}=\left[\begin{array}{l}
1,15970311,13683181,05273930,7546776 \\
1,13683181,58172321,53962401,3209349 \\
1,05273931,53962401,89165881,7056839 \\
0,75467761,32093491,70568391,8957967
\end{array}\right] \\
& \left(C S C^{\prime}\right)^{2}=\left[\begin{array}{l}
4,31509635,73424346,24982375,6032386 \\
5,73424347,90954608,79758728,0776307 \\
6,24982378,79758729,96643269,2884236 \\
5,60323868,07763079,28842368,8178098
\end{array}\right] \\
& \hat{\varepsilon}_{g g}=\frac{\left[\operatorname{tr}\left(C S C^{\prime}\right)\right]^{2}}{(p-1) \operatorname{tr}\left[\left(C S C^{\prime}\right)^{2}\right]}=\frac{(6,5288818)^{2}}{4 \times 31,008885}=0,3437
\end{aligned}
$$

c) Utilizando a expressão:

$$
\hat{\varepsilon}_{g g}=\frac{\left(\sum_{i=1}^{q} a_{i i}\right)^{2}}{(p-1) \sum_{i=1}^{q} \sum_{j=1}^{q} a_{i j}^{2}}=\frac{6,5288818^{2}}{4 \times 31,0088847}=0,3437
$$


Sendo $\left(C S C^{\prime}\right)=\left[a_{i j}\right]$. Note que: $1 \geq \varepsilon_{g g} \geq \frac{1}{p-1}=0,25$.

\subsubsection{CORREÇÃO HUYNH E FELDT $(H-F)$}

Collier et al. (1967) e Stoloff (1970) efetuando simulações verificaram que a correção $\varepsilon_{g g}$ é negativamente viesada para valores próximos de um e também negativamente viesada para valores baixos de $\varepsilon_{g g}$, principalmente quando o número de observações é pequeno. Huynh \& Feldt (1976) desenvolveram a correção $\varepsilon_{h f}$ que é menos viesada do que quando $\varepsilon_{g g} \geq 0,75$. Esta correção pode atingir valores maiores do que um, mas, neste caso, se considera $\varepsilon_{h f}=1$.

$$
\varepsilon_{h f}=\frac{N(p-1) \in_{g g}-2}{(p-1)\left[N-g-(p-1) \in_{g g}\right]}
$$

Exercício 3.4.5.1. Seja a matriz de covariância S, citada no exercício 3.4.4.2, originada de um inventário florestal contínuo com cinco ocasiões e trinta parcelas permanentes. Obter a correção de Huynh e Feldt $(h-f)$. Dado que $\hat{\varepsilon}_{g g}=0,3436619662$. Então:

$$
\hat{\varepsilon}_{h f}=\frac{120 \times(5-1) \times 0,3436619662-2}{(5-1)[120-4-(5-1) \times 0,3436619662}=\frac{162,95774378}{458,50140854}=0,3554
$$




\section{Capítulo 4}

\section{Análise de variância multivariada}

Este capítulo aborda definições, conceitos e aplicações da análise de experimentos multidimensionais que são importantes subsídios no estudo de análise perfil. Apresenta também os seguintes temas: análise multivariada para testar um vetor de médias de tratamento versus um vetor padrão populacional; análise para testar vetores de médias de dois tratamentos independentes; análise de variância multivariada do ensaio inteiramente ao acaso; princípio da união-intersecção de Roy; estatísticas para testar a hipótese linear geral $H: C \beta U=\phi$, lambda de Wilks, a maior raiz característica de Roy, traço de Pillai e o traço de Hotelling-Lawley; análise de variância univariada e teste de Tukey usando a função discriminante ou variável canônica. 


\section{INTRODUÇÃO}

A estatística multivariada é o ramo da estatística devotado ao estudo das amostras e distribuições multidimensionais, consistindo em planejar a coleta de dados das parcelas, analisar e interpretar as observações, de modo que em cada unidade amostral são feitas mensurações para duas ou mais variáveis respostas.

$$
\langle P A R C E L A\rangle \Rightarrow y_{v}^{\prime}=\left[\begin{array}{llll}
y_{1} & y_{2} & \cdots & y_{p}
\end{array}\right]
$$

$y_{v}^{\prime}$ : é o vetor resposta considerando $p$ variáveis.

KENDALL (1950) classifica as técnicas multivariadas em dois grupos:

a) Técnicas que estudam as interdependências de um conjunto de variáveis:

a1) Análise de agrupamento;

a2) Análise de componentes principais;

a3) Análise de fatores.

b) Técnicas que estudam as dependências de uma ou mais variáveis em relação às outras:

b1) Análise discriminante;

b2) Análise de variância multivariada;

b3) Análise de medidas repetidas;

b4) Análise de regressão multivariada;

b5) Análise de correlação canônica.

4.1. ANÁLISE MULTIVARIADA PARA TESTAR UM VETOR DE MÉDIAS DE TRATAMENTO VERSUS UM VETOR PADRÃO POPULACIONAL

Dado um vetor $y_{v}$ de variáveis com distribuição normal $p$-dimensional, apresentando vetor de médias $\mu_{v}$ e matriz de covariância não singular $\sum$.

Seja $H_{0}: \mu_{v}=\mu_{0 v}$ vs $H_{A}: \mu_{v} \neq \mu_{0 v}$ :

$$
H_{0}:\left[\begin{array}{c}
\mu_{1} \\
\vdots \\
\mu_{p}
\end{array}\right]=\left[\begin{array}{c}
\mu_{01} \\
\vdots \\
\mu_{0 p}
\end{array}\right] \text { versus } H_{A}:\left[\begin{array}{c}
\mu_{1} \\
\vdots \\
\mu_{p}
\end{array}\right] \neq\left[\begin{array}{c}
\mu_{01} \\
\vdots \\
\mu_{0 p}
\end{array}\right]
$$

Seja uma combinação linear arbitrária $\boldsymbol{a}_{v}^{\prime} \boldsymbol{y}_{v}$ das $p$ variáveis, sendo $\boldsymbol{a}_{v} \neq \mathbf{0}_{v}$, donde $\boldsymbol{a}_{v} \in$ $\boldsymbol{R}$. 
Então:

$$
\begin{gathered}
E\left(a_{v}^{\prime} y_{v}\right)=a_{v}^{\prime} \mu_{v} \\
V\left(a_{v}^{\prime} y_{v}\right)=a_{v}^{\prime} \sum a_{v} \\
a_{v}^{\prime} y_{v} \sim N\left(a_{v}^{\prime} \mu_{v}, a_{v}^{\prime} \sum a_{v}\right)
\end{gathered}
$$

Na construção do teste para verificar se o vetor de médias de interesse é diferente de um vetor padrão, pode-se usar o princípio de união-intersecção de Roy $(1953,1957)$.

$$
H_{0}\left(a_{v}\right): a_{v}^{\prime} \mu_{v}=a_{v}^{\prime} \mu_{v 0}
$$

$a_{v}^{\prime} \mu_{v 0}$ : é uma combinação linear das médias populacionais (padrão).

$$
t\left(a_{v}\right)=\frac{a_{v}^{\prime} \bar{y}_{v}-a_{v}^{\prime} \mu_{v 0}}{\sqrt{\frac{a_{v}^{\prime} S a_{v}}{n}}}=\frac{a_{v}^{\prime}\left(\bar{y}_{v}-\mu_{v 0}\right) \sqrt{n}}{\sqrt{a_{v}^{\prime} S a_{v}}}
$$

A significância ocorrerá quando: $t_{\left(a_{v}\right)}^{2}>t_{\left(\frac{\alpha}{2} ; n-1\right)}^{2}$, tal que $n$ é o número de repetições.

No princípio da união-intersecção de Roy, a hipótese $H_{0}: a_{v}^{\prime} \mu_{v}=a_{v}^{\prime} \mu_{v 0}$ será verdadeira se, somente se, $H_{0}: a_{v}^{\prime} \mu_{v}=a_{v}^{\prime} \mu_{v 0}$ for verdadeira para todo $a_{v} \neq 0$. A não rejeição de $H_{0}: a_{v}^{\prime} \mu_{v}=a_{v}^{\prime} \mu_{v 0}$ é equivalente a aceitar todas as possíveis hipóteses univariadas efetuadas, variando os elementos que constituem o vetor $a_{v}$, ou seja, consiste em procurar a combinação linear das variáveis que melhor discrimine os tratamentos. A combinação linear melhor indicada é aquela que produz o maior valor para a estatística univariada utilizada, por exemplo, $t$ de Student ou $F$ de Snedecor.

Morrison (1976) cita que pelo princípio de união-intersecção de Roy, a região multivariada de aceitação de $H_{0}$ é a interseção de todas as possíveis regiões de aceitação das hipóteses univariadas e simbolicamente representada por: $\cap_{a_{v}}\left[t_{\left(a_{v}\right)}^{2} \leq t_{\left(\frac{\alpha}{2} ; n-1\right)}^{2}\right]$, significando que todas as estatísticas $t_{\left(a_{v}\right)}^{2}$ pertencem à região de aceitação de $H_{0}$ para qualquer vetor $a_{v}$, o que equivale a dizer que o máximo valor de $t_{\left(a_{v}\right)}^{2}$ também pertence à região de aceitação de $H_{0}$, isto é, $T^{2}=\max \left[t_{\left(a_{v}\right)}^{2} \leq t_{\left(\frac{\alpha}{2} ; n-1\right)}^{2}\right]$. Esta estatística é chamada de $\mathrm{T}^{2}$ de Hotelling.

Dado que, na expressão 4.1.1, $t_{\left(a_{v}\right)}^{2}$ é invariante a mudança de escala do vetor $a_{v}$, então, utilizando o método dos multiplicadores de Lagrange com a restrição $a_{v}^{\prime} S a_{v}=1$, para obter o valor máximo, tem-se a equação 4.1.2. 


$$
\begin{aligned}
& t^{2}\left(a_{v}\right)=\frac{a_{v}^{\prime}\left(\bar{y}_{v}-\mu_{v 0}\right)\left(\bar{y}_{v}-\mu_{v 0}\right)^{\prime} a_{v} \times n}{a_{v}^{\prime} S a_{v}} \\
& Z=a_{v}^{\prime}\left(\bar{y}_{v}-\mu_{v 0}\right)\left(\bar{y}_{v}-\mu_{v 0}\right)^{\prime} a_{v} \times n-\lambda\left(a_{v}^{\prime} S a_{v}-1\right)
\end{aligned}
$$

Diferenciando 4.1.2 em relação ao vetor $a_{v}$ e anulando a equação resultante, tem-se:

$$
\left[\left(\bar{y}_{v}-\mu_{v 0}\right)\left(\bar{y}_{v}-\mu_{v 0}\right)^{\prime} n-\lambda S\right] a_{v}=0
$$

Pré-multiplicando por $a_{v}^{\prime}$, então:

$$
\lambda=\frac{a_{v}^{\prime}\left(\bar{y}_{v}-\mu_{v 0}\right)\left(\bar{y}_{v}-\mu_{v 0}\right)^{\prime} a_{v} n}{a_{v}^{\prime} S a_{v}}=\frac{\left[a_{v}^{\prime}\left(\bar{y}_{v}-\mu_{v 0}\right)\right]^{2} n}{a_{v}^{\prime} S a_{v}}=t^{2}\left(a_{v}\right)
$$

MORRISON (1976) mostra que $\lambda$ é também a única raiz não nula da equação determinantal.

$$
\left|S^{-1}\left(\bar{y}_{v}-\mu_{v 0}\right)\left(\bar{y}_{v}-\mu_{v 0}\right)^{\prime} n-\lambda I\right|=0
$$

Então, desde que $\lambda$ é a única raiz não nula, o máximo para a estatística $t_{\left(a_{v}\right)}^{2}$, é:

$$
\lambda=\operatorname{tr}\left[S^{-1}\left(\bar{y}_{v}-\mu_{v 0}\right)\left(\bar{y}_{v}-\mu_{v 0}\right)^{\prime} n\right]
$$

Com efeito, têm-se:

$$
\begin{gathered}
\lambda=n\left(\bar{y}_{v}-\mu_{v 0}\right)^{\prime} S^{-1}\left(\bar{y}_{v}-\mu_{v 0}\right) \\
T^{2}=n\left(\bar{y}_{v}-\mu_{v 0}\right)^{\prime} S^{-1}\left(\bar{y}_{v}-\mu_{v 0}\right)
\end{gathered}
$$

A forma quadrática 4.1.3 é denominada de estatística de Hotelling. Da estatística multivariada quando a hipótese $H_{0}$ é verdadeira, tem-se:

$$
F=\frac{n-p}{p(n-1)} T^{2}, \text { tal que } F_{(\alpha ; p, n-p)}
$$

A natureza da estatística $T^{2}$ de Hotelling permite verificar quais variáveis possibilitam a rejeição de $H_{0}$.

$$
\left[a_{v}^{\prime} \bar{y}_{v}-\sqrt{\left.\frac{1}{n} a_{v}^{\prime} S a_{v} \cdot T_{(\alpha ; p, n-p}^{2}\right)} \leq a_{v}^{\prime} \mu_{v} \leq a_{v}^{\prime} \bar{y}_{v}+\sqrt{\frac{1}{n} a_{v}^{\prime} S a_{v} \cdot T_{(\alpha ; p, n-1)}^{2}}\right]
$$

$a_{v}^{\prime}=[0,0, \ldots, 1, \ldots, 0]$, tal que, a localização do valor 1 (um) no vetor $a_{v}$ caracteriza a $i$ ésima variável resposta. 
Se o intervalo incluir o valor correspondente à combinação linear das médias em estudo, há razão para concluir que a mesma não contribui para a rejeição de $H_{0}$.

Morrison (1976) demonstra que a estatística $T^{2}$ de Hotelling não é afetada apenas por mudanças de unidade da variável resposta, mas é invariante sob todas as transformações da forma $y=C x+d_{v}$ das observações e de hipóteses, donde $C$ é qualquer matriz não singular $p \times p$ e $d_{v}$ é um vetor de ordem $p \times 1$.

4.1.1. Exercício. Uma determinada espécie florestal (E1) possui em seu país de origem, com respeito às variáveis $\mathrm{y} 1=$ volume de madeira em $\mathrm{m}^{3} / 0,10$ ha e $\mathrm{y} 2=$ produção de sementes em kg, o comportamento aos sete anos de idade de $\mu_{0}^{\prime}=\left[\bar{y}_{1}, \bar{y}_{2}\right]=[4,50,9]$. 0 objetivo é verificar o seu desempenho em outro país (teste de procedência). Foram instaladas dez parcelas de 0,10 ha que após sete anos apresentaram os resultados (tabela 4.1.1). Testar o vetor de médias do tratamento contra o vetor padrão $\mu_{0}^{\prime}=[4,50,9]$. Mostrar que $\lambda$ é a única raiz não nula da equação determinantal: $\left|S^{-1}\left(\bar{y}_{v}-\mu_{v 0}\right)\left(\bar{y}_{v}-\mu_{v 0}\right)^{\prime} n-\lambda I\right|=0$.

Tabela 4.1.1 - Volume de madeira e produção de sementes de E1.

\begin{tabular}{c|c|c|c|c|c} 
Parc. & \multicolumn{2}{c}{ E1 } & Parc. & \multicolumn{2}{c}{ E1 } \\
& $\mathrm{y} 1$ & $\mathrm{y} 2$ & & $\mathrm{y} 1$ & $\mathrm{y} 2$ \\
\hline 1 & 4,59461 & 1,01091 & 6 & 4,49093 & 1,03807 \\
2 & 4,83847 & 1,09984 & 7 & 4,34527 & 1,01440 \\
3 & 4,63485 & 1,36393 & 8 & 4,76123 & 1,24293 \\
4 & 4,13427 & 0,75682 & 9 & 4,85951 & 1,28071 \\
5 & 4,41401 & 0,99222 & 10 & 4,54754 & 0,96189 \\
\hline
\end{tabular}

a) Testar $H_{0}: \mu_{v}=\mu_{v 0}$ versus $H_{A}: \mu_{v} \neq \mu_{v 0}$ :

Dado que $T^{2}=n\left(\bar{y}_{v}-\mu_{v 0}\right)^{\prime} S^{-1}\left(\bar{y}_{v}-\mu_{v 0}\right)$ e $F=\frac{n-p}{(n-1) p} T^{2}$ que sob $H o$ possui $(p, n-p)$ graus de liberdade, tem-se:

$$
\begin{gathered}
\bar{y}_{v}=\left[\begin{array}{l}
4,5621 \\
1,0762
\end{array}\right] \quad S=\left[\begin{array}{ll}
0,0520108 & 0,0318025 \\
0,0318025 & 0,0316255
\end{array}\right] \quad \bar{y}_{v}-\mu_{v 0}=\left[\begin{array}{l}
0,0621 \\
0,1762
\end{array}\right] \\
\operatorname{det} S=0,0006334685 \\
S^{-1}=\frac{1}{0,0006334685}\left[\begin{array}{cc}
0,0316255055 & -0,0318025028 \\
-0,0318025028 & 0,0520107626
\end{array}\right]=\left[\begin{array}{cc}
49,924341 & -50,20376 \\
-50,20376 & 82,04787
\end{array}\right] \\
T^{2}=n\left(\bar{y}_{v}-\mu_{v 0}\right)^{\prime} S^{-1}\left(\bar{y}_{v}-\mu_{v 0}\right)
\end{gathered}
$$




$$
T^{2}=10[0,0621 \quad 0,1762]\left[\begin{array}{ll}
49,924341 & -50,20376 \\
-50,20376 & 82,104787
\end{array}\right]\left[\begin{array}{c}
0,0621 \\
0,1762
\end{array}\right]=16,429291
$$

$F=\frac{n-p}{p(n-1)} T^{2}$ que sob $\mathrm{H}_{0}$ possui $p$ e $n-p$ graus de liberdade.

$$
F=\frac{10-2}{2(10-1)} \times 16,429291=7,3019
$$

Conclui-se pela rejeição de $H_{0}$, pois $F_{2 ; 8 ; 0,05}=4,46$.

b) Verificar se $\lambda$ é a única raiz não nula da equação determinantal:

$$
\left|S^{-1}\left(\bar{y}_{v}-\mu_{v 0}\right)\left(\bar{y}_{v}-\mu_{v 0}\right)^{\prime} n-\lambda I\right|=0
$$

Seja $A=S^{-1}\left(\bar{y}_{v}-\mu_{v 0}\right)\left(\bar{y}_{v}-\mu_{v 0}\right)^{\prime} n$, então:

$$
A=\left[\begin{array}{ll}
49,924341 & -50,20376 \\
-50,20376 & 82,104787
\end{array}\right]\left[\begin{array}{l}
0,0621 \\
0,1762
\end{array}\right] \times\left[\begin{array}{ll}
0,178 & 0,104
\end{array}\right] \times 10=\left[\begin{array}{cc}
-3,568018 & -10,12375 \\
7,0478595 & 19,997308
\end{array}\right]
$$

Tem-se que $\operatorname{det}(A)=0$ e $\operatorname{tr}(A)=-3,568018+19,997308=16,42929$. Então: $T^{2}=16,42929$.

Seja $|A-\lambda I|=0$, logo:

$$
\begin{aligned}
& \operatorname{det}\left\{\left[\begin{array}{cc}
-3,568018 & -10,12375 \\
7,0478595 & 19,997308
\end{array}\right]-\lambda\left[\begin{array}{ll}
1 & 0 \\
0 & 1
\end{array}\right]\right\}=\operatorname{det}\left[\begin{array}{cc}
-3,568018-\lambda & -10,12375 \\
7,0478595 & 19,997308-\lambda
\end{array}\right]=0 \\
& -71,35076+3,568018 \lambda-19,997308 \lambda+\lambda^{2}+71,35076=0 \\
& \lambda^{2}-16,42929 \lambda=0 \rightarrow \lambda-16,42929=0 \quad \rightarrow \quad T^{2}=\lambda=16,42929
\end{aligned}
$$

Conclui-se que a estatística $T^{2}$ de Hotelling é igual ao único autovalor da matriz $A$ e, obviamente, é igual ao seu traço.

\subsection{ANÁliSE MULTIVARIADA PARA TESTAR VETORES DE MÉDIAS PARA DOIS TRATAMENTOS INDEPENDENTES}

Sejam duas amostras aleatórias independentes, observações de $p$ variáveis mensuradas em uma mesma unidade amostral, originárias de duas populações multinormais e com matrizes de covariâncias homogêneas.

Muitas vezes durante as pesquisas desenvolvidas na engenharia florestal, o pesquisador pode ter interesse em comparar se dois povoamentos possuem características semelhantes, com respeito a um conjunto de $p$ variáveis, como por exemplo, as variáveis florísticas, dendrométricas, produção de sementes, de resinas e etc, 
Seja a hipótese: $H_{0}: \mu_{v 1}=\mu_{v 2}$ versus $H_{A}: \mu_{v 1} \neq \mu_{v 2}$. Para testar a igualdade de dois vetores de médias provenientes de duas populações independentes, aplica-se a estatística:

$$
T^{2}=\frac{n_{1} \times n_{2}}{n_{1}+n_{2}}\left(\bar{y}_{v 1}-\bar{y}_{v 2}\right)^{\prime} S^{-1}\left(\bar{y}_{v 1}-\bar{y}_{v 2}\right)
$$

A estatística $T^{2}$ pode ser escrita em função da estatística $F$, que sob $H_{0}: \mu_{v 1}=\mu_{v 2}$ possui distribuição $F \operatorname{com} p$ e $\left(n_{1}+n_{2}-p-1\right)$ graus de liberdade, através da expressão:

$$
F=\frac{n_{1}+n_{2}-p-1}{\left(n_{1}+n_{2}-2\right) p} T^{2}
$$

A verificação da contribuição de cada variável na significância dos tratamentos, no caso da rejeição de $H_{0}: \mu_{v 1}=\mu_{v 2}$, pode de obtida pelo intervalo de confiança, através da seguinte expressão.

$$
\begin{aligned}
& {\left[a_{v}^{\prime}\left(\bar{y}_{v 1}-\bar{y}_{v 2}\right)-\sqrt{\left.a_{v}^{\prime} S a_{v} \frac{n_{1}+n_{2}}{n_{1}+n_{2}} T_{\left(\alpha ; p, n_{1}+n_{2}-p-1\right.}^{2}\right)} \leq a_{v}^{\prime}\left(\mu_{v 1}-\mu_{v 2}\right) \leq\right.} \\
& a_{v}^{\prime}\left(\bar{y}_{v 1}-\bar{y}_{v 2}\right)+\sqrt{\left.a_{v}^{\prime} S a_{v} \frac{n_{1}-n_{2}}{n_{1} \cdot n_{2}} T_{\left(\alpha: p, n_{1}+n_{2}-p-1\right)}^{2}\right]}
\end{aligned}
$$

$a_{v}^{\prime}=[0,0, \ldots, 1, \ldots, 0]$, tal que, a localização do valor 1 (um) no vetor $a_{v}$ caracteriza a $i$ ésima variável resposta.

Se o intervalo incluir o valor correspondente à combinação linear das médias em estudo, há razão para concluir que a mesma não contribui para a rejeição de $H o$.

Exercício 4.2.1. Comparar as espécies florestais E1 e E2, com 10 anos de idade, com respeito às variáveis $\mathrm{y} 1=$ produção de sementes em $\mathrm{kg} / \mathrm{ha}$ e y2= produção de resinas em

\begin{tabular}{|c|c|c|c|c|c|c|c|c|c|}
\hline \multirow[t]{2}{*}{ Parc. } & \multicolumn{2}{|c|}{ E1 } & \multicolumn{2}{|c|}{ E2 } & \multirow[t]{2}{*}{ Parc. } & \multicolumn{2}{|c|}{ E1 } & \multicolumn{2}{|c|}{ E2 } \\
\hline & y1 & y2 & y1 & y2 & & y1 & y2 & y1 & y2 \\
\hline 1 & 4,5946 & 1,0109 & 4,6276 & 1,1316 & 6 & 4,4909 & 1,0381 & 4,5962 & 0,8952 \\
\hline 2 & 4,8385 & 1,0998 & 4,5779 & 0,8095 & 7 & 4,3453 & 1,0144 & 4,7396 & 1,1269 \\
\hline 3 & 4,6348 & 1,3639 & 4,6314 & 0,9600 & 8 & 4,7612 & 1,2429 & 4,5621 & 0,9993 \\
\hline 4 & 4,1343 & 0,7568 & 4,3747 & 0,8520 & 9 & 4,8595 & 1,2807 & 4,7066 & 0,9983 \\
\hline 5 & 4,4140 & 0,9922 & 4,5553 & 0,8244 & 10 & 4,5475 & 0,9619 & 4,9366 & 0,8054 \\
\hline
\end{tabular}
$\mathrm{kg} /$ ha. 0 ensaio consistiu de 10 parcelas que apresentaram os resultados mostrados na tabela 4.2.1.

Tabela 4.2.1 - Produção de sementes e de resina de E1 e E2. 


$$
\begin{aligned}
& H_{0}: \mu_{v 1}=\mu_{v 2} \text { versus } H_{A}: \mu_{v 1} \neq \mu_{v 2} \\
& \bar{y}_{v 1}=\left[\begin{array}{l}
4,5621 \\
1,0762
\end{array}\right] \quad \bar{y}_{v 1}=\left[\begin{array}{c}
4,6308 \\
0,9403
\end{array}\right] \quad\left(\bar{y}_{v 1}-\bar{y}_{v 2}\right)=\left[\begin{array}{c}
-0,0687 \\
0,1359
\end{array}\right] \\
& S_{1}=\left[\begin{array}{ll}
0,0520108 & 0,0318025 \\
0,0318025 & 0,0316255
\end{array}\right] \quad S_{2}=\left[\begin{array}{ll}
0,0212346 & 0,0020701 \\
0,0020701 & 0,0152506
\end{array}\right] \\
& S=\frac{S_{1}+S_{2}}{2}=\left[\begin{array}{ll}
0,0366227 & 0,0169363 \\
0,0169363 & 0,0234381
\end{array}\right] \quad S^{-1}=\left[\begin{array}{rr}
41,009566 & -29,63345 \\
-29,63345 & 64,078753
\end{array}\right] \\
& T^{2}=\frac{n_{1} \times n_{2}}{n_{1}+n_{2}}\left(\bar{y}_{v 1}-\bar{y}_{v 2}\right)^{\prime} S^{-1}\left(\bar{y}_{v 1}-\bar{y}_{v 2}\right) \\
& T^{2}=\left(\frac{10 \times 10}{10+10}\right)\left[\begin{array}{ll}
-0,0687 & 0,1359
\end{array}\right] \times\left[\begin{array}{cc}
41,009566 & -29,63345 \\
-29,63345 & 64,078753
\end{array}\right] \times\left[\begin{array}{c}
-0,0687 \\
0,1359
\end{array}\right] \\
& T^{2}=9,6517305
\end{aligned}
$$

$F=\frac{n_{1}+n_{2}-p-1}{\left(n_{1}+n_{2}-2\right) p} T^{2}$ que sob $H_{0}$ possui p, $n_{1}+n_{2}-2$ graus de liberdade:

$$
F=\frac{(10+10-2-1)}{(10+10-2) 2} \times 9,6517305=4,56 * \quad\left(F_{2,18 ; 0,05}=3,55\right)
$$

Conclui-se que as espécies E1 e E2 apresentam vetores de médias diferentes.

\subsection{MODELO LINEAR UNIVARIADO PARA MAIS DE DOIS TRATAMENTOS}

Sejam $y_{i j}$ observações independentes de um ensaio inteiramente ao caso com $g$ tratamentos, apresentando efeitos fixos, com distribuição normal com médias $\mu_{1}, \mu_{2}, \cdots, \mu_{g}$ e variância comum desconhecida.

Seja o modelo linear: $y_{i j}=\mu+\tau_{i}+e_{i j}$.

$\mu$ : média geral comum a todos os tratamentos;

$\tau_{i}$ : efeito devido ao $i=$ ésimo tratamento $(i=1, \cdots, g)$;

$e_{i j}$ : erro residual com distribuição normal com média zero e variância $\sigma^{2}$. 
Seja a hipótese de efeitos iguais de tratamentos para o modelo $y_{i j}=\mu+\tau_{i}+e_{i j}$ :

$$
H_{0}: \tau_{1}=\tau_{2}=\cdots=\tau_{g}
$$

Reparametrizado o modelo para $y_{i j}=\mu_{i}+e_{i j}$, tal que $\mu_{i}=\mu+\tau_{i}$, a hipótese passa a ser escrita como: $H_{0}: \mu_{1}=\mu_{2}=\cdots=\mu_{g}$. Matricialmente, o modelo reparametrizado, apresenta a seguinte forma: $Y_{v}=X \beta_{v}+\varepsilon_{v}$.

$$
{ }_{N} X_{g}=\left[\begin{array}{cccc}
1 & 0 & \cdots & 0 \\
\cdots & \cdots & \cdots & \cdots \\
1 & 0 & \cdots & 0 \\
\cdots & \cdots & \cdots & \cdots \\
0 & 0 & \cdots & 1 \\
\cdots & \cdots & \cdots & \cdots \\
0 & 0 & \cdots & 1
\end{array}\right] \quad \beta_{v}^{\prime}=\left[\mu_{1}, \mu_{2}, \cdots \mu_{g}\right] \quad \varepsilon_{v}^{\prime}=\left[\varepsilon_{1}, \varepsilon_{2}, \cdots \varepsilon_{N}\right]
$$

Considerando $n_{i}$ repetições por tratamentos e $N=n_{1}+n_{2}+\cdots+n_{g}$, tem-se:

$$
{ }_{N} Y_{1}={ }_{N} X_{g} \times{ }_{g} \beta_{1}+{ }_{N} \varepsilon_{1}
$$

${ }_{N} Y_{1}$ : vetor dos dados;

${ }_{N} X_{g}$ : denominada de matriz de planejamento.

A combinação linear dos parâmetros $a_{v}^{\prime} \beta_{v}$, considerando o modelo reparametrizado, é estimável se existir uma função linear $a_{v}^{\prime} \hat{\beta}_{v}$ das observações, tal que: $E\left(a_{v}^{\prime} \hat{\beta}_{v}\right)=a_{v}^{\prime} \beta_{v}$. Seja $\hat{\beta}_{v}=\left(X^{\prime} X\right)^{-1} X^{\prime} Y_{v}$ o estimador de mínimos quadrados ou de máxima verossimilhança de $\beta_{v}$. A estimativa do componente $a_{v}^{\prime} \beta_{v}$ de variância mínima pelos métodos de quadrados mínimos e de máxima verossimilhança é:

$$
a_{v}^{\prime} \hat{\beta}_{v}=a_{v}^{\prime}\left(X^{\prime} X\right)^{-1} X^{\prime} Y_{v} \quad \hat{\beta}_{v}^{\prime}=\left[\bar{y}_{1}, \bar{y}_{2}, \cdots, \bar{y}_{g}\right]
$$

Para o modelo reparametrizado, tem-se:

$$
\left(X^{\prime} X\right)^{-1}=\left[\begin{array}{cccc}
\frac{1}{n_{1}} & 0 & \cdots & 0 \\
0 & \frac{1}{n_{2}} & \cdots & 0 \\
\cdots & \cdots & \cdots & \cdots \\
0 & 0 & \cdots & \frac{1}{n_{g}}
\end{array}\right]
$$

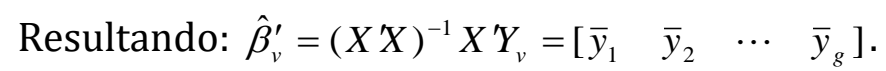


Portanto, considerando $a_{v}^{\prime}$ como um vetor unitário, sendo $\hat{\beta}_{v}$ o vetor das médias amostrais dos $g$ tratamentos, então, se $a_{v}^{\prime} \beta_{v}$ é uma função qualquer estimável, logo sua estimativa não tendenciosa de variância mínima será a combinação linear $\left(a_{v}^{\prime} \hat{\beta}_{v}\right)$ das médias amostrais dos $(g)$ tratamentos. Se o vetor $a_{v}^{\prime}$ tiver a configuração $a_{v}^{\prime}=[0, \cdots, 1, \cdots 0]$, ou seja, com o número 1 em uma posição caracterizando o $i$-ésimo tratamento, então a média $\mu_{i}$ populacional é estimável, tendo $\bar{y}_{i}$ como sua estimativa de variância mínima.

Destarte, então, qualquer contraste $\theta=c_{1} \mu_{1}+c_{2} \mu_{2}+\cdots+c_{g} \mu_{g}$ é estimável e sua estimativa de variância mínima é $\hat{\theta}=c_{1} \bar{y}_{1}+c_{2} \bar{y}_{2}+\cdots+c_{g} \bar{y}_{g}$. 0 contraste $\theta=c_{1} \mu_{1}+c_{2} \mu_{2}+\cdots+c_{g} \mu_{g}=0$, sob $H_{0}$, pode ser escrito matricialmente como: $H_{0}: C \beta_{v}=0$, donde $C(r \times g)$ de posto $r$ é a matriz responsável pelo estabelecimento dos contrastes. A hipótese alternativa é dada por $H_{A}: C \beta_{v} \neq 0$. A estatística para testar $H_{0}: C \beta_{v}=0$, será:

$$
F=\frac{(N-g)\left[Y_{v}^{\prime} X\left(X^{\prime} X\right)^{-1} C^{\prime}\right]\left[C\left(X^{\prime} X\right)^{-1} C^{\prime}\right]^{-1}\left[C\left(X^{\prime} X\right)^{-1} X^{\prime} Y_{v}\right]}{r Y_{v}^{\prime}\left[I-X\left(X^{\prime} X\right)^{-1} X^{\prime}\right] Y_{v}}
$$

$\mathrm{O}$ valor calculado $\mathrm{F}$, se a hipótese $\mathrm{H}_{0}$ é verdadeira, terá distribuição $\mathrm{F}$ com r e N-g graus de liberdade. Então, não se rejeita $\mathrm{H}_{0}$ se $F_{(a)} \leq F_{(\alpha ; r, N-g)}$.

A hipótese $H_{0}$ pode se escrita nas formas: $H_{0}: \mu_{1}=\mu_{2}=\cdots=\mu_{g}$, ou, $H_{0}: C \beta_{v}=0_{v}$. Os valores de $F_{(a)} \leq F_{(\alpha ; r, N-g)}$ serão os mesmos considerando as seguintes matrizes $C$.

a) Usando contrastes entre tratamentos consecutivos:

$$
C=\left[\begin{array}{cccccc}
1 & -1 & 0 & \cdots & 0 & 0 \\
0 & 1 & -1 & \cdots & 0 & 0 \\
\cdots & \vdots & \cdots & \cdots & \cdots & \vdots \\
0 & 0 & 0 & \cdots & 1 & -1
\end{array}\right]
$$

b) Considerando os contrastes sempre em relação ao primeiro tratamento:

$$
C=\left[\begin{array}{cccccc}
1 & -1 & 0 & \cdots & 0 & 0 \\
1 & 0 & -1 & \cdots & 0 & 0 \\
\cdots & \vdots & \cdots & \cdots & \cdots & \vdots \\
1 & 0 & 0 & \cdots & 0 & -1
\end{array}\right]
$$

c) Utilizando os contrastes sempre em relação ao último tratamento:

$$
C=\left[\begin{array}{cccccc}
1 & 0 & 0 & \cdots & 0 & -1 \\
0 & 1 & 0 & \cdots & 0 & -1 \\
\cdots & \vdots & \cdots & \cdots & \cdots & \vdots \\
0 & 0 & 0 & \cdots & 1 & -1
\end{array}\right]
$$


Para a estatística $F$, considerando as três matrizes citadas, será usado na expressão 4.3.1 o valor $r=g-1$, haja vista que os resultados serão os mesmos qualquer que seja a maneira de construir os contrastes.

\subsection{ANÁliSE DE VARIÂNCIA MULTIVARIADA DO ENSAIO INTEIRAMENTE AO ACASO}

A análise de variância multivariada (MANAVA) é uma generalização da análise de variância univariada (ANAVA), então, para facilitar o entendimento, é importante estudar o caso do ensaio inteiramente ao acaso. 0 objetivo da análise de variância multivariada é efetuar a comparação de tratamentos experimentais, onde cada tratamento é composto por $n_{i}$ parcelas ou repetições que são medidas considerando $p$ variáveis ou respostas.

Os tratamentos devem ser estabelecidos de forma independentes e apresentar uma matriz comum de covariância $\sum$ de ordem $p \times p$, assim como atender a pressuposição básica de que os vetores das observações devem possuir uma distribuição multinormal $p$-dimensional.

Seja o modelo matemático tal que $y_{i j k}$ representa cada observação:

$$
y_{i j k}=\mu_{j}+t_{i j}+e_{i j k}
$$

$i=1,2, \cdots, g$ : indicando o $i$-ésimo tratamento ( $g$ : número de tratamentos);

$j=1,2, \cdots, n_{i}$ : caracterizando a $j$-ésima repetição dentro do $i$-ésimo tratamento $\left(n_{i}\right.$ : número de repetições em $g_{i}$;

$k=1,2, \cdots, p$ : representando a $k$-ésima variável resposta ( $p$ : número de variáveis);

$N=n_{1}+n_{2}+\cdots+n_{g}$.

Seja o modelo na forma matricial para o conjunto de todas as observações:

$$
{ }_{N} Y_{p}={ }_{N} X_{g} \beta_{g} \beta_{p}+{ }_{N} \varepsilon_{p}
$$

$Y(N \times p)$ : matriz das observações dos $g$ tratamentos, onde cada linha representa uma parcela ou individuo e as colunas as $p$ variáveis respostas;

$X(N \times g)$ matriz de planejamento formada por números 1 e 0 que associam cada parcela com seu respectivo tratamento;

$\beta(g \times p)$ : matriz dos parâmetros desconhecidos, onde cada linha representa o $i$-ésimo tratamento e cada coluna a $k$-ésima variável resposta;

$\varepsilon(N \times p)$ : é a matriz dos efeitos residuais, onde os elementos representam o desvio entre os valores esperados de acordo com o modelo e os valores observados no experimento. 


$$
X=\left[\begin{array}{cccc}
1 & 0 & \cdots & 0 \\
\cdots & \cdots & \cdots & \cdots \\
1 & 0 & \cdots & 0 \\
0 & 1 & \cdots & 0 \\
\cdots & \cdots & \cdots & \cdots \\
0 & 1 & \cdots & 0 \\
\cdots & \cdots & \cdots & \cdots \\
0 & 0 & \cdots & 1 \\
\cdots & \cdots & \cdots & \cdots \\
0 & 0 & \cdots & 1
\end{array}\right] \quad \beta=\left[\begin{array}{cccc}
\mu_{11} & \mu_{12} & \cdots & \mu_{1 P} \\
\mu_{21} & \mu_{22} & \cdots & \mu_{2 P} \\
\cdots & \cdots & \cdots & \cdots \\
\mu_{g 1} & \mu_{g 2} & \cdots & \mu_{g p}
\end{array}\right]
$$

As hipóteses de nulidade serão construídas na forma matricial $H_{0}: C \beta U=\phi$.

$$
C=\left[\begin{array}{cccccc}
1 & -1 & 0 & \cdots & 0 & 0 \\
0 & 1 & -1 & \cdots & 0 & 0 \\
\cdots & \cdots & \cdots & \cdots & \cdots & \cdots \\
0 & 0 & 0 & \cdots & 1 & -1
\end{array}\right]
$$

$C(c \times g)$ : matriz de posto $c$ responsável para efetuar os contrastes entre os tratamentos; $U(p \times u)$ : matriz de posto $u$ encarregada de estabelecer os contrastes entre as variáveis respostas.

No caso da hipótese $H_{0}: \mu_{v 1}=\mu_{v 2}=\cdots=\mu_{v g}$, a matriz $U$ será uma identidade $U=I_{p}$.

$$
H_{0}:\left[\begin{array}{l}
\mu_{11} \\
\mu_{12} \\
\cdots \\
\mu_{1 p}
\end{array}\right]=\left[\begin{array}{c}
\mu_{21} \\
\mu_{22} \\
\cdots \\
\mu_{2 p}
\end{array}\right]=\cdots=\left[\begin{array}{c}
\mu_{g 1} \\
\mu_{g 2} \\
\cdots \\
\mu_{g p}
\end{array}\right]
$$

Para testar $H_{0}: C \beta U=\phi$ calcula-se a matriz $H$ que corresponde às somas de quadrados $\mathrm{e}$ de produtos cruzados de tratamentos e a matriz $E$ que corresponde às somas de quadrados e de produtos cruzados devido ao resíduo.

Pelo princípio da união-intersecção de Roy $(1953,1957)$, a hipótese $H_{0}: C \beta U=\phi$, será verdadeira, se e somente se, todas as hipóteses univariadas da forma $H_{0}: C \beta U a_{v}=0$ também o forem. 0 vetor $a_{v}=[0, \cdots, 1, \cdots, 0]$ composto por um número 1 na posição $k$ $(k=1, \ldots, p)$ será o responsável para escolher a variável resposta.

A estatística $F_{(a)}$ para testar essas hipóteses univariadas, $H_{0}: C \beta U a_{v}=0$, é:

$$
F_{(a)}=\frac{\left.(N-g) a_{v}^{\prime} U^{\prime} Y^{\prime} X\left(X^{\prime} X\right)^{-1} C^{\prime}\left[C^{\prime} X^{\prime} X\right)^{-1} C\right]^{-1} C\left(X^{\prime} X\right)^{-1} X^{\prime} Y U a_{v}}{(g-1) a_{v}^{\prime} U^{\prime} Y^{\prime}\left[I-X\left(X^{\prime} X\right)^{-1} X^{\prime}\right] Y U a_{v}}
$$


A hipótese univariada, $H_{0}: C \beta U a_{v}=0$, não será rejeitada se $F_{(a)} \leq F_{(\alpha ; g-1, N-g)}$.

Então a análise de variância multivariada para um fator consiste em procurar uma combinação linear das variáveis que melhor discrimine os $g$ grupos (tratamentos). A combinação linear melhor indicada é aquela que produz o maior valor para a estatística univariada $F$. De acordo com o princípio da união-intersecção de Roy, tem-se:

$$
F_{\left(a_{v}\right)}=\frac{a_{v}^{\prime} H a_{v} /(g-1)}{a_{v}^{\prime} E a_{v} /(N-g)}=\frac{a_{v}^{\prime} H a_{v}}{a_{v}^{\prime} E a_{v}}\left(\frac{N-g}{g-1}\right)
$$

Tal que: $H(p \times p)$ e $E(p \times p)$.

Dada restrição, $a_{v}^{\prime} E a_{v}=1$, para que $a_{v}$ seja único. Pelo método dos multiplicadores de Lagrange:

$$
\begin{aligned}
& Z=a_{v}^{\prime} H a_{v}-\lambda\left(a_{v}^{\prime} E a_{v}-1\right) \\
& d Z=\left(2 H a_{v}-2 \lambda E a_{v}\right) d a_{v}
\end{aligned}
$$

Dado que $d Z \equiv 0$, então: $2\left(H a_{v}-\lambda E a_{v}\right)=0_{v}$, tal que: $(H-\lambda E) a_{v}=0_{v}$.

Pré-multiplicando $(H-\lambda E) a_{v}=0_{v}$ por $E^{-1}$, tem-se $\left[E^{-1} H-\lambda I\right] a_{v}=0_{v}$. Pós-multiplicando $(H-\lambda E) a_{v}=0_{v}$ por $E^{-1}$, resulta $\left[H E^{-1}-\lambda I\right] a_{v}=0_{v}$. A solução não trivial é obtida por $\left|E^{-1} H-\lambda I\right|=0$ ou $\left|H E^{-1}-\lambda I\right|=0$.

Pré-multiplicando $H a_{v}-\lambda E a_{v}=0_{v}$ por $a_{v}^{\prime}$ :

$$
\begin{gathered}
a_{v}^{\prime} H a_{v}-\lambda a_{v}^{\prime} E a_{v}=0 \rightarrow \lambda=\frac{a_{v}^{\prime} H a_{v}}{a_{v}^{\prime} E a_{v}} \\
F_{\left(a_{v}\right)}=\frac{a_{v}^{\prime} H a_{v}}{a_{v}^{\prime} E a_{v}}\left(\frac{N-g}{g-1}\right) \rightarrow \lambda=\frac{a_{v}^{\prime} H a_{v}}{a_{v}^{\prime} E a_{v}}=\frac{g-1}{N-g} F\left(a_{v}\right) \rightarrow F_{\left(a_{v}\right)}=\lambda\left(\frac{N-g}{g-1}\right)
\end{gathered}
$$

Conclui-se que a maior raiz característica da matriz $E^{-1} H$ ou de $H E^{-1}$ corresponde à solução de máximo. Qualquer que seja o vetor não nulo, o máximo valor de $F_{\left(a_{v}\right)}$ também será proporcional à maior raiz característica de $|H-\lambda E|=\left|H E^{-1}-\lambda I\right|=0$.

$$
H_{p \times p}=U^{\prime} Y^{\prime} X\left(X^{\prime} X\right)^{-1} C^{\prime}\left[C\left(X^{\prime} X\right)^{-1} C^{\prime}\right]^{-1} C\left(X^{\prime} X\right)^{-1} X^{\prime} Y U \quad E_{p \times p}=U^{\prime} Y^{\prime}\left[I-X\left(X^{\prime} X\right)^{-1} X^{\prime}\right] Y U
$$

$H(p \times p)$ : matriz de soma de quadrados e de produtos cruzados de tratamentos devido à hipótese $H_{0}$;

$E$ ( $p \times \mathrm{p}):$ matriz das somas de quadrados e de produtos cruzados do resíduo devido à hipótese $H_{0}$. 
Tabela 4.4.1 - Análise de variância multivariada inteiramente ao acaso.

\begin{tabular}{l|c|c}
\multicolumn{1}{c|}{ CV } & GL & Matrizes (SQPC) \\
Tratamentos & $g-1$ & $H=\sum_{i=1}^{g} n_{i}\left(\bar{y}_{v i}-\bar{y}_{v}\right)\left(\bar{y}_{v i}-\bar{y}_{v}\right)^{\prime}$ \\
\hline Resíduo & $N-g$ & $E=\sum_{i=1}^{g} \sum_{j=1}^{n_{i}}\left(y_{v i j}-\bar{y}_{v i}\right)\left(y_{v i j}-\bar{y}_{v i}\right)^{\prime}$ \\
\hline Total & $N-1$ & $H+E=\sum_{i=1}^{g} \sum_{j=1}^{n_{i}}\left(y_{v i j}-\bar{y}_{v}\right)\left(y_{v i j}-\bar{y}_{v}\right)^{\prime}$ \\
\hline
\end{tabular}

$i=1,2, \cdots, g$ : indicando o $i$-ésimo tratamento ( $g$ : número de tratamentos);

$k=1,2, \cdots, p$ : representando a $k$-ésima variável resposta ( $p$ : número de variáveis);

$j=1,2, \cdots, n_{i}$ : caracterizando a $j$-ésima repetição dentro do $i$-ésimo tratamento.

A hipótese $H_{0}: C \beta_{v} U=0$ não será rejeitada para um nível $\alpha$ de significância, se a maior raiz característica $\lambda_{h}$ de $|H-\lambda E|=\left|H E^{-1}-\lambda I\right|=0$ for $\lambda_{h} \leq \lambda_{(\alpha)}$. A maior raiz característica $\lambda_{(\alpha)}$ é o valor crítico ao nível $\alpha$ da distribuição da maior raiz característica quando a hipótese de nulidade for verdadeira. 0 teste fica mais bem definido por $\theta_{k}=\lambda_{k} /\left(1+\lambda_{k}\right)$, tal que $\lambda_{k}$ corresponde a $k$-ésima raiz característica de $H E^{-1}$.

\subsection{TESTES DE HIPÓTESES MULTIVARIADOS E APROXIMAÇÕES PARA A DISRIBUIÇÃO F}

Ferreira (2011) cita as estatísticas para testar as hipóteses do modelo linear geral multivariado $H: C \beta U=\phi$, que são as baseadas no princípio da união-intersecção de Roy e da razão de verossimilhança de Wilks, respectivamente, critério do máximo autovalor de Roy $\left(\theta_{R}\right)$ e de lambda de Wilks $(\Lambda)$. Outras duas estatísticas muito utilizadas são o critério do traço de Lawley-Hotelling $\left(T_{L H}\right)$ e o critério de Pillai $\left(V_{P}\right)$. Também apresenta as suas aproximações para a estatística $F$.

As distribuições exatas dessas estatísticas, sob a hipótese nula, dependem unicamente dos parâmetros $\left(M, N^{*}\right.$ e $S$ ) e são encontradas em Morrison (1976). Anderson (1958) apresenta as aproximações assintóticas para as distribuições $\chi^{2}$ e $F$.

$$
M=\frac{(|u-c|-1)}{2} \quad N^{*}=\frac{(N-g-u-1)}{2} \quad S=\min (u, c)
$$

$u$ : é posto da matriz da matriz $U$;

c: é o posto da matriz $C$;

$g$ : é o posto da matriz $X$. 
No caso da hipótese $H_{0}: \mu_{v 1}=\mu_{v 2}=\cdots=\mu_{v g}$, a matriz $U$ será uma identidade $U=I_{p}$, então o posto de $U$ será $p$ que é o número de variáveis. Sejam as estatísticas usadas para testar a hipótese de igualdade de vetores de médias de tratamentos, onde $\lambda_{k}$ $(k=1,2, \cdots, p)$ satisfazem as equações $|H-\lambda(E+H)|=0$ e $|H-\lambda E|=\left|H E^{-1}-\lambda I\right|=0$ :

a) Critério lambda de Wilks ( $\Lambda)$ :

$$
\Lambda=\prod_{k=1}^{p}\left(1-\theta_{k}\right)=\frac{|E|}{|E+H|}
$$

Onde: $\theta_{k}=\lambda_{k} /\left(1+\lambda_{k}\right)$, tal que $\lambda_{k}$ corresponde a $k$-ésima raiz característica de $H E^{-1}$.

Valor aproximado da transformação de $\Lambda$ em $F$ :

$p$ : número de variáveis;

$v_{H}$ : graus de liberdade (tratamentos);

$v_{E}$ : graus de liberdade (resíduo).

a1) Caso1: $p=2, v_{H}$ e $v_{E}$ quaisquer:

$$
F\left[2 v_{H}, 2\left(v_{E}-1\right)\right]=\frac{v_{E}-1}{v_{H}} \times \frac{1-\sqrt{\Lambda}}{\sqrt{\Lambda}}
$$

a2) Caso 2: $v_{H}=1, p$ e $v_{E}$ quaisquer:

$$
F\left(p, v_{E}-p+1\right)=\frac{v_{E}-p+1}{p} \cdot \frac{1-\Lambda}{\Lambda}
$$

a3) Caso 3: $v_{H}=2$, $p$ e $v_{E}$ quaisquer:

$$
F\left[2 p, 2\left(v_{E}-p+1\right)\right]=\frac{v_{E}-p+1}{p} \cdot \frac{1-\sqrt{\Lambda}}{\sqrt{\Lambda}}
$$

a4) Caso 4 (geralmente aproximado):

$F\left(p v_{H}, a b-c\right)=\left[\left(\frac{1}{\Lambda}\right)^{\frac{1}{b}}-1\right] \frac{a b-c}{p v_{H}} \quad a=v_{E}-\frac{1}{2}\left(p-v_{H}+1\right) \quad c=\frac{1}{2}\left(p v_{H}-2\right)$

Se $p^{2}+v_{H}^{2}-5>0 \rightarrow b=\sqrt{\frac{p^{2} v_{H}^{2}-4}{p^{2}+v_{H}^{2}-5}}$, caso contrário $\rightarrow b=1$

b) Critério de Pillai ( $V p)$ :

$$
V_{P}=\sum_{k=1}^{p} \theta_{k}=\sum_{k=1}^{p} \frac{\lambda_{k}}{1+\lambda_{k}}=\operatorname{tr}\left[H(H+E)^{-1}\right]
$$

Valor aproximado da transformação de $V_{P}$ em F: 


$$
\begin{gathered}
F\left(v_{1}, v_{2}\right)=\left(\frac{V_{p}}{s-V_{P}}\right)\left(\frac{2 f+s+1}{2 m+s+1}\right) \\
f=\left(v_{E}-p-1\right) / 2 \quad s=\min \left(p, v_{H}\right) \quad m=\left(\left|p-v_{H}\right|-1\right) / 2 \\
v_{1}=s(2 m+s+1) \quad v_{2}=s(2 f+s+1)
\end{gathered}
$$

c) Critério do traço de Lawley-Hotelling $\left(\mathrm{T}_{\mathrm{LH}}\right)$ :

$$
T_{L H}=\operatorname{tr}\left(E^{-1} H\right)=\sum_{k=1}^{p} \lambda_{k}, \text { ou } T_{T L}=\sum_{k=1}^{p} \theta_{k}\left(1-\theta_{k}\right)^{-1}
$$

Valor aproximado da transformação de $T_{L H}$ em $F$ :

$$
\begin{aligned}
F\left(v_{1}=a, v_{2}=b\right)=\frac{T_{L H}}{c} \quad c & =\frac{a(b-2)}{b\left(v_{E}-p-10\right)} \quad a=p v_{H} \quad b=4+\frac{(a+2)}{\varphi-1} \\
\varphi & =\frac{\left(v_{E}+v_{H}-p-1\right)\left(v_{E}-1\right)}{\left[\left(v_{E}-p-3\right)\left(v_{E}-p\right)\right]}
\end{aligned}
$$

d) Critério do máximo autovalor de $\operatorname{Roy}\left(\theta_{\mathrm{R}}\right): \theta_{R}=\operatorname{máx}\left(\lambda_{k}\right)$ (Roy, 1953).

Valor aproximado da transformação de $\theta_{R}$ em $F$ :

$$
F\left(v_{1}=d, v_{2}=v_{E}-d+v_{H}\right)=\frac{\left(v_{E}-d+v_{H}\right) \lambda_{1}}{d} \quad d=\max \left(p, v_{H}\right)
$$

Godoi (1985) enfatiza que não existe um teste melhor para todas as situações, acrescentando que o tipo de alternativa vai decidir qual teste é o mais poderoso.

\subsection{A FUNÇÃO DISCRIMINANTE OU VARIÁVEL CANÔNICA $\left(Z_{1}\right)$}

Quando a hipótese $H_{0}: \mu_{v 1}=\mu_{v 2}=\cdots=\mu_{v g}$ é rejeitada, então significa que pelo menos um dos vetores de médias é diferente dos demais. Neste caso, é aconselhável obter uma função linear das variáveis respostas originais, a qual pode ser usada, dentro do modelo de uma análise de variância univariada, como uma nova variável resposta representando o contexto multivariado. Esta função é denominada de função discriminante ou variável canônica, sendo obtida maximizando o valor da estatística $F$.

0 procedimento consiste, então, na obtenção da função linear das variáveis respostas, $Z_{1}=a_{1} y_{1}+a_{2} y_{2}+\cdots+a_{p} y_{p}$, que será aplicada a cada parcela do experimento. A função discriminante ou variável canônica $Z_{1}$ é a função cujos valores de $a_{v}^{\prime}=\left[a_{1}, a_{2}, \cdots, a_{p}\right]$ maximizam o valor do teste $F$ devido a tratamento. A função $Z_{1}=a_{1} y_{1}+a_{2} y_{2}+\cdots+a_{p} y_{p}$ terá 
como coeficientes os valores do autovetor correspondente ao maior autovalor da matriz $H E^{-1}$.

\subsection{ANÁLISE DE UM EXPERIMENTO INTEIRAMENTE AO ACASO BIVARIADO}

Exercício 4.7.1 Seja um ensaio de comparação de crescimento de três espécies E1, E2 e E3, cujas variáveis respostas são y1 (volume de madeira) e y2 (produção de sementes). Considerando parcelas de 0,10 ha e os dados apresentados na tabela 4.7.1. As análises foram realizadas pelo programa, desenvolvido na linguagem do SAS, apresentado na tabela C4 do apêndice C.

a) Testar a hipótese de igualdade dos vetores de médias e obter a função discriminante ou variável canônica $\left(Z_{1}\right)$ no caso de sua rejeição;

b) No caso de rejeição da igualdade dos vetores de médias efetuar, considerando a variável canônica ou função discriminante $\left(Z_{1}\right)$, efetuar a análise de variância univariada e o teste de Tukey.

Tabela 4.7.1 - Dados: y1(volume de madeira) e y2 (produção de sementes).

\begin{tabular}{c|c|c|c|c|c|c} 
Parc. & \multicolumn{2}{c|}{ E1 } & \multicolumn{2}{c}{ E2 } & \multicolumn{2}{c}{ E3 } \\
& $\mathrm{y} 1$ & $\mathrm{y} 2$ & $\mathrm{y} 1$ & $\mathrm{y} 2$ & $\mathrm{y} 1$ & $\mathrm{y} 2$ \\
\hline 1 & 4,5946 & 1,0109 & 5,8933 & 1,3060 & 4,6276 & 1,1316 \\
2 & 4,8385 & 1,0998 & 6,3927 & 1,0245 & 4,5779 & 0,8095 \\
3 & 4,6348 & 1,3639 & 6,0489 & 0,9511 & 4,6314 & 0,9600 \\
4 & 4,1343 & 0,7568 & 5,8370 & 1,1856 & 4,3746 & 0,8520 \\
5 & 4,4140 & 0,9922 & 5,9015 & 0,8214 & 4,5553 & 0,8244 \\
6 & 4,4909 & 1,0381 & 6,2968 & 1,2887 & 4,5962 & 0,8952 \\
7 & 4,3453 & 1,0144 & 5,7785 & 1,0970 & 4,7396 & 1,1269 \\
8 & 4,7612 & 1,2429 & 5,5181 & 0,9897 & 4,5621 & 0,9993 \\
9 & 4,8595 & 1,2807 & 5,6938 & 1,2463 & 4,7066 & 0,9983 \\
10 & 4,5475 & 0,9619 & 6,1735 & 1,1230 & 4,9366 & 0,8054 \\
\hline
\end{tabular}

a) Teste de homogeneidade das matrizes de covariãncias

$$
\begin{gathered}
S_{1}=\left[\begin{array}{ll}
0,052010726 & 0,0318025(28 \\
0,0318025(28) & 0,0316255055
\end{array}\right] \quad S_{2}=\left[\begin{array}{ll}
0,0751199608 & 0,002785892 \\
0,002785892 & 0,024983475
\end{array}\right] \\
S_{3}=\left[\begin{array}{ll}
0,0212345752 & 0,0020700814 \\
0,0020700814 & 0,0152507080
\end{array}\right] \quad S=\left[\begin{array}{ll}
0,0494551 & 0,0122195 \\
0,0122195 & 0,0239532
\end{array}\right] \\
\operatorname{Det}_{1}=-7,36430 \text { Det } S_{2}=-6,28235 \operatorname{Det}_{3}=-8,04857 \text { Det } S=-6,87307 \\
M=\sum_{i=1}^{g}\left(n_{i}-1\right) \operatorname{Ln}|S|-\sum_{i=1}^{g}\left(n_{i}-1\right) \operatorname{Ln}\left|S_{i}\right|
\end{gathered}
$$




$$
\begin{gathered}
M=-185,57289+195,25698=9,68409 \\
C^{-1}=1-\frac{2 p^{2}+3 p-1}{6(p+1)(g-1)}\left[\sum_{i=1}^{g} \frac{1}{n_{i}-1}-\frac{1}{\sum_{i=1}^{g}\left(n_{i}-1\right)}\right] \\
C^{-1}=1-\frac{\left(2 p^{2}+3 p-1\right)(g+1)}{6(p+1) g(n-1)}=1-\frac{52}{486}=0,893004115 \quad\left(n_{i}=n\right)
\end{gathered}
$$

Dado $M C^{-1}=8,647932219$ e $\chi_{\frac{1}{2}(g-1) p(p+1) ; 0,01}^{2}=\chi_{6 ; 0,01}^{2}=16,8$ conclui-se que, como $\chi_{6 ; 0,01}^{2}<M C^{-1}$ ( $p$-valor $\left.=0,1944\right)$, tabela 4.7.2, não há razão para rejeitar a hipótese $H_{0}: \Sigma_{1}=\Sigma_{2}=\Sigma_{3}=\sum$, isto é, as matrizes de covariâncias das espécies E1, E2 e E3 são homogêneas.

Tabela 4.7.2 - Teste de homogeneidade de matrizes de covariâncias (SAS).

\begin{tabular}{c|c|c}
\hline Qui-quadrado & GL & Pr $>\chi^{2}$ \\
\hline 8,648003 & 6 & 0,1944 \\
\hline
\end{tabular}

\section{b) Testes de multinormalidade}

A análise de multinormalidade foi realizada pelo programa SAS, denominado \% Multinorm Macro: Mardia tests of multivariate normality, version 1.1, disponibilizado pelo SAS Institute Inc., através do link:

http://homepages.wmich.edu/ applegab/EMR675/SASFiles/multnorm.sas

Tabela 4.7.3 - Teste de multinormalidade de Mardia.

\begin{tabular}{l|c|c} 
Testes Estatísticos & Valores & Prob. \\
Mardia Skewness & 3,78 & 0,4360 \\
\hline Mardia Kurtosis & $-1,15$ & 0,2486 \\
\hline
\end{tabular}

Os resultados do teste de multinormalidade de Márdia para simetria ( $p$-valor $=0,4360$ ) e para curtose ( $p$-valor $=0,2486$ ) deram não significativos, tabela 4.7.3, então, conclui-se que há razão afirmar que as variáveis y1 e y2 aderem a uma distribuição normal bivariada. A Figura 4.7.1 mostra o "Gráfico Qui-Quadrado Q-Q", apresentando uma configuração linear sem valores discrepantes dos "Quantil Qui-Quadrado" versus as "Distâncias Quadradas", indicando uma aderência dos dados à normalidade multivariada. 


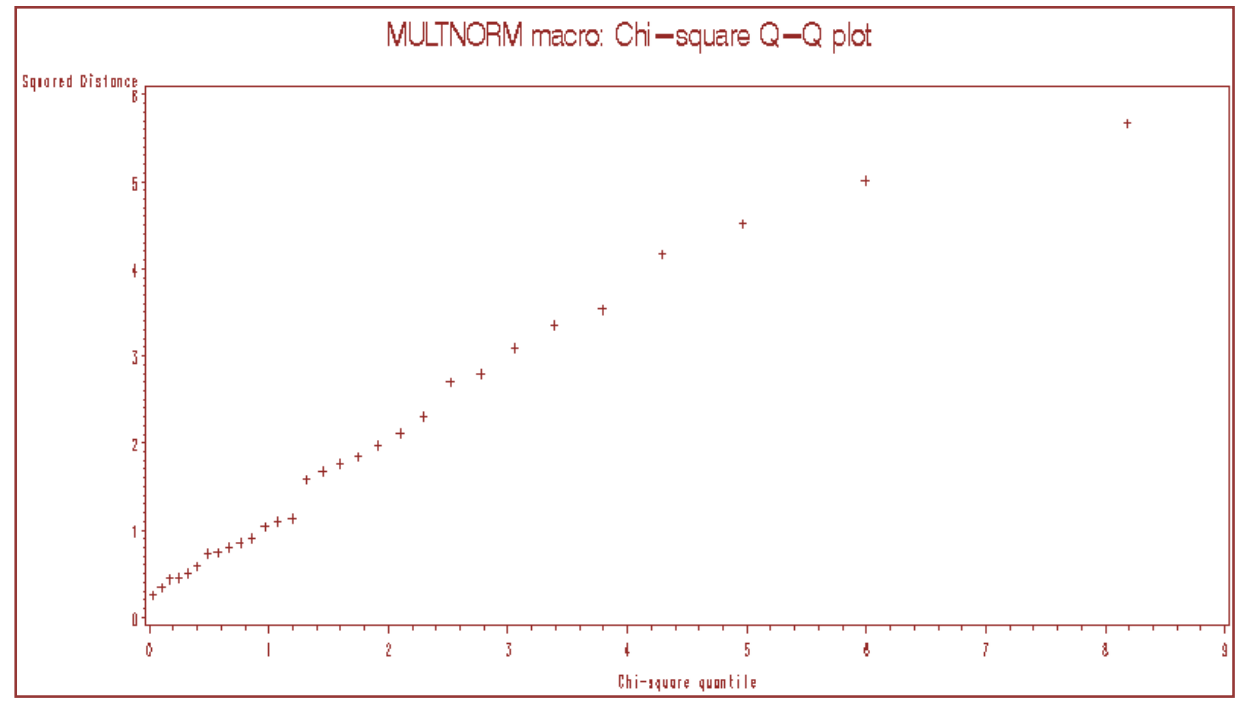

Figura 4.7.1 - Gráfico de macro multnorm Qui-Quadrado Q-Q

c) Análise de variância multivariada do experimento

$$
H_{0}: \mu_{v 1}=\mu_{v 2}=\mu_{v 3} \text { versus } H_{A}: \text { Não } H_{0}
$$

Vetores das médias e matrizes de somas de quadrados e de produtos cruzados:

$$
\begin{gathered}
\hat{\mu}_{v 1}=\left[\begin{array}{l}
4,5621 \\
1,0762
\end{array}\right] \quad \hat{\mu}_{v 2}=\left[\begin{array}{l}
5,9533 \\
1,1033
\end{array}\right] \quad \hat{\mu}_{v 3}=\left[\begin{array}{l}
4,6308 \\
0,9403
\end{array}\right] \\
\left\{\begin{array}{c}
S Q_{\text {TOTAL }}(y 1)=13,633173 B \\
S Q_{\text {TOTAL }}(y 2)=0,79940037 \\
S Q P_{\text {TOTAL }}(y 1, y 2)=1,1436397766
\end{array} \quad T=\left[\begin{array}{cc}
13,6331737 & 1,1436397766 \\
1,1436397766 & 0,79940037
\end{array}\right]\right.
\end{gathered}
$$

Matriz de somas de quadrados e de produtos cruzados de tratamentos $(H)$ :

$$
\left\{\begin{array}{c}
S Q_{T R A T}(y 1)=12,2978860 \\
S Q_{\text {TRAT }}(y 2)=0,15266318 \\
S Q P_{T R A T}(y 1 y 2)=0,15266318
\end{array} \quad H=\left[\begin{array}{cc}
12,29788601 & 0,8137134816 \\
0,8137134816 & 0,152663178
\end{array}\right]\right.
$$

Matriz de somas de quadrados e de produtos cruzados do resíduo $(E)$.

$$
E=T-H=\left[\begin{array}{cc}
1,33528769 & 0,3299262965 \\
0,3299262965 & 0,6467371922
\end{array}\right]
$$


Tabela 4.7.4 - Análise de variância multivariada.

\begin{tabular}{l|c|c}
\multicolumn{1}{c|}{ CV } & GL & Matrizes (SQPC) \\
Tratamentos & $v_{H}=2$ & $H=\left[\begin{array}{cc}12,2978860 & 0,8137134816 \\
0,8137134826 & 0,1526631758\end{array}\right]$ \\
\hline Resíduo & $v_{E}=27$ & $E=\left[\begin{array}{cc}1,33528769 & 0,3299262965 \\
0,3299262965 & 0,6467371922\end{array}\right]$ \\
\hline Total & $N-1=29$ & $T=\left[\begin{array}{cc}13,63317373 & 1,1436397766 \\
1,1436397766 & 0,79940037\end{array}\right]$ \\
\hline
\end{tabular}

d) Cálculo das raízes características da matriz $\mathrm{HE}^{-1}$

$$
\begin{array}{cc}
H=\left[\begin{array}{cc}
12,2978860 & 0,8137134816 \\
0,8137134826 & 0,1526631758
\end{array}\right] & E=\left[\begin{array}{cc}
1,33528769 & 0,3299262965 \\
0,3299262965 & 0,6467371922
\end{array}\right] \\
E^{-1}=\left[\begin{array}{cc}
0,8569133 & -0,437145 \\
-0,437145 & 1,7692284
\end{array}\right] & E^{-1} H=\left[\begin{array}{cc}
10,182511 & 0,6305459 \\
-3,93632 & -0,085615
\end{array}\right]
\end{array}
$$

Então $\left|E^{-1} H-\lambda I\right|=\left|\begin{array}{cc}10,182511-\lambda & 0,6305459 \\ -3,93632 & -0,085615-\lambda\end{array}\right|=0$

$$
(10.182511-\lambda) \times(-0.085615-\lambda)-(0.6305459 \times-3.93632)=0
$$

$\lambda^{2}-10,096896 \lambda+1,610254=0$, resultando: $\lambda_{1}=9,934814$ e $\lambda_{2}=0,16208$

\section{e) Testes multivariados e aproximações para distribução F}

\section{e1) Critério de Wilks ( $\Lambda$ ):}

Dado que $|E|=0,7547288$ e $|T|=|H+E|=9,5904522$. Então:

$$
\Lambda=\frac{\operatorname{det} E}{\operatorname{det}(H+E)}=\frac{\operatorname{det} E}{\operatorname{det} T}=\frac{0,7547288}{9,5904522}=0,07869585 \quad \Lambda=\prod_{k=1}^{p}\left(1-\theta_{k}\right)=0,07869585
$$

Donde $\theta_{k}=\lambda_{k} /\left(1+\lambda_{k}\right)$, tal que $\lambda_{k}$ é $k$-ésima raiz característica de $H E^{-1}$, ou seja, $\lambda_{1}=9,934814$ e $\lambda_{2}=0,16208$. Então:

$$
\theta_{1}=9,934814 \div(1+9,934814)=0,908549 \quad \theta_{2}=0,16208 \div(1+0,16208)=0,139474
$$

Valor aproximado da transformação de $\Lambda$ em $F$ :

$p$ = número de variáveis;

$v_{H}=2:$ graus de liberdade (tratamentos);

$v_{E}=27$ : graus de liberdade (resíduo). 
Caso1: $p=2, v_{H}$ e $v_{E}$ quaisquer:

$$
F\left[2 v_{H}, 2\left(v_{E}-1\right)\right]=\frac{v_{E}-1}{v_{H}} \times \frac{1-\sqrt{\Lambda}}{\sqrt{\Lambda}} \quad F_{(4 ; 52)}=\frac{26}{2} \times \frac{1-\sqrt{0,0786958512}}{\sqrt{0,0786958512}}=33,3412 * *
$$

Caso 2: $v_{H}=1$, p e $v_{E}$ quaisquer (não aplicado):

$$
F\left(p, v_{E}-p+1\right)=\frac{v_{E}-p+1}{p} \cdot \frac{1-\Lambda}{\Lambda}
$$

Caso 3: $v_{H}=2$, p e $v_{E}$ quaisquer:

$$
F\left[2 p, 2\left(v_{E}-p+1\right)\right]=\frac{v_{E}-p+1}{p} \cdot \frac{1-\sqrt{\Lambda}}{\sqrt{\Lambda}} \quad F(4 ; 52)=\frac{26}{2} \times \frac{1-\sqrt{0,0786958512}}{\sqrt{0,0786958512}}=33,3412^{* *}
$$

Caso 4: (geralmente aproximado):

$$
\begin{gathered}
F\left(p v_{H}, a b-c\right)=\left[\left(\frac{1}{\Lambda}\right)^{\frac{1}{b}}-1\right] \frac{a b-c}{p v_{H}} \\
a=v_{E}-\frac{1}{2}\left(p-v_{E}+1\right)=27-\frac{1}{2}(2-2+1)=26,5 \quad c=\frac{1}{2}\left(p v_{H}-2\right)=\frac{1}{2}(2 \times 2-2)=1 \\
\text { Se } p^{2}+v_{H}^{2}-5>0 \rightarrow b=\sqrt{\frac{p^{2} v_{H}^{2}-4}{p^{2}+v_{H}^{2}-5}}, \text { caso contrário } \rightarrow b=1 \\
p^{2}+v_{H}^{2}-5=4+4-5=3>0 \rightarrow b=\sqrt{\frac{2^{2} \times 2^{2}-4}{2^{2}+2^{2}-5}}=2 \\
F(4 ; 52)=\left[\left(\frac{1}{0,0786958512}\right)^{\frac{1}{2}}-1\right] \frac{52}{4}=33,3412^{* *}
\end{gathered}
$$

e2) Critério do traço de Lawley-Hotelling $\left(\mathrm{T}_{\mathrm{LH}}\right)$ :

$$
\begin{gathered}
T_{L H}=\operatorname{tr}\left(E^{-1} H\right)=\sum_{k=1}^{p} \lambda_{k} \\
H E^{-1}=\left[\begin{array}{cc}
10,182511 & -3,93632 \\
0,6305459 & -0,085615
\end{array}\right] \quad \operatorname{tr}\left(H E^{-1}\right)=10,182511-0,085615=10,09689 \\
T_{L H}=\sum_{k=1}^{p} \theta_{k}\left(1-\theta_{k}\right)^{-1}
\end{gathered}
$$


Sendo $\theta_{k}=\lambda_{k} /\left(1+\lambda_{k}\right)$, tal que $\lambda_{k}$ é $k$-ésima raiz característica de $H E^{-1}$, donde: $\lambda_{1}=9,934814$ e $\lambda_{2}=0,16208$. Então:

$$
\begin{gathered}
\theta_{1}=9,934814 \div(1+9,934814)=0,908549 \quad \theta_{2}=0,16208 \div(1+0,16208)=0,139474 \\
\theta_{1} \div\left(1-\theta_{1}\right)=9,93481 \quad \theta_{2} \div\left(1-\theta_{2}\right)=0,162080 \\
T_{L H}=9,93481+0,162080=10,09689
\end{gathered}
$$

Valor aproximado da transformação de $T_{L H}$ em $F$ :

$$
\begin{gathered}
F\left(v_{1}=a, v_{2}=b\right)=\frac{T_{L H}}{c} \\
a=p v_{H}=2 \times 2=4 \quad b=4+\frac{(a+2)}{\varphi-1}=4+\frac{(4+2)}{(1,22909090-1)}=30,19047723 \\
\varphi=\frac{\left(v_{E}+v_{H}-p-1\right)\left(v_{E}-1\right)}{\left(v_{E}-p-3\right)\left(v_{E}-p\right)}=\frac{(27+2-2-1)(27-1)}{(27-2-3)(27-2)}=\frac{26 \times 26}{550}=1,22909090 \\
c=\frac{a(b-2)}{b\left(v_{E}-p-1\right)}=\frac{4(30,19047723-2)}{30,1904772327-2-1)}=0,15562566 \\
F\left(v_{1}=4, v_{2}=30,19\right)=\frac{T_{L H}}{c}=\frac{10,096896}{0,155625661}=64,88
\end{gathered}
$$

e3) Roy Greatest $\operatorname{Root}\left(\theta_{R}\right)$ :

$$
\theta_{R}=\operatorname{máx}\left(\lambda_{k}\right)
$$

Autovalores de $H E^{-1}: \lambda_{1}=9,934814$ e $\lambda_{2}=0,16208$. Então: $\theta_{R}=9,934814$

Valor aproximado da transformação de $\theta_{R}$ em $F$ :

$$
\begin{gathered}
F\left(v_{1}=d, v_{2}=v_{E}-d+v_{H}\right)=\frac{\left(v_{E}-d+v_{H}\right) \lambda_{1}}{d} \\
d=\max \left(p, v_{H}\right)=\max (2,2) \quad v_{2}=v_{E}-d+v_{H}=27-2+2 \\
F(2,27)=\frac{\left(v_{E}-d+v_{H}\right) \lambda_{1}}{d}=\frac{(27-2+2)}{2} \times 9,934814=134,12
\end{gathered}
$$




\section{e4) Pillai Trace (Tp):}

Sendo $\theta_{i}=\lambda_{k} /\left(1+\lambda_{k}\right)$, tal que $\lambda_{k}$ é i-ésima raiz característica de $H E^{-1}$, donde:

$$
\lambda_{1}=9,934814 \quad \lambda_{2}=0,162089,934814
$$

$$
\theta_{1}=9,934814 \div(1+9,934814)=0,908549 \quad \theta_{2}=0,16208 \div(1+0,16208)=0,139474
$$

$$
\begin{gathered}
T_{P}=\sum_{k=1}^{p} \theta_{k}=0,908549+0,139474=1,048024 \\
T_{P}=\operatorname{tr}\left[H(E+H)^{-1}\right]=\operatorname{tr}\left[\begin{array}{ll}
0,9280417 & -0,309772 \\
0,0496213 & 0,1199827
\end{array}\right]=1,048024
\end{gathered}
$$

Valor aproximado da transformação de $T_{P}$ em $F$ :

$$
\begin{gathered}
F\left(v_{1}, v_{2}\right)=\left(\frac{V}{s-V}\right)\left(\frac{2 f+s+1}{2 m+s+1}\right)=\frac{1,0480244}{2-1,0480244}\left(\frac{2 \times 12+2+1}{2 \times-0,5+2+1}\right)=14,86 \\
f=\left(v_{E}-p-1\right) / 2=(27-2-1) / 2=12
\end{gathered}
$$

$s=\min \left(p, v_{H}\right)=\min (2,2)$

$m=\left(\left|p-v_{H}\right|-1\right) / 2=(|2-2|-1) / 2=-0,5$

$v_{1}=s(2 m+s+1)=2(2 \times-0,5+2+1)=4$

$v_{2}=s(2 f+s+1)=2(2 \times 12+2+1)=54$

$F(4,54)=14,86$

Na tabela 4.7.5 estão os resultados dos testes multivariados e das aproximações para a estatística $F$ realizados pelo programa desenvolvido no SAS.

$$
\begin{gathered}
M=\frac{(u-c \mid-1)}{2}=-0,5 \\
N^{*}=\frac{(N-g-u-1)}{2}=\frac{(30-3-2-1)}{2}=12 \\
S=\min (u, c)=\min (2,2)=2
\end{gathered}
$$

$u=p=2$ : é posto da matriz da matriz $U$;

$c=g-1=2$ : é o posto da matriz $C$. 
Tabela 4.7.5 - Resultados dos testes para $H_{0}: \mu_{v 1}=\mu_{v 2}=\mu_{v 3}$.

\begin{tabular}{|c|c|c|c|c|c|}
\hline \multicolumn{6}{|c|}{$\begin{array}{l}\text { Testes e aproximações de } F \text { para a hipótese de ausência de efeito de tratamento } \\
\qquad\left(\mathrm{S}=2 ; M=-0,5 ; N^{*}=12\right) \quad \mathrm{H}=\text { Tratamento SQPC } \quad \mathrm{E}=\text { Error SQPC }\end{array}$} \\
\hline Testes multivariados & Valores & Valor F & Num GL & Den GL & $\mathrm{Pr}>\mathrm{F}$ \\
\hline Wilks Lambda & 0,07869586 & 33,34 & 4 & 52 & $<0,0001$ \\
\hline Pillai Trace & 1,04802442 & 14,86 & 4 & 54 & $<0,0$ \\
\hline Hotelling-Lawley Trace & 10,09689587 & 64,88 & 4 & 30,19 & $<0,0001$ \\
\hline Roy Greatest Root & 9,93481398 & 134,12 & 2 & 27 & $<0,0001$ \\
\hline
\end{tabular}

De acordo com a tabela 4.7.5, conclui-se que os testes foram significativos ( $p$-valor < 0,0001 ), assim como, sendo $F_{4,52 ; 0,01} \cong 3,65$, então se rejeita $H_{0}: \mu_{v 1}=\mu_{v 2}=\mu_{v 3}$.

\section{f) Função discriminante ou variável canônica $\mathrm{Z}_{1}$}

Como a hipótese $H_{0}: \mu_{v 1}=\mu_{v 2}=\mu_{v 3}$ foi rejeitada, então ocorre que pelo menos um dos vetores de médias é diferente dos demais. Neste caso, serão calculados os valores de uma nova variável resposta $Z_{1}=a_{1} y_{1}+a_{2} y_{2}$, denominada de função discriminante ou variável canônica, que é função linear das variáveis respostas originais $y_{1}$ e $y_{2}$. Esta variável $Z_{1}$ maximiza o valor da estatística $F$.

0 procedimento consistirá na obtenção da função linear $Z_{1}=a_{1} y_{1}+a_{2} y_{2}$ das variáveis respostas que será aplicada a cada parcela do experimento. Esta função terá como coeficientes os valores do autovetor correspodente ao maior autovalor $\left(\lambda_{1}=9,934814\right)$ da matriz $H E^{-1}$.

f1) Cálculo da primeira variável canônica $\mathrm{Z}_{1}$ ou Can1:

$$
\begin{gathered}
E^{-1} H=\left[\begin{array}{cc}
10,182511 & 0,6305459 \\
-3,93632 & -0,085615
\end{array}\right] \\
{\left[E^{-1} H-\lambda_{1} I\right] a_{v}=0_{v} \rightarrow\left[E^{-1} H-9,934814\left[\begin{array}{ll}
1 & 0 \\
0 & 1
\end{array}\right]\right] a_{v}=\left[\begin{array}{l}
0 \\
0
\end{array}\right]} \\
{\left[\begin{array}{cc}
0,247697 & 0,630559 \\
-3,93632 & -10,020429
\end{array}\right] \times\left[\begin{array}{l}
a_{1} \\
a_{2}
\end{array}\right]=\left[\begin{array}{l}
0 \\
0
\end{array}\right]} \\
\left\{\begin{array}{c}
0,247697 a_{1}+0,630559 a_{2}=0 \\
-3,93642 a_{1}-10,020429 a_{2}=0
\end{array}\right.
\end{gathered}
$$

Se $a_{2}=1 \rightarrow a_{1}=\frac{-0,630559}{0,247697}=-2,545687$ 
0 ideal é obter uma função normalizada $Z_{1}^{*}=a_{1}^{*} y_{1}+a_{2}^{*} y_{2}$ que significa que a soma dos quadrados dos coeficientes seja igual a um. Então:

$$
\begin{aligned}
& a_{1}^{*}=\frac{a_{1}}{\sqrt{a_{1}^{2}+a_{2}^{2}}}=\frac{-2,545687}{\sqrt{\left.(-2,545687)^{2}+1^{2}\right)}}=-0,930763 \\
& a_{2}^{*}=\frac{a_{2}}{\sqrt{a_{1}^{2}+a_{2}^{2}}}=\frac{1}{\left.\sqrt{(-2,545687)^{2}+1^{2}}\right)}=0,365623
\end{aligned}
$$

$Z_{1}^{*}=$ Can $1=-0,930763 y 1+0,365623 y 2$ que é a primeira variável discriminante ou canônica, também, pode ser escrita na forma: $Z_{1}^{*}=\operatorname{Can} 1=0,930763 y 1-0,365623 y 2$.

f2) Cálculo da segunda variável canônica $Z_{2}$ ou Can2:

Os coeficientes de $Z_{2}^{*}=b_{1}^{*} y_{1}+b_{2}^{*} y_{2}$ correspondem aos elementos do autovetor referente ao segundo autovalor $\left(\lambda_{2}=0,162082\right)$. 0 interesse em obter essa segunda variável é construir o gráfico 4.7.2 (Can1 versus Can2). Ele mostra a tendência da representacão da discriminação dos tratamentos.

$$
\begin{gathered}
E^{-1} H=\left[\begin{array}{cc}
10,182511 & 0,6305459 \\
-3,93632 & -0,085615
\end{array}\right] \\
{\left[E^{-1} H-\lambda_{2} I\right] a_{v}=0_{v} \rightarrow\left[E^{-1} H-0,16208 \times\left[\begin{array}{ll}
1 & 0 \\
0 & 1
\end{array}\right]\right] a_{v}=\left[\begin{array}{l}
0 \\
0
\end{array}\right]} \\
{\left[\begin{array}{cc}
10,020429 & 0,630559 \\
-3,93632 & -0,247697
\end{array}\right] \cdot\left[\begin{array}{l}
b_{1} \\
b_{2}
\end{array}\right]=\left[\begin{array}{l}
0 \\
0
\end{array}\right] \quad\left\{\begin{array}{l}
10,020429 b_{1}+0,630559 b_{2}=0 \\
-3,93632 b_{1}-10,020429 b_{2}=0
\end{array}\right.}
\end{gathered}
$$

Se $b_{1}=1 \rightarrow b_{2}=\frac{-10,020429}{0,630559}=-15,891342$

Normalizando o vetor dos coeficientes:

$$
\begin{gathered}
b_{1}^{*}=\frac{b_{1}}{\sqrt{b_{1}^{2}+b_{2}^{2}}}=\frac{1}{\left.\sqrt{(-15,891342)^{2}+1^{2}}\right)}=0,062803 \\
b_{2}^{*}=\frac{b_{2}}{\sqrt{b_{1}^{2}+b_{2}^{2}}}=\frac{-15,891342}{\sqrt{(-15,89134)^{2}+1^{2}}}=-0,998026
\end{gathered}
$$


$Z_{2}^{*}=$ Can2 = 0,062803y1-0,998026y2 é a segunda variável discriminante ou canônica.

Tabela 4.7.6 - Resultados dos coeficientes das duas variáveis canônicas.

\begin{tabular}{l|c|c|c|c}
\multicolumn{5}{c}{ Coeficientes Canônicos } \\
\multicolumn{4}{c}{ Estandartizados } & \multicolumn{2}{c}{ Não Estandartizados } \\
& Can1 & Can2 & Can1 & Can2 \\
\hline X1 & 3,28548871 & $-0,28692473$ & 4,79181998 & $-0,41847402$ \\
\hline X2 & $-0,31252716$ & 1,10413276 & $-1,88236826$ & 6,65025230 \\
\hline
\end{tabular}

A tabela 4.7.6 apresenta os resultados, calculados pelo programa desenvolvido no SAS, dos coeficientes para as duas variáveis canônicas. É importante verificar que normalizando os autovetores obtidos, resultam os mesmos valores alcançados anteriormente.

a) Primeira variável canônica Can1: normalização do vetor (dados não estandartizados):

$$
\begin{gathered}
a_{1}^{*}=\frac{a_{1}}{\sqrt{a_{1}^{2}+a_{2}^{2}}}=\frac{4,79181998}{\sqrt{(4,7918199)^{2}+\left(-1,88236826^{2}\right)}}=0,930763 \\
a_{2}^{*}=\frac{a_{2}}{\sqrt{a_{1}^{2}+a_{2}^{2}}}=\frac{-1,88236826}{\sqrt{\left(4,79181998^{2}+\left(-1,88236826^{2}\right)\right.}}=-0,365623
\end{gathered}
$$

b) Segunda variável canônica Can2: normalização do vetor (dados não estandartizados):

$$
\begin{aligned}
& b_{1}^{*}=\frac{b_{1}}{\sqrt{b_{1}^{2}+b_{2}^{2}}}=\frac{-0,41847402}{\sqrt{\left(-0,418474(2)^{2}+(6,65025230)^{2}\right)}}=-0,062803 \\
& b_{2}^{*}=\frac{b_{2}}{\sqrt{b_{1}^{2}+b_{1}^{2}}}=\frac{6,65025230}{\sqrt{\left(-0,418474(\mathbb{R})^{2}+(6,65025230)^{2}\right)}}=0,998026
\end{aligned}
$$

g) Análise de variância da função discriminante variável canônica $\mathrm{Z}_{1}^{*}$ (Can1)

Tabela 4.7.7 - Valores para $\operatorname{Can} 1=0,930763 y 1-0,365623 y 2$.

\begin{tabular}{c|c|c|c|c|c|c|c} 
Parc. & E1 & E2 & E3 & Parc. & E1 & E2 & E3 \\
\hline 1 & 3,90688 & 5,00777 & 3,89346 & 6 & 3,80044 & 5,38966 & 3,95062 \\
\hline 2 & 4,10134 & 5,57551 & 3,96502 & 7 & 3,67353 & 4,97733 & 3,99940 \\
\hline 3 & 3,81526 & 5,28235 & 3,95974 & 8 & 3,97714 & 4,77420 & 3,88083 \\
\hline 4 & 3,57131 & 4,99840 & 3,76026 & 9 & 4,05480 & 4,84387 & 4,01569 \\
\hline 5 & 3,74562 & 5,19262 & 3,93852 & 10 & 3,88099 & 5,33548 & 4,30031 \\
\hline
\end{tabular}


A tabela 4.7.7 apresenta os valores para a variável $\operatorname{Can} 1=0,930763 y 1-0,365623 y 2$. Estes foram obtidos a partir dos dados das variáveis respostas $y_{1}$ e $y_{2}$ que estão disponíveis na tabela 4.7.1.

Tabela 4.7.8 - ANAVA da variável Can1 =0,930763y1-0,365623y2.

\begin{tabular}{l|c|c|c|c}
\hline \multicolumn{1}{c}{ Causas } & Gl & SQ & QM & $F$ \\
Tratamentos & 2 & 10,1205 & 5,0602 & 134,12 \\
\hline Resíduo & 27 & 1,0187 & 0,0377 & \\
\hline Total & 29 & 11,1391 & & \\
\hline
\end{tabular}

O valor da estatística $F(134,12)$ obtido na ANAVA para a variável Can1, tabela 4.7.8, é o mesmo calculado pela equação deduzida pelo princípio da união-intersecção de Roy.

$$
F_{\left(a_{v}\right)}=\lambda_{1}\left(\frac{N-g}{g-1}\right)=9,934814 \times \frac{30-3}{3-1}=134,12
$$

Como $F_{2 ; 27 ; 0,01=3,40}$, então se rejeita a hipotése $H_{0}: \mu_{E 1}=\mu_{E 2}=\mu_{E 3}$. A função Can1 $=0,930763 y 1-0,365623 y 2$ maximiza $F$, atendendo ao princípio da uniãointercecção de Roy. Resultado importante é que a variável y1 tem uma significativa contribuição na construção da função $Z_{1}^{*}$, haja vista o valor absoluto de seu coeficiente ser igual a 0,930763, enquanto a variável y2 possui um coeficiente igual a 0,365623.

\section{i) Teste de comparação multipla de Tukey para a variável resposta (Can1)}

Tabela 4.7.10 - Teste de Tukey para $(\operatorname{Can} 1=-0,930763 y 1+0,365623 y 2)$.

\begin{tabular}{c|c|c|c}
\hline Tukey (Grupos) & Médias & N & Tratamentos \\
\hline G1 & 5,13772 & 10 & B \\
\hline G2 & 3,96638 & 10 & C \\
\hline G2 & 3,85273 & 10 & A \\
\hline
\end{tabular}

A figura 4.7.2 apresenta a distribuição dos tratamentos, segundo as variáveis Can1 e Can2, confirmando o resultado do teste de Tukey, ou seja, o tratamentos B forma o grupo (G1), enquanto que os tratamentos A e C representam o grupo (G2). 0 teste de Tukey mostra a existência de não significância entre os tratamentos A e C. 


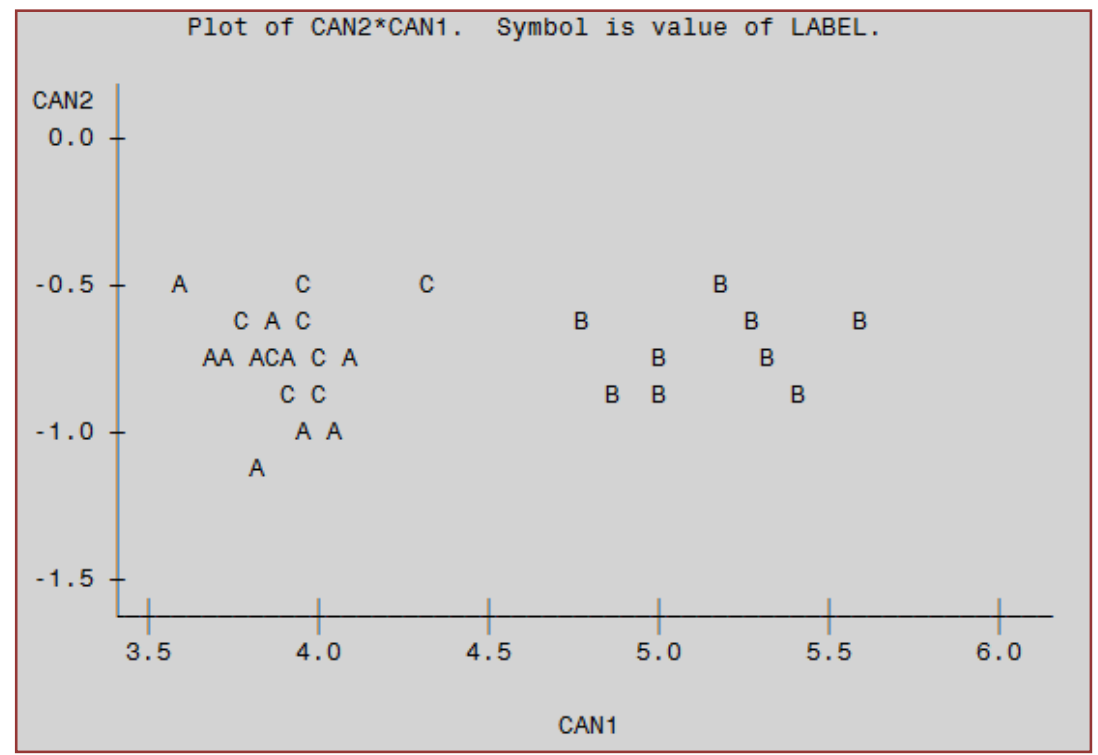

Figura 4.7.2 - Distribuição dos tratamentos: Can1 vs Can2. Nota SAS: 3 obs. ocultas. 


\section{Capítulo 5}

\section{Noções sobre regressão não linear}

Neste capítulo é dada uma breve abordagem sobre a teoria de regressão não linear com o objetivo de obter curvas de crescimento para os métodos de manejo e de espécies. Aborda os seguintes conteúdos: teorema de Taylor; estimadores de máxima verossimilhança; teste da razão de verossimilhança; teste para verificar a igualdade de parâmetros e de identidade de modelos de regressão não linear; análise de resíduos; regressão não linear pelo método de Gauss-Newton; avaliação da adequacidade dos modelos testados; validação da predição do modelo de regressão pelo teste de Graybill. 


\section{INTRODUÇÃO}

Considerando que será apresentado o emprego do modelo de regressão não linear de Mitscherlich para ajustar os perfis de crescimento de florestas e de espécies, então, para um melhor entendimento dos procedimentos, serão apresentados alguns temas que são importantes no ajuste de modelos não lineares, tendo como enfoque principal a aplicação do método de Gauss-Newton.

\subsection{TEOREMA DE TAYLOR}

Qualquer função $f(X)$ que seja contínua e derivável, e que tenha uma derivada de $n$-ésima ordem, pode ser aproximada em torno de um ponto $X=X_{0}$ por uma função polinomial e um resto $(R)$. A equação 5.1 .1 é denominada de expansão da série de Taylor $f(X)$ em torno do ponto $X=X_{0}$. Quanto maior a ordem, melhor será a aproximação da função original.

$$
f(X)=\frac{f\left(X_{0}\right)}{0 !}+\frac{f^{\prime}\left(X_{0}\right)\left(X-X_{0}\right)}{1 !}+\frac{f^{\prime \prime}\left(X_{0}\right)\left(X-X_{0}\right)^{2}}{2 !}+\cdots+\frac{f^{n}\left(X_{0}\right)\left(X-X_{0}\right)^{n}}{n !}+\cdots+R
$$

$f^{\prime}\left(X_{0}\right), f^{\prime \prime}\left(X_{0}\right), \cdots, f^{n}\left(X_{0}\right)$ : são as derivadas da função $f(X)$ de ordem de $1,2, \cdots, n$ avaliadas em $X=X_{0}$.

Se $n=1$ tem-se uma aproximação linear;

Se $n=2$ tem-se uma aproximação polinomial de segundo grau.

Seja o exemplo da função $f(X)=e^{X}$, tal que $X_{0}=0$, aproximar até o polinômio de sexto grau.

$$
\begin{gathered}
f^{\prime}\left(X_{0}\right)=1 \quad f^{\prime \prime}(X)=e^{X} \quad f^{\prime \prime}\left(X_{0}\right)=1 \quad f^{\prime \prime \prime}(X)=e^{X} \quad f^{\prime \prime \prime}\left(X_{0}\right)=1 \\
f^{4}(X)=e^{X} \quad f^{4}\left(X_{0}\right)=1 \quad f^{5}(X)=e^{X} \quad f^{5}\left(X_{0}\right)=1 \quad f^{6}(X)=e^{X} \quad f^{6}\left(X_{0}\right)=1
\end{gathered}
$$

$P(X)=1+X+\frac{X^{2}}{2 !}+\frac{X^{3}}{3 !}+\frac{X^{4}}{4 !}+\frac{X^{5}}{5 !}+\frac{X^{6}}{6 !}$ é o polinômio do sexto grau que aproxima $f(X)=e^{X}$. Se $f(X=2)=e^{2}=7,3890561$, então:

$$
f(X=2)=1+2+\frac{2^{2}}{2 !}+\frac{2^{3}}{3 !}+\frac{2^{4}}{4 !}+\frac{2^{5}}{5 !}+\frac{2^{6}}{6 !}+R=7,3555555+0,0335005(R=0,0335005)
$$

O objetivo do uso da expansão de Taylor na regressão não linear é aproximar, em alguns casos, a função a ser ajustada para uma função linear excluindo os termos de ordens elevadas. 0 método de Gauss-Newton consiste na linearização da equação não linear em torno de alguns valores iniciais dos parâmetros. A equação linearizada é, então, estimada pelo método dos Mínimos Quadrados Ordinários (MQO) e os valores escolhidos inicialmente são ajustados. Esses valores ajustados são usados para relinearizar o modelo e novamente são estimados por MQO. 0 processo continua até que, a partir das 
últimas iterações, não haja mais alterações substanciais nos valores estimados. A principal técnica usada para a linearização de uma equação não linear é a expansão de Taylor.

Gujarati \& Porter (2011) citam que a estimação de modelos intrinsecamente não lineares está basicamente sistematizada em dois algoritmos, o método iterativo de Gauss-Newton e o método de Newton-Raphson, os quais já estão incorporados a vários programas de análise estatística.

\subsection{ESTIMADORES DE MÁXIMA VEROSSIMILHANÇA}

0 método da máxima verossimilhança adota como estimativas dos parâmetros os valores que maximizam a probabilidade (no caso de variável discreta) ou a densidade de probabilidade (no caso de variável contínua) de ser obtida a amostra observada. É necessário conhecer a distribuição da variável em estudo.

Seja uma variável aleatória contínua com função densidade de probabilidade $f(x, \theta)$, sendo $\theta$ o parâmetro desconhecido. Considere uma amostra aleatória simples de $X$ de tamanho $n$ e $x_{1}, x_{2, \cdots}, x_{n}$ os valores observados.

A função de verossimilhança $L$ é definida como:

$$
L\left(x_{1}, x_{2}, \cdots, x_{n} ; \theta\right)=f\left(x_{1} ; \theta\right) \times f\left(x_{2} ; \theta\right) \times \cdots \times f\left(x_{n} ; \theta\right)=\prod_{i=1}^{n} f\left(x_{i} ; \theta\right)
$$

O estimador de máxima verossimilhança de $\theta$ é o valor que maximiza a função 5.2 .1 e pode ser encontrado, na maioria dos casos, seguindo as seguintes etapas:

a) Encontrar a função de verossimilhança;

b) Obter o logaritmo natural (neperiano) da função;

c) Derivar em relação ao parâmetro $\theta$;

d) Igualar o resultado a zero;

e) Verificar se o estimador representa um ponto de máximo.

Seja a determinação dos estimadores de máxima verossimilhança da média $\mu$ e da variância $\sigma^{2}$ de uma variável aleatória $(X)$ com distribuição normal, com base em uma amostra de $n$ elementos.

$$
f\left(X_{i}\right)=\frac{1}{\sqrt{2 \pi \sigma^{2}}} \exp \left[-\frac{\left(X_{i}-\mu\right)^{2}}{2 \sigma^{2}}\right]
$$

Considerando que as observações são independentes, a densidade de probabilidade de obter os valores $X_{1}, X_{2}, \cdots, X_{n}$ da amostra é: 


$$
\begin{gathered}
L\left(X_{1}, X_{2}, \cdots, X_{n} ; \mu, \sigma^{2}\right)=f\left(X_{1}\right) \cdot f\left(X_{2}\right) \cdots \cdot f\left(X_{n}\right)=\prod_{i=1}^{n} \frac{1}{\sqrt{2 \pi \sigma^{2}}} \exp \left[-\frac{\sum_{i=1}^{n}\left(X_{i}-\mu\right)^{2}}{2 \sigma^{2}}\right] \\
L\left(X_{1}, X_{2}, \cdots, X_{n} ; \mu, \sigma^{2}\right)=\left(2 \pi \sigma^{2}\right)^{-\frac{n}{2}} \exp \left[-\frac{\sum_{i=1}^{n}\left(X_{i}-\mu\right)^{2}}{2 \sigma^{2}}\right]
\end{gathered}
$$

A função 5.2.2 é denominada de função de verossimilhança da amostra e os estimadores de máxima verossimilhança de $\mu$ e de $\sigma^{2}$ são os valores que maximizam esta função.

Como o logaritmo é uma função monotônica crescente, os valores de $\mu$ e $\sigma^{2}$ que maximizam 5.2.2 também maximizam $\operatorname{Ln}\left[L\left(X_{1}, X_{2}, \cdots, X_{n} ; \mu, \sigma^{2}\right)\right]$.

$$
\operatorname{Ln}\left[L\left(X_{1}, X_{2}, \cdots, X_{n} ; \mu, \sigma^{2}\right)\right]=-\frac{n}{2} \operatorname{Ln} 2 \pi-\frac{n}{2} \operatorname{Ln} \sigma^{2}-\frac{\sum_{i=1}^{n}\left(X_{i}-\mu\right)^{2}}{2 \sigma^{2}}
$$

Derivando e igualando a zero as derivadas parciais em relação a $\mu$ e a $\sigma^{2}$, obtêm-se o sistema de equações:

$$
\frac{2 \sum_{i=1}^{n}\left(X_{i}-\hat{\mu}\right)}{2 \hat{\sigma}^{2}}=0 \quad-\frac{n}{2 \hat{\sigma}^{2}}+\frac{\sum_{i=1}^{n}\left(X_{i}-\hat{\mu}\right)^{2}}{2 \hat{\sigma}^{4}}=0
$$

Resultando:

$$
\hat{\mu}=\frac{\sum_{i=1}^{n} X_{i}}{n}=\bar{X} \quad \hat{\sigma}^{2}=\frac{\sum_{i=1}^{n}\left(X_{i}-\mu\right)^{2}}{n}
$$

Seja $\bar{X}$ o estimador de mínimos quadrados não tendencioso e de variância mínima, então como $\bar{X}=\hat{\mu}$ e a variável $X$ tem distribuição normal, logo $\bar{X}$ também é um estimador de máxima verossimilhança.

Nota-se que o estimador $\hat{\sigma}^{2}$ de máxima verossimilhança da variância é tendencioso (viesado), uma vez que o estimador não tendencioso é:

$$
s^{2}=\frac{\sum_{i=1}^{n}\left(X_{i}-\mu\right)^{2}}{n-1}
$$




\subsection{TESTE DA RAZÃO DE VEROSSIMILHANÇA}

Seja $X_{1}, X_{2}, \cdots, X_{N}$ uma amostra aleatória independente e igualmente distribuída de uma população com função densidade de probabilidade $f(x / \theta)$, donde $\theta$ é o parâmetro ou vetor paramétrico de interesse. A função de verossimilhança é dada por:

$$
L\left(\theta ; x_{1}, x_{2}, \cdots, x_{N}\right)=f\left(x_{1} ; \theta\right) \times f\left(x_{2} ; \theta\right) \times \cdots \times f\left(x_{N} ; \theta\right)=\prod_{i=1}^{N} f\left(x_{i} ; \theta\right)
$$

De acordo com Regazzi \& Silva (2004), denotando $\hat{\theta}$ como o estimador de máxima verossimilhança de $\theta$ sobre o espaço $\Omega$ e $\hat{\theta}_{0}$ o estimador de máxima verossimilhança de $\theta$ sobre o espaço de $\omega$, e considerando o problema geral de testar a hipótese de nulidade dada por $H_{0}: \theta \in \omega$ versus $H_{a}: \theta \in \omega^{c}$, donde $\omega$ é um subconjunto do espaço paramétrico $\Omega$ e $\omega^{\mathrm{c}}$ é o complemento de $\omega$, tal que $\Omega=\omega \cup \omega^{C}$, a estatística do teste da razão de verossimilhanças terá a seguinte forma:

$$
L=\left(\frac{\hat{\sigma}_{\Omega}^{2}}{\hat{\sigma}_{\omega}^{2}}\right)^{\frac{N}{2}}
$$

$\Omega$ : é o espaço paramétrico irrestrito e $\omega$ é o espaço paramétrico restrito à hipótese nula; $\hat{\sigma}_{\Omega}^{2}$ : é a estimativa de máxima verossimilhança de $\sigma^{2}$, sem restrição, no espaço paramétrico (modelo completo);

$\hat{\sigma}_{\omega}^{2}$ : é a estimativa de máxima verossimilhança de $\sigma^{2}$, com as restrições, definidas em $H_{0}$ (modelo reduzido).

Conforme Rao (1973), para grandes amostras, tem-se:

$$
-2 \operatorname{Ln} L=-N \operatorname{Ln}\left(\frac{\hat{\sigma}_{\Omega}^{2}}{\hat{\sigma}_{\omega}^{2}}\right) \underset{N \rightarrow \infty}{\stackrel{d}{\rightarrow}} \chi_{v}^{2}
$$

E a estatística de máxima verossimilhança para testar $H_{0}$ será:

$$
\chi_{\text {cal }}^{2}=-N \operatorname{Ln}\left(\frac{\hat{\sigma}_{\Omega}^{2}}{\hat{\sigma}_{\omega}^{2}}\right)=-N \operatorname{Ln}\left[\left(\frac{\operatorname{SQRR}(\Omega)}{\operatorname{SQRR}(\omega)}\right]\right.
$$

Com efeito, rejeita-se $H_{0}$ se $\chi_{\text {cal }}^{2} \geq \chi_{v}^{2}$, sendo $v=p_{\Omega}-p_{\omega}$ o número de graus de liberdade, onde $p_{\Omega}$ é o número de parâmetros estimados no modelo completo, enquanto $\mathrm{p}_{\omega}$ é o número de parâmetros estimados no modelo reduzido. SQRR é a soma de quadrados do resíduo da regressão. 


\subsection{TESTE PARA VERIFICAR A IGUALDADE DE PARÂMETROS E A IDENTIDADE DE MODELOS DE REGRESSÃO NÃO LINEAR}

Na análise de regressão aplicada, por exemplo, em estudos de florestas naturais, na obtenção de equações de crescimento, geralmente se considera uma equação para cada espécie, ou seja, um número de equações correspondente à quantidade de espécies existentes é ajustado, resultando na necessidade de agrupar as espécies com perfis de crescimentos semelhantes.

Graybill (1976) apresentou um modelo geral para testar a igualdade de um conjunto de modelos lineares usando o teste $F$.

Steel \& Torrie (1980) apresentaram testes para verificar a igualdade entre dois coeficientes de regressão e, também, entre mais de dois coeficientes de regressão simples.

Neter et al. (1996) testaram, utilizando o teste $F$, se duas equações de regressão simples eram idênticas.

Regazzi (2003) usou o teste da razão de máxima verossimilhança para verificar a igualdade de parâmetros e a identidade de modelos de regressão não linear, aplicando o modelo logístico para dois tratamentos em uma cultura de milho, medindo a variável matéria seca total em nove ocasiões equidistantes de 15 dias.

Regazzi \& Silva (2004) aplicaram, usando o modelo Michaelis-Menten, o teste da razão de máxima verossimilhança para verificar a igualdade de parâmetros e a identidade de modelos de regressão não linear para dados de um delineamento inteiramente ao acaso. Estudaram a velocidade da reação versus seis doses equidistantes de concentração de substrato de um experimento com Puromycin, considerando dois tratamentos: enzima tratada e não tratada.

Sejam, por exemplo, as hipóteses considerando $g$ tratamentos (espécies ou métodos de manejo):

a) $H_{0}$ : as $g$ equações são idênticas versus $H_{1}: N a \tilde{o} H_{0}$;

b) $H_{0}$ : um determinado subconjunto de parâmetros apresenta igualdade nos $g$ tratamentos versus $H_{1}: N a \tilde{o} H_{0}$.

Seja o modelo de Mitscherlich com erro aditivo e a seguinte parametrização:

$$
Y_{i j}=\beta_{0 i}\left[1-10^{-\beta_{1 i}\left(X_{i j}+\beta_{2 i}\right)}\right]+e_{i j} \quad(i=1, \cdots, g) \quad\left(j=1, \cdots, n_{i}\right)
$$

$Y_{i j}$ : valor observado na $j$-ésima parcela permanente do $i$-ésimo tratamento;

$X_{i j}$ : valor da variável independente (tempo ou ocasião);

$\beta_{0 i}, \beta_{1 i}, \beta_{2 i}$ : parâmetros do modelo e $\hat{\beta}_{0 i}, \hat{\beta}_{1 i}, \hat{\beta}_{2 i}$ seus estimadores; 
$e_{i j}$ : erro aleatório com as pressuposições usuais: $e_{i j} \sim N I D\left(0, \sigma^{2}\right)$;

$N=n_{1}+n_{2}+\cdots+n_{g}$ : é o número total de observações dos $g$ tratamentos.

Algumas hipóteses que podem ser consideradas:

a) $H_{0}^{(1)}: \beta_{01}=\beta_{02}=\cdots=\beta_{0 g}=B_{0}$ vs. $H_{a}^{(1)}$ :nemtodos $\beta_{0 i}$ sãoiguais;

b) $H_{0}^{(2)}: \beta_{11}=\beta_{12} \cdots=\beta_{1 g}=\beta_{1}$ vs. $H_{a}^{(2)}:$ nemtodos $\beta_{1 i}$ são iguais;

c) $H_{0}^{(3)}: \beta_{21}=\beta_{22}=\cdots=\beta_{2 g}=\beta_{2}$ vs. $H_{a}^{(3)}:$ nemtodos $\beta_{2 i}$ sãoiguais;

d) $H_{0}^{(4)}: \beta_{01}=\beta_{02}=\cdots=\beta_{0 g}=\beta_{0} ; \beta_{11}=\beta_{12}=\cdots=\beta_{1 g}=\beta_{1} ; \beta_{21}=\beta_{22}=\cdots=\beta_{2 g}=\beta_{2}$

vs. $H_{a}^{(4)}$ : pelo menos uma igualdadenão ocorre.

Variáveis dummy são as variáveis binárias que só assumem dois valores distintos, normalmente 0 e 1 . Elas são importantes na representação de variáveis qualitativas; existência de mudanças na análise; ocorrência de estratos; etc.

$$
D_{i}=\left\{\begin{array}{c}
D_{i}=1: \text { se a observação } Y_{i j} \text { pertence tratamento }(i) \\
D_{i}=0: \quad(\text { em caso contrário })
\end{array} \quad(i=1, \cdots, g)\right.
$$

Tem-se que: $Y_{i j}=\sum_{i=1}^{g} D_{i} \beta_{0 i}\left[1-10^{-\beta_{i i}\left(X_{i j}+\beta_{2 i}\right)}\right]+e_{i j}$.

As hipóteses formuladas acima podem ser testadas, usando o teste da razão de verossimilhança, pela estatística:

$$
L=\left(\frac{\hat{\sigma}_{\Omega}^{2}}{\hat{\sigma}_{\omega}^{2}}\right)^{\frac{N}{2}}
$$

$\hat{\sigma}_{\Omega}^{2}$ : estimativa de máxima verossimilhança de $\sigma^{2}$ quando nenhuma restrição é feita no espaço paramétrico;

$\hat{\sigma}_{\omega}^{2}$ : estimativa de verossimilhança de $\sigma^{2}$ quando as restrições lineares colocadas em $H_{0}$ são impostas no espaço paramétrico.

A distribuição de $-2 \operatorname{Ln} L$, para grandes amostras, é aproximadamente um qui-quadrado com $v=p_{\Omega}-p_{\omega}$ graus de liberdade, tal que $p_{\Omega}$ é o número de parâmetros estimados em $\Omega$, e $p_{\omega}$ é o número de parâmetros estimados em $\omega$.

Para grandes amostras, Rao (1973):

$$
-2 \operatorname{Ln} L=-N \operatorname{Ln}\left(\frac{\hat{\sigma}_{\Omega}^{2}}{\hat{\sigma}_{\omega}^{2}}\right) \stackrel{{ }^{d}}{\underset{N \rightarrow \infty}{\longrightarrow}} \chi_{v}^{2}
$$

Sejam os seguintes passos para testar a identidade de modelos: 
a) Ajustar o modelo completo $\Omega$ e obter $\hat{\sigma}_{\Omega}^{2}=\frac{S Q R R_{\Omega}}{N}$, sendo $S Q R R_{\Omega}$ a soma de quadrados residual para o modelo completo $\Omega$, onde $p_{\Omega}$ é o número de parâmetros estimados em $\Omega$;

b) Ajustar o modelo reduzido $\omega$, sob a restrição dada por $H_{0}$, e obter $\hat{\sigma}_{\omega}^{2}=\frac{S Q R R_{\omega}}{N}$, sendo $S Q R R \omega$ a soma de quadrados residual para o modelo reduzido $\omega$, donde $p_{\omega}$ é o número de parâmetros estimados em $\omega$;

c) Obter a estatística $\chi_{\text {Cal }}^{2}=-N \operatorname{Ln}\left(\frac{\hat{\sigma}_{\Omega}^{2}}{\hat{\sigma}_{\omega}^{2}}\right)$, ou seja:

$$
\chi_{\text {Cal }}^{2}=-N \operatorname{Ln}\left(\frac{S Q R R_{\Omega} / N}{S Q R R_{\omega} / N}\right)=-N \operatorname{Ln}\left(\frac{S Q R R_{\Omega}}{S Q R R_{\omega}}\right) ;
$$

d) Estabelecer a regra de decisão: se $\chi_{C a l}^{2} \geq \chi_{T a b}^{2} \Rightarrow$ rejeitar $H_{0}$, caso contrário, não rejeitar $H_{0}$. $O$ valor tabelado é função do nível de significância $\alpha$ e do número de graus de liberdade $v=p_{\Omega}-p_{\omega}$.

A estimativa de máxima verossimilhança de $\sigma^{2}$, isto é, $\hat{\sigma}^{2}$, é obtida pela soma de quadrados residual dividida por $\left(N=n_{1}+n_{2}+\cdots+n_{g}\right)$.

\subsection{ANÁLISE DE RESÍDUOS}

Na análise de regressão linear simples ou múltipla, as pressuposições assumidas precisam ser validadas para que os resultados sejam confiáveis. A análise de resíduo pode ser definida como um conjunto de técnicas que objetiva investigar a adequabilidade de um modelo de regressão com base nos resíduos.

A análise de resíduos é um procedimento fundamental na verificação da qualidade do ajuste do modelo e objetiva avaliar a influência das observações no modelo ajustado. A análise de resíduo permite diagnosticar possíveis evidências de violações nas suposições estabelecidas, tais como:

a) Falta de algum componente no modelo;

b) Existência de homocedasticidade, ou seja, se a variância $\left(\sigma^{2}\right)$ é a mesma para todos os $Y_{j}$;

c) Se as suposições de normalidade e independência são válidas para os erros.

Seja o modelo de regressão com $p$ variáveis:

$$
Y_{j}=\beta_{0}+\beta_{1} X_{1 j}+\beta_{2} X_{2 j}+\cdots+\beta_{p} X_{p j}+e_{j} \quad(j=1,2, \cdots, n)
$$


0 resíduo é definido como a diferença entre o valor observado da variável resposta $Y_{j}$ e o seu valor estimado pela regressão $\hat{Y}_{j}$, ou seja, $e_{j}=Y_{j}-\hat{Y}_{j}$.

$$
e_{j}=Y_{j}-\left(\hat{\beta}_{0}+\hat{\beta}_{1} X_{1 j}+\hat{\beta}_{2} X_{2 j}+\cdots+\hat{\beta}_{p} X_{p j}\right)
$$

A concepção básica é que, se o modelo for adequado, os resíduos devem refletir as propriedades impostas pelo componente denominado erro do modelo. Seja o modelo na forma matricial $Y_{v}=X \beta_{v}+e_{v}$, donde:

$Y_{v}^{\prime}=\left[\begin{array}{llll}Y_{1} & Y_{2} & \cdots & Y_{n}\end{array}\right]:$ vetor das $n$ observações;

$X$ : matriz $n \times(p+1)$ do modelo das derivadas parciais de $Y_{j}$ em relação aos parâmetros; $e_{v}^{\prime}=\left[e_{1}, e_{2}, \ldots, e_{n}\right]$ : vetor dos erros;

$\beta_{v}^{\prime}=\left[\beta_{0}, \beta_{1}, \ldots, \beta_{p}\right]$ : vetor dos parâmetros.

Pressuposições:

a) $e_{j^{\prime}}$ e $e_{j}$ são independentes $\left(j^{\prime} \neq j\right)$;

b) $\operatorname{Var}\left(e_{j}\right)=\sigma^{2}$ (constante);

c) $e_{j} \sim N\left(0, \sigma^{2}\right)$;

d) 0 modelo é linear;

e) Não existem dados discrepantes (outliers).

No caso do uso de um modelo de regressão múltipla, também, é necessário diagnosticar a existência de colinearidade e de multicolinearidade entre as variáveis regressoras. As técnicas para diagnosticar as suposições sobre o modelo podem ser gráficas ou através de testes. As técnicas gráficas são subjetivas. 0 ideal é combinar técnicas gráficas com testes estatísticos para verificar a existência de problemas nas suposições sobre o modelo.

Ferreira (2013) cita que na literatura existem os seguintes tipos de resíduos: ordinário, padronizado, estudentizado internamente e estudentizado externamente (resíduo Jackknife).

0 resíduo ordinário, que é definido como $e_{j}=Y_{j}-\hat{Y}_{j}$, não é recomendado para construção dos gráficos. É mais adequado utilizar os resíduos padronizados ou os estudentizados.

O problema básico dos resíduos ordinários é que, por possuírem variâncias distintas, eles não são comparáveis entre si, então, para obter a mesma dispersão em todos os $n$ resíduos preditos, há a necessidade de estabelecer alguma forma de padronização. 
Seja $\hat{\sigma}^{2}=Q M_{\mathrm{Res}}$ o estimador da variância $\sigma^{2}$. 0 resíduo padronizado ( $\mathrm{z}$ ) é a divisão do resíduo ordinário pelo desvio padrão. Este resíduo reduz a variabilidade dentro de uma determinada faixa, mas não elimina o problema de ocorrência de variâncias diferentes.

$$
z_{j}=\frac{e_{j}}{\sqrt{Q M_{\mathrm{Res}}}}=\frac{e_{j}}{\hat{\sigma}}
$$

Os resíduos estudentizados internamente são obtidos por meio da razão entre o resíduo ordinário e o seu estimador do erro padrão específico, ou seja:

$$
z_{j}=\frac{e_{j}}{\sqrt{\left(1-h_{j j}\right) Q M_{\mathrm{Re} s}}} \quad H=\left[h_{j j^{\prime}}\right] \quad H=X\left(X^{\prime} X\right)^{-1} X^{\prime}
$$

O uso do resíduo estudentizado internamente é mais recomendável do que o resíduo padronizado, haja vista considerar a variância individual de cada resíduo ordinário. Por outro lado, se ocorrer da j-ésima observação ser uma observação discrepante, a estimativa da variância poderá ser afetada por esse valor.

0 resíduo estudentizado externamente, também denominado de resíduo de jackknife, foi proposto com o propósito de contornar o problema acima citado, além de possuir algumas propriedades mais interessantes do que as demais formas de padronização. 0 procedimento consiste em eliminar a j-ésima observação e obter o estimador da variância $\hat{\sigma}_{(j)}^{2}$. 0 subscrito $j$ escrito entre parênteses significa tratar-se de um estimador aplicado para as $(n-1)$ observações resultantes da eliminação da $j$-ésima da amostra.

$$
z_{j}=\frac{e_{j}}{\sqrt{\left(1-h_{j j}\right) \hat{\sigma}_{(j)}^{2}}}
$$

De acordo com Ferreira (2013), a matriz $X\left(X^{\prime} X\right)^{-1} X^{\prime}$ é denominada de projetor e representada por $P$, pois projeta o vetor $n$-dimensional das observações $\left(Y_{v}\right)$ no subespaço $(p+1)$-dimensional. Aplicando esta matriz ao vetor das observações $\left(Y_{v}\right)$ é obtido o vetor dos valores preditos $\left(\hat{Y}_{v}\right)$, ou seja, $\hat{Y}_{v}=P Y_{v}$. A matriz $P$ é denominada na análise de regressão simples de matriz Hat e representada por $H$. Os elementos da diagonal da matriz $H$ são designados por $\left(h_{j j}\right)$.

Seja o modelo na forma matricial $Y_{v}=X \beta_{v}+e_{v}$. As equações preditoras de erros, $e_{v}=Y_{v}-X \hat{\beta}_{v}$ e $e_{v}=(I-H) Y_{v}$, são equivalentes.

Dado que: $\hat{\beta}_{v}=\left(X^{\prime} X\right)^{-1} X^{\prime} Y_{v}$ e $\hat{Y}_{v}=X \hat{\beta}_{v}$. Então: $e_{v}=Y_{v}-X \hat{\beta}_{v}$, logo:

$$
\begin{gathered}
e_{v}=Y_{v}-X\left(X^{\prime} X\right)^{-1} X^{\prime} Y_{v}=(I-H) Y_{v} \\
\operatorname{Var}\left(e_{v}\right)=(I-H) \operatorname{Var}\left(Y_{v}\right)(I-H)^{\prime}
\end{gathered}
$$




$$
\begin{gathered}
\operatorname{Var}\left(e_{v}\right)=(I-H)\left(I \sigma^{2}\right)(I-H)^{\prime} \\
\operatorname{Var}\left(e_{v}\right)=(I-H)(I-H)^{\prime} \sigma^{2} \\
\operatorname{Var}\left(e_{v}\right)=\left[(I-H)-(I-H) H^{\prime}\right] \sigma^{2} \\
\operatorname{Var}\left(e_{v}\right)=\left(I-H-H^{\prime}+H H^{\prime}\right) \sigma^{2}
\end{gathered}
$$

Dado que $H H^{\prime}=H^{\prime}$, então: $\operatorname{Var}\left(e_{v}\right)=(I-H) \sigma^{2}$.

$\operatorname{Var}\left(\hat{e}_{v}\right)=(I-H) \hat{\sigma}^{2}$

$\operatorname{Var}\left(\hat{e}_{j}\right)=\left(1-h_{j j}\right) \hat{\sigma}^{2}$ (para j-ésima observação).

Prudente (2009) cita que a distribuição dos resíduos ordinários, no modelo não linear, é matematicamente intratável, principalmente para pequenas amostras. Em geral, esses resíduos possuem esperanças diferentes de zero e distribuição dependente, grandemente dos valores ajustados, o que pode conduzi-los a não refletirem exatamente a distribuição dos erros. Então, nesse caso, os critérios de diagnósticos da regressão normal linear podem falhar quando utilizados na regressão não linear.

As técnicas utilizadas no diagnóstico na regressão não linear, com exceção dos resíduos, são simples adaptações da regressão linear. Na regressão não linear, os resíduos usuais (ordinário, padronizado e estudentizado) formam uma base de utilização significativa na construção de métodos de diagnósticos, mas é necessário cautela ao usá-los.

De acordo com Cook \& Tsai (1985), pode-se empregar uma expansão em série de Taylor até a segunda ordem, expandindo $\mu\left(\hat{\beta}_{v}\right)$, tal que, $Y=\mu\left(\beta_{v}\right)+e_{v}$ e $\hat{e}_{j}=y_{j}-\mu_{j}\left(\hat{\beta}_{v}\right)$, em torno de $\beta_{v}$ para investigar o comportamento dos resíduos na regressão não linear. $\mathrm{A}$ função $\mu\left(\beta_{v}\right)=g\left(\beta_{v} ; X\right)$ é diferenciável em $\beta_{v}$ e $X$ representa a matriz dos valores das variáveis explicativas ou regressoras. Particularmente, derivam aproximações quadráticas para a média e variância dos resíduos ordinários, e para as covariâncias entre os resíduos ordinários e os valores ajustados.

A conclusão da pesquisa é que os resíduos comumente usados nas análises de regressão linear podem produzir resultados enganosos, quando aplicados na regressão não linear, não refletindo de forma correta a distribuição dos erros, então sugerem um novo tipo de resíduo, denominado resíduo projetado, o qual supera muitas das deficiências potenciais dos resíduos ordinários.

Martins (2013) utiliza a análise de resíduos projetados, desenvolvida por Cook \& Tsai (1985), em modelos não lineares de regressão, em uma aplicação no estudo de crescimento de frutos, utilizando o modelo logístico e de Gompertz na análise dos resíduos ordinários e dos resíduos projetados, a fim de verificar as suas vantagens. Os resíduos projetados se mostraram viáveis para o diagnóstico dos modelos e descartaram possíveis tendências apresentadas pelos resíduos padronizados.

Em geral, na regressão não linear, os resíduos projetados apresentam maior precisão nos diagnósticos do que os resíduos ordinários. Prudente (2009) e Martins (2013) 
mostram com mais profundidade a teoria e os procedimentos para a obtenção dos resíduos projetados.

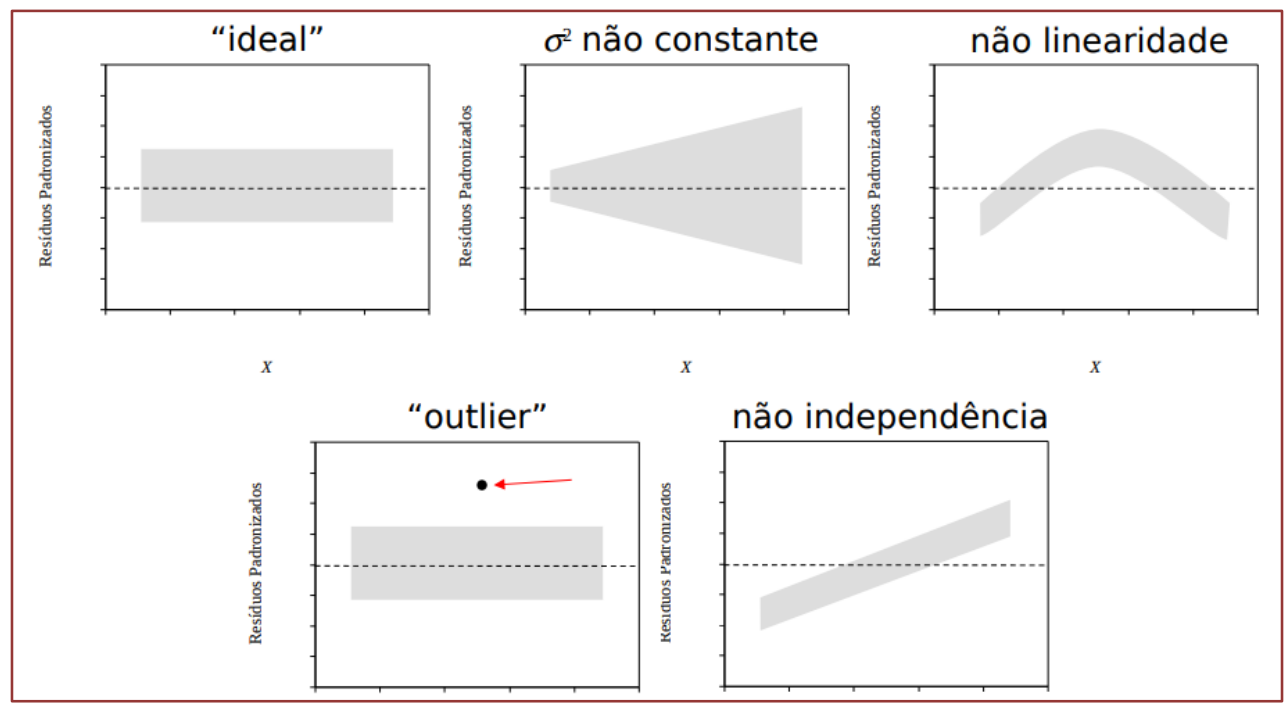

Figura 5.5.1 - Algumas formas de gráfico de distribuição de resíduos. Fonte:

https://www.ime.unicamp.br/ nancy/Cursos/me104/regressao.pdf (17 mar. 2021)

O gráfico dos resíduos versus valores ajustados (valores preditos) é uma das principais técnicas utilizadas para verificar as suposições sobre os resíduos. Os gráficos, além de detectar heteroscedasticidade, podem indicar a não existência de uma relação linear entre as variáveis regressoras ou explicativas com a variável dependente ou resposta. A figura 5.5.1 mostra diversas formas de gráficos configurando vários tipos de distribuições de resíduos em torno do valor zero.

Caso o gráfico apresente uma distribuição dos resíduos aleatória, não demonstrando nenhum tipo de tendência aparente, ou seja, mostrando que a faixa de variação dos resíduos ao longo dos valores ajustados é constante, há indícios de que a variância dos resíduos é homocedástica. Entretanto, se o gráfico apresentar uma forma de funil há o indicativo da presença de heterocedasticidade.

No caso de a variância do erro não ser constante, a solução é fazer uma transformação na variável dependente $Y$ ou usar o método dos mínimos quadrados ponderados.

No caso de uma regressão linear, por exemplo, se os pontos do gráfico formam uma parábola, então há indicativo de seja necessário incluir termos de segundo grau.

\subsection{REGRESSÃO NÃO LINEAR}

A classificação de um modelo como linear, muitas vezes, é interpretada de acordo com o tipo de curva matemática que o modelo descreve.

Ferreira (2013) sugere utilizar um conceito filosófico para esclarecer e definir se um modelo é linear ou não linear, ou seja, classificar se é linear ou não linear nos parâmetros. Formalmente, diz que um modelo é linear se as derivadas parciais da 
variável dependente, em relação a cada parâmetro, não são funções dos próprios parâmetros.

Na verificação, para melhor entendimento, se o modelo é linear ou não linear, sejam os seguintes modelos para estimar o volume $(V)$ de uma árvore em função do seu $D A P(D)$ :

$$
\begin{array}{ll}
\text { a) } V_{j}=\beta_{0}+\beta_{1} D_{j}+\beta_{2} D_{j}^{2}+e_{j} & \text { b) } V_{j}=\beta_{0} D_{j}^{\beta_{1}}+e_{j}
\end{array}
$$

Examinando o modelo (a):

$$
\frac{\partial V_{j}}{\partial \beta_{0}}=1 \quad \frac{\partial V_{j}}{\partial \beta_{1}}=D_{j} \quad \frac{\partial V_{j}}{\partial \beta_{2}}=D_{j}^{2}
$$

Denotando que o modelo (a) é linear, haja vista que nenhuma das derivadas parciais depende dos próprios parâmetros.

Considerando o modelo (b):

$$
\frac{\partial V_{j}}{\partial \beta_{0}}=D_{j}^{\beta_{1}} \quad \frac{\partial V_{j}}{\partial \beta_{1}}=\beta_{0} D_{j}^{\beta_{1}}
$$

0 modelo (b) é não linear nos parâmetros, pois as duas derivadas parciais são funções dos próprios parâmetros.

As equações normais do modelo não linear, ao contrário das equações do modelo de regressão linear, apresentam incógnitas nos dois lados das equações, impossibilitando obter as soluções explicitas para as incógnitas com base nos valores conhecidos. De acordo com Gujarati \& Porter (2011), existem vários procedimentos ou algoritmos para estimar os parâmetros para os modelos não lineares. Destacam-se os seguintes:
a) Método da busca direta ou da tentativa e erro ou método da livre derivada;
b) Método da otimização direta;
c) Método da linearização iterativa.

Neste livro, será utilizado o método de linearização de Gauss-Newton, o qual consiste em linearizar uma função não linear em torno de alguns valores iniciais dos parâmetros. Pelo método dos mínimos quadrados ordinários (MQO) é estimada a função linear e os valores escolhidos inicialmente são ajustados. Estes valores ajustados são utilizados para relinearizar o modelo e novamente são estimados pelo MQO e os valores estimados são reajustados. 0 processo continua até que não haja mais alterações substanciais nos valores estimados a partir das últimas iterações. 0 método de Gauss Newton usa a expansão de séries de Taylor para a linearização da função não linear. 


\subsubsection{DETERMINAÇÃO DAS ESTIMATIVAS DOS PARÂMETROS, PARA O MODELO DE MITSCHERLICH, PELO MÉTODO DOS MÍNIMOS QUADRADOS ORDINÁRIOS}

Seja $X$ a variável, simbolizando as ocasiões, para caracterizar unidades de tempo no modelo de regressão de Mitscherlich.

$$
Y_{j}=\beta_{0}\left[1-10^{-\beta_{1}\left(X_{j}+\beta_{2}\right)}\right]+e_{j}
$$

Supondo que os erros $e_{j}(j=1, \cdots, n)$ sejam não correlacionados entre si e possuindo distribuição normal com média zero e variância $\sigma^{2}$. Usando o método dos mínimos quadrados ordinários (MQO), para obter os estimadores de mínimos quadrados, têm-se:

$$
Z=\sum_{j=1}^{n} e_{j}^{2}=\sum_{j=1}^{n}\left\{Y_{j}-\beta_{0}\left[1-10^{-\beta_{1}\left(X_{j}+\beta_{2}\right)}\right]\right\}^{2}
$$

a) $\frac{\partial Z}{\partial \beta_{0}}=2 \sum_{j=1}^{n}\left\{Y_{j}-\beta_{0}\left[1-10^{-\beta_{1}\left(X_{j}+\beta_{2}\right)}\right]\right\} \times-\left[1-10^{-\beta_{1}\left(X_{j}+\beta_{2}\right)}\right] \quad \frac{\partial Z}{\partial \beta_{0}}=0$

$$
\sum_{j=1}^{n} Y_{j}\left[1-10^{-\hat{\beta}_{1}\left(X_{j}+\hat{\beta}_{2}\right)}\right]=\hat{\beta}_{0} \sum_{j=1}^{n}\left[1-10^{-\hat{\beta}_{1}\left(X_{j}+\hat{\beta}_{2}\right)}\right]^{2}
$$

b) $\frac{Z}{\partial \beta_{1}}=2 \sum_{j=1}^{n}\left\{Y_{j}-\beta_{0}\left[1-10^{-\beta_{1}\left(X_{j}+\beta_{2}\right)}\right]\right\}\left[\beta_{0} 10^{-\beta_{1}\left(X_{j}+\beta_{2}\right)}\right] \times-\left(X_{j}+\beta_{2}\right) \ln 10 \quad \frac{\partial Z}{\partial \beta_{1}}=0$

$$
\sum_{j=1}^{n}\left[Y_{j}\left(X_{j}+\hat{\beta}_{2}\right) 10^{-\hat{\beta}_{1}\left(X_{j}+\hat{\beta}_{2}\right)}\right]=\hat{\beta}_{0} \sum_{j=1}^{n}\left(X_{j}+\hat{\beta}_{2}\right)\left[1-10^{-\hat{\beta}_{1}\left(X_{j}+\hat{\beta}_{2}\right)}\right] \times 10^{-\beta_{1}\left(X_{j}+\hat{\beta}_{2}\right)}
$$

c) $\frac{\partial Z}{\partial \beta_{2}}=2 \sum_{j=1}^{n}\left\{Y_{j}-\beta_{0}\left[1-10^{-\beta_{1}\left(X_{j}+\beta_{2}\right)}\right]\right\}\left[\beta_{0} 10^{-\beta_{1}\left(X_{j}+\beta_{2}\right)}\right]\left(-\beta_{1}\right) \operatorname{Ln} 10 \quad \frac{\partial Z}{\partial \beta_{2}}=0$

$$
\sum_{j=1}^{n} Y_{i} 10^{-\hat{\beta}_{1}\left(X_{j}+\hat{\beta}_{2}\right)}=\hat{\beta}_{0} \sum_{J=1}^{n}\left[1-10^{-\hat{\beta}_{1}\left(X_{j}+\hat{\beta}_{2}\right)}\right] 10^{-\hat{\beta}_{1}\left(X_{j}+\hat{\beta}_{2}\right)}
$$

Resultando: $\left\{\begin{array}{c}\sum_{j=1}^{n} Y_{j}\left[1-10^{-\hat{\beta}_{1}\left(X_{j}+\hat{\beta}_{2}\right)}\right]=\hat{\beta}_{0} \sum_{j=1}^{n}\left[1-10^{-\hat{\beta}_{1}\left(X_{j}+\hat{\beta}_{2}\right)}\right]^{2} \\ \sum_{j=1}^{n}\left[Y_{j}\left(X_{j}+\hat{\beta}_{2}\right) 10^{-\hat{\beta}_{1}\left(X_{j}+\hat{\beta}_{2}\right)}\right]=\hat{\beta}_{0} \sum_{j=1}^{n}\left(X_{j}+\hat{\beta}_{2}\right) 10^{-\beta_{1}\left(X_{j}+\hat{\beta}_{2}\right)}\left[1-10^{-\hat{\beta}_{1}\left(X_{j}+\hat{\beta}_{2}\right)}\right] \\ \sum_{j=1}^{n} Y_{j} 10^{-\hat{\beta}_{1}\left(X_{j}+\hat{\beta}_{2}\right)}=\hat{\beta}_{0} \sum_{j=1}^{n} 10^{-\hat{\beta}_{1}\left(X_{j}+\hat{\beta}_{2}\right)}\left[1-10^{-\hat{\beta}_{1}\left(X_{j}+\hat{\beta}_{2}\right)}\right]\end{array}\right.$ 


\subsubsection{DETERMINAÇÃO DAS ESTIMATIVAS DOS PARÂMETROS, PELO MÉTODO DOS} MÍNIMOS QUADRADOS ORDINÁRIOS, USANDO A FORMA MATRICIAL PARA 0 MODELO DE MITSCHERLICH.

Seja o modelo não linear $F$ definido para o vetor de parâmetros $\beta_{v}^{\prime}=\left[\begin{array}{llll}\beta_{1} & \beta_{1} & \cdots & \beta_{m}\end{array}\right]$ e para o vetor de variáveis regressoras da $j$-ésima unidade amostral $Z_{v j}^{\prime}=\left[\begin{array}{llll}Z_{1 j} & Z_{2 j} & \cdots & Z_{m j}\end{array}\right]$.

$$
Y_{j}=F_{j}\left(\beta_{v}, Z_{v j}\right)+e_{j}
$$

Na forma matricial: $Y_{v}=F\left(\beta_{v}\right)+e_{v}$.

A equação de Mitscherlich possui uma única variável regressora $X$.

$$
Y_{j}=\beta_{0}\left[1-10^{-\beta_{1}\left(X_{j}+\beta_{2}\right)}\right]+e_{j}
$$

$\beta_{v}^{\prime}=\left[\beta_{0}, \beta_{1}, \beta_{2}\right]$ : é o vetor de parâmetros;

$e_{v}^{\prime}=\left[e_{1}, e_{2}, \cdots, e_{n}\right]$ : é o vetor dos erros.

Obtenção do vetor $F\left(\beta_{v}\right)$ que será representado por $F_{v}$ :

$F_{v}^{\prime}=\left\{\beta_{0}\left[1-10^{-\beta_{1}\left(X_{1}+\beta_{2}\right)}\right] \quad \beta_{0}\left[1-10^{-\beta_{1}\left(X_{2}+\beta_{2}\right)}\right] \quad \cdots \quad \beta_{0}\left[1-10^{-\beta_{1}\left(X_{n}+\beta_{2}\right)}\right]\right\}$ : é o vetor do modelo.

Na forma matricial: $Y_{v}=F\left(\beta_{v}\right)+e_{v}$, onde $Y_{v}^{\prime}=\left[\begin{array}{llll}Y_{1} & Y_{2} & \cdots & Y_{n}\end{array}\right]$ é o vetor das $n$ observações.

$$
\left[\begin{array}{c}
Y_{1} \\
Y_{2} \\
\vdots \\
Y_{n}
\end{array}\right]=\left[\begin{array}{c}
\beta_{0}-10^{-\beta_{1}\left(X_{1}+\beta_{2}\right)} \\
\beta_{0}-10^{-\beta_{1}\left(X_{2}+\beta_{2}\right)} \\
\vdots \\
\beta_{0}-10^{-\beta_{1}\left(X_{n}+\beta_{2}\right)}
\end{array}\right]+\left[\begin{array}{c}
e_{1} \\
e_{2} \\
\vdots \\
e_{n}
\end{array}\right]
$$

De acordo com Ferreira (2013), utilizando o método de mínimos quadrados, tem-se: $L\left(\beta_{v}\right)=e_{v}^{\prime} e_{v}$. Substituindo $e_{v}=Y_{v}-F_{v}$ e derivando em relação ao vetor $\beta_{v}$.

$$
\begin{gathered}
L\left(\beta_{v}\right)=e_{v}^{\prime} e_{v}=\left(Y_{v}-F_{v}\right)^{\prime}\left(Y_{v}-F_{v}\right)=Y_{v}^{\prime} Y_{v}-2 Y_{v}^{\prime} F_{v}+F_{v}^{\prime} F_{v} \\
\frac{\partial L}{\partial \beta_{v}}=\frac{-2 \partial Y_{v}^{\prime} F_{v}}{\partial \beta_{v}}+\frac{\partial F_{v}^{\prime} F_{v}}{\partial \beta_{v}}
\end{gathered}
$$

Dado que:

$$
\frac{-2 \partial Y_{v}^{\prime} F_{v}}{\partial \beta_{v}}=\frac{-2 \partial Y_{v}^{\prime} F_{v}}{\partial F_{v}} \times \frac{\partial F_{v}}{\partial \beta_{v}}=-2 Y_{v}^{\prime} X
$$


Tal que $X=\frac{\partial F_{v}}{\partial \beta_{v}}$ é a matriz de derivadas parciais, em que cada coluna é formada pela derivada da função linear em relação aos parâmetros.

Simplificando $\frac{\partial F_{v}^{\prime} F_{v}}{\partial \beta_{v}}$ para: $\frac{\partial F_{v}^{\prime} F_{v}}{\partial \beta_{v}}=\frac{\partial F_{v}^{\prime} F_{v}}{\partial F_{v}} \times \frac{\partial F_{v}}{\partial \beta_{v}}=2 F_{v}^{\prime} X$

Portanto: $\frac{\partial L}{\partial \beta_{v}}=-2 Y_{v}^{\prime} X+2 F_{v}^{\prime} X$

$$
\frac{\partial L}{\partial \beta_{v}}=0 \rightarrow X^{\prime} F_{v}=X^{\prime} Y_{v}
$$

Tal que, $X^{\prime} F_{v}=X^{\prime} Y_{v}$, é o sistema de equações normais para os modelos não lineares. No entanto, como $F_{v}$ e $X$ são funções de $\beta_{v}$, um procedimento iterativo deve ser utilizado para obter $\hat{\beta}_{v}$. No caso da equação de Mitscherlich, têm-se:

$$
\begin{aligned}
& X=\left[\begin{array}{ccc}
{\left[1-10^{-\beta_{1}\left(X_{1}+\beta_{2}\right)}\right]} & \beta_{0} 10^{-\beta_{1}\left(X_{1}+\beta_{2}\right)}\left(X_{1}+\beta_{2}\right) \ln 10 & \beta_{0} 10^{-\beta_{1}\left(X_{1}+\beta_{2}\right)}\left(\beta_{1}\right) \operatorname{Ln} 10 \\
{\left[1-10^{-\beta_{1}\left(X_{2}+\beta_{2}\right)}\right]} & \beta_{0} 10^{-\beta_{1}\left(X_{2}+\beta_{2}\right)}\left(X_{2}+\beta_{2}\right) \operatorname{Ln} 10 & \beta_{0} 10^{-\beta_{1}\left(X_{2}+\beta_{2}\right)}\left(\beta_{1}\right) \operatorname{Ln} 10 \\
\cdots & \cdots & \cdots \\
\cdots & \cdots & \cdots \\
{\left[1-10^{-\beta_{1}\left(X_{n}+\beta_{2}\right)}\right]} & \beta_{0} 10^{-\beta_{1}\left(X_{n}+\beta_{2}\right)}\left(X_{n}+\beta_{2}\right) \operatorname{Ln} 10 & \beta_{0} 10^{-\beta_{1}\left(X_{n}+\beta_{2}\right)}\left(\beta_{1}\right) \operatorname{Ln} 10
\end{array}\right] \\
& Y_{v}^{\prime}=\left[\begin{array}{llll}
Y_{1} & Y_{2} & \cdots & Y_{n}
\end{array}\right] \\
& F_{v}^{\prime}=\left\{\begin{array}{llll}
\beta_{0}\left[1-10^{-\beta_{1}\left(X_{1}+\beta_{2}\right)}\right] & \beta_{0}\left[1-10^{-\beta_{1}\left(X_{2}+\beta_{2}\right)}\right] & \cdots & \beta_{0}\left[1-10^{-\beta_{1}\left(X_{n}+\beta_{2}\right)}\right]
\end{array}\right\} \\
& X^{\prime} F_{v}=\left[\begin{array}{c}
\hat{\beta}_{o} \sum_{j=1}^{n}\left[1-10^{-\hat{\beta}_{1}\left(X_{j}+\hat{\beta}_{2}\right)}\right]^{2} \\
\hat{\beta}_{0}^{2} \operatorname{Ln} 10 \sum_{j=1}^{n} 10^{-\hat{\beta}_{1}\left(X_{j}+\hat{\beta}_{2}\right)}\left[1-10^{-\hat{\beta}_{1}\left(X_{j}+\hat{\beta}_{2}\right)}\left(X_{j}+\hat{\beta}_{2}\right)\right. \\
\hat{\beta}_{0}^{2} \hat{\beta}_{1} \operatorname{Ln} 10 \sum_{j=1}^{n} 10^{-\hat{\beta}_{1}\left(X_{j}+\hat{\beta}_{2}\right)}\left[1-10^{-\hat{\beta}_{1}\left(X_{j}+\hat{\beta}_{2}\right)}\right]
\end{array}\right] \\
& X^{\prime} Y_{v}=\left[\begin{array}{c}
\sum_{j=1}^{n} Y_{j}\left[1-10^{-\hat{\beta}_{1}\left(X_{j}+\hat{\beta}_{2}\right)}\right] \\
\beta_{0} \operatorname{Ln} 10 \sum_{j=1}^{n} Y_{j}\left(X_{j}+\hat{\beta}_{2}\right) 10^{-\hat{\beta}_{1}\left(X_{j}+\hat{\beta}_{2}\right)} \\
\beta_{0} \beta_{1} \operatorname{Ln} \sum_{j=1}^{n} Y_{j} 10^{-\hat{\beta}_{1}\left(X_{j}+\hat{\beta}_{2}\right)}
\end{array}\right]
\end{aligned}
$$




$$
X^{\prime} Y_{v}=\left[\begin{array}{c}
\sum_{j=1}^{n} Y_{j}\left[1-10^{-\hat{\beta}_{1}\left(X_{j}+\hat{\beta}_{2}\right)}\right] \\
\sum_{j=1}^{n} Y_{j}\left(X_{j}+\hat{\beta}_{2}\right) 10^{-\hat{\beta}_{1}\left(X_{j}+\hat{\beta}_{2}\right)} \\
\sum_{j=1}^{n} Y_{j} 10^{-\hat{\beta}_{1}\left(X_{j}+\hat{\beta}_{2}\right)}
\end{array}\right]=X^{\prime} F_{v}=\left[\begin{array}{c}
\hat{\beta}_{o} \sum_{j=1}^{n}\left[1-10^{-\hat{\beta}_{1}\left(X_{j}+\hat{\beta}_{2}\right)}\right]^{2} \\
\hat{\beta}_{0} \sum_{j=1}^{n}\left(X_{j}+\hat{\beta}_{2}\right) 10^{-\hat{\beta}_{1}\left(X_{j}+\hat{\beta}_{2}\right)}\left[1-10^{-\hat{\beta}_{1}\left(X_{j}+\hat{\beta}_{2}\right)}\right] \\
\hat{\beta}_{0} \sum_{j=1}^{n} 10^{-\hat{\beta}_{1}\left(X_{j}+\hat{\beta}_{2}\right)}\left[1-10^{-\hat{\beta}_{1}\left(X_{j}+\hat{\beta}_{2}\right)}\right]
\end{array}\right]
$$

Tem-se, então, o sistema de equações normais para o modelo de Mitscherlich:

$$
\left\{\begin{array}{c}
\sum_{j=1}^{n} Y_{j}\left[1-10^{-\hat{\beta}_{1}\left(X_{j}+\hat{\beta}_{2}\right)}\right]=\hat{\beta}_{0} \sum_{j=1}^{n}\left[1-10^{-\hat{\beta}_{1}\left(X_{j}+\hat{\beta}_{2}\right)}\right]^{2} \\
\sum_{j=1}^{n}\left[Y_{j}\left(X_{j}+\hat{\beta}_{2}\right) 10^{-\hat{\beta}_{1}\left(X_{j}+\hat{\beta}_{2}\right)}\right]=\hat{\beta}_{0} \sum_{j=1}^{n}\left(X_{j}+\hat{\beta}_{2}\right) 10^{-\beta_{1}\left(X_{j}+\hat{\beta}_{2}\right)}\left[1-10^{-\hat{\beta}_{1}\left(X_{j}+\hat{\beta}_{2}\right)}\right] \\
\sum_{j=1}^{n} Y_{j} 10^{-\hat{\beta}_{1}\left(X_{j}+\hat{\beta}_{2}\right)}=\hat{\beta}_{0} \sum_{j=1}^{n} 10^{-\hat{\beta}_{1}\left(X_{j}+\hat{\beta}_{2}\right)}\left[1-10^{-\hat{\beta}_{1}\left(X_{j}+\hat{\beta}_{2}\right)}\right]
\end{array}\right.
$$

Como não existe uma solução explicita, haja vista, que o vetor $F_{v}$ e a matriz $X$ são funções do vetor de parâmetros $\beta_{v}$, então há a necessidade de iniciar um processo iterativo. Os métodos iterativos precisam do estabelecimento de um vetor $\beta_{v}^{*}$ com valores iniciais para os parâmetros, tal que, de acordo com o método a ser utilizado, deve ser melhorado até que a soma dos quadrados dos resíduos seja minimizada.

Considerando que $S Q_{\mathrm{Res}}(k)=e_{v}^{\prime} e_{v}$ é a soma de quadrados dos resíduos na $k$-ésima iteração, então a matriz $X$ e o vetor $Y_{v}$ são usados para calcular o vetor $\Delta_{v}$, tal que, para um valor constante $\lambda$, tem-se: $S Q_{\operatorname{Re} s}\left(\beta_{v k}^{*}+\lambda \Delta_{v}\right)<S Q_{\operatorname{Re} s}\left(\beta_{v k}^{*}\right)$.

No SAS existem quatros métodos disponíveis que diferem na forma de obter o vetor $\Delta_{v}$, o qual é calculado para propiciar as trocas no vetor de parâmetros. De uma forma geral, de acordo com Ferreira (2013), os critérios básicos para os métodos: Gradiente, GaussNewton, Newton e Marquardt, são:

Gradiente: $\Delta_{v}=X^{\prime} e_{v}$

Gauss-Newton: $\Delta_{v}=\left(X^{\prime} X\right)^{-} X^{\prime} e_{v}$

Newton: $\Delta_{v}=G^{-} X^{\prime} e_{v}$

Marquardt: $\Delta_{v}=\left[X^{\prime} X+\delta \operatorname{diag}\left(X^{\prime} X\right)\right]^{-} X^{\prime} e_{v}$

$\left(X^{\prime} X\right)^{-}$: é uma matriz inversa generalizada que pode ser uma reflexiva (g2). 0 ideal é usar uma matriz inversa generalizada de Moore-Penrose. 
Os métodos Gauss-Newton e Marquardt realizam a regressão de resíduos em relação às primeiras derivadas do modelo não linear em relação aos parâmetros, até que haja convergência. 0 método de Newton faz a regressão dos resíduos em relação a uma função das segundas derivadas do modelo não linear com relação aos parâmetros $\left(G^{-}\right)$.

0 método de Marquardt é uma combinação entre os métodos de Gauss-Newton e o método do gradiente, onde a fórmula para atualização do vetor dos parâmetros é: $\Delta_{v}=\left[X^{\prime} X+\delta \operatorname{diag}\left(X^{\prime} X\right)\right]^{-} X^{\prime} e_{v}$. Quando $\delta \rightarrow 0$ aproxima-se do método de GaussNewton e se $\delta \rightarrow \infty$ tende ao método de gradiente.

\subsection{MÉTODO DE GAUSS-NEWTON}

O método de Gauss-Newton usa a expansão em séries de Taylor do vetor de funções $F\left(\beta_{v}\right)=F\left(\beta_{v}^{*}\right)+X\left(\beta_{v}-\beta_{v}^{*}\right)+\cdots$, donde a matriz das primeiras derivadas $X$ é avaliada no ponto $\beta_{v}^{*}$.

De acordo com Ferreira (2013), substituindo os dois termos dessa expansão nas equações normais $X^{\prime} F_{v}=X^{\prime} Y_{v}$, têm-se:

$$
\begin{gathered}
X^{\prime} F_{v}\left(\beta_{v}\right)=X^{\prime} Y_{v} \\
X^{\prime}\left[F\left(\beta_{v}^{*}\right)+X\left(\beta_{v}-\beta_{v}^{*}\right)\right]=X^{\prime} Y_{v} \\
X^{\prime} X\left(\beta_{v}-\beta_{v}^{*}\right)=X^{\prime} Y_{v}-X^{\prime} F_{v}\left(\beta_{v}^{*}\right) \\
X^{\prime} X \Delta_{v}=X^{\prime} e_{v}
\end{gathered}
$$

Portanto: $\Delta_{v}=\left(X^{\prime} X\right)^{-} X^{\prime} e_{v}$.

Obtido o valor de $\Delta_{v}=\left(X^{\prime} X\right)^{-} X^{\prime} e_{v}$ para $\beta_{v}^{*}$, aplica-se as equações $\beta_{v(k+1)}^{*}=\beta_{v k}^{*}+\lambda \Delta_{v} \mathrm{e}$ $S Q_{\text {Res }}\left(\beta_{v k}^{*}+\lambda \Delta_{v}\right)<S Q_{\text {Res }}\left(\beta_{v k}^{*}\right)$, para obter o vetor das estimativas dos parâmetros do primeiro passo. 0 procedimento é repetido até que o vetor não se altere (convergência) dentro da precisão estipulada.

Para melhor entender o método interativo de Gauss-Newton, seja o modelo para estimar a altura de árvore (ALT), em função do DAP, utilizando 55 observações da espécie.

$$
A L T_{j}=\beta_{0} e^{\beta_{1} D A P}+u_{j} \quad j=1, . ., n
$$

Para facilitar o desenvolvimento seja $Y_{j}=A L T$ e $X_{j}=D A P$.

$$
\begin{gathered}
Y_{j}=\beta_{0} e^{\beta_{1} X_{j}}+u_{j} \quad j=1, . ., n \\
Z=\sum_{j=1}^{n} u_{j}^{2}=\sum_{j=1}^{n}\left(Y_{j}-\beta_{0} e^{\beta_{1} X_{j}}\right)^{2} \\
\frac{\partial Z}{\partial \beta_{0}}=2 \sum_{j=1}^{n}\left(Y_{j}-\beta_{0} e^{\beta_{1} X_{j}}\right)\left(-e^{\beta_{1} X_{j}}\right) \\
\frac{Z}{\partial \beta_{1}}=2 \sum_{j=1}^{n}\left(Y_{j}-\beta_{0} e^{\beta_{1} X_{j}}\right)\left(-\beta_{0} e^{\beta_{1} X_{j}}\right)\left(X_{j}\right)
\end{gathered}
$$


Sejam as equações normais considerando $\hat{\beta}_{0}$ e $\hat{\beta}_{1}$, como estimadores MMO dos parâmetros $\beta_{0}$ e $\beta_{1}$.

$$
\left\{\begin{array}{c}
\sum_{j=1}^{n} Y_{j} e^{\hat{\beta}_{1} X_{j}}=\hat{\beta}_{0} \sum_{j=1}^{n} e^{2 \hat{\beta}_{1} X_{j}} \\
\sum_{j=1}^{n} Y_{j} X_{j} e^{\hat{\beta}_{1} X_{j}}=\hat{\beta}_{0} \sum_{j=1}^{n} X_{j} e^{2 \hat{\beta}_{1} X_{j}}
\end{array}\right.
$$

Devido à existência de incógnitas em ambos os lados das equações não é possível obter soluções explícitas. Seja a obtenção da aproximação linear dessa função não linear pela expansão de Taylor considerando dois parâmetros. De acordo com Gujarati \& Porter (2011), considerando a expansão em torno de $X=a$ e $Z=b$, o teorema de Taylor mostra que:

Tal que:

$$
\begin{aligned}
& f(X, Z)=f(a, b)+f_{X}(a, b)(X-a)+f_{Z}(a, b) f(Z-b)+\frac{1}{2 !} f_{X X}(a, b)(X-a)^{2} \\
& -2 f_{X Z}(a, b)(X-a)(Z-b)+f_{Z Z}(a, b)(Z-b)^{2}+\ldots
\end{aligned}
$$

$f_{X}$ : derivada parcial da função em relação a $X$ e $f_{X X}$ é a segunda derivada da função em relação a $X$;

$f_{Z}$ : derivada parcial da função em relação a $Z$ e $f_{Z Z}$ é a segunda derivada da função em relação a $Z$;

$f_{X Z}$ : derivada da função em relação a $X$ e $Z$.

Como o interesse, de acordo com o método de Gauss-Newton, é obter a aproximação linear da função $Y_{j}=\beta_{0} e^{\beta_{1} X_{j}}+u_{j}$, então serão utilizados os termos lineares de $f(X, Z)$ :

$$
f(X, Z)=f(a, b)+f_{X}(a, b)(X-a)+f_{Z}(a, b) f(Z-b)
$$

Linearizando a função em $\beta_{0}=\beta_{0}^{*}$ e $\beta_{1}=\beta_{1}^{*}$, e definindo os valores de $\beta_{0}^{*}$ e de $\beta_{1}^{*}$ como valores fixos dados, ou seja, soluções preliminares, então de 5.7.1:

$$
f\left(\beta_{0}, \beta_{1}\right)=f\left(\beta_{0}^{*}, \beta_{1}^{*}\right)+f_{\beta_{0}}\left(\beta_{0}^{*}, \beta_{1}^{*}\right)\left(\beta_{0}-\beta_{0}^{*}\right)+f_{B_{1}}\left(\beta_{0}^{*}, \beta_{1}^{*}\right) f\left(\beta_{1}-\beta_{1}^{*}\right)
$$

Supondo $\beta_{0}^{*}=6,6$ e $\beta_{1}^{*}=3,1$, como soluções iniciais propostas, para estimar os valores dos coeficientes, têm-se:

$$
f\left(\beta_{0}^{*}, \beta_{1}^{*}\right)=6,6 e^{3,1 X_{i}}
$$

Dado que: $f_{\beta_{0}}=e^{\beta_{1} X_{j}}$ e $f_{\beta_{1}}=\beta_{0} X_{j} e^{\beta_{1} X_{j}}$. Então:

$$
f\left(\beta_{0}, \beta_{1}\right)=6,6 e^{3,1 X_{j}}+e^{3,1 X_{j}}\left(\beta_{0}-6,6\right)+6,6 X_{j} e^{3,1 X_{j}}\left(\beta_{1}-3,1\right)
$$


Fazendo $Y_{j}^{*}=f\left(\beta_{0}, \beta_{1}\right)-6,6 e^{3,1 X_{j}}$. Resulta:

$$
Y_{j}^{*}=e^{3,1 X_{j}}\left(\beta_{0}-6,6\right)+6,6 X_{j} e^{3,1 X_{j}}\left(\beta_{1}-3,1\right)
$$

Fazendo também: $X_{1 j}=e^{3,1 X_{j}} \quad X_{2 j}=6,6 X_{j} e^{3,1 X_{j}} \quad \alpha_{1}=\beta_{0}-6,6 \quad \alpha_{2}=\beta_{1}-3,1$

Tem-se, então, o modelo de regressão linear $Y_{j}^{*}=\alpha_{1} X_{1 j}+\alpha_{2} X_{2 j}+u_{j}$.

Dado que $Y_{j}^{*}, X_{1 j}$ e $X_{2 j}$ podem ser obtidos através do banco de dados, logo os valores de $\alpha_{1}$ e $\alpha_{2}$ podem ser obtidos pelo MQO, possibilitando obter: $\beta_{0}=\hat{\alpha}_{1}+6,6$ e $\beta_{1}=\hat{\alpha}_{2}+3,1$.

Definindo esses valores como $\beta_{0}^{* * *}=\hat{\alpha}_{1}+6,6$ e $\beta_{1}^{* * *}=\hat{\alpha}_{2}+3,1$, o procedimento é aplicado sucessivamente na equação 5.7.2 até que haja convergência.

Tabela 5.7.1 - Resultados (SAS) para os valores iniciais $\beta_{0}^{*}=6,0$ e $\beta_{1}^{*}=3,1$

\begin{tabular}{c|c|c|c}
\multicolumn{4}{c}{ Método: Gauss-Newton/Iterações } \\
Iteração & $\widehat{\beta_{0}}$ & $\widehat{\beta_{1}}$ & Soma de Quadrados \\
\hline 0 & 6,0000 & 3,1000 & 370,1 \\
\hline 1 & 6,6726 & 3,0961 & 303,8 \\
\hline 2 & 6,6723 & 3,0967 & 303,8 \\
\hline
\end{tabular}

Na tabela 5.7.1 estão os resultados da regressão não linear pelo método de GaussNewton, usando os valores iniciais $\beta_{0}^{*}=6,6$ e $\beta_{1}^{*}=3,1$, onde foram suficientes apenas duas iterações para atingir os valores das estimativas MQO dos parâmetros.

Tabela 5.7.2 - Resultados considerando os valores iniciais $\left(\beta_{0}^{*}=9.0\right.$ e $\left.\beta_{1}^{*}=5.0\right)$.

\begin{tabular}{c|c|c|c}
\multicolumn{4}{c}{ Método: Gauss-Newton $/$ Iterações } \\
Iteração & $\widehat{\beta_{0}}$ & $\widehat{\beta_{1}}$ & Soma de Quadrados \\
\hline 0 & 9,0000 & 5,0000 & 4971,7 \\
\hline 1 & 6,5489 & 3,8747 & 391,7 \\
\hline 2 & 6,6776 & 3,1262 & 304,0 \\
\hline 3 & 6,6742 & 3,0951 & 303,8 \\
\hline 4 & 6,6723 & 3,0968 & 303,8 \\
\hline 5 & 6,6724 & 3,0967 & 303,8 \\
\hline
\end{tabular}

Verifica-se que os valores iniciais escolhidos estão muito próximos das estimativas MQO, mas caso fosse utilizado os seguintes valores mais distantes $\left(\beta_{0}^{*}=9,0\right.$ e $\left.\beta_{1}^{*}=5,0\right)$, ver tabela 5.7.2, o número de iterações necessário passa para cinco.

0 método iterativo de Gauss-Newton encontra-se disponível no Sistema de Análise Estatística (SAS). Para usar o procedimento NLIN do SAS é necessário estabelecer, 
através da opção PARMS, os valores iniciais das estimativas dos parâmetros. No exemplo não linear apresentado, os valores iniciais dos parâmetros estimados poderiam ser os obtidos pela linearização do modelo, ou seja, $L n A L T_{j}=\operatorname{Ln} \beta_{0}+\beta_{1} D A P_{j}+L n u_{j}$.

\subsection{AVALIAÇÃO DA ADEQUACIDADE DOS MODELOS TESTADOS}

Em modelos de regressão linear que incluem o termo constante (intercepto), o coeficiente de determinação $R^{2}$ representa a proporção da variação explicada pelo modelo. Neste caso, o quadrado do coeficiente de correlação entre os valores observados e preditos é exatamente o valor do $R^{2}$. Se o modelo é linear e o termo constante não está presente (sem intercepto), o $R^{2}$ deve ser redefinido conforme Searle (1971). Muito cuidado deve-se ser tomado na sua interpretação, porque ele não é igual ao quadrado do coeficiente de correlação entre os valores observados e preditos. Pode ocorrer que o valor do coeficiente de determinação na versão sem intercepto, domine em muito, em modelos equivalentes, o valor correspondente ao caso com intercepto.

O coeficiente de determinação (ajustado ou não ajustado) não tem nenhum significado no caso de modelos não lineares, pois muitas vezes ocorrem valores altos mesmo havendo enorme discrepância entre os valores observados e estimados. Segundo Ratkowsky (1990) independentemente de haver ou não termo constante no modelo, o $R^{2}$ não tem nenhum significado para modelos não lineares. SOUZA (1998) recomenda que no caso de regressão não linear, atendidas as pressuposições da análise de variância, a adequacidade do ajustamento do modelo pode ser medida pelo quadrado do coeficiente de correlação entre os valores observados e preditos, pela análise gráfica dos resíduos e pela verificação da magnitude da variância residual obtida por máxima verossimilhança.

Para decidir sobre o ajustamento de um modelo não linear é fundamental verificar a discrepância entre os valores observados e preditos. É importante fazer uma análise do gráfico de resíduos e verificar a magnitude da variância residual obtida por máxima verossimilhança. Evidentemente que quanto menor for o valor da variância residual melhor será o ajuste.

Atendidas as pressuposições usuais da análise de variância, pode ser usado o coeficiente de variação (CV\%) considerando a raiz quadrada da estimativa de máxima verossimilhança da variância residual $(R Q M R)$.

$$
\begin{gathered}
\mathrm{RQMR}=\sqrt{\frac{\sum_{i=1}^{N}\left(Y_{i}-\hat{Y}_{i}\right)^{2}}{N}} \\
\mathrm{CV} \%=\frac{R Q M R}{\bar{Y}}
\end{gathered}
$$




\subsection{VALIDAÇÃO DA PREDIÇÃO DO MODELO DE REGRESSÃO PELO TESTE DE GRAYBILL}

Este procedimento de validação da predição consiste em ajustar um modelo linear de primeiro grau, mas considerando os valores estimados $\left(\hat{Y}_{j}\right)$ como variável dependente e os valores observados no experimento $\left(Y_{j}\right)$ como variável independente, isto é, $\hat{Y}_{j}=\beta_{0}+\beta_{1} Y_{j}+e_{j}$. Conforme Graybill (1976), a significância da regressão é avaliada aplicando o teste $F\left(H_{0}\right)$ para as estimativas dos parâmetros, conforme o seguinte teste de hipótese:

$$
H_{0}: C^{\prime} \beta_{v}=\left[\begin{array}{ll}
1 & 0 \\
0 & 1
\end{array}\right]\left[\begin{array}{l}
\beta_{0} \\
\beta_{1}
\end{array}\right]=\left[\begin{array}{l}
0 \\
1
\end{array}\right] \text {, versus, } H_{A}: C^{\prime} \beta_{v}=\left[\begin{array}{ll}
1 & 0 \\
0 & 1
\end{array}\right]\left[\begin{array}{l}
\beta_{0} \\
\beta_{1}
\end{array}\right] \neq\left[\begin{array}{l}
0 \\
1
\end{array}\right] \quad \quad C^{\prime}=\left[\begin{array}{ll}
1 & 0 \\
0 & 1
\end{array}\right]
$$

$H_{0}$ : hipótese nula significando que $\beta_{0}=0$ e $\beta_{1}=1$, ou seja, o intercepto é igual a zero e a inclinação é igual a 1 e $H_{A}$ : hipótese alternativa de rejeição de $H_{0}$. Não rejeitar $H_{0}$ quando $F\left(H_{0}\right)$ é menor do que $F_{\alpha ; m ; n-p}$.

$$
\begin{gathered}
F\left(H_{0}\right)=\frac{\left(C^{\prime} \hat{\beta}_{v}-\theta_{v}\right)^{\prime}\left[C^{\prime}\left(Y^{\prime} Y\right)^{-1} C\right]^{-1}\left(C^{\prime} \hat{B}_{v}-\theta_{v}\right)}{m \hat{\sigma}^{2}} \\
\theta_{v}=\left[\begin{array}{l}
0 \\
1
\end{array}\right] \quad \hat{\beta}_{v}=\left(Y^{\prime} Y\right)^{-1}\left(Y^{\prime} \hat{Y}_{v}\right) \quad \hat{\sigma}^{2}=Q M R_{\operatorname{Res}}=\frac{\hat{Y}_{v}^{\prime} \hat{Y}_{v}-\hat{\beta}_{v}^{\prime} Y^{\prime} \hat{Y}_{v}}{n-p} \quad Y=\left[\begin{array}{cc}
1 & Y_{1} \\
1 & Y_{2} \\
\cdots & \cdots \\
1 & Y_{n}
\end{array}\right]
\end{gathered}
$$

$Y$ : é a matriz do modelo tendo o número de colunas igual ao número de elementos de $\beta_{v}$ e o número de linhas igual ao tamanho da amostra $(n)$;

$\hat{Y}_{v}$ : vetor dos valores estimados pela regressão;

$Y_{v}=\left[Y_{1}, Y_{2}, \cdots, Y_{n}\right]:$ vetor dos dados observados no experimento;

$p=2$ : número de parâmetros estimados;

$m=2$ : número de linhas da matriz $\mathrm{C}$;

$C$ : é a matriz com $m$ linhas e $p$ colunas;

$Q M_{\mathrm{Res}}$ : é o quadrado médio do resíduo. 


\section{Capítulo 6}

\section{Ciclo de corte economicamente aconselhável}

Neste capítulo, antes de tratar especificamente sobre o tema em foco, preliminarmente serão abordados alguns tópicos fundamentais de matemática, com o intuito de melhor embasar os estudantes e/ou leitores para o tema principal, que será obter a equação de crescimento da floresta ou das espécies, com base na função de Mitscherlich, visando predizer a rotação economicamente aconselhável. Desse modo, os seguintes temas são apresentados: derivadas; máximo e mínimos de uma função com uma variável; máximo e mínimo de uma função com duas variáveis; exemplo de máximo e mínimo de uma função com três variáveis; máximos e mínimos condicionados; multiplicadores de Lagrange; rotação economicamente aconselhável pela equação de Mitscherlich e pela equação de Von-Bertalanffy. 


\subsection{DERIVADAS}

Dada uma função $y=f(x)$ e $x_{0}$ um ponto de seu domínio. Tomando a diferença $\Delta y=f(x)-f\left(x_{0}\right)$, onde $x \neq x_{0}$, é o acréscimo ou incremento da função e $\Delta x=x-x_{0}$ como o acréscimo da variável independente $x$, tem-se a razão incremental definida pelo quociente:

$$
\frac{\Delta y}{\Delta x}=\frac{f(x)-f\left(x_{0}\right)}{x-x_{0}}
$$

Define-se a derivada de $y=f(x)$ no ponto $x_{0}$ e representada por $f^{\prime}\left(x_{0}\right)$ ou $y^{\prime}$, ao limite da razão incremental quando $x$ tende para o ponto $x_{0}$.

$$
f^{\prime}\left(x_{0}\right)=\lim _{x \rightarrow x_{0}} \frac{f(x)-f\left(x_{0}\right)}{x-x_{0}}
$$

Desde $\Delta x=x-x_{0}$, se $x \rightarrow x_{0}$, então $\Delta x \rightarrow 0$, logo: $f^{\prime}\left(x_{0}\right)=\lim _{\Delta x \rightarrow 0} \frac{\Delta y}{\Delta x}$.

0 valor numérico da derivada de uma função $y=f(x)$, no ponto $\left(x_{0}, y_{0}\right)$, é o coeficiente angular (inclinação) da reta tangente no gráfico da função nesse ponto com o eixo das abscissas. Derivadas de algumas funções, sendo $u=f(x)$ e $v=z(x)$ :
a) $y=k$ (constante)
$y^{\prime}=0$
b) $y=a x^{n}$
$y^{\prime}=n \times a x^{n-1}$
c) $y=u v$
$y^{\prime}=u v^{\prime}+v u^{\prime}$
d) $y=\frac{u}{v}$
$y^{\prime}=\frac{v u^{\prime}-u v^{\prime}}{v^{2}}$
e) $y=u^{n}$
$y^{\prime}=n u^{n-1} u^{\prime}$
f) $y=\sqrt[n]{u}$
$y^{\prime}=\frac{u^{\prime}}{n \sqrt[n]{u^{n-1}}}$
g) $y=\log _{a} x$
$y^{\prime}=\frac{1}{x} \log _{a} e$
h) $y=\log _{e} x$
$y^{\prime}=\frac{1}{x}$
i) $y=\log _{a} u$
$y^{\prime}=\frac{1}{u} u^{\prime} \log _{a} e$
j) $y=\log _{e} u$
$y^{\prime}=\frac{u^{\prime}}{u}$
k) $y=a^{x}$
$y^{\prime}=a^{x} \operatorname{Ln} a$ 
l) $y=e^{x}$

$$
y^{\prime}=e^{x}
$$

m) $y=a^{u}$

$y^{\prime}=a^{u} u^{\prime} \operatorname{Ln} a$

n) $y=e^{u}$

$y^{\prime}=e^{u} u^{\prime}$

\subsection{MÁXIMO E MÍNIMO DE UMA FUNÇÃO COM UMA VARIÁVEL}

A figura 6.1 mostra os pontos de abscissas $x_{1}, x_{2}, x_{3}, x_{4}$. Estes pontos são chamados de pontos extremos da função. Os valores $f\left(x_{1}\right)$ e $f\left(x_{3}\right)$ são chamados de máximos relativos e $f\left(x_{2}\right)$ e $f\left(x_{4}\right)$ são denominados de mínimos relativos.

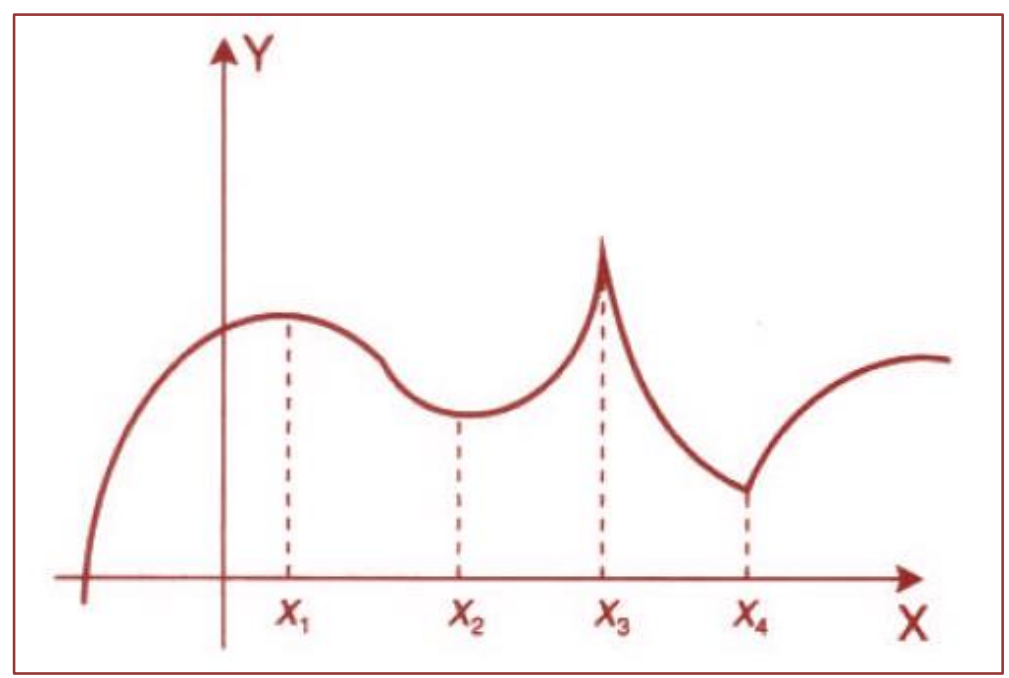

Figura 6.1 - Fonte: Flemming \& Gonçalves (2007).

São chamados de pontos de máximos relativos, os pontos onde a função deixa de ser crescente e passa a ser decrescente, enquanto que os pontos denominados de mínimos relativos são os pontos onde a função passa de decrescente para crescente.

O Teorema de Rolle mostra que uma função $f(x)$ definida e contínua em $[a, b]$, derivável em $(a, b)$ e se anula em suas extremidades [ $f(a)=f(b)=0$ ], então existe pelo menos um ponto intermediário $x=x_{0}, a<x_{0}<b$, tal que a derivada $f^{\prime}(x)$ se anula, ou seja, $f^{\prime}\left(x_{0}\right)=0$.

a) $(a, b)$ : significa intervalo aberto: $\{x / a<x<b\}$;

b) $[\mathrm{a}, \mathrm{b}]$ : significa intervalo fechado: $\{x / a \leq x \leq b\}$. 
De acordo com Piskounov (1978):

a) Se a função $f(x)$ derivável sobre o segmento [a, b] é crescente sobre este segmento, então, a sua derivada não é negativa sobre $[\mathrm{a}, \mathrm{b}]$, isto é, $f^{\prime}(x) \geq 0$;

b) Se a função $f(x)$ é contínua sobre [a, b], derivável no intervalo (a,b) e, mais, se $f^{\prime}(x)>0$ para $a<x<b$, então, $f(x)$ é uma função crescente sobre [a, b];

c) Se $f(x)$ é uma função decrescente sobre [a, b], então, $f^{\prime}(x) \leq 0$ sobre este segmento. Se $f^{\prime}(x)<0$ no intervalo (a,b), então, $f(x)$ é decrescente sobre [a, b].

Uma função $f(x)$ admite um máximo no ponto $x_{0}$, se o valor da função $f(x)$ é neste ponto maior que em qualquer outro ponto de um determinado intervalo contendo o ponto $x_{0}$, ou seja, $f(x)$ admite um máximo no ponto $x=x_{0}$, se $f\left(x_{0}+\Delta x\right)<f\left(x_{0}\right)$ para todos os $\Delta x$ (positivos ou negativos) rigorosamente pequenos em valor absoluto.

Uma função $f(x)$ admite um mínimo no ponto $x=x_{0}$, se $f\left(x_{0}+\Delta x\right)>f\left(x_{0}\right)$ para todos os $\Delta x$ (positivos ou negativos) rigorosamente pequenos em valor absoluto.

Se a função derivável $f(x)$ tem um máximo ou um mínimo no ponto $x=x_{0}$, então a sua derivada se anula nesse ponto, ou seja, $f^{\prime}(x)=0$, significando que a tangente é paralela ao eixo das abscissas nos pontos de máximo ou de mínimo. Entretanto, ressalta-se que, essa condição não é suficiente para concluir se o ponto é máximo ou mínimo.

Piskounov (1979) cita que dada uma função contínua num intervalo contendo o ponto crítico $x_{0}$ e derivável em qualquer ponto desse intervalo (exceto, possivelmente, no ponto $x_{0}$ ). Se a derivada muda de sinal de mais para menos quando passa pelo ponto crítico da esquerda para a direita, a função tem um máximo para $x=x_{0}$. Se a derivada muda de sinal de menos para mais quando passa pelo ponto $x_{0}$ da esquerda para direita, a função tem um mínimo nesse ponto, ou seja:

$$
\begin{gathered}
\text { Se }\left\{\begin{array}{lll}
f^{\prime}(x)>0 & \text { para } & x<x_{0} \\
f^{\prime}(x)<0 & \text { para } & x>x_{0}
\end{array} \text { : a função admite um máximo no ponto } x_{0} .\right. \\
\text { Se }\left\{\begin{array}{lll}
f^{\prime}(x)<0 & \text { para } & x<x_{0} \\
f^{\prime}(x)>0 & \text { para } & x>x_{0}
\end{array} \text { : a função admite um mínimo no ponto } x_{0} .\right.
\end{gathered}
$$

Gomes \& Nogueira (1980) definem os pontos de inflexão de uma função como os pontos de máximo ou de mínimo da sua derivada primeira.

Quando existe $f^{\prime}(x)$, a condição $f^{\prime}(x)=0$ é necessária para a existência de um extremo relativo em $x_{0}$, mas não é suficiente. 0 ponto $x_{0}$ pertencente ao domínio da função tal que $f^{\prime}(x)=0$ é chamado ponto crítico. 
Seja uma função $f(x)$ derivável no intervalo (a,b) e $x_{0}$ um ponto crítico de $f(x)$ nesse intervalo, isto é, $f^{\prime}\left(x_{0}\right)=0$, com $a<x_{0}<b$, se $f(x)$ admite $f^{\prime \prime}\left(x_{0}\right)$, em $(a, b)$, pelo critério da segunda derivada, têm-se:

a) Se $f^{\prime \prime}\left(x_{0}\right)<0, f(x)$ tem um valor máximo relativo em $x_{0}$;

b) Se $f^{\prime \prime}\left(x_{0}\right)>0, f(x)$ tem um valor mínimo relativo em $x_{0}$.

Para visualizar os pontos extremos de uma função é muito importante observar o gráfico da função.

Uma função definida em um determinado intervalo pode admitir vários pontos extremos relativos. 0 maior valor é denominado de máximo absoluto da função nesse intervalo. De forma análoga, o menor valor é chamado de mínimo absoluto.

\subsection{MÁXIMO E MÍNIMO DE UMA FUNÇÃO COM DUAS VARIÁVEIS}

Diz-se que a função $z=f(x, y)$ admite um máximo no ponto $\left(x=x_{0}\right.$ e $\left.y=y_{0}\right)$, se $f\left(x_{0}, y_{0}\right)>f(x, y)$ para todos os pontos $(x, y)$ rigorosamente vizinhos do ponto $\left(x_{0}, y_{0}\right)$, mas diferentes deste ponto.

Analogamente, a função $z=f(x, y)$ admite um mínimo no ponto $\left(x_{0}, y_{0}\right)$ se $f\left(x_{0}, y_{0}\right)<f(x, y)$, para todos os pontos $(x, y)$ suficientemente vizinhos do ponto $\left(x_{0}, y_{0}\right)$, mas diferentes deste ponto.

Se $z=f(x, y)$ admite um ponto extremo (máximo ou mínimo) para os valores ( $x=x_{0} \mathrm{e}$ $\left.y=y_{0}\right)$, então, cada derivada parcial de primeira ordem de $z=f(x, y)$ anula-se para esses valores das variáveis independentes ou não existe neste ponto.

$$
f_{x}^{\prime}\left(x_{0}, y_{0}\right)=0 \quad f_{y}^{\prime}\left(x_{0}, y_{0}\right)=0
$$

No caso de uma função $z=f(x, y)$ com duas variáveis cujas derivadas parciais de $1^{a}$ e $2^{a}$ ordem são contínuas em um conjunto aberto que contém $\left(x_{0}, y_{0}\right)$, sendo $\left(x_{0}, y_{0}\right)$ um ponto crítico de $z=f(x, y)$, e considerando det $H(x, y)$ como o determinante da matriz Hessiana $H$, tem-se:

$$
\operatorname{det} H(x . y)=\left|\begin{array}{ll}
f_{x x} & f_{x y} \\
f_{y x} & f_{y y}
\end{array}\right|=f_{x x} f_{y y}-f_{x y}^{2}
$$


a) Se $\operatorname{det} H\left(x_{0}, y_{0}\right)>0$ e $f_{x x}\left(x_{0}, y_{0}\right)>0$, então $f\left(x_{0}, y_{0}\right)$ é um mínimo de $f(x, y)$;

b) Se det $H\left(x_{0}, y_{0}\right)>0$ e $f_{x x}\left(x_{0}, y_{0}\right)<0$, então $f\left(x_{0}, y_{0}\right)$ é um máximo de $f(x, y)$;

c) Se det $H\left(x_{0}, y_{0}\right)<0$, então $f(x, y)$ não têm máximo nem mínimo. Neste caso é um ponto de sela;

d) Se $\operatorname{det} H\left(x_{0}, y_{0}\right)=0$, nada se pode afirmar (pode ser um ponto de máximo, de mínimo ou de sela).

De acordo com Gonçalves \& Flemming (2007), a matriz $H(x, y)$ é denominada de matriz Hessiana e o seu determinante $\operatorname{det} H(x, y)$ é chamado de determinante hessiano da função $f(x, y)$ ou hessiano da função.

$$
H(x, y)=\left[\begin{array}{cc}
\frac{\rho^{2} f(x, y)}{\rho x^{2}} & \frac{\rho^{2} f(x, y)}{\rho y \rho x} \\
\frac{\rho^{2} f(x, y)}{\rho x \rho y} & \frac{\rho^{2} f(x, y)}{\rho y^{2}}
\end{array}\right]
$$

Exercício 6.3.1. Seja a função $Z=f(x, y)=x^{2}-2 x+3 y^{2}-12 y+3$, por meio da qual se deseja obter o ponto crítico.

a) Tomar as derivadas parciais de primeira ordem e obter o ponto $\left(x_{0}, y_{0}\right)$ :

$$
\frac{\partial Z}{\partial x}=2 x-2=0 \quad \frac{\partial Z}{\partial y}=6 y-12=0 \quad\left(x_{0}, y_{0}\right)=(1,2)
$$

b) Obter a matriz Hessiana $H$ e definir a natureza de $\left(x_{0}, y_{0}\right)=(1,2)$ :

$$
f_{x x}=2 \quad f_{y y}=6 \quad f_{x y}=f_{y x}=0 \quad H=\left[\begin{array}{ll}
2 & 0 \\
0 & 6
\end{array}\right] \quad \operatorname{det} H=12>0
$$

Como $\operatorname{det} H=12>0$ e $f_{x x}=2$, ou seja, $f_{x x}>0$, então $\left(x_{0}, y_{0}\right)=(1,2)$ é um ponto de mínimo. 


\subsection{EXEMPLO DE OBTENÇÃO DE MÁXIMO E MÍNIMO DE UMA FUNÇÃO COM TRÊS VARIÁVEIS}

Exercício 6.4.1. Seja $Z=f(x, y, z)=x^{2}-2 x y+3 y^{2}+2 z^{2}+y z+6 x-2 y$. Obter os pontos críticos.

a) Tomar as derivadas parciais de primeira ordem e obter o ponto $\left(x_{0}, y_{0}, z_{0}\right)$ :

$$
\begin{array}{lrl}
\frac{\partial Z}{\partial x}=2 x-2 y+6=0 & x=-\frac{61}{15} \\
\frac{\partial Z}{\partial y}=-2 x+6 y+z-2=0 & y=-\frac{16}{15} \\
\frac{\partial Z}{\partial z}=4 z+y=0 & z=\frac{4}{15}
\end{array}
$$

b) Obter a matriz Hessiana $H$ e definir a natureza do ponto $\left(x_{0}, y_{0}, z_{0}\right)$ :

$$
\begin{gathered}
f_{x x}=2 f_{y y}=6 \quad f_{z z}=4 \quad f_{x y}=-2 \quad f_{x z}=0 \quad f_{y z}=1 \\
H=\left[\begin{array}{ccc}
2 & -2 & 0 \\
-2 & 6 & 1 \\
0 & 1 & 4
\end{array}\right] \quad \operatorname{det} H=30
\end{gathered}
$$

b1) Diagonalizar a matriz Hessiana $(H)$, através de operações elementares, para definir a natureza do ponto $\left(x_{0}, y_{0}, z_{0}\right)$. Resultando:

$$
D=\left[\begin{array}{ccc}
2 & 0 & 0 \\
0 & 4 & 0 \\
0 & 0 & 15 / 4
\end{array}\right]
$$

Dado que os elementos da diagonal principal são todos positivos, conclui-se que a matriz Hessiana é definida positiva, então a função passa por um mínimo relativo no ponto:

$$
\left(x_{0}, y_{0}, z_{0}\right)=\left(-\frac{61}{15},-\frac{16}{15}, \frac{4}{15}\right)
$$

Entende-se como operações elementares: permutar entre si quaisquer duas linhas (colunas); multiplicar os elementos de uma linha (coluna) por um escalar (constante); adicionar a uma linha (coluna) outra linha (coluna) cujos elementos sejam multiplicados por um escalar (constante). 
b2) Diagonalizar a matriz Hessiana, pela decomposição espectral de $H$, ver capítulo 2, seção 2.8, para definir a natureza do ponto $\left(x_{0}, y_{0}, z_{0}\right)$. Têm-se da álgebra de matrizes: $H=P D P^{\prime}$ e $D=P^{\prime} H P$.

$P$ : é a matriz composta pelos autovetores associados aos autovalores segundo a sequência $\left(\lambda_{1}>\lambda_{2}>\lambda_{3}\right)$ e $D$ é matriz diagonal composta pelos autovalores de $H$. Usando o programa de operações com vetores e matrizes (Proc IML), tabela C1 do apêndice C, então:

$$
P=\left[\begin{array}{ccc}
-0,349382 & 0,238434 & 0,9061356 \\
0,8918807 & -0,211794 & 0,3996151 \\
0,2871955 & 0,947783 & -0,138658
\end{array}\right] \quad D=\left[\begin{array}{ccc}
7,1054826 & 0 & 0 \\
0 & 3,7765379 & 0 \\
0 & 0 & 1,1179795
\end{array}\right]
$$

A decomposição espectral mostra que a matriz Hessiana $H$ é definida positiva, haja vista os seus autovalores serem todos positivos, então a função passa por um mínimo relativo no ponto $\left(x_{0}, y_{0}, z_{0}\right)=\left(-\frac{61}{15},-\frac{16}{15}, \frac{4}{15}\right)$.

\subsection{MÁXIMOS E MÍNIMOS CONDICIONADOS}

É frequente a ocorrência de problemas de determinação de máximos e mínimos condicionados de funções, como por exemplo, obter os pontos críticos da função $f(x, y)$, mas condicionada a uma (ou várias) restrição sobre os valores das variáveis, tal como a restrição $g(x, y)=c$.

Cita-se, como exemplo, o planejamento de amostragem para a realização de um levantamento florestal, onde a amostra é dimensionada visando a maximizar a precisão, mas condicionada a atender a uma determinada função custo. É também o caso da análise econômica para determinar a data da exploração de uma floresta a ser manejada, mas condicionando obter a maximização da receita líquida.

Marchand (2016) cita que problemas de otimização restrita podem ser muito complexos, não havendo um método geral para determinar a solução de todas essas classes de questões. Em algumas situações simples é possível obter um resultado explicitando uma variável em função das outras na restrição, substituindo o resultado na função objetivo e resolvendo o problema de otimização irrestrita (ou não restrita) resultante.

Gonçalves \& Flemming (2007) citam que entre os métodos que permitem analisar situações mais gerais, está o método dos Multiplicadores de Lagrange. Por meio desse método, um problema de otimização restrita com $p$ variáveis e $m$ variáveis de igualdade é transformado em uma solução de otimização não restrita com $(p+m)$ variáveis. Então, o método dos multiplicadores de Lagrange consiste em transformar problemas de otimização com restrição em resoluções sem restrição, através da inserção de um novo parâmetro, denominado de multiplicador de Lagrange $(\lambda)$. 
Um problema de otimização com restrição pode ser escrito da seguinte forma: minimize (ou maximize) uma função real submetida a uma função limitadora (ou restritiva).

\subsection{MÉTODO DOS MULTIPLICADORES DE LAGRANGE (JOSEPH LOUIS LAGRANGE 1736-1813)}

0 método dos multiplicadores de Lagrange permite encontrar extremos (máximos e mínimos) de uma função com uma ou mais variáveis submetidas a uma ou mais restrições. 0 método consiste em construir a função $(L)$ de Lagrange, originada pela introdução de novas variáveis $\left(\lambda_{\mathrm{i}}\right)$ chamadas de multiplicadores de Lagrange. Então, as análises são feitas a partir da função de Lagrange.

Seja um problema de uma função com $p$ variáveis $f\left(x_{1}, x_{2}, \cdots, x_{p}\right)$ e $m$ funções de restrição, $g_{1}\left(x_{1}, x_{2}, \cdots, x_{p}\right), \ldots, g_{m}\left(x_{1}, x_{2}, \cdots, x_{p}\right)$, definidas e com derivadas parciais contínuas. Então, o método dos multiplicadores de Lagrange se baseia no fato que todo extremo relativo de uma função $f\left(x_{1}, x_{2}, \cdots, x_{p}\right)$ sujeita às restrições: $g_{i}\left(x_{1}, x_{2}, \cdots, x_{p}\right)=c_{i}$, tal que $c_{i}(i=1, m)$ são constantes, ocorre em um ponto crítico da função de Lagrange (L).

Seja a função $L$ definida para as variáveis $x_{1}, x_{2}, \cdots, x_{p}$ :

$$
L=f\left(x_{1}, x_{2}, \cdots, x_{p}\right)+\lambda_{1} g_{1}\left(x_{1}, x_{2}, \cdots, x_{p}\right)+\cdots+\lambda_{m} g_{m}\left(x_{1}, x_{2}, \cdots, x_{p}\right)
$$

Os números reais $\lambda_{\mathrm{i}}$ que tornam compatível o sistema são chamados multiplicadores de Lagrange. Os termos $\lambda_{i}$ podem ser adicionados ou subtraídos.

Com efeito, considerando o exemplo da função com duas variáveis $f(x, y)$ com a restrição $g(x, y)=c$, tem-se que, os pontos de máximo e/ou mínimo condicionados de $f(x, y)$ devem satisfazer o sistema de equações 6.6.1:

$$
\begin{aligned}
& L=L(x, y, \lambda)=f(x, y)+\lambda g[(x, y)-c] \\
& \frac{\partial L}{\partial x}=0 \quad \frac{\partial L}{\partial y}=0 \quad \frac{\partial L}{\partial \lambda}=0
\end{aligned}
$$

Então, resolvendo o sistema de equações 6.6.1, diversas soluções podem ser obtidas. 0 ponto $\left(x_{0}, y_{0}\right)$ desejado, onde a função $f(x, y)$ tem seus extremos sujeitos à restrição, estão entre essas soluções.

O método dos multiplicadores de Lagrange apenas fornece os pontos críticos da função sem definir a sua natureza, ou seja, se é de mimo ou de máximo. Assim sendo, as suas classificações devem ser feitas usando outros argumentos, entre eles os meios geométricos. 0 método de Lagrange é aplicável também a funções não lineares. 
Exercício 6.6.1 - Seja a função $f(x, y)=x^{2}-2 x+3 y^{2}-12 y+3$, vista no exercício 6.3.1, através da qual se deseja obter o ponto crítico, mas respeitando-se a condição $g(x, y)=x+2 y=7$.

a) Como visto no exercício 6.3.1, ocorreu que $\operatorname{det} H=12>0$ e $f_{x x}=2$, então $\left(x_{0}, y_{0}\right)=(1,2)$ é um ponto de mínimo;

b) Usando o procedimento clássico de substituição de $x=7-2 y$ em $f(x, y)$, obter o mínimo condicionado de $f(x, y)$ sob a condição $x+2 y=7$ :

$$
\begin{gathered}
f(x, y)=(7-2 y)^{2}-2(7-2 y)+3 y^{2}-12 y+3 \\
f(x, y)=49-28 y+4 y^{2}-14+4 y+3 y^{2}-12 y+3 \\
f(x, y)=7 y^{2}-36 y+38
\end{gathered}
$$

$\frac{d f(x, y)}{d y}=14 y-36=0$, onde implica: $y=\frac{36}{14}=\frac{18}{7}$

Como $x=7-2 y$, então: $x=7-2 \times \frac{18}{7}=\frac{13}{7}$

0 mínimo da função $f(x, y)=x^{2}-2 x+3 y^{2}-12 y+3$, sob a restrição $g(x, y)=x+2 y=7$, ocorrerá para $x=\frac{13}{7}$ e $y=\frac{18}{7}$.

c) Obter o mínimo condicionado para $g(x, y)=x+2 y=7$, mas através do método dos multiplicadores de Lagrange.

Seja, então, a função escrita na forma:

$$
\begin{aligned}
& L=L(x, y, \lambda)=f(x, y)+\lambda g(x, y) \\
& L=x^{2}-2 x+3 y^{2}-12 y+3+\lambda(x+2 y-7) \\
& \frac{\partial L}{\partial x}=2 x-2+\lambda=0 \quad \frac{\partial L}{\partial y}=6 y-12+2 \lambda=0 \quad \frac{\partial L}{\partial \lambda}=x+2 y-7=0 \\
& \left\{\begin{array}{l}
2 x-2=-\lambda \\
3 y-6=-\lambda
\end{array} \rightarrow 2 x-3 y=-4\right. \\
& \left\{\begin{array} { c } 
{ x + 2 y = 7 } \\
{ 2 x - 3 y = - 4 }
\end{array} \rightarrow \left\{\begin{array}{c}
-2 x-4 y=-14 \\
2 x-3 y=-4
\end{array} \rightarrow \quad y=\frac{18}{7} \quad x=\frac{13}{7}\right.\right.
\end{aligned}
$$


Os resultados confirmam o obtido usando a metodologia convencional vista anteriormente.

Exercício 6.6.2 - Seja a função $Z=f(x, y)=x^{2}-2 x y+3 y^{2}-6 x-2 y$, pela qual se deseja obter o ponto crítico, mas respeitando-se a condição $g(x, y)=x+2 y=2$.

a) Estudar natureza do ponto crítico da função $Z=f(x, y)=x^{2}-2 x y+3 y^{2}-6 x-2 y$ :

$$
\begin{gathered}
\frac{\partial Z}{\partial x}=2 x-2 y-6=0 \quad \frac{\partial Z}{\partial y}=-2 x+6 y-2=0 \quad(x, y)=(5,2) \\
H=\left[\begin{array}{cc}
2 & -2 \\
-2 & 6
\end{array}\right] \quad \operatorname{det} H=8>0
\end{gathered}
$$

Como $\operatorname{det} H=8>0$ e $f_{x x}=2>0$, então $\left(x_{0}, y_{0}\right)=(5,2)$ é um ponto de mínimo.

b) Obter o mínimo condicionado sob a condição $g(x, y)=x+2 y=2$, pelo método dos multiplicadores de Lagrange:

$$
\begin{gathered}
L=x^{2}-2 x y+3 y^{2}-6 x-2 y+\lambda(x+2 y-2) \\
\frac{\partial L}{\partial x}=2 x-2 y-6+\lambda=0 \quad \rightarrow \quad \lambda=-2 x+2 y+6 \quad(1) \\
\frac{\partial L}{\partial y}=-2 x+6 y-2+2 \lambda=0 \quad \rightarrow \quad \lambda=x-3 y+1 \quad(2) \\
\frac{\partial L}{\partial \lambda}=x+2 y-2=0 \\
(1)=(2) \quad \rightarrow-2 x+2 y+6=x-3 y+1 \\
\left\{\begin{array}{l}
-3 x+5 y=-5 \\
x+2 y=2
\end{array} \quad \begin{array}{l}
-3 x+5 y=-5 \\
3 x+6 y=6
\end{array} \quad \begin{array}{l}
\rightarrow \\
x=\frac{1}{11} \quad x=\frac{20}{11}
\end{array}\right.
\end{gathered}
$$

Então sob a condição $g(x, y)=x+2 y=2$, o ponto $\left(x_{0}, y_{0}\right)=\left(\frac{20}{11}, \frac{1}{11}\right)$ minimiza a função $f(x, y)=x^{2}-2 x y+3 y^{2}-6 x-2 y$. 
Exercício 6.6.3 - Seja a função $f(x, y, z)$. Obter pelo método dos multiplicadores de Lagrange o mínimo sob a restrição: $g(x, y, z)=x+2 y+z=4$.

$$
f(x, y, z)=x^{2}-2 x y+3 y^{2}+2 z^{2}+y z+6 x-2 y
$$

a) No exercício 6.4.1 é mostrado que $f(x, y, z)$ possui um ponto de mínimo;

b) Obter o mínimo condicionado da função $f(x, y, z)$ pelo método dos multiplicadores de Lagrange, sob a restrição $g(x, y, z)=x+2 y+z=4$.

Seja a função de $L$ de Lagrange:

$$
\begin{gathered}
L=x^{2}-2 x y+3 y^{2}+2 z^{2}+y z+6 x-2 y+\lambda(x+2 y+z-4) \\
\frac{\partial L}{\partial x}=2 x-2 y+6+\lambda=0 \quad \frac{\partial L}{\partial y}=-2 x+6 y+z-2+2 \lambda=0 \\
\frac{\partial L}{\partial z}=4 z+y+\lambda=0 \quad \rightarrow \quad \lambda=-4 z-y \\
\frac{\partial L}{\partial \lambda}=x+2 y+z-4=0
\end{gathered}
$$

Como $\lambda=-4 z-y$, então:

$$
\begin{gathered}
\left\{\begin{array}{c}
2 x-2 y+(-4 z-y)=-6 \\
-2 x+6 y+z+2(-4 z-y)=2 \\
x+2 y+z=4
\end{array}\right. \\
z=\frac{42}{83} \quad y=11 z-4=\frac{130}{83} \quad x=-2 y-z+4=\frac{30}{83} \\
-2 x+4 y-7 z=2 \\
x+2 y+z=4
\end{gathered} \rightarrow\left\{\begin{array}{c}
2 x-3 y-4 z=-6 \\
8 y-5 z=10
\end{array}\right.
$$

Sob a condição $x+2 y+z=4$, o ponto $\left(x_{0}, y_{0}, z_{0}\right)=\left(\frac{30}{83}, \frac{130}{83}, \frac{42}{83}\right)$ minimiza a função:

$$
f(x, y, z)=x^{2}-2 x y+3 y^{2}+2 z^{2}+y z+6 x-2 y
$$




\subsection{EQUAÇÃO DE MITSCHERLICH PARA ESTIMAR O CRESCIMENTO DE UMA FLORESTA.}

Nas ciências biológicas, na maioria dos casos, é mais recomendável usar equações não lineares para a modelagem de estudos de crescimento de espécies. Neste caso, são referências: Draper \& Smith (1981), Ratkowsky (1990), Gallant (1987), Hoffmann (2011), Souza (1998), dentre outros.

Prudente (2009) cita alguns modelos mostrados na tabela 6.7.1. Nesses modelos o parâmetro $\alpha$ é o valor máximo esperado ou assíntota da variável resposta. 0 parâmetro $\beta$ está relacionado com o intercepto, isto é, o valor correspondente quando $X=0$ (ocasião). 0 parâmetro $\gamma$ está relacionado com a taxa de crescimento da curva e, finalmente, o parâmetro $\delta$ apresentado em alguns modelos é utilizado para aumentar a flexibilidade dos mesmos nos ajustes dos dados. Neste livro, para estabelecer o ciclo de corte de uma floresta manejada e considerando as suas características matemáticas, será usada a equação de Mitscherlich. Esta equação pela sua formulação é muito utilizada no estudo da variação de crescimento de vegetais em ensaios de adubação.

Tabela 6.7.1 - Alguns modelos normais não lineares aplicáveis ao estudo dos processos de recomposição de florestas manejadas.

\begin{tabular}{l|l} 
Gallant & \multicolumn{1}{c}{ Modelo } \\
\hline Von-Bertalanffy & $\beta_{1} X_{1}+\beta_{2} X_{2}+\beta_{3} \exp \left(\gamma X_{3}\right)$ \\
\hline Mitscherlich & $\alpha\{1-\exp [-\gamma(X-\beta)]\}$ \\
\hline Logístico & $\alpha\left[1-10^{-\gamma(X+\beta)}\right]$ \\
\hline Gompertz & $\alpha /[1+\exp (\beta-\gamma X)]$ \\
\hline Richards & $\alpha \exp [-\exp (\beta-\gamma X)]$ \\
\hline Morgan-Mercer-Flodin & $\alpha /\left\{[1+\exp (\beta-\gamma X)]^{\frac{1}{\delta}}\right\}$ \\
\hline Weibull & $\left(\beta \gamma+\alpha X^{\delta}\right) /\left(\gamma+X^{\delta}\right)$ \\
\hline
\end{tabular}

Fonte: Prudente (2009).

Vários tipos de modelos matemáticos podem ser usados para estudar os processos de dinâmica de recomposição de florestas manejadas, mas os modelos sigmoidais possuem propriedades que podem melhor atender aos objetivos da análise de perfis de crescimento da floresta e das espécies ocorrentes. Os parâmetros dos modelos não lineares são muito mais fáceis de serem interpretados biologicamente.

Seja o modelo não linear:

$$
Y_{j}=\alpha+\beta \rho^{X_{j}}+e_{j}
$$


Tal que $\alpha, \beta, \rho$ são os parâmetros e os $e_{j}$ são os erros aleatórios independentes com distribuição normal de média zero e variância $\sigma^{2}$.

A equação $f(X)=\alpha+\beta \rho^{X}$ (6.7.1), quando $\alpha>0, \beta<0$ e $|\rho|<1$ é denominada de função de Spillman. Substituindo, na equação 6.7.1, $\alpha=\beta_{0}, \beta=-\beta_{0} 10^{-\beta_{1} \beta_{2}}$ e $\rho=10^{-\beta_{1}}$, resulta:

$$
f(X)=\beta_{0}\left[1-10^{-\beta_{1}\left(X+\beta_{2}\right)}\right]
$$

A relação 6.7.2 passa a ser denominada de equação de Mitscherlich.

Na equação 6.7.1, como $|\rho|<1$, tem-se que: $\lim _{X \rightarrow \infty} f(X)=\alpha$. Destarte, também na equação 6.7.2, como $\beta_{1}>0$, resulta $\lim _{X \rightarrow \infty} f(X)=\beta_{0}$.

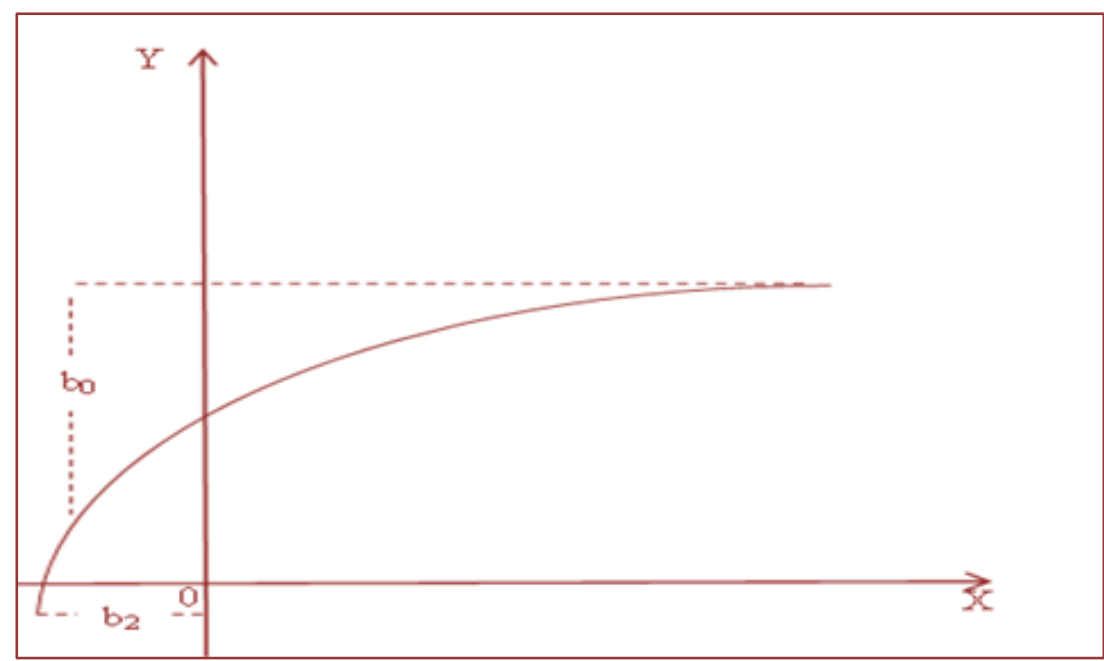

Figura 6.7.1 - Equação de Mitscherlich $\left(\beta_{0}=b_{0}\right.$ e $\left.\beta_{2}=b_{2}\right)$.

0 valor da função se aproxima assintoticamente de $\beta_{0}$ quando a variável independente $X$ se aproxima do infinito. Analisando o gráfico da figura 6.7.1, dentro do contexto do crescimento de uma floresta manejada, sendo $X=$ unidades de tempo, o parâmetro $\beta_{0}$ representado por $\left(\mathrm{b}_{0}\right)$, corresponderia à produção máxima alcançada considerando um período de tempo muito longo. 0 parâmetro $\beta_{2}$, representado por $\left(\mathrm{b}_{2}\right)$, representaria a quantidade de madeira remanescente logo após a exploração florestal, pois se $f(X)=0$, então, resulta $X=-\beta_{2}$. Em um experimento de adubação o parâmetro $\beta_{1}$ é denominado de coeficiente de eficácia e está relacionado com a intensidade do efeito do nutriente no desenvolvimento da planta.

No caso de manejo florestal, o coeficiente $\left(\beta_{1}\right)$ poderia ser expresso como uma característica de eficácia da floresta em termos de crescimento, ou mesmo no caso de espécies, poderia ser dito que esse valor seria o coeficiente de eficácia específico da espécie, ou seja, no manejo de uma floresta natural cada espécie teria o seu respectivo 
coeficiente de eficácia. Este coeficiente refletiria uma medida do grau de aceleração do crescimento.

Hoffmann (2011) cita algumas características matemáticas da função $f(X)=\alpha+\beta \rho^{X}$ que também são válidas para a de Mitscherlich.

Como $f(X)-\alpha=\beta \rho^{X}$ :

$$
\frac{d f(X)}{d X}=\beta \rho^{X}(\operatorname{Ln} \rho)
$$

$$
\begin{gathered}
\frac{d f(X)}{d X}=[f(X)-\alpha](\operatorname{Ln} \rho) \\
\frac{d f(X)}{d X}=(-\operatorname{Ln} \rho)[\alpha-f(X)]
\end{gathered}
$$

Como $|\rho|<1$, tem-se $(-\operatorname{Ln} \rho)>0$.

A expressão $(-\operatorname{Ln} \rho)[\alpha-f(X)]$ mostra que à medida que $X$ cresce, a declividade da curva decresce por que o valor de $f(X)$ se aproxima de $\alpha$. 0 valor $[\alpha-f(X)]$, denominado de "fator de contenção", diminui com o aumento de $X$ (ocasião).

Hoffmann (2011) também mostra que, se for dado acréscimos constantes à variável $X$, na equação $f(X)=\alpha+\beta \rho^{X}$, os valores das sucessivas variações no valor da função apresentam modificações porcentuais constantes:

$$
\begin{gathered}
f\left(X_{0}\right)=\alpha+\beta \rho^{X_{0}} \\
f\left(X_{0}+\Delta X\right)=\alpha+\beta \rho^{X_{0}+\Delta X} \\
f\left(X_{0}+2 \Delta X\right)=\alpha+\beta \rho^{X_{0}+2 \Delta X}
\end{gathered}
$$

Resultando:

$$
\begin{gathered}
f\left(X_{0}+\Delta X\right)-f\left(X_{0}\right)=\beta \rho^{X_{0}+\Delta X}-\beta \rho^{X_{0}}=\beta \rho^{X_{0}}\left(\rho^{\Delta X}-1\right) \\
f\left(X_{0}+2 \Delta X\right)-f\left(X_{0}+\Delta X\right)=\beta \rho^{X_{0}+2 \Delta X}-\beta \rho^{X_{0}+\Delta X}=\beta \rho^{X_{0}+\Delta X}\left(\rho^{\Delta X}-1\right)
\end{gathered}
$$

Consequentemente:

$$
\frac{f\left(X_{0}+2 \Delta X\right)-f\left(X_{0}+\Delta X\right)}{f\left(X_{0}+\Delta X\right)-f\left(X_{0}\right)}=\frac{\beta \rho^{X_{0}+\Delta X}\left(\rho^{\Delta X}-1\right)}{\beta \rho^{X_{0}}\left(\rho^{\Delta X}-1\right)}=\rho^{\Delta X}
$$

Demonstrando que, dado um acréscimo $\Delta X$ em $X$, a relação de mudanças sucessivas em $f(X)$ é constante.

Sejam, por exemplo, $X_{1}, X_{2}, X_{3}$ três valores equiespaçados de $X$, ou seja, $X_{3}-X_{2}=X_{2}-X_{1}=\Delta X$, e sejam $Y_{1}, Y_{2}, Y_{3}$ os correspondentes valores de $Y$. Os valores $Y_{1}, Y_{2}, Y_{3}$ podem fazer parte da amostra ou não, pois, alternativamente, pode-se obtê-los traçando uma curva e ler no gráfico as coordenadas dos três pontos escolhidos. 
De acordo com:

$$
\frac{f\left(X_{0}+2 \Delta X\right)-f\left(X_{0}+\Delta X\right)}{f\left(X_{0}+\Delta X\right)-f\left(X_{0}\right)}=\rho^{\Delta X}
$$

Pode-se ter uma estimativa preliminar de $\rho$ :

$$
\hat{\rho}=\left(\frac{Y_{3}-Y_{2}}{Y_{2}-Y_{1}}\right)^{\frac{1}{\Delta X}}
$$

\subsection{CICLO DE CORTE ECONOMICAMENTE ACONSELHÁVEL COM A EQUAÇÃO DE MITSCHERLICH.}

Para estimar o ciclo ótimo de corte de uma floresta manejada será usada a equação de Mitscherlich (6.7.2). Esta função é sempre crescente, pois sua derivada dada pela fórmula 6.8.1 é sempre positiva, denotando que não tem máximo. No caso florestal, significaria que a produção será sempre crescente no decorrer do tempo.

$$
V^{\prime}=\operatorname{Ln} 10 \times \beta_{0} \beta_{1}\left[10^{-\beta_{1}\left(t+\beta_{2}\right)}\right]
$$

Por outro lado, do ponto de vista do manejo florestal, muitas vezes, não é suficiente apenas calcular o máximo de uma função, mas, também levar em consideração o fator econômico. Desta maneira, é importante obter a produção que maximiza a renda liquida.

Gomes \& Nogueira (1980) mostram, usando a função de Mitscherlich, a equação para a obtenção da dose economicamente aconselhável de fertilizantes em experimentos de adubação.

Considerando $W$ o preço da madeira $\left(\mathrm{m}^{3} / \mathrm{ha}\right)$ e $V$ o volume madeira, por hectare, estimado pela função de crescimento, a partir da equação de Mitscherlich, então $W V$ representará a receita bruta. Considerando as despesas, compostas de uma parte fixa $(D F)$ e outras referentes às despesas na execução do plano de manejo, denominada de Custo do Manejo ( $C M$ ) no tempo $t$ (ocasiões/ano), a fórmula da receita líquida será dada pela função Z.

$$
Z=W V-D F-C M \times t
$$

Tomando a primeira derivada da função $Z$ em relação a variável tempo $(t)$, tem-se:

$$
Z^{\prime}=\frac{d Z}{d t}=W V^{\prime}-C M
$$

Seja, $y^{\prime}=c k^{u} \times L n k \times u^{\prime}$, a derivada da função $y=c k^{u}$, tal que $u=f(t)$.

Dado que $V=\beta_{0}\left[1-10^{-\beta_{1}\left(t+\beta_{2}\right)}\right]=\beta_{0}-\beta_{0} 10^{-\beta_{1}\left(t+\beta_{2}\right)}$ 


$$
\begin{gathered}
\frac{d V}{d t}=V^{\prime}=0-\beta_{0} 10^{-\beta_{1}\left(t+\beta_{2}\right)} \times \operatorname{Ln} 10 \times-\beta_{1} \\
V^{\prime}=\operatorname{Ln} 10 \times \beta_{0} \beta_{1}\left[10^{-\beta_{1}\left(t+\beta_{2}\right)}\right]
\end{gathered}
$$

Então:

$$
Z^{\prime}=2,302585 W\left[\beta_{0} \beta_{1} 10^{-\beta_{1}\left(t+\beta_{2}\right)}\right]-C M
$$

Anulando a primeira derivada $Z^{\prime}=0$, então:

$$
Z^{\prime}=2,302585 W\left[\hat{\beta}_{0} \hat{\beta}_{1} 10^{-\hat{\beta}_{1}\left(t_{o t i}+\hat{\beta}_{2}\right)}\right]-C M=0
$$

Sendo $t_{o t i}$ definido como tempo ótimo para o corte da floresta, ou seja, tempo economicamente aconselhável. Logo:

$$
\begin{gathered}
2,302585 W \hat{\beta}_{0} \hat{\beta}_{1} 10^{-\hat{\beta}_{1}\left(t_{o i t}+\hat{\beta}_{2}\right)}=C M \\
10^{-\hat{\beta}_{1}\left(t_{o t i}+\hat{\beta}_{2}\right)}=\frac{C M}{2,302585 \times W \times \hat{\beta}_{0} \times \hat{\beta}_{1}}
\end{gathered}
$$

Tomando o logaritmo na base dez de ambos os membros, têm-se:

$$
\begin{gathered}
-\hat{\beta}_{1}\left(t_{o t i}+\hat{\beta}_{2}\right)=\log _{10}\left(\frac{C M}{2,302585 \times W \times \hat{\beta}_{0} \times \hat{\beta}_{1}}\right) \\
-\hat{\beta}_{1} t_{o t i}-\hat{\beta}_{1} \hat{\beta}_{2}=\log _{10}\left(\frac{C M}{2,302585 \times W \times \hat{\beta}_{0} \times \hat{\beta}_{1}}\right) \\
-\hat{\beta}_{1} t_{o t i}=\log _{10}\left(\frac{C M}{2,302585 \times W \times \hat{\beta}_{0} \times \hat{\beta}_{1}}\right)+\hat{\beta}_{1} \hat{\beta}_{2} \\
\hat{\beta}_{1} t_{o t i}=-\log _{10}\left(\frac{C M}{2,302585 \times W \times \hat{\beta}_{0} \times \hat{\beta}_{1}}\right)-\hat{\beta}_{1} \hat{\beta}_{2} \\
t_{o t i}=-\frac{1}{\hat{\beta}_{1}}\left[\log _{10}\left(\frac{C M}{2,302585 \times W \times \hat{\beta}_{0} \times \hat{\beta}_{1}}\right)\right]-\hat{\beta}_{2}
\end{gathered}
$$

Definindo $R$ como uma razão de custo entre a receita obtida pela venda da madeira $(W)$ e o custo do manejo florestal por hectare $(C M)$, tem-se:

$$
R=\frac{W}{C M} \rightarrow t_{\text {oti }}=-\frac{1}{\hat{\beta}_{1}}\left[\log _{10}\left(\frac{1}{2,302585 \times R \times \hat{\beta}_{0} \times \hat{\beta}_{1}}\right)\right]-\hat{\beta}_{2}
$$

Dada a seguinte propriedade dos logaritmos: $\log _{a}\left(\frac{b}{c}\right)=\log _{a} b-\log _{a} c$, tem-se: 


$$
\begin{gathered}
\log _{10}\left(\frac{1}{2,302585 \times R \times \hat{\beta}_{0} \times \hat{\beta}_{1}}\right)=\log _{10} 1-\log \left(2,302585 \times R \times \hat{\beta}_{0} \times \hat{\beta}_{1}\right. \\
\log _{10}\left(\frac{1}{2,302585 \times R \times \hat{\beta}_{0} \times \hat{\beta}_{1}}\right)=-\log \left(2,302585 \times R \times \hat{\beta}_{0} \times \hat{\beta}_{1}\right.
\end{gathered}
$$

Resultando a equação 6.8.2 para estimar, utilizando a equação de Mitscherlich, o ciclo de corte economicamente aconselhável de uma floresta manejada em função da razão de custo $R$.

$$
t_{\text {oti }}=\frac{1}{\hat{\beta}_{1}} \log _{10}\left(2,302585 R \hat{\beta}_{0} \hat{\beta}_{1}\right)-\hat{\beta}_{2}
$$

Exercício 6.8.1. Seja uma área de floresta natural onde foram aplicadas técnicas de manejo. 0 monitoramento do crescimento foi realizado por meio de inventário florestal contínuo com 30 parcelas permanentes. As medições foram realizadas em cinco ocasiões equidistantes de cinco anos, ou seja, 0 ano, 5 anos, 10 anos, 15 anos e 20 anos. 0 ano zero $(0)$ corresponde à data logo após a exploração florestal. Obter o ciclo de corte economicamente aconselhável $\left(t_{o t i}\right)$ e a corresponde produção de madeira $\left(V_{o t i}\right)$, considerando uma razão de custo $\mathrm{R}=3$. A equação de crescimento obtida foi a seguinte:

$$
\begin{gathered}
V_{\text {oti }}=145,85\left[1-10^{-0,07475(t+6,377)}\right] \\
t_{\text {oti }}=\left(\frac{1}{0,07475}\right) \log (2,302585 \times 3 \times 145,85 \times 0,07475)-6,377=18,7 \text { anos } \\
V_{o t i}=145,85\left[1-10^{-0,07475(18,7+6,377)}\right]=143,90 \mathrm{~m}^{3} / \mathrm{ha}
\end{gathered}
$$

O ciclo de corte economicamente aconselhável, para uma razão de custo igual $\mathrm{R}=3$, é de 18,7 anos, correspondendo a uma produção de 143,9 m³ /ha.

\subsection{CICLO DE CORTE ECONOMICAMENTE ACONSELHÁVEL PELA EQUAÇÃO DE VON- BERTALANFFY}

No caso do uso da equação de Von-Bertalanffy, têm-se:

$$
\begin{gathered}
V=\beta_{0}\left[1-e^{-\beta_{1}\left(t-\beta_{2}\right)}\right] \quad \rightarrow \quad V=\beta_{0}-\beta_{0} e^{-\beta_{1}\left(t-\beta_{2}\right)} \\
\frac{d V}{d t}=-\beta_{0} e^{-\beta_{1}\left(t-\beta_{2}\right)} \times-\beta_{1} \\
\frac{d V}{d t}=\beta_{0} \beta_{1} e^{-\beta_{1}\left(t-\beta_{2}\right)}
\end{gathered}
$$




$$
\begin{gathered}
Z^{\prime}=\frac{d Z}{d t}=W V^{\prime}-C M \\
Z^{\prime}=W\left[\beta_{0} \beta_{1} e^{-\beta_{1}\left(t-\beta_{2}\right)}\right]-C M
\end{gathered}
$$

Anulando a primeira derivada $Z^{\prime}=0$, então:

$$
\begin{aligned}
& W \hat{\beta}_{0} \hat{\beta}_{1} e^{-\hat{\beta}_{1}\left(t_{o t i}-\hat{\beta}_{2}\right)}=C M \\
& e^{-\hat{\beta}_{1}\left(t_{o t i}-\hat{\beta}_{2}\right)}=\frac{C M}{W \times \hat{\beta}_{0} \times \hat{\beta}_{1}}
\end{aligned}
$$

Tomando o logaritmo neperiano de ambos os membros:

$$
\begin{gathered}
-\hat{\beta}_{1}\left(t_{o t i}-\hat{\beta}_{2}\right)=\operatorname{Ln}\left(\frac{C M}{W \times \hat{\beta}_{0} \times \hat{\beta}_{1}}\right) \\
-\hat{\beta}_{1} t_{o t i}+\hat{\beta}_{1} \hat{\beta}_{2}=\operatorname{Ln}\left(\frac{C M}{W \times \hat{\beta}_{0} \times \hat{\beta}_{1}}\right) \\
-\hat{\beta}_{1} t_{o t i}=\operatorname{Ln}\left(\frac{C M}{W \times \hat{\beta}_{0} \times \hat{\beta}_{1}}\right)-\hat{\beta}_{1} \hat{\beta}_{2} \\
\hat{\beta}_{1} t_{o t i}=-\operatorname{Ln}\left(\frac{C M}{W \times \hat{\beta}_{0} \times \hat{\beta}_{1}}\right)+\hat{\beta}_{1} \hat{\beta}_{2} \\
t_{o t i}=-\frac{1}{\hat{\beta}_{1}} \operatorname{Ln}\left(\frac{C M}{W \times \hat{\beta}_{0} \times \hat{\beta}_{1}}\right)+\hat{\beta}_{2}
\end{gathered}
$$

Definindo $R$ como uma razão de custo entre a receita obtida pela venda da madeira $(W)$ e o custo do manejo florestal por hectare $(C M)$, resulta a equação 6.9.1 para estimar, utilizando a equação de Von-Bertalanffy, o ciclo de corte economicamente aconselhável de uma floresta manejada em função da razão de custo $R$.

$$
\begin{aligned}
& R=\frac{W}{C M} \quad \rightarrow \quad t_{o t i}=-\frac{1}{\hat{\beta}_{1}} \operatorname{Ln}\left(\frac{1}{R \times \hat{\beta}_{0} \times \hat{\beta}_{1}}\right)+\hat{\beta}_{2} \\
& t_{\text {oti }}=\frac{1}{\hat{\beta}_{1}} \operatorname{Ln} R \hat{\beta}_{0} \hat{\beta}_{1}+\hat{\beta}_{2}
\end{aligned}
$$

No capítulo 7, seção 7.2.1, item f, está apresentado um exemplo de aplicação, usando a equação de Von-Bertalanffy, para obter o ciclo de corte economicamente aconselhável de uma floresta manejada. 


\section{Capítulo 7}

\section{Análise de inventários florestais contínuos}

Este capítulo apresenta a análise multivariada de inventários florestais contínuos (IFC) com parcelas permanentes, expondo exemplos de aplicação, considerando os perfis de crescimento com relação a cinco ocasiões de quatro métodos de manejo florestal. Comenta sobre o uso da análise univariada em inventários florestais com parcelas permanentes pelo método de análise de parcelas subdivididas. Na análise multivariada ou análise de perfil do IFC são apresentados os seguintes tópicos: análise de inventários florestais com parcelas permanentes com apenas um tratamento; análise de inventários florestais com parcelas permanentes considerando dois tratamentos; análise de inventários florestais com parcelas permanentes considerando mais de dois tratamentos. No exemplo de aplicação, considerando os quatro métodos de manejo, os seguintes assuntos são abordados: teste de esfericidade de matriz de covariância pelo critério de Mauchly; cálculo dos valores das correções de Greenhouse-Geisser Epsilon $(\varepsilon g g)$ e de Huynh-Feldt Epsilon ( $\left.\varepsilon_{h f}\right)$; teste de homogeneidade de matrizes de covariâncias; teste de multinormalidade de assimetria e de curtose de Mardia; teste de normalidade multivariada de Henze-Zirkler; análise de agrupamento pelo método de Ward; teste de paralelismo e de coincidência de perfil de crescimento segundo a hipótese linear geral $H$ : $C \beta U=\phi$; teste de coincidência de perfil de crescimento pelo teste " $t$ " de Student; determinação do ciclo de corte economicamente aconselhável com o uso de regressão não linear de Mitscherlich; análise de resíduos; teste de validade de regressão de Graybill; teste da razão da máxima verossimilhança para verificar a igualdade de parâmetros e identidade de modelos de regressão não linear. 


\section{INTRODUÇÃO}

Serão discutidos dois métodos para testar as hipóteses sobre o efeito dos tratamentos e a correspondente tendência de crescimento ao longo do tempo. 0 primeiro é o procedimento univariado, que trata as observações medidas no tempo como se fossem originadas de subdivisões das unidades experimentais (split-plot), analisado conforme o delineamento em parcelas subdivididas. 0 segundo método, denominado de análise de perfil "profile analysis", que adota um procedimento multivariado, ou seja, considera as observações repetidas sobre cada unidade experimental como um vetor de respostas.

Descreve-se o método de Wilks para testar se a matriz de covariância entre as ocasiões é uniforme e o critério de Mauchly (1940) para verificar a ocorrência de esfericidade, assim como as correções de Geisser \& Greenhouse (1958) e de Huynh \& Heldt (1976) para corrigir os graus de liberdade do teste $F$, para a igualdade de efeitos de ocasiões e de ocorrência de paralelismo de perfis de crescimento, caso haja a opção pela escolha da análise univariada do delineamento em parcelas divididas.

Apresenta-se, também, o procedimento de análise de inventários florestais com parcelas permanentes considerando apenas um, dois ou vários tratamentos. Em um exemplo aplicativo, analisa-se o problema da multinormalidade dos dados, homogeneidade de matrizes de covariâncias, paralelismo e coincidência de perfis de crescimento, assim como, trata-se do ajuste pela função de Mitscherlich de curvas de crescimento, possibilitando a obtenção do ciclo de corte economicamente aconselhável.

Os programas desenvolvidos na linguagem do Sistema de Análise Estatística (SAS) para as análises realizadas estão apresentados no apêndice $C$.

O inventário florestal contínuo com parcelas permanentes enquadra-se, no contexto da análise estatística, no grupo dos denominados experimentos com medidas repetidas (EMR). Os EMR constituem um problema de população multivariada, em que as variáveis aleatórias, como por exemplo, o volume de madeira em $p$-ocasiões, que possui uma distribuição de probabilidade com vetor de médias $\mu^{\prime}=\left[\mu_{1}, \mu_{2}, \cdots, \mu_{p}\right]$ e matriz de covariâncias $\sum$.

$$
\Sigma=\left[\begin{array}{cccc}
\sigma_{11} & \sigma_{12} & \cdots & \sigma_{1 p} \\
\sigma_{21} & \sigma_{22} & \cdots & \sigma_{2 p} \\
\cdots & \cdots & \cdots & \cdots \\
\sigma_{p 1} & \sigma_{p 2} & \cdots & \sigma_{p p}
\end{array}\right]
$$

0 procedimento adotado é selecionar e medir as parcelas na primeira ocasião, tornando as unidades de amostra permanentes, sendo remedidas nas ocasiões seguintes. Neste procedimento ocorrerão correlações entre as medições nas diversas ocasiões, significando, em termos da ciência florestal, importante mecanismo para a interpretação do comportamento dos parâmetros envolvidos na dinâmica da floresta.

A aplicação da análise univariada pelo método de parcelas subdivididas, em experimentos de medidas repetidas no tempo, somente é válida sob a pressuposição de uniformidade da matriz de covariância. A matriz uniforme ocorre quando as ocasiões 
apresentarem homogeneidade de variâncias e forem igualmente correlacionadas $(\rho)$, ou seja:

$$
\sum=\sigma^{2}\left[\begin{array}{cccc}
1 & \rho & \cdots & \rho \\
\rho & 1 & \cdots & \rho \\
\cdots & \cdots & \cdots & \cdots \\
\rho & \rho & \cdots & 1
\end{array}\right]
$$

Onde $\sigma^{2}$ é a variância comum entre as unidades dentro das ocasiões e $\rho$ é a correlação comum entre as ocasiões combinadas aos pares. Por outro lado, no caso de não ser atendida essa pressuposição e o pesquisador tiver o interesse em analisar pela modelagem univariada do delineamento em parcelas divididas, a correção $\varepsilon_{g g}$ de Geisser \& Greenhouse (1958) ou a correção $\varepsilon_{h f}$ de Huynh \& Heldt (1976) podem ser usadas para corrigir os graus de liberdade do teste $F$, para a igualdade de efeitos de ocasiões e de ocorrência de paralelismo de perfis de crescimento.

\subsection{ANÁLISE UNIVARIADA DE INVENTÁRIOS FLORESTAIS COM PARCELAS PERMANENTES PELO MÉTODO DE PARCELAS SUBDIVIDIDAS}

Seja um experimento com o delineamento inteiramente ao acaso, em que $g$ métodos de manejo são aplicados em $N$ unidades experimentais, de modo que $n_{i}$ seja o número de parcelas submetidas ao $i$-ésimo tratamento $(i=1,2, \cdots, g)$ e as observações sejam feitas, periodicamente, em $p$ ocasiões distintas $(k=1,2, \cdots, p)$, onde $N=n_{1}+n_{2}+\cdots+n_{g}$ é o número total de parcelas permanentes.

Seja o modelo misto univariado 7.1.1 representando cada uma das observações $y_{i j k}$, onde os tratamentos e as ocasiões são dois fatores fixos completamente cruzados.

$$
y_{i j k}=\mu+\tau_{i}+\delta_{j(i)}+\gamma_{k}+(\tau \gamma)_{i k}+e_{i j k}
$$

$y_{i j k}$ : é a medida efetuada na $j$-ésima unidade de amostra de tamanho $n_{i}\left(j=1,2, \cdots, n_{i}\right)$, submetida ao $i$-ésimo tratamento e correspondente a $k$-ésima ocasião $(k=1,2, \cdots, p)$;

$\mu$ : média geral populacional;

$\tau_{i}$ : é o efeito do $i$-ésimo tratamento;

$\delta_{j(i)}$ : é o efeito aleatório da $j$-ésima unidade experimental dentro do $i$-ésimo tratamento, supostos homocedásticos, independentes e normalmente distribuídos;

$\gamma_{k}$ : efeito da $k$-ésima ocasião;

$(\tau \gamma)_{i k}$ : é o efeito da interação entre o $i$-ésimo tratamento e a $k$-ésima ocasião;

$e_{i j k}$ : é o erro aleatório associado às observações da $k$-ésima ocasião do i-ésimo tratamento da $j$-ésima parcela experimental, supostos homocedásticos, independentes e normalmente distribuídos. 
Denota-se que os tratamentos são aplicados sobre cada unidade experimental, enquanto que as ocasiões são consideradas como subdivisões dessas unidades. Neste caso, os efeitos principais de tratamentos são calculados sobre os totais de unidades, enquanto que, os efeitos da interação tratamento × ocasião são calculados a partir das diferenças entre observações dentro das unidades. As somas de quadrados, pressupondo normalidade dos erros, apresentam distribuição $\chi^{2}$ e são mutuamente independentes.

A Tabela 7.1.1 apresenta a análise de variância usual para experimentos inteiramente ao acaso para tratamentos em parcelas subdivididas, considerando o mesmo número de repetições por ocasião dentro de cada tratamento. A presença ou ausência de interação entre os tratamentos e as ocasiões definirá o procedimento adequado para estabelecer os testes de comparações múltiplas. O procedimento de ajustamento de curvas depende da ausência ou presença de interação tratamento×ocasião. No caso de a interação ser significativa, implica que os tratamentos se comportam de forma diferente em relação às ocasiões. Porém, se não existir interação significa que os tratamentos, no tempo, apresentam a mesma tendência de crescimento (perfis paralelos).

Seja uma área florestal submetida a $g$ tratamentos (intensidades de manejo), onde foi realizado um inventário florestal contínuo, com uma amostragem simples ao acaso, considerando $p$ ocasiões equidistantes. Seja $n$ o mesmo número de parcelas em cada tratamento, então $N=n g$ é o total de parcelas permanentes medidas em $p$ ocasiões e $N p$ é o total de observações realizadas.

Tabela 7.1.1 - Delineamento inteiramente ao acaso de parcelas subdivididas ( $p$ ocasiões).

\begin{tabular}{l|l|l|l}
\multicolumn{1}{c|}{ C. V. } & \multicolumn{1}{c}{$\mathrm{G} . \mathrm{L}}$. & $\mathrm{SQ}$ & $\mathrm{F}$ \\
Trat. & $g-1$ & $S Q_{1}$ & $F_{1}=\frac{N-g}{g-1} \frac{S Q_{1}}{S Q_{2}}$ \\
\hline Erro (a) & $N-g$ & $S Q_{2}$ & \\
\hline Ocasiões & $p-1$ & $S Q_{3}$ & $F_{2}=(N-g) \frac{S Q_{3}}{S Q_{5}}$ \\
\hline Trat× Ocasião & $(g-1)(p-1)$ & $S Q_{4}$ & $F_{3}=\frac{g-1}{N-g} \frac{S Q_{4}}{S Q_{5}}$ \\
\hline Erro (b) & $(N-g)(p-1)$ & $S Q_{5}$ & \\
\hline Total & $N p-1$ & $S Q_{6}$ & \\
\hline$S Q_{1}=\frac{1}{n p} \sum_{i=1}^{g} y_{i . .}^{2}-C$ & $C=\frac{1}{N p}\left(\sum_{i=1}^{g} \sum_{j=1}^{n} \sum_{k=1}^{p} y_{i j k}\right)^{2}=\frac{y_{\ldots}^{2}}{N p}$ \\
$S Q_{2}=\frac{1}{p} \sum_{i=1}^{g} \sum_{j=1}^{n} y_{i j .}^{2}-\frac{1}{n p} \sum_{i=1}^{g} y_{i . .}^{2}$ & $S Q_{3}=\frac{1}{N} \sum_{k=1}^{p} y_{. k}^{2}-C$ \\
$S Q_{4}=\frac{1}{n} \sum_{i=1}^{g} \sum_{k=1}^{p} y_{i . k}^{2}-C-S Q_{1}-S Q_{3}$ & $S Q_{6}=\sum_{i=1}^{g} \sum_{j=1}^{n} \sum_{k=1}^{p} y_{i j k}^{2}-C$
\end{tabular}


Normalmente, em experimentos com parcelas divididas, o interesse é testar as seguintes hipóteses:

a) $H_{01}$ : Não existência de efeito diferencial de tratamentos:

$$
\begin{gathered}
H_{01}: \tau_{i}=0 \text {, versus, } H_{1}: N \tilde{a} o H_{01} \quad(i=1, \cdots, g) \\
F_{1}=\frac{Q M_{1}}{Q M_{2}}
\end{gathered}
$$

b) $H_{02}$ : Não existência de efeito diferencial de ocasiões:

$$
\begin{gathered}
H_{01}: \gamma_{k}=0 \text {, versus, } H_{2}: \text { Não } H_{02} \quad(k=1, \cdots, p) \\
F_{2}=\frac{Q M_{3}}{Q M_{5}}
\end{gathered}
$$

c) $H_{03}$ : Não existência de efeito de interação entre tratamentos e ocasiões:

$$
\begin{gathered}
H_{03}: \tau \gamma_{11}=\tau \gamma_{12}=\cdots=\tau \gamma_{g p} \text {, versus, } H_{3}: N \tilde{a} o H_{03} \quad[(i=1, \cdots, g),(k=1, \cdots, p)] \\
F_{3}=\frac{Q M_{4}}{Q M_{5}}
\end{gathered}
$$

Se $H_{03}$ denota que o efeito da interação é não significativo, resulta que todos os tratamentos apresentam a mesma tendência ou perfil de crescimento. No entanto, se o efeito da interação é significativo, verifica-se que pelo menos um tratamento apresenta uma tendência de crescimento diferente (não paralela).

\subsection{INVENTÁRIOS FLORESTAIS COM PARCELAS PERMANENTES COM UM TRATAMENTO}

Considerando a aplicação de um método de manejo medindo, em $p$ ocasiões, o volume em $n$ parcelas permanentes. Seja o modelo matemático $y_{k j}=\mu+\tau_{k}+e_{k j}$ inerente a esse levantamento, tal que $k=1, \cdots, p$ caracterizando as ocasiões e $j=1, \cdots, n$ especificando a parcela, então $\mu_{k}=\mu+\tau_{k}$ mede o efeito inerente a $k$-ésima ocasião e $e_{k j}$ é o erro aleatório correspondente a $k$-ésima ocasião da j-ésima unidade amostral.

Dado que as $n$ unidades amostrais são independentes entre si, onde o vetor dos erros aleatórios das repetições possui distribuição normal multivariada com vetor de médias nulo e matriz de covariância $\sum$, resultando, então, que o vetor $y_{v j}$ de ordem $p \times 1$ das observações da $j$-ésima unidade amostral, com vetor de médias $\mu_{v}^{\prime}=\left[\begin{array}{llll}\mu_{1} & \mu_{2} & \cdots & \mu_{p}\end{array}\right]$ e matriz de covariâncias também $\sum$, também apresenta distribuição normal multivariada. 
A formulação da hipótese, considerando o exposto, para testar a ausência do efeito de tempo é $H_{0}: \mu_{1}=\mu_{2}=\cdots=\mu_{p}$, ou mais explicitamente na forma:

$$
H_{0}:\left[\begin{array}{c}
\mu_{1}-\mu_{2} \\
\mu_{2}-\mu_{3} \\
\cdots \\
\mu_{(p-1)}-\mu_{p}
\end{array}\right]=\left[\begin{array}{c}
0 \\
0 \\
\vdots \\
0
\end{array}\right]
$$

Existem outras maneiras de explicitar a hipótese $H_{0}$, como por exemplo, considerar os contrastes em relação à primeira ou em relação à última ocasião, ou ainda, construir em relação a uma ocasião particular.

Seja $\bar{y}_{v}^{\prime}=\left[\begin{array}{llll}\bar{y}_{1} & \bar{y}_{2} & \cdots & \bar{y}_{p}\end{array}\right]$ o vetor de médias amostrais por ocasião e o vetor das $p-1$ diferenças, $\left(\bar{y}_{v}^{*}\right)^{\prime}=\left[\bar{y}_{1}-\bar{y}_{2}, \bar{y}_{2}-\bar{y}_{3}, \ldots, \bar{y}_{(p-1)}-\bar{y}_{p}\right]$, entre as ocasiões consecutivas. Então, a estatística multivariada para testar a hipótese da nulidade de efeito de ocasião é a seguinte:

$$
\left.T^{2}=n\left(\bar{y}_{v}^{*}\right)^{\prime}\left(S^{*}\right)^{-1}\right) \bar{y}_{v}^{*}
$$

Onde $S^{*}$ é a matriz de covariância das diferenças consecutivas. A estatística $T^{2}$ pode ser transformada na estatística $F$ de Snedecor, pela seguinte relação:

$$
F=\frac{n-p+1}{(n-1)(p-1)} T^{2} \text { que sob } H_{0} \text { possui }(p-1) \text { e }(n-p-1) \text { graus de liberdade. }
$$

A estatística $\left.T^{2}=n\left(\bar{y}_{v}^{*}\right)^{\prime}\left(S^{*}\right)^{-1}\right) \bar{y}_{v}^{*}$ pode ser escrita na forma $T^{2}=n \bar{y}_{v}^{\prime} C^{\prime}\left(C S C^{\prime}\right)^{-1} C \bar{y}_{v}$, a qual não requer a construção do vetor de médias das diferenças $\left(\bar{y}_{v}^{*}\right)$ e a sua respectiva matriz de covariância $\left(\mathrm{S}^{*}\right)$, tal que a matriz $\mathrm{C}$ caracteriza os contrastes. No caso de os contrastes serem entre ocasiões consecutivas, tem-se:

$$
C_{(p-1) p}=\left[\begin{array}{cccccc}
1 & -1 & 0 & \cdots & 0 & 0 \\
0 & 1 & -1 & \cdots & 0 & 0 \\
\cdots & \cdots & \cdots & \cdots & \cdots & \cdots \\
0 & 0 & 0 & \cdots & 1 & -1
\end{array}\right]
$$

A estatística $T^{2}$ apresenta o mesmo resultado qualquer que seja a forma de organizar os $(p-1)$ contrastes. Essa unicidade do teste é consequência da invariância de $T^{2}$. Sejam as formas da matriz $C$, considerando as comparações, respectivamente, em relação à primeira e à última ocasião.

$$
C_{(p-1) p}=\left[\begin{array}{cccccc}
1 & -1 & 0 & \cdots & 0 & 0 \\
1 & 0 & -1 & \cdots & 0 & 0 \\
\cdots & \cdots & \cdots & \cdots & \cdots & \cdots \\
1 & 0 & 0 & \cdots & 0 & -1
\end{array}\right] \quad C_{(p-1) p}=\left[\begin{array}{cccccc}
1 & 0 & 0 & \cdots & 0 & -1 \\
0 & 1 & 0 & \cdots & 0 & -1 \\
\cdots & \cdots & \cdots & \cdots & \cdots & \cdots \\
0 & 0 & 0 & \cdots & 1 & -1
\end{array}\right]
$$


No próximo passo após a comprovação da rejeição de $H_{0}$, ou seja, existe crescimento da floresta em relação ao tempo, pode-se estimar a curva de crescimento e a partir da equação obtida, então, calcular a idade economicamente ideal para a colheita da madeira.

\subsubsection{ANÁLISE DE UM INVENTÁRIO FLORESTAL CONTÍNUO COM UM MÉTODO DE MANEJO}

Seja uma área florestal de 1000 ha onde se aplicou um método de manejo M1. 0 monitoramento do crescimento foi realizado por um inventário florestal contínuo com 30 parcelas permanentes. As medições foram realizadas em cinco ocasiões equidistantes de cinco anos, ou seja, 0 ano, 5 anos, 10 anos, 15 anos e 20 anos. 0 ano zero (0) corresponde à data logo após a exploração florestal. Os dados $V$ (volume por ha) foram simulados e estão apresentados na Tabela B1 do apêndice B. O programa construído usando a linguagem SAS, para a análise de inventários florestais com parcelas permanentes para um tratamento, está apresentado na tabela C5 do apêndice C.

a) Gráfico com o perfil de crescimento do método de manejo M1

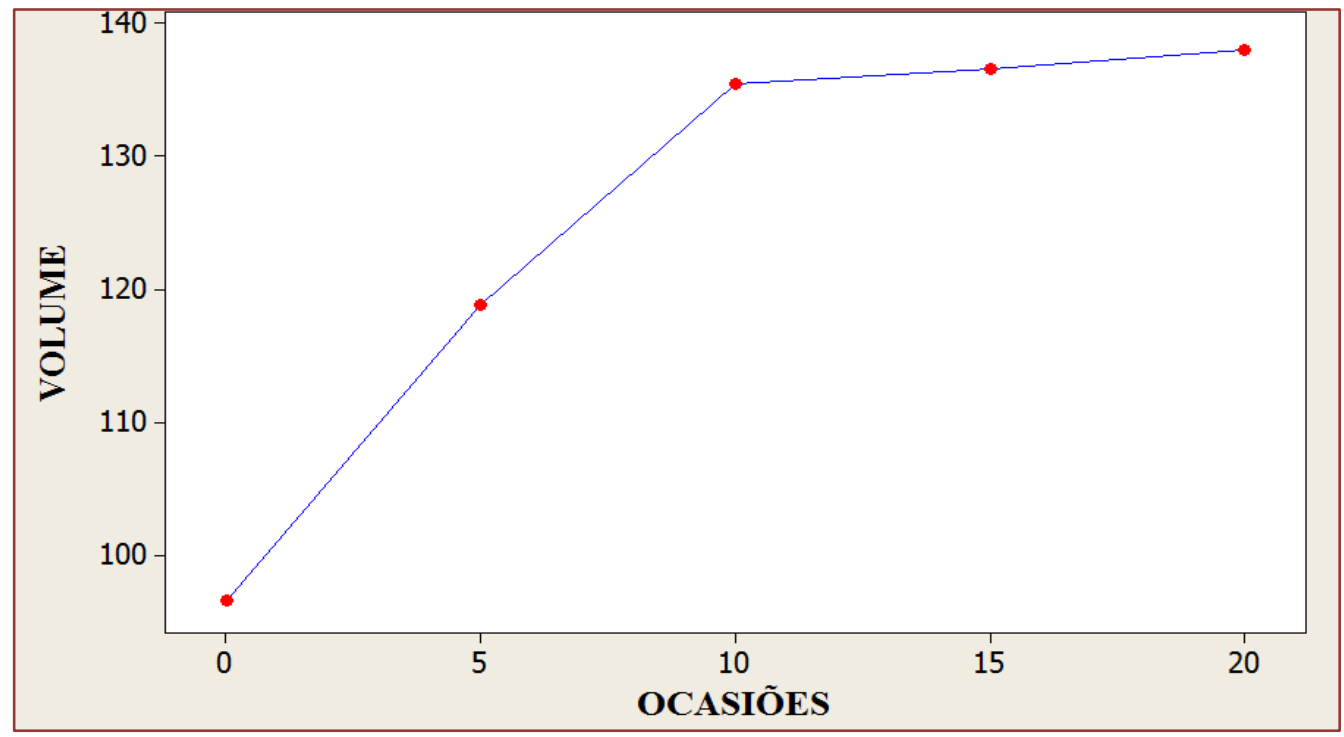

Figura 7.2.1.1 - Gráfico do perfil de crescimento para (M1).

b) Vetor de médias e matriz de covariância para o método de manejo M1

$$
\begin{aligned}
& \bar{y}_{v 1}^{\prime}=\left[\begin{array}{lllll}
96,65589 & 118,86703 & 135,42923 & 136,51993 & 137,96803
\end{array}\right] \\
& S_{1}=\left[\begin{array}{lllll}
1,84061681 & 1,76852089 & 1,88189246 & 2,01479114 & 2,04154190 \\
1,76852089 & 3,46728879 & 3,78614806 & 4,05873545 & 4,04560596 \\
1,88189246 & 3,78614806 & 4,57316908 & 4,85074615 & 4,85566809 \\
2,01479114 & 4,05873545 & 4,85074615 & 5,47236765 & 5,51975907 \\
2,04154190 & 4,04560596 & 4,85566809 & 5,51975907 & 5,96583927
\end{array}\right]
\end{aligned}
$$


c) Formulação da hipótese para testar a ausência do efeito de ocasião

$$
H_{0}: \mu_{0}=\mu_{5}=\mu_{10}=\mu_{15}=\mu_{20} \rightarrow H_{0}:\left[\begin{array}{c}
\mu_{0}-\mu_{5} \\
\mu_{5}-\mu_{10} \\
\mu_{10}-\mu_{15} \\
\mu_{15}-\mu_{20}
\end{array}\right]=\left[\begin{array}{c}
0 \\
0 \\
\vdots \\
0
\end{array}\right]
$$

d) Aplicação da estatística $\mathrm{T}^{2}$ de Hotelling e da estatística F

$$
\begin{gathered}
C=\left[\begin{array}{ccccc}
1 & -1 & 0 & 0 & 0 \\
0 & 1 & -1 & 0 & 0 \\
0 & 0 & 1 & -1 & 0 \\
0 & 0 & 0 & 1 & -1
\end{array}\right] \rightarrow C S_{1} C^{\prime}=\left[\begin{array}{c}
1,77086380,20548770,1396887-0,0398800 \\
0,20548770,46816180,00498970,0180514 \\
0.13968870 .00498970 .34404440 .0424695 \\
-0,03988000,01805140,04246950,3986888
\end{array}\right] \\
\left(C S_{1} C^{\prime}\right)^{-1}=\left[\begin{array}{c}
0,6191751-0,2729320-0,26002900,1019917 \\
-0,27293202,26018490,0952918-0,1397860 \\
-0,26002900,09529183,0547070-0,3557210 \\
0,1019917-0,1397860-0,35572102,5626457
\end{array}\right] \\
T_{1}^{2}=n \bar{y}_{v 1}^{\prime} C^{\prime}\left(C S_{1} C^{\prime}\right)^{-1} C \bar{y}_{v 1}=21.696,694 \quad F=\frac{n-p+1}{(n-1)(p-1)} T^{2}=4863,0521
\end{gathered}
$$

Que sob $H_{0}$ possui $p-1=4$ e $n-p+1=26$ graus de liberdade.

Dado que $F_{4 ; 26 ; 0,05} \cong 2,75$, conclui-se que o crescimento foi significativo. Então, haja vista a rejeição de $H_{0}$, pode-se ajustar um modelo de crescimento para obter o ciclo de corte economicamente aconselhável.

\section{e) Ciclo de corte economicamente aconselhável pela equação de Mitscherlich}

Seja a equação de Mitscherlich $V=\beta_{0}\left[1-10^{-\beta_{1}\left(t+\beta_{2}\right)}\right]$. As estimativas dos parâmetros estão inseridas na tabela 7.2.1.1 e foram obtidas, pelo método de Gauss-Newton, a partir do desenvolvimento do programa SAS, citado na tabela C12 do apêndice C.

$$
V_{M 1}=140,6\left[1-10^{-0,0717(t+6,9767)}\right] \quad(0 \leq t \leq 20 \text { anos })
$$


Dado que: $t_{\text {oti }}=\frac{1}{\hat{\beta}_{1}} \log _{10}\left[2,302585 R \hat{\beta}_{0} \hat{\beta}_{1}\right]-\hat{\beta}_{2}$, considerando $(R=3)$ a razão entre a receita obtida pela venda da madeira (w) e o custo do manejo florestal por hectare (CM), têmse:

$$
\begin{gathered}
t_{\text {Oti }(M 1)}=\left(\frac{1}{0,0717}\right) \log (2,302585 \times 3 \times 140,6 \times 0,0717)-6,9767=18,7 \text { anos } \\
V_{\text {oti }(M 1)}=140,6\left[1-10^{-0,0717(18,7+6,9767)}\right]=138,57 \mathrm{~m}^{3} / \mathrm{ha} \\
\mathrm{RQMR}=\sqrt{\frac{\sum_{\frac{j=1}{N}\left(Y_{j}-\hat{Y}_{j}\right)^{2}}^{N}}{N}=\sqrt{\frac{1150,6}{30 \times 5}}=2,7696 \quad(N=n \times p=150)} \\
\mathrm{CV}=\frac{R Q M R}{\bar{Y}} \times 100=\frac{2,7696}{125,09} \times 100=2,21 \%
\end{gathered}
$$

\begin{tabular}{|c|c|c|c|c|}
\hline Parâmetros & Estimativas & Error Padrão Aprox. & \multicolumn{2}{|c|}{ Limites de Confiança (95\%) } \\
\hline$\hat{\beta}_{0}$ & 140,6 & 0,6164 & 139,4 & 141,9 \\
\hline$\hat{\beta}_{1}$ & 0,0717 & 0,00321 & 0,0654 & 0,0781 \\
\hline$\hat{\beta}_{2}$ & 6,9767 & 0,2971 & 6,3896 & 7,5638 \\
\hline Fonte & GL & Soma de Quadrados & Quadrado Médio & Valor de F \\
\hline Erro & 147 & 1150,6 & 7,8275 & 2362,55 \\
\hline \multicolumn{5}{|c|}{ Teste de validade de Graybill $\left(F_{G}\right)$} \\
\hline Numerador & 2 & 17,35852 & 2,30 & 0,1036 \\
\hline Denominador & 148 & 7,54007 & & \\
\hline \multicolumn{5}{|c|}{ Coeficiente de correlação de Pearson, $\mathrm{N}=150$, Prob $>|\mathrm{r}|$ Sob H0: Rho $=0$} \\
\hline \multicolumn{3}{|c|}{$r_{M 1}=0,9848(<0,0001)$} & \multicolumn{2}{|c|}{$r_{M 1}^{2}=0,9698$} \\
\hline
\end{tabular}

Tabela 7.2.1.1 - Análise de regressão da equação de Mitscherlich para M1.

Os indicadores utilizados para avaliar o ajustamento do modelo de Mitscherlich para M1, $C V \%, r^{2}, F_{G}$ e a distribuição dos resíduos, dentro do contexto esperado, apresentaram resultados satisfatórios. É recomendável, haja vista o resultado da análise do ciclo de corte economicamente aconselhável, usando a equação de Mitscherlich, efetuar a colheita da madeira quando o povoamento florestal atingir 18,7 anos $\left(138,57 \mathrm{~m}^{3} / \mathrm{ha}\right)$. 


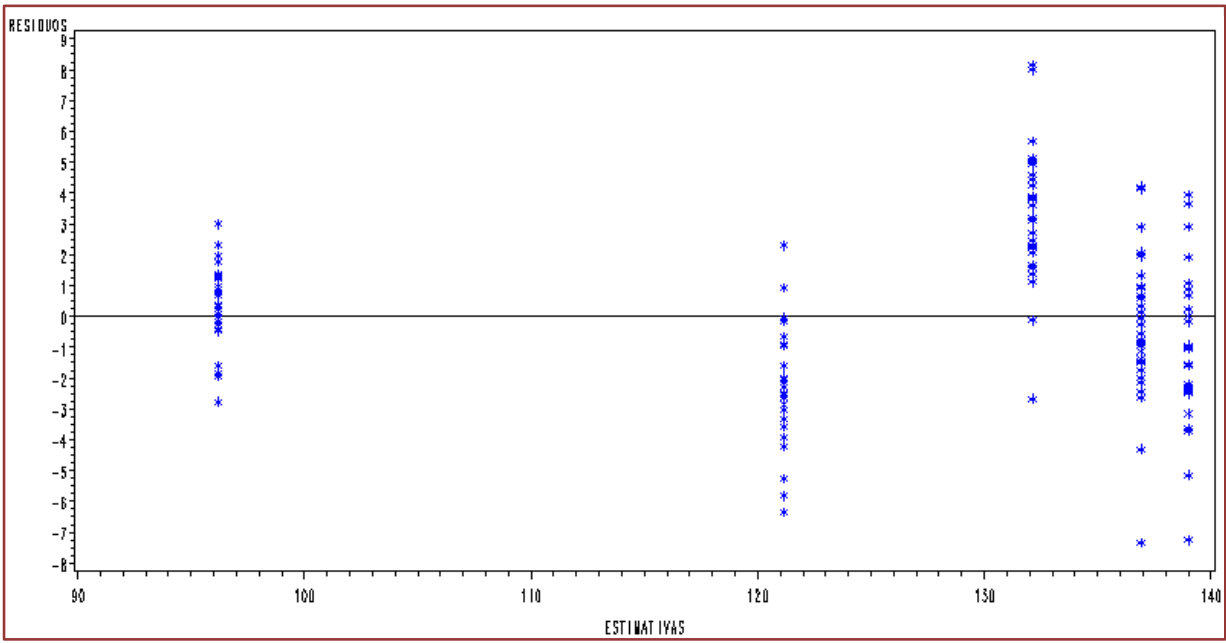

Figura 7.2.1.2 - Gráfico dos resíduos para M1.

f) Ciclo de corte economicamente aconselhável usando a equação de VonBertalanffy

A tabela 7.2.1.2 mostra a análise de regressão da equação de Von-Bertalanffy para o método de manejo $M 1$.

$$
\begin{gathered}
V=\beta_{0}\left[1-e^{-\beta_{1}\left(t-\beta_{2}\right)}\right] \rightarrow V_{M 1}=140,6\left[1-e^{-0,1651(t+6,9767)}\right] \\
t_{o t i}=\frac{1}{\hat{\beta}_{1}} \operatorname{LnR} \hat{\beta}_{0} \hat{\beta}_{1}+\hat{\beta}_{2}=\frac{1}{0,1651} \operatorname{Ln}(3 \times 140,6 \times 0,1651)-6,9767=18,7
\end{gathered}
$$

Os resultados da rotação economicamente aconselhável, para as equações de Mitscherlich e de Von-Bertalanffy, foram os mesmos.

\begin{tabular}{|c|c|c|c|c|}
\hline Parâmetros & Estimativas & Error Padrão Aprox. & \multicolumn{2}{|c|}{ Limites de Confiança (95\%) } \\
\hline$\hat{\beta}_{0}$ & 140,6 & 0,6164 & 139,4 & 141.9 \\
\hline$\hat{\beta}_{1}$ & 0,1651 & 0,00739 & 0,1505 & 0.1797 \\
\hline$\hat{\beta}_{2}$ & $-6,9767$ & 0,2971 & $-7,5638$ & $-6,3896$ \\
\hline Fonte & GL & Soma de Quadrados & Quadrado Médio & Valor de $F$ \\
\hline Erro & 147 & 1150,6 & 7,8275 & 2362,55 \\
\hline & & \multicolumn{3}{|c|}{ Teste de validade Graybill $\left(F_{G}\right)$} \\
\hline Fonte & GL & Quadrado Médio & Valor de $F_{G}$ & $\operatorname{Pr}>\mathrm{F}$ \\
\hline Numerador & 2 & 17,35852 & 2,30 & 0,1036 \\
\hline Denominador & 148 & \multirow{2}{*}{$\frac{7,54007}{\text { Pearson } \mathrm{N}=150 . \text { Prol }}$} & & \\
\hline \multicolumn{4}{|c|}{ Coeficiente de correlação de Pearson, $\mathrm{N}=150$, Prob $>|\mathrm{r}|$ Sob H0: Rho=0 } & \\
\hline \multicolumn{2}{|c|}{$r_{M 1}=0,9795(<0,0001)$} & \multicolumn{2}{|c|}{\begin{tabular}{|l|l|} 
& $r^{2}{ }_{M 1}=0,9594$ \\
\end{tabular}} & \\
\hline
\end{tabular}

Tabela 7.2.1.2 - Análise de regressão da equação de Von-Bertalanffy para M1. 


\subsection{INVENTÁRIOS FLORESTAIS COM PARCELAS PERMANENTES COM DOIS TRATAMENTOS}

Seja um inventário florestal contínuo com parcelas permanentes com dois tratamentos. As seguintes hipóteses podem ser formuladas:

a) Hipótese $H_{01}$ : os dois perfis médios populacionais dos tratamentos apresentam comportamentos similares (paralelismo)?

Testar a hipótese $H_{01}$ para verificar a existência de interação (tratamento×ocasião). A ausência dessa interação implica em um paralelismo estatístico entre os perfis.

b) Hipótese $H_{02}$ aceito o paralelismo entre os dois perfis populacionais, perguntar-se-ia? São os mesmos coincidentes?

A comprovação da hipótese $H_{02}$ de coincidência dos dois perfis é verificada, para as $p$ ocasiões, testando a existência de igualdade dos efeitos dos dois tratamentos. Aceito o paralelismo dos perfis, para obter a função de crescimento dos dois tratamentos, utilizase o mesmo modelo matemático de regressão. No caso de coincidência dos perfis obtémse uma única equação para expressar a função de crescimento.

No caso da interação entre os dois tratamentos ser significativa, depreende-se que o modelo de regressão para estimar as funções de crescimento pode não ser o mesmo.

c) Hipótese $\mathrm{H}_{03}$ : os perfis médios das respostas correspondentes aos tratamentos são paralelos ao eixo das abscissas, ou seja, não existe efeito do fator que define as ocasiões?

A verificação da existência de diferença entre as ocasiões considerando os dois grupos, concernente à referida hipótese, significaria testar se a floresta está crescendo.

Seja $y_{v i}$ o vetor das variáveis respostas observadas nas $p$ ocasiões, em cada parcela permanente, apresentando distribuição normal multivariada $p$-dimensional com vetor de médias $\mu_{v i}^{\prime}=\left[\begin{array}{llll}\mu_{i 1} & \mu_{i 2} & \cdots & \mu_{i p}\end{array}\right],(i=1,2)$, e matriz de covariância comum não singular $\sum$. Então:

A primeira hipótese a ser testada é verificar a ausência de interação tratamento×ocasião:

Ho1: não existência de interação tratamento×ocasião

A hipótese $H_{01}$ pode ser escrita na forma matricial $H_{01}: C \mu_{v 1}=C \mu_{v 2}$, que corresponde a testar o paralelismo entre os dois perfis de crescimento. A matriz $\mathrm{C}$ apresenta a seguinte formatação e a estatística para testar $H_{01}$ é a de Hotelling. 


$$
\begin{gathered}
H_{01}:\left[\begin{array}{l}
\mu_{11}-\mu_{12} \\
\mu_{12}-\mu_{13} \\
\mu_{13}-\mu_{14} \\
\mu_{14}-\mu_{15}
\end{array}\right]=\left[\begin{array}{l}
\mu_{21}-\mu_{22} \\
\mu_{22}-\mu_{23} \\
\mu_{23}-\mu_{24} \\
\mu_{24}-\mu_{25}
\end{array}\right] \quad C_{(p-1) p}=\left[\begin{array}{cccccc}
1 & -1 & 0 & \cdots & 0 & 0 \\
0 & 1 & -1 & \cdots & 0 & 0 \\
\cdots & \cdots & \cdots & \cdots & \cdots & \cdots \\
0 & 0 & 0 & \cdots & 1 & -1
\end{array}\right] \\
T^{2}=\frac{n_{1} n_{2}}{n_{1}+n_{2}}\left(\bar{y}_{v 1}-\bar{y}_{v 2}\right)^{\prime} C^{\prime}\left(C S C^{\prime}\right)^{-1} C\left(\bar{y}_{v 1}-\bar{y}_{v 2}\right) \quad S=\frac{\left(n_{1}-1\right) S_{1}+\left(n_{2}-1\right) S_{2}}{n_{1}+n_{2}-2}
\end{gathered}
$$

Onde $\bar{y}_{v i}^{\prime}=\left[\begin{array}{llll}\bar{y}_{i 1} & \bar{y}_{i 2} & \cdots & \bar{y}_{i p}\end{array}\right]$ é o vetor estimado das médias, considerando $n_{1}$ e $n_{2}$, o número de parcelas permanentes para os dois tratamentos e $S$ a matriz de covariância comum. Onde $S_{i}(i=1,2)$ são as matrizes de covariâncias dos tratamentos. A estatística $T^{2}$ pode transformada na estatística $F$ de Snedecor obedecendo a seguinte relação:

$$
F=\frac{n_{1}+n_{2}-p}{\left(n_{1}+n_{2}-2\right)(p-1)} T^{2}
$$

Que sob $H_{01}$ apresenta $(p-1)$ e $\left(n_{1}+n_{2}-p\right)$ graus de liberdade.

Caso a hipótese de paralelismo não seja rejeitada, a hipótese $H_{02}$ (coincidência dos dois perfis) deve ser testada. Esta hipótese consiste na aplicação do teste " $t$ de Student" para dois tratamentos independentes dentro de cada ocasião ( $p$ testes). Caso todos forem não significativos implica que os dois perfis são coincidentes.

Então, como visto anteriormente, aceito o paralelismo dos perfis, pode-se obter a equação de crescimento dos tratamentos utilizando o mesmo modelo matemático de regressão, e se houver coincidência dos perfis, então uma única equação deve ser obtida para expressar a curva de crescimento.

No caso da interação entre os dois tratamentos ser significativa, então o modelo de regressão para estimar as funções de crescimento pode não ser o mesmo, entretanto as equações de crescimento serão diferentes se aplicado o mesmo modelo.

\subsubsection{ANÁLISE DE UM INVENTÁRIO FLORESTAL CONTÍNUO COM DOIS MÉTODOS DE MANEJO}

Seja uma área florestal onde foram aplicados dois métodos de manejo, denominados M1 e M2. O monitoramento do crescimento, em cada método de manejo, foi realizado por um inventário florestal contínuo com 30 parcelas permanentes. As medições foram realizadas em cinco ocasiões equidistantes de cinco anos, ou seja, 0 ano, 5 anos, 10 anos, 15 anos e 20 anos. 0 ano zero (0) correspondeu à data logo após a exploração florestal. Os dados V (volume por ha) são simulados e estão apresentados na tabela B1 do apêndice B. 0 programa SAS, para a análise de inventários florestais com parcelas permanentes para dois tratamentos, está apresentado na tabela C6 do apêndice C. 
a) Gráfico com os perfis de crescimentos dos métodos M1 e M2

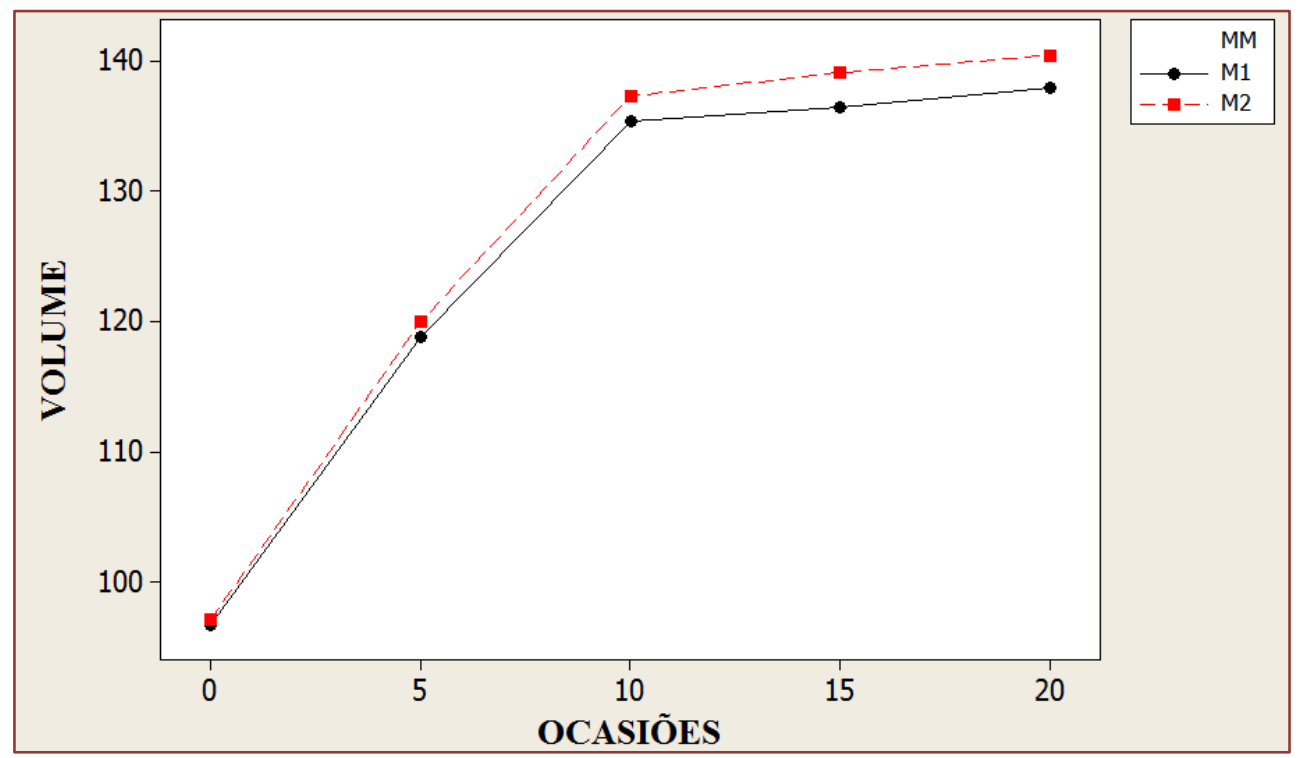

Figura 7.3.1.1 - Gráfico com os perfis de crescimentos para M1 e M2.

b) Vetor de médias e matrizes de covariâncias dos métodos M1 e M2

$$
\begin{gathered}
\bar{y}_{v 1}^{\prime}=\left[\begin{array}{lllll}
96,65589 & 118,86703 & 135,42923 & 136,51993 & 137,96803
\end{array}\right] \\
\bar{y}_{v 2}^{\prime}=\left[\begin{array}{llllll}
97,07460 & 120,00173 & 137,35973 & 139,15100 & 140,50200
\end{array}\right] \\
S_{1}=\left[\begin{array}{llllll}
1,84061681 & 1,76852089 & 1,88189246 & 2,01479114 & 2,04154190 \\
1,76852089 & 3,46728879 & 3,78614806 & 4,05873545 & 4,04560596 \\
1,88189246 & 3,78614806 & 4,57316908 & 4,85074615 & 4,85566809 \\
2,01479114 & 4,05873545 & 4,85074615 & 5,47236765 & 5,51975907 \\
2,04154190 & 4,04560596 & 4,85566809 & 5,51975907 & 5,96583927
\end{array}\right] \\
S_{2}=\left[\begin{array}{llllll}
2,17477232 & 2,59689899 & 2,68184199 & 2,71630059 & 2,85198576 \\
2,59689899 & 5,76460937 & 7,16915586 & 7,72605210 & 7,74732466 \\
2,68184199 & 7,16915586 & 9,78160489 & 10,7380652 & 11,0652238 \\
2,71630059 & 7,72605210 & 10,7380652 & 12,3077877 & 12,7868027 \\
2,85198576 & 7,74732466 & 11,0652238 & 12,7868027 & 13,8031213
\end{array}\right]
\end{gathered}
$$

c) Teste de hipótese para não existência de interação entre Método de Manejo×0casião

Ho1: não existência de interação método de manejo×ocasião.

$$
H_{01}:\left[\begin{array}{l}
\mu_{11}-\mu_{12} \\
\mu_{12}-\mu_{13} \\
\mu_{13}-\mu_{14} \\
\mu_{14}-\mu_{15}
\end{array}\right]=\left[\begin{array}{l}
\mu_{21}-\mu_{22} \\
\mu_{22}-\mu_{23} \\
\mu_{23}-\mu_{24} \\
\mu_{24}-\mu_{25}
\end{array}\right]
$$




\section{d) Aplicação da estatística $T^{2}$ de Hotelling e da estatística $F$}

A hipótese $H_{01}$ pode ser escrita na seguinte na forma matricial $H_{01}: C \mu_{v 1}=C \mu_{v 2}$, que corresponde a testar o paralelismo entre os dois perfis de crescimento. A matriz $C$ apresenta a seguinte formatação e a estatística para testar $H_{01}$ é a de Hotelling.

$$
\begin{aligned}
& S=\frac{\left(n_{1}-1\right) S_{1}+\left(n_{2}-1\right) S_{2}}{n_{1}+n_{2}-2} \quad\left(n_{1}=n_{2}=30\right) \\
& S=\left[\begin{array}{l}
2,00769462,18270992,28186722,3655459 \\
2,182709944,61594915,47765205,89239385,8964653 \\
2,28186725,47765207,17738707,79440577,9604459 \\
2,36554595,89239387,79440578,89007779,1532809 \\
2,44676385,89646537,96044599,15328099,8844803
\end{array}\right] \\
& S=\frac{S_{1}+S_{2}}{2} \quad C_{(p-1) p}=\left[\begin{array}{cccccc}
1 & -1 & 0 & \cdots & 0 & 0 \\
0 & 1 & -1 & \cdots & 0 & 0 \\
\cdots & \cdots & \cdots & \cdots & \cdots & \cdots \\
0 & 0 & 0 & \cdots & 1 & -1
\end{array}\right] \\
& C S C^{\prime}=\left[\begin{array}{cccc}
2,2582238 & 0,7625456 & 0,3310632 & -0,0771460 \\
0,7625456 & 0,8380321 & 0,2022769 & 0,1619687 \\
0,3310632 & 0,2022769 & 0,4786533 & 0,0971629 \\
-0,0771460 & 0,1619687 & 0,0971629 & 0,4679963
\end{array}\right] \\
& \left(C S C^{\prime}\right)^{-1}=\left[\begin{array}{ccrr}
0,7273679 & -0,668400 & -0,3047660 & 0,4145026 \\
-0,6684000 & 2,003218 & -0,2308800 & -0,7555410 \\
-0,3047660 & -0,230880 & 2,4967583 & -0,4886980 \\
0,4145026 & -0,755541 & -0,4886980 & 2,5680434
\end{array}\right] \\
& T_{12}^{2}=\frac{n_{1} n_{2}}{n_{1}+n_{2}}\left(\bar{y}_{v 1}-\bar{y}_{v 2}\right)^{\prime} C^{\prime}\left(C S C^{\prime}\right)^{-1} C\left(\bar{y}_{v 1}-\bar{y}_{v 2}\right)=25,378072
\end{aligned}
$$

A estatística de Hotelling $T_{12}^{2}$ pode transformada na estatística $F$ de Snedecor que, sob $H_{01}$, apresenta $(p-1)=4$ e $\left(n_{1}+n_{2}-p\right)=55$ graus de liberdade $(p=5$ e $n=30)$.

$$
F=\frac{n_{1}+n_{2}-p}{\left(n_{1}+n_{2}-2\right)(p-1)} T^{2}=6,0163532
$$

Haja vista o valor de $F_{4 ; 55 ; 0,01} \cong 3,65$, conclui-se que os perfis de crescimento dos métodos de manejo M1 e M2 não são paralelos, ou seja, foi rejeitada a hipótese de não existência de interação método de manejo×ocasião, então se deve obter a equação e o ciclo de corte ideal, separadamente, para cada método. 
Por outro lado, considerando o presente exemplo de aplicação, foi realizada a análise de variância multivariada de perfil, que será abordada na seção 7.4. A tabela 7.3.1.1, considerando os dois métodos M1 e M2, apresenta a verificação da interação entre método de manejo×ocasião. Pode ser verificado, como o esperado, que o resultado foi o mesmo para a estatística $F(6,02)$. Análise realizada pelo programa desenvolvido no SAS, apresentado na tabela C8 do apêndice $C$, usando o arquivo com os métodos M1 e M2.

Tabela 7.3.1.1 - Teste da interação dos perfis de crescimento para M1 e M2.

\begin{tabular}{l|c|c|c|c|c}
\multicolumn{5}{c}{$\begin{array}{c}\text { Testes multivariados e as estatísticas exatas de F para a hipótese de não existência de } \\
\text { efeito da interação Ocasião×Método de Manejo (S=1; M=1; N*26,5) }\end{array}$} \\
\begin{tabular}{l|c|c|c|c|c} 
Testes multivariados \\
Telores
\end{tabular} Valor F & Num GL & Den GL & Prob. \\
Wilks Lambda & 0,69562666 & 6,02 & 4 & 55 & 0,0004 \\
\hline Pillai Trace & 0,30437334 & 6,02 & 4 & 55 & 0,0004 \\
\hline Hotelling-Lawley Trace & 0,43755272 & 6,02 & 4 & 55 & 0,004 \\
\hline Roy Greatest Root & 0,43755272 & 6,02 & 4 & 55 & 0,0004 \\
\hline
\end{tabular}

\section{e) Ciclo de corte economicamente aconselhável para os métodos M1 e M2}

0 método M1 é o mesmo considerado no exemplo de aplicação 7.2.1, onde o ciclo de corte economicamente aconselhável foi 18,7 anos.

Dada a equação $V=\beta_{0}\left[1-10^{-\beta_{1}\left(t+\beta_{2}\right)}\right]$, para obter a duração do ciclo de corte ideal para o método de manejo M2, têm-se as estimativas dos parâmetros, na tabela 7.3.1.2, que foram obtidas pelo programa SAS citado na tabela C12 do apêndice C.

Tabela 7.3.1.2 - Análise de regressão da equação de Mitscherlich para M2.

\begin{tabular}{c|c|c|c|c} 
Parâmetros & Estimativas & Erro Padrão Aprox. & Limites de Confiança (95\%) \\
$\hat{\beta}_{0}$ & 143,6 & 0,8096 & 142,0 & 145,2 \\
\hline$\hat{\beta}_{1}$ & 0,0692 & 0,00375 & 0,0618 & 0,0767 \\
\hline$\hat{\beta}_{2}$ & 7,0005 & 0,3591 & 6,2908 & 7,7103 \\
Fonte & GL & Soma de Quadrados & Quadrado Médio & Valor de F \\
Erro & 147 & 1821,8 & 12,3934 & 1649,98 \\
\hline \multicolumn{5}{c}{ Teste de validade de Graybill $\left(F_{G}\right)$} \\
Fonte & GL & Qualor de $F_{G}$ & Pr $>$ F \\
Numerador & 2 & 38,84695 & 3,30 & 0,0398 \\
\hline Denominador & 148 & 11,78467 & Q & \\
\hline Coeficiente de correlação de Pearson, N =150, Prob > |r| Sob H0: Rho=0 \\
\hline$r_{M 2}=0,97844(<0,0001)$ & $r_{M 2}{ }_{M 2}=0,9573$ \\
\hline
\end{tabular}




$$
V_{M 2}=143,6\left[1-10^{-0,0692(t+7,0005)}\right] \quad(0 \leq t \leq 20 \text { anos })
$$

Dado que $t_{o t i}=\frac{1}{\hat{\beta}_{1}} \log _{10}\left[2,302585 R \hat{\beta}_{0} \hat{\beta}_{1}\right]-\hat{\beta}_{2}$ e considerando $R=3$, então:

$$
\begin{gathered}
t_{\text {oti }(M 2)}=\left(\frac{1}{0,0692}\right) \log (2,302585 \times 3 \times 143,6 \times 0,0692)-7,0005=19,5 \text { anos } \\
V_{\text {oti }(M 2)}=143,6\left[1-10^{-0,0692(19,5+7,0005)}\right]=141,49 \mathrm{~m}^{3} / \mathrm{ha}
\end{gathered}
$$

$$
\begin{aligned}
& \mathrm{RQMR}=\sqrt{\frac{\sum_{i=1}^{N}\left(Y_{i}-\hat{Y}_{i}\right)^{2}}{N}}=\sqrt{\frac{1821,8}{30 \times 5}}=3,4850 \\
& \mathrm{CV}=\frac{R Q M R}{\bar{Y}} \times 100=\frac{3,4850}{126,82} \times 100=2,7489 \%
\end{aligned}
$$

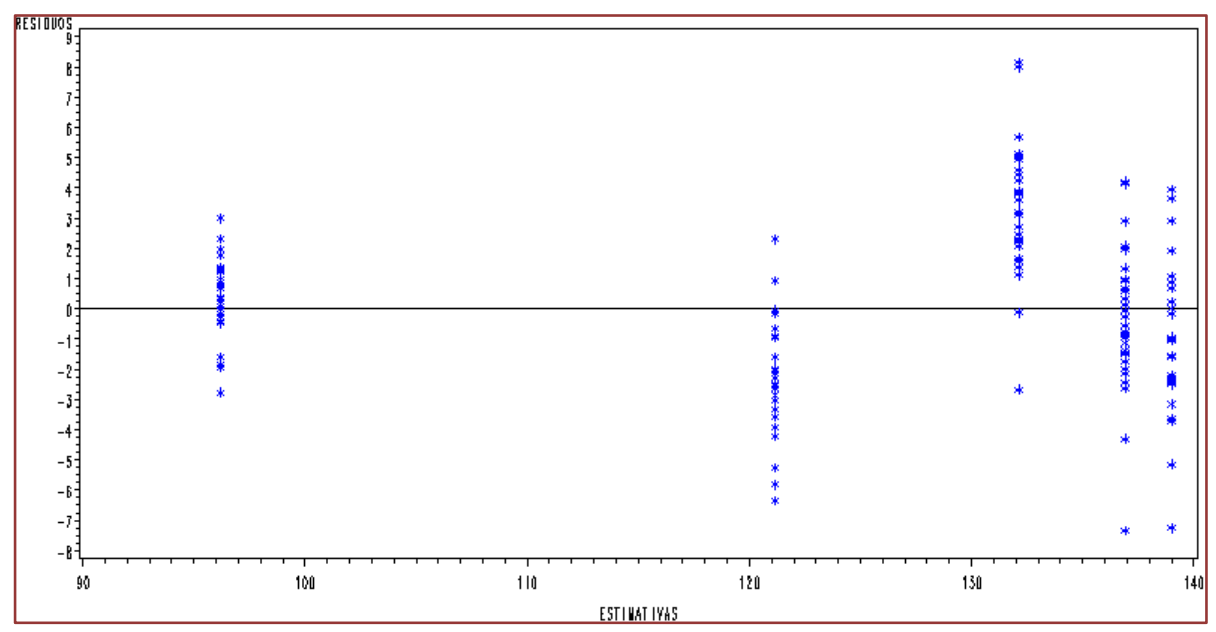

Figura 7.3.1.2 - Gráfico dos resíduos para o método de manejo M2.

Os indicadores CV\%, $r^{2}{ }_{M 1}, F_{G}$ e a distribuição dos resíduos, para avaliação do ajustamento do modelo de Mitscherlich para o método M2, apresentaram resultados satisfatórios dentro do esperado.

Verifica-se pelos resultados, que a floresta, quando aplicado o método de manejo M1, o ciclo de corte economicamente aconselhável foi de 18,7 anos $\left(138,57 \mathrm{~m}^{3} / \mathrm{ha}\right)$, enquanto que, pelo método de manejo M2, a duração do ciclo foi de 19,5 anos $\left(141,49 \mathrm{~m}^{3} / \mathrm{ha}\right)$. Portanto, considerando o ciclo de corte, o método de manejo M1 seria o recomendável, entretanto, analisando a produção de madeira, o método de manejo M2 apresenta um acréscimo de produção de 2,92 m³ /ha em comparação com M1, concluindo-se, então, que a escolha do método de manejo dependerá do preço da madeira no mercado. 
Por outro lado, supondo que os resultados apresentassem paralelismo entre os perfis de M1 e M2, então poderia ser aplicado o teste " $t$ " para verificar a existência de coincidência dos mesmos, e em sendo confirmada, uma única equação de crescimento seria ajustada para os dois métodos.

\subsection{INVENTÁRIOS FLORESTAIS COM PARCELAS PERMANENTES COM MAIS DE DOIS TRATAMENTOS}

Os inventários florestais com parcelas permanentes devem ser analisados pela técnica de análise de perfil. Este procedimento corresponde a um problema multivariado que objetiva analisar dados oriundos de experimentos com medidas repetidas. A análise de perfil tem como característica ser um método alternativo mais geral, haja vista não fazer restrições quanto à estrutura da matriz de covariância.

O objetivo da análise de perfil é estudar a variação dos efeitos dos tratamentos ao longo do tempo, por isso, mais modernamente, este método é denominado de análise de levantamento longitudinal. A análise de perfil, normalmente, visa responder às seguintes questões:

a) Hipótese $H_{01}$ : os perfis médios populacionais dos tratamentos apresentam comportamentos similares (paralelismo)?

Testar essa hipótese para verificar a existência de interação entre os tratamentos e as ocasiões. A ausência dessa interação implica em um paralelismo estatístico entre os perfis.

b) Hipótese Ho2: aceito o paralelismo entre os perfis médios populacionais, perguntar-seia? São os mesmos coincidentes?

A comprovação da hipótese de coincidência dos perfis, para o conjunto das $p$ ocasiões, é verificada testando a existência de igualdade dos efeitos dos $g \geq 3$ tratamentos.

c) Hipótese $H_{03}$ : os perfis médios das respostas correspondentes aos tratamentos são paralelos ao eixo das abscissas, ou seja, não existe efeito do fator que define as ocasiões?

A verificação da existência de diferença entre as ocasiões considerando os $g \geq 3$ grupos, concernente a referida hipótese, significaria testar se a floresta está crescendo.

Seja um experimento florestal consistindo de $g$ métodos de manejo florestal, tal que são medidas $n_{i}$ parcelas permanentes por tratamento e em $p$ ocasiões. Seja $\left(y_{i j 1}, \ldots, y_{i j k}, \ldots, y_{i j p}\right)$ o perfil correspondente a $j$-ésima parcela permanente, do $i$-ésimo tratamento, da $k$-ésima ocasião, onde $j=1, \ldots, n_{i}, i=1, \ldots, g$ e $k=1, \ldots, p$.

A análise de perfil, ou análise de dados longitudinais, tem por objetivo estudar a variação dos efeitos de tratamentos ao longo do tempo. Seja $Y(N \times p)$ a matriz que constitui o conjunto de todas as observações, onde $\mathrm{N}=n_{1}+n_{2}+\cdots+n_{g}$. Os vetores $y_{v i j}$, correspondentes aos perfis das $N$ unidades experimentais permanentes, são 
considerados provenientes de amostras de uma distribuição normal multivariada com $p$ ocasiões, apresentando vetor de médias $\mu_{\mathrm{vi}}=\left[\mu_{\mathrm{i} 1}, \ldots, \mu_{\mathrm{ip}}\right]$ e matriz de covariância comum aos $N$ vetores $\sum$.

O modelo linear geral multivariado, para o conjunto de dados, tem a forma: $\mathrm{Y}=X \beta+\varepsilon$. A matriz $Y$ é a matriz das observações com dimensão $N \times p$, ou seja, $p$ medidas para cada uma das $N$ unidades experimentais. A matriz $X$ é denominada de planejamento com dimensão $N \times g$, sendo construída pelos números 0 e 1 de acordo com delineamento inteiramente ao acaso.

$$
\left[\begin{array}{cccc}
y_{111} & y_{112} & \cdots & y_{11 p} \\
\cdots & \cdots & \cdots & \cdots \\
y_{n_{1} 11} & y_{n_{1} 12} & \cdots & y_{n_{1} 1 p} \\
y_{121} & y_{122} & \cdots & y_{12 p} \\
\cdots & \cdots & \cdots & \cdots \\
y_{n_{2} 21} & y_{n_{2} 22} & \cdots & y_{n_{2} 2 p} \\
\cdots & \cdots & \cdots & \cdots \\
\cdots & \cdots & \cdots & \cdots \\
y_{1 g 1} & y_{1 g 2} & \cdots & y_{1 g p} \\
\cdots & \cdots & \cdots & \cdots \\
y_{n_{g} g 1} & y_{n_{g} g} & \cdots & y_{n_{g} g p}
\end{array}\right]=\left[\begin{array}{cccc}
1 & 0 & \cdots & 0 \\
\cdots & \cdots & \cdots & \cdots \\
1 & 0 & \cdots & 0 \\
0 & 1 & \cdots & 0 \\
\cdots & \cdots & \cdots & \cdots \\
0 & 1 & \cdots & 0 \\
\cdots & \cdots & \cdots & \cdots \\
\cdots & \cdots & \cdots & \cdots \\
0 & 0 & \cdots & 1 \\
\cdots & \cdots & \cdots & \cdots \\
0 & 0 & \cdots & 1
\end{array}\right] \times\left[\begin{array}{cccc}
\beta_{11} & \beta_{12} & \cdots & \beta_{1 p} \\
\beta_{21} & \beta_{22} & \cdots & \beta_{2 p} \\
\cdots & \cdots & \cdots & \cdots \\
\beta_{g 1} & \beta_{g 2} & \cdots & \beta_{g p}
\end{array}\right]+\left[\begin{array}{cccc}
\varepsilon_{111} & \varepsilon_{112} & \cdots & \varepsilon_{11 p} \\
\cdots & \cdots & \cdots & \cdots \\
\varepsilon_{n_{1} 11} & \varepsilon_{n_{1} 12} & \cdots & \varepsilon_{n_{1} 1 p} \\
\varepsilon_{121} & \varepsilon_{122} & \cdots & \varepsilon_{12 p} \\
\cdots & \cdots & \cdots & \cdots \\
\varepsilon_{n_{2} 21} & \varepsilon_{n_{2} 22} & \cdots & \varepsilon_{n_{2} 2 p} \\
\cdots & \cdots & \cdots & \cdots \\
\cdots & \cdots & \cdots & \cdots \\
\varepsilon_{1 g 1} & \varepsilon_{1 g 2} & \cdots & \varepsilon_{1 g p} \\
\cdots & \cdots & \cdots & \cdots \\
\varepsilon_{n_{g} g 1} & \varepsilon_{n_{g} g 2} & \cdots & \varepsilon_{n_{g} g p}
\end{array}\right]
$$

A matriz $\beta$, de dimensão $g \times p$, é denominada de parâmetros desconhecidos (médias populacionais), correspondentes aos efeitos dos tratamentos envolvidos no experimento. Cada linha representa os valores esperados em cada ocasião.

A matriz $\varepsilon$ de dimensão $N \times p$ é composta pelos componentes aleatórios das observações.

Sejam $\hat{\beta}$ e $S$ as estimativas não tendenciosas de quadrados mínimos ou de máxima verossimilhança das matrizes $\beta$ e $\sum$ do modelo $\mathrm{Y}=X \beta+\varepsilon$ :

$$
\hat{\beta}=\left(X^{\prime} X\right)^{-1} X^{\prime} Y S=Y^{\prime}\left[I-X\left(X^{\prime} X\right)^{-1} X^{\prime}\right] Y /(N-g)
$$

Seja $H_{01}$ a hipótese para verificar a ocorrência de paralelismo entre os perfis. Em termos dos parâmetros do modelo $\mathrm{Y}=X \beta+\varepsilon$, ou seja, $\mathrm{E}(\mathrm{Y})=X \beta$, pode-se escrever $H_{01}$ da forma:

$$
H_{01}:\left[\begin{array}{c}
\mu_{11}-\mu_{12} \\
\mu_{12}-\mu_{13} \\
\vdots \\
\mu_{1(p-1)}-\mu_{1 p}
\end{array}\right]=\left[\begin{array}{c}
\mu_{21}-\mu_{22} \\
\mu_{22}-\mu_{23} \\
\vdots \\
\mu_{2(p-1)}-\mu_{2 p}
\end{array}\right]=\cdots=\left[\begin{array}{c}
\mu_{g 1}-\mu_{g 2} \\
\mu_{g 2}-\mu_{g 3} \\
\vdots \\
\mu_{g(p-1)}-\mu_{g p}
\end{array}\right]
$$

Andrade \& Singer (1986) citam que a hipótese $H_{01}$ é fundamental, haja vista que a sua validade pode determinar a forma das hipóteses $H_{02}$ e $H_{03}$. No caso de não rejeição de $H_{01}$, ou seja, o paralelismo dos perfis médios de resposta é aceitável, então as hipóteses $\mathrm{H}_{02}$ e $\mathrm{H}_{03}$ podem ser expressas como: 


$$
H_{02}: \sum_{k=1}^{p} \mu_{1 k}=\sum_{k=1}^{p} \mu_{2 k}=\cdots=\sum_{k=1}^{p} \mu_{g k} \quad H_{03}: \sum_{i=1}^{g} \mu_{i 1}=\sum_{i=1}^{g} \mu_{i 2}=\cdots=\sum_{i=1}^{g} \mu_{i p}
$$

Com efeito, $\mathrm{H}_{02}$ : coincidência dos perfis de crescimento equivale à igualdade das respostas médias globais considerando todas as $p$ ocasiões, enquanto $H_{03}$ : os perfis médios das respostas correspondentes aos tratamentos são paralelos ao eixo das abscissas, corresponde à igualdade das respostas médias globais, mas considerando todos os $g$ tratamentos.

As hipóteses $\mathrm{H}_{02}$ e $\mathrm{H}_{03}$, independentemente do conhecimento a priori da não rejeição de $H_{01}$, podem ser escritas das seguintes formas alternativas:

$$
H_{02}^{*}:\left[\begin{array}{c}
\mu_{11} \\
\mu_{12} \\
\vdots \\
\mu_{1 p}
\end{array}\right]=\left[\begin{array}{c}
\mu_{21} \\
\mu_{22} \\
\vdots \\
\mu_{2 p}
\end{array}\right]=\cdots=\left[\begin{array}{c}
\mu_{g 1} \\
\mu_{g 2} \\
\vdots \\
\mu_{g p}
\end{array}\right] \quad H_{03}^{*}:\left[\begin{array}{c}
\mu_{11} \\
\mu_{21} \\
\vdots \\
\mu_{g 1}
\end{array}\right]=\left[\begin{array}{c}
\mu_{12} \\
\mu_{22} \\
\vdots \\
\mu_{g 2}
\end{array}\right]=\cdots=\left[\begin{array}{c}
\mu_{1 p} \\
\mu_{2 p} \\
\vdots \\
\mu_{g p}
\end{array}\right]
$$

Por outro lado, objetivando a unificação dos resultados estatísticos, as hipóteses de nulidades podem ser apresentadas na forma da hipótese linear geral, $H: C \beta U=\phi$, tal que $C(c \times g)$ e $U(p \times u)$ são matrizes de constantes com postos $c$ e $u$. A matriz $C$ de ordem $c \times g$ é construída para definir os contrastes entre os tratamentos, enquanto a matriz $U$ de ordem $p \times u$ é a matriz responsável pelos contrastes entre ocasiões.

No caso de $H_{01}: C_{1} \beta U_{1}=\phi$, têm-se:

$$
C_{1}=\left[\begin{array}{cccccc}
1 & -1 & 0 & \cdots & 0 & 0 \\
1 & 0 & -1 & \cdots & 0 & 0 \\
\cdots & \cdots & \cdots & \cdots & \cdots & \cdots \\
1 & 0 & 0 & \cdots & 0 & -1
\end{array}\right]_{g} \quad U_{1}=\left[\begin{array}{cccc}
1 & 0 & \cdots & 0 \\
-1 & 1 & \cdots & 0 \\
0 & -1 & \cdots & 0 \\
\cdots & \cdots & \cdots & \cdots \\
0 & 0 & \cdots & 1 \\
0 & 0 & \cdots & -1
\end{array}\right]_{(p-1)}
$$

$H_{02}: C_{2} \beta U_{2}=\phi$, tal que $\mathrm{C}_{2}=\mathrm{C}_{1}$ e $U_{2}^{\prime}=\left[\begin{array}{llll}1 & 1 & \cdots & 1\end{array}\right]$ é um vetor unitário $p$-dimensional;

$H_{03}: C_{3} \beta U_{3}=\phi$, tal que $C_{3}^{\prime}=\left[\begin{array}{llll}1 & 1 & \cdots & 1\end{array}\right]$ é um vetor unitário $g$-dimensional e $U_{3}=U_{1}$;

$H_{02}^{*}: C_{2} \beta U_{2}=\phi$, tal que $C_{2}=\mathrm{C}_{1}$ e $U_{2}^{\prime}=\left[\begin{array}{llll}1 & 1 & \cdots & 1\end{array}\right]$ é um vetor unitário $p$-dimensional;

$H_{03}^{*}: C_{3} \beta U_{3}=\phi$, tal que $C_{3}^{\prime}=\left[\begin{array}{llll}1 & 1 & \cdots & 1\end{array}\right]$ é um vetor unitário $g$-dimensional e $U_{3}=U_{1}$; 
É importante esclarecer que as representações dessas matrizes não são únicas. Por exemplo, as matrizes $C_{1}^{*}$ e $C_{1}$ são equivalentes na definição de $H_{01}, H_{02}$ ou $H_{02}^{*}$.

$$
C_{1}^{*}=\left[\begin{array}{cccccc}
1 & -1 & 0 & \cdots & 0 & 0 \\
0 & 1 & -1 & \cdots & 0 & 0 \\
\cdots & \cdots & \cdots & \cdots & \cdots & \cdots \\
0 & 0 & 0 & \cdots & 1 & -1
\end{array}\right]_{g}
$$

As hipóteses expressas pelo modelo linear geral $H: C \beta U=\phi$ podem ser testadas por diversos critérios heurísticos que, geralmente, são estatísticas das raízes características da matriz $H E^{-1}$, tal que $H=(C \hat{\beta} U)^{\prime}\left[C\left(X^{\prime} X\right)^{-1} C^{\prime}\right]^{-1}(C \hat{\beta} U)$ para $\hat{\beta}=\left(X^{\prime} X\right)^{-1} X^{\prime} Y$ e $E=U^{\prime} Y^{\prime}\left[I-X\left(X^{\prime} X\right)^{-1} X^{\prime}\right] Y U$, sendo que $H$ de ordem $p \times p$ é a matriz de soma de quadrados e produtos cruzados devido à hipótese nula e $E$, de ordem $p \times p$, a matriz de soma de quadrados e produtos cruzados devido ao erro.

As estatísticas mais conhecidas para testar $H: C \beta U=\phi$ são as baseadas no princípio da união-intersecção de Roy e da razão de verossimilhança de Wilks. Outras estatísticas muito utilizadas são Lawley-Hotelling e de Pillai.

$$
\begin{gathered}
H=U^{\prime} Y^{\prime} X\left(X^{\prime} X\right)^{-1} C^{\prime}\left(C\left(X^{\prime} X\right)^{-1} C^{\prime}\right)^{-1} C\left(X^{\prime} X^{-1} X^{\prime} Y U\right. \\
E=U^{\prime} Y^{\prime}\left[I-X\left(X^{\prime} X\right)^{-1} X^{\prime}\right] Y U
\end{gathered}
$$

Seja uma combinação linear arbitrária $a_{v}^{\prime} y_{v}$ das $p$ variáveis, sendo $a_{v} \neq 0_{v}$ e $a_{v} \in R^{p}$. A hipótese $H_{0}$ será verdadeira se, e se somente se, $H_{0}$ for verdadeira para todo $a_{v} \neq 0$. A aceitação de $H_{0}$ é equivalente aceitar todas as hipóteses univariadas possíveis efetuadas variando os elementos que constituem o vetor $a_{v}$. Diz-se, então, que a região multivariada de aceitação de $H_{0}$ é a interseção de todas as possíveis regiões de aceitação de todas as hipóteses univariadas.

A análise de variância multivariada para um fator consiste em procurar uma combinação linear das variáveis que melhor discrimine os $g$ grupos (tratamentos). A combinação linear melhor indicada é aquela que produz o maior valor para a estatística univariada F.

De acordo com o princípio da união-interseção de Roy, tem-se:

$$
F_{(a)}=\frac{a_{v}^{\prime} H a_{v} /(g-1)}{a^{\prime} E a_{v} /(N-g)}=\frac{a_{v}^{\prime} H a_{v}}{a_{v}^{\prime} E a_{v}}\left(\frac{N-g}{g-1}\right) \text {. Tal que } H(p \times p) e E(p \times p) .
$$

Dada restrição para que $a_{v}$ seja único: $a_{v}^{\prime} E a_{v}=1$. Pelo método dos multiplicadores de Lagrange, resulta: $\left|E^{-1} H-\lambda I\right|=0$ e $F\left(a_{v k}\right)=\frac{N-g}{g-1} \lambda_{k}$. A maior raiz característica $\lambda_{k}$ da matriz $E^{-1} H$ ou de $H E^{-1}$ corresponde à solução de máximo. 


\subsection{TESTES DE HIPÓTESE MULTIVARIADOS E AS APROXIMAÇÕES PARA A DISTRIBUIÇÃO F}

$H_{0}$ : não existe interação método de manejo×ocasião: $H_{01}: C_{1} \beta U_{1}=\phi$.

Seja $\theta_{k}=\lambda_{k} /\left(1+\lambda_{k}\right)$, tal que $\lambda_{k}$ corresponde a $k$-ésima raiz característica de $E^{-1} H$ ou $H E^{-1}$.

As estatísticas de testes:

a) Critério lambda de Wilks $(\Lambda)$ :

$$
\Lambda=\prod_{k=1}^{p}\left(1-\theta_{k}\right)=\frac{|E|}{|E+H|}
$$

b) Critério de Pillai $\left(V_{P}\right)$ :

$$
V_{P}=\sum_{k=1}^{p} \theta_{k}=\sum_{k=1}^{p} \frac{\lambda_{k}}{1+\lambda_{k}}=\operatorname{tr}\left[H(H+E)^{-1}\right]
$$

c) Critério do traço de Lawley-Hotelling Pillai $\left(T_{L H}\right)$ :

$$
T_{L H}=\operatorname{tr}\left(E^{-1} H\right)=\sum_{k=1}^{p} \lambda_{k} \text { ou } T_{L H}=\sum_{k=1}^{p} \theta_{k}\left(1-\theta_{k}\right)^{-1}
$$

d) Critério do máximo autovalor de $\operatorname{Roy}\left(\theta_{R}\right)$ :

$$
\theta_{R}=\operatorname{máx}\left(\lambda_{k}\right)
$$

As fórmulas para obter os valores aproximados das transformações de $\Lambda, V_{P}, T_{L H}$ e $\theta_{R}$, para a estatística $F$, estão apresentadas no capítulo 4 , seção 4.5 .

As distribuições exatas dessas estatísticas sob a hipótese nula dependem unicamente dos parâmetros $M, N^{*}$ e $S$, e são encontradas em Morrison (1976). Anderson (1958) apresenta as aproximações assintóticas para as distribuições $\chi^{2}$ e $F$.

$$
M=\frac{(|u-c|-1)}{2} \quad N^{*}=\frac{(N-g-u-1)}{2} \quad S=\min (u, c)
$$

$u$ : é o posto da matriz da matriz $U$;

$c$ : é o posto da matriz $C$;

$g$ : é o posto da matriz $X$ 


\subsection{ANÁLISE DE AGRUPAMENTO PELO MÉTODO DE WARD}

A análise de agrupamento é a técnica multivariada que consiste na aplicação de uma série de técnicas e algoritmos, objetivando identificar e separar objetos ou indivíduo em grupos similares. A análise de agrupamento também é conhecida como análise de conglomerados, classificação ou cluster.

Seja uma amostra de $g$ objetos, onde foram observadas $p$ características ou variáveis. 0 problema a ser resolvido pela análise de agrupamento é estabelecer um esquema de classificação, visando congregar esses indivíduos em um determinado número de grupos, os mais homogêneos possíveis, e que sejam dimensionados e caracterizados, tal que o pesquisador possa eleger representantes típicos para a população e assim poder descrevê-la com a máxima precisão.

Os algoritmos utilizados nas análises de agrupamento podem ser classificados:
a) Técnicas hierárquicas;
b) Técnicas de partição;
c) Técnicas de cobertura.

Nas técnicas hierárquicas os objetos são classificados em grupos em diferentes etapas, tendo como resultado uma árvore de classificação (dendrograma ou fenograma). 0 que caracteriza o processo hierárquico é que a reunião numa determinada etapa de dois grupos produzirá um dos agrupamentos da etapa superior.

Os algoritmos hierárquicos podem ser classificados em aglomerativos e divisivos. 0 agrupamento é definido como aglomerativo quando através de fusões sucessivas dos $g$ indivíduos ou objetos são produzidos $g-1, g$-2, etc. grupos, e assim, sucessivamente, até reunir todos os indivíduos em um único grupo. Divisivos quando a partir de um único grupo e por subdivisões sucessivas são obtidos 2,3 ,..., etc. grupos.

Nas técnicas de partição os agrupamentos são resultantes de uma partição do conjunto de objetos. Nos métodos de cobertura os agrupamentos resultantes recobrem o conjunto de objetos, inclusive podem se sobrepor um ao outro.

Os algoritmos também podem ser sequenciais ou não sequenciais. São algoritmos sequenciais quando o mesmo conjunto de operações é aplicado nas várias etapas do agrupamento.

Seja $y_{v i}$ o vetor que representa os valores observados da variável $y_{i j}$ correspondente ao método de manejo florestal $M_{i}(i=1,2, \cdots, g)$.

$y_{i j}$ : é o volume de madeira, concernente a $j$-ésima ocasião $(j=1,2, \cdots, p)$, correspondente ao método de manejo $M_{i}(i=1,2, \cdots, g)$, isto é, $y_{v i}^{\prime}=\left[\begin{array}{llll}y_{i 1} & y_{i 2} & \cdots & y_{i p}\end{array}\right]$.

Segundo Ferreira (2011), o método de Ward consiste em um procedimento de agrupamento hierárquico baseado na análise de variância, ou seja, as somas de 
quadrados entre e dentro dos grupos, em relação às $p$ variáveis, são utilizadas como critério de agrupamento.

O método de Ward é também conhecido como o método do incremento das somas de quadrados, no qual a medida de similaridade usada para reunir grupos (agrupamento ou clusters) é calculada como a soma de quadrados, sobre todas as variáveis, entre os dois agrupamentos. Em cada fase, combinam-se os dois grupos que apresentarem menor aumento, dentro dos agrupamentos, na soma total de quadrados.

Montada a matriz de soma de quadrados, considerando pares de indivíduos, considerase como um grupo formado o par que apresentar a mínima soma de quadrados. 0 processo continua seguindo o princípio da soma de quadrados mínima. Mingoti (2005) cita que o método de Ward é também chamado de variância mínima e fundamenta-se nos princípios:

a) Considerar, inicialmente, que cada elemento (indivíduos ou objetos) é um único grupo;

b) Calcular, em cada passo do algoritmo de agrupamento, a soma de quadrados dentro de cada grupo. Esta soma é o quadrado da distância Euclidiana de cada elemento amostral pertencente ao grupo ou cluster em relação ao correspondente vetor de médias do grupo, ou seja:

$$
S Q_{i}=\sum_{j=1}^{n_{i}}\left(y_{v i j}-\bar{y}_{v i .}\right)^{\prime}\left(y_{v i j}-\bar{y}_{v i .}\right)
$$

$n_{i}$ : é o número de elementos do conglomerado $C_{i}$ no $k$-ésimo passo do processo;

$y_{v i j}$ : é o vetor de observações do $j$-ésimo elemento amostral que pertence ao $i$-ésimo conglomerado;

$\bar{y}_{v i .}$ : é o centroide do conglomerado $C_{i}$;

$S Q_{i}$ : é a soma de quadrados correspondente ao conglomerado $C_{i}$.

No $k$-ésimo passo, a soma de quadrados total dentro dos grupos é definida, como:

$$
S Q R=\sum_{i=1}^{g_{k}} S Q_{i}
$$

$g_{k}$ : é o numero de grupos existentes no $k$-ésimo passo.

A distância entre os conglomerados $C_{l}$ e $C_{i}$ é dada pela expressão:

$$
\begin{gathered}
d\left(C_{l}, C_{i}\right)=S Q_{l, i}-\left(S Q_{l}+S Q_{i}\right) \\
d\left(C_{l}, C_{i}\right)=\left(\frac{n_{l} n_{i}}{n_{l}+n_{i}}\right)\left[\left(\bar{y}_{v l .}-\bar{y}_{v i .}\right)^{\prime}\left(y_{v l .}-\bar{y}_{v i .}\right)\right]
\end{gathered}
$$


Esta expressão é a soma de quadrados entre os conglomerados $C_{l}$ e $C_{i}$. Em cada etapa da aplicação do algoritmo de agrupamento, os dois conglomerados que tornam a distância mínima $d\left(C_{l}, C_{i}\right)$ são reunidos.

Mingoti (2005) cita também que a medida de distância $d\left(C_{l}, C_{i}\right)$ é a diferença entre o valor de $S Q R$ depois e antes de se combinar os conglomerados $C_{l}$ e $C_{i}$ em um único grupo. Desta forma, em cada passo do agrupamento, o método de Ward combina os dois grupos que resultam no menor valor de $S Q R$.

Com efeito, considerando o conjunto de todos os grupos, a soma dos quadrados dos erros é dada pela soma de quadrados dos erros dos grupos individuais em relação a todos os grupos. É importante considerar, no método de Ward, que:

$$
S Q_{l i}=0,5 d\left(C_{l}, C_{i}\right)
$$

\subsection{ANÁLISE DE UM INVENTÁRIO FLORESTAL CONTÍNUO COM QUATRO MÉTODOS DE MANEJO}

Seja uma área florestal de 1000 ha onde foram aplicados quatro métodos de manejo M1, M2, M3, M4. O monitoramento do crescimento, em cada método de manejo, foi realizado por um inventário florestal contínuo com 30 parcelas permanentes. As medições foram realizadas em cinco ocasiões equidistantes de cinco anos, ou seja, o ano 0 (zero), 5 anos, 10 anos, 15 anos e 20 anos. 0 ano zero (0) corresponde à data logo após a exploração florestal. Os dados (volume por ha) foram simulados, dentro de um contexto próximo ao real de uma floresta natural, e estão apresentados na tabela B1 do apêndice B. Os cálculos foram realizados pelo sistema de análise estatística SAS e os programas desenvolvidos para efetuar as análises estão apresentados nas tabelas C7, C8, C9, C10, C11, C12 e C13 do apêndice $C$.

\subsubsection{ANÁLISE DA INTERAÇÃO OCASIÃO×MÉTODO DE MANEJO}

a) Vetores de médias, dos métodos de manejo M1, M2, M3 e M4, considerando as cinco ocasiões $0,5,10,15$ e 20 anos

$$
\begin{aligned}
\bar{y}_{v 1}^{\prime} & =\left[\begin{array}{lllll}
96,65589 & 118,86703 & 135,42923 & 136,51993 & 137,96803
\end{array}\right] \\
\bar{y}_{v 2}^{\prime} & =\left[\begin{array}{lllll}
97,07460 & 120,00173 & 137,35973 & 139,15100 & 140,50200
\end{array}\right] \\
\bar{y}_{v 3}^{\prime} & =\left[\begin{array}{lllll}
96,73433 & 130,93337 & 142,36860 & 149,35633 & 150,12670
\end{array}\right] \\
\bar{y}_{v 4}^{\prime} & =\left[\begin{array}{lllll}
97,17653 & 131,25127 & 143,12620 & 150,28670 & 150,97080
\end{array}\right]
\end{aligned}
$$


b) Gráfico dos perfis de crescimentos dos métodos de manejo M1, M2, M3 e M4

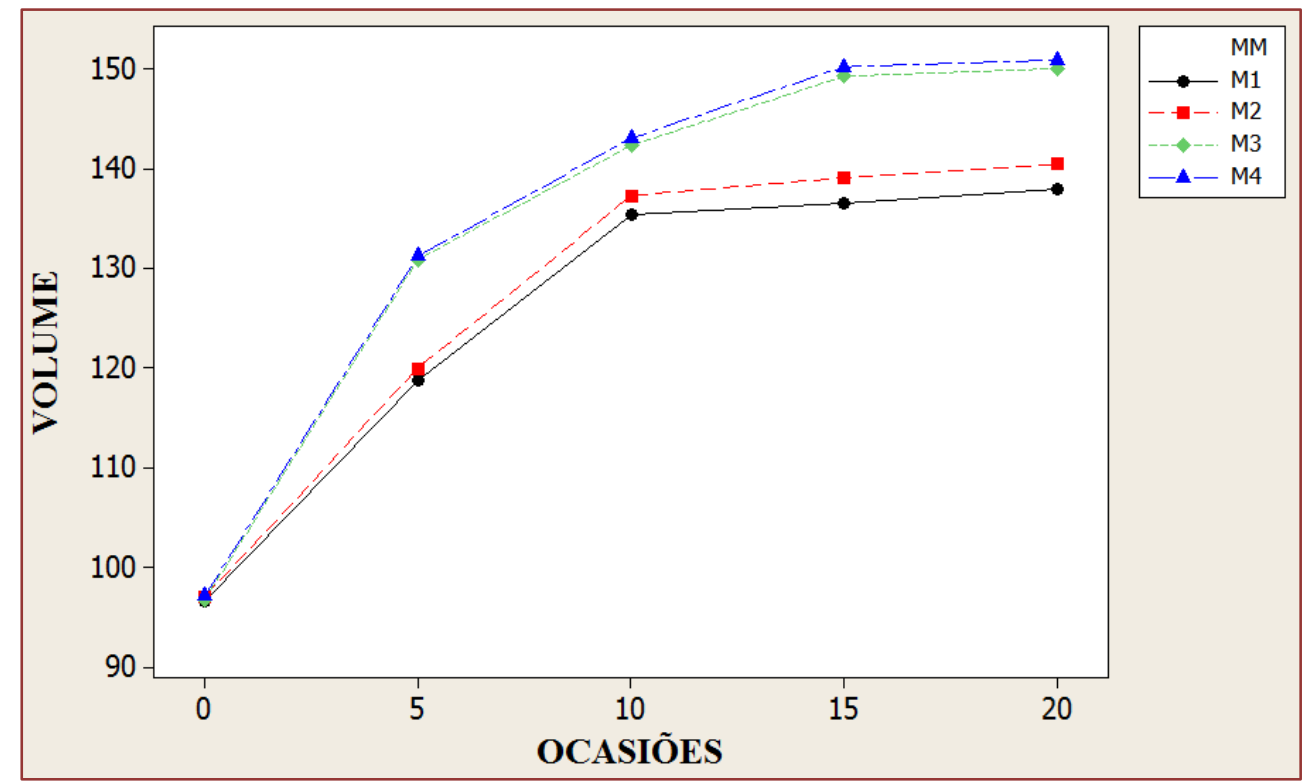

Figura 7.7.1.1 - Perfis de crescimentos dos métodos M1, M2, M3 e M4.

c) Matrizes de covariâncias dos métodos M1, M2, M3 e M4 considerando as cinco ocasiões 0, 5, 10, 15 e 20 anos

$$
\begin{aligned}
S_{1} & =\left[\begin{array}{lllll}
1,84061681 & 1,76852089 & 1,88189246 & 2,01479114 & 2,04154190 \\
1,76852089 & 3,46728879 & 3,78614806 & 4,05873545 & 4,04560596 \\
1,88189246 & 3,78614806 & 4,57316908 & 4,85074615 & 4,85566809 \\
2,01479114 & 4,05873545 & 4,85074615 & 5,47236765 & 5,51975907 \\
2,04154190 & 4,04560596 & 4,85566809 & 5,51975907 & 5,96583927
\end{array}\right] \\
S_{2} & =\left[\begin{array}{llllll}
2,17477232 & 2,59689899 & 2,68184199 & 2,71630059 & 2,85198576 \\
2,59689899 & 5,76460937 & 7,16915586 & 7,72605210 & 7,74732466 \\
2,68184199 & 7,16915586 & 9,78160489 & 10,7380652 & 11,0652238 \\
2,71630059 & 7,72605210 & 10,7380652 & 12,3077877 & 12,7868027 \\
2,85198576 & 7,74732466 & 11,0652238 & 12,7868027 & 13,8031214
\end{array}\right] \\
S_{3} & =\left[\begin{array}{llllll}
1,71308108 & 1,14810639 & 0,69754194 & 0,25827404 & -0,0956008 \\
1,14810639 & 3,47591569 & 3,96563570 & 4,17305767 & 3,99328305 \\
0,69754194 & 3,96563570 & 5,56337459 & 6.49248559 & 6,73230947 \\
0,25827404 & 4,17305767 & 6,49248559 & 8,46297747 & 9,04132329 \\
-0,0956008 & 3,99328305 & 6,, 3230947 & 9,04132329 & 10,0929095
\end{array}\right] \\
S_{4} & {\left[\begin{array}{llllll}
2,69608384 & 3,60369934 & 3,69112813 & 3,55789403 & 3,08094366 \\
3,60369934 & 6,37970758 & 7,23914367 & 7,42276691 & 6,54165978 \\
3,69112813 & 7,23914367 & 9,24803313 & 9,87512234 & 9,14563949 \\
3,55789403 & 7,42276691 & 9,87512234 & 11,19773456 & 10,7082246 \\
3,08094366 & 6,54165978 & 9,14563949 & 10,70822456 & 10,7512713
\end{array}\right] }
\end{aligned}
$$


d) Cálculo da matriz (comum) do erro

$$
S=\frac{S_{1}+S_{2}+S_{3}+S_{4}}{4}=\left[\begin{array}{rrrrr}
2,1061385 & 2,2793064 & 2,2381011 & 2,1368150 & 1,9697176 \\
2,2793064 & 4,7718804 & 5,5400208 & 5,8451530 & 5,5819684 \\
2,2381011 & 5,5400208 & 7,2915454 & 7,9891048 & 7,9497102 \\
2,1368150 & 5,8451530 & 7,9891048 & 9,3602169 & 9,5140274 \\
1,9697176 & 5,5819684 & 7,9497102 & 9,5140274 & 10,1532850
\end{array}\right]
$$

e) Análise de esfericidade da matriz de covariância $(S$ ) pelo critério de Mauchly e as correções de Greenhouse-Geisser Epsilon $\left(\varepsilon_{g g}\right)$ e de Huynh-Feldt Epsilon $\left(\varepsilon_{h f}\right)$

Tabela 7.7.1.1 - Teste de esfericidade pelo critério de Mauchly e os valores das correções de Greenhouse-Geisser Epsilon $\left(\varepsilon_{g g}\right)$ e de Huynh-Feldt Epsilon $\left(\varepsilon_{h f}\right)$.

\begin{tabular}{l|c|c|c|l} 
Variáveis & GL & Critério de Mauchly & Qui-Quadrado & Pr $>\chi^{2}$ \\
Variáveis Transformadas & 9 & 0,0084525 & 546,1448 & $<0,0001$ \\
\hline Componentes ortogonais & 9 & 0,0084525 & 546,1448 & $<0,0001$ \\
\hline Greenhouse-Geisser Epsilon $\hat{\varepsilon}_{g g}=0,3437$ \\
Huynh-Feldt Epsilon $\hat{\varepsilon}_{h f}=0,3554$
\end{tabular}

A Tabela 7.7.1.1 mostra o resultado do teste de esfericidade pelo critério de Mauchly, o qual apresentou um resultado altamente significativo, o que denota que a matriz do erro $(S)$ não é esférica, concluindo-se pela não realização da análise univariada de parcelas subdivididas (split-plot), mas caso seja o desejo do pesquisador, então pode ser usada a correção de Greenhouse-Geisser Epsilon $\left(\varepsilon_{g g}\right)$ ou a de Huynh-Feldt Epsilon $\left(\varepsilon_{h f}\right)$ para corrigir os graus de liberdade do teste $F$, para a igualdade de efeitos de ocasiões e para igualdade de paralelismo (interação de ocasião×método de manejo).

f) Teste de homogeneidade das matrizes de covariâncias

$$
H_{0}: \sum_{M 1}=\sum_{M 2}=\sum_{M 3}=\sum_{M 4}=\sum
$$

Pelo método da razão de máxima verossimilhança generalizada, Anderson (1958), temse:

$$
\begin{gathered}
M=\sum_{i=1}^{g}\left(n_{i}-1\right) \operatorname{Ln}|S|-\sum_{i=1}^{g}\left(n_{i}-1\right) \operatorname{Ln}\left|S_{i}\right| . \text { Então: } \\
M=4 \times 29 \times-0,49478-29 \times-4,10116=61,53916 \\
\operatorname{Ln}\left(\operatorname{det} S_{1}\right)=-1,72499 \quad \operatorname{Ln}\left(\operatorname{det} S_{2}\right)=-0,87645 \quad \operatorname{Ln}\left(\operatorname{det} S_{3}\right)=-0,64480 \\
\operatorname{Ln}\left(\operatorname{det} S_{4}\right)=-0,85492 \quad \operatorname{Ln}(\operatorname{det} S)=-0,49478
\end{gathered}
$$


De acordo com BOX (1949), citado por MORRISON (1976), a estatística $M C^{-1}$ é aproximadamente distribuída como uma variável qui-quadrado $\left(\chi^{2}\right)$ assintótica com $(v)$ graus de liberdade quando $n_{i}$ tende para infinito. Donde:

$$
v=\frac{1}{2}(g-1) p(p+1) \quad C^{-1}=1-\frac{2 p^{2}+3 p-1}{6(p+1)(g-1)}\left[\sum_{i=1}^{g} \frac{1}{n_{i}-1}-\frac{1}{\sum_{i=1}^{g}\left(n_{i}-1\right)}\right]
$$

Caso os tratamentos apresentem o mesmo número de repetições, $n=n_{1}=n_{2}=\cdots=n_{g}$, a fórmula de $C^{-1}$ será dada por:

$$
\begin{gathered}
C^{-1}=1-\frac{\left(2 p^{2}+3 p-1\right)(g+1)}{6(p+1) g \times(n-1)} \\
C^{-1}=1-\frac{\left(2 p^{2}+3 p-1\right)(g+1)}{6(p+1) g \times(n-1)}=1-\frac{\left(2 \times 5^{2}+3 \times 5-1\right)(4+1)}{6(5+1) \times 4 \times(29)}=0,92337 \\
M C^{-1}=61,53916 \times 0,92337=56,8234 \\
v=\frac{1}{2}(g-1) p(p+1)=\frac{1}{2}(4-1) \times 5 \times(5+1)=45
\end{gathered}
$$

Tabela 7.7.1.2 - Estatística de qui-quadrado $\chi^{2}$ (assintótico).

\begin{tabular}{c|c|c}
\hline Qui-Quadrado & \multicolumn{1}{c}{ GL } & Pr $>\chi^{2}$ \\
\hline 56,823368 & 45 & 0,1112 \\
\hline
\end{tabular}

Considerando o valor de $\operatorname{Pr}>$ Qui-Quadrado $=0,1112$ (tabela 7.7.1.2), então se conclui pela não rejeição de $H_{0}: \sum_{M 1}=\sum_{M 2}=\sum_{M 3}=\sum_{M 4}=\sum$, ou seja, as matrizes de covariâncias dos tratamentos M1, M2, M3, M4 podem ser consideradas homogêneas.

g) Testes de multinormalidade de Mardia e de Henze-Zirkler para M1, M2, M3 e M4

Seja a estatística do teste amostral para a assimetria de Mardia (1970):

$$
\hat{\beta}_{1, p}=\frac{1}{n^{2}} \sum_{i=1}^{n} \sum_{j=1}^{n}\left[\left(y_{v i}-\bar{y}_{v}\right)^{\prime} S^{-1}\left(y_{v j}-\bar{y}_{v}\right)\right]^{3}
$$

Tal que $A=n \hat{\beta}_{1, p} / 6$ é assintoticamente distribuída como uma variável aleatória quiquadrado com $p(p+1)(p+2) / 6$ graus de liberdades. 
Seja a estatística do teste amostral para a curtose de Mardia (1970):

$$
\left.\hat{\beta}_{2, p}=\frac{1}{n} \sum_{i=1}^{n}\left(y_{v i}-\bar{y}_{v}\right)^{\prime} S_{n}^{-1}\left(y_{v i}-\bar{y}_{v}\right)\right]^{2}
$$

Tal que $\quad B=\left[\hat{\beta}_{2, p}-p(p+2)\right] / \sqrt{8 p(p+2)}$ é assintoticamente distribuída segundo uma distribuição normal padrão.

De acordo Korkmaz, Goksuluk e Zararsiz (2014), a estatística do teste de multinormalidade de Henze-Zirkler, ver seção 3.2, é dada por:

$$
H Z=\frac{1}{n} \sum_{i}^{n} \sum_{j}^{n} e^{-\frac{\beta^{2}}{2} D_{i j}}-2\left(1+\beta^{2}\right)^{-\frac{p}{2}} \sum_{i=1}^{n} e^{-\frac{\beta^{2}}{2\left(1+\beta^{2}\right)} D_{i}}+n\left(1+2 \beta^{2}\right)^{-\frac{p}{2}}
$$

Se os dados são multivariados normais, a estatística do teste (HZ) é aproximadamente log-normalmente distribuída com média $\mu$ e variância $\sigma^{2}$. Usando os parâmetros da distribuição log-normal, $\mu$ e $\sigma$, testa-se a normalidade multivariada dos dados pela estatística:

$$
Z=\frac{\log (H Z)-\log (\mu)}{\log (\sigma)}
$$

A tabela 7.7.1.3 mostra os resultados dos testes de multinormalidade de Mardia Skewness, de Mardia Kurtosis e de Henze-Zirkler, para o conjunto das cinco ocasiões, considerando os métodos de manejo M1, M2, M3 e M4. A análise foi realizada através do programa: \% Multinorm Macro: Mardia Tests of Multivariate Normality, Version 1.1, disponível pelo SAS Institute Inc. como um serviço para seus usuários, em:

http://homepages.wmich.edu/ applegab/EMR675/SASFiles/multnorm.sas

Tabela 7.7.1.3 - Testes de multinormalidade para o conjunto das cinco ocasiões.

\begin{tabular}{c|l|c|c} 
Equações & Estatística dos Testes & Valores & Prob. \\
System (SAS) & Mardia Skewness & 28,01 & 0,7933 \\
\hline & Mardia Kurtosis & $-2,15$ & 0,0317 \\
\hline & Henze-Zirkler T & 6,75 & $<0,0001$ \\
\hline
\end{tabular}

0 teste de Henze-Zirkler apresentou resultado significativo ( $p$-valor $<0,0001)$. 0 teste de Mardia Skewness foi não significativo ( $p$-valor $=0,7933)$ e Mardia Kurtosis ( $p$-valor $=$ $0,0317)$ também para o nível de significância $(\alpha=0,01)$, mostrando que há razão para considerar que as populações em estudo são multinormais. A Figura 7.7.1.2 mostra o "Gráfico Qui-Quadrado Q-Q", apresentando uma configuração linear sem valores discrepantes dos "Quantil Qui-Quadrado" versus as "Distâncias Quadradas", indicando uma aderência dos dados à normalidade multivariada. 


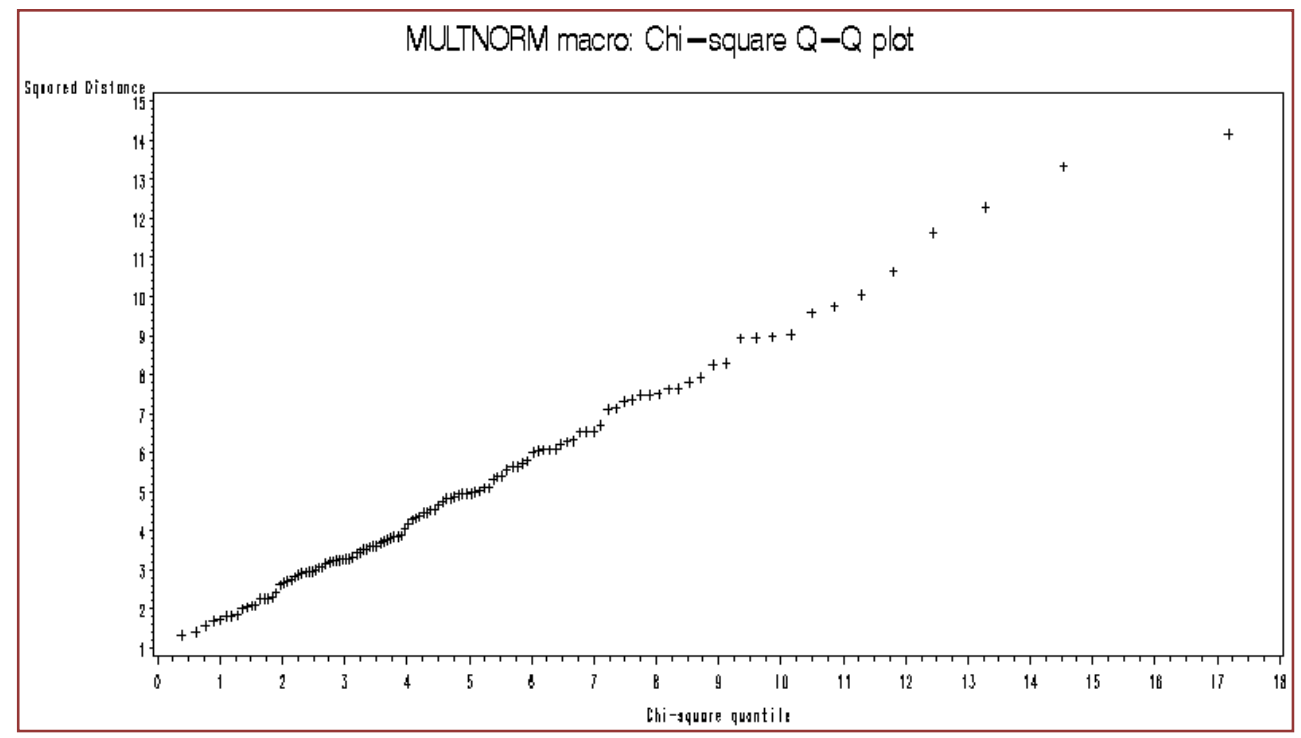

Figura 7.7.1.2 - Gráfico Multnorm Macro Qui-Quadrado Q-Q para M1, M2, M3 e M4.

\section{h) Teste da interação Método de Manejo×Ocasião para M1, M2, M3 e M4}

Tabela 7.7.1.4 - Teste da existência de interação entre os perfis de crescimento dos métodos.

\begin{tabular}{|c|c|c|c|c|c|}
\hline Testes multivariados & Valores & Valores $F$ & Num GL & Den GL & $\operatorname{Pr}>\mathrm{F}$ \\
\hline Wilks Lambda & 0,01112936 & 111,59 & 12 & 299,26 & $<0,0001$ \\
\hline Pillai Trace & 1,15886102 & 18,10 & 12 & 345 & $<0,0001$ \\
\hline Hotelling-Lawley Trace & 73,61405339 & 688,04 & 12 & 193,48 & $<0,0001$ \\
\hline Roy Greatest Root & 73,40695894 & 2110,45 & 4 & 115 & $<0,0001$ \\
\hline
\end{tabular}

A tabela 7.7.1.4 apresenta os resultados dos testes para a hipótese da interação ocasião×método de manejo. Os testes de Wilks Lambda, Pillai Trace, Hotelling-Lawley Trace e de Roy Greatest Root apresentaram resultados significativos ( $p$-valor $<0,0001$ ), denotando que existem pelo menos dois métodos de manejo que apresentam perfis de crescimento não paralelos. Essas estatísticas estão transformadas na $F$ de Snedecor.

$$
\begin{gathered}
M=\frac{(u-c \mid-1)}{2}=\frac{|4-3|-1}{2}=0 \quad N^{*}=\frac{(N-g-u-1)}{2}=\frac{(120-4-4-1)}{2}=55,5 \\
\mathrm{~S}=\min (\mathrm{u}, \mathrm{c})=(4,3)
\end{gathered}
$$

$u=p-1=4$ : é posto, no caso desta hipótese, da matriz $U_{1}$;

$c=g-1=3$ : é o posto da matriz $C$;

$N$ : número de parcelas (120). 
Com efeito, como a interação foi significativa considerando todos os quatro métodos de manejo, então o próximo passo será verificar quais perfis de crescimento são paralelos, ou coincidentes. Pode-se, para obter uma discriminação preliminar dos métodos de manejo, testar, por exemplo, cada ocasião separadamente pelo teste $F$, ou realizar um teste de comparação múltipla, por exemplo, o teste de Tukey. Optou-se pelo uso da técnica de análise de agrupamento de Ward.

i) Análise de agrupamento (método de Ward) dos quatro métodos de manejo (objetos)

Tabela 7.7.1.5 - Matriz das médias dos quatro métodos de manejo.

\begin{tabular}{l|c|c|c|c|c}
\multicolumn{1}{l}{ MMF } & OC1 & OC2 & OC 3 & OC4 & OC5 \\
M1 & 96,6558900 & 118,867033 & 135,429233 & 136,519933 & 137,968033 \\
\hline M2 & 97,0746000 & 120,001733 & 137,359733 & 139,151000 & 140,502000 \\
\hline M3 & 96,7343267 & 130,933367 & 142,368600 & 149,356333 & 150,126967 \\
\hline M4 & 97,1765333 & 131,251267 & 143,126200 & 150,286700 & 150,970800 \\
\hline
\end{tabular}

Os métodos de manejo foram agrupados utilizando as ocasiões como variáveis. Matriz de distâncias ao quadrado considerando os $g(g-1) / 2=6$ pares de objetos:

$$
\begin{gathered}
M 1 \\
M 2 \\
M 3
\end{gathered}\left[\begin{array}{cccc}
0 & 18,533195 & 506,37022 & 571,47945 \\
& 0 & 341,49397 & 393,41414 \\
& \text { Simétrica } & 0 & 2,4482017 \\
& & & 0
\end{array}\right]
$$

De acordo com a matriz de distâncias ao quadrado, considera-se como o primeiro grupo formado o par (M3, M4) que apresentou a mínima distância ao quadrado $(2,4482017)$. 0 processo continua seguindo o princípio da soma de quadrados mínima. Dois programas desenvolvidos no SAS, Proc IML e Proc cluster method, estão na tabela C9 do apêndice C.

Tabela 7.7.1.6 - Análise de agrupamento no SAS (Proc IML).

\begin{tabular}{l|l|l|l|l|l}
\multicolumn{1}{l}{ ETAPA } & \multicolumn{1}{c|}{ FUSÃ0 } & \multicolumn{1}{c}{ SQEli } & \multicolumn{1}{c}{ SQE (Ac.) } & PPI & $d\left(C_{l}, C_{i}\right)$ \\
\hline 1 & $(\mathrm{M} 3)$ e (M4) & 1,2241 & 1,2241 & 0,0027 & 2,4482 \\
\hline 2 & $(\mathrm{M} 1, \mathrm{M} 2)$ & 9,2666 & 10,4907 & 0,0202 & 18,5332 \\
\hline 3 & $(\mathrm{M} 3, \mathrm{M} 4)$ e (M1, M2) & 447,9441 & 458,4348 & 0,9771 & 895,8882 \\
\hline
\end{tabular}

É importante observar, na tabela 7.7.1.6, que $S Q E_{l i}=0,5 d\left(C_{l}, C_{i}\right) .0$ gráfico 7.7.1.3 foi elaborado usando as medidas de distâncias, ou seja, $\sqrt{d\left(C_{l}, C_{i}\right)}$.

$P P I=\mathrm{SPRSQ}=\frac{S Q E_{l i}}{S Q T o t a l}=\frac{S Q E_{l i}}{458,4348}$ (é a porcentagem de perda de informação). 
Tabela 7.7.1.7 - Análise de agrupamento no SAS (Proc cluster method).

\begin{tabular}{c|c|c|c|c|c|c}
\multicolumn{7}{c}{ Resultados do Agrupamento - SAS } \\
NCL & \multicolumn{2}{c}{ FUSÃO } & FREQ & SPRSQ & SPRSR (Ac.) & RSQ \\
\hline 3 & M3 & M4 & 2 & 0,0027 & 0,0027 & 0,997 \\
\hline 2 & M1 & M2 & 2 & 0,0202 & 0,0229 & 0,977 \\
\hline 1 & CL2 & CL3 & 4 & 0,9771 & 1,0000 & 0,000 \\
\hline
\end{tabular}

$$
R S Q=1-\operatorname{SPRSR}(A c .)
$$

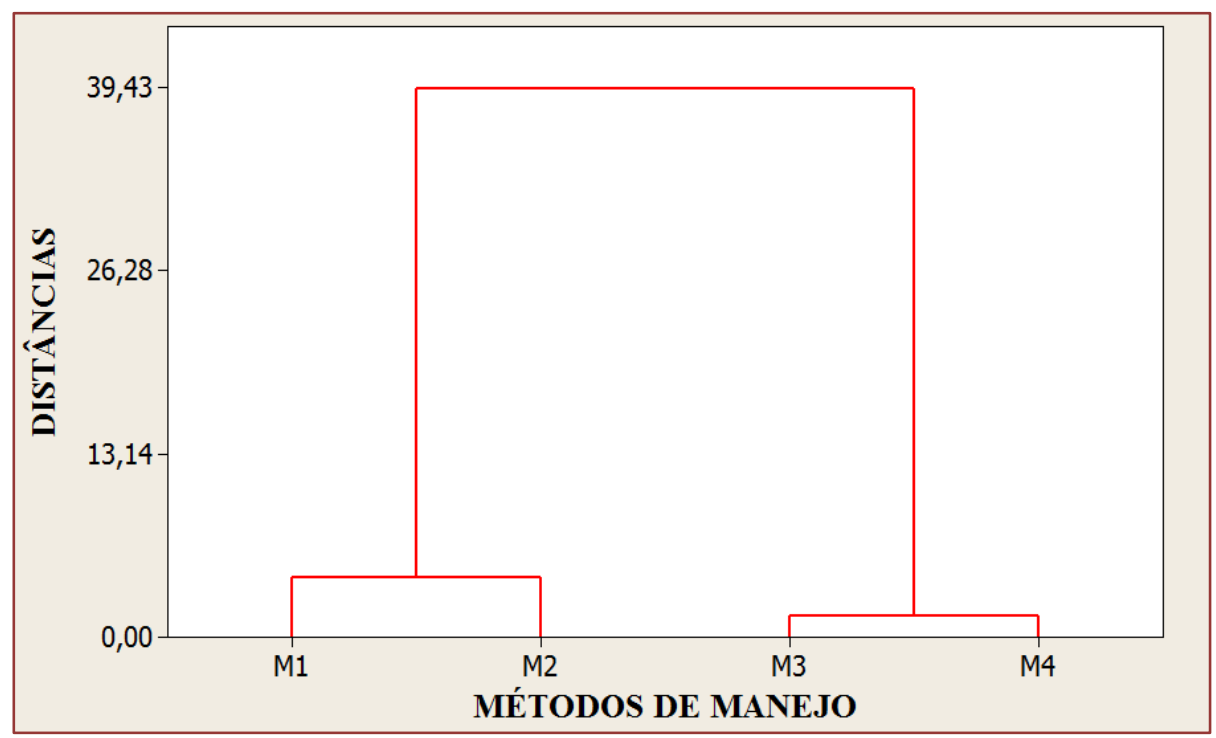

Figura 7.7.1.3 - Dendrograma dos quatro métodos de manejo.

\section{j) Teste de paralelismo entre grupos (M1 e M2) e (M3 e M4)}

Considerando os resultados da análise de agrupamento, apresentados nas tabelas 7.7.1.6, 7.7.1.7 e no gráfico 7.7.1.3, é recomendável testar a existência de interação dos perfis de crescimentos entre os métodos M3 e M4, assim como entre os métodos M1 e M2. Os resultados para os métodos M3 e M4 estão mostrados na tabela 7.7.1.8. Os testes para verificação de existência de interação, entre M1 e M2, estão apresentados no exemplo de aplicação da seção 7.3.1, o qual mostrou existir interação entre os perfis de M1 e M2.

Tabela 7.7.1.8 - Teste de paralelismo entre os perfis de crescimento de M3 e M4.

Testes e estatísticas exatas de $F$ para a hipótese de não existência de efeito de interação
Ocasião $\begin{gathered}\text { Método de Manejo }\left(\mathrm{S}=1 ; \mathrm{M}=1 ; \mathrm{N}^{*}=26,5\right) \\
\text { Testes multivariados }\end{gathered}$ Valores
\begin{tabular}{l|c|c|c|c|c} 
Wilks Lambda & 0,88048940 & 1,87 & 4 & 55 & 0,1294 \\
\hline Pillai Trace & 0,11951060 & 1,87 & 4 & 55 & 0,1294 \\
\hline Hotelling-Lawley Trace & 0,13573201 & 1,87 & 4 & 55 & 0,1294 \\
\hline Roy Greatest Root & 0,13573201 & 1,87 & 4 & 55 & 0,1294 \\
\hline
\end{tabular}


A tabela 7.7.1.8 mostra que os testes de Wilks Lambda, Pillai Trace, Hotelling-Lawley Trace e de Roy Greatest Root apresentaram resultados não significativos ( $p$-valor = 0,1294 ) para a interação ocasião×método de manejo, denotando que os perfis de crescimento dos métodos M3 e M4 são paralelos. Programa SAS para verificação de existência de interação, ver tabela C8 do apêndice C, usando o arquivo com os métodos M3 e M4. Destarte, o próximo passo, considerando que os perfis de crescimento entre os métodos M3 e M4 são paralelos, então será verificar se os mesmos são coincidentes.

k) Teste de coincidência entre os perfis de crescimento entre M3 e M4 pelo teste " $t$ " de Student

A verificação da coincidência entre os métodos de manejo M3 e M4 poderá ser feito pelo teste $t$ de Student, segundo a hipótese:

$H_{02}=j^{\prime} \mu_{v 3}=j^{\prime} \mu_{v 4}$, tal que $j^{\prime}=\left[\begin{array}{lllll}1 & 1 & 1 & 1 & 1\end{array}\right]$. Esta hipótese também pode ser escrita da seguinte forma: $H_{02}=\mu_{31}+\mu_{32}+\mu_{33}+\mu_{34}+\mu_{35}=\mu_{41}+\mu_{42}+\mu_{43}+\mu_{44}+\mu_{45}$.

$$
\begin{aligned}
& t=\frac{j^{\prime}\left(\bar{y}_{v 3}-\bar{y}_{v 4}\right)}{\sqrt{j^{\prime} S j\left(\frac{1}{n_{3}}+\frac{1}{n_{4}}\right)}}, \text { com } n_{3}+n_{4}-2=58 \text { graus de liberdade. } \\
& \left.j^{\prime}\left(\bar{y}_{v 3}-\bar{y}_{v 4}\right)=3,2919066 \quad j^{\prime} S j\right)=136,06318
\end{aligned}
$$

$$
\begin{gathered}
\mathrm{S}=\frac{S_{3}+S_{4}}{2}=\left[\begin{array}{l}
2,20458252,37590292,19433501,90808401,4926714 \\
2,37590294,92781165,60238975,79791235,2674714 \\
2,19433505,60238977,40570398,18380407,9389745 \\
1,90808405,79791238,18380409,83035609,8747739 \\
1,49267145,26747147,93897459,874773910,422090
\end{array}\right] \\
t_{C A L}=\frac{j^{\prime}\left(\bar{x}_{v 3}-\bar{\mu}_{v 4}\right)}{\sqrt{j^{\prime} S j\left(\frac{1}{n_{3}}+\frac{1}{n_{4}}\right)}}=\frac{3.2919066}{\left.\sqrt{136.06318\left(\frac{1}{30}+\frac{1}{30}\right.}\right)}=\frac{3,2919066}{3,0117896}=1,0930
\end{gathered}
$$

Sendo $t_{58 ; 0,05} \cong 2,0$, conclui-se pela não rejeição de $H_{02}$, então há razão para aceitar a coincidência dos perfis de crescimento dos métodos de manejo M3 e M4. Neste caso, será estimada uma única equação de crescimento para ambos os métodos. Programa desenvolvido no SAS apresentado na tabela C10 (Proc IML) do apêndice C. 
1) Teste de coincidência dos perfis de crescimento entre M3 e M4 pela análise de perfil

Teste de coincidência dos perfis segundo a hipótese linear: $H_{02}: C_{2} \beta U_{2}=\phi$.

$$
\begin{gathered}
C_{2}=\left[\begin{array}{ccccc}
1 & -1 & 0 & 0 & 0 \\
0 & 1 & -1 & 0 & 0 \\
0 & 0 & 1 & -1 & 0 \\
0 & 0 & 0 & 1 & -1
\end{array}\right] \\
U_{2}^{\prime}=\left[\begin{array}{lllll}
1 & 1 & 1 & 1 & 1
\end{array}\right]
\end{gathered}
$$

Tabela 7.7.1.9 - Testes de coincidência entre os perfis de crescimento de (M3, M4).

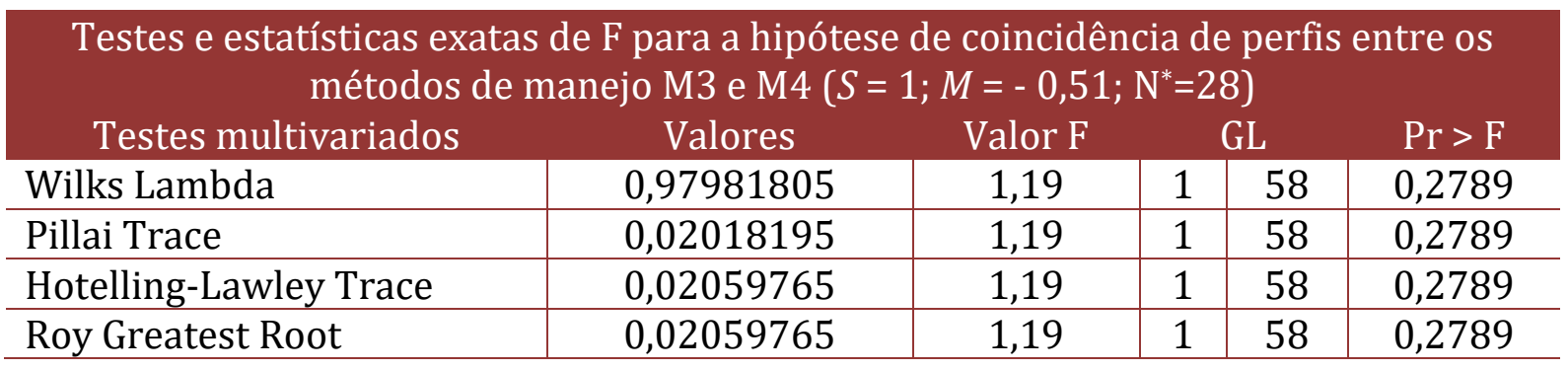

A tabela 7.7.1.9 mostra que os testes de Wilks Lambda, Pillai Trace, Hotelling-Lawley Trace e de Roy Greatest Root apresentaram resultados não significativos ( $p$-valor = 0,2789 ) para o teste de coincidência, mostrando que os perfis de crescimento dos métodos de manejo M3 e M4 são coincidentes, assim sendo, pode-se obter uma equação única de crescimento para os dois métodos. Programa desenvolvido no SAS (Proc GML) está apresentado na tabela C11 do apêndice C.

É importante observar que, no teste de coincidência dos perfis, segundo a hipótese linear $H_{02}: C_{2} \beta U_{2}=\phi$, o valor da estatística $F_{1 ; 58}$ foi 1,19 , enquanto que o valor " $t_{58}$ " foi 1,0930 , ou seja, $F_{1,58}=t_{58}^{2}$. Este resultado é devido à relação, $F_{1 ; n ; \alpha}=t_{n ; \alpha}^{2}$, existente entre as funções densidades de $F \mathrm{e}$ " $\mathrm{t}$ " de Student.

A aceitação de coincidência dos perfis de crescimento dos métodos de manejo M3 e M4, obtidas pelo teste " $t$ " de Student e pela hipótese linear $H_{02}: C_{2} \beta U_{2}=\phi$, também foi confirmada, na seção 7.7.3, pelo teste da razão da máxima verossimilhança que comprovou a igualdade de parâmetros e a identidade de modelos de regressão para as equações de crescimento dos métodos de manejo M3 e M4.

\subsubsection{ESTIMATIVA DO CICLO DE CORTE ECONOMICAMENTE ACONSELHÁVEL PARA M1, M2, M3 E M4}

Seja a equação de Mitscherlich $V=\beta_{0}\left[1-10^{-\beta_{1}\left(t+\beta_{2}\right)}\right]$. As análises foram realizadas a partir do programa desenvolvido no SAS e disponível na tabela C12 do apêndice $\mathrm{C}$. 
Seja a fórmula desenvolvida e mostrada no capítulo 6, seção 6.8, para obter o ciclo de corte baseada na função de Mitscherlich: $t_{o t i}=\frac{1}{\hat{\beta}_{1}} \log _{10}\left[2,302585 R \hat{\beta}_{0} \hat{\beta}_{1}\right]-\hat{\beta}_{2}$.

O objetivo é obter a produção máxima de madeira, mas condicionado a maximizar a renda líquida. Neste exemplo, será utilizada uma razão de custo $R=3$, que é o quociente entre a receita obtida da venda da madeira e o custo do manejo florestal. Nos exemplos analisados, nas seções 7.2.1 e 7.3.1, foram apresentados os ciclos de cortes obtidos pelos métodos M1 e M2, que foram respectivamente $t_{\text {Oti }(M 1)}=18,7$ anos $\left(138,57 \mathrm{~m}^{3} / \mathrm{ha}\right)$ e $t_{\text {Oti }(M 2)}=19,5$ anos $\left(141,49 \mathrm{~m}^{3} / \mathrm{ha}\right)$. Os métodos M3 e M4 têm perfis de crescimento coincidentes, então, falta obter o ciclo de corte conjunto para M3 e M4.

\section{a) Cálculo do ciclo de corte economicamente aconselhável conjunto para M3 e M4}

A tabela 7.7.2.1 apresenta os resultados da análise de regressão da equação de Mitscherlich, pelo método de Gauss-Newton, considerando conjuntamente os métodos M3 e M4.

$$
\begin{gathered}
V_{M 34}=152,1\left[1-10^{-0,0819(t+5,3838)}\right] \quad(0 \leq t \leq 20 \text { anos }) \\
t_{\text {oti }(M 34)}=\left(\frac{1}{0,0819}\right) \log (2,302585 \times 3 \times 152,1 \times 0,0819)-5,3838=18,2 \text { anos } \\
V_{\text {oti }(M 34)}=152,1\left[1-10^{-0,0819(18,2+5,3838)}\right]=150,32 \mathrm{~m}^{3} / \mathrm{ha} \\
\operatorname{RQMR}_{\mathrm{M} 34}=\sqrt{\frac{\sum_{i=1}^{N}\left(Y_{i}-\hat{Y}_{i}\right)^{2}}{N}}=\sqrt{\frac{2188,2}{2 \times 150}}=2,70074 \quad C V_{M 34}=\frac{R Q M R}{\bar{Y}_{M 34}} \times 100=\frac{2,70074}{133,90} \times 100=2,02 \%
\end{gathered}
$$

Tabela 7.7.2.1 - Análise de regressão da equação de Mitscherlich para M3 e M4.

\begin{tabular}{l|c|c|c|c}
$\begin{array}{c}\text { Parâmetros } \\
\hat{\beta}_{0}\end{array}$ & Estimativas & Erro Padrão Aprox. & \multicolumn{3}{c}{ Limites de Confiança (95\%) } \\
\hline$\hat{\beta}_{1}$ & 0,0819 & 0,3640 & 151,4 & 152,8 \\
\hline$\hat{\beta}_{2}$ & 5,3838 & 0,00192 & 0,0781 & 0,0857 \\
\hline Fonte & GL & 0,1241 & 5,1396 & 5,6281 \\
\multicolumn{1}{c|}{ Erro } & 297 & Soma de Quadrados & Quadrado Médio & Valor F \\
\multicolumn{5}{c|}{ Teste de validade Graybill $F_{G}$} \\
Fonte & GL & Quadrado Médio & Valor $F_{G}$ & Pr > F \\
Numerador & 2 & 19,79871 & 2,75 & 0,0658 \\
\hline Denominador & 298 & 7,21017 & & \\
\hline
\end{tabular}

Coeficiente de correlação de Pearson, $\mathrm{N}=281$, Prob > $|\mathrm{r}|$ Sob H0: Rho=0

\begin{tabular}{l|l}
\hline$r_{M 43}=0,99091(<0,0001)$ & $r_{M 43}^{2}=0,9819$
\end{tabular}




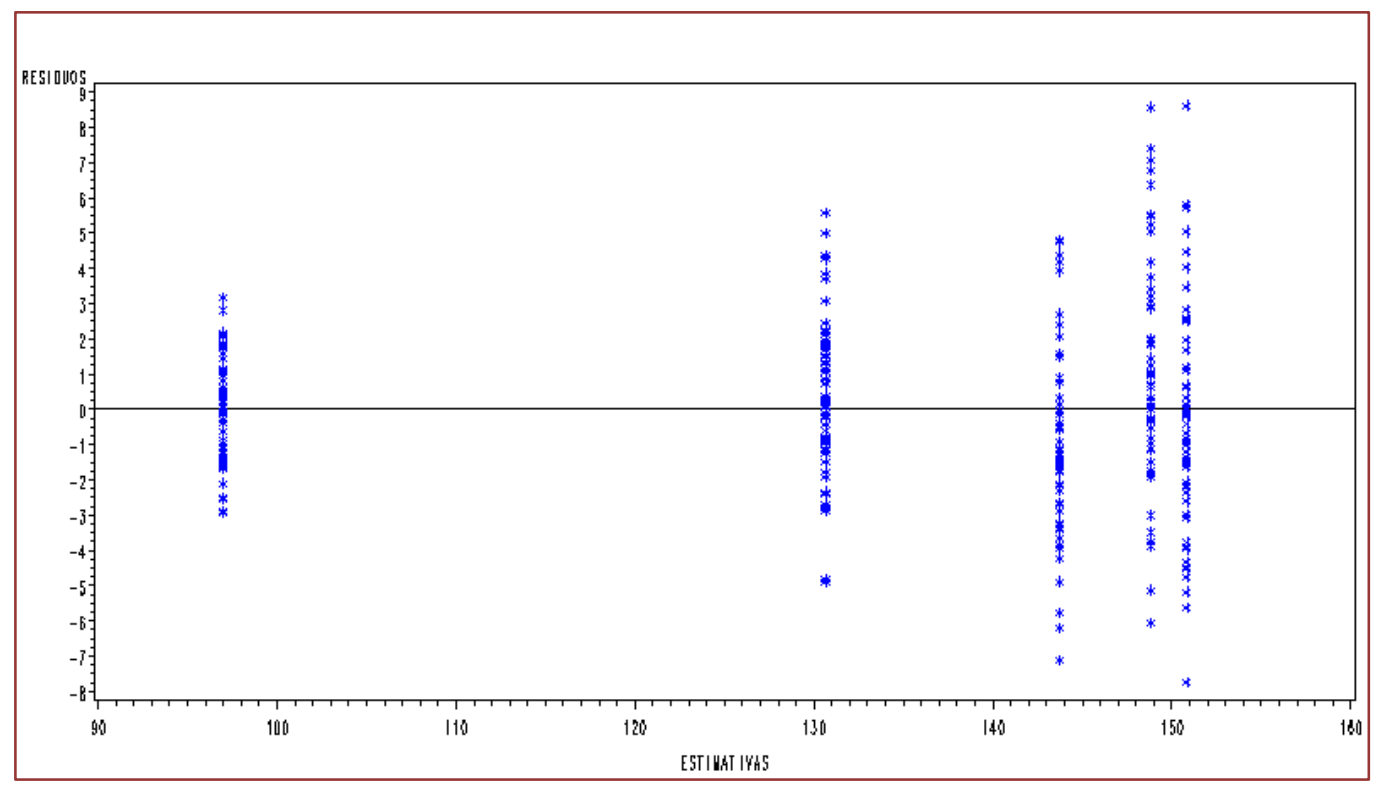

Figura 7.7.2.1 - Gráfico dos resíduos para o conjunto dos métodos (M3 e M4).

Os indicadores CV\%, $r^{2}{ }_{M 1}, F_{G}$ e a distribuição dos resíduos, utilizados para avaliar o ajustamento do modelo de Mitscherlich do grupo (M3,M4), apresentaram resultados satisfatórios dentro do contexto esperado.

b) Ciclo de corte economicamente aconselhável para grupo (M1, M2, M3, M4)

Da tabela 7.7.2.2, têm-se:

$$
\begin{gathered}
V_{M 1234}=147,1\left[1-10^{-0.0762(t+6,1052)}\right] \quad(0 \leq t \leq 20 \text { anos }) \\
t_{\text {oti }(M 1234)}=\left(\frac{1}{0,0762}\right) \log (2,302585 \times 3 \times 147,1 \times 0,0762)-6,1052=18,7 \text { anos } \\
V_{\text {oti }(M 1234)}=147,1\left[1-10^{-0.0762(18,7+6,1052)}\right]=145,21 \mathrm{~m}^{3} / \mathrm{ha} \\
\operatorname{RQMR}_{1234}=\sqrt{\frac{\sum_{i=1}^{N}\left(Y_{i}-\hat{Y}_{i}\right)^{2}}{N}}=\sqrt{\frac{18000,2}{4 \times 150}}=5,4773 \quad C V_{M 1234}=\frac{R Q M R}{\bar{Y}_{M 1234}} \times 100=\frac{5,4773}{134,60} \times 100=4,07 \%
\end{gathered}
$$


Tabela 7.7.2.2 - Análise de regressão conjunta para grupo (M1, M2, M3, M4).

\begin{tabular}{l|c|c|c|c}
\hline \multicolumn{1}{c|}{ Parâmetros } & Estimativas & Erro Padrão Aprox. & \multicolumn{2}{c}{ Limites de Confiança (95\%) } \\
\multicolumn{1}{c|}{$\hat{\beta}_{0}$} & 147,1 & 0,5633 & 146,0 & 148,2 \\
\hline \multicolumn{1}{c|}{$\hat{\beta}_{1}$} & 0,0762 & 0,00287 & 0,0706 & 0,0818 \\
\hline \multicolumn{1}{c|}{$\hat{\beta}_{2}$} & 6,1052 & 0,2223 & 5,6685 & 6,5419 \\
Fonte & GL & Soma de Quadrados & Quadrado Médio & Valor F \\
Erro & 597 & 18000,2 & 30,1511 & 114410,0 \\
\hline \multicolumn{5}{|c|}{ Teste de validade Graybill $F_{G}$} \\
Fonte & GL & Quadrado Médio & Valor $F_{G}$ & Pr $>$ F \\
Numerador & 2 & 763,12500 & 27,70 & $<0,0001$ \\
\hline Denominador & 598 & 27,54843 & & \\
\hline
\end{tabular}

Coeficiente de correlação de Pearson, $\mathrm{N}=600$, Prob $>|\mathrm{r}|$ Sob H0: Rho=0

\begin{tabular}{l|l}
$r_{M 1234}=0,95667 \quad(<0,0001)$ & $r_{M 1234}^{2}=0,9152$
\end{tabular}

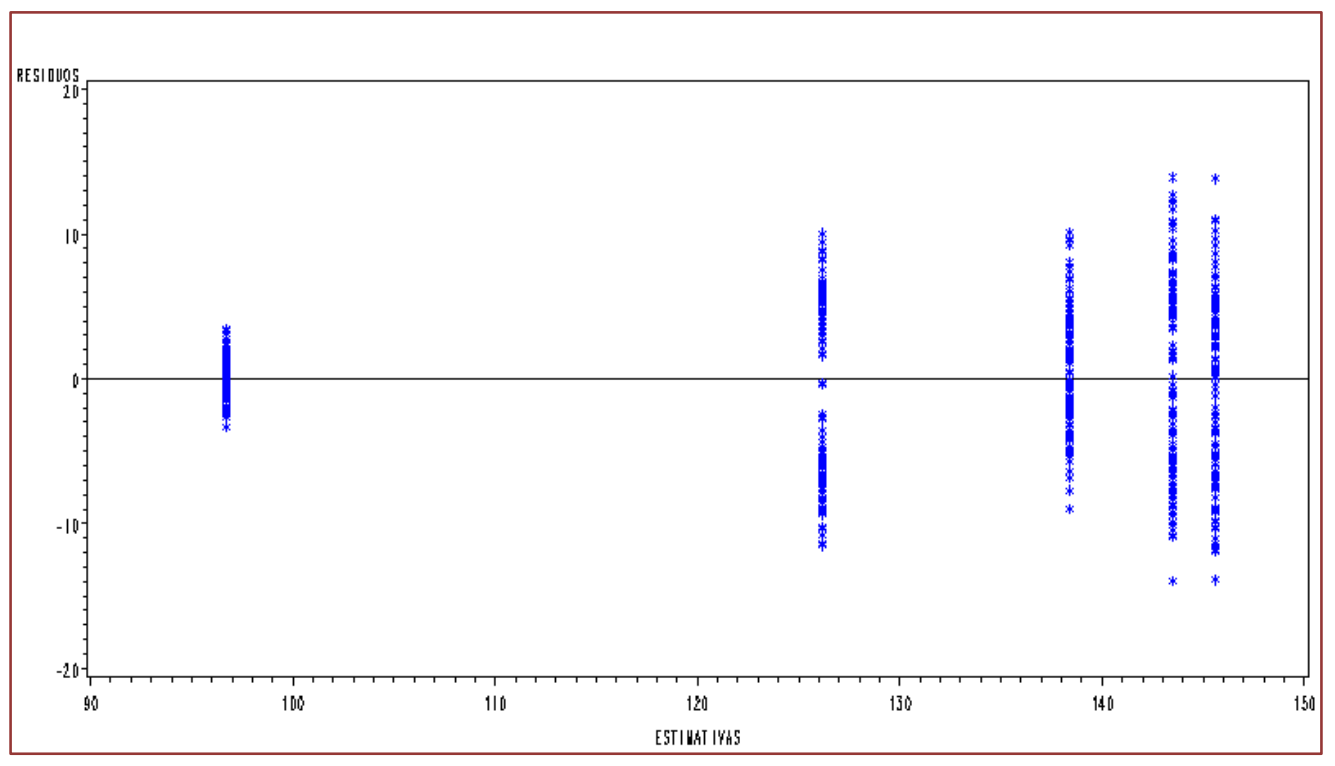

Figura 7.7.2.2 - Gráfico dos resíduos para o grupo (M1, M2, M3, M4).

Os indicadores CV\%, $r^{2}{ }_{M 1234}$ e a distribuição dos resíduos, utilizados para avaliar o ajustamento do modelo de Mitscherlich para o grupo (M1, M2, M3, M4), apresentaram resultados satisfatórios dentro do contexto esperado, mas o teste de validade de Graybill apresentou o valor $F_{G}=27,70(<0,0001)$, o que não recomenda usar uma única equação para o conjunto dos quatro métodos de manejo. 


\subsubsection{TESTE DA RAZÃO DA MÁXIMA VEROSSIMILHANÇA, DE VERIFICAÇÃO DE IGUALDADE DE PARÂMETROS E A IDENTIDADE DE MODELOS DE REGRESSÃO NÃO LINEAR, PARA M3 E M4}

A tabela 7.7.3.1 apresenta para o modelo completo $(\Omega)$, as estimativas dos parâmetros e os seus correspondentes intervalos de confiança. A tabela 7.7.3.2 mostra para o modelo com restrições $(\omega)$, as estimativas dos parâmetros e seus respectivos intervalos de confiança. 0 programa SAS está apresentado na tabela C13 do apêndice C.

Tabela 7.7.3.1 - Modelo completo ômega $(\Omega)$ para M3 e M4.

\begin{tabular}{cc|c|c|c|c}
\multicolumn{2}{c}{ Parâmetros } & Estimativas & Erro Padrão Aprox. & \multicolumn{2}{c}{ Limites de Confiança (95\%) } \\
\hline$\hat{\beta}_{03}$ & 151,6 & 0,5076 & 150,6 & 152,6 \\
\hline$\hat{\beta}_{13}$ & 0,0828 & 0,00275 & 0,0774 & 0,0882 \\
\hline & $\hat{\beta}_{23}$ & 5,3412 & 0,1742 & 4,9983 & 5,6841 \\
\hline & $\hat{\beta}_{04}$ & 152,7 & 0,5185 & 151,6 & 153,7 \\
\hline & $\hat{\beta}_{14}$ & 0,0811 & 0,00268 & 0,0758 & 0,0864 \\
\hline & $\hat{\beta}_{24}$ & 5,4260 & 0,1756 & 5,0804 & 5,7717 \\
Fonte & GL & Soma de Quadrados & Quadrado Médio & Valor F \\
Error & 294 & 2152,1 & 7,3200 & 3245,16 \\
\hline \multicolumn{5}{r|r|r}{}
\end{tabular}

Da tabela 7.7.3.1 que mostra a análise de variância referente ao modelo completo $(\Omega)$ :

$$
S Q R R_{\Omega}=2152,1 \quad Q M R R_{\Omega}=7,3200 \quad \mathrm{~N}=300
$$

$p_{\Omega}=6$ (número de parâmetros do modelo sem restrição), então:

$$
\hat{\sigma}_{\Omega}^{2}=\frac{S Q R R_{\Omega}}{N}=\frac{N-p_{\Omega}}{N} \times M Q R R_{\Omega}=\frac{2152,1}{300}=\left(\frac{300-6}{300}\right) \times 7,3200=7,1737
$$

Tabela 7.7.3.2 - Modelo com restrição ( $\omega)$ para M3 e M4.

\begin{tabular}{ccc|c|c|c}
\multicolumn{5}{c}{$H_{0}^{4}: \beta_{03}=\beta_{04}=\beta_{0} ; \beta_{13}=\beta_{14}=\beta_{1 ;} \beta_{23}=\beta_{24}=\beta 2$} \\
Parâmetros & Estimativas & Erro Padrão Aprox. & \multicolumn{2}{c}{ Limites de Confiança (95\%) } \\
\hline$\hat{\beta}_{0}$ & 152,1 & 0,3640 & 151,4 & 152,8 \\
\hline$\hat{\beta}_{1}$ & 0,0819 & 0,00192 & 0,0781 & 0,0857 \\
\hline & $\hat{\beta}_{2}$ & 5,3838 & 0,1241 & 5,1396 & 5.6280 \\
Fonte & GL & Soma de Quadrados & Quadrado Médio & Valor F \\
Error & 297 & 2188,2 & 7,3678 & 8057,88 \\
\hline
\end{tabular}

Da tabela 7.7.3.2, que apresenta a análise de variância referente ao modelo com restrição $(\omega)$ :

$$
S Q R R_{\omega}=2188,2 \quad M Q R R_{\omega}=7,3678 \quad N=300
$$


$p_{\omega}=3$ (número de parâmetros do modelo com restrição), então:

$$
\hat{\sigma}_{\omega}^{2}=\frac{S Q R R_{\omega}}{N}=\frac{N-p_{\omega}}{N} \times M Q R R_{\omega}=\frac{2188,2}{300}=\left(\frac{300-3}{300}\right) \times 7,3678=7,2941
$$

Do teste da razão de máxima verossimilhança, conforme Rao (1973), para grandes amostras, têm-se:

$$
\begin{gathered}
-2 \operatorname{LnL}=-N \operatorname{Ln}\left(\frac{\hat{\sigma}_{\Omega}^{2}}{\hat{\sigma}_{\omega}^{2}}\right) \underset{N \rightarrow \infty}{\stackrel{d}{\rightarrow}} \chi_{v}^{2} \\
\chi_{\text {Cal }}^{2}=-N \operatorname{Ln}\left(\frac{S Q R R_{\Omega} / N}{S Q R R_{\omega} / N}\right)=-N \operatorname{Ln}\left(\frac{S Q R R_{\Omega}}{S Q R R_{\omega}}\right)=-300 \operatorname{Ln}\left(\frac{7,1737}{7,2941}\right)=4,9933 \\
\chi_{v ; 0,01}^{2}=\chi_{3,0,05}^{2}=7,81, \text { tal que: } v=p_{\Omega}-p_{\omega}=3 .
\end{gathered}
$$

Como $\chi_{\text {Cal }}^{2}>\chi_{3,0,05}^{2}$, a hipótese $H_{0}^{(4)}$ foi não significativa, conclui-se ser recomendável usar uma única equação para estimar a tendência de crescimento para os métodos M3 e M4, assim sendo, a equação $V_{M 34}$, cujas estimativas dos parâmetros estão apresentadas na tabela 7.7.3.2, poderá ser usada, considerando esses dois métodos de manejo, para estimar o perfil de crescimento.

7.7.4. TESTE DA RAZÃO DA MÁXIMA VEROSSIMILHANÇA, CONSIDERANDO OS MÉTODOS DE MANEJOS M1, M2, M3 E M4, PARA VERIFICAR A IGUALDADE DE PARÂMETROS E A IDENTIDADE DE MODELOS DE REGRESSÃO NÃO LINEAR

A tabela 7.7.4.1 apresenta as estimativas dos parâmetros e os seus correspondentes intervalos de confiança, considerando o modelo completo $(\Omega)$. Na tabela 7.7.4.2. são apresentados os valores das estimativas dos parâmetros e seus respectivos intervalos de confiança, para o modelo $(\omega)$ com as restrições $\left(H_{0}^{(4)}\right)$. Programa construído no SAS está apresentado na tabela C12 do apêndice C.

Da Tabela 7.7.4.1 que mostra a análise de variância referente ao modelo completo $(\Omega)$ :

$$
S Q R R_{\Omega}=5124,6 \quad Q M R R_{\Omega}=8,7152 \quad \mathrm{~N}=600
$$

$p_{\Omega}=12$ (número de parâmetros do modelo sem restrição), então:

$$
\hat{\sigma}_{\Omega}^{2}=\frac{S Q R R_{\Omega}}{N}=\frac{N-p_{\Omega}}{N} \times M Q R R_{\Omega}=\frac{5124,6}{600}=\left(\frac{600-12}{600}\right) \times 8,7152=8,541
$$


Tabela 7.7.4.1 - Modelo completo ômega $(\Omega)$ para M1, M2, M3 e M4.

\section{Parâmetros Estimativas Erro Padrão Aprox. Limites de Confiança (95\%)}

\begin{tabular}{c|c|c|c|c}
$\hat{\beta}_{01}$ & 140,6 & 0,6504 & 139,4 & 141,9 \\
\hline$\hat{\beta}_{11}$ & 0,0717 & 0,00339 & 0,0651 & 0,0784 \\
\hline$\hat{\beta}_{21}$ & 6,9768 & 0.3135 & 6,3611 & 7,5924 \\
\hline$\hat{\beta}_{02}$ & 143,6 & 0,6790 & 142,3 & 145,0 \\
\hline$\hat{\beta}_{12}$ & 0,0692 & 0,00315 & 0,0631 & 0,0754 \\
\hline$\hat{\beta}_{22}$ & 7,0005 & 0,3012 & 6,4090 & 7,5920 \\
\hline$\hat{\beta}_{03}$ & 151,6 & 0,5539 & 150,5 & 152,7 \\
\hline$\hat{\beta}_{13}$ & 0,0828 & 0,00300 & 0,0769 & 0,0886 \\
\hline$\hat{\beta}_{23}$ & 5,3412 & 0,1901 & 4,9678 & 5,7145 \\
\hline$\hat{\beta}_{04}$ & 152,7 & 0,5658 & 151,5 & 153,8 \\
\hline$\hat{\beta}_{14}$ & 0,0811 & 0,00292 & 0,0754 & 0,0868 \\
\hline$\hat{\beta}_{24}$ & 5,4260 & 0,1916 & 5,0496 & 5,8024 \\
Fonte & GL & Soma de Quadrados & Quadrado Médio & Valor F \\
Error & 588 & 5124,6 & 8,7152 & 2160,95 \\
\hline \multicolumn{5}{|c|}{}
\end{tabular}

Tabela 7.7.4.2 - Modelo com restrição ( $\omega$ ) para M1, M2, M3 e M4.

\begin{tabular}{c|c|c|c|c}
\multicolumn{5}{c}{$H_{0}^{4}: \beta_{01}=\beta_{02}=\beta_{03}=\beta_{04}=\beta_{0} ; \beta_{11}=\beta_{12}=\beta_{13}=\beta_{14}=\beta_{1} ; \beta_{21}=\beta_{22}=\beta_{23}=\beta_{24}=\beta_{2}$} \\
Parâmetros & Estimativas & Erro Padrão Aprox. & \multicolumn{1}{l}{ Limites de Confiança $(95 \%)$} \\
\hline$\hat{\beta}_{0}$ & 147,1 & 0,5633 & 146,0 & 148,2 \\
\hline$\hat{\beta}_{1}$ & 0,0762 & 0,00287 & 0,0706 & 0,0818 \\
\hline$\hat{\beta}_{2}$ & 6,1052 & 0,2223 & 5,6685 & 6,5419 \\
Fonte & GL & Soma de Quadrados & Quadrado Médio & Valor F \\
Erro & 597 & 18000,2 & 30,1511 & 114410,0 \\
\hline
\end{tabular}

Da Tabela 7.7.4.2 que mostra a análise de variância referente ao modelo com restrição $(\omega)$ :

$$
S Q R R_{\omega}=18000,2 \quad M Q R R_{\omega}=30,1511 N=600
$$

$p_{\omega}=3$ (número de parâmetros do modelo com restrição), então:

$$
\hat{\sigma}_{\omega}^{2}=\frac{S Q R R_{\omega}}{N}=\frac{N-p_{\omega}}{N} \times M Q R R_{\omega}=\frac{18000,2}{600}=\left(\frac{600-3}{600}\right) \times 30,1511=30,0003
$$

Do teste da razão de máxima verossimilhança, conforme Rao (1973), para grandes amostras, tem-se:

$$
-2 \operatorname{Ln} L=-N \operatorname{Ln}\left(\frac{\hat{\sigma}_{\Omega}^{2}}{\hat{\sigma}_{\omega}^{2}}\right) \underset{N \rightarrow \infty}{\stackrel{d}{\rightarrow}} \chi_{v}^{2}
$$

Então: $\chi_{\text {Cal }}^{2}=-N \operatorname{Ln}\left(\frac{S Q R R_{\Omega} / N}{S Q R R_{\omega} / N}\right)=-N \operatorname{Ln}\left(\frac{S Q R R_{\Omega}}{S Q R R_{\omega}}\right)=-600 \operatorname{Ln}\left(\frac{8,541}{30,0003}\right)=753,80$ 
Dado $\chi_{v ; 0,01}^{2}=\chi_{9,0,01}^{2}=21,7$, tal que: $v=p_{\Omega}-p_{\omega}=9$.

Como $\chi_{C a l}^{2}>\chi_{9,0,01}^{2}$, a hipótese $H_{0}^{(4)}$ foi significativa, conclui-se não ser recomendável usar uma única equação para estimar a tendência de crescimento para os quatro métodos, assim sendo, a equação $V_{M 1234}$, cujas estimativas dos parâmetros estão apresentadas na tabela 7.7.4.2, considerando conjuntamente os quatro métodos de manejo, não pode ser usada para estimar o perfil de crescimento.

\subsubsection{ANÁLISE DOS RESULTADOS E CONCLUSÕES DO EXEMPLO DO INVENTÁRIO FLORESTAL CONTÍNUO}

Na tabela 7.7.5.1 estão os valores das estatísticas calculadas com o objetivo de medir o grau de precisão, adequação e validade das equações de crescimento dos métodos de manejo.

Dentre os resultados importantes para aferir conclusões, têm-se:

Tabela 7.7.5.1 - Valores das estatísticas indicativas de precisão e validade.

\begin{tabular}{l|l|l|l|l|c|c|c|c}
\multicolumn{1}{c|}{ MMF } & SQerro & $\bar{Y}_{M M F}$ MMF & \multicolumn{1}{c}{$R Q M R$} & \multicolumn{1}{c}{ CV\% } & $t_{\text {oti }}$ & $\mathrm{r}^{2} \mathrm{Mi}$ & $F_{g}\left(P_{r}>F\right)$ & $V_{\text {oti }}$ \\
M1 & 1150,6 & 125,088 & 2,7696 & 2,21 & 18,7 & 0,9698 & $2,30(0,1036)$ & 138,57 \\
\hline M2 & 1821,8 & 126,818 & 3,4850 & 2,75 & 19,5 & 0,9573 & $3,30(0,0398)$ & 141,49 \\
\hline M34 & 2188,2 & 133,900 & 2,7007 & 2,02 & 18,2 & 0,9819 & $2,75(0,0658)$ & 150,32 \\
\hline M1234 & 18000,2 & 134,600 & 5,4773 & 4,07 & 18,7 & 0,9152 & $27,70(0,0001)$ & 145,21 \\
\hline
\end{tabular}

a) Concernentes aos testes de existência de interação entre os métodos de manejo e as ocasiões verificou-se os seguintes resultados:

a1) Os métodos de manejo M3 e M4 são coincidentes;

a2) Os métodos de manejo M1 e M2 não são paralelos

É recomendável estabelecer equações especificas $V_{M 1}$ e $V_{M 2}$ para os métodos M1 e M2 e uma equação única $V_{M 34}$ para os métodos M3 e M4.

b) Em relação aos valores dos ciclos de corte economicamente aconselhável, têm-se:

Os métodos de manejo M3 e M4 apresentaram o menor valor (18,2 anos) e 150,32 $\mathrm{m}^{3} /$ ha, enquanto que o método M2 o valor (19,5 anos) e $141,49 \mathrm{~m}^{3} /$ ha. 0 método M1 apresentou o valor $\left(18,7\right.$ anos) e $138,57 \mathrm{~m}^{3} / \mathrm{ha}$. Considerando todos os métodos (M1234), ou seja, uma equação única, o valor foi de 18,7 anos e 145,21 m³/ha. Então, é recomendável escolher, considerando o critério dos valores da rotação economicamente aconselhável, o método de manejo M3 ou o M4 que apresentaram perfis coincidentes. 
c) Dado os valores dos coeficientes de variação (CV\%) e das correlações ao quadrado ( $r_{M i}^{2}$ ) entre os valores estimados e observados, os métodos de manejo M3 e M4, conjuntamente apresentaram o menor valor de CV\% e o maior de $r_{M i}^{2}$. O ideal é escolher o método de manejo M3 ou o M4, haja vista os valores do CV\% e do $r_{M i}^{2}$.

d) No contexto do teste de validade de Graybill $\left(F_{G}\right)$, o método M1 apresentou o valor não significativo de $F_{G}=2,30$ ( $p$-valor $\left.=0,1036\right)$. Os métodos de manejos M3 e M4, considerados de forma conjunta, apresentaram $F_{G}=2,75(p$-valor $=0,0658)$ sendo também não significativos. 0 método $\mathrm{M} 2$, com $F_{G}=3,30$ ( $p$-valor $=0,0398$ ), pode ser considerado não significativo ao nível $\alpha=0,01$.

Por outro lado, os métodos M1, M2, M3, M4 apresentam o valor de $F_{G}=27,70$ ( $p$-valor < 0,0001), quando considerados de forma conjunta, denotando não ser recomendável estabelecer uma equação única para todos os métodos de manejo.

e) Os gráficos dos resíduos, relativos aos comportamentos das equações de crescimentos dos métodos de manejo estudados, apresentaram distribuições dos resíduos aleatórias, não mostrando nenhum tipo de tendência aparente, ou seja, revelando que a faixa de variação dos resíduos ao longo dos valores ajustados é constante, não havendo indícios de que as variâncias dos resíduos dos métodos de manejo sejam heteroscedásticas.

f) Uma equação única $\left(V_{M 1234}\right)$ não é recomendável, conforme o teste de validade de Graybill, assim como apresentou também, pelo teste da razão da máxima verossimilhança, para verificação de igualdade de parâmetros e identidade de modelos de regressão não linear, um valor altamente significativo de $\chi_{\text {Cal }}^{2}=753,80$.

g) É recomendável escolher o método M3 ou o M4, com perfis coincidentes, em função de apresentarem, conjuntamente, os seguintes indicadores:

i) 0 menor ciclo de corte (18,2 anos) e a maior produção $\left(150,32 \mathrm{~m}^{3} / \mathrm{ha}\right)$;

ii) $\mathrm{O}$ menor $\mathrm{CV}=2,02 \%$ e o maior coeficiente de correlação ao quadrado $\left(r_{M 34}^{2}=0,9819\right)$ entre os valores estimados e observados;

iii) resultado não significativo ( $\alpha=0,05)$ para o teste de Graybill. 


\section{Capítulo 8}

\section{Análise de perfil de espécies em florestas naturais}

Este capítulo aborda a análise do perfil de crescimento de espécies em inventários florestais com parcelas permanentes, expondo um exemplo de aplicação considerando os perfis de crescimento de quatro espécies florestais, envolvendo os seguintes temas: teste de esfericidade de matriz de covariância pelo critério de Mauchly; cálculo dos valores das correções de Greenhouse-Geisser Epsilon $\left(\varepsilon_{g g}\right)$ e de Huynh-Feldt Epsilon $\left(\varepsilon_{h f}\right)$; teste de homogeneidade de matrizes de covariâncias; testes de multinormalidade de assimetria e de curtose de Mardia; teste de normalidade multivariada de HenzeZirkler; análise de agrupamento pelo método de Ward; testes de paralelismo e de coincidência de perfis de crescimento pela hipótese linear geral $H$ : $C \beta U=\phi$; análise de coincidência de perfis pelo teste " $t$ " de Student; determinação do ciclo de corte economicamente aconselhável; análise de resíduos; teste de validade de Graybill; teste da razão da máxima verossimilhança para verificar a igualdade de parâmetros e identidade de modelos de regressão não linear. 


\section{INTRODUÇÃO}

No exemplo de aplicação apresentado no capítulo 7, considerando os quatro métodos de manejo M1, M2, M3 e M4, foi verificado que o método de manejo M3 (ou o M4) deve ser o indicado para manejar a floresta em estudo.

É importante ressaltar que, para definição do melhor método de manejo, na análise do exemplo citado no capítulo anterior, os valores simulados dos volumes foram obtidos considerando várias espécies. Entretanto, como cada espécie dentro de uma floresta natural tem a sua própria dinâmica de crescimento, o recomendável é estudar o perfil de todas elas e, usando a análise multivariada de perfil construir grupos de espécies com perfis semelhantes, ou seja, coincidentes, e assim estabelecer um plano de corte policíclico. Neste capítulo, objetivando ter um melhor entendimento do tema, será apresentada uma aplicação com quatro espécies.

\subsection{ANÁLISE DE PERFIL DE CRESCIMENTO DE QUATRO ESPÉCIES FLORESTAIS}

Foram escolhidas, nesta aplicação, considerando tratar-se de uma floresta natural, quatro espécies caracterizadas como de boa ocorrência e de rápido crescimento, denominadas de E1, E2, E3 e E4, para estudar os seus perfis de crescimento e calcular os seus respectivos ciclos de cortes economicamente aconselháveis. Os dados (volume em $\mathrm{m}^{3} / \mathrm{ha}$ ) foram simulados, dentro de um contexto próximo ao real, e estão apresentados na tabela B2 do apêndice B.

Os programas usados nas análises são os mesmos usados no capítulo 7, obviamente adaptados, ou seja, trocando o banco de dados (B1) dos métodos de manejo M1, M2, M3 e M4 para o (B2) das espécies E1, E2, E3 e E4. Os programas construídos no SAS estão dispostos nas tabelas C7, C8, C9, C10, C11, C12 e C13 do apêndice C.

\subsubsection{ANÁLISE DA INTERAÇÃO OCASIÃO×ESPÉCIE}

a) Vetores de médias, para E1, E2, E3 e E4, considerando as ocasiões 0, 5, 10, 15 e 20 anos

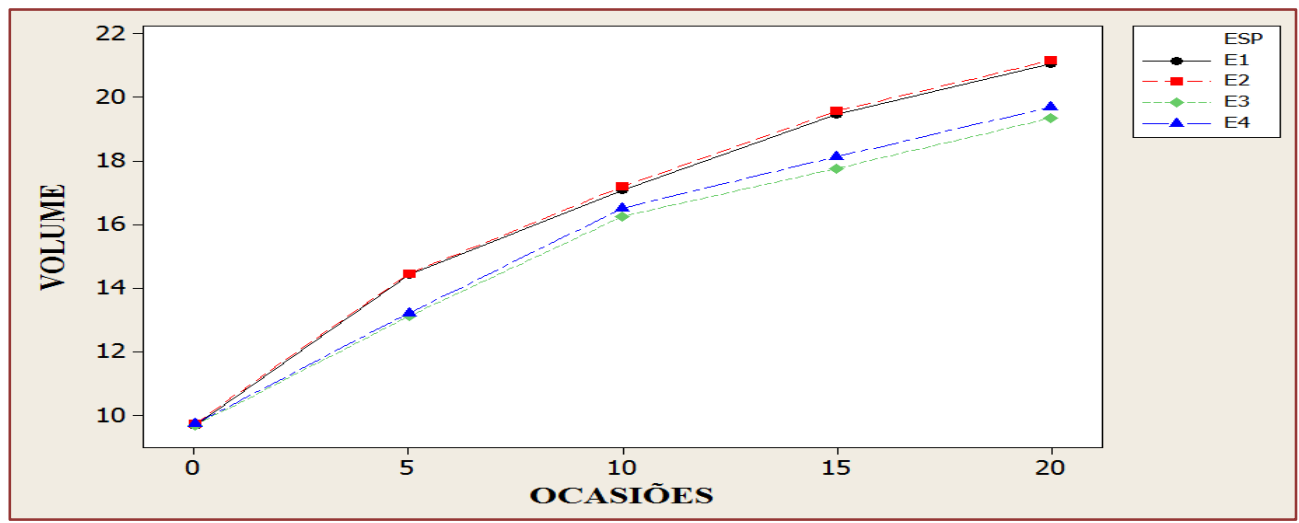

Figura 8.1.1.1 - Perfis de crescimento das espécies E1, E2, E3 e E4. 


$$
\begin{aligned}
& \bar{y}_{v 1}^{\prime}=[9,6844966714,415266717,089190019,445480021,0381800 \\
& \bar{y}_{v 2}^{\prime}=[9,7430533314,446093317,192783319,560786721,1482133] \\
& \bar{y}_{V 3}^{\prime}=\left[\begin{array}{lllll}
9,67315333 & 13,1066700 & 16,253233 & 17,7585900 & 19,3376867
\end{array}\right] \\
& \bar{y}_{v 4}^{\prime}=[9,7248333313,205866716,507890018,122250019,6947067]
\end{aligned}
$$

b) Matrizes de covariâncias, para as espécies E1, E2, E3 e E4, considerando as ocasiões 0, 5, 10, 15 e 20 anos

$$
\begin{aligned}
S_{1} & =\left[\begin{array}{llllll}
0,02116377 & 0,01284834 & 0,00953389 & 0,00545315 & 0,00246514 \\
0,01284834 & 0,03637208 & 0,04591649 & 0,05407382 & 0,05468861 \\
0,00953389 & 0,04591649 & 0,07636850 & 0,09784063 & 0,10911020 \\
0,00545315 & 0,05407382 & 0,09784063 & 0,13976266 & 0,15863031 \\
-0,00246514 & 0,05468861 & 0,10911020 & 0,15863031 & 0,19214423
\end{array}\right] \\
S_{2} & {\left[\begin{array}{llllll}
0,03646297 & 0,04560458 & 0,05474387 & 0,05517822 & 0,05146438 \\
0,04560458 & 0,07333821 & 0,09644924 & 0,10125858 & 0,09653067 \\
0,05474387 & 0,09644924 & 0,14576223 & 0,15577815 & 0,15673012 \\
0,05517822 & 0,10125858 & 0,15577815 & 0,18148101 & 0,18653086 \\
0,05146438 & 0,09653067 & 0,15673012 & 0,18653086 & 0,20608829
\end{array}\right] } \\
S_{3} & =\left[\begin{array}{llllll}
0,02139458 & 0,02052219 & 0,02527012 & 0,03050280 & 0,03191725 \\
0,02052219 & 0,04193350 & 0,04921580 & 0,05883239 & 0,06282835 \\
0,02527012 & 0,04921580 & 0,06604163 & 0,07798079 & 0,08242055 \\
0,03050280 & 0,05883239 & 0,07798079 & 0,09932675 & 0,10520135 \\
0,03191725 & 0,06282835 & 0,08242055 & 0,10520135 & 0,12296290
\end{array}\right] \\
S_{4}= & {\left[\begin{array}{llllll}
0,02750088 & 0,03008828 & 0,03433776 & 0,03940853 & 0,04354558 \\
0,03008828 & 0,06821718 & 0,09510694 & 0,10908753 & 0,11828130 \\
0,03433776 & 0,09510694 & 0,15067941 & 0,17067383 & 0,18980145 \\
0,03940853 & 0,10908753 & 0,17067383 & 0,20539926 & 0,22770458 \\
0,04354558 & 0,11828130 & 0,18980145 & 0,22770458 & 0,26214444
\end{array}\right] }
\end{aligned}
$$


c) Teste de esfericidade da matriz de covariância pelo critério de Mauchly e as correções de Greenhouse-Geisser Epsilon ( $\varepsilon_{g g}$ ) e de Huynh-Feldt Epsilon $\left(\varepsilon_{h f}\right)$.

A Tabela 8.1.1.1 mostra o resultado do teste de esfericidade pelo critério de Mauchly, o qual apresentou um resultado altamente significativo ( $p$-valor $<0,0001$ ), denotando que a matriz do erro $(S)$ não é esférica, concluindo-se pela não realização da análise univariada de parcelas subdivididas (split-plot), mas caso seja o desejo do pesquisador, então deve ser usado a correção de Greenhouse-Geisser Epsilon $\left(\hat{\varepsilon}_{g g}=0,3336\right)$ ou a de Huynh-Feldt Epsilon ( $\hat{\varepsilon}_{h f}=0,3448$ ), e assim corrigir os graus de liberdade do teste $F$, para a igualdade de efeitos de ocasião e igualdade de paralelismo (interação de ocasião×espécie).

Tabela 8.1.1.1 - Teste de esfericidade pelo critério de Mauchly e os valores das correções de Greenhouse-Geisser Epsilon $\left(\varepsilon_{g g}\right)$ e de Huynh-Feldt Epsilon $\left(\varepsilon_{h f}\right)$.

\begin{tabular}{l|c|c|c|c} 
Variáveis & GL & Critério de Mauchly & Qui-Quadrado & Pr $>\chi^{2}$ \\
Variáveis Transformadas & 9 & 0,0135191 & 492,40987 & $<0,0001$ \\
\hline Componentes Ortogonais & 9 & 0,0135191 & 492,40987 & $<0,0001$ \\
\hline Greenhouse-Geisser Epsilon & \multicolumn{5}{c}{$\hat{\varepsilon}_{g g}=0,3336$} \\
\hline Huynh-Feldt Epsilon & \multicolumn{6}{c}{$\hat{\varepsilon}_{h f}=0,3448$} \\
\hline
\end{tabular}

d) Teste de homogeneidade das matrizes de covariâncias para E1, E2, E3 e E4

$$
M=\sum_{i=1}^{g}\left(n_{i}-1\right) \operatorname{Ln}|S|-\sum_{i=1}^{g}\left(n_{i}-1\right) \operatorname{Ln}\left|S_{i}\right|
$$

$$
\begin{gathered}
\operatorname{Ln}\left(\operatorname{det} S_{1}\right)=-20,96756 \quad \operatorname{Ln}\left(\operatorname{det} S_{2}\right)=-20,39542 \quad \operatorname{Ln}\left(\operatorname{det} S_{3}\right)=-21,8735 \mathrm{C} \\
\operatorname{Ln}\left(\operatorname{det} S_{4}\right)=-20,49219 \quad \operatorname{Ln}(\operatorname{det} S)=-20,40534 \\
M=4 \times 29 \times-20,40534-29(-20,96756-20,39542-21,87350-20,49219) \\
M=4 \times 29 \times-20,40534-29(-83,72867)=61,11169 \\
C^{-1}=1-\frac{\left(2 p^{2}+3 p-1\right)(g+1)}{6(p+1) g \times(n-1)}=1-\frac{\left(2 \times 5^{2}+3 \times 5-1\right)(4+1)}{6(5+1) 4(29)}=0,9233716475 \\
M C^{-1}=61,11169 \times 0,9233716475=56,4288 \\
\frac{1}{2}(g-1) p(p+1)=\frac{1}{2}(4-1) \times 5 \times(5+1)=45
\end{gathered}
$$


Tabela 8.1.1.2 - Estatística de qui-quadrado $\chi^{2}$ (assintótico).

\begin{tabular}{c|c|c}
\hline Qui-Quadrado & DF & Pr $>\chi^{2}$ \\
56,428804 & 45 & 0,1181 \\
\hline
\end{tabular}

Desde que $\chi_{C A L}^{2}=56,43$ ( $p$-valor $=0,1181$ ), tabela 8.1.1.2, conclui-se pela não rejeição de $H_{0}: \sum_{E 1}=\sum_{E 2}=\sum_{E 3}=\sum_{E 4}=\sum$, ou seja, as matrizes de covariâncias das espécies E1, E2, E3 e E4 podem ser consideradas homogêneas.

\section{e) Testes de multinormalidade de Mardia e de Henze-Zirkler}

A tabela 8.1.1.3 expõe os resultados dos testes de multinormalidade de Mardia Skewness, de Mardia Kurtosis e de Henze-Zirkler, para o conjunto das cinco ocasiões, considerando as espécies E1, E2, E3 e E4. A análise foi realizada através do programa: \% Multinorm Macro: Mardia Tests of Multivariate Normality, Version 1.1, disponível pelo SAS Institute Inc. como um serviço para seus usuários, em:

http://homepages.wmich.edu/ applegab/EMR675/SASFiles/multnorm.sas

Os testes de Mardia Skewness ( $p$-valor $=0,3625$ ) e de Kurtosis ( $p$-valor $=0,1026$ ) foram não significativos, enquanto que o de Henze-Zirkler foi significativo ( $p$-valor $<0,0001$ ). Usando como critério os testes de multinormalidade de Mardia Skewness e de Kurtosis, as populações das quatro espécies, com respeito às cinco ocasiões, serão consideradas multinormais. A Figura 8.1.1.2 do "Gráfico Qui-Quadrado Q-Q" apresenta uma configuração linear sem valores discrepantes dos "Quantil Qui-Quadrado" versus as "Distâncias Quadradas", indicando aderência dos dados à normalidade multivariada.

Tabela 8.1.1.3 - Testes de multinormalidade para as cinco ocasiões (variáveis).

\begin{tabular}{c|c|c|c} 
Equações & Estatísticas do Teste & Valores & Prob. \\
System & Mardia Skewness & 37,33 & 0,3625 \\
\hline & Mardia Kurtosis & $-1,63$ & 0,1026 \\
\hline & Henze-Zirkler T & 7,50 & $<0,0001$ \\
\hline
\end{tabular}

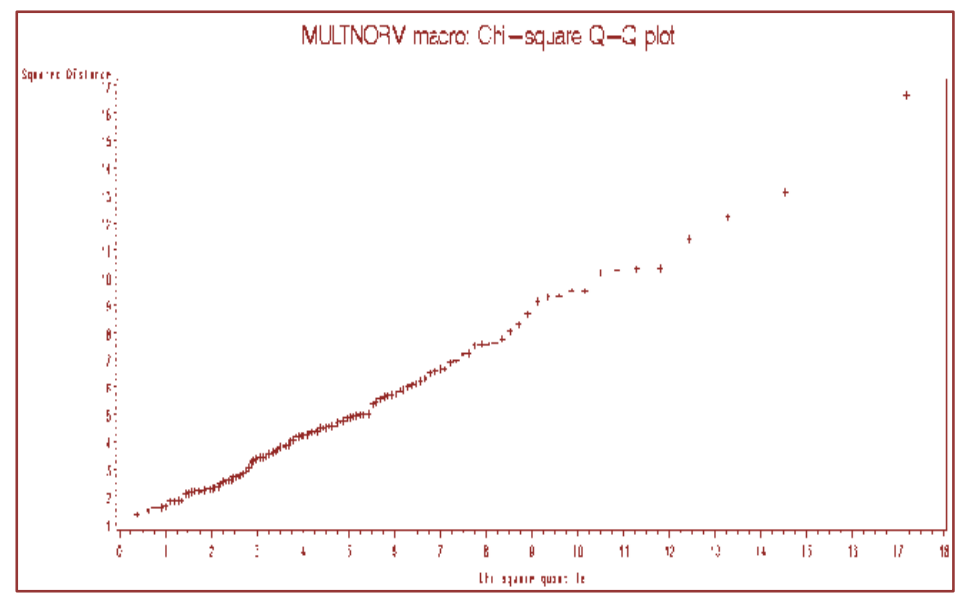

Figura 8.1.1.2 - Gráfico Multnorm Macro Qui-Quadrado Q-Q para E1, E2, E3 e E4. 


\section{f) Teste de existência de interação Ocasião×Espécie para E1, E2, E3 e E4}

Tabela 8.1.1.4 - Teste da interação Ocasião×Espécie para E1, E2, E3 e E4.

$\begin{aligned} & \text { Testes e aproximações das estatísticas F para a hipótese de não existência de efeito da } \\
& \text { interação OcasiãoxEspécie (S = 3; M = 0; N*55,5) }\end{aligned}$
\begin{tabular}{l|c|c|c|c|c} 
Estatísticas & Valores & Valor F & Num GL & Den GL & Pr > F \\
Wilks Lambda & 0,01633596 & 93,16 & 12 & 299,26 & $<0,0001$ \\
\hline Pillai Trace & 1,20021028 & 19,17 & 12 & 345,00 & $<0,0001$ \\
\hline $\begin{array}{l}\text { Hotelling-Lawley } \\
\text { Trace }\end{array}$ & 46,9596533 & 438,91 & 12 & 193,48 & $<0,0001$ \\
\hline Roy Greatest Root & 46,67569000 & 1341,93 & 4 & 115,00 & $<0,0001$ \\
\hline
\end{tabular}

Os testes de Wilks Lambda, Pillai Trace, Hotelling-Lawley Trace e de Roy Greatest Root apresentaram resultados significativos ( $p$-valor $<0,0001)$ para a interação ocasiãoxespécie, mostrando que existem pelo menos duas espécies que apresentam perfis de crescimento não paralelos. Essas estatísticas estão transformadas na $F$ de Snedecor. Os valores, respectivamente, estão expostos na tabela 8.1.1.4 com os seus graus de liberdade.

Com efeito, como a interação foi significativa, considerando todas as quatro espécies florestais, então o próximo passo será verificar quais perfis de crescimento são paralelos ou coincidentes. Pode-se realizar um estudo preliminar testando, por exemplo, cada ocasião separadamente pelo teste $F$, ou realizar um teste de comparação múltipla, por exemplo, o teste de Tukey. Optou-se pelo uso da técnica de análise de agrupamento de Ward. Os grupos foram agrupados utilizando as ocasiões como variáveis.

g) Análise de agrupamento (método de Ward) das quatro espécies florestais (objetos)

Tabela 8.1.1.5 - Vetores de médias das quatro espécies E1, E2, E3 e E4.

\begin{tabular}{c|c|c|c|c|c}
\hline ESP. & OC1 & OC2 & OC3 & OC4 & OC5 \\
E1 & 9,68449667 & 14,4152667 & 17,0891900 & 19,4454800 & 21,0381800 \\
\hline E2 & 9,74305333 & 14,4460933 & 17,1927833 & 19,5607867 & 21,1482133 \\
\hline E3 & 9,67315333 & 13,1066700 & 16,2523233 & 17,7585900 & 19,3376867 \\
\hline E4 & 9,72483333 & 13,2058667 & 16,5078900 & 18,1222500 & 19,6947067 \\
\hline
\end{tabular}

Cálculo da matriz de distância ao quadrado considerando os $g(g-1) / 2=6$ pares de objetos ou indivíduos. As espécies foram agrupadas utilizando as ocasiões como variáveis.

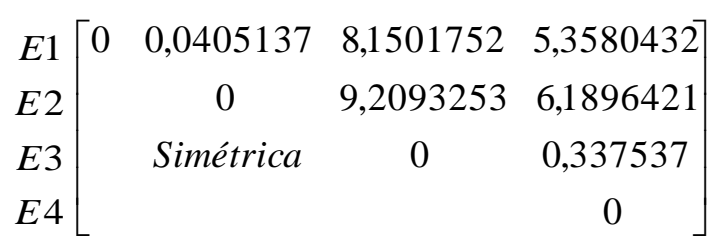


Da matriz de distâncias ao quadrado, considera-se o par (E1, E2) como o primeiro grupo formado, pois apresentou a menor distância ao quadrado (0,0405137). 0 processo continua seguindo o princípio da soma de quadrados mínima. É importante observar, na tabela 8.1.1.6, que $S Q E_{l i}=0,5 d\left(C_{l}, C_{i}\right)$. O gráfico 8.1.1.3 foi construído considerando as distâncias, isto é, $\sqrt{d\left(C_{l}, C_{i}\right)}$. PPI (SPRSQ) é a porcentagem de perda de informação.

Tabela 8.1.1.6 - Análise de agrupamento de Ward no SAS (Proc IML).

\begin{tabular}{c|c|c|c|c|c}
\hline ETAPA & FUSÃ0 & SQE ${ }_{i i}$ & SQE $(A c)$. & PPI & $d\left(C_{l}, C_{i}\right)$ \\
1 & (E1 e E2) & 0,0202568 & 0,0202568 & 0,0028 & 0,0405137 \\
\hline 2 & (E3, E4) & 0,1687685 & 0,1890253 & 0,0231 & 0,337537 \\
\hline 3 & (E1, E2) e (E3, E4) & 7,1322838 & 7,3213091 & 0,9742 & 14,264568 \\
\hline
\end{tabular}

$$
P P I=\frac{S Q E_{l i}}{S Q T o t a l}=\frac{S Q E_{l i}}{7,3213091}
$$

Tabela 8.1.1.7 - Análise de agrupamento de Ward no SAS (Proc cluster method)

\begin{tabular}{c|c|c|c|c|c|c}
\hline NCL & FUSÃO & FREQ & SPRSQ & SPRSR (Ac.) & RSQ \\
\hline 3 & E1 & E2 & 2 & 0,0028 & 0,0028 & 0,9972 \\
\hline 2 & E3 & E4 & 2 & 0,0231 & 0,0259 & 0,9741 \\
\hline 1 & CL3 & CL2 & 4 & 0,9742 & 1,000 & 0,0000 \\
\hline \multicolumn{6}{c}{$R S Q=1-\operatorname{SPRSR}($ Ac.) }
\end{tabular}

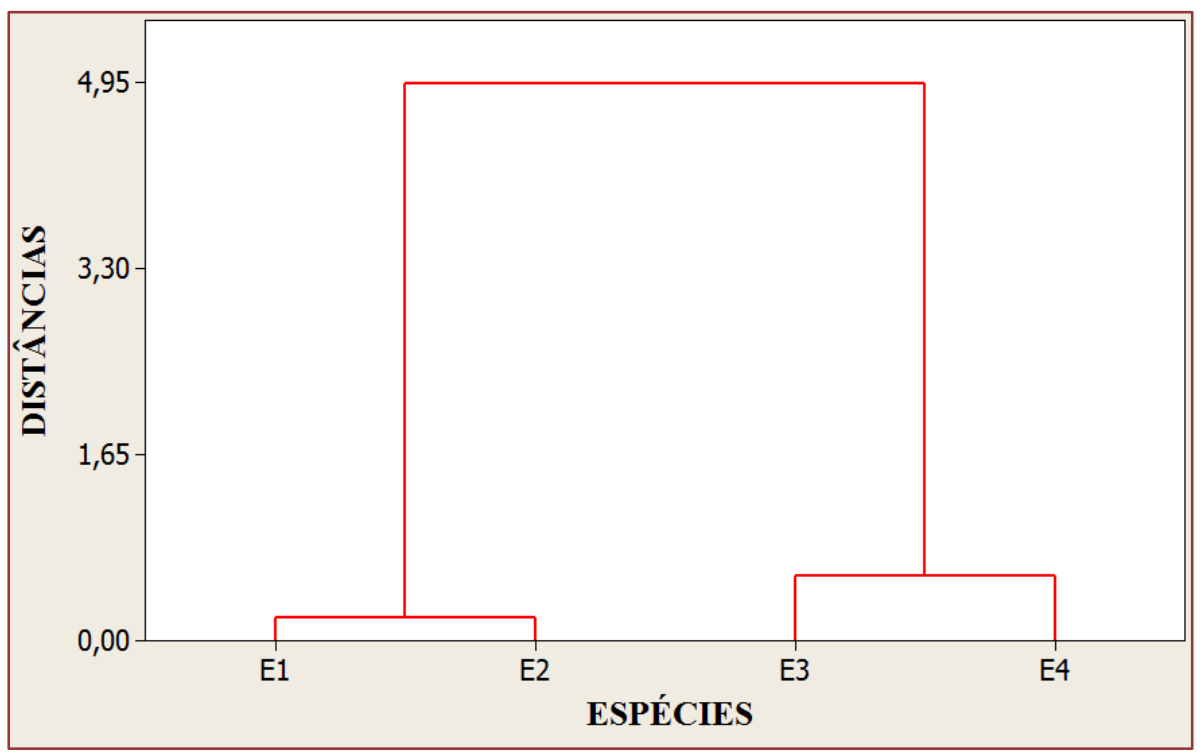

Figura 8.1.1.3 - Dendrograma das quatro espécies florestais.

\section{h) Testes de existência de interação Ocasião×Espécie para E1 e E2}

A partir do dendrograma ou fenograma, resultado da análise de agrupamento, foi realizado o teste para verificar se os perfis de crescimentos das espécies E1 e E2 são paralelos. 
A Tabela 8.1.1.8 mostra os resultados dos testes de Wilks Lambda, Pillai Trace, Hotelling-Lawley Trace e de Roy Greatest Root, os quais deram não significativos ( $p$ valor $=0,0904)$, denotando não existir interação entre os perfis de crescimento das espécies E1 e E2, ou seja, os seus perfis são paralelos. 0 próximo passo será testar se os perfis de crescimento são coincidentes.

Tabela 8.1.1.8 - Teste de existência de interação de Ocasião×Espécie para E1 e E2.

$\begin{aligned} & \text { Testes e estatísticas exatas de } F \text { para a hipótese de não existência de efeito da interação } \\
& \left.\text { Ocasião×Espécie (S=1; M=1; } N^{*}=26,5\right) .\end{aligned}$
\begin{tabular}{l|c|c|c|c|c} 
Estatísticas & Valores & Valor F & Num GL & Den GL & Pr > F \\
Wilks Lambda & 0,86631212 & 2,12 & 4 & 55 & 0,0904 \\
\hline Pillai Trace & 0,13368788 & 2,12 & 4 & 55 & 0,0904 \\
\hline $\begin{array}{l}\text { Hotelling-Lawley } \\
\text { Trace }\end{array}$ & 0,15431838 & 2,12 & 4 & 55 & 0,0904 \\
\hline Roy Greatest Root & 0,15431838 & 2,12 & 4 & 55 & 0,0904 \\
\hline
\end{tabular}

i) Teste de coincidência dos perfis de crescimento de E1 e E2 pelo teste " $t$ " de Student

A verificação da coincidência, entre as espécies E1 e E2, poderá ser feito pelo teste " $t$ " de Student segundo a hipótese $\mathrm{H}_{02}$ :

$H_{02}=j^{\prime} \mu_{v 1}=j^{\prime} \mu_{v 2}$, tal que $j^{\prime}=\left[\begin{array}{lllll}1 & 1 & 1 & 1 & 1\end{array}\right]$. Esta hipótese pode ser escrita da forma:

$$
\begin{aligned}
& H_{02}: \mu_{11}+\mu_{12}+\mu_{13}+\mu_{14}+\mu_{15}=\mu_{21}+\mu_{22}+\mu_{23}+\mu_{24}+\mu_{25} \\
& t=\frac{j^{\prime}\left(\bar{y}_{v 1}-\bar{y}_{v 2}\right)}{\sqrt{j^{\prime} S j\left(\frac{1}{n_{1}}+\frac{1}{n_{2}}\right)}}, \text { com } n_{1}+n_{2}-2=58 \text { graus de liberdade. } \\
& \bar{y}_{v 1}^{\prime}=[9,6844966714,415266717,089190019,445480021,0381809 \\
& \bar{y}_{v 2}^{\prime}=[9,7430533314,446093317,192783319,560786721,1482133]
\end{aligned}
$$

$$
\begin{aligned}
& \left.j^{\prime}\left(\bar{y}_{v 2}-\bar{y}_{v 3}\right)=0,418316 \quad j^{\prime} S j\right)=2,10037
\end{aligned}
$$

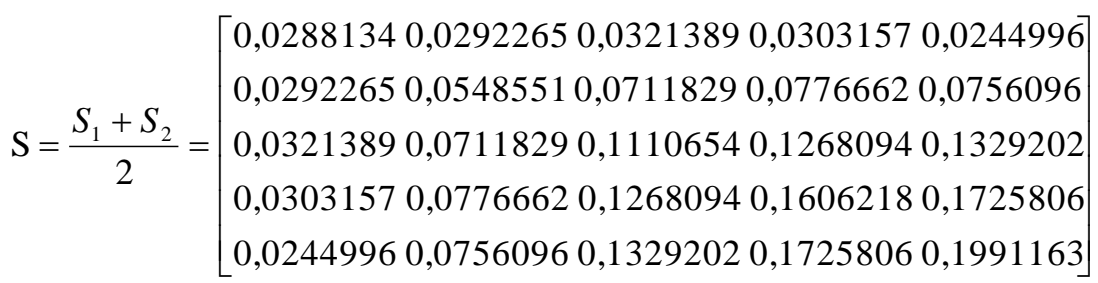

As matrizes $S_{1}$ e $S_{2}$ estão apresentadas na seção 8.1.1.

$$
t_{C A L}=\frac{j^{\prime}\left(\bar{x}_{v 2}-\bar{\mu}_{v 3}\right)}{\sqrt{j^{\prime} S j\left(\frac{1}{n_{2}}+\frac{1}{n_{3}}\right)}}=\frac{0,418316}{\sqrt{2,10037 \times\left(\frac{1}{30}+\frac{1}{30}\right)}}=\frac{0,418316}{0,3741987}=1,1178993
$$


Dado que $t_{58 ; 0,05} \cong 2,0$, conclui-se pela não rejeição de $H_{02}$, então há razão para aceitar a coincidência dos perfis de crescimento das espécies E1 e E2. Neste caso, será estimada uma única equação de crescimento para ambos os métodos.

j) Teste de coincidência dos perfis de E1 e E2 pela hipótese linear $\left(\mathrm{H}_{02}: \mathrm{C}_{2} \beta \mathrm{U}_{2}=\phi\right)$

$$
\begin{gathered}
C_{2}=\left[\begin{array}{ccccc}
1 & -1 & 0 & 0 & 0 \\
0 & 1 & -1 & 0 & 0 \\
0 & 0 & 1 & -1 & 0 \\
0 & 0 & 0 & 1 & -1
\end{array}\right] \\
U_{2}^{\prime}=\left[\begin{array}{lllll}
1 & 1 & 1 & 1 & 1
\end{array}\right]
\end{gathered}
$$

De acordo com a tabela 8.1.1.9, sendo $F_{\text {Cal }}=1,25<F_{1 ; 58 ; 0,05} \cong 4,0$, conclui-se pela não rejeição de $H_{02}$, então há razão para aceitar a coincidência dos perfis de crescimento das espécies E1 e E2.

É importante observar que, no teste de coincidência dos perfis, para a hipótese linear $H_{02}: C_{2} \beta U_{2}=\phi$, o valor da estatística $F_{1 ; 58}$ foi 1,25 , enquanto que o valor de $t_{58}$, no teste de coincidência " $t$ " de Student, foi de 1,1178993, ou seja, $F_{1,58}=t_{58}^{2}=1,25$. Este resultado é devido à relação, $F_{1 ; n ; \alpha}=t_{n ; \alpha}^{2}$, existente entre as funções densidades de $F \mathrm{e}$ " $t$ " de Student.

Tabela 8.1.1.9 - Teste de coincidência de perfis $\left(H_{02}: C_{2} \beta U_{2}=\phi\right)$ para E1 e E2.

\begin{tabular}{l|c|c|c|c|c}
\multicolumn{5}{c}{ Testes e estatísticas exatas de $F$ para a hipótese de coincidência de perfis das espécies E1 } \\
\cline { 2 - 6 } & e E2 $\left(\mathrm{S}=1 ; \mathrm{M}=-0,5 ; N^{*}=28\right)$ & & \\
\multicolumn{1}{c|}{ Estatísticas } & Valores & Valor F & Num GL & Den GL & Pr > F \\
\hline Wilks Lambda & 0,97890792 & 1,25 & 1 & 58 & 0,2682 \\
\hline Pillai Trace & 0,02109208 & 1,25 & 1 & 58 & 0,2682 \\
\hline Hotelling-Lawley trace & 0,02154654 & 1,25 & 1 & 58 & 0,2682 \\
\hline Roy Greatest Root & 0,02154654 & 1,25 & 1 & 58 & 0,2682 \\
\hline
\end{tabular}

A aceitação de coincidência dos perfis de crescimento das espécies E1 e E2, obtidas pelo teste " $t$ " de Student e pela hipótese linear $H_{02}: C_{2} \beta U_{2}=\phi$, também foram confirmadas, na seção 8.1.3, pelo teste da razão da máxima verossimilhança para verificar a igualdade de parâmetros e a identidade de modelos de regressão, para as equações de crescimento das espécies E1 e E2.

\section{k) Testes de existência de interação Ocasião×Espécie para E3 e E4}

A Tabela 8.1.1.10 apresenta os resultados dos testes de Wilks Lambda, Pillai Trace, Hotelling-Lawley Trace e de Roy Greatest Root, os quais deram significativos ( $p$-valor < 0, 0001), denotando existir interação entre os perfis de crescimento das espécies E3 e 
E4, ou seja, os seus perfis não são paralelos. Destarte, as equações de crescimento desses métodos devem ser obtidas separadamente.

Tabela 8.1.1.10 - Teste de existência de interação Ocasião×Espécie para E3 e E4.

\begin{tabular}{|c|c|c|c|c|c|}
\hline Estatísticas & Valor & Valor F & Num GL & Den GL & $\operatorname{Pr}>\mathrm{F}$ \\
\hline Wilks Lambda & 0.61481030 & 8.61 & 4 & 55 & $<0,0001$ \\
\hline Pillai Trace & 0.38518970 & 8.61 & 4 & 55 & $<0,0001$ \\
\hline $\begin{array}{l}\text { Hotelling-Lawley } \\
\text { Trace }\end{array}$ & 0.62651797 & 8.61 & 4 & 55 & $<0,0001$ \\
\hline Roy Greatest Root & 0.62651797 & 8.61 & 4 & 55 & $<0,0001$ \\
\hline
\end{tabular}

\subsubsection{CICLO DE CORTE ECONOMICAMENTE ACONSELHÁVEL PARA E1, E2, E3 E E4}

\section{a) Estimativa do ciclo de corte do grupo formado pelas espécies (E1, E2)}

Como houve coincidência dos perfis de crescimento das espécies E1 e E2, então será obtida uma única equação para ambas as espécies ( $\left.V_{E 12}\right)$.

Seja a equação de Mitscherlich ( $V$ ) e a fórmula para obter o ciclo de corte economicamente aconselhável $\left(t_{o t i}\right)$ :

$$
V=\beta_{0}\left[1-10^{-\beta_{1}\left(t+\beta_{2}\right.}\right] \quad t_{\text {oti }}=\frac{1}{\hat{\beta}_{1}} \log _{10}\left[2,302585 R \hat{\beta}_{0} \hat{\beta}_{1}\right]-\hat{\beta}_{2}
$$

Nesta análise será utilizada uma razão de custo $R=3$ que é o quociente entre a receita obtida pela venda da madeira e o custo do manejo florestal (por ha). Da Tabela 8.1.2.1 da análise de regressão para o grupo formado pelas espécies E1 e E2:

$$
\begin{gathered}
V_{E 12}=24,591\left[1-10^{-0,0310(t+7,0978)}\right] \\
t_{\text {Oti }(E 12)}=\left(\frac{1}{0,0310}\right) \log (2,302585 \times 3 \times 24,5911 \times 0,0310)-7,0978=16,2 \text { anos } \\
V_{\text {oti }(E 12)}=24,591\left[1-10^{-0,0310(16,2+7,0978}\right]=19,929 m^{3} / \mathrm{ha} \\
\operatorname{RQMR}_{\mathrm{E} 12}=\sqrt{\frac{\sum_{i=1}^{N}\left(Y_{i}-\hat{Y}_{i}\right)^{2}}{N}}=\sqrt{\frac{38,0422}{2 \times 150}}=0,3561 \\
\mathrm{CV}_{\mathrm{E} 12}=\frac{R Q M R}{\bar{Y}_{E 12}} \times 100=\frac{0,3561}{16,3764} \times 100=2,17 \%
\end{gathered}
$$


Tabela 8.1.2.1 - Análise de regressão do grupo formado (E1, E2).

\begin{tabular}{|c|c|c|c|c|}
\hline Parâmetros & Estimativas & Erro Padrão Aprox. & \multicolumn{2}{|c|}{ Limites de Confiança (95\%) } \\
\hline$\hat{\beta}_{0}$ & 24,5911 & 0,2105 & 24,1769 & 25,0053 \\
\hline$\hat{\beta}_{1}$ & 0,0310 & 0,000824 & 0,0294 & 0,0326 \\
\hline$\hat{\beta}_{2}$ & 7,0978 & 0,1406 & 6,8212 & 7,3745 \\
\hline Fonte & GL & Soma de Quadrados & Quadrado Médio & Valor F \\
\hline Erro & 297 & 38,0422 & 0,1281 & 221975,0 \\
\hline \multicolumn{5}{|c|}{ Teste de validade de Graybill $F_{G}$} \\
\hline Fonte & GL & Quadrado Médio & Valor $F_{G}$ & $\operatorname{Pr}>\mathrm{F}$ \\
\hline Numerador & 2 & 0,14828 & 1,17 & 0,3116 \\
\hline Denominador & 298 & 0,12666 & & \\
\hline
\end{tabular}

Coeficiente de correlação de Pearson, $\mathrm{N}=300$, Prob $>|\mathrm{r}|$ Sob H0: Rho=0

$$
\begin{array}{l|l}
r_{E 12}=0,99609(<0,0001) & r_{E 12}^{2}=0,9922 \\
\hline
\end{array}
$$

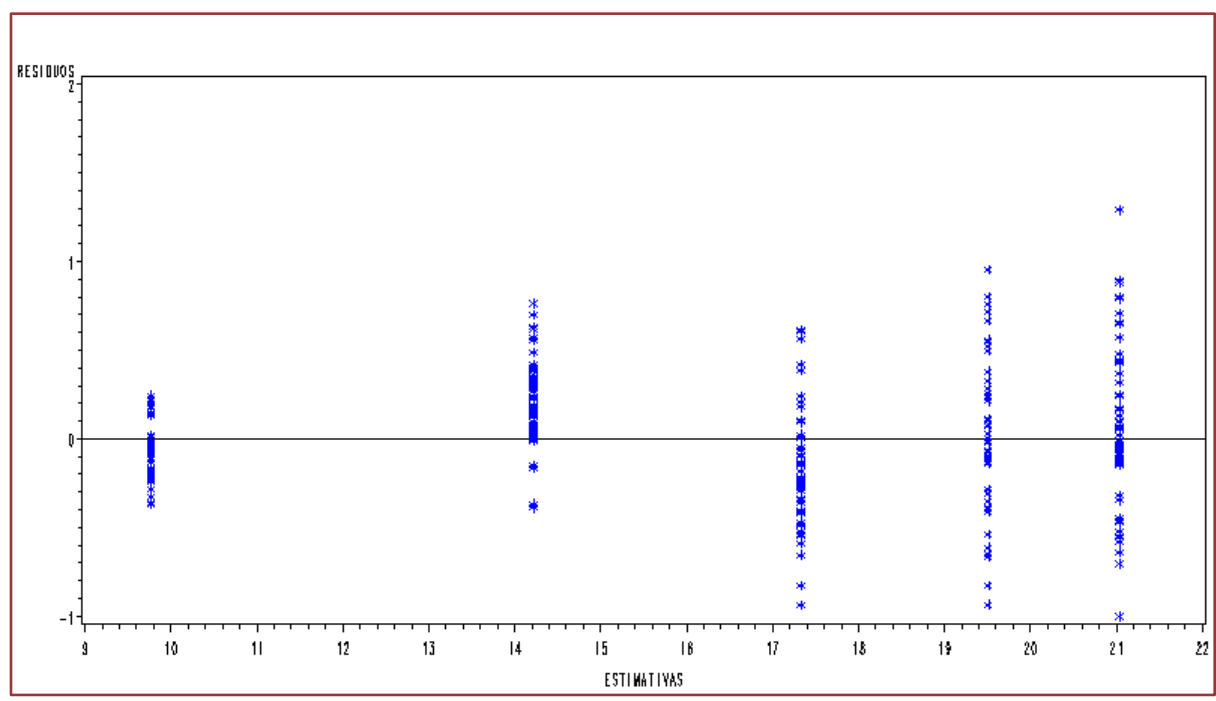

Figura 8.1.2.1 - Distribuição dos resíduos da equação conjunta do grupo (E1, E2).

b) Estimativa do ciclo de corte para a espécie E3

De acordo com a tabela 8.1.2.2:

$$
\begin{gathered}
V_{E 3}=23,0163\left[1-10^{-0,0279\left(t_{i}+8,4227\right)}\right] \\
t_{\text {Oti }(E 3)}=\left(\frac{1}{0,0279}\right) \log (2,302585 \times 3 \times 23,0163 \times 0,0279)-8,4227=14,8 \text { anos } \\
V_{o t i(E 3)}=23,0163\left[1-10^{-0,0279(14,8+8,4227)}\right]=17,839 \mathrm{~m}^{3} / \mathrm{ha} \\
\operatorname{RQMR}_{\mathrm{E} 3}=\sqrt{\frac{\sum_{i=1}^{N}\left(Y_{i}-\hat{Y}_{i}\right)^{2}}{N}}=\sqrt{\frac{14,4743}{150}}=0,310637
\end{gathered}
$$




$$
\mathrm{CV}_{\mathrm{E} 3}=\frac{R Q M R}{\bar{Y}_{E 3}} \times 100=\frac{0,310637}{15,2257} \times 100=2,04 \%
$$

Tabela 8.1.2.2 - Análise de regressão da equação de Mitscherlich para E3.

\section{Parâmetros Estimativas $\quad$ Erro Padrão Aprox. Limites de Confiança (95\%)}

\begin{tabular}{c|c|c|c|c}
$\hat{\beta}_{0}$ & 23,0163 & 0,3167 & 22,3904 & 23,6422 \\
\hline$\hat{\beta}_{1}$ & 0,0279 & 0,00117 & 0,0256 & 0,0302 \\
\hline$\hat{\beta}_{2}$ & 8,4227 & 0,2463 & 7,9360 & 8,9094 \\
\hline
\end{tabular}

\begin{tabular}{|c|c|c|c|c|}
\hline Fonte & GL & Soma de Quadrados & Quadrado Médio & Valor F \\
\hline Erro & 147 & 14,4743 & 0,0985 & 9072,78 \\
\hline \multicolumn{5}{|c|}{ Teste de validade de Graybill $F_{G}$} \\
\hline Fonte & GL & Quadrado Médio & Valor $F_{G}$ & $\operatorname{Pr}>\mathrm{F}$ \\
\hline Numerador & 2 & 0,05816 & 0,60 & 0,5504 \\
\hline Denominador & 148 & 0,09701 & & \\
\hline
\end{tabular}

Coeficiente de correlação de Pearson, $\mathrm{N}=150$, Prob $>|\mathrm{r}|$ Sob H0: Rho=0

$$
\begin{array}{l|l}
r_{E 3}=0,99597(<0,0001) & r_{E 3}^{2}=0,9920
\end{array}
$$

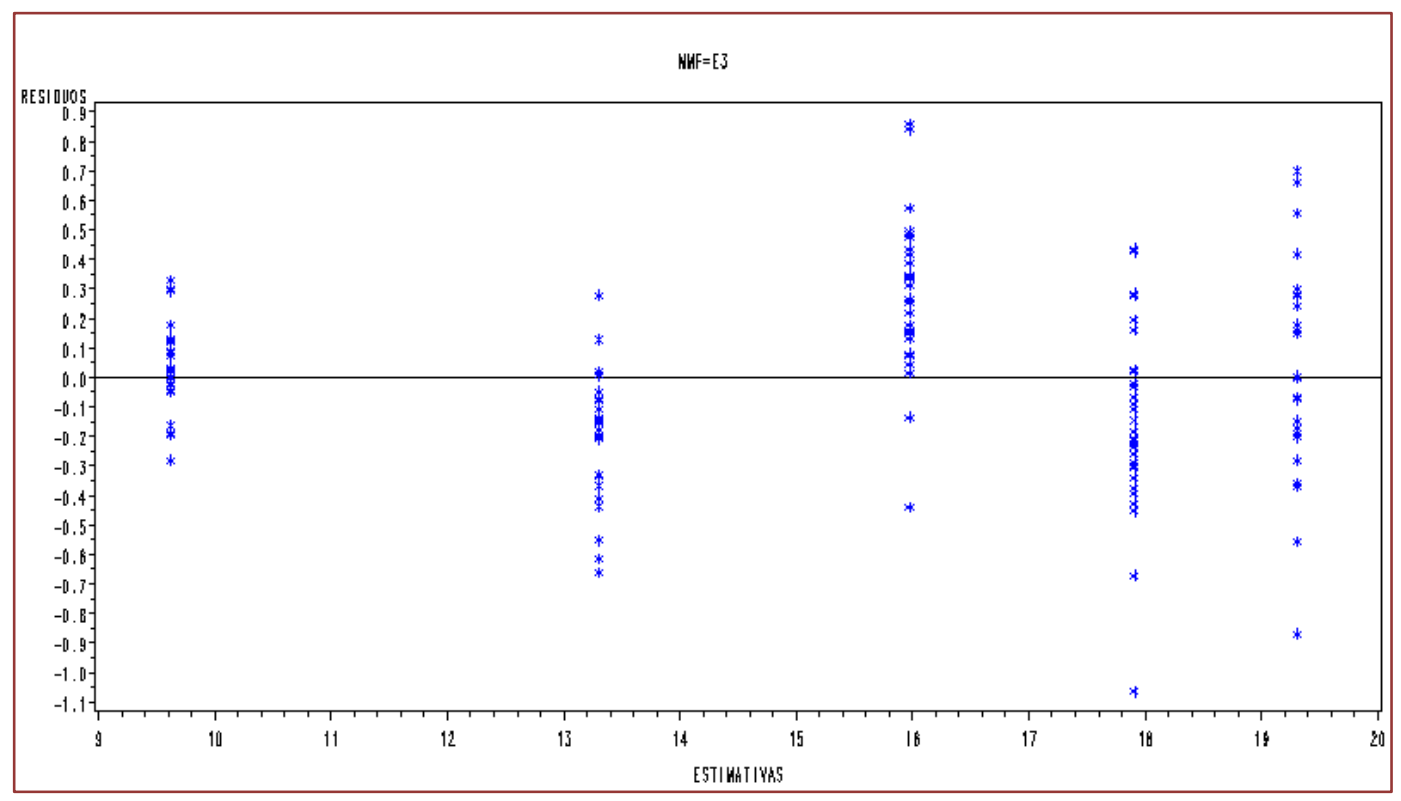

Figura 8.1.2.2 - Gráfico dos resíduos da equação de Mitscherlich para E3.

c) Determinação do ciclo de corte para a espécie E4

De acordo com a tabela 8.1.2.3:

$$
\begin{gathered}
V_{E 4}=23,6604\left[1-10^{-0,0274(t+8,3068}\right] \\
t_{\text {Oti }(E 4)}=\left(\frac{1}{0,0274}\right) \log (2,302585 \times 3 \times 23,6604 \times 0,0274-8,3068=15,5 \text { anos }
\end{gathered}
$$




$$
\begin{gathered}
V_{\text {oti(E4) }}=23,6604\left[1-10^{-0,0274(15,5+8,3068}\right]=18,392 \mathrm{~m}^{3} / \mathrm{ha} \\
\mathrm{RQMR}_{\mathrm{E} 4}=\sqrt{\frac{\sum_{i=1}^{N}\left(Y_{i}-\hat{Y}_{i}\right)^{2}}{N}}=\sqrt{\frac{25,5569}{150}}=0,41277 \\
\mathrm{CV}_{\mathrm{E} 4}=\frac{R Q M R}{\bar{Y}_{E 4}} \times 100=\frac{0,41277}{15,4511} \times 100=2,67 \%
\end{gathered}
$$

\begin{tabular}{|c|c|c|c|c|}
\hline Parâmetros & Estimativas & Erro Padrão Aprox. & \multicolumn{2}{|c|}{ Limites de Confiança (95\%) } \\
\hline$\hat{\beta}_{0}$ & 23,6604 & 0,4351 & 22,8006 & 24,5203 \\
\hline$\hat{\beta}_{1}$ & 0,0274 & 0,00150 & 0,0245 & 0,0304 \\
\hline$\hat{\beta}_{2}$ & 8,3068 & 0,3137 & 7,6869 & 8,9268 \\
\hline Fonte & GL & Soma de Quadrados & Quadrado Médio & Valor F \\
\hline Erro & 147 & 25,5569 & 0,1739 & 5515,72 \\
\hline \multicolumn{5}{|c|}{ Teste de validade de Graybill $F_{G}$} \\
\hline Fonte & GL & Quadrado Médio & Valor $F_{G}$ & $\operatorname{Pr}>\mathrm{F}$ \\
\hline Numerador & 2 & 0,16804 & 0,99 & 0,3755 \\
\hline Denominador & 148 & 0,17041 & & \\
\hline \multicolumn{5}{|c|}{ Coeficiente de correlação de Pearson, $\mathrm{N}=150$, Prob $>|\mathrm{r}|$ Sob H0: Rho=0 } \\
\hline \multicolumn{3}{|c|}{$r_{E 4}=0,99340(<0,0001)$} & \multicolumn{2}{|c|}{$r_{E 4}^{2}=0,9868$} \\
\hline
\end{tabular}

Tabela 8.1.2.3 - Análise de regressão da equação de Mitscherlich para E4.

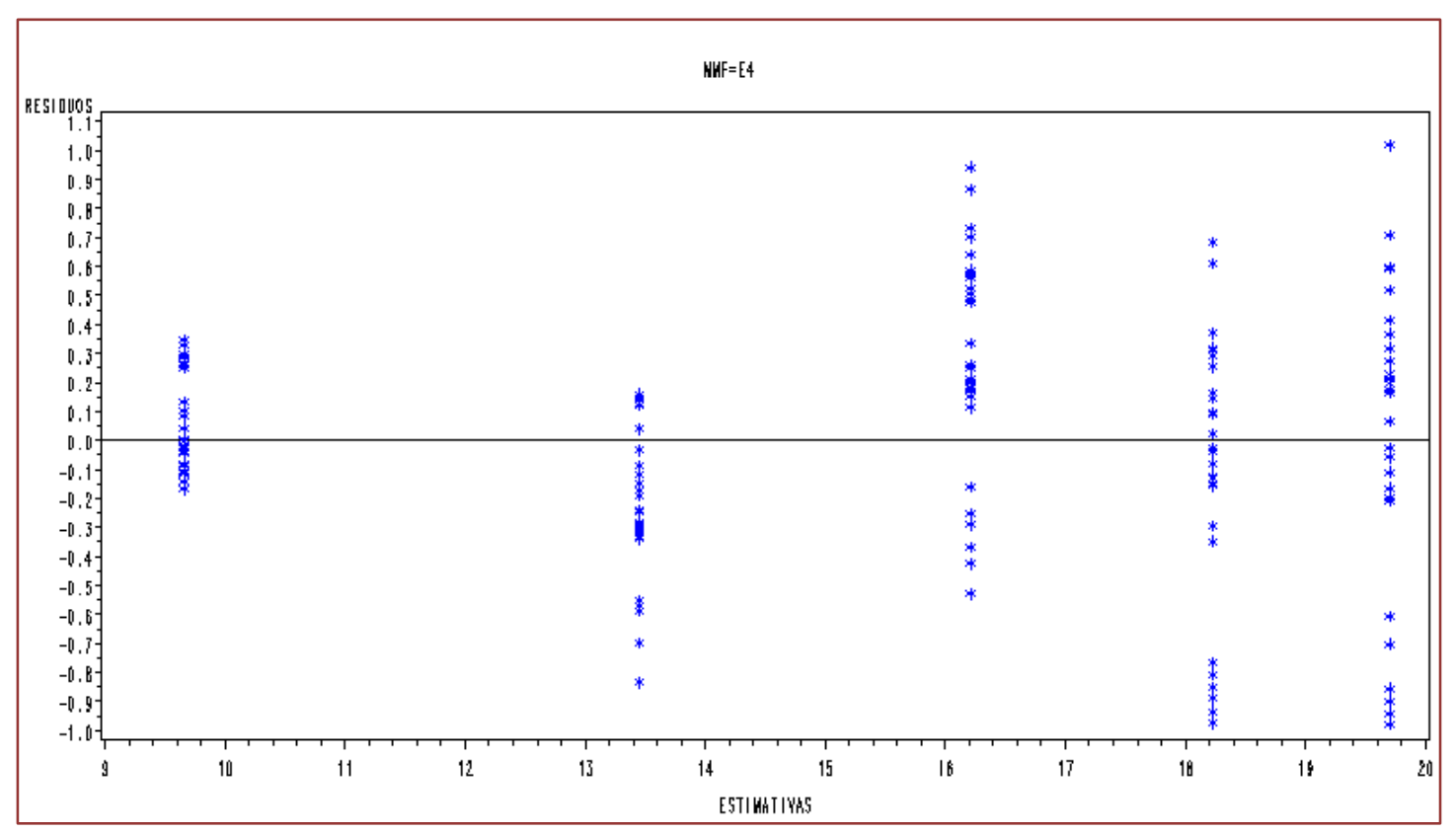

Figura 8.1.2.3 - Gráfico dos resíduos da equação de Mitscherlich para (E4). 
d) Estimativa do ciclo de corte do grupo formado pelas espécies (E1, E2, E3, E4)

De acordo com a tabela 8.1.2.4:

$$
\begin{gathered}
V_{E 1234}=23,9520\left[1-10^{-0,0294(t+7,6796)}\right] \\
t_{\text {Oti }(1234)}=\left(\frac{1}{0,0294}\right) \log (2,302585 \times 3 \times 23,9520 \times 0,0294)-7,6796=15,7 \text { anos } \\
V_{\text {oti }(M 1234)}=23,9520\left[1-10^{-0,0294(15,7+7,6796)}\right]=19,032 m^{3} / \mathrm{ha} \\
\operatorname{RQMR}_{\mathrm{E} 1234}=\sqrt{\frac{\sum_{i=1}^{N}\left(Y_{i}-\hat{Y}_{i}\right)^{2}}{N}}=\sqrt{\frac{283,7}{4 \times 150}}=0,68763 \\
\mathrm{CV}_{\mathrm{E} 1234}=\frac{R Q M R}{\bar{Y}_{E 1234}} \times 100=\frac{0,68763}{15,8574} \times 100=4,34 \%
\end{gathered}
$$

\begin{tabular}{|c|c|c|c|c|}
\hline Parâmetros & Estimativas & Erro Padrão Aprox. & \multicolumn{2}{|c|}{ Limites de Confiança (95\%) } \\
\hline$\hat{\beta}_{0}$ & 23,9520 & 0,3161 & 23,3311 & 24,5729 \\
\hline$\hat{\beta}_{1}$ & 0,0294 & 0,00119 & 0,0271 & 0,0317 \\
\hline$\hat{\beta}_{2}$ & 7,6796 & 0,2235 & 7,2406 & 8,1185 \\
\hline Fonte & GL & Soma de Quadrados & Quadrado Médio & Valor F \\
\hline Erro & 597 & 283,7 & 0,4752 & 8950,77 \\
\hline \multicolumn{5}{|c|}{ Teste de validade de Graybill $F_{G}$} \\
\hline Numerador & 2 & 4,57751 & 9,97 & $<0,0001$ \\
\hline Denominador & 598 & 0,45906 & & \\
\hline \multicolumn{5}{|c|}{ Coeficiente de correlação de Pearson, $\mathrm{N}=600$, Prob $>|\mathrm{r}|$ Sob H0: Rho=0 } \\
\hline \multicolumn{3}{|c|}{$r_{E 4}=0,98373(<0,0001)$} & \multicolumn{2}{|c|}{$r_{E 4}^{2}=0,9677$} \\
\hline
\end{tabular}

Tabela 8.1.2.4 - Análise de regressão da equação de Mitscherlich para (E1, E2, E3, E4). 


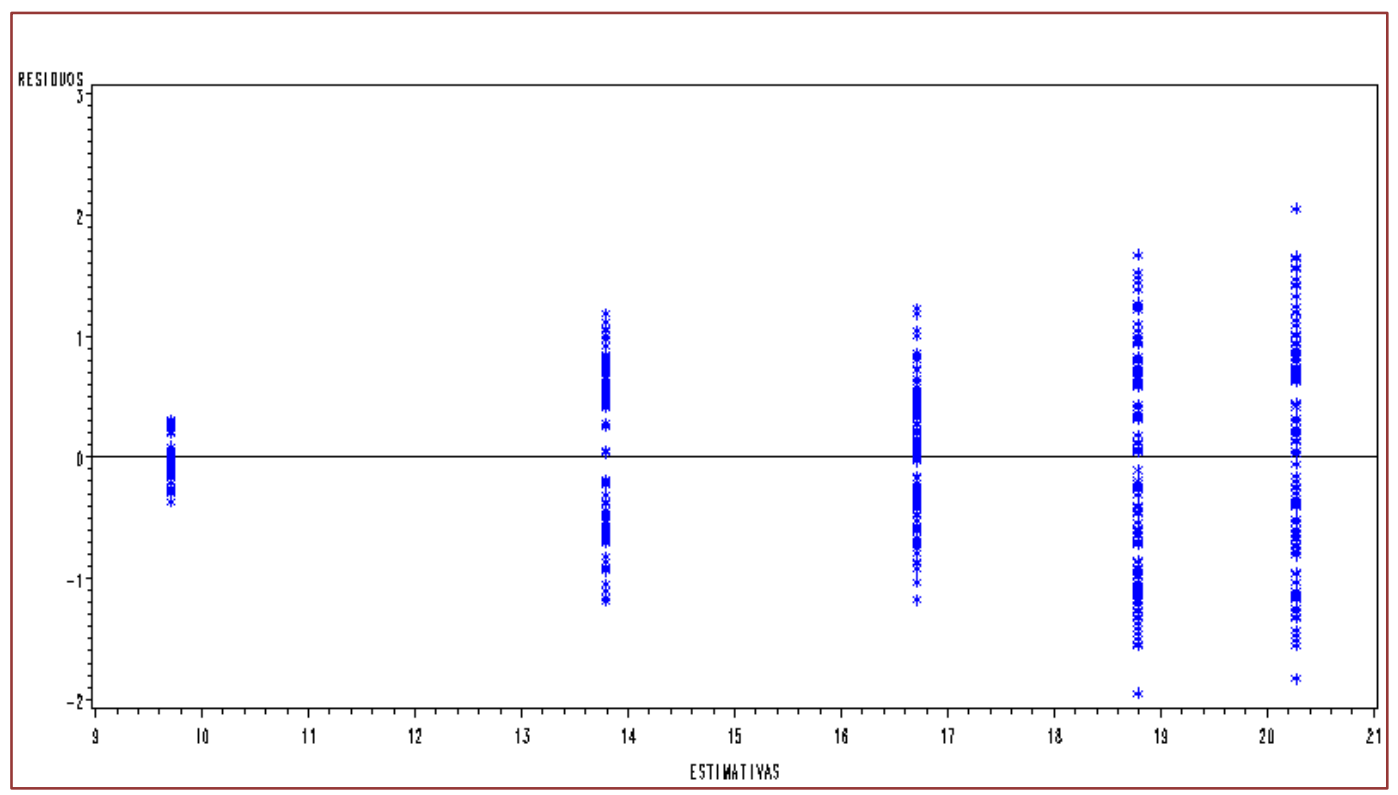

Figura 8.1.2.4 - Gráfico dos resíduos considerando (E1, E2, E3 e E4).

\section{e) Observações sobre os resultados dos ciclos de corte para as quatro espécies}

Os ciclos de cortes foram os seguintes para as quatro espécies consideradas de crescimento rápido, grupo $(E 1+\mathrm{E} 2)=16,2$ anos, $\mathrm{E} 3=14,8$ anos e $E 4=15,5$ anos. No capítulo 7 foi recomendado o uso do método de manejo (M3 ou o M4), os quais apresentaram, considerando todas as espécies ocorrentes na área, um ciclo de corte economicamente recomendável de 18,2 anos.

Esses resultados denotam a necessidade de estabelecer várias colheitas em diferentes datas. Evidentemente que, usando dados reais de uma floresta natural amazônica e considerando todas as espécies, em diferentes idades ou estágios de desenvolvimento, obviamente que os resultados seriam equivalentes a essa situação exposta, o que cria a necessidade de estabelecer um sistema de manejo policíclico, ou seja, manejar a floresta por grupo de espécies com perfis de crescimento semelhantes.

\subsubsection{TESTE DA RAZÃO DA MÁXIMA VEROSSIMILHANÇA PARA VERIFICAR A IGUALDADE DE PARÂMETROS E A IDENTIDADE DE MODELOS DE REGRESSÃO NÃO LINEAR}

a) Verificar se as duas equações de crescimento das espécies E1 e E2 diferem estatisticamente, ou seja, se os seus perfis de crescimento são coincidentes.

$$
\begin{aligned}
& H_{0}^{(4)}: \beta_{01}=\beta_{02}=\beta_{0}, \beta_{11}=\beta_{12}=\beta_{1}, \beta_{21}=\beta_{22}=\beta_{2} \\
& \text { versus } H_{A}^{(4)} \text { : pelo menos uma igualdade não ocorre. }
\end{aligned}
$$

No caso da hipótese $H_{0}^{4}$ não ser rejeitada, uma única equação de crescimento deverá ser obtida considerando, conjuntamente, as duas espécies E1 e E2. 
Da Tabela 8.1.3.1 que expõe a análise de variância referente ao modelo completo $(\Omega)$ :

$$
S Q R R_{\Omega}=37,4623 \quad Q M R R_{\Omega}=0,1274 \quad N=300
$$

$p_{\Omega}=6$ (número de parâmetros do modelo sem restrição), então:

$$
\hat{\sigma}_{\Omega}^{2}=\frac{S Q R R_{\Omega}}{N}=\frac{N-p_{\Omega}}{N} \times M Q R R_{\Omega}=\frac{37,4623}{300}=\left(\frac{300-6}{300}\right) \times 0,1274=0,1249
$$

Tabela 8.1.3.1 - Modelo completo ômega $(\Omega)$ para E1 e E2.

\begin{tabular}{c|c|c|c|c} 
Parâmetros & Estimativas & Erro Padrão Aprox. & Limites de Confiança (95\%) \\
$\hat{\beta}_{01}$ & 24,4837 & 0,2941 & 23,9048 & 25,0625 \\
\hline$\hat{\beta}_{11}$ & 0,0312 & 0,00117 & 0,0289 & 0,0335 \\
\hline$\hat{\beta}_{21}$ & 7,0789 & 0,1981 & 6,6891 & 7,4687 \\
\hline$\hat{\beta}_{02}$ & 24,6987 & 0,2996 & 24,1090 & 25,2884 \\
\hline$\hat{\beta}_{12}$ & 0,0308 & 0,00116 & 0,0286 & 0,0331 \\
\hline$\hat{\beta}_{22}$ & 7,1166 & 0,1985 & 6,7260 & 7,5072 \\
\hline Fonte & GL & Soma de Quadrados & Quadrado Médio & Valor F \\
Erro & 294 & 37,4623 & 0,1274 & 7600.59 \\
\hline \multicolumn{5}{|c|}{}
\end{tabular}

Da Tabela 8.1.3.2 que mostra a análise de variância do modelo com restrição $(\omega)$ :

$$
S Q R R_{\omega}=38,0422 \quad M Q R R_{\omega}=0,1281 \quad \mathrm{~N}=300
$$

$p_{\omega}=3$ (número de parâmetros do modelo com restrição), então:

$$
\hat{\sigma}_{\omega}^{2}=\frac{S Q R R_{\omega}}{N}=\frac{N-p_{\omega}}{N} \times M Q R R_{\omega}=\frac{38,0422}{300}=\left(\frac{300-3}{300}\right) \times 0,1281=0,1268
$$

Tabela 8.1.3.2 - Modelo com restrição $(\omega)$ para E1 e E2.

\begin{tabular}{cc|c|c|c}
\multicolumn{5}{c}{$H_{0}^{4}: \beta_{01}=\beta_{02}=\beta_{0} ; \beta_{11}=\beta_{12=} \beta_{1 ;} \beta_{21}=\beta_{22}=\beta_{2}$} \\
Parâmetros & Estimativas & Erro Padrão Aprox. & \multicolumn{1}{l}{ Limites de Confiança (95\%) } \\
$\hat{\beta}_{0}$ & 24,5911 & 0,2105 & 24,1769 & 25,0053 \\
\hline$\hat{\beta}_{1}$ & 0,0310 & 0,000824 & 0,0294 & 0,0326 \\
\hline$\hat{\beta}_{2}$ & 7,0978 & 0,1406 & 6,8212 & 7,3745 \\
Fonte & GL & Soma de Quadrados & Quadrado Médio & Valor F \\
Erro & 297 & 38,0422 & 0,1281 & 18900,5 \\
\hline
\end{tabular}

Do teste da razão de máxima verossimilhança, conforme Rao (1973), para grandes amostras, tem-se: 


$$
\begin{gathered}
-2 \operatorname{LnL}=-N \operatorname{Ln}\left(\frac{\hat{\sigma}_{\Omega}^{2}}{\hat{\sigma}_{\omega}^{2}}\right) \underset{N \rightarrow \infty}{\stackrel{d}{\rightarrow}} \chi_{v}^{2} \\
\chi_{C a l}^{2}=-N \operatorname{Ln}\left(\frac{S Q R R_{\Omega} / N}{S Q R R_{\omega} / N}\right)=-N \operatorname{Ln}\left(\frac{S Q R R_{\Omega}}{S Q R R_{\omega}}\right)=-300 \operatorname{Ln}\left(\frac{0,1249}{0,1268}\right)=4,5293
\end{gathered}
$$

Dado $\chi_{v ; 0,01}^{2}=\chi_{3,0,01}^{2}=11,3$, donde $v=p_{\Omega}-p_{\omega}=3$.

Como $\chi_{\text {Cal }}^{2}<\chi_{3,0,01}^{2}$, a hipótese $H_{0}^{4}$ foi não significativa, conclui-se que as duas equações não diferem estatisticamente, assim sendo, a equação comum, cujas estimativas estão mostradas na tabela 8.1.3.2, deverá ser usada para estimar a curva de crescimento para o grupo (E1, E2).

$$
V_{E 12}=15,2298\left[1-10^{-0,0816(t+5,4118)}\right]
$$

b) Verificar se as duas equações de crescimento das espécies E3 e E4 diferem estatisticamente, ou seja, se os seus perfis de crescimento são coincidentes.

Da Tabela 8.1.3.3 que mostra a análise de variância referente ao modelo completo $(\Omega)$ :

$$
S Q R R_{\Omega}=40,0312 \quad Q M R R_{\Omega}=0,1362 \quad \mathrm{~N}=300
$$

$p_{\Omega}=6$ (número de parâmetros do modelo sem restrição), então:

$$
\hat{\sigma}_{\Omega}^{2}=\frac{S Q R R_{\Omega}}{N}=\frac{N-p_{\Omega}}{N} \times M Q R R_{\Omega}=\frac{40,0312}{300}=\left(\frac{300-6}{300}\right) \times 0,1362=0,1334
$$

Tabela 8.1.3.3 - Modelo completo ômega $(\Omega)$ para E3 e E4.

\section{Parâmetros Estimativas Erro Padrão Aprox. Limites de Confiança (95\%)}

\begin{tabular}{c|c|c|c|c}
$\hat{\beta}_{03}$ & 23,0163 & 0,3724 & 22,2833 & 23,7493 \\
\hline$\hat{\beta}_{13}$ & 0,0279 & 0,00138 & 0,0252 & 0,0306 \\
\hline$\hat{\beta}_{23}$ & 8,4227 & 0,2896 & 7,8527 & 8,9927 \\
\hline$\hat{\beta}_{04}$ & 23,6604 & 0,3850 & 22,9027 & 24,4182 \\
\hline$\hat{\beta}_{14}$ & 0,0274 & 0,00133 & 0,0248 & 0,0300 \\
\hline$\hat{\beta}_{24}$ & 8,3068 & 0,2776 & 7,7605 & 8,8532 \\
Fonte & GL & Soma de Quadrados & Quadrado Médio & Valor F \\
Erro & 294 & 40,0312 & 0,1362 & 5447,09 \\
\hline
\end{tabular}


Da Tabela 8.1.3.4 que expõe a análise de variância referente ao modelo com restrição $(\omega)$ :

$$
S Q R R_{\omega}=45,0135 \quad M Q R R_{\omega}=0,1516 \quad \mathrm{~N}=300
$$

$p_{\omega}=3$ (número de parâmetros do modelo com restrição), então:

$$
\hat{\sigma}_{\omega}^{2}=\frac{S Q R R_{\omega}}{N}=\frac{N-p_{\omega}}{N} \times M Q R R_{\omega}=\frac{45,0135}{300}=\left(\frac{300-3}{300}\right) \times 0,1516=0,1516
$$

Tabela 8.1.3.4 - Modelo com restrição ( $\omega)$ para (E3, E4).

\begin{tabular}{c|c|c|c|c}
\multirow{5}{*}{$H_{0}^{4}: \beta_{03}=\beta_{04}=\beta_{0} ; \beta_{13}=\beta_{14}=\beta_{1} ; \beta_{23}=\beta_{24}=\beta_{2}$} \\
Parâmetros & Estimativas & Erro Padrão Aprox. & Limites de Confiança (95\%) \\
& 23,3381 & 0,2826 & 22,7820 & 23,8942 \\
\hline$\hat{\beta}_{0}$ & 0,0277 & 0,00101 & 0,0257 & 0,0297 \\
\hline$\hat{\beta}_{1}$ & 8,3648 & 0,2115 & 7,9485 & 8,7811 \\
\hline$\hat{\beta}_{2}$ & GL & Soma de Quadrados & Quadrado Médio & Valor F \\
\hline Fonte & 297 & 45,0135 & 0,1516 & 163374,0 \\
\hline Erro & \multicolumn{4}{|c}{}
\end{tabular}

Do teste da razão de máxima verossimilhança, conforme Rao (1973), para grandes amostras, tem-se:

$$
\begin{gathered}
-2 \operatorname{Ln} L=-N \operatorname{Ln}\left(\frac{\hat{\sigma}_{\Omega}^{2}}{\hat{\sigma}_{\omega}^{2}}\right) \underset{N \rightarrow \infty}{\stackrel{d}{\rightarrow}} \chi_{v}^{2} \\
\chi_{C a l}^{2}=-N \operatorname{Ln}\left(\frac{S Q R R_{\Omega} / N}{S Q R R_{\omega} / N}\right)=-N \operatorname{Ln}\left(\frac{S Q R R_{\Omega}}{S Q R R_{\omega}}\right)=-300 \operatorname{Ln}\left(\frac{0,1362}{0,1516}\right)=32,14
\end{gathered}
$$

Dado que $\chi_{v ; 0,01}^{2}=\chi_{3,0,01}^{2}=11,3$, onde $v=p_{\Omega}-p_{\omega}=3$, e como $\chi_{\text {Cal }}^{2}>\chi_{3,0,01}^{2}$, o teste $H_{0}^{4}$ foi significativo, então conclui-se que as duas equações diferem estatisticamente, assim sendo, não é recomendável ajustar uma equação única para as espécies E3 e E4. 
c) Verificar se as equações de crescimento das espécies E1, E2, E3 e E4 diferem estatisticamente, isto é, os seus perfis de crescimento são coincidentes.

Tabela 8.1.3.5 - Modelo completo ômega $(\Omega)$ para grupo (E1, E2, E3, E4).

\begin{tabular}{c|c|c|c|c} 
Parâmetros & Estimativas & Erro Padrão Aprox. & \multicolumn{2}{c}{ Limite de Confiança (95\%) } \\
$\hat{\beta}_{01}$ & 24,4837 & 0,2991 & 23,8962 & 25,0711 \\
\hline$\hat{\beta}_{11}$ & 0,0312 & 0,00119 & 0,0288 & 0,0335 \\
\hline$\hat{\beta}_{21}$ & 7,0789 & 0,2014 & 6,6832 & 7,4746 \\
\hline$\hat{\beta}_{02}$ & 24,6987 & 0,3047 & 24,1002 & 25,2971 \\
\hline$\hat{\beta}_{12}$ & 0,0308 & 0,00118 & 0,0285 & 0,0332 \\
\hline$\hat{\beta}_{22}$ & 7,1166 & 0,2018 & 6,7202 & 7,5130 \\
\hline$\hat{\beta}_{03}$ & 23,0163 & 0,3664 & 22,2966 & 23,7359 \\
\hline$\hat{\beta}_{13}$ & 0,0279 & 0,00135 & 0,0253 & 0,0306 \\
\hline$\hat{\beta}_{23}$ & 8,4227 & 0,2849 & 7,8631 & 8,9823 \\
\hline$\hat{\beta}_{04}$ & 23,6604 & 0,3788 & 22,9165 & 24,4044 \\
\hline$\hat{\beta}_{14}$ & 0,0274 & 0,00130 & 0,0249 & 0,0300 \\
\hline$\hat{\beta}_{24}$ & 8,3068 & 0,2731 & 7,7704 & 8,8432 \\
\hline Fonte & GL & Soma de Quadrados & Quadrado Médio & Valor F \\
\hline Erro & 588 & 77,4935 & 0,1318 & 6009,79 \\
\hline
\end{tabular}

Da Tabela 8.1.3.5 que mostra a análise de variância referente ao modelo completo $(\Omega)$ :

$$
S Q R R_{\Omega}=77,4935 \quad Q M R R_{\Omega}=0,1318 \quad \mathrm{~N}=600
$$

$p_{\Omega}=12$ (número de parâmetros do modelo sem restrição). Então:

$$
\hat{\sigma}_{\Omega}^{2}=\frac{S Q R R_{\Omega}}{N}=\frac{N-p_{\Omega}}{N} \times M Q R R_{\Omega}=\frac{77,4935}{600}=\left(\frac{600-12}{600}\right) \times 0,1318=0,12916
$$

Tabela 8.1.3.6 - Modelo com restrição ( $\omega$ ) para o grupo (E1, E2, E3, E4).

\section{$H_{0}^{4}: \beta_{01}=\beta_{02}=\beta_{03}=\beta_{04}=\beta_{0} ; \beta_{11}=\beta_{12}=\beta_{13}=\beta_{14}=\beta_{1} ; \beta_{21}=\beta_{22}=\beta_{23}=\beta_{24}=\beta_{2}$}

Parametros Estimativas Erro Padrão Aprox. Limite de Confiança (95\%)

\begin{tabular}{c|c|c|c|c}
$\hat{\beta}_{0}$ & 23,9520 & 0,3161 & 23,3311 & 24,5729 \\
\hline$\hat{\beta}_{1}$ & 0,0294 & 0,00119 & 0,0271 & 0,0317 \\
\hline$\hat{\beta}_{2}$ & 7,6795 & 0,2235 & 7,2406 & 8,1185 \\
\hline Fonte & GL & Soma de quadrados & Quadrado Médio & Valor F \\
Erro & 597 & 283,7 & 0,4752 & 111806.0 \\
\hline
\end{tabular}


Da Tabela 8.1.3.6 que apresenta a análise de variância referente ao modelo com restrição $(\omega)$ :

$$
S Q R R_{\omega}=283,7 \quad M Q R R_{\omega}=0, .4752 \quad \mathrm{~N}=600
$$

$p_{\omega}=3$ (número de parâmetros do modelo com restrição), então:

$$
\hat{\sigma}_{\omega}^{2}=\frac{S Q R R_{\omega}}{N}=\frac{N-p_{\omega}}{N} \times M Q R R_{\omega}=\frac{283,7}{600}=\left(\frac{600-3}{600}\right) \times 0,4752=0,4728 \text { Do teste da razão de }
$$

máxima verossimilhança, conforme Rao (1973), para grandes amostras:

$$
\begin{gathered}
-2 \operatorname{Ln} L=-N \operatorname{Ln}\left(\frac{\hat{\sigma}_{\Omega}^{2}}{\hat{\sigma}_{\omega}^{2}}\right) \underset{N \rightarrow \infty}{\stackrel{d}{\rightarrow}} \chi_{v}^{2} \\
\chi_{\text {Cal }}^{2}=-N \operatorname{Ln}\left(\frac{S Q R R_{\Omega} / N}{S Q R R_{\omega} / N}\right)=-N \operatorname{Ln}\left(\frac{S Q R R_{\Omega}}{S Q R R_{\omega}}\right)=-600 \times \operatorname{Ln}\left(\frac{0,12916}{0,4752}\right)=390,81
\end{gathered}
$$

Dado que $\chi_{v ; 0,01}^{2}=\chi_{9,0,01}^{2}=21,7$, onde $v=p_{\Omega}-p_{\omega}=12-3=9$, e como $\chi_{\text {Cal }}^{2}>\chi_{9,0,01}^{2}$, então $H_{0}^{(4)}$ foi significativa, conclui-se não ser recomendável usar uma equação única para estimar a tendência de crescimento para as quatro espécies, assim sendo a equação $V_{E 1234}$, cujas estimativas dos parâmetros estão apresentadas na tabela 8.1.3.6, não deverá ser usada para estimar o perfil de crescimento considerando as quatro espécies.

$$
V_{E 1234}=23,9520\left[1-10^{-0.0294(t+7,6795)}\right]
$$

\subsubsection{CONSIDERAÇõES FINAIS DA ANÁLISE DO IFC PARA AS ESPÉCIES E1, E2, E3 E E4}

Na tabela 8.1.4.1 estão os valores das estatísticas calculadas com o objetivo de medir o grau de precisão, adequação e validade das equações de crescimento das espécies.

Tabela 8.1.4.1 - Valores das estatísticas de precisão, adequação e validade.

\begin{tabular}{l|c|c|c|c|c|c|c|c}
\multicolumn{1}{c}{ ESP } & SQerro & $\bar{Y}_{E i}$ & $R Q M R$ & \multicolumn{1}{c}{ CV\% } & $t_{\text {oti }}$ & $\mathrm{r}^{2} \mathrm{Ei}$ & $F_{g}\left(P_{r}>F\right)$ & $V_{\text {oti }}$ \\
E12 & 38,0422 & 16,3764 & 0,3561 & 2,17 & 16,2 & 0,9922 & $1,17(0,3116)$ & 19,929 \\
\hline E3 & 14,4743 & 15,2257 & 0,3106 & 2,04 & 14,8 & 0,9920 & $0,60(0,5504)$ & 17,839 \\
\hline E4 & 25,5569 & 15,4511 & 0,4128 & 2,67 & 15,5 & 0,9868 & $0,99(0,3755)$ & 18,392 \\
\hline E1234 & 283,700 & 15,8574 & 0,6876 & 4,34 & 15,7 & 0,9677 & $9,97(0,0001)$ & 19,032 \\
\hline
\end{tabular}


a) Como citado no capítulo 6, seção 6.7, sobre a interpretação, no caso do manejo florestal, do coeficiente $\left(\beta_{1}\right)$ da equação de Mitscherlich, o qual poderia ser expresso como uma característica específica da eficácia da espécie, ou seja, o coeficiente $\beta_{1}$ refletiria uma medida própria do grau de aceleração do crescimento da espécie.

Pode ser observado na tabela 8.1.4.2 que a equação conjunta de (E1, E2), espécies com perfis de crescimento coincidentes, apresentou o maior valor para o coeficiente $\beta_{1}$ $(0,0310)$, resultando na maior produção de madeira $\left(19,929 \mathrm{~m}^{3} / \mathrm{ha}\right)$ no ciclo de corte economicamente aconselhável, correspondendo a um crescimento de $203 \%$ em relação ao volume medido logo após a exploração florestal (tempo zero). Destarte, as espécies E1 e E2 possuem um crescimento mais acelerado que as espécies E3 e E4. A mesma interpretação pode ser feita comparando a espécie E4 (190,6\%) com a espécie E3 (185,5 \%), onde a espécie E4 cresce mais rapidamente que a espécie E3.

Tabela 8.1.4.2 - Análise do comportamento do coeficiente $\left(\hat{\beta}_{1}\right)$.

\begin{tabular}{c|c|c|c|c} 
Espécies & \multicolumn{1}{|c}{$V(t=0)$} & \multicolumn{1}{c}{$V_{\text {oti }}$} & $\hat{\beta}_{1}$ & Crescimento $(\%)$ \\
\hline E12 & 9,775 & 19,929 & 0,0310 & 203,9 \\
\hline E3 & 9,618 & 17,839 & 0,0279 & 185,5 \\
\hline E4 & 9,651 & 18,392 & 0,0294 & 190,6 \\
\hline
\end{tabular}

b) No que concerne aos testes de existência de interação (espéciexocasião), têm-se:

b1) Os perfis de crescimento das espécies E1 e E2 são coincidentes;

b2) Os perfis de crescimento das espécies E3 e E4 não são paralelos.

É recomendável estabelecer equações específicas para as espécies E3 e E4 ( $V_{E 3}$ e $\left.V_{E 4}\right)$. Por outro lado, para as espécies E1 e E2 é factível obter uma equação única $\left(V_{E 12}\right)$.

c) Em relação aos valores dos ciclos de corte economicamente aconselhável, têm-se:

As espécies E1 e E2 apresentaram um ciclo de corte de 16,2 anos, enquanto que as espécies E3 e E4 requerem 14,8 e 15,5 anos, respectivamente. Por outro lado, considerando a equação única, reunindo as quatro espécies, o ciclo recomendável é de 15,7 anos.

Sob o aspecto dos valores da rotação economicamente aconselhável seria recomendável efetuar, na idade de 15 anos, a exploração dirigida das espécies E3 e E4, dentro do contexto de um trato silvicultural especifico. Repetir o procedimento, na idade de 16 anos, para as espécies E1 e E2. Obviamente, considerando que uma floresta natural possui um número grande de espécies, as decisões sobre as datas das intervenções, considerando o sistema de manejo policlíco, teriam que estar baseadas nos ciclos de cortes economicamente aconselháveis dos diversos grupos. Estes originados de acordo com a similaridade dos perfis de crescimento das espécies. 
d) Levando em consideração os valores dos coeficientes de variação (CV\%) e das correlações ao quadrado $\left(r_{M i}^{2}\right)$ entre os valores estimados e observados. As equações $V_{12}$, $V_{3}$ e $V_{4}$ apresentaram valores razoáveis de CV\% e de $\left(r_{M i}^{2}\right)$ que recomendam os seus usos.

e) Em se tratando dos valores do teste de validade de Graybill $\left(F_{G}\right)$, as equações $V_{12}, V_{3}$ e $V_{4}$ apresentaram $F_{G}$ não significativo ao nível de significância de $\alpha=0,05$. No caso do uso de uma equação única $\left(V_{E 1234}\right)$ ocorreu um valor de $F_{G}=9,97$ ( $p$-valor $<0,0001$ ), o que não recomenda o seu uso.

f) Os gráficos dos resíduos, relativos aos comportamentos das equações de crescimentos das espécies, apresentaram aproximadamente distribuições dos resíduos aleatórias, não demonstrando nenhum tipo de tendência aparente, ou seja, mostraram que a faixa de variação dos resíduos ao longo dos valores ajustados é constante, não havendo indícios de que as variâncias dos resíduos das equações das espécies sejam heteroscedásticas.

g) Uma equação única $\left(V_{E 1234}\right)$ não é recomendável, conforme o teste de validade de Graybill, assim como apresentou pelo teste da razão da máxima verossimilhança, para verificação de igualdade de parâmetros e a identidade de modelos de regressão não linear, um valor altamente significativo de $\chi_{\text {Cal }}^{2}=390,81$. 


\section{Capítulo 9}

\section{Técnicas multivariadas em inventário florestal}

O conteúdo principal proposto para esta obra era tratar da análise de perfil de inventário florestal contínuo. Entretanto, considerando a enorme importância da análise multivariada, no contexto da disciplina de Inventário Florestal, considerou-se importante escrever este capítulo apresentando algumas noções sobre os métodos de análise multivariada aplicáveis em inventário florestal. Serão abordadas as técnicas: análise de componentes principais; analise discriminante; análise de correlação canônica; análise de fatores pelos métodos da máxima verossimilhança e de componentes principais. Para os exercícios de componentes principais, de análise discriminante e de análise de fatores foram usados os dados das espécies E1, E2, E3 e E4 denotadas como quatro populações, e as ocasiões OC1, OC2, OC3, OC4 e OC5 caracterizadas como variáveis. No exercício sobre a análise de correlação canônica, considerando os métodos de manejo M1, M2, M3, M4 versus as quatro espécies E1, E2, E3, E4, foram investigadas as inter-relações das variáveis OC1, OC2, OC3, OC4, OC5 entre os métodos de manejo e as espécies. 


\section{INTRODUÇÃO}

É comum em inventários florestais a medição de diversas variáveis em uma mesma unidade amostral. Neste caso, é recomendável proceder a uma exploração das informações, visando diminuir a dimensão da matriz dos dados, porém conservando ao máximo as informações inerentes às variáveis originais.

Para tratar do problema da diminuição da dimensão do vetor de variáveis respostas são recomendados, na análise multivariada, principalmente, os métodos de análises de componentes principais e de fatores. Estes métodos objetivam gerar um número menor de variáveis hipotéticas, combinações lineares das variáveis originais, tal que, mesmo em menor número expliquem adequadamente a interdependência do conjunto original multidimensional dos dados.

A análise de componentes principais consiste em dado um conjunto de variáveis aleatórias, obter por transformação linear um número igual de novas variáveis não correlacionadas (componentes principais), tal que a soma das variâncias seja invariante.

Por outro lado, no caso de os primeiros componentes principais explicarem parte significativa da variância total, pode-se então avaliá-los para cada indivíduo ("score") ou unidade de amostra e processá-los em outras análises em substituição às variáveis originais.

A análise de fatores estuda as relações internas de um conjunto de variáveis aleatórias, substituindo-as por um número menor de variáveis hipotéticas denominadas de fatores, que podem ser correlacionados (fatores oblíquos) ou não correlacionados (fatores ortogonais). Estes fatores devem explicar parte significativa da variância do conjunto das variáveis originais.

Existem algumas similaridades entre as análises de fatores e de componentes principais. Ambas iniciam com um modelo linear e encerram com a fatoração da matriz de covariância ou de correlação. No entanto, na análise dos componentes principais a fatoração da matriz é única, se todos os componentes de variância forem diferentes. Na análise de fatores a fatoração não será única, pois, por transformação ortogonal (rotação), um número infinito de matrizes de pesos fatoriais pode ser obtido para reproduzir a matriz de covariância ou de correlação das variáveis respostas.

No que diz respeito à aplicação em inventários florestais pode-se, considerando os escores dos componentes principais ou os pesos fatoriais relativos, interpretar os efeitos das variáveis respostas envolvidas e suas inter-relações. Por outro lado, a partir da interpretação do significado florestal dos componentes ou dos fatores é possível, usando a amostragem sistemática no levantamento, produzir mapas, os quais podem ser importantes para orientar o planejamento de diversas atividades, principalmente, na elaboração de planos de manejo florestal e na análise do Inventário Florestal Nacional (IFN).

Esses mapas, definidos como originários de um processo denominado de pósestratificação multidimensional, podem ser construídos usando índices que expressem os efeitos e as inter-relações do conjunto de variáveis respostas. De acordo com Queiroz 
(1984), um índice que tem se mostrado eficiente e consistente é o definido, no caso do uso de componentes principais, como o módulo da resultante da soma vetorial dos escores ponderados pela contribuição dos componentes principais à variância total. Por outro lado, no caso da análise de fatores, a definição seria o módulo da resultante da soma vetorial dos escores dos fatores ponderados pela contribuição de cada fator à comunalidade total.

É muito comum na engenharia florestal, principalmente na botânica sistemática e dendrologia que tratam da classificação de vegetais, a existência de $k$ populações e a mensuração de $p$ variáveis respostas, a necessidade de classificar um novo indivíduo, com base em observações dessas $p$ características, com chance mínima de erro, em uma (e somente uma) das $k$ populações. A aplicação da análise discriminante é muito importante na classificação de vegetais.

A aplicação geral e usual da análise de correlação canônica é investigar as relações existentes entre dois conjuntos distintos de variáveis. Por exemplo, em uma área florestal pode-se pesquisar qual o seu perfil florístico de acordo com o padrão edafoclimático ocorrente na floresta. 0 método foi desenvolvido por Hotelling (1935).

A análise de correlação canônica ajuda a responder a esse questionamento, determinando combinações lineares das características florísticas que mais se correlacionam com outras combinações lineares envolvendo as características edafoclimáticas.

É importante no manejo florestal estudar as inter-relações entre grupos de variáveis, por exemplo, um grupo pode ser formado por variáveis florísticas e o outro pelas variáveis que refletem a fertilidade de solo. A análise de correlação canônica poderá explicar essas correlações, inclusive fornecendo mapas que podem mostrar as estratificações das diversas relações solo-vegetação.

As técnicas de análises de agrupamento, de discriminante e de análise de variância multivariada podem ser usadas para delimitar, classificar (discriminar) e comparar tipos florestais e, assim, obter mapas de vegetação baseados nas variáveis mais importantes na explicação das estruturas florísticas e ecológicas da vegetação.

\subsection{ANÁLISE DE COMPONENTES PRINCIPAIS}

Seja o vetor com $p$ variáveis aleatórias $x_{v}=\left[x_{1}, x_{2}, \cdots, x_{p}\right]$, apresentando uma determinada distribuição multivariada com vetor de médias $\mu_{v}$ e matriz de covariância $\sum$ de posto $r \leq p$, tal que, $\lambda_{1}>\lambda_{2}>\ldots>\lambda_{\mathrm{q}}$ são as $q$ maiores raízes características distintas $\operatorname{de} \sum$.

Seja a matriz de dados $X(n \times p)$, donde $p$ é o número de variáveis e $n$ é o número de unidades de amostra. 


$$
{ }_{n} X_{p}=\left[\begin{array}{cccc}
x_{11} & x_{21} & \cdots & x_{p 1} \\
x_{12} & x_{22} & \cdots & x_{p 2} \\
\cdots & \cdots & \cdots & \cdots \\
x_{1 n} & x_{2 n} & \cdots & x_{p p}
\end{array}\right]
$$

O primeiro componente principal das observações, da matriz de dados $X(n \times p)$, é definido como o componente linear $Y_{1}=a_{11} x_{1}+a_{21} x_{2}+\ldots+a_{p 1} x_{p}=a_{v 1}^{\prime} x_{v}$ que apresenta como variância:

$$
V\left(Y_{1}\right)=\sum_{i=1}^{p} \sum_{j=1}^{p} a_{i 1} a_{j 1} s_{i j}=a_{v 1}^{\prime} S a_{v 1} \quad S=\left[s_{i j}\right]: \text { é a matriz de covariância amostral }
$$

A variância $V\left(Y_{1}\right)$ é a maior para todos os vetores normalizados da forma $a_{v 1}^{\prime} a_{v 1}=1$. Seja $Y_{1}$ o primeiro componente principal das observações da matriz de dados $X(n \times p)$, onde $p$ é o número de variáveis e $n$ é o número de unidades de amostra.

Para obter o primeiro componente principal, calcula-se $a_{v 1}^{\prime}=\left[a_{11}, a_{21}, \ldots, a_{p 1}\right]$, tal que, a $\operatorname{Var}\left(Y_{1}\right)=a_{v 1}^{\prime} S a_{v 1}$ atinja o maior valor possível para a condição: $a_{v 1}^{\prime} a_{v 1}=1$.

Pelo método dos multiplicadores de Lagrange, diferenciando com respeito ao vetor a $a_{v 1}$, têm-se:

$$
F_{1}=\left[a_{v 1}^{\prime} S a_{v 1}+\lambda_{1}\left(1-a_{v 1}^{\prime} a_{v 1}\right)\right]
$$

$\frac{\partial F_{1}}{\partial a_{v 1}}=\mathrm{O}_{v}$, resultando: $2 S a_{v 1}-2 \lambda_{1} a_{v 1}=0_{v}$.

Os coeficientes do primeiro componente principal devem satisfazer o sistema de equações homogêneas $\left(S-\lambda_{1} I\right) a_{v 1}=0_{v}$. As soluções não nulas devem satisfazer $\left|S-\lambda_{1} I\right|=0$. Então, $\lambda_{1}$ é a maior raiz característica (autovalor) da matriz de covariância e $a_{v 1}$ o seu vetor característico (autovetor) associado.

O primeiro componente principal de um conjunto de valores amostrais das variáveis respostas $x_{1}, x_{2}, \cdots x_{p}$ é definido como componente linear $Y_{1}=a_{11} x_{1}+a_{21} x_{2}+\ldots+a_{p 1} x_{p}$, cujos coeficientes $a_{i j}$ são os elementos do vetor característico associado à raiz característica $\lambda_{1}$ da matriz de covariância ou de correlação. Se, $a_{v 1}^{\prime} a_{v 1}=1$, então a raiz característica $\lambda_{1}$ é interpretada como a variância amostral de $Y_{1}$.

0 segundo componente principal é o componente linear $Y_{2}=a_{12} x_{1}+a_{22} x_{2}+\ldots+a_{p 2} x_{p}$, cujos coeficientes $a_{v 2}=\left[a_{12}, a_{22}, \cdots, a_{p 2}\right]$ atendem à condição de valor máximo da variância dadas as restrições: $a_{v 2}^{\prime} a_{v 2}=1$ e $a_{v 1}^{\prime} a_{v 2}=0$. Então $\operatorname{Var}\left(Y_{2}\right)=a_{v 2}^{\prime} S a_{v 2}$. 
Aplicando o método de Lagrange e diferenciando em relação $a_{v 2}$, tem-se:

$$
\begin{gathered}
F_{2}=\left[a_{v 2}^{\prime} S a_{v 2}+\lambda_{2}\left(1-a_{v 2}^{\prime} a_{v 2}\right)+\mu_{2} a_{v 1}^{\prime} a_{v 2}\right] \\
\frac{\partial F_{2}}{\partial a_{v 2}}=2\left[S-\lambda_{2} I\right] a_{v 2}+\mu_{2} a_{v 1}=0_{v}
\end{gathered}
$$

Pré-multiplicando (9.1.1) por $a_{v 1}^{\prime}$ resulta $2 a_{v 1}^{\prime} S a_{v 2}+\mu_{2}=0$, mas como $a_{v 1}^{\prime} S a_{v 2}=0$, então $\mu_{2}=0$, concluindo-se que $a_{v 2}$ deve satisfazer: $\left(S-\lambda_{2} I\right) a_{v 2}=0_{v}$.

Assim, o j-ésimo componente principal de uma amostra $p$-dimensional é o conjunto linear $Y_{j}=a_{1 j} x_{1}+a_{2 j} x_{2}+\ldots+a_{p j} x_{p}$, cujos coeficientes são os elementos do vetor característico da matriz de covariância amostral correspondente a $j$-ésima maior raiz característica $\lambda_{j}$. Se $\lambda_{i} \neq \lambda_{j}$, os coeficientes dos $i$-ésimo e $j$-ésimo componentes são necessariamente ortogonais. Se $\lambda_{i}=\lambda_{j}$, os coeficientes podem ser definidos ortogonais, entretanto, infinitos vetores existirão. A variância amostral do $j$-ésimo componente é $\lambda_{j}$ e a variância total será $\lambda_{1}+\lambda_{2}+\cdots+\lambda_{p}=t r S$. A importância do $j$-ésimo componente é dado por $\frac{\lambda_{j}}{t r S}$.

O sinal algébrico e a magnitude de $a_{i j}$ indicam a direção e a importância da contribuição da $i$-ésima variável resposta ao $j$-ésimo componente.

As covariâncias amostrais das respostas com o $j$-ésimo componente são dadas pelo vetor coluna $S a_{v j}$, pois dado que $\left(S-\lambda_{j} I\right) a_{v j}=0_{v}$, então $S a_{v j}=\lambda_{j} a_{v j}$, de onde, se conclui que a covariância da $i$-ésima variável resposta com o componente $Y_{j}$ é, meramente, $\lambda_{j} a_{i j}$.

A correlação entre a i-ésima variável resposta e o j-ésimo componente será:

$$
\operatorname{corr}\left(x_{i}, Y_{j}\right)=\frac{a_{i j} \sqrt{\lambda_{j}}}{\sigma_{i i}} \quad i, j=1,2, \cdots, p
$$

Por outro lado, caso seja utilizada a matriz de correlação para obter os componentes principais, a correlação entre a $i$-ésima variável resposta e o j-ésimo componente será:

$$
\operatorname{corr}\left(x_{i}, Y_{j}\right)=a_{i j} \sqrt{\lambda_{j}} \quad i, j=1,2, \cdots, p
$$


No caso dos primeiros componentes principais explicarem parte significativa da variância total, pode-se então avaliá-los para cada indivíduo ("score") ou unidade de amostra, e processá-los em outras análises em substituição às variáveis originais.

De acordo com Morrison (1976), para calcular a matriz dos scores $Y(n \times r)$ dos componentes principais para o i-ésimo individuo, quando os componentes são extraídos da matriz de covariância, têm-se:

$$
y_{i 1}=a_{v 1}^{\prime}\left(x_{v i}-\bar{x}_{v}\right), y_{i 2}=a_{v 2}^{\prime}\left(x_{v i}-\bar{x}_{v}\right), \cdots, y_{i r}=a_{v r}^{\prime}\left(x_{v i}-\bar{x}_{v}\right)
$$

Onde $r$ é o número de componentes principais utilizados, $x_{v i}$ é o vetor das observações originais e $\overline{\boldsymbol{x}}_{v}$ é o vetor das médias.

Generalizando para Y $(n \times r)$, tem-se:

$$
{ }_{n} \mathrm{Y}_{r}=\left[I_{n}-\frac{1}{n}\left({ }_{n} E_{n}\right)\right]\left({ }_{n} X_{p} A_{r}\right)
$$

$X(n \times p)$ : matriz dos dados originais;

$E(n \times n)$ : matriz de uns em todas as posições;

A $(p \times r)$ : matriz formada cujas colunas são formadas pelos $r$ vetores característicos.

A equação básica do modelo linear dos componentes principais não possui componente residual. Esta propriedade o diferencia marcadamente do modelo da análise de fatores. $\mathrm{Na}$ análise de componentes principais, por ser um método matemático, não há obrigatoriedade de multinormalidade dos dados.

Uma grande desvantagem da análise de componentes principais é que os componentes oriundos da matriz de correlação não são os mesmos computados da matriz de covariância. Por outro lado, as estimativas de máxima verossimilhança dos parâmetros da análise de fatores possuem uma importantíssima propriedade, ou seja, "mudança nas escalas das variáveis originais somente implica modificação na escala dos pesos fatoriais". Particularmente, os valores dos pesos fatoriais da matriz de correlação diferem dos pesos computados da matriz de covariância pelo fator $1 / s_{i}(i=1, \cdots, p)$, tal que $s_{i}$ é o desvio padrão da variável resposta $x_{i}$ e $p$ é o número de variáveis originais. Esta propriedade não é apresentada pelos componentes principais.

\subsubsection{SIGNIFICADO GEOMÉTRICO DOS COMPONENTES PRINCIPAIS}

De acordo com Morrison (1976), os componentes principais de uma amostra de $n$ observações $p$-dimensionais são as novas variáveis especificadas pelos eixos de uma rotação rígida do sistema de coordenadas das variáveis originais, tendo as orientações correspondentes às direções da variância máxima da configuração (nuvem) de pontos da amostra. Os cosenos diretivos dos novos eixos são os vetores característicos normalizados correspondentes às sucessivas maiores raízes características da matriz de covariância. Se duas ou mais raízes são iguais, as direções dos eixos associados não serão únicas e os componentes podem ser escolhidos de um número infinito de posições 
ortogonais. Se usada a matriz de correlação o sistema de coordenadas das respostas é expresso em unidades padrão de média zero e variância unitária.

A Figura 9.1.1.1 mostra a interpretação geométrica dos componentes principais de uma população trivariada. Supondo $Y_{1}$ centrado no ponto médio e $a_{11}^{2}+a_{21}^{2}+a_{31}^{2}=1$, tem-se $a_{21}=\cos \alpha_{2}$ e $a_{31}=\cos \alpha_{3}$. Onde as novas coordenadas do eixo $Y_{1}$, por exemplo, seriam dadas por:

$$
y_{i 1}=a_{11}\left(X_{i 1}-\bar{X}_{1}\right)+a_{21}\left(X_{i 2}-\bar{X}_{2}\right)+a_{31}\left(X_{i 3}-\bar{X}_{3}\right)
$$

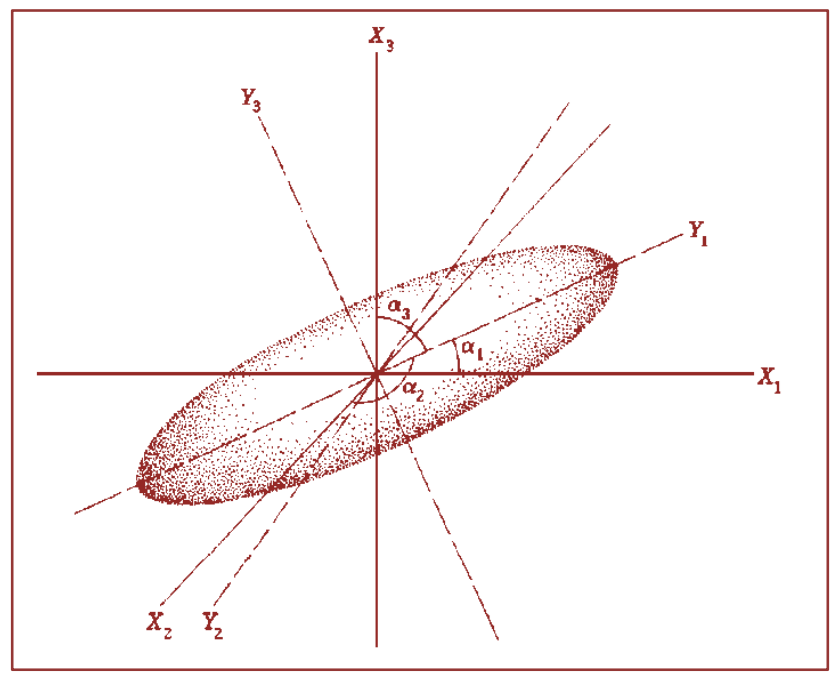

Figura 9.1.1.1 - Interpretação geométrica dos componentes principais de uma população trivariada.

Fonte: Morrison (1976).

\subsubsection{METODOLOGIA DOS ÍNDICES NORMALIZADOS}

São definidos, equação 9.1.2.1, de acordo com Queiroz (1984), como o módulo da resultante da soma vetorial dos escores dos componentes principais, ponderados pela contribuição em porcentagem $\left(p_{j}\right)$ do respectivo componente à variância total $(\operatorname{trS})$, os quais são divididos pelo de maior magnitude, implicando numa normalização entre 0 e 1. A justificativa da aplicação desses índices resulta do fato dos componentes principais serem ortogonais. A Figura 9.1.2.1 mostra a representação gráfica do índice normalizado.

$$
I_{i}=\sqrt{\sum_{j=1}^{r} p_{j} Y_{i j}^{2}} \quad i=1,2, \cdots, N
$$




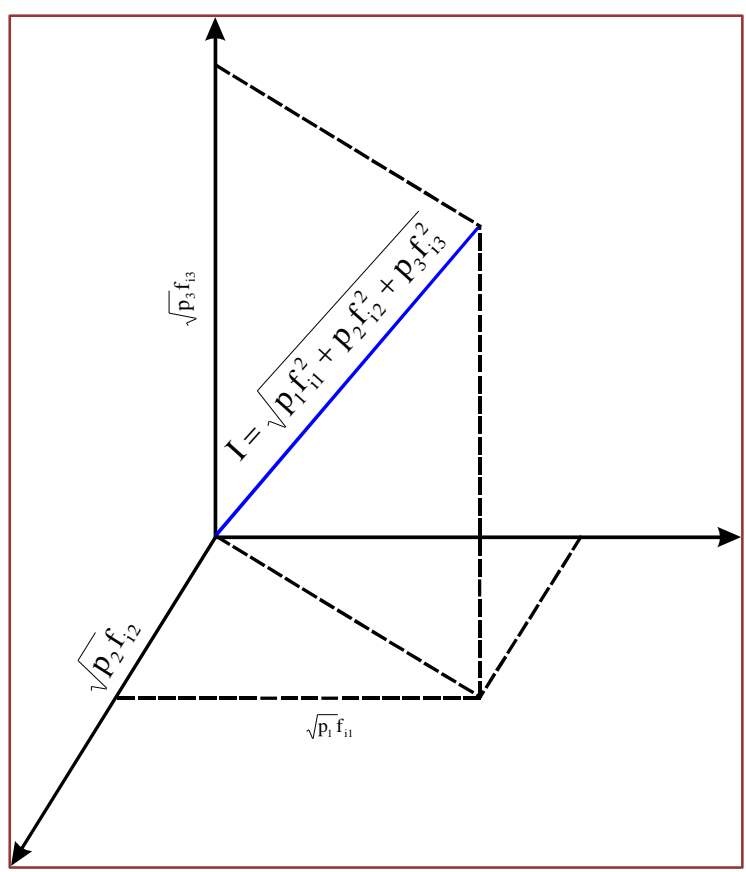

Figura 9.1.2.1 - Representação gráfica do índice normalizado.

Na fitossociologia florestal é recomendável usar, como o índice de valor de importância, o primeiro componente principal, em substituição a expressão 9.1.2.2, a qual é inadequada de acordo com Queiroz et al. (2017), pois não considera as inter-relações entre as variáveis: $F R_{i}, D R_{i}$ e $D O R_{i}$.

$$
I V I_{i}=F R_{i}+D R_{i}+D O R_{i}
$$

$I V I_{i}=$ Índice de Valor de Importância da i-ésima espécie;

$F R_{i}=$ Frequência Relativa da i-ésima espécie em \%;

$D R_{i}=$ Densidade Relativa da i-ésima espécie em \%;

$D O R_{i}=$ Dominância Relativa da i-ésima espécie em \%.

Por outro lado, se pode usar os primeiros componentes principais e construir como definido na expressão 9.1.2.1, um índice de valor de importância mais preciso.

\subsubsection{ANÁLISE DE COMPONENTES PRINCIPAIS DAS ESPÉCIES E1, E2, E3, E4, CONSIDERANDO COMO VARIÁVEIS AS OCASIÕES OC1, OC2, OC3, OC4 E OC5}

A tabela C14, do apêndice C, apresenta o programa SAS construído para efetuar as análises de componentes principais e de fatores para as espécies E1, E2, E3, E4. 
a) Matriz de covariância comum das espécies para as variáveis $0 \mathrm{C} 1,0 \mathrm{C} 2,0 \mathrm{C}$, 0C4, OC5 (dados da tabela B2 do apêndice B)

$$
S=\left[\begin{array}{lllll}
0,02678314 & 0,03220126 & 0,03545548 & 0,04085641 & 0,03934563 \\
0,03220126 & 0,46438298 & 0,31791906 & 0,58583988 & 0,59253834 \\
0,03545548 & 0,31791906 & 0,26248354 & 0,43538079 & 0,44656015 \\
0,04085641 & 0,58583988 & 0,43538079 & 0,78654179 & 0,80448517 \\
0,03934563 & 0,59253834 & 0,44656015 & 0,80448517 & 0,83544778
\end{array}\right]
$$

b) Obtenção dos autovalores (eigenvalues) da matriz de covariância (S)

Tabela 9.1.3.1 - Autovalores (Eigenvalues) e suas contribuições à variância total.

\begin{tabular}{l|l|l|l|l}
\multicolumn{5}{c}{ Autovalores da matriz de covariância } \\
\multicolumn{1}{c}{ Autovalor } & Diferença & Proporção & Acumulado \\
\hline 1 & 2,29644092 & 2,25953403 & 0,9667 & 0,9667 \\
\hline 2 & 0,03690689 & 0,00643365 & 0,0155 & 0,9822 \\
\hline 3 & 0,03047324 & 0,02217336 & 0,0128 & 0,9950 \\
\hline 4 & 0,00829988 & 0,00478156 & 0,0035 & 09985 \\
\hline 5 & 0,00351832 & & 0,0015 & 1,0000 \\
\hline Total & 2,37563925 & & & \\
\hline
\end{tabular}

A tabela 9.1.3.1 mostra que os dois maiores autovalores, $\lambda_{1}=2,29644092 \mathrm{e}$ $\lambda_{2}=0,03690689$, explicam 98,22 \% da variância total, significando que os dois primeiros componentes principais explicam 98,22 \% da estrutura de dispersão.

c) Obtenção dos autovetores associados aos cinco autovetores (eigenvectors)

Tabela 9.1.3.2 - Autovetores: coeficientes dos componentes principais.

\begin{tabular}{l|l|l|l|l|l}
\multicolumn{5}{c}{ Autovetores associados aos autovalores da matriz de covariância } \\
\multicolumn{1}{c|}{ Prin1 } & Prin2 & Prin3 & Prin4 & Prin5 \\
\hline OC1 & 0,032216 & 0,573660 & 0,551355 & 0,596831 & $-0,098371$ \\
\hline OC2 & 0,437907 & $-0,515112$ & 0,674295 & $-0,105689$ & 0,277583 \\
\hline OC3 & 0,325754 & 0,633589 & 0,076852 & $-0,660716$ & 0,223602 \\
\hline OC4 & 0,584268 & $-0,045499$ & $-0,089009$ & $-0,038174$ & $-0,804475$ \\
\hline OC5 & 0,599762 & 0,045484 & $-0,476974$ & 0,441157 & 0,464858 \\
\hline
\end{tabular}

A tabela 9.1.3.2 apresenta os autovetores relativos aos cinco autovalores, tal que, os seus coeficientes correspondem aos cinco componentes principais. Sejam os dois primeiros componentes principais Prin1 e Prin2: 


\section{$\operatorname{Pr} i n 1=0,032216 \mathrm{OCl}+0,437907 \mathrm{OC}+0,325754 \mathrm{OC} 3+0,584268 \mathrm{OC} 4+0,599762 \mathrm{OC}$ \\ Prin $2=0,5736600 \mathrm{OC}-0,515112 \mathrm{OC} 2+0,6335890 \mathrm{OC}-0,045499 \mathrm{OC} 4+0,045484 \mathrm{OC}$}

Prin1 mostra um panorama de contribuição com relativa igualdade dos coeficientes com respeito às variáveis $0 \mathrm{C} 2,0 \mathrm{C} 3,0 \mathrm{C} 4$ e $0 \mathrm{C} 5$, enquanto que a variável $0 \mathrm{C} 1$ pouco contribui na construção do primeiro componente principal.

Por outro lado, o segundo componente principal Prin2 mostra uma boa contribuição em termos de valores absolutos dos coeficientes para as variáveis 0C1, OC2 e OC3, entretanto, a variável OC3 apresenta o sinal diferente. As variáveis OC4 e OC5 contribuem relativamente pouco na construção do componente principal Prin2.

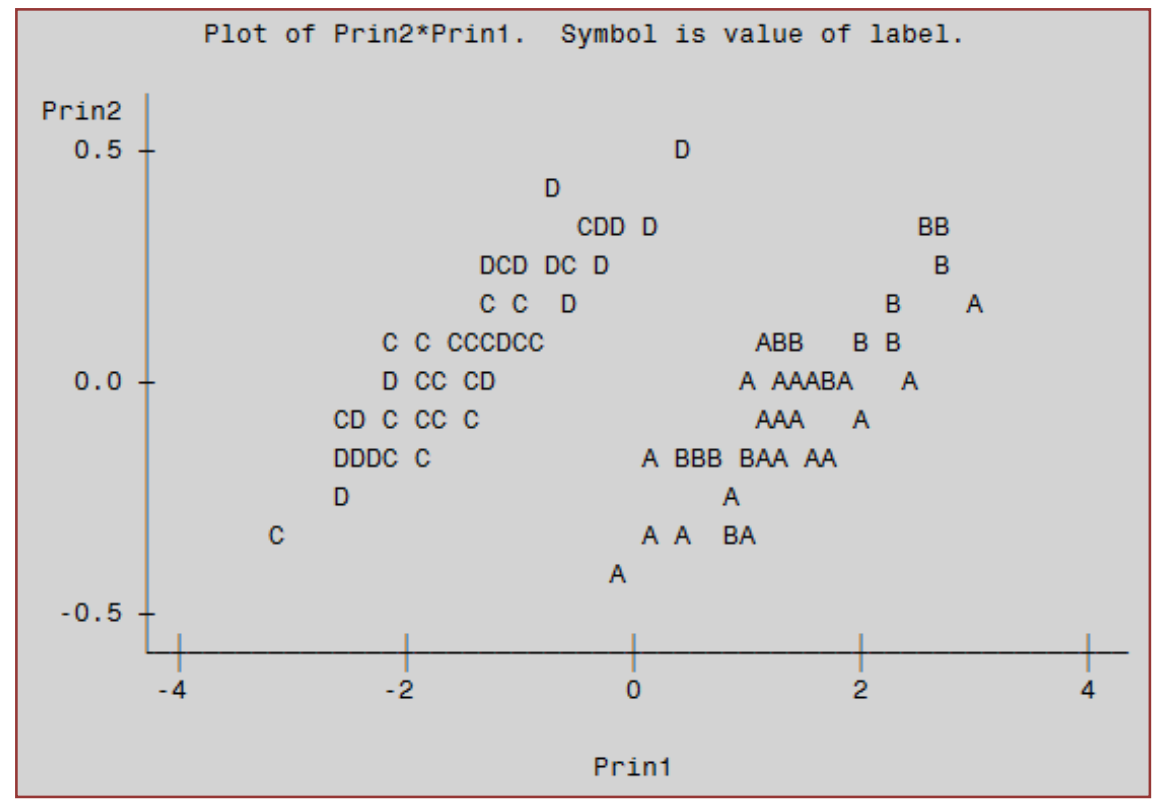

Figura 9.1.3.1 - Distribuição das quatro espécies E1=A, E2=B, E3=C, E4=D considerando os dois primeiros componentes principais. Nota do SAS: 43 obs. ocultas.

As letras A, B, C e D, denominadas de label no programa desenvolvido no SAS, foram criadas para possibilitar a criação do gráfico (Prin1×Prin2).

A figura 9.1.3.1 confirma, de forma precisa, o resultado da análise de agrupamento produzido para as espécies E1, E2, E3, e E4, mostrado na figura 8.1.1.3, capítulo 8, seção 8.1, item g, dendrograma do agrupamento de Ward, o qual demonstra que as espécies E1 e E2 formam um grupo distinto do composto pelas espécies E3 e E4.

Não estão apresentados os valores dos índices normalizados, conforme a expressão 9.1.2.1, já que a estrutura do banco de dados está baseada nas variáveis aleatórias ocasiões, as quais não apresentam um interesse prático para montagem de um mapa de pós-estratificação multidimensional. Entretanto, caso fosse aplicada a equação 9.1.2.1, ela teria a seguinte formulação: 


$$
\begin{gathered}
I_{i}=\sqrt{\sum_{j=1}^{r} p_{j} Y_{j i}^{2}}=\sqrt{0,966662 \times \mathrm{Y}_{1 \mathrm{i}}^{2}+0,015536 \times \mathrm{Y}_{2 \mathrm{i}}^{2}} \quad(i=1, \ldots, N=120) \\
p_{1}=\frac{\lambda_{1}}{\operatorname{Tr}(S)}=\frac{2,29644092}{2,37563925}=0,966662 \quad p_{2}=\frac{\lambda_{2}}{\operatorname{Tr}(S)}=\frac{0,03690689}{2,37563925}=0,015536
\end{gathered}
$$

$Y_{1 i}$ : valor de prin1 obtido na i-ésima parcela $(i=1, \ldots, N)$;

$Y_{2 i}$ : valor de prin2 obtido na i-ésima parcela $(i=1, \ldots, N)$;

$\operatorname{Tr}(S)=\lambda_{1}+\lambda_{2}+\lambda_{3}+\lambda_{3}+\lambda_{3}=2,37563925$

\subsection{ANÁLISE DISCRIMINANTE}

\subsubsection{FUNÇÃO LINEAR DISCRIMINANTE DE FISHER}

Sejam $k$ populações $\pi_{1}, \pi_{2}, \cdots, \pi_{k}$ e $y_{v}^{\prime}=\left[y_{1}, y_{2}, \cdots, y_{p}\right]$ um vetor aleatório com função densidade de probabilidade $f_{j}\left(y_{v j}\right)$ pertencente, unicamente, a uma população $\pi_{j}$ onde: $j=1,2, \cdots, k$ e $i=1,2, \cdots, p$.

0 problema básico da análise discriminante é o de classificar um novo indivíduo $y_{v}$, com base em observações de $p$ características $y_{1}, y_{2}, \cdots, y_{p}$, com chance mínima de erro, em uma (e somente uma) das populações $\pi_{1}, \pi_{2}, \cdots, \pi_{k}$.

Seja a regra de decisão: classifique $y_{v} \in \pi_{j}$ se $y_{v} \in w_{j}$, onde $w_{j}$ é a região que contém $y_{v}$. Em geral, tem-se que $w=\bigcup_{j=1}^{k} w_{j}$ (união disjunta), ou seja, $w_{j} \cap w_{j^{\prime}}=\phi$ para $j \neq j^{\prime}$. As regiões $w_{j}$ são construídas de modo a maximizar a probabilidade de classificar corretamente.

FISHER (1936) sem usar o critério da razão das funções de densidade de probabilidade e sem suposição de multinormalidade obteve a função linear $a_{v}^{\prime} y_{v}$, derivada das populações $\pi_{1}$ e $\pi_{2}$, maximizando o quociente:

$$
\underset{a_{v} \neq 0}{\operatorname{Máx}} \frac{\left[E\left(a_{v}^{\prime} y_{v}\right) / \pi_{1}-E\left(a_{v}^{\prime} y_{v}\right) / \pi_{2}\right]^{2}}{\operatorname{Var}\left(a_{v}^{\prime} y_{v}\right)}
$$

O numerador corresponde ao quadrado da distância entre as médias amostrais de $a_{v}^{\prime} y_{v}$, em relação à variância amostral de $a_{v}^{\prime} y_{v}$, considerando $\pi_{1}$ e $\pi_{2}$. Definindo que $\Sigma_{1}=\Sigma_{2}=\sum$, têm-se:

$$
\phi=\frac{\left(a_{v}^{\prime} \mu_{v 1}-a_{v}^{\prime} \mu_{v 2}\right)^{2}}{a_{v}^{\prime} \sum a_{v}}=\frac{\left[a_{v}^{\prime}\left(\mu_{v 1}-\mu_{v 2}\right)\right]^{2}}{a_{v}^{\prime} \sum a_{v}}
$$

Obter $a_{v}$ que maximize a expressão 9.2.1.1: 


$$
\frac{\partial \phi}{\partial a_{v}}=\frac{2\left(\mu_{v 1}-\mu_{v 2}\right) a_{v}^{\prime} \sum a_{v}-2 \sum a_{v}\left(a_{v}^{\prime} \mu_{v 1}-a_{v 2}^{\prime}\right)}{\left(a_{v}^{\prime} \sum a_{v}\right)^{2}}=0
$$

Resultando: $\mu_{v 1}-\mu_{v 2}=\sum a_{v}\left(\frac{a_{v}^{\prime} \mu_{v 1}-a_{v 2}^{\prime} \mu_{v 2}}{a_{v}^{\prime} \sum a_{v}}\right)$

Lachenbruch (1975) cita que como $a_{v}$ será usado somente para separar as populações, então se pode multiplicar $a_{v}$ por qualquer constante diferente de zero. Portanto, $a_{v}$ será proporcional a $\Sigma^{-1}\left(\mu_{v 1}-\mu_{v 2}\right)$, resultando a função $f=\left(\mu_{v 1}-\mu_{v 2}\right)^{\prime} \Sigma^{-1} y_{v}$ que é denominada de função linear discriminante de Fisher, donde:

$$
\begin{aligned}
& \mu_{v 1}=E\left(y_{v} / \pi_{1}\right) ; \\
& \mu_{v 2}=E\left(y_{v} / \pi_{2}\right) ; \\
& \operatorname{Var}\left(y_{v j}\right)=\sum=E\left(y_{v j}-\mu_{v j}\right)\left(y_{v j}-\mu_{v j}\right)^{\prime} \quad(j=1,2) .
\end{aligned}
$$

No caso de os parâmetros serem desconhecidos, então as estimativas $\bar{y}_{v 1}, \bar{y}_{v 2}$ e $S$ devem ser utilizadas. 0 procedimento para classificar um indivíduo em $\pi_{1}$, é: se $f=\left(\mu_{v 1}-\mu_{v 2}\right)^{\prime} S^{-1} y_{v} \quad$ é mais próximo de $f_{1}=\left(\mu_{v 1}-\mu_{v 2}\right)^{\prime} S^{-1} y_{v 1}$ do que de $f_{2}=\left(\mu_{v 1}-\mu_{v 2}\right)^{\prime} S^{-1} y_{v 2}$, caso contrário, classificar em $\pi_{2}$. Seja o ponto médio do intervalo entre $f_{1}$ e $f_{2}$ :

$$
\frac{f_{1}+f_{2}}{2}=\frac{1}{2}\left(\bar{y}_{v 1}-\bar{y}_{v 2}\right)^{\prime} S^{-1}\left(\bar{y}_{v 1}+\bar{y}_{v 2}\right)
$$

Destarte, $f$ está mais próximo de $f_{1}$ se: $\left|f-f_{1}\right|<\left|f-f_{2}\right|$, o que ocorrerá quando $f>\frac{1}{2}\left(f_{1}+f_{2}\right)$, desde que $f_{1}>f_{2}$.

Deve ser observado que: $\left(f_{1}-f_{2}\right)=\left(\bar{y}_{1}-\bar{y}_{2}\right)^{\prime} S^{-1} \bar{y}_{1}-\left(\bar{y}_{1}-\bar{y}_{2}\right)^{\prime} S^{-1} \bar{y}_{2}$.

$\left(f_{1}-f_{2}\right)=\left(\bar{y}_{1}-\bar{y}_{2}\right)^{\prime} S^{-1}\left(\bar{y}_{1}-\bar{y}_{2}\right)$ é denominada distância $D^{2}$ de Mahalanobis (1936).

Por outro lado:

$$
\operatorname{Var}(f)=a_{v}^{\prime} S a_{v}=\left(\bar{y}_{1}-\bar{y}_{2}\right)^{\prime} S^{-1}\left(\bar{y}_{1}-\bar{y}_{2}\right)=D^{2}
$$

Pode-se usar a distribuição de $D^{2}$ para testar, entre os dois grupos, a existência de diferenças significativas. Neste caso, tem-se a estatística:

$$
F=\frac{n_{1} n_{2}\left(n_{1}+n_{2}-p-1\right)}{\left(n_{1}+n_{2}\right)\left(n_{1}+n_{2}-2\right) p} D^{2}
$$


Tal que $n_{1}$ e $n_{2}$ correspondem aos tamanhos das amostras, respectivamente, de $\pi_{1}$ e $\pi_{2}$ e $p$ é o número de variáveis. A estatística $F$ tem distribuição com $p$ e $n_{1}+n_{2}-p-1$ graus de liberdade.

\subsubsection{CASO DE DUAS POPULAÇõES MINIMIZANDO A PROBABILIDADE TOTAL DE CLASSIFICAÇÃO}

Welch (1939) solucionou o problema da minimização da probabilidade total de classificação. Seja $f_{1}\left(y_{v}\right)$ a função densidade de probabilidade de $y_{v}$ pertencer a $\pi_{1} \mathrm{e}$ $f_{2}\left(y_{v}\right)$ de $y_{v}$ pertencer a $\pi_{2}$. Considerando $p_{1}$ a proporção de ocorrência na população $\pi_{1}$ e $p_{2}=1-p_{1}$ a ocorrência na população $\pi_{2}$. Supondo classificar $y_{v}$ em $\pi_{1}$, se $y_{v}$ está em alguma região de $\mathrm{R}_{1}$, e em $\pi_{2}$, se $y_{v}$ está na região $R_{2}$. Assume-se que $R_{1}$ e $R_{2}$ são mutuamente exclusivas e que a união delas abrange todo espaço $R$.

Lachenbruch (1975) define a probabilidade total de classificação errada, como:

$$
\begin{aligned}
& T_{\left[R, f\left(y_{v}\right)\right]}=p_{1} \int_{R 2} f_{1}\left(y_{v}\right) d y_{v}+p_{2} \int_{R 1} f_{2}\left(y_{v}\right) d y_{v} \\
& T_{\left[R, f\left(y_{v}\right)\right]}=p_{1}\left[1-\int_{R 1} f_{1}\left(y_{v}\right) d y_{v}\right]+p_{2} \int_{R 1} f_{2}\left(y_{v}\right) d y_{v} \\
& T_{\left[R, f\left(y_{v}\right)\right]}=p_{1}+\int_{R 1}\left[p_{2} f_{2}\left(y_{v}\right)-p_{1} f_{1}\left(y_{v}\right)\right] d y_{v}
\end{aligned}
$$

A quantidade 9.2.2.1 é minimizada se $R_{1}$ é definida, onde $p_{2} f_{2}\left(y_{v}\right)-p_{1} f_{1}\left(y_{v}\right)<0$ para todos os pontos em $R_{1}$. A regra de classificação é: atribuir $y_{v}$ para $\pi_{1}$ se $\frac{f_{1}\left(y_{v}\right)}{f_{2}\left(y_{v}\right)}>\frac{p_{2}}{p_{1}}$, caso contrário atribuir em $\pi_{2}$. Esta regra minimiza a probabilidade total de classificação errada. Seja a probabilidade de classificação errada dentro de cada grupo:

$$
P_{1}=\int_{R_{2}} f_{1}\left(y_{v}\right) d_{v} \quad P_{2}=\int_{R_{1}} f_{2}\left(y_{v}\right) d_{v}
$$

\subsubsection{CASO DE DUAS POPULAÇÕES MULTINORMAIS COM DISPERSÕES CONHECIDAS E IGUAIS}

Seja a função densidade de probabilidade (f.d.p.) da distribuição multinormal:

$$
f_{j}\left(y_{v}\right)=\frac{1}{(2 \pi)^{\frac{p}{2}} \sqrt{\left|\sum_{j}\right|}} \exp \left[-\frac{1}{2}\left(y_{v}-\mu_{v j}\right)^{\prime} \Sigma^{-1}\left(y_{v}-\mu_{v j}\right)\right]
$$


Considerando como critério o quociente entre as duas densidades $f_{1}\left(y_{v}\right)$ e $f_{2}\left(y_{v}\right)$ :

$$
\begin{gathered}
\frac{f_{1}\left(y_{v}\right)}{f_{2}\left(y_{v}\right)}=\frac{\frac{1}{(2 \pi)^{\frac{p}{2}} \sqrt{\left|\sum_{1}\right|}} \exp \left[-\frac{1}{2}\left(y_{v}-\mu_{v 1}\right)^{\prime} \sum_{1}^{-1}\left(y_{v}-\mu_{v 1}\right)\right]}{\frac{1}{(2 \pi)^{\frac{p}{2}} \sqrt{\left|\sum_{2}\right|}} \exp \left[-\frac{1}{2}\left(y_{v}-\mu_{v 2}\right)^{\prime} \sum_{2}^{-1}\left(y_{v}-\mu_{v 2}\right)\right]} \\
\frac{f_{1}\left(y_{v}\right)}{f_{2}\left(y_{v}\right)}=\frac{\left|\sum_{2}\right|^{\frac{1}{2}}}{\left|\sum_{1}\right|^{\frac{1}{2}}} \exp \left[\frac{1}{2}\left(y_{v}-\mu_{v 2}\right)^{\prime} \sum_{2}^{-1}\left(y_{v}-\mu_{v 2}\right)-\frac{1}{2}\left(y_{v}-\mu_{v 1}\right)^{\prime} \sum_{1}^{-1}\left(y_{v}-\mu_{v 1}\right)\right]
\end{gathered}
$$

Sendo $\frac{f_{1}\left(y_{v}\right)}{f_{2}\left(y_{v}\right)}=\frac{p_{2}}{p_{1}}$ e aplicando a função crescente logaritmo neperiano a ambos os lados:

$$
\operatorname{Ln} \frac{f_{1}\left(y_{v}\right)}{f_{2}\left(y_{v}\right)}=\frac{1}{2}\left[y_{v}^{\prime}\left(\sum_{2}^{-1}-\sum_{1}^{-1}\right) y_{v}-2\left(\mu_{v 2}^{\prime} \Sigma_{2}^{-1}-\mu_{v 1}^{\prime} \Sigma_{1}^{-1}\right) y_{v}+\mu_{v 2}^{\prime} \Sigma_{1}^{-1} \mu_{v 2}-\mu_{v 1}^{\prime} \sum_{2}^{-1} \mu_{v 1}\right] \geq \operatorname{Ln}\left(\frac{p_{2}}{p_{1}} \frac{\left|\sum_{1}\right|^{\frac{1}{2}}}{\left|\sum_{2}\right|^{\frac{1}{2}}}\right)
$$

Considerando $\Sigma_{1}=\sum_{2}=\sum$ e a regra: classifique $y_{v}$ em $\pi_{1}$, se $y_{v} \in w_{1}$, donde $w_{1}$ será o conjunto:

$$
w_{1}=\left\{y_{v} \in R^{p}: \frac{1}{2}\left[-2\left(\mu_{v 2}-\mu_{v 1}\right)^{\prime} \Sigma^{-1} y_{v}+\mu_{v 2}^{\prime} \Sigma^{-1} \mu_{v 2}-\mu_{v 1}^{\prime} \Sigma^{-1} \mu_{v 1}\right] \geq \operatorname{Ln}\left(\frac{p_{2}}{p_{1}}\right)\right\}
$$

Somando $\mu_{v 1}^{\prime} \Sigma^{-1} \mu_{v 2}-\mu_{v 1}^{\prime} \Sigma^{-1} \mu_{v 2}$, têm-se:

$$
\begin{gathered}
w_{1}=\left\{y_{v} \in R^{p}: \frac{1}{2}\left[-2\left(\mu_{v 2}-\mu_{v 1}\right)^{\prime} \sum^{-1} y_{v}-\left(\mu_{v 1}-\mu_{v 2}\right)^{\prime} \Sigma^{-1}\left(\mu_{v 1}+\mu_{v 2}\right)\right] \geq \operatorname{Ln}\left(\frac{p_{2}}{p_{1}}\right)\right\} \\
w_{1}=\left\{y_{v} \in R^{p}:\left(\mu_{v 1}-\mu_{v 2}\right)^{\prime} \sum^{-1}\left[y_{v}-\frac{1}{2}\left(\mu_{v 1}+\mu_{v 2}\right)\right] \geq \operatorname{Ln} \frac{p_{2}}{p_{1}}\right\} \\
w_{1}=\left[y_{v} \in R^{p}:\left(\mu_{v 1}-\mu_{v 2}\right)^{\prime} \Sigma^{-1} y_{v} \geq \frac{1}{2}\left(\mu_{v 1}-\mu_{v 2}\right)^{\prime} \Sigma^{-1}\left(\mu_{1}+\mu_{2}\right)+\operatorname{Ln} \frac{p_{2}}{p_{1}}\right]
\end{gathered}
$$

0 termo $\left(\mu_{v 1}-\mu_{v 2}\right)^{\prime} \sum^{-1} y_{v}$ é chamado de função linear discriminante de Fisher e $\left(\mu_{v 1}-\mu_{v 2}\right)^{\prime} \Sigma^{-1}$ são os seus coeficientes.

Considerando $p_{1}=p_{2}$, isto é, as probabilidades a priori são iguais de $y_{v}$ pertencer à população $\pi_{1}$ ou $\pi_{2}$. Então:

$$
\begin{aligned}
& w_{1}=\left[y_{v} \in R^{p}:\left(\mu_{v 1}-\mu_{v 2}\right)^{\prime} \Sigma^{-1} y_{v} \geq \frac{1}{2}\left(\mu_{v 1}-\mu_{v 2}\right)^{\prime} \Sigma^{-1}\left(\mu_{v 1}+\mu_{v 2}\right)\right] \\
& w_{2}=\left[y_{v} \in R^{p}:\left(\mu_{v 1}-\mu_{v 2}\right)^{\prime} \Sigma^{-1} y_{v}<\frac{1}{2}\left(\mu_{v 1}-\mu_{v 2}\right)^{\prime} \Sigma^{-1}\left(\mu_{v 1}+\mu_{v 2}\right)\right]
\end{aligned}
$$




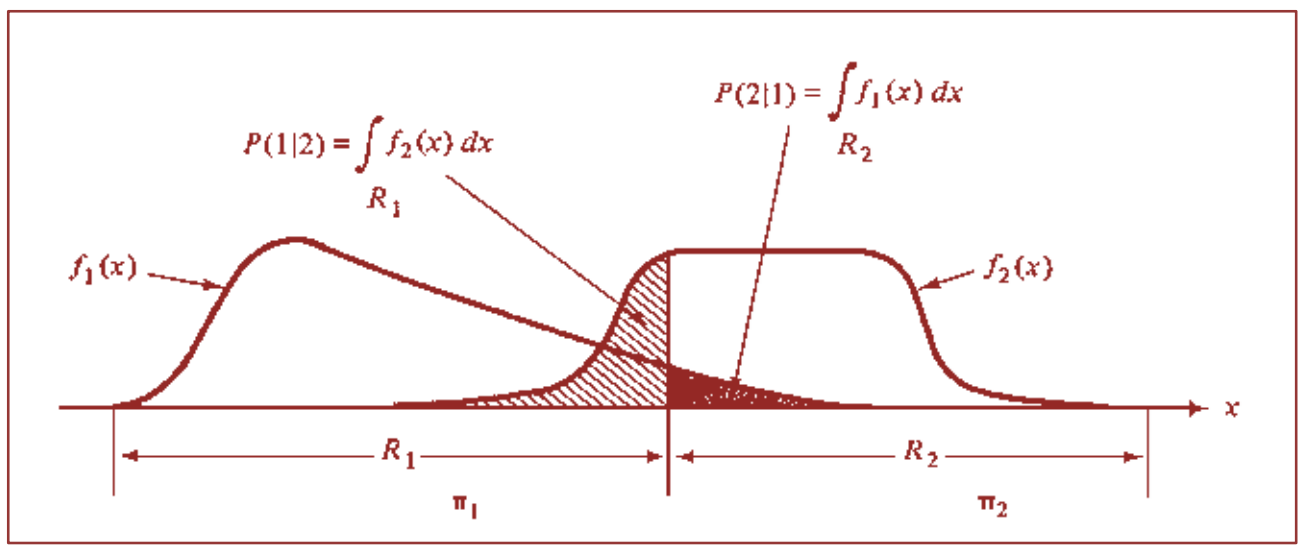

Figura 9.2.3.1 - Probabilidades de classificação incorreta.

Fonte: Johnson \& Wichern (1992)

De acordo com a figura 9.2.3.1, têm-se:

$P(1 / 2)$ : probabilidade de $y_{v}$ pertencer à população $\pi_{2}$ e ser classificado na $\pi_{1}$. $P(2 / 1)$ : probabilidade de $y_{v}$ pertencer à população $\pi_{1}$ e ser classificado na $\pi_{2}$.

\subsubsection{CASO DE TRÊS POPULAÇÕES MULTINORMAIS COM DISPERSÕES CONHECIDAS E IGUAIS, ASSIM COMO $\left(P_{1}=P_{2}\right)$}

$$
\begin{gathered}
w_{1}=\left[y_{v} \in R^{p}: p_{1} f_{1}\left(y_{v}\right) \geq p_{2} f_{2}\left(y_{v}\right) \text { e } p_{1} f_{1}\left(y_{v}\right) \geq p_{3} f_{3}\left(y_{v}\right)\right] \rightarrow \\
\text { Classifique } y_{v}, \text { em } \pi_{1} \text {, se } \frac{f_{1}\left(y_{v}\right)}{f_{2}\left(y_{v}\right)} \geq \frac{p_{2}}{p_{1}} \text { e } \frac{f_{1}\left(y_{v}\right)}{f_{3}\left(y_{v}\right)} \geq \frac{p_{3}}{p_{1}} . \\
w_{2}=\left[y_{v} \in R^{p}: p_{2} f_{2}\left(y_{v}\right) \geq p_{1} f_{1}\left(y_{v}\right) \text { e } p_{2} f_{2}\left(y_{v}\right) \geq p_{3} f_{3}\left(y_{v}\right)\right] \rightarrow \\
\text { Classifique } y_{v}, \text { em } \pi_{2}, \text { se } \frac{f_{1}\left(y_{v}\right)}{f_{2}\left(y_{v}\right)} \leq \frac{p_{2}}{p_{1}} \text { e } \frac{f_{2}\left(y_{v}\right)}{f_{3}\left(y_{v}\right)} \geq \frac{p_{3}}{p_{2}} . \\
w_{3}=\left[y_{v} \in R^{p}: p_{3} f_{3}\left(y_{v}\right) \geq p_{1} f_{1}\left(y_{v}\right) \text { e } p_{3} f_{3}\left(y_{v}\right) \geq p_{2} f_{2}\left(y_{v}\right)\right] \rightarrow \\
\text { Classifique } y_{v}, \text { em } \pi_{3}, \text { se } \frac{f_{1}\left(y_{v}\right)}{f_{3}\left(y_{v}\right)} \leq \frac{p_{3}}{p_{1}} \text { e } \frac{f_{2}\left(y_{v}\right)}{f_{3}\left(y_{v}\right)} \leq \frac{p_{3}}{p_{2}} .
\end{gathered}
$$

Seja o exemplo para três populações apresentado por Morrison (1976), donde as estatísticas discriminantes citadas são:

$$
\begin{aligned}
& w_{12}=y_{v}^{\prime} S^{-1}\left(\bar{y}_{v 1}-\bar{y}_{v 2}\right)-\frac{1}{2}\left(\bar{y}_{v 1}+\bar{y}_{v 2}\right)^{\prime} S^{-1}\left(\bar{y}_{v 1}-\bar{y}_{v 2}\right) \\
& w_{13}=y_{v}^{\prime} S^{-1}\left(\bar{y}_{v 1}-\bar{y}_{v 3}\right)-\frac{1}{2}\left(\bar{y}_{v 1}+\bar{y}_{v 3}\right)^{\prime} S^{-1}\left(\bar{y}_{v 1}-\bar{y}_{v 3}\right) \\
& w_{23}=y_{v}^{\prime} S^{-1}\left(\bar{y}_{v 2}-\bar{y}_{v 3}\right)-\frac{1}{2}\left(\bar{y}_{v 2}+\bar{y}_{v 3}\right)^{\prime} S^{-1}\left(\bar{y}_{v 2}-\bar{y}_{v 3}\right)
\end{aligned}
$$


0 autor citado observa que, devido $w_{23}=w_{13}-w_{12}$, é somente necessário usar as estatísticas $w_{12}$ e $w_{13}$, estabelecendo as seguintes regras de classificação: classificar $y_{v}$ a partir de:

$$
\begin{aligned}
& \text { Na população } \pi_{1} \text { se } w_{12}>0 \text { e } w_{13}>0 \\
& \text { Na população } \pi_{2} \text { se } w_{12}<0 \text { e } w_{13}>w_{12} \\
& \text { Na população } \pi_{3} \text { se } w_{13}>0 \text { e } w_{12}>w_{13}
\end{aligned}
$$

\subsubsection{MÉTODO NÃO PARAMÉTRICO PARA CALCULAR A PROBABILIDADE DE MÁ CLASSIFICAÇÃO}

Este método é não tendencioso e independe da distribuição, não precisando de homogeneidade de matrizes de covariâncias. Godoi (1985) cita que se $n_{1}$ e $n_{2}$ forem grandes, toma-se uma parte dos dados para construir a função linear de discriminação, sendo a classificação feita somente para os indivíduos pertencentes à outra parte dos dados, resultando em uma tabela de duas entradas, possibilitando obter as estimativas não tendenciosas para $P(1 / 2)$ e $P(2 / 1)$.

\begin{tabular}{l|c|c}
\multicolumn{1}{c|}{ Classificação } & $\pi_{1}$ & $\pi_{2}$ \\
Classificação correta & $\mathrm{a}$ & $\mathrm{B}$ \\
\hline Classificação errada & $\mathrm{c}$ & $\mathrm{d}$ \\
\hline & $\mathrm{a}+\mathrm{c}$ & $\mathrm{b}+\mathrm{d}$ \\
\hline
\end{tabular}

Por conseguinte:

$$
\hat{P}(1 / 2)=\frac{d}{b+d} \quad \hat{P}(2 / 1)=\frac{c}{a+c}
$$

$P(1 / 2)$ : probabilidade de $y_{v}$ pertencer à população $\pi_{2}$ e ser classificado na $\pi_{1}$. $P(2 / 1)$ : probabilidade de $y_{v}$ pertencer à população $\pi_{1}$ e ser classificado na $\pi_{2}$.

\subsubsection{ANÁLISE DE DISCRIMINANTE, CONSIDERANDO AS VARIÁVEIS (OC1, OC2, OC3, OC4, OC5), PARA AS QUATRO POPULAÇÕES (E1, E2, E3, E4)}

No caso exposto, têm-se duas alternativas para efetuar a análise discriminante. A primeira é obter a função discriminante utilizando o total das 30 parcelas medidas por espécie (população). A segunda é considerar uma parte dos dados para obter as funções discriminantes e efetuar a classificação a partir dos dados restantes. Optou-se em usar 15 parcelas por espécie para construir as funções discriminantes e 15 parcelas para efetuar as classificações, e assim, obter estimativas de classificações erradas não tendenciosas. A tabela C15 do apêndice C, mostra o programa SAS desenvolvido para análise discriminante para as espécies E1, E2, E3 e E4. 
a) Vetores de médias e matrizes de covariâncias, considerando 15 parcelas, para as espécies (E1, E2, E3, E4)

$$
\begin{aligned}
& \bar{Y}_{V 1}^{\prime}=[9,6746200014,387580017,047633319,388426720,9754800 \\
& \bar{Y}_{V 2}^{\prime}=[9,7488400014,429413317,150926719,544320021,1748200 \\
& \bar{Y}_{V 3}^{\prime}=[9,7072066713,168826716,310940017,807106719,417386] \\
& \bar{Y}_{V 4}^{\prime}=[9,6904000013,193166716,487840018,111346719,6555800] \\
& S_{1}=\left[\begin{array}{l}
0,01850860,01513720,01262080,01485830,0072885 \\
0,01513720,05143980,06821040,08654010,0910265 \\
0.01262080 .06821040 .11795070 .16263730,1833055 \\
0,01485830,08654010,16263730,23600230,2682735 \\
0,00728850,09102650,18330550,26827350,3210484
\end{array}\right]
\end{aligned}
$$

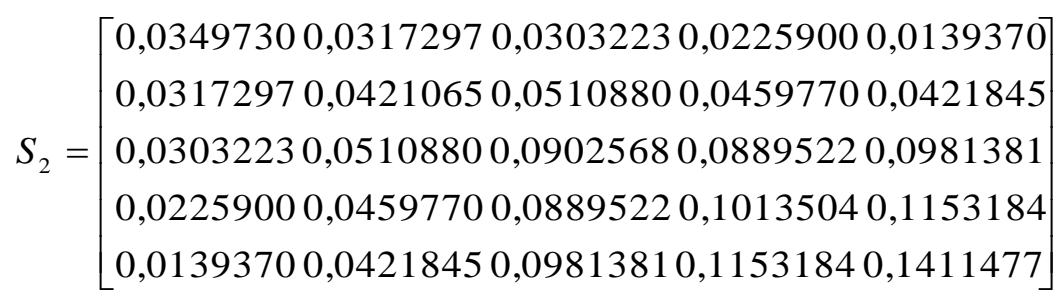

$$
\begin{aligned}
& S_{3}=\left[\begin{array}{l}
0,01939650,02185760,02942820,03682080,0352956 \\
0,02185760,04294400,06041610,07211560,0721124 \\
0,02942820,06041610,09271850,10979900,1107514 \\
0,03682080,07211560,10979900,13984350,1462786 \\
0,03529560,07211240,11075140,14627860,1634582
\end{array}\right] \\
& S_{4}=\left[\begin{array}{l}
0,01679830,00897210,00679750,00797260,0047087 \\
0,00897210,03231210,04780390,05568400,0550067 \\
0,00679750,04780390,08405710,09468170,0983453 \\
0,00797260,05568400,09468170,12003990,1235505 \\
0,00470870,05500670,09834530,12355050,1359118
\end{array}\right] \\
& S=\frac{S_{1}+S_{2}+S_{3}+S_{4}}{4}=\left[\begin{array}{l}
0,02241910,01942410,01979220,02056040,0153075 \\
0,01942410,04220060,05687960,06507920,0650825 \\
0,01979220,05687960,09624580,11401750,1226351 \\
0,02056040,06507920,11401750,14930900,1633553 \\
0,01530750,06508250,12263510,16335530,1903915
\end{array}\right]
\end{aligned}
$$




\section{b) Função Linear discriminante por espécie}

Considerando, por exemplo, as espécies E1 e E2, classifique em E1, se:

$$
\begin{gathered}
w_{E 1}=\left[y_{v} \in R^{p}:\left(\mu_{v 1}-\mu_{v 2}\right)^{\prime} \Sigma^{-1} y_{v} \geq \frac{1}{2}\left(\mu_{v 1}-\mu_{v 2}\right)^{\prime} \Sigma^{-1}\left(\mu_{1}+\mu_{2}\right)\right] \\
w_{E 1}=\left\{y_{v} \in R^{p}:\left[f=\mu_{v 1}^{\prime} \Sigma^{-1} y_{v}-\frac{1}{2} \mu_{v 1}^{\prime} \Sigma^{-1} \mu_{v 1}-\left(\mu_{v 2}^{\prime} \Sigma^{-1} y_{v}-\frac{1}{2} \mu_{v 2}^{\prime} \Sigma^{-1} \mu_{v 2}\right)\right] \geq 0\right\} \\
w_{E 1}=\left[y_{v} \in R^{p}: f_{E 1}-f_{E 2} \geq 0\right]
\end{gathered}
$$

Considerando: $\sum=E(S)$ e $\mu_{v i}=E\left(\bar{y}_{v i}\right)$, têm-se:

$$
\begin{array}{cc}
f_{E 1}=\bar{y}_{v 1}^{\prime} S^{-1} y_{v}-\frac{1}{2} \bar{y}_{v 1}^{\prime} S^{-1} \bar{y}_{v 1} & f_{E 2}=\bar{y}_{v 2}^{\prime} S^{-1} y_{v}-\frac{1}{2} \bar{y}_{v 2}^{\prime} S^{-1} \bar{y}_{v 2} \\
f_{E 3}=\bar{y}_{v 3}^{\prime} S^{-1} y_{v}-\frac{1}{2} \bar{y}_{v 3}^{\prime} S^{-1} \bar{y}_{v 3} & f_{E 4}=\bar{y}_{v 4}^{\prime} S^{-1} y_{v}-\frac{1}{2} \bar{y}_{v 4}^{\prime} S^{-1} \bar{y}_{v 4}
\end{array}
$$

$y_{v}$ : indivíduo a ser classificado, de acordo com o seu desempenho no tempo, com base em uma (e somente uma) das populações E1, E2, E3, E4 com chance mínima de erro.

A tabela 9.2.6.1 apresenta os coeficientes das funções lineares discriminantes das espécies E1, E2, E3, E4.

Tabela 9.2.6.1 - Funções discriminantes das espécies E1, E2, E3 e E4.

\begin{tabular}{l|l|l|l|l}
\multicolumn{5}{c}{ Funções lineares discriminantes por ESPÉCIE } \\
Variável & \multicolumn{1}{c}{ FE1 $_{\text {E1 }}$} & \multicolumn{1}{c}{ FE2 $_{\text {E }}$} & \multicolumn{1}{c}{ FE3 $_{\text {FE4 }}$} \\
\hline Constante & -3383 & -3419 & -3091 & -3044 \\
\hline OC1 & 277,24113 & 284,37115 & 349,80662 & 348,49615 \\
\hline OC2 & 462,44627 & 456,58042 & 286,36560 & 270,56530 \\
\hline OC3 & $-157,49399$ & $-156,90469$ & $-1,83273$ & 1,25295 \\
\hline OC4 & $-388,41390$ & $-390,49938$ & $-445,89852$ & $-420,93560$ \\
\hline OC5 & 364,50245 & 368,39111 & 359,73214 & 343,08402 \\
\hline
\end{tabular}

c) Classificação das espécies E1, E2, E3 e E4

Tabela 9.2.6.2 - Número de observações e porcentagens de classificação na ESPÉCIE.

\begin{tabular}{|l|l|l|l|l|l|}
\multicolumn{1}{c}{ Da ESP } & \multicolumn{1}{c}{ E1 } & \multicolumn{1}{c}{ E2 } & \multicolumn{1}{c|}{ E3 } & Total \\
\hline E1 & $9(60,00)$ & $6(40,00)$ & $0(0,00)$ & $0(0,00)$ & $15(100,00)$ \\
\hline E2 & $4(26,67)$ & $11(73,33)$ & $0(0,00)$ & $0(0,00)$ & $15(100,00)$ \\
\hline E3 & $0(0,00)$ & $0(0,00)$ & $14(93,33)$ & $1(6,67)$ & $15(100,00)$ \\
\hline E4 & $0(0,00)$ & $0(0,00)$ & $3(20,00)$ & $12(80,00)$ & $15(100,00)$ \\
\hline Total & $13(21,67)$ & $17(28,33)$ & $17(28,33)$ & $13(21,67)$ & $60(100,00)$ \\
\hline Prob. a priori & 0,25 & 0,25 & 0,25 & 0,25 & \\
\hline
\end{tabular}


Na tabela 9.2.6.2, observa-se que das 15 observações da espécie E1, seis (40\%) foram classificadas como E2, enquanto que das 15 observações da espécie E2 quatro $(26,67 \%)$ foram classificadas em E1, assim como, das 15 observações da espécie E3, apenas uma $(6,67 \%)$ foi classificada como E4, enquanto que das 15 observações da espécie E4 três (20\%) foram classificadas em E3. É importante verificar que nenhuma observação de E1 ou de E2 foi classificada em E3 ou em E4, e como também, nenhuma da espécie E3 ou de E4 foi classificada em E1 ou em E2. Estes resultados da análise discriminante confirmam os resultados obtidos no capítulo 8, seção 8.1., item g, dendrograma do agrupamento de Ward para as quatro espécies, ou seja, as espécies E1 e E2 formam um grupo distinto do cluster formado pelas espécies E3 e E4, o que pode ser confirmado na figura 9.2.6.1.

Tabela 9.2.6.3 - Erros de classificação por espécie e para o total.

\begin{tabular}{c|c|c|c|c|c} 
& E1 & E2 & E3 & E4 & Total \\
Observado & 0,4000 & 0,2667 & 0,0667 & 0,2000 & 0,2333 \\
\hline Pr. a priori & 0,2500 & 0,2500 & 0,2500 & 0,2500 & \\
\hline
\end{tabular}

A Tabela 9.2.6.3 mostra as estimativas, por espécie e para o total, dos erros de classificação. As espécies E1 e E2 apresentaram, respectivamente, classificações erradas de $40 \%$ e 26,67 \%. As espécies E3 e E4 tiveram, respectivamente, classificações erradas de $6,67 \%$ e $20 \%$. 0 total de classificações erradas foi de $23,33 \%$, ou seja, 14 observações, de um total de 60 , foram classificadas erroneamente.

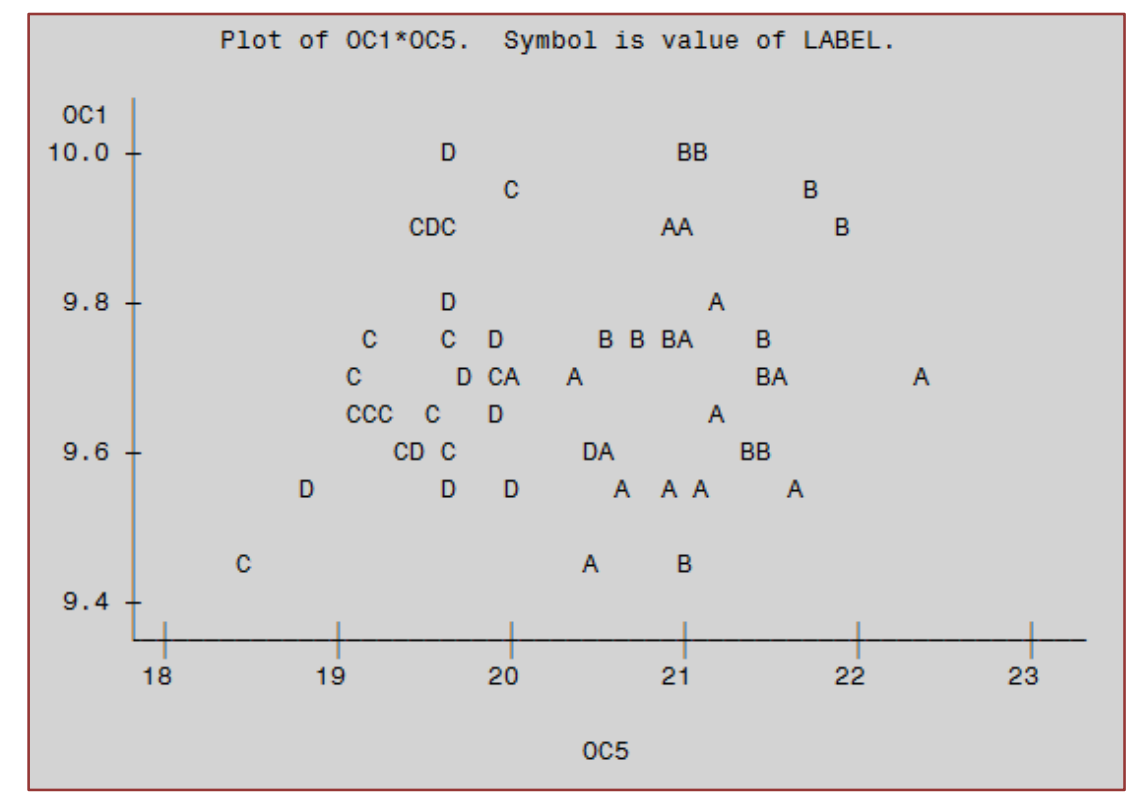

Figura 9.2.6.1 - Mostra a discriminação, considerando as variáveis OC1 e 0C5, para as quatro espécies $(A=E 1, B=E 2, C=E 3$ e $D=E 4)$. Nota do programa SAS: 8 obs. ocultas.

As letras A, B, C e D, denominadas de label no programa SAS, foram criadas para possibilitar a criação do gráfico. 
Apesar do exemplo apresentado não ser propriamente uma pesquisa de classificação de espécies com referência às suas características florísticas, mas sim aos seus perfis de crescimento no tempo, os resultados revelam conclusões interessantes que confirmam os resultados da análise de perfil para as espécies E1, E2, E3 e E4 vista no capítulo 8.

\subsection{ANÁLISE DE CORRELAÇÃo CANÔNICA}

A aplicação geral e usual da análise de correlação canônica é investigar as relações existentes entre dois conjuntos distintos de variáveis. Por exemplo, em uma área florestal pode-se pesquisar qual o seu perfil florístico de acordo com o padrão edafoclimático ocorrente. 0 método foi desenvolvido por Hotelling (1935).

A análise de correlação canônica ajuda a responder a esses questionamentos, determinando combinações lineares das características florísticas que mais se correlacionam com outras combinações lineares envolvendo as características edafoclimáticas.

Supondo que o primeiro conjunto das variáveis (florísticas) seja definido por $X_{1}, X_{2}, \cdots, X_{p}$, e as inerentes ao segundo conjunto de variáveis (edafoclimáticas) por $Y_{1}, Y_{2}, \cdots, Y_{q}$. A técnica consiste em determinar as combinações lineares $W$ e $V$, ou seja, obter $u_{v}^{\prime}=\left[u_{1}, u_{2}, \cdots, u_{p}\right]$ e $v_{v}^{\prime}=\left[v_{1}, v_{2}, \cdots, v_{q}\right]$, tal que a correlação $\left(r_{Z W}\right)$ entre as combinações lineares $W$ e $V$ atinja o máximo valor possível. Então:

$$
W=u_{1} X_{1}+u_{2} X_{2}+\cdots+u_{p} X_{p} \quad V=v_{1} Y_{1}+v_{2} Y_{2}+\cdots+v_{q} Y_{q}
$$

Da definição de correlação linear simples:

$$
r_{W_{i} V_{i}}=\frac{\sum w_{i j} v_{i j}}{\sqrt{\sum w_{i j}^{2} \times \sum v_{i j}^{2}}}=\frac{u_{v}^{\prime} S_{x y} v_{v}}{\sqrt{\left(u_{v}^{\prime} S_{x x} u_{v}\right)\left(v_{v}^{\prime} S_{y y} v_{v}\right)}}
$$

$i=1,2, \cdots,(q$ ou $p)$, donde: $(q$ se $q<p$ ou $p$ se $p<q)$;

$j=1,2, \cdots, n$ (número de parcela).

Estabelecendo as condições, $u_{v}^{\prime} S_{x x} u_{v}=1$ e $v_{v}^{\prime} S_{y y} v_{v}=1$, têm-se pelo método dos multiplicadores de Lagrange:

$$
\begin{gathered}
F\left(u_{v}, v_{v}\right)=u_{v}^{\prime} S_{x y} v_{v}-\frac{\lambda}{2}\left(u_{v}^{\prime} S_{x x} u_{v}-1\right)-\frac{\mu}{2}\left(v_{v}^{\prime} S_{y y} v_{v}-1\right) \\
\frac{\partial F}{\partial u_{v}}=S_{x y} v_{v}-\lambda S_{x x} u_{v}=0_{v} \rightarrow 1 \\
\frac{\partial F}{\partial v_{v}^{\prime}}=u_{v}^{\prime} S_{x y}-\mu v_{v}^{\prime} S_{y y}=0_{v}^{\prime} \rightarrow 2
\end{gathered}
$$


Pré-multiplicando (1) por $u_{v}^{\prime}$ e pós-multiplicando (2) por $v_{v}$, resulta:

$$
u_{v}^{\prime} S_{x y} v_{v}=\lambda u_{v}^{\prime} S_{x x} u_{v}=\mu v_{v}^{\prime} S_{y y} v_{v}
$$

Dado que $u_{v}^{\prime} S_{x x} u_{v}=v_{v}^{\prime} S_{y y} v_{v}=1$, então $u_{v}^{\prime} S_{x y} v_{v}=\lambda=\mu$. Logo, $\lambda=\mu$ é o valor máximo possível para $r_{Z W}$.

De (1) e (2), têm-se:

$$
\begin{aligned}
& S_{x y} v_{v}=\mu S_{x x} u_{v} \\
& S_{y x} u_{v}=\mu S_{y y} v_{v}
\end{aligned}
$$

Sendo $S_{y y}$ uma matriz não singular, tem-se de (4):

$$
v_{v}=\frac{1}{\mu} S_{y y}^{-1} S_{y x} u_{v}
$$

Substituindo (5) em (3), tem-se: $S_{x y} \frac{1}{\mu} S_{y y}^{-1} S_{y x} u_{v}=\mu S_{x x} u_{v}$

Pré-multiplicando ambos os membros de (6) por $\mu S_{x x}^{-1}$, tem-se:

$$
\left[S_{x x}^{-1} S_{x y} S_{y y}^{-1} S_{y x}-\mu^{2} I\right] u_{v}=0_{v}
$$

Com efeito, o maior autovalor $\mu_{1}^{2}$ do produto matricial $S_{x x}^{-1} S_{x y} S_{y y}^{-1} S_{y x}$, corresponde ao quadrado do máximo valor de $r_{Z W}$. Os elementos do autovetor, associado ao autovalor $\mu_{1}^{2}$, são os coeficientes da função linear do conjunto das variáveis $X_{1}, X_{2}, \cdots, X_{p}$. Os elementos do autovetor $v_{v 1}=\frac{1}{\mu_{1}} S_{y y}^{-1} S_{y x} u_{v 1}$ são os coeficientes da função linear das variáveis $Y_{1}, Y_{2}, \cdots, Y_{q}$. 0 valor máximo possível para $r_{Z W}$ é $\mu_{1}$.

Os pares das combinações lineares $W_{i}$ e $V_{i}$ são denominados variáveis canônicas. 0 número de variáveis canônicas será igual ao posto da matriz $S_{x x}^{-1} S_{x y} S_{y y}^{-1} S_{y x}$, ou seja, o menor valor entre $p$ e $q$.

As combinações lineares $W_{i}$ e $W_{j}$ para $(i \neq j)$ são não correlacionadas, assim como para $V_{i}$ e $V_{j}(i \neq j)$, estendendo-se a independência para $W_{i}$ e $V_{j}$ para $(i \neq j)$.

Godoi (1985) cita que as variáveis canônicas, ao contrário dos componentes principais, não variam pela mudança de escala.

Tatsuoka (1971) mostra que o teste de significância das variáveis canônicas, usando o critério $(\Lambda)$ de Wilks (a), pode ser escrito em função de $\mu_{i}^{2}(\mathrm{~b})$, donde $r$ é o menor valor entre $p$ eq. 


$$
\Lambda=\frac{1}{\prod_{i=1}^{r}\left(1+\lambda_{i}\right)}(\mathrm{a}) \quad \Lambda=\prod_{i=1}^{r}\left(1-\mu_{i}^{2}\right)
$$

\subsubsection{ANÁLISE DE CORRELAÇÃO CANÔNICA CONSIDERANDO OS MÉTODOS DE MANEJO (M1, M2, M3, M4) E AS QUATRO ESPÉCIES (E1, E2, E3, E4)}

Considerando a existência de cinco variáveis que são os volumes medidos, nas cinco ocasiões OC1, OC2, OC3, OC4, OC5, nas duas populações de espécies e de métodos de manejo, foram obtidos os cinco pares de variáveis canônicas. A tabela C16, do apêndice C, apresenta o programa desenvolvido no sistema SAS para análise de correlação canônica, considerando as inter-relações entre os grupos (M1, M2, M3 e M4) versus (E1, E2, E3, E4).

A tabela 9.3.1.1 mostra que o teste geral de significância apresentou uma relação linear altamente significativa ( $p$-valor $<0,0001$ ) das ocasiões entre duas as populações.

Tabela 9.3.1.1 - Teste de significância geral da análise de correlação canônica.

\begin{tabular}{|c|c|c|c|c|c|}
\hline \multicolumn{6}{|c|}{ Estatísticas multivariadas e aproximações da estatística $F\left(S=5 ; M=-0,5 ; N^{*}=54\right)$. } \\
\hline Estatística & Valor & Valor F & Num GL & Den GL & $\operatorname{Pr}>\mathrm{F}$ \\
\hline Wilks Lambda & 0,02709784 & 26,93 & 25 & 410,13 & $<0,0001$ \\
\hline Pillai Trace & 1,18189381 & 7,06 & 25 & 570 & $<0,0001$ \\
\hline Hotelling-Lawley Tra & 28,68651913 & 124,88 & 25 & 260,76 & $<0,0001$ \\
\hline Roy Greatest Root & 28,45051946 & 648,67 & 5 & 114 & $<0,0001$ \\
\hline
\end{tabular}

Tabela 9.3.1.2 - Coeficientes de correlação dos cinco pares de variáveis canônicas.

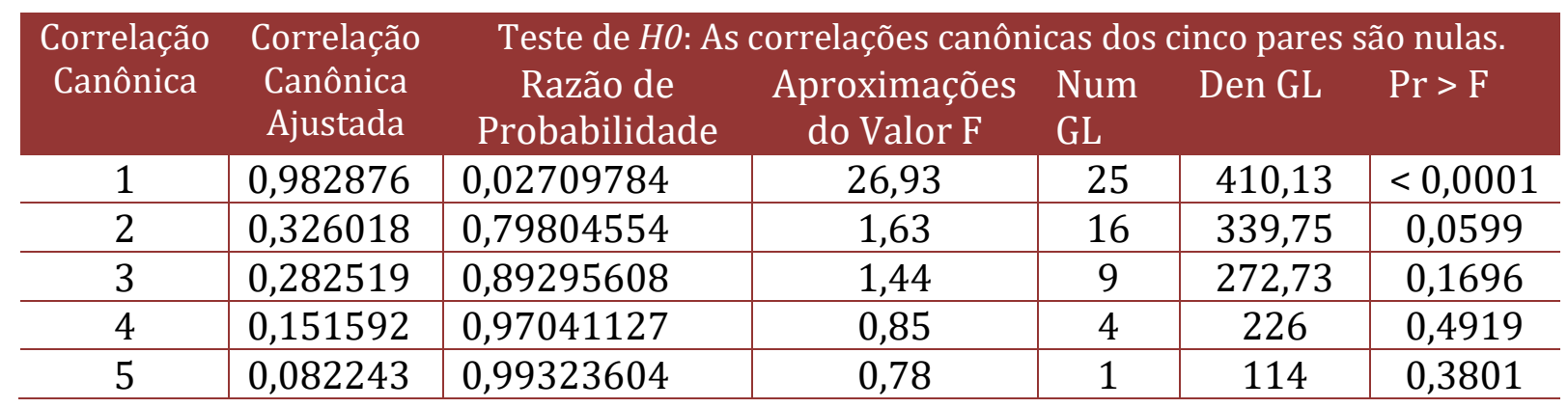

Na tabela 9.3.1.2 são apresentados os valores dos coeficientes de correlação dos cinco pares de variáveis canônicas e os respectivos testes de nulidade $H_{0}: r_{V_{i} W_{i}}=0$. Do resultado altamente expressivo do valor do coeficiente da correlação canônica apresentado pelas funções lineares $V_{1}$ e $W_{1}\left(r_{V_{1} W_{1}}=0,982876\right)$, depreende-se que o primeiro par de variáveis canônicas deve ser usado para explicar as inter-relações, concernentes ao comportamento dentro das ocasiões, entre o grupo das espécies versus o grupo dos métodos de manejo. Os demais pares de variáveis canônicas não apresentam importância, pois foram não significativos $(\alpha=0,05)$ para o teste de hipótese $H_{0}: r_{V_{i} W_{i}}=0$. 
Tabela 9.3.1.3 - Coeficientes das cinco variáveis canônicas da população Espécie.

\begin{tabular}{l|l|l|l|l|l}
\hline ESP & \multicolumn{1}{|c}{ V1 } & \multicolumn{1}{c}{ V2 } & \multicolumn{1}{c}{ V3 } & \multicolumn{1}{c}{ V4 } & V5 \\
OC1 & $-0,64226575$ & 5,108813586 & $-4,952066114$ & 2,258133900 & 2,902715309 \\
\hline OC2 & 1,75069585 & $-4,59027763$ & $-3,597833545$ & $-2,812345386$ & 0,671023990 \\
\hline OC3 & $-1,65797666$ & $-2,18489350$ & 2,842566157 & $-7,892334625$ & $-0,18043754$ \\
\hline OC4 & 0,410219689 & 8,496290285 & 1,832121098 & 4,6451865293 & $-9,34327408$ \\
\hline OC5 & 0,2334971928 & $-3,68806721$ & $-0,22616514$ & 1,4392687648 & 8,761691969 \\
\hline
\end{tabular}

Na tabela 9.3.1.3 são apresentados os coeficientes das cinco variáveis canônicas, V1, V2, V3, V4, V5, da população ESPÉCIE, onde se observa que para V1 os coeficientes das ocasiões OC2 e OC3 apresentaram valores absolutos superiores às das demais ocasiões, significando que contribuem mais significativamente na construção de V1. As ocasiões OC2 e OC3 apresentam sinais diferentes formando um contraste.

Tabela 9.3.1.4 - Coeficientes das cinco variáveis canônicas da população Manejo Florestal.

\begin{tabular}{l|l|l|l|l|l} 
MM & \multicolumn{1}{|c}{ W1 } & \multicolumn{1}{c}{ W2 } & \multicolumn{1}{c}{ W3 } & \multicolumn{1}{c}{ W4 } & \multicolumn{1}{c}{ W5 } \\
OC1 & 0,060216817 & 0,354471296 & 0,883643286 & 0,0834517268 & 0,0768368829 \\
\hline OC2 & $-0,19072233$ & $-0,81376746$ & 0,028547624 & 0,0726938549 & $-0,168580501$ \\
\hline OC3 & 0,25078963 & $-0,40408516$ & $-0,661500325$ & 0,026470001 & $-0,922437684$ \\
\hline OC4 & $-0,07918303$ & 1,70977664 & 0,310897736 & $-1,462769623$ & 0,6193247797 \\
\hline OC5 & $-0,03564363$ & $-0,75020581$ & 0,005366130 & 1,4576417761 & 0,0307107047 \\
\hline
\end{tabular}

Na tabela 9.3.1.4 são mostrados os coeficientes das cinco variáveis canônicas, W1, W2, W3, W4, W5, da população manejo florestal, onde se observa que para W1 os coeficientes das ocasiões OC2 e OC3 apresentaram também valores absolutos superiores às das demais ocasiões, significando que 0C2 e 0C3 contribuem mais significativamente na construção de W1. As ocasiões OC2 e OC3 apresentam sinais diferentes (contraste).

Do exposto, tem-se o primeiro par $\left(\mathrm{V}_{1} * W_{1}\right)$ de variáveis canônicas:

$$
\begin{aligned}
& V 1=-0,642266 \propto C 1+1,750696 \mathrm{OC}-1,657977 \mathrm{OC} 3+0,41022 \mathrm{OC} 4+0,233497190 \mathrm{C} 5 \\
& W 1=0,0602168 \mathrm{OC} 1-0,190722 \mathrm{OC} 2+0,2507896 \propto C 3-0,079183 \mathrm{OC}-0,035644 \mathrm{OC} 5
\end{aligned}
$$

A figura 9.3.1.1 (gráfico $\mathrm{V}_{1} * V_{2}$ ) confirma o resultado da análise de agrupamento produzido para as espécies E1, E2, E3, E4, mostrado na figura 8.1.1.3, capítulo 8, seção 8.1., item g, dendrograma do agrupamento de Ward, o qual demonstra que as espécies E1 e E2 formam um grupo distinto do estruturado pelas espécies E3 e E4. 


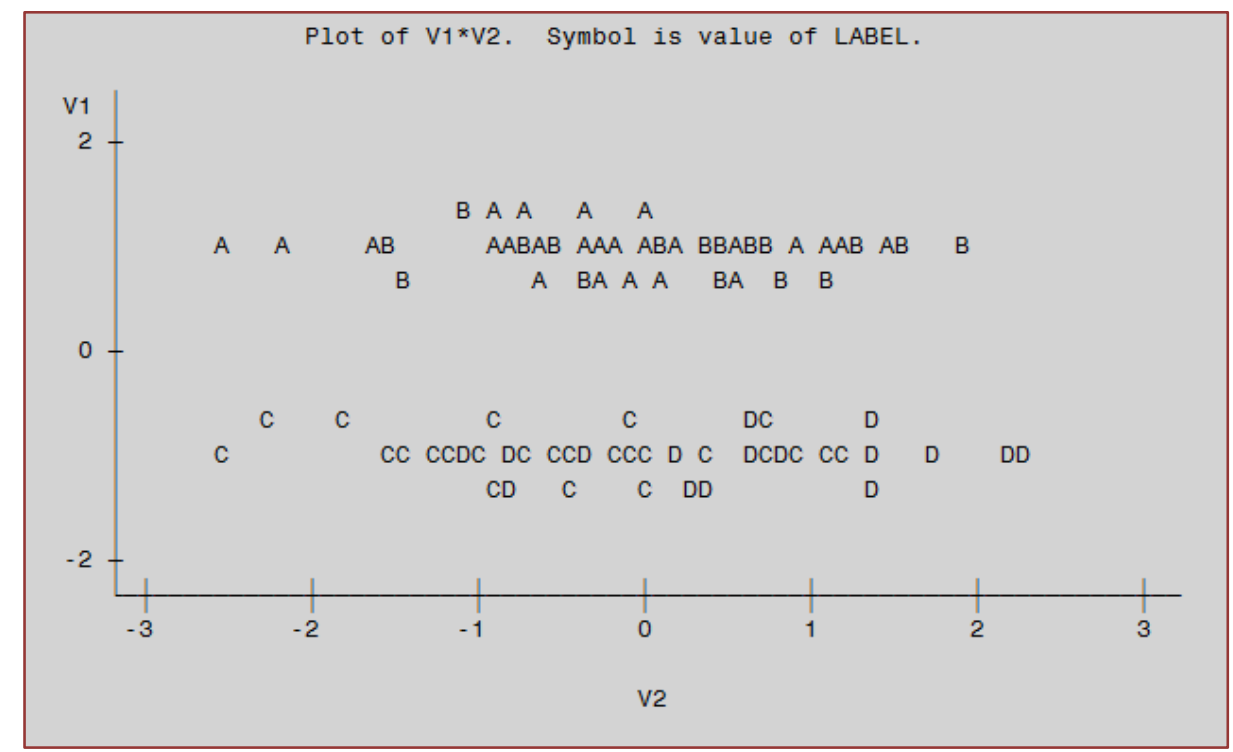

Figura 9.3.1.1 - Agrupamento da população de Espécies ( $A=E 1, B=E 2, C=E 3, D=E 4)$. Nota do programa SAS: 37 obs. ocultas.

A figura 9.3.1.2 (gráfico $\mathrm{W}_{1} * W_{2}$ ) confirma o resultado da análise de agrupamento realizada para os métodos de manejo $M 1, M 2$, M3 e M4, mostrado na figura 7.7.1.3, capítulo 7, seção 7.7.1., item $i$, dendrograma do agrupamento de Ward, o qual demonstra que os métodos de manejo M1 e M2 formam um grupo distinto do organizado pelos métodos M3 e M4.

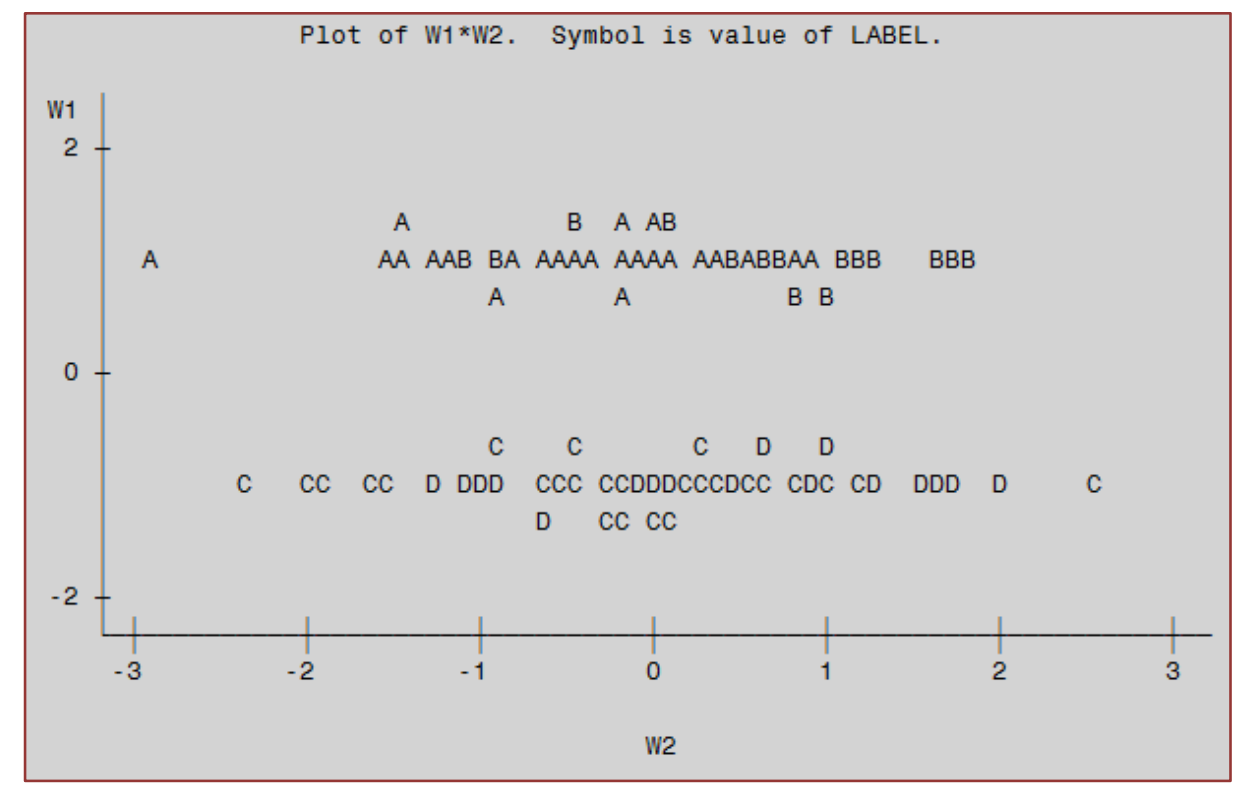

Figura 9.3.1.2 - Agrupamento da população de Métodos de Manejo (A= M1, B= M2, C= M3, D= M4). Nota do programa SAS: 38 obs. ocultas. 


\subsection{ANÁLISE DE FATORES (AF)}

Com esta análise estudam-se as relações internas de um conjunto de variáveis, substituindo-as por um número menor de variáveis hipotéticas denominadas fatores, que podem ser correlacionados (fatores oblíquos) ou não correlacionados (fatores ortogonais). Esses fatores devem explicar parte significativa da variância do conjunto das variáveis originais.

Spearman (1904) publicou pela primeira vez uma teoria sobre análise de fatores. Este autor obteve um fator " $g$ " que explicaria as inter-relações de uma série de características cognitivas. Bartlett (1962) cita que a fatoração da matriz de dispersão proposta por Spearman (1904) é inadequada, pois está estruturada somente a partir de um fator comum, quando na realidade deveria ser introduzidos mais fatores no modelo.

Seja o modelo $m$-dimensional linear $x_{i}=\sum_{j=1}^{m} a_{i j} F_{j}+e_{i}(i=1, \cdots, p)$ que na forma matricial apresenta a seguinte formulação:

$$
x_{v}=A f_{v}+e_{v}
$$

A é a matriz dos pesos correspondentes a $i$-ésima variável resposta e ao $j$-ésimo fator, sendo $m$ o número de fatores do modelo, onde $m \leq p$.

$$
A=\left[\begin{array}{cccc}
a_{11} & a_{12} & \ldots & a_{1 m} \\
a_{21} & a_{22} & \ldots & a_{2 m} \\
\ldots & \ldots & \ldots & \ldots \\
a_{p 1} & a_{p 2} & \ldots & a_{p m}
\end{array}\right]
$$

$x_{v}^{\prime}=\left[x_{1}, x_{2}, \cdots, x_{p}\right]:$ vetor correspondente às $p$ variáveis respostas observadas; $f_{v}^{\prime}=\left[F_{1}, F_{2}, \ldots, F_{m}\right]$ : vetor correspondente aos fatores comuns (variáveis hipotéticas) aleatórios, independentes e de variâncias iguais a um;

$e_{v}^{\prime}=\left[e_{1}, e_{2}, \cdots, e_{p}\right]:$ vetor inerente aos resíduos específicos aleatórios de média zero.

Seja $\Sigma=E\left(x_{v} x_{v}^{\prime}\right)=\left[\sigma_{i j}\right]$ a matriz populacional de covariância das $p$ variáveis respostas $x_{1}, x_{2}, \cdots, x_{p}$, considerando as seguintes pressuposições:

$$
\begin{gathered}
E\left(f_{v} e_{v}^{\prime}\right)=\phi \\
E\left(f_{v} f_{v}^{\prime}\right)={ }_{m} I_{m} \\
E\left(e_{v} e_{v}^{\prime}\right)={ }_{p} \psi_{p}=\operatorname{diag}\left(\psi_{1}, \psi_{2}, \cdots, \psi_{p}\right)
\end{gathered}
$$

Pode-se escrever que: 


$$
\begin{gathered}
\Sigma=E\left(x_{v} x_{v}^{\prime}\right)=E\left[\left(A f_{v}+e_{v}\right)\left(A f_{v}+e_{v}\right)^{\prime}\right] \\
\Sigma=E\left[\left(A f_{v}+e_{v}\right)\left(f_{v}^{\prime} A^{\prime}+e_{v}^{\prime}\right)\right] \\
\Sigma=A E\left(f_{v} f_{v}^{\prime}\right) A^{\prime}+E\left(e_{v} e_{v}^{\prime}\right) \\
\sum=A A^{\prime}+\psi
\end{gathered}
$$

Portanto, conclui-se que a matriz de covariância de $x_{v}^{\prime}=\left[x_{1}, x_{2}, \cdots, x_{p}\right]$ sob o modelo fatorial $x_{v}=A f_{v}+e_{v}$, é:

$$
\Sigma=A A^{\prime}+\psi
$$

Denotando que:

$$
\begin{gathered}
V\left(x_{i}\right)=\sigma_{i i}=a_{i 1}^{2}+a_{i 2}^{2}+\cdots+a_{i m}^{m}+\psi_{i} \\
\operatorname{Cov}\left(x_{i}, x_{j}\right)=\sigma_{i j}=a_{i 1} a_{j 1}+a_{i 2} a_{j 2}+\cdots+a_{i m} a_{j m}
\end{gathered}
$$

Os elementos diagonais da matriz $A A^{\prime}$ são denominados de comunalidades das variáveis respostas $x_{1}, x_{2}, \cdots, x_{p}$.

Da expressão 9.4.2., tem-se: $\sum_{j=1}^{m} a_{i j}^{2}=\sigma_{i i}-\hat{\psi}_{i}$.

Desta maneira, se as pressuposições citadas anteriormente forem atendidas, então os parâmetros $a_{i j}$ corresponderão às covariâncias entre $x_{i}$ e $F_{j}$, entretanto, se $\sum$ exprimir a matriz das correlações entre as variáveis observáveis, então os valores de $a_{i j}$ expressarão os coeficientes de correlação entre $x_{i}$ e $F_{j}$, pois:

$$
\begin{gathered}
\operatorname{Cov}\left(x_{v}, f_{v}^{\prime}\right)=E\left[\left(A f_{v}+e_{v}\right) f_{v}^{\prime}\right] \\
\operatorname{Cov}\left(x_{v}, f_{v}^{\prime}\right)=A E\left(f_{v} f_{v}^{\prime}\right)+E\left(e_{v} f_{v}^{\prime}\right) \\
\operatorname{Cov}\left(x_{v}, f_{v}^{\prime}\right)=A
\end{gathered}
$$

Quando os fatores comuns forem correlacionados (oblíquos) ou apresentarem variâncias não unitárias, a matriz de covariância de $x_{v}$ sob o modelo fatorial 9.4.1 será dada por:

$$
\begin{gathered}
\Sigma=A \Gamma A^{\prime}+\psi \\
\Gamma=E\left(f_{v} f_{v}^{\prime}\right)
\end{gathered}
$$

As equações 9.4.1, 9.4.2 e 9.4.3 são invariantes para transformações ortogonais não singulares dos fatores. Essas expressões não são alteradas quando da substituição de:

$$
f_{v} \text { por } M f_{v}, \text { A por } A M^{\prime}, \Gamma \text { por } M \Gamma M^{\prime}
$$

M: é uma matriz ortogonal de ordem correspondente ao número de fatores.

Comprovações: 


$$
\begin{gathered}
x_{v}=A M^{\prime}\left(M f_{v}\right)+e_{v}=A\left(M^{\prime} M\right) f_{v}+e_{v}=A f_{v}+e_{v} \\
\Sigma=\left(A M^{\prime}\right)\left(M A^{\prime}\right)+\psi=A A^{\prime}+\psi \\
\Sigma=A M^{\prime}\left(M \Gamma M^{\prime}\right) M A^{\prime}+\psi=A^{\prime}\left(M^{\prime} M\right) \Gamma\left(M^{\prime} M\right) A^{\prime}+\psi=A \Gamma A^{\prime}+\psi
\end{gathered}
$$

No uso da análise de fatores (AF), após verificar a multinormalidade dos dados, é importante efetuar os seguintes procedimentos:

0 primeiro consiste em identificar na matriz de correlação o número de correlações que são estatisticamente diferentes de zero. Hair et al. (2009) citam que para aplicar a análise de fatores, o número de correlações com valores menores que 0,30 não deve ser grande. Por outro lado, Fávero et al. (2009) observam que as variáveis com alta correlação tendem a ser incluídas no mesmo fator.

O segundo passo é obter a estatística de Kaiser-Meyer-Olkin (KMO) que de acordo com Maroco (2007) compara as correlações simples com as correlações parciais entre as variáveis. A estatística de KMO é dada pela fórmula 9.4.4, onde $r_{i j}$ é o coeficiente de correlação entre as variáveis e $a_{i j}$ é o coeficiente de correlação parcial.

$$
K M O=\frac{\sum_{i \neq j} \sum r_{i j}^{2}}{\sum_{i \neq j} \sum r_{i j}^{2}+\sum_{i \neq j} \sum a_{i j}^{2}}
$$

\begin{tabular}{|c|c|c|c|}
\hline KMO & Análise de Fatores & KMO & Análise de Fatores \\
\hline $1,0-0,9$ & Muito boa & $0,6-0,7$ & Razoável \\
\hline $0,8-0,9$ & Boa & $0,5-0,6$ & Má \\
\hline $0,7-0,8$ & Média & $<0,5$ & Inaceitável \\
\hline
\end{tabular}

Tabela 9.4.1 - Estatística KMO (Kaiser-Meyer-Olkin)

Os valores da estatística KMO variam de 0 a 1 e, segundo Fávero et al. (2009), quanto mais próximo da unidade mais recomendável é a aplicação da análise de fatores. Citam os intervalos de análise dos valores de KMO, tabela 9.4.1, e concluem que os valores iguais ou inferiores a 0,6 indicam que aplicação da análise de fatores pode ser inadequada.

O terceiro procedimento é aplicar o teste de esfericidade de Bartlett. Este critério mostra a probabilidade estatística da matriz de correlação de apresentar correlações significantes, em pelo menos algumas variáveis, pois, se a matriz for igual à matriz identidade, então não tem sentido usar a técnica de análise de fatores. Mingoti (2005) apresenta a estatística $T$ de Bartlett para testar a esfericidade da matriz de correlação. Sob a hipótese nula, para grandes amostras de tamanho $N$, a estatística $T$ tem uma distribuição aproximadamente qui-quadrado com $v=1 / 2 p(p-1)$ graus de liberdade. 


$$
T=-\left[N-\frac{1}{6}(2 p+11)\right]\left(\sum_{i=1}^{p} \lambda_{i}\right)
$$

O quarto passo, de acordo com Fávero et al. (2009), é verificar se a matriz de correlação anti-imagem contém os valores negativos das correlações parciais. É uma forma de obter indícios sobre a necessidade de eliminar variáveis do modelo. A diagonal da matriz antiimagem fornece os valores de MSA (Measure of Sampling Adequacy):

$$
M S A=\frac{\sum_{i \neq j} r_{i j}^{2}}{\sum_{i \neq j} r_{i j}^{2}+\sum_{i \neq j} a_{i j}^{2}}
$$

Fávero et al. (2009) citam que quanto maiores forem os valores de MSA, melhor será o uso da análise de fatores. Se uma variável apresentar baixo valor na diagonal principal e alto valor fora dela, há indicativo de excluí-la do modelo, mas também ressaltam que, por vezes, a baixa correlação de determinada variável com as demais, não necessariamente, implica na sua eliminação, uma vez que essa variável pode representar um fator isoladamente.

\subsubsection{ANÁLISE DE FATORES PELO MÉTODO DA MÁXIMA VEROSSIMILHANÇA}

Lawley (1940) aborda originalmente o problema da estimação na análise de fatores, tornando-a um método estatístico propriamente dito. Este autor emprega o método da máxima verossimilhança para estimar os parâmetros do modelo fatorial $x_{v}=A f_{v}+e_{v}$, mostrando que as estimativas dos pesos fatoriais $\hat{\mathrm{A}}$ e as estimativas das variâncias específicas $\hat{\psi}$, tal que $E\left(e_{v}^{\prime} e_{v}\right)$, são soluções das seguintes equações matriciais implícitas:

$$
\left\{\begin{array}{c}
\hat{\psi}=\operatorname{diag}\left(S-A^{\prime} A\right) \\
S \hat{\psi}^{-1} A=\hat{A}\left(I+\hat{A}^{\prime} \hat{\psi}^{-1} \hat{A}\right)
\end{array}\right.
$$

O método de estimadores de máxima verossimilhança consiste em adotar como estimativas dos parâmetros, os valores que maximizam a probabilidade (variável discreta) ou a densidade de probabilidade (variável contínua) de ser obtida a amostra observada. É necessário para obter os estimadores de máxima verossimilhança conhecer a distribuição da variável de interesse. Para obter os estimadores de máxima verossimilhança é necessário obter a função de máxima verossimilhança.

Seja uma variável aleatória contínua com função densidade de probabilidade $f(x, \theta)$, sendo $\theta$ o parâmetro desconhecido. Considere uma amostra aleatória simples da variável $X$ de tamanho $n$ e $x_{1}, x_{2, \cdots} x_{n}$ os valores observados. A função de verossimilhança $L$ é definida como: 


$$
L\left(x_{1}, x_{2}, \cdots, x_{n} ; \theta\right)=f\left(x_{1} ; \theta\right) \times f\left(x_{2} ; \theta\right) \times \cdots \times f\left(x_{n} ; \theta\right)=\prod_{i=1}^{n} f\left(x_{i} ; \theta\right)
$$

Na aplicação do método da máxima verossimilhança na análise de fatores, considera-se que o vetor dos fatores comuns $f_{v}$ e o vetor dos resíduos específicos $e_{v}$ são independentes e multinormalmente distribuídos, com vetor de médias nulas (sem perda de generalidade), e respectivamente, com matrizes de covariâncias ${ }_{m} I_{m} \mathrm{e}_{p} \hat{\psi}_{p}$.

Não obstante, sendo $x_{v}$ formado por funções lineares de $f_{v}$ e $e_{v}$, então $x_{v}$ é multinormalmente distribuído com matriz de covariâncias $\Sigma=A A^{\prime}+\psi$ e, sem perda de generalidade, com vetor de médias nulo.

Seja $S=\left[s_{i j}\right]$ a matriz de covariância amostral, tal que $E(S)=\Sigma$, originada de uma amostra aleatória com $N>p$ observações de $x_{v}$. Então, segundo Anderson (1958), os elementos de $S$ ou de $n S$ seguem a distribuição de Wishart, equação 9.4.1.1, $\operatorname{com} n=N-1$ graus de liberdade.

$$
f(S)=c|S|^{\frac{1}{2}(n-p-1)}\left|\sum\right|^{-\frac{1}{2} n} \exp \left[-\frac{1}{2} n \operatorname{tr}\left(S \sum^{-1}\right)\right]
$$

Como o logaritmo é uma função monotônica crescente, os valores dos parâmetros que maximizam a função de máxima verossimilhança $L\left(x_{1}, x_{2}, \ldots, x_{n} ; \theta\right)$, também maximizam a função $\operatorname{LnL}\left(x_{1}, x_{2}, \ldots, x_{n} ; \theta\right)$.

Seja o logaritmo natural (neperiano) da função de verossimilhança, somente com informações denotadas por $S$, omitindo-se os termos independentes A e $\Psi$ :

$$
\operatorname{LnL}(A, \psi)=-\frac{1}{2} n\left[\operatorname{Ln}\left|\sum\right|+\operatorname{tr}\left(S \Sigma^{-1}\right)\right]
$$

De acordo com Lawley e Maxwell (1971), maximizar $\operatorname{LnL}(A, \psi)$ é equivalente a minimizar $F_{m}$, pois $\operatorname{LnL}(A, \psi)$ é $-\frac{1}{2} n$ vezes $F_{m}$ mais uma função (constante) de observações. 0 índice $m$ indica o número de fatores.

$$
F_{m}=\operatorname{Ln}\left|\sum\right|+\operatorname{tr}\left(S \Sigma^{-1}\right)-\operatorname{Ln}|S|-p
$$

A diferenciação da expressão 9.4.1.3 com respeito a $A$ e $\Psi$ resulta no seguinte sistema de equação matricial:

$$
\begin{aligned}
& \frac{\partial F_{m}}{\partial A}=2 \Sigma^{-1}\left(\sum-S\right) \Sigma^{-1} A \\
& \frac{\partial F_{m}}{\partial \psi}=\operatorname{Diag}\left[\Sigma^{-1}(\Sigma-S) \Sigma^{-1}\right]
\end{aligned}
$$


Lawley e Maxwell (1971) recomendam a utilização de um procedimento em duas etapas para a determinação do mínimo da função $F_{m}$. A primeira etapa consiste em calcular o mínimo condicional para $F_{m}(\psi)$, onde $\Psi$ é uma matriz fixa e sob a condição de que $\Delta=A^{\prime} \psi^{-1} A$ seja diagonal.

Igualando a zero a expressão 9.4.1.4 e pré-multiplicando pela matriz $\sum$, tem-se:

$$
\left(\sum-S\right) \Sigma^{-1} A=\phi
$$

Seja o lema definido pela expressão 9.4.1.7, onde $\Delta=A^{\prime} \psi^{-1} A$, o qual pode ser comprovado pós- multiplicando 9.4.1.7 por $A A^{\prime}+\psi$.

$$
\Sigma^{-1}=\psi^{-1}-\psi^{-1} A(I+\Delta)^{-1} A^{\prime} \psi^{-1}
$$

Pós-multiplicando, 9.4.1.7, pela matriz $A$, resulta:

$$
\sum^{-1} A=\psi^{-1} A(I+\Delta)^{-1}
$$

Logo, pode-se escrever a expressão 9.4.1.6 da seguinte forma:

$$
\left(\sum-S\right) \psi^{-1} A(I+\Delta)^{-1}=\phi
$$

Pós-multiplicando a expressão 9.4.1.9 pela matriz $(I+\Delta)$ e substituindo a matriz $\sum$ por $A A^{\prime}+\psi$, tem-se:

$$
S \psi^{-1} A=A(I+\Delta)
$$

Finalmente, pré-multiplicando 9.4 .1 .10 por $\Psi^{-\frac{1}{2}}$, resulta:

$$
{ }_{p}\left(\psi^{-\frac{1}{2}} S \psi^{-\frac{1}{2}}\right)_{p}\left(\psi^{-\frac{1}{2}} A\right)_{m}={ }_{p}\left(\psi^{-\frac{1}{2}} A\right)_{m}(I+\Delta)_{m}
$$

Impondo a condição de que a matriz $\Delta=A^{\prime} \psi^{-1} A$ seja diagonal, então a expressão 9.4.1.11 é um sistema de equações, donde ${ }_{p}\left(\psi^{-\frac{1}{2}} A\right)_{m}$ é a matriz com colunas iguais aos vetores característicos da matriz $S^{*}={ }_{p}\left(\psi^{-\frac{1}{2}} S \psi^{-\frac{1}{2}}\right)_{p}$, assim como ${ }_{m}(I+\Delta)_{m}$ é a matriz diagonal correspondente aos seus maiores valores característico (próprios).

Seja $\theta=\operatorname{diag}\left(\theta_{1}, \theta_{2}, \cdots, \theta_{m}\right)$ a matriz das $m$ maiores raízes características de $S^{*}=_{p}\left(\psi^{-\frac{1}{2}} S \psi^{-\frac{1}{2}}\right)_{p}$, supondo-as distintas e maiores que a unidade, arranjadas na ordem decrescente e associadas respectivamente à matriz de vetores característicos $W=\left(w_{v 1}, w_{v 2}, \cdots, w_{v m}\right)$, na forma normalizada $\left(W^{\prime} W=I\right)$, satisfazendo $S^{*} W=W \theta$. 
Fazendo-se:

$$
\psi^{-\frac{1}{2}} A_{0}=W(\theta-I)^{\frac{1}{2}} \text { e } \Delta=A_{0}^{\prime} \psi^{-1} A_{0}, \text { obtém-se de 9.4.1.11: }
$$

$$
S^{*} W(\theta-I)^{\frac{1}{2}}=W(\theta-I)^{\frac{1}{2}} \theta
$$

Pós-multiplicando 9.4.1.12 pela matriz diagonal $(\theta-I)^{-\frac{1}{2}}$, tem-se:

$$
S^{*} W=W \theta
$$

Portanto, pode-se escrever que:

$$
A_{0}=\psi^{\frac{1}{2}} W(\theta-I)^{\frac{1}{2}}
$$

Essa conceituação não é válida quando pelo menos dois valores próprios são iguais, pois os correspondentes vetores próprios são indeterminados, e também quando pelo menos uma das raízes característica é menor do que a unidade, o que corresponderia a ter elementos imaginários na matriz $A$ o.

Lawley e Maxwell (1971) mostram que $A_{0}=\psi^{\frac{1}{2}} W(\theta-I)^{\frac{1}{2}}$ minimiza a função 9.4.1.3, dada por: $F_{m}=\operatorname{Ln}|\Sigma|+\operatorname{tr}\left(S \Sigma^{-1}\right)-\operatorname{Ln}|S|-p$. A segunda etapa consiste em determinar $\hat{\psi}$ que a minimize.

Igualando a zero a expressão 9.4.1.5 e usando a identidade 9.4.1.7, tem-se:

$$
\hat{\psi}=\operatorname{diag}\left(S-A_{0} A_{0}^{\prime}\right)
$$

Resume-se que, o método de análise de fatores de máxima verossimilhança, onde $F_{m}(\psi)$ seja mínimo, consiste em calcular:

$$
\begin{gathered}
\hat{A}=\hat{\psi}^{1 / 2} W(\theta-I)^{1 / 2} \\
\hat{\psi}=\operatorname{diag}\left(S-A^{\prime} A\right)
\end{gathered}
$$

Os procedimentos e demonstrações nas obtenções de $\hat{A}$ e $\hat{\psi}$ mostram que é operacionalmente mais fácil minimizar a expressão $F_{m}=\operatorname{Ln}|\Sigma|+\operatorname{tr}\left(S \Sigma^{-1}\right)-\operatorname{Ln}|S|-p$ do que maximizar $\operatorname{Ln} L(A, \psi)=-\frac{1}{2} n\left[\operatorname{Ln}|\Sigma|+\operatorname{tr}\left(S \Sigma^{-1}\right)\right]$.

Usando a restrição de que $A^{\prime} \psi^{-1} A$ seja diagonal, o sistema é resolvido de maneira iterativa. Do ponto de vista computacional, as estimativas de $\hat{A}$ e $\hat{\psi}$ são obtidas por procedimentos iterativos, ressaltando que nem sempre ocorrem convergências. 
Queiroz (1984) desenvolveu um programa computacional, em linguagem FORTRAN, para obter as estimativas de máxima verossimilhança dos pesos fatoriais e variâncias específicas, utilizando o processo de Newton-Raphson, para minimizar a função 9.4.1.3, posta em função das $m$ maiores raízes características da matriz $\psi^{-\frac{1}{2}} S \psi^{-\frac{1}{2}}$.

\subsubsection{TESTE DE HIPÓTESE PARA DETERMINAR O NÚMERO DE FATORES (M) ADEQUADO PARA REPRODUZIR O MODELO (MÉTODO DE MÁXIMA VEROSSIMILHANÇA)}

A mais importante vantagem do método de máxima verossimilhança é verificar se um determinado número de fatores $m$ está adequado para reproduzir o modelo.

Segundo Bartlett (1954), quando $H_{0}$ é verdadeiro a variável:

$$
\begin{gathered}
U_{m}=\left(n-\frac{2 p+5}{6}-\frac{2}{3} m\right)\left(\log _{e}|\hat{\Sigma}|-\log _{e}|S|\right) \text { é aproximadamente } \chi_{(\mathrm{v}, \alpha)}^{2}, \text { onde: } \\
\hat{\Sigma}=A A^{\prime}+\hat{\psi} \quad \mathrm{v}=\frac{1}{2}\left[(\mathrm{p}-\mathrm{m})^{2}-\mathrm{p}-\mathrm{m}\right]
\end{gathered}
$$

\subsubsection{ESTIMAÇÃO DOS ESCORES DOS FATORES}

Os escores fatoriais não são estimados no sentido estatístico usual, pois não são parâmetros do modelo de fatores, mas valores atribuídos às variáveis hipotéticas. Há a necessidade de atribuir um princípio de quadrados mínimos para predizer os escores dos fatores. 0 método de THOMSON (1951) consiste em obter $m$ regressões lineares de $f_{v}$ sobre $x_{v}$ e escolher o estimador linear $\hat{f}_{v}=A x_{v}$ que minimize $E\left(\hat{f}_{v}-f_{v}\right)^{2}$ :

$$
\hat{f}_{v}=\left(I_{m}+A^{\prime} \psi^{-1} A\right)^{-1} A^{\prime} \psi^{-1} x_{v} \quad x_{v}^{\prime}=\left[x_{1}, x_{2}, \ldots, x_{p}\right]: \text { vetor das observações }
$$

Generalizando para a matriz $F$ de ordem $N \times n$ dos escores fatoriais e $X$ a matriz $n \times p$ das observações, tem-se: $F=X \psi^{-1} A\left(I_{m}+A^{\prime} \psi^{-1} A\right)^{-1}$.

\subsubsection{METODOLOGIA DOS ÍNDICES NORMALIZADOS}

De acordo com Queiroz (1984), o índice normalizado é definido como o módulo da resultante da soma vetorial dos escores fatoriais ponderados pela contribuição $\left(p_{j}\right)$ do respectivo fator à comunalidade total. Os valores obtidos são divididos pelo de maior magnitude, implicando numa normalização entre 0 e 1 . A justificativa da aplicação desses índices deve-se ao fato dos fatores serem ortogonais. A Figura 9.4.4.1 mostra a representação gráfica do índice normalizado. A expressão 9.4.4.1 representa o exposto: 


$$
I_{i}=\sqrt{\sum_{j=1}^{m} p_{j} f_{i j}^{2}} \quad i=1,2, \ldots, N
$$

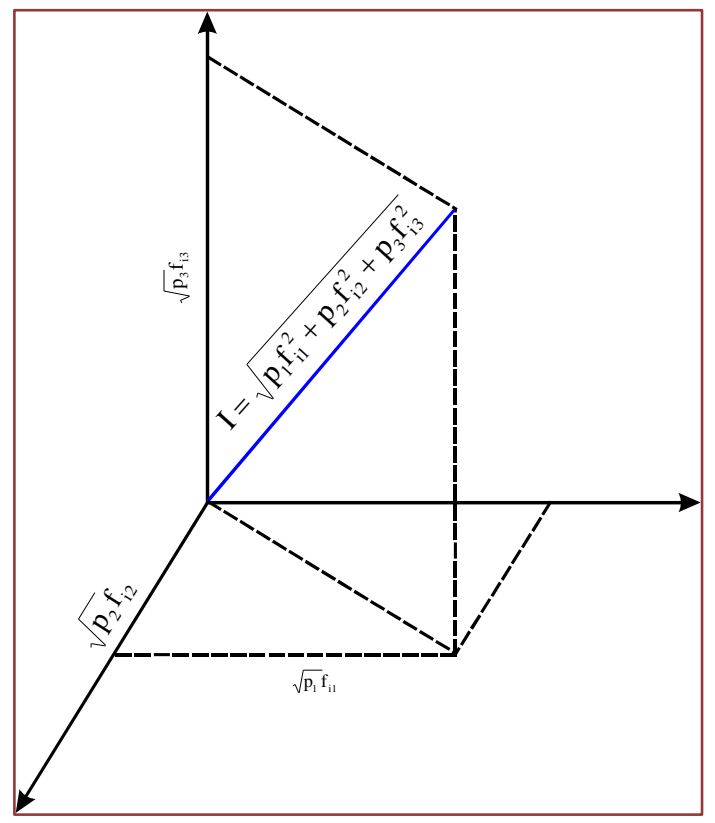

Figura 9.4.4.1 - Representação gráfica do índice normalizado

\subsubsection{ANÁLISE DE FATORES POR COMPONENTES PRINCIPAIS}

São as combinações lineares, componentes principais, das variáveis $x_{1}, x_{2}, \cdots, x_{p}$. Tal que $b_{i j}$ são os valores dos autovetores da matriz de correlações ou de covariâncias.

$$
\begin{aligned}
& Z_{1}=b_{11} x_{1}+b_{12} x_{2}+\ldots+b_{1 p} x_{p} \\
& Z_{2}=b_{21} x_{1}+b_{22} x_{2}+\ldots+b_{2 p} x_{p} \\
& \begin{array}{lllll}
\cdots & \ldots & \ldots & \ldots & \ldots
\end{array} \\
& Z_{p}=b_{p 1} x_{1}+b_{p 2} x_{2}+\ldots+b_{p p} x_{p}
\end{aligned}
$$

A transformação dos valores de $x_{v}$ para os valores de $Z_{v}$ é ortogonal. Manly (2008) cita que o relacionamento inverso é simplesmente:

$$
\begin{aligned}
& x_{1}=b_{11} Z_{1}+b_{21} Z_{2}+\ldots+b_{p 1} Z_{p} \\
& x_{2}=b_{12} Z_{1}+b_{22} Z_{2}+\ldots+b_{p 2} Z_{p} \\
& \ldots \quad \ldots \quad \ldots \ldots \quad \ldots \quad \ldots \\
& x_{p}=b_{1 p} Z_{1}+b_{2 p} Z_{2}+\ldots+b_{p p} Z_{p}
\end{aligned}
$$


0 método considera os $m$ primeiros componentes. 0 valor de $e_{i}$ se torna uma combinação linear dos componentes principais de $Z_{m+1}$ a $Z_{p}$.

$$
\begin{aligned}
& x_{1}=b_{11} Z_{1}+b_{21} Z_{2}+\ldots+b_{m 1} Z_{m}+e_{1} \\
& x_{2}=b_{12} Z_{1}+b_{22} Z_{2}+\ldots+b_{m 2} Z_{m}+e_{2} \\
& \ldots \quad \ldots \quad \ldots \quad \ldots \quad \ldots \quad \ldots \\
& x_{p}=b_{1 p} Z_{1}+b_{2 p} Z_{2}+\ldots+b_{m p} Z_{m}+e_{p}
\end{aligned}
$$

0 próximo passo é escalonar os componentes $Z_{1}, Z_{2}, \cdots, Z_{m}$ para, como exigido pela análise de fatores (AF), ter variâncias unitárias. Manly (2008) cita que a divisão de $Z_{i}$ pelo seu desvio padrão, raiz quadrada do correspondente autovalor da matriz de correlações, por exemplo, escalona os componentes $Z_{1}, Z_{2}, \cdots, Z_{m}$ para terem variâncias unitárias e atender a (AF).

$$
\begin{gathered}
x_{1}=\sqrt{\lambda_{1}} b_{11} F_{1}+\sqrt{\lambda_{2}} b_{21} F_{2}+\ldots+\sqrt{\lambda_{m}} b_{m 1} F_{m}+e_{1} \\
x_{2}=\sqrt{\lambda_{1}} b_{12} F_{1}+\sqrt{\lambda_{2}} b_{22} F_{2}+\ldots+\sqrt{\lambda_{m}} b_{m 2} F_{m}+e_{2} \\
\ldots \quad \ldots \quad \ldots \quad \ldots \ldots \ldots+\cdots \\
x_{p}=\sqrt{\lambda_{1}} b_{1 p} F_{1}+\sqrt{\lambda_{2}} b_{2 p} F_{2}+\ldots+\sqrt{\lambda_{m}} b_{m p} F_{m}+e_{p} \\
F_{i}=\frac{Z_{i}}{\sqrt{\lambda_{i}}} \rightarrow Z_{i}=\sqrt{\lambda_{i}} F_{i} ;
\end{gathered}
$$

$\sqrt{\lambda_{i}}$ : é a raiz quadrada do correspondente autovalor, por exemplo, da matriz de correlação que é o desvio padrão de $Z_{i}$.

Fazendo-se $a_{i j}=\sqrt{\lambda_{j}} b_{j i}$, tem-se o modelo não rotacionado:

$$
\begin{aligned}
& x_{1}=a_{11} F_{1}+a_{12} F_{2}+\ldots+a_{1 m} F_{m}+e_{1} \\
& x_{2}=a_{21} F_{1}+a_{22} F_{2}+\ldots+a_{2 m} F_{m}+e_{2} \\
& x_{p}=a_{p 1} F_{1}+a_{p 2} F_{2}+\ldots+a_{p m} F_{m}+e_{p}
\end{aligned}
$$

Aplicando-se uma determinada rotação, por exemplo, varimax, tem-se uma nova solução:

$$
\begin{aligned}
& x_{1}=g_{11} F_{1}^{*}+g_{12} F_{2}^{*}+\ldots+g_{1 m} F_{m}^{*}+e_{1} \\
& x_{2}=g_{21} F_{1}^{*}+g_{22} F_{2}^{*}+\ldots+g_{2 m} F_{m}^{*}+e_{2} \\
& \text { ․ } \ldots \ldots \ldots \ldots \\
& x_{p}=g_{p 1} F_{1}^{*}+g_{p 2} F_{2}^{*}+\ldots+g_{p m} F_{m}^{*}+e_{p}
\end{aligned}
$$


Os valores do $i$-ésimo fator, não rotacionado, são justamente os valores do $i$-ésimo componente principal após eles terem sido escalonados para terem uma variância igual a um.

De acordo com Manly (2008), os valores dos fatores rotacionados são mais complicados de obter, mas podem ser dados pela equação matricial $F^{*}=X G\left(G^{\prime} G\right)^{-1}$.

$F^{*}$ : é uma matriz $n \times m$ contendo os valores para os $m$ fatores rotacionados em suas colunas;

$X$ : é uma matriz $n \times p$ dos dados originais para as $p$ variáveis das $n$ observações, após transformar as variáveis $x_{1}, x_{2}, \cdots, x_{p}$ para média zero e variância unitária;

$G$ : é uma matriz $p \times m$ das cargas fatoriais rotacionados de acordo com 9.4.5.1.

\subsubsection{MÉTODOS DE ROTAÇÃO DOS FATORES}

Um procedimento importante na interpretação de fatores é a rotação fatorial. Por este método, os eixos de referência dos fatores são rotacionados em torno da origem até que alguma outra posição seja alcançada. 0 caso mais simples é a rotação ortogonal, na qual os eixos são mantidos a 90․ Também é possível rotacionar os eixos sem manter o ângulo de $90^{\circ}$ entre os eixos de referência. Este procedimento de rotação se chama rotação oblíqua.

Hair et al. (2009) citam que o efeito final de rotacionar a matriz fatorial é redistribuir a variância dos primeiros fatores para os últimos com o objetivo de atingir um padrão fatorial mais simples e teoricamente mais significativo. Comentam também que as soluções de fatores não-rotacionadas extraem fatores na ordem de sua importância. 0 primeiro fator tende a ser um fator geral com quase todas as variáveis com carga significativa, explicando a quantia maior de variância. 0 segundo fator e os seguintes são então baseados na quantia residual de variância. Cada fator explica porções sucessivamente menores de variância.

Fávero et al. (2009) citam que dos métodos rotacionais ortogonais, merecem destaque o varimax, o quartimax e o equamax. 0 método varimax minimiza a ocorrência de uma variável possuir altas cargas fatoriais para diferentes fatores. Ele simplifica a interpretação dos fatores. A rotação varimax é o método mais utilizado em análise de fatores.

0 método quartimax objetiva minimizar o número de fatores necessários para explicar cada variável. Corrar et al. (2007) citam que o quartimax tende a concentrar grande parte das variáveis em um único fator, o que pode resultar na produção de estruturas de difícil interpretação.

O equamax é também um método que busca uma combinação dos outros (varimax e quartimax). Fávero et al. (2009) citam que o equamax tem como objetivo simplificar as linhas e as colunas simultaneamente, ou seja, resulta na simplificação dos fatores e das variáveis. 


\subsubsection{ANÁLISE DE FATORES, PELO MÉTODO DOS COMPONENTES PRINCIPAIS,} PARA QUATRO ESPÉCIES (E1, E2, E3, E4) EM RELAÇÃO ÀS VARIÁVEIS (OC1, OC2, OC3, 0C4, 0C5).

Não foi possível, no exemplo, como era o objetivo deste livro, analisar os dados pelo método da máxima verossimilhança, pela sua grande característica de ser um método estatístico, devido ter ocorrido soluções impróprias, tendo em vista o fato de ocorrer variâncias específicas negativas. Usando o comando (proc factor nfactors $=1$ method=ml mineigen $=0.0001$ out=prova3;) no programa desenvolvido, o resultado da análise expediu a mensagem: (ERROR: Communality greater than 1.0), ou seja, comunalidade maior do que 1,0 .

A opção mineigen $=\lambda$ especifica o valor do critério, ou seja, os autovalores menores que $\lambda$ não serão considerados na análise. A tabela C14, do apêndice $C$, apresenta o programa desenvolvido no SAS para as análises de componentes principais e de fatores das espécies.

\section{a) Matriz de correlação das variáveis (0C1, 0C2, 0C3, 0C4, 0C5)}

$\mathrm{Na}$ tabela 9.4.7.1 é mostrado que todas as correlações apresentaram resultados significativos ( $p$-valor $>0,01$ ), sendo as correlações entre as ocasiões diferentes de zero.

Tabela 9.4.7.1 - Matriz de correlação de (OC1, OC2, OC3, OC4, OC5).

\begin{tabular}{|c|c|c|c|c|c|}
\hline \multicolumn{6}{|c|}{ Coeficiente de correlação de Pearson, $N=120$, Prob $>|r|$ Sob H0: Rho=0 } \\
\hline & OC1 & OC2 & OC3 & OC4 & OC5 \\
\hline OC1 & 1,00000 & $\begin{array}{l}0,28874 \\
0,0014\end{array}$ & $\begin{array}{l}0,42286 \\
<0,0001\end{array}$ & $\begin{array}{l}0,28149 \\
0,0018\end{array}$ & $\begin{array}{l}0,26303 \\
0,0037\end{array}$ \\
\hline OC2 & $\begin{array}{l}0,28874 \\
0,0014\end{array}$ & 1,00000 & $\begin{array}{l}0,91060 \\
<0,0001\end{array}$ & $\begin{array}{l}0,96935 \\
<0,0001\end{array}$ & $\begin{array}{l}0,95130 \\
<0,0001\end{array}$ \\
\hline OC3 & $\begin{array}{l}0,42286 \\
<0,0001\end{array}$ & $\begin{array}{l}0,91060 \\
<0,0001\end{array}$ & 1,00000 & $\begin{array}{l}0,95820 \\
<0,0001\end{array}$ & $\begin{array}{l}0,95361 \\
<0,0001\end{array}$ \\
\hline OC4 & $\begin{array}{l}0,28149 \\
0,0018\end{array}$ & $\begin{array}{l}0,96935 \\
<0,0001\end{array}$ & $\begin{array}{l}0,95820 \\
<0,0001\end{array}$ & 1,00000 & $\begin{array}{l}0,99242 \\
<0,0001\end{array}$ \\
\hline 0C5 & $\begin{array}{l}0,26303 \\
0,0037\end{array}$ & $\begin{array}{l}0,95130 \\
<0,0001\end{array}$ & $\begin{array}{l}0,95361 \\
<0,0001\end{array}$ & $\begin{array}{l}0,99242 \\
<0,0001\end{array}$ & 1,00000 \\
\hline
\end{tabular}

\section{b) Autovalores da matriz de correlação}

A tabela 9.4.7.2 mostra que o primeiro autovalor explica 79,99 \% da variação total e o segundo $17,78 \%$, totalizando $97,77 \%$, enquanto que os demais somam apenas $2,23 \%$. 
Tabela 9.4.7.2 - Autovalores da matriz de correlação.

\begin{tabular}{|c|c|c|c|c|c|}
\hline & \multicolumn{5}{|c|}{ Autovalores da matriz de correlação: Total = 5 e Média $=1$} \\
\hline & Autovalor $\left(\lambda_{\mathrm{i}}\right)$ & $\operatorname{Ln}\left(\lambda_{\mathrm{i}}\right)$ & Diferença & Proporção & Acumulado \\
\hline 1 & 3,99949056 & 1,386167 & 3,11034875 & 0,7999 & 0,7999 \\
\hline 2 & 0,88914181 & $-0,117499$ & 0,80876697 & 0,1778 & 0,9777 \\
\hline 3 & 0,08037485 & $-2,521054$ & 0,05405550 & 0,0161 & 0,9938 \\
\hline 4 & 0,02631935 & $-3,637451$ & 0,02164592 & 0,0053 & 0,9991 \\
\hline 5 & 0,00467343 & $-5,365862$ & & 0,0009 & 1,0000 \\
\hline Total & & $-10,255699$ & & & \\
\hline
\end{tabular}

\section{c) Cálculo do KMO e do teste de esfericidade de Bartlett.}

O teste de Kaiser-Meyer-Olkin (Measure of Sampling Adequacy-MSA) mostra o grau de explicação dos dados considerando os fatores encontrados. Um grau de explicação menor que $(0,6)$ indica que os fatores obtidos não conseguiram explicar adequadamente as variações dos dados originais. Os valores da estatística KMO variam de 0 a 1 e segundo Fávero et al. (2009) quanto mais próximo da unidade mais recomendável é a aplicação da análise de fatores. 0 valor obtido de 0,74910545, tabela 9.4.7.3, mostra uma classificação média de acordo com a tabela 9.4.1.

Tabela 9.4.7.3 - Medida de Adequação da Amostragem de Kaiser (MSA).

\begin{tabular}{|c|c|c|c|c|}
\hline \multicolumn{5}{|c|}{ Medida de Adequação da Amostragem de Kaiser: MSA Geral = 0,74910545 } \\
\hline OC1 & OC2 & OC3 & OC4 & OC5 \\
\hline 0,4788306 & 0,79052135 & 0,81752638 & 0,68364487 & 0,78 \\
\hline
\end{tabular}

A matriz Anti-imagem indica o poder de explicação dos fatores em cada uma das variáveis. Os valores de MSA para cada variável, apresentados na tabela 9.4.7.3, correspondem aos valores diagonais dessa matriz. Observa-se que o valor de MSA da variável OC1 é igual a 0,47883069 . Os valores menores que $(0,5)$ indicam pouco poder de explicação dos fatores e podem ser retirados da análise. Entretanto, por tratar-se de um levantamento longitudinal, tal procedimento não seria recomendável.

De acordo com Mingoti (2005), seja a estatística T de Bartlett para testar a esfericidade da matriz de correlação, que sob $H_{0}$, para grandes amostras de tamanho $N$, tem uma distribuição aproximadamente qui-quadrado com $v=1 / 2 p(p-1)$ graus de liberdade.

$$
\begin{gathered}
T=-\left[N-\frac{1}{6}(2 p+11)\right]\left(\sum_{i=1}^{p} \lambda_{i}\right) \\
T=-\left[120-\frac{1}{6}(2 \times 5+11)\right] \times 10,255699=1.194,7886 \\
v=1 / 2 p(p-1)=10 \quad \chi_{10 ; 0,01}=23,2
\end{gathered}
$$


Conclusão: rejeita-se a hipótese de que a matriz de correlação seja esférica.

\section{d) Pesos fatoriais padrão e rotacionados pelo método Varimax}

Os valores dos pesos do primeiro fator padrão (Factor1), tabela 9.4.7.4, mostram que o fator1 é explicado pelas ocasiões OC2, OC3, OC4 e OC5, enquanto que o segundo fator (Factor2), expressa a ocasião 0C1. Em relação aos fatores rotacionados, pelo método de rotação varimax, verifica-se a mesma interpretação dos fatores padrão.

Tabela 9.4.74 - Pesos fatoriais padrão e rotacionado (Varimax)

\begin{tabular}{|c|c|c|c|c|c|}
\hline & \multicolumn{2}{|c|}{ Fator padrão } & \multicolumn{2}{|c|}{ Fator padrão rotacionado } & \multirow{2}{*}{$\begin{array}{c}\text { Estimativas } \\
\text { comunalidade }\end{array}$} \\
\hline & Factor1 & Factor2 & Factor1 & Factor2 & \\
\hline OC1 & 0,40982 & 0,91155 & 0,15523 & 0,98731 & 0,99888264 \\
\hline OC2 & 0,96700 & $-0,12314$ & 0,96529 & 0,13593 & 0,95025738 \\
\hline OC3 & 0,97882 & 0,03120 & 0,93603 & 0,28794 & 0,95906634 \\
\hline OC4 & 0,98816 & $-0,13644$ & 0,98920 & 0,12868 & 0,99506893 \\
\hline OC5 & 0,98076 & $-0,15317$ & 0,98647 & 0,11059 & 0,98535709 \\
\hline Total & & & & & 4,88863238 \\
\hline \multicolumn{6}{|c|}{ Variância explicada por cada fator } \\
\hline & 3,999490 & 0,889141 & 3,78367 & 1,1049585 & \\
\hline
\end{tabular}

A figura 9.4.7.1 apresenta, e de modo similar ao que ocorreu nos resultados das análises multivariadas já realizadas, uma discriminação dos dados, mostrando um perfeito agrupamento das espécies em dois clusters, o primeiro grupo formado pelas espécies E1 e E2, e o segundo pelas espécies E3 e E4. Estes resultados são os mesmos apresentados na figura 8.1.1.3, capítulo 8, seção 8.1, item g, dendrograma do agrupamento de Ward, onde as espécies E1 e E2 formam um grupo distinto do grupo das espécies E3 e E4.

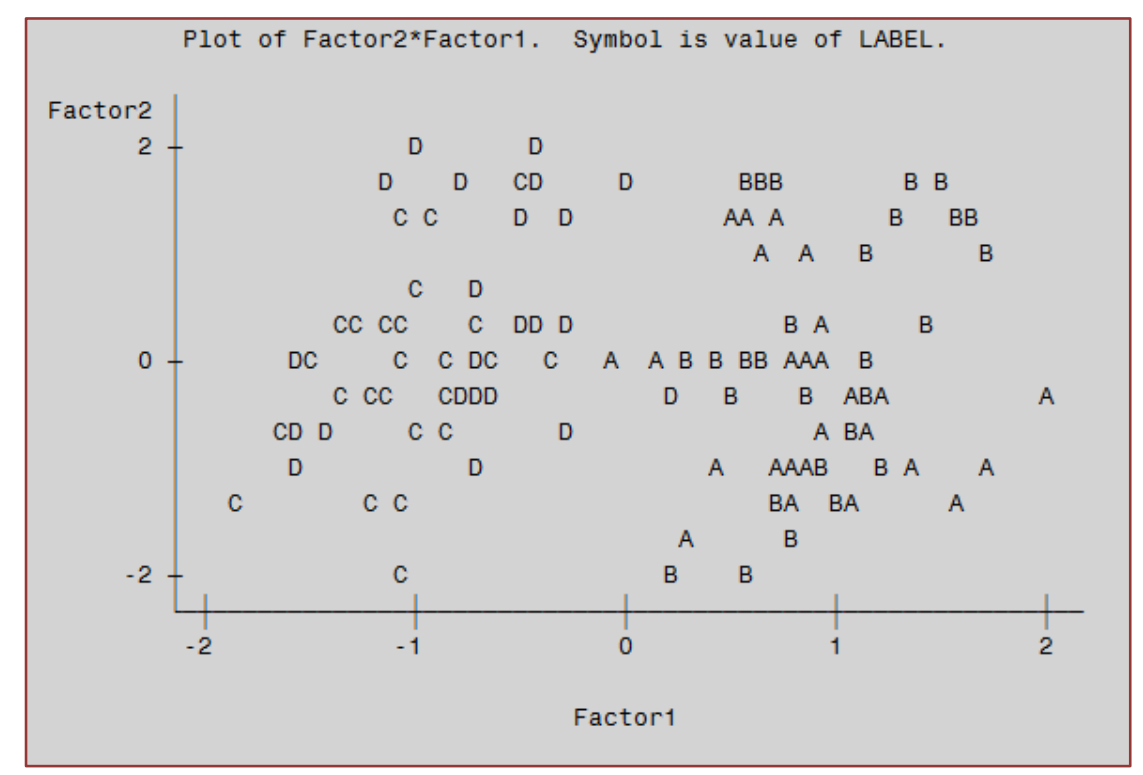

Figura 9.4.7.1 - Distribuição das espécies, ( $\mathrm{A}=\mathrm{E} 1, \mathrm{~B}=\mathrm{E} 2, \mathrm{C}=\mathrm{E} 3, \mathrm{D}=\mathrm{E} 4)$, de acordo com os fatores 1 e 2 (Factor Pattern). 
Os valores dos índices normalizados podem ser obtidos conforme a expressão 9.4.4.1, mas considerando que a estrutura do banco está baseada nas variáveis ocasiões, e que estas não apresentam um interesse prático na montagem de um mapa de pósestratificação multidimensional, então os valores dos índices não foram calculados. Entretanto, caso fosse aplicada essa metodologia, a equação considerando os fatores factor1 e factor2 teria a seguinte formulação:

$$
\begin{gathered}
I_{i}=\sqrt{\sum_{j=1}^{m} p_{j} f_{j i}^{2}}=\sqrt{\left(0,8181 \times f_{1 i}^{2}+0,1819 \times f_{2 i}^{2}\right)} \\
p_{1}=\frac{3,9994906}{3,9994906+0,8891418}=0,8181 \quad p_{2}=\frac{0,8891418}{3,9994906+0,8891418}=0,1819
\end{gathered}
$$

$f_{1 i}$ : valor de factor1 obtido na $i$-ésima parcela;

$f_{2 i}$ : valor de factor 2 obtido na i-ésima parcela;

$j=1,2, \cdots, m=2$ e $i=1,2, \cdots, N=120$.

Queiroz (1984) efetuou a análise de fatores, pelo método da máxima verossimilhança, usando o modulo da resultante da soma vetorial dos escores dos fatores, na pósestratificação multidimensional de uma área localizada na Floresta Nacional do Tapajós, no Estado do Pará. 0 estudo envolveu seis variáveis, três dendrométricas e três referentes ao potencial madeireiro ocorrente de três classes de espécies de acordo com a sua importância econômica. De acordo com o teste de verificação da suficiência do número de fatores, quatro fatores foram aceitáveis para reprodução do modelo. 0 fator2 evidenciou a distribuição volumétrica das espécies pertencentes ao grupo ou classe I, o fator3 explicou a ocorrência volumétrica das espécies da classe II, enquanto que o fator4 caracterizou a distribuição do potencial madeireiro do grupo de espécies III. Esses resultados propiciaram a elaboração de um mapa com a localização (estratificação) desses três grupos de espécies, o que possibilitaria, por exemplo, a elaboração do planejamento da exploração desses tipos florestais. 


\section{Considerações Finais}

O foco principal deste livro foi discutir técnicas de análise de perfil de inventário florestal contínuo. Contudo, considerando a enorme importância da análise multivariada, no contexto da disciplina Inventario Florestal, principalmente em florestas naturais, optou-se em acrescentar mais um capítulo abrangendo algumas noções sobre os métodos de componentes principais, análise discriminante, análise de correlação canônica e análise de fatores. A execução do Inventário Florestal Nacional (IFN) do Brasil e as necessidades de informações multidimensionais, na elaboração de planos de manejo florestal, exigem dos estudantes uma formação mais aprimorada em métodos estatísticos, tendo em vista que, além das variáveis dendrométricas e florísticas, observações sobre as condições edafoclimáticas, em especial sobre as características do solo, incluindo o uso da terra, assim como as informações socioeconômicas da região e outras variáveis de interesse coletadas, resultam na necessidade de analisar um diversificado banco de dados multidimensional.

Neste livro, as observações analisadas foram simuladas procurando uma adequação com a realidade de uma floresta natural, devido à impossibilidade de acessar um banco de dados compatível com as exigências da modelagem matemática requerida pelos levantamentos longitudinais, e assim atender as especificações demandadas pelos exemplos apresentados nos capítulos 7 e 8 . Mas, por outro lado, considerando o caráter didático a que se propõe esta obra, acredita-se que os exemplos citados com os quatro métodos de manejo e as quatro espécies, atenderam aos objetivos estabelecidos.

No entanto, apesar de os dados utilizados não apresentarem características ideais, para produzir exercícios práticos mais apropriados sobre as técnicas multidimensionais de componentes principais, análise de fatores, análise de correlação canônica e análise discriminante, pois foram estruturados para realizar as análises de perfil dos exemplos citados nos capítulos 7 e 8, os exercícios apresentados no capítulo 9, considerando o caráter didático proposto neste livro, abrangendo os quatro métodos de manejo e as quatro espécies, atenderam aos objetivos propostos, pois resultados interessantes ocorreram nas análises.

As análises foram realizadas por meio de programas desenvolvidos usando a linguagem de programação de análise estatística SAS, dadas às facilidades operacionais e à amplitude nas análises oferecidas por este sistema.

Espera-se que com o desenvolvimento do Inventário Florestal Nacional (IFN-BR) e o avanço do manejo florestal em concessões florestais nas florestas públicas, e outras iniciativas no campo dos inventários florestais contínuos, essas contribuições possam ser úteis, assim como contribuam para o aprendizado dos estudantes e profissionais que labutam na área de inventário florestal. 


\section{REFERÊNCIAS BIBLIOGRÁFICAS}

ANDRADE, D. F. \& SINGER, J. M. Análise de dados longitudinais. VII Simpósio Nacional de Probabilidade e Estatística. Campinas - SP, 1986. 106 p.

ANDERSON, T.W. An introduction to multivariate Statiscal Analysis. New York: John Wiley e Sons, 1958. 374 p.

BARTLETT, M.S. Factor analysis in psychology as a statistician see it, in essays on probability and Statistics. New York, Wiley. 1962.

BARTLETT, M.S. 'A note on the multiplying factor for various $\chi^{2}$ approximations', J. R. Static. Soc., B 16, 296-298. 1954.

BOX, G. E. P. A general distribution theory for a class of likelihood criteria. Biometrika. 36, 317-346. 1949.

BOX, G. E. P. Problems in the analysis of growth and wear curves. Biometrics, 6: 362389. 1950.

BOX, G. E. P. Some theorems on quadratic forms applied to the study of analysis of variance problems. Annals of the Mathematical Statistics. 25, 290-302, $484-498$. 1954.

COLLIER, R.O.; BAKER, F.B.; MANDEVILLE, G.R.; HAYES,T.F. Estimates of test size for several test procedures based on convention variance ratios in the repeated measures design. Psychometrika, 32, 339-353. 1967.

COOK, R. D. \& TSAI, C. L. Residual in nonlinear regression. Biometrika. London, v.72, p. 23-29, 1985.

CORRAR, L. J.; PAULO, E.; DIAS FILHO, J. M.(Coordenadores). Análise multivariada para os cursos de administração, ciências contábeis e economia. São Paulo: Atlas, 2007.

DRAPER, N. R. \& SMITH, H. Applied regression analysis. 2nd. ed. New York: John Wiley, 1981. $709 \mathrm{p}$.

DUARTE, J. B. Introdução às inversas generalizadas de matrizes de aplicação comum em estatística experimental. Texto didático produzido durante o curso de Álgebra de Matrizes, na Escola Superior de Agricultura 'Luiz de Queiroz' / USP, Piracicaba, SP, em 1996.

DRAPER, N. R \& SMITH, H. Applied regression analysis. 2. ed. New York: John Wiley \& Sons, 1981. 709 p.

FARHADIAN, R. \& ASADIAN, N. On the Helmert and application in stochastic processes. International Journal of Mathematics and Computer Science, 12, n. 2, 107-115. 2017. 
FÁVERO, L. P.; BELFIORE, P.; SILVA, F. L.; CHAM, B. L. Análise de dados: modelagem multivariada para tomada de decisão. São Paulo: Campus, 2009. 646 p.

FERREIRA, F. F. Recursos computacionais utilizando R. Departamento de ciências exatas da Universidade Federal de Lavras. Lavras-Minas Gerais- Brasil, 2013. 240 p.

FERREIRA, F. F. Estatística multivariada. 2. ed. rev. e ampliada- Lavras: Ed. UFLA, 2011. $675 \mathrm{p}$.

FISHER, R. A. The use of multiple measurements in taxonomic problems, Annals of Eugenics, 7 (1936),179-188.

FLEMMING, D. M \& GONÇALVES, M. B. Cálculo A: Funções, limites, derivação e integração. 6. ed. São Paulo: Pearson Prentice Hall, 2007. 464 p.

GALLANT, A. R. Nonlinear statistical models. New York: John Wiley, 1987. 611p.

GONÇALVES, M. B \& FLEMMING, D. M. Cálculo B: Funções de várias variáveis, integrais múltiplas, integrais curvilíneas e de superfície. 2. Ed. São Paulo: Pearson, Prentice Hall. 2007. 435 p.

GEISSER, S. \& GREENHOUSE, S. W. An extension of Box's results on the use of F distribution in multivariate analysis. Ann. Math. Statist., 29, 855-891. 1958.

GODOI, C. R. M. Análise estatística multidimensional. 1ำ Simpósio de Estatística Aplicada à Experimentação Agronômica. 3ํㅡㄹ Reunião Anual da Região Brasileira da Sociedade Internacional de Biometria. Piracicaba- São Paulo. 1985.

GOMES, F. P. \& NOGUEIRA, I. R. Análise matemática. 2. ed. Piracicaba: Augegraf: produções gráficas, 1980.371 P.

GRAYBIL, F. A. Theory and application of linear model. Belmont: Duxbury Press, 1976. $704 \mathrm{p}$.

GREENHOUSE, S. W. \& GEISSER, S. On methods in the analysis of profile data. Psychometrika, 24: 95-112, 1959.

GUJARATI, D. \& PORTER, D. C. Econometria básica. 5. ed. Porto Alegre: AMGH. 2011. $924 \mathrm{p}$.

HAIR Jr., J. F.; ANDRESON, R. E.; TATHAM, R. L.; BLACK, W. C. Análise multivariada de dados. 6. Ed. São Paulo: Bookman, 2009. 688 p.

HOFFMANN, R. Análise estatística de relações lineares e não lineares. 1. ed. São Paulo: LP-Books, 2011. 270 p.

HOTELling, H. The most predictable criterion. Journal of Educational Psychology, 26,139 -142, 1935. 
HUYNH, H. \& FELDT, L.S. Estimation of the Box correction for degrees of freedom from sample data in randomized block and split-plot designs. J. Ed. Statist., 1, 69-82. 1976.

HUYNH, H. \& FELDT, L.S. Conditions under which means square rations in repeated measurements designs have exact F-distributions. Journal of the American Statistical Association, Boston, v.65, n. 322, 1582-1589. 1970.

IEMMA, A. F. Valores singulares de matrizes uniformes. Ciência e Natura, 4: 21 - 26. 1982.

JOHNSON, R.A. \& WICHERN, D.W. Applied multivariate analysis. New Jersey: Prantice Hall, 816 p. 1992.

KANKAINEN, A; TASKINEN, S.; OJA, H. On Mardia's test of multinormality. Mathematics Subject Classification. 1991.

KENDALL, M. G. Factor analysis as statistical techniques. J. R. Statist. Soc., B, 22: 60-73, 1950.

KOIZUMI, K; SUMIKAWA, T; PAVLENKO, T. Measures of multivariate skewness and kurtosis in high-dimensional framework. Sut Journal of mathematics, v.50, n. 2, 483511. 2014.

KOOLO, T. Multivariate skewness and kurtosis measures with an application in ICA. Journal of Multivariate Analysis. 99: 2328-2338. 2008.

KORKMAZ, S.; GOKSULUK, D.; ZARARSIZ, G. MVN: An R package for assessing multivariate normality. The R Journal. Vol. 6/2, 2014.

LACHENBRUCH, P. A. Discriminant analysis. Hafner Press, Division of Macmillan Publishing Co., Inc. 1975.

LANCASTER, H. O. The Helmert matrices. Am. Math. Monthly, 72 (1): 4-12, 1965.

LAWLEY, D.N. The estimation of factor loading by the method of maximum likelihood. Proceedings of the Royal Society of Edinburger, 60: 64-82. 1940.

LAWLEY, D. N. \& MAXWELL, A. E. Factor analysis as statistical method. Butterworths, London. 1971.

LEAL, M. L. S. Análise de dados de experimentos com medidas repetidas. Brasília. (Mestrado - Universidade de Brasília). 99 p. 1979.

MARCHAND, L. Multiplicadores de Lagrange: uma aplicação em problemas de otimização global restrita. Trabalho de Conclusão de Curso, Matemática Aplicada Bacharelado. Instituto de Matemática, Estatística e Física da Universidade Federal do Rio Grande. 2016. 
MAHALANOBIS, P. C. On the generalized distance in statistics. Proc. Nat. Inst. Sci., India,2: 49-55, 1936.

MANLY, B. F. J. Métodos estatísticos multivariados: uma introdução. Tradução: Sara Landa Carmona- 3. Ed.- Porto Alegre, Bookman. 229p. 2008.

MARDIA, K. V. Measures of multivariate skewness and kurtosis with applications. Biometrika, v. 57, p. 519 - 530. 1970.

MARDIA, K. V. Applications of some measures of multivariate skewness and kurtosis in testing normality and robustness studies. Sankhyã, Ser B 36(2), 115-128. 1974.

MARDIA, K. V. Mardia, Tests of univariate and multivariate normality. In: P.R. Krishnaiah (ed.), Handbook of Statistics, vol 1, 279-320, North Holland, 1980.

MARTINS, M. C. Análise de resíduo projetado em modelos lineares de regressão: uma aplicação em crescimento de frutos. 90 p. Tese de mestrado. ESALQ-USP. Piracicaba-SP. 2013.

MAUCHLY, J. W. Significance Test for Sphericity of a Normal n-Variate Distribution. The Annals of Mathematical Statistics. Baltimore, v.11, n.6, p. 204-209, 1940.

MAROCO, J. Análise estatística com utilização do SPSS. 3. ed. Lisboa: Edições Sílabo, 2007.

MELO, J. M. Proposta de um teste exato para avaliar a normalidade multivariada baseado em uma transformação $t$ de Student. 86 p. Dissertação (mestrado acadêmico). Departamento de Ciências Exatas, Universidade Federal de Lavras: UFLA. Lavras, 2016.

MINGOTI, S.A. Análise de Dados Através de Métodos de Estatística Multivariada: Uma abordagem aplicada. Belo Horizonte: Editora UFMG, 2005.

MORRISON, D.F., Multivariate Statiscal Methods. 2. ed. Tokyo, McGraw-Hill, Inc. 1976. $415 \mathrm{p}$.

NETER, J.; KUTNER, M. H.; NACHTSHEIM, C. J. \& WASSERMAN, W. Applied linear statistical models. 4. ed. USA, Richard D. Irwin, 1996. 1408 p.

PISKOUNOV, N. Cálculo diferencial e integral. 7. ed. Porto, Lopes da Silva, v. I, 1979, $515 \mathrm{p}$.

PONTES (2005), A. C. F. Análise de variância multivariada com a utilização de testes não paramétricos e componentes principais baseados em matrizes de postos. $106 \mathrm{p}$. Tese (Doutorado em Estatística e Experimentação Agronômica)-Escola Superior de Agricultura Luiz de Queiroz/USP, Piracicaba, 2005.

PRUDENTE, A. A. Modelos não lineares de regressão: alguns aspectos de teoria assintótica. 108 p. Dissertação de mestrado, Departamento de Estatística e informática. Universidade Federal Rural de Pernambuco, Recife. 2009. 
QUEIROZ, W. T. Análise de fatores ("Factor analysis") pelo método de máxima verossimilhança: aplicação ao estudo de estrutura de florestas tropicais. 112 p. Tese (Doutorado em Estatística e Experimentação Agronômica) - Escola Superior de Agricultura Luiz de Queiroz/USP, Piracicaba, 1984.

QUEIROZ, W. T. Amostragem em Inventário Florestal. Belém: Universidade Federal Rural da Amazônia, 2012. 441 p.

QUEIROZ, W. T. Análise de Perfil Multivariada de um Inventário Contínuo com Parcelas Permanentes. Atualidades em Mensuração florestal. Ed. Ana Paula Dalla Corte [et al.] . 1. Ed. - Curitiba, 2014. 407 p.

QUEIROZ, W. T.; SILVA, M. L.; JARDIM, F. C. S.; VALE, R.; VALENTE, M. D. R.; PINHEIRO, J. G. Índice de valor de importância de espécies arbóreas da Floresta Nacional do Tapajós via análise de componentes principais e de fatores. Ciência Florestal-UFSM, v. 27, p. 4759, 2017.

RAO, C. R. Linear statistical inference and its applications. New York: John Wiley, 1973. $522 \mathrm{p}$.

RATKOWSKY, D. A. Handbook of nonlinear regression models. New York: Marcel Dekker, 1990. 241 p.

REGAZZI, A. J. Teste para verificar a igualdade de parâmetros e a identidade de modelos de regressão não linear. Revista Ceres, v.50, n. 287, p. 9-26, 2003.

REGAZZI, A. J. \& SILVA, C. H. O. Teste para verificar a igualdade de parâmetros e a identidade de modelos de regressão não linear. I. Dados no delineamento inteiramente casualizado. Rev. Mat. Estat., São Paulo, v.22, n.3, p.33-45, 2004

ROY, S.N. On a heuristic method of test construction and its uses in multivariate analysis. The Annals of Mathematical Statistics. Baltimore, 24: 220-238, 1953.

ROY, S.N. Some aspects of multivariate analysis, John Wiley \& Sons, Inc., New York. 1957.

SAS INSTITUTE INC. SAS/STAT. Version 9.00, Cary, NC, USA, 2002.

SEARLE, S. R. 1971. Linear models. New York: John Wiley \& Sons. 420 p.

SILVA, R. B. V. Extensão do teste de normalidade de Shapiro-Francia para o caso multivariado. 59p. Tese (Doutorado em Estatística e Experimentação Agropecuária), Departamento de Ciências Exatas, Universidade Federal de Lavras: UFLA. Lavras, 2009.

SPEARMAN, C. General intelligence objectively determined and measured. Amer. J. Psychol.,15: 201-293. 
SOUZA, G. S. Introdução aos modelos de regressão linear e não linear. Brasília, DF: Embrapa, 1998. 505 p.

STEEL, R. G. D. \& TORRIE, J. H. Principles and procedures of statistics: with special reference to the biological sciences. New York: McGraw-Hill, 1960. 481 p.

STOLOFF, P.H. Correcting for heterogeneity of covariance for repeated measures designs of the analysis of variance. Educ. and Psych. Measurements, 30, 909-924. 1970.

TATSUOKA, M.M. Multivariate analysis: techniques for educational and psychological research. New York, John Wiley e Sons. 310 p.

THOMSON, G.H. The factorial analysis of human ability. London, University of London. 1951.

WELCH, B. L. 'Note on discriminant functions ', Biometrika, 31, 218-220. 1939.

WILKS, S. S. Sample criteria for equality of means, equality of variances and equality of covariances in a normal multivariate distribution. The Annals Mathematical Statistics, London, 17: 257-281, 1946.

YARED, J. A. G. Comportamento e viabilidade de procedimento de Cordia alliodora (Ruiz e pav.) Oken, no Planalto do Tapajós - Belterra, PA. 1983. 108 p. (Dissertação Mestrado em Engenharia Florestal)-Escola Superior de Agricultura Luiz de Queiroz, Piracicaba, 1983. 


\title{
Apêndice A
}

\author{
Distribuições Estatísticas
}

Tabela A1 - Distribuição " $t$ ” de Student.

Tabela A2 - Distribuição F de Snedecor para $\alpha=5 \%$.

Tabela A3 - Distribuição F de Snedecor para $\alpha=1 \%$.

Tabela A4 - Distribuição de $\chi^{2}$. 


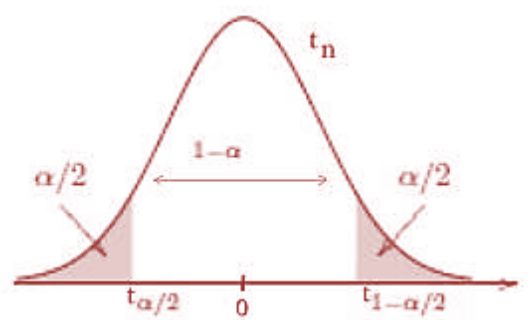

Tabela A1 - Distribuição “ $t$ ” de Student.

\begin{tabular}{|c|c|c|c|c|c|c|c|}
\hline & 0,50 & 0,25 & 0,10 & 0,05 & 0,025 & 0,01 & 0,005 \\
\hline 1 & 1,00000 & 2,4142 & 6,3138 & 12,706 & 25,542 & 63,657 & 127,32 \\
\hline 2 & 0,81650 & 1,6036 & 2,9200 & 4,3127 & 6,2053 & 9,9248 & 14,089 \\
\hline 3 & 0,76489 & 1,4226 & 2,3534 & 3,1825 & 4,1765 & 5,8409 & 7,4533 \\
\hline 4 & 0,74070 & 1,3444 & 2,1318 & 2,7764 & 3,4954 & 4,6041 & 5,5976 \\
\hline 5 & 0,72669 & 1,3009 & 2,0150 & 2,5706 & 3,1634 & 4,0321 & 4,7733 \\
\hline 6 & 0,71756 & 1,2733 & 1,9432 & 2,4469 & 2,9687 & 3,7074 & 4,3168 \\
\hline 7 & 0,71114 & 1,2543 & 1,8946 & 2,3646 & 2,8412 & 3,4995 & 4,0293 \\
\hline 8 & 0,70639 & 1,2403 & 1,8595 & 2,3060 & 2,7515 & 3,3554 & 3,8325 \\
\hline 9 & 0,70272 & 1,2297 & 1,8331 & 2,2622 & 2,6850 & 3,2498 & 3,6897 \\
\hline 10 & 0,69981 & 1,2213 & 1,8125 & 2,2281 & 2,6338 & 3,1693 & 3,5814 \\
\hline 11 & 0,69745 & 1,2145 & 1,7959 & 2,2010 & 2,5931 & 3,1058 & 3,4966 \\
\hline 12 & 0,69548 & 1,2089 & 1,7823 & 2,1788 & 2,5600 & 3,9545 & 3,4284 \\
\hline 13 & 0,69384 & 1,2041 & 1,7709 & 2,1604 & 2,5326 & 3,0123 & 3,3725 \\
\hline 14 & 0,69200 & 1,2001 & 1,7613 & 2,1448 & 2,5096 & 5,9768 & 3,3257 \\
\hline 15 & 0,69120 & 1,1967 & 1,7530 & 2,1315 & 2,4899 & 2,9467 & 3,2860 \\
\hline 16 & 0,69013 & 1,1937 & 1,7459 & 2,1199 & 2,4729 & 2,9208 & 3,2520 \\
\hline 17 & 0,68919 & 1,1910 & 1,7396 & 2,1098 & 2,4581 & 2,8982 & 3,2225 \\
\hline 18 & 0,68837 & 1,1887 & 1,7341 & 2,1009 & 2,4450 & 2,8784 & 3,1966 \\
\hline 19 & 0,68763 & 1,1866 & 1,7291 & 2,0930 & 2,4334 & 2,8609 & 3,1737 \\
\hline 20 & 0,68696 & 1,1848 & 1,7247 & 2,0860 & 2,4231 & 2,8453 & 3,1534 \\
\hline 21 & 0,68635 & 1,1831 & 1,7207 & 2,0796 & 2,4138 & 2,8314 & 3,1352 \\
\hline 22 & 0,68580 & 1,1816 & 1,7171 & 2,0739 & 2,4055 & 2,8188 & 3,1188 \\
\hline 23 & 0,68531 & 1,1802 & 1,7139 & 2,0687 & 2,3979 & 2,8073 & 3,1040 \\
\hline 24 & 0,68485 & 1,1789 & 1,7109 & 2,0639 & 2,3910 & 2,7969 & 3,0905 \\
\hline 25 & 0,68443 & 1,1777 & 1,7081 & 2,0595 & 2,3846 & 2,7874 & 3,0782 \\
\hline 26 & 0,68405 & 1,1766 & 1,7056 & 2,0555 & 2,3788 & 2,7787 & 3,0669 \\
\hline 27 & 0,68370 & 1,1757 & 1,7033 & 2,0518 & 2,3734 & 2,7707 & 3,0565 \\
\hline 28 & 0,68335 & 1,1748 & 1,7011 & 2,0484 & 2,3685 & 2,7633 & 3,0469 \\
\hline 29 & 0,68304 & 1,1739 & 1,6991 & 2,0452 & 2,3638 & 2,7564 & 3,0380 \\
\hline 30 & 0,68276 & 1,1731 & 1,6973 & 2,0423 & 2,3596 & 2,7500 & 3,0298 \\
\hline 40 & 0,68066 & 1,1673 & 1,6839 & 2,0211 & 2,3289 & 2,7045 & 2,9712 \\
\hline 60 & 0,67862 & 1,1616 & 1,6707 & 2,0003 & 2,2991 & 2,6603 & 2,9146 \\
\hline 120 & 0,67656 & 1,1559 & 1,6577 & 1,9799 & 2,2699 & 2,6174 & 2,8599 \\
\hline$\infty$ & 0,67449 & 1,1503 & 1,6449 & 1,9600 & 2,2414 & 2,5758 & 2,8070 \\
\hline
\end{tabular}




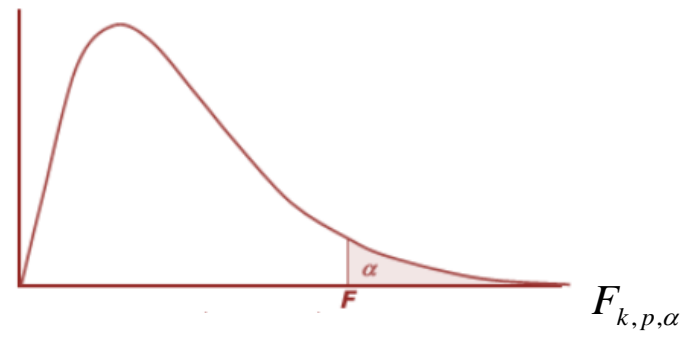

Tabela A2 - Distribuição $F$ de Snedecor para $\alpha=5 \%$.

\begin{tabular}{|c|c|c|c|c|c|c|c|c|c|c|c|c|c|}
\hline e & 1 & 2 & 3 & 4 & 5 & 6 & 7 & 8 & 9 & 10 & 20 & 30 & 120 \\
\hline 1 & 161,4 & 199,5 & 215,7 & 224,6 & 230,2 & 234,0 & 236,8 & 238,9 & 240,5 & 241,9 & 248,0 & 250,1 & 253,3 \\
\hline 2 & 18,51 & 19,00 & 9,16 & 19,25 & 19,30 & 19,33 & 19,35 & 19,37 & 19,38 & 19,40 & 19,45 & 19,46 & 19,49 \\
\hline 3 & 10,13 & 9,55 & 9,28 & 9,12 & 9,01 & 8,94 & 8,89 & 8,85 & 8,81 & 8,79 & 8,66 & 8,62 & 8,55 \\
\hline 4 & 7,71 & 6,94 & 6,59 & 6,39 & 6,26 & 6,16 & 6,09 & 6,04 & 6,00 & 5,96 & 5,80 & 5,75 & 5,66 \\
\hline 5 & 6,61 & 5,79 & 5,41 & 5,19 & 5,05 & 4,95 & 4,88 & 4,82 & 4,77 & 4,74 & 4,56 & 4,50 & 4,40 \\
\hline 6 & 5,99 & 5,14 & 4,76 & 4,53 & 4,39 & 4,28 & 4,21 & 4,15 & 4,10 & 4,06 & 3,87 & 3,81 & 3,70 \\
\hline 7 & 5,59 & 4,74 & 4,35 & 4,12 & 3,97 & 3,87 & 3,79 & 3,73 & 3,68 & 3,64 & 3,44 & 3,38 & 3,27 \\
\hline 8 & 5,32 & 4,46 & 4,07 & 3,84 & 3,69 & 3,58 & 3,50 & 3,44 & 3,39 & 3,35 & 3,15 & 3,08 & 2,97 \\
\hline 9 & 5,12 & 4,26 & 3,86 & 3,63 & 3,48 & 3,37 & 3,29 & 3,23 & 3,18 & 3,14 & 2,94 & 2,86 & 2,75 \\
\hline 10 & 4,96 & 4,10 & 3,71 & 3,48 & 3,33 & 3,22 & 3,14 & 4,07 & 3,02 & 2,98 & 2,77 & 2,70 & 2,58 \\
\hline 11 & 4,84 & 3,98 & 3,59 & 3,36 & 3,20 & 3,09 & 3,01 & 2,95 & 2,90 & 2,85 & 2,65 & 2,57 & 2,45 \\
\hline 12 & 4,75 & 3,89 & 3,49 & 3,26 & & 3,00 & 2,90 & 2,85 & 2,80 & 2,75 &, 54 & 47 & 2,34 \\
\hline 13 & 4,67 & 3,81 & 3,41 & 3,18 & 3,03 & 2,92 & 2,83 & 2,77 & 2,71 & 2,67 & 2,46 & 2,38 & 2,25 \\
\hline 14 & 4,60 & 3,74 & 3,34 & 3,11 & 2,96 & 2,85 & 2,76 & 2,70 & 2,65 & 2,60 & 2,39 & 3,31 & 2,18 \\
\hline 15 & 4,54 & 3,68 & 3,29 & 3,06 & 2,90 & 2,79 & 2,71 & 2,64 & 2,59 & 2,54 & 2,33 & 2,25 & 2,11 \\
\hline 16 & 4,49 & 3,63 & 3,24 & 3,01 & 2,85 & 2,74 & 2,66 & 2,59 & 2,54 & 2,49 & 2,28 & 2,19 & 2,06 \\
\hline 17 & 4,45 & 3,59 & 3,20 & 2,96 & 2,81 & 2,70 & 2,61 & 2,55 & 2,49 & 2,45 & 2,23 & 2,15 & 2,01 \\
\hline 18 & 4,41 & 3,55 & 3,16 & 2,93 & 2,77 & 2,66 & 2,58 & 2,51 & 2,46 & 2,41 & 2,19 & 2,11 & 1,97 \\
\hline 19 & 4,38 & 3,52 & 3,13 & 2,90 & 2,74 & 2,63 & 2,54 & 2,48 & 2,42 & 2,38 & 2,16 & 2,07 & 1,93 \\
\hline 20 & 4,35 & 3,49 & 3,10 & 2,87 & 2,71 & 2,60 & 2,51 & 2,45 & 2,39 & 2,35 & 2,12 & 2,04 & 1,90 \\
\hline 21 & 4,32 & 3,47 & 3,07 & 2,84 & 2,68 & 2,57 & 2,49 & 2,42 & 2,37 & 2,32 & 2,10 & 2,01 & 1,87 \\
\hline 22 & 4,30 & 3,44 & 3,05 & 2,82 & 2,66 & 2,55 & 2,46 & 2,40 & 2,34 & 2,30 & 2,07 & 1,98 & 1,84 \\
\hline 23 & 4,28 & 3,42 & 3,03 & 2,80 & 2,64 & 2,53 & 2,44 & 2,37 & 2,32 & 2,27 & 2,05 & 1,96 & 1,81 \\
\hline 24 & 4,26 & 3,40 & 3,01 & 2,78 & 2,62 & 2,51 & 2,42 & 2,36 & 2,30 & 2,25 & 2,03 & 1,94 & 1,79 \\
\hline 30 & 4,17 & 3,32 & 2,92 & 2,69 & 2,53 & 2,42 & 2,33 & 2,27 & 2,21 & 2,16 & 1,93 & 1,84 & 1,68 \\
\hline 40 & 4,08 & 3,23 & 2,84 & 2,61 & 2,45 & 2,34 & 2,21 & 2,18 & 2,12 & 2,08 & 1,84 & 1,74 & 1,58 \\
\hline 60 & 4,00 & 3,15 & 2,76 & 2,53 & 2,37 & 2,25 & 2,17 & 2,10 & 2,04 & 1,99 & 1,75 & 1,65 & 1,47 \\
\hline 120 & 3,92 & 3,07 & 2,68 & 2,45 & 2,29 & 2,17 & 2,09 & 2,02 & 1,96 & 1,91 & 1,66 & 1,55 & 1,35 \\
\hline$\infty$ & 3,84 & 3,00 & 2,60 & 2,37 & 2,21 & 2,10 & 2,01 & 1,94 & 1,88 & 1,83 & 1,57 & 1,46 & 1,22 \\
\hline
\end{tabular}




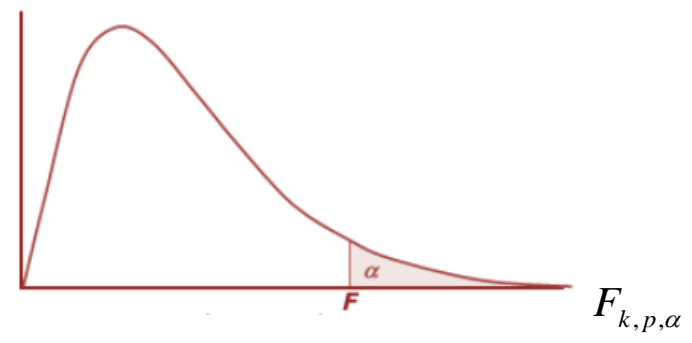

Tabela A3 - Distribuição $F$ de Snedecor para $\alpha=1 \%$.

\begin{tabular}{|c|c|c|c|c|c|c|c|c|c|c|c|c|c|}
\hline & 1 & 2 & 3 & 4 & 5 & 6 & 7 & 8 & 9 & 10 & 20 & 30 & 120 \\
\hline 1 & 4052 & 4999 & 5403 & 5625 & 5764 & 5859 & 5928 & 5982 & 6022 & 6056 & 6209 & 6261 & 6339 \\
\hline 2 & & 9,00 & & 9,25 & & 99,33 & 99,36 & & & & 9,45 & & 9,49 \\
\hline 3 & & 0,82 & 9,46 & 28,71 & 28,24 & 27,91 & 27,67 & 27,49 & 27,35 & & 26,69 & & 26,22 \\
\hline 4 & & 3,00 & 5,69 & 5,98 & & & & & & & 14,02 & 13,84 & 13,56 \\
\hline 5 & & 3,27 & 2,06 & 11,39 & 10,97 & 10,67 & 10,46 & 10,29 & 10,16 & & 9,55 & 9,38 & 9,11 \\
\hline 6 &, 75 & ),92 & 9,78 & 9,15 & 8,75 & 8,47 & 8,26 & 8,10 & 7,98 & 7,87 & 7,40 & 7,23 & 6,97 \\
\hline 7 & 12,25 & 9,55 & 8,45 & 7,85 & 7,46 & 7,19 & 6,99 & 6,84 & 6,72 & 5,62 & 16 & & 5,74 \\
\hline 8 & & 8,65 & 7,59 & 7,01 & 63 & 6,37 & 6,1 & & 5,91 & 81 & ,36 & 20 & 4,95 \\
\hline 9 & & 8,02 & 6,99 & 6,42 & 06 & 5,80 & 5,61 & 5,47 & 5,35 & 5,26 & 81 &, 65 & 4,40 \\
\hline 10 & & 7,56 & & & & 5,39 & & & & & & & 00 \\
\hline 11 & & 7,21 & & 5,67 & & 5,07 & 4,89 & 4,74 & 4,63 & 4,54 & 10 & 94 & 3,69 \\
\hline 12 & & & & & & & & & & & & & 3,45 \\
\hline 13 & & 6,70 & 5,74 & 5,21 & 4,86 & 4,62 & 4,44 & 4,30 & 4,19 & 4,10 & 3,66 &, 51 & 3,25 \\
\hline 14 & & & & & & & & & & & & & 3,09 \\
\hline 15 & 8,68 & 6,36 & 42 & 4,89 & 4,56 & 4,32 & & & & & ,37 & 1 & 2,96 \\
\hline 16 & & & & & & & & & & & & & \\
\hline 17 & 8,40 & 6,11 & & & 4,34 & 4,10 & 3,9 & & 8 & & 6 & 0 & 2,75 \\
\hline 18 & 29 & & & & & & & & & & & 2 & 2,66 \\
\hline 19 & 18 & 5,93 & 5,01 & 4,50 & & 3,94 & 3,77 & 3,63 & 3,52 & 3,43 & 00 & 84 & 2,58 \\
\hline 20 & & & & & & & & & & & 94 & 78 & 2,52 \\
\hline 21 & & 5,78 & 4,82 & 4,37 & 04 & 81 & 3,64 & 3,51 & 3,40 & & 88 & 72 & 2,46 \\
\hline 22 & 7,95 & 5,72 & & & & 3,76 & & & & & 2,78 & 67 & 2,40 \\
\hline 23 & 7,88 & 5,66 & 4,76 & 4,26 & 94 & 3,71 & 3,54 & 3,41 & 3,30 & 3,21 & 2,74 & 62 & 2,35 \\
\hline 24 & & & 4,72 & & & & & & & & & & 2,31 \\
\hline 25 & 7,77 & & & & & & & & & & & & 2,27 \\
\hline 26 & & & & & & & & & & & & & 2,23 \\
\hline 27 & & & & & & & & & & & & 47 & 2,20 \\
\hline 28 & & & & & & & & & & & &, 44 & 2,17 \\
\hline 29 & & & & & & & & & & & 2,55 & & 2,14 \\
\hline 30 & & & & & & & & & & & & 2,39 & 2,11 \\
\hline 40 & & & & & & & & & & & 2,37 & 2,20 & 1,92 \\
\hline 60 & & & & & & & & & & & & 2,03 & 1,73 \\
\hline 120 & & & & & & 2,96 & 2,79 & 2,66 & 2,56 & 2,47 & 2,03 & 1,86 & 1,53 \\
\hline$\infty$ & 6,63 & 4,61 & 3,78 & 3,32 & 3,02 & 2,80 & 2,64 & 2,51 & 2,41 & 2,32 & 1,88 & 1,70 & 1,32 \\
\hline
\end{tabular}




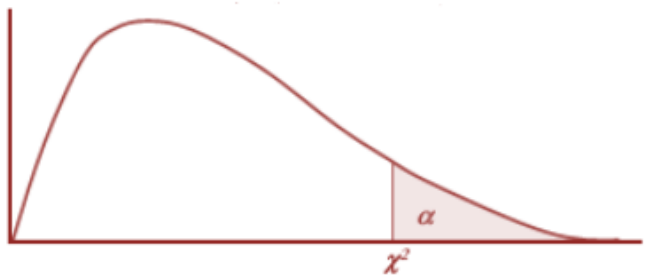

Tabela A4 - Distribuição de $\chi^{2}$.

\begin{tabular}{|c|c|c|c|c|c|c|c|c|c|c|c|c|c|}
\hline${ }_{k}^{\alpha}$ & 0,995 & 0,990 & 0,975 & 0,95 & 0,90 & 0,75 & 0,50 & 0,25 & 0,10 & 0,05 & 0,025 & 0,01 & 0,005 \\
\hline 1 & 0,000 & 0,0002 & 0,0010 & 0,0039 & 0,0158 & 0,102 & 0,455 & 1,32 & 2,71 & 3,84 & 5,02 & 6,63 & 7,88 \\
\hline 2 & 0,010 & 0,0001 & 0,0506 & 0,103 & 0,211 & 0,575 & 1,39 & 2,77 & 4,61 & 5,99 & 7,38 & 9,21 & 10,6 \\
\hline 3 & 0,0717 & 0,115 & 0,216 & 0,352 & 0,584 & 1,021 & 2,37 & 4,11 & 6,25 & 7,81 & 9,25 & 11,3 & 12,8 \\
\hline 4 & 0,207 & 0,297 & 0,484 & 0,711 & 1,06 & 1,92 & 3,36 & 5,39 & 7,78 & 9,49 & 11,1 & 13,3 & 14,9 \\
\hline 5 & 0,412 & 0,554 & 0,831 & 1,15 & 1,61 & 2,67 & 4,35 & 6,63 & 9,24 & 11,1 & 12,8 & 15,1 & 16,7 \\
\hline 6 & 0,676 & 0,872 & 1,24 & 1,64 & 2,20 & 3,45 & 5,35 & 7,84 & 10,6 & 12,6 & 14,4 & 16,8 & 18,5 \\
\hline 7 & 0,989 & 1,24 & 1,69 & 2,17 & 2,83 & 4,25 & 6,35 & 9,04 & 12,0 & 14,1 & 16,0 & 18,5 & 20,3 \\
\hline 8 & 1,34 & 1,65 & 2,18 & 2,73 & 3,49 & 5,07 & 7,34 & 10,2 & 13,4 & 15,5 & 17,5 & 20,1 & 22,0 \\
\hline 9 & 1,73 & 2,09 & 2,70 & 3,33 & 4,17 & 5,90 & 8,34 & 11,4 & 14,7 & 16,9 & 19,0 & 21,7 & 23,6 \\
\hline 10 & 2,16 & 2,56 & 3,25 & 3,94 & 4,87 & 6,74 & 9,34 & 12,5 & 16,0 & 18,3 & 20,5 & 23,2 & 25,2 \\
\hline 11 & 2,60 & 3,05 & 3,82 & 4,57 & 5,58 & 7,58 & 10,3 & 13,7 & 17,3 & 19,7 & 21,9 & 24,7 & 26,8 \\
\hline 12 & 3,07 & 3,57 & 4,40 & 5,23 & 6,30 & 8,44 & 11,3 & 14,8 & 18,5 & 21,0 & 23,3 & 26,2 & 28,3 \\
\hline 13 & 3,57 & 4,11 & 5,01 & 5,89 & 7,04 & 9,30 & 12,3 & 16,0 & 19,8 & 22,4 & 24,7 & 27,7 & 29,8 \\
\hline 14 & 4,07 & 4,66 & 5,63 & 6,57 & 7,79 & 10,2 & 13,3 & 17,1 & 21,1 & 23,7 & 26,1 & 29,1 & 31,3 \\
\hline 15 & 4,60 & 5,23 & 6,23 & 7,26 & 8,55 & 11,0 & 14,3 & 18,2 & 22,3 & 25,0 & 27,5 & 30,6 & 32,8 \\
\hline 16 & 5,14 & 5,80 & 6,91 & 7,96 & 8,31 & 11,9 & 15,3 & 19,4 & 23,5 & 26,3 & 28,4 & 32,0 & 34,3 \\
\hline 17 & 5,70 & 6,41 & 7,56 & 8,67 & 10,1 & 12,8 & 16,3 & 20,5 & 24,8 & 27,6 & 30,2 & 53,4 & 35,7 \\
\hline 18 & 6,26 & 7,01 & 8,23 & 9,39 & 10,9 & 13,7 & 17,3 & 21,6 & 26,0 & 28,9 & 31,5 & 34,8 & 37,2 \\
\hline 19 & 6,84 & 7,63 & 8,91 & 10,1 & 11,7 & 14,6 & 18,3 & 22,7 & 27,2 & 30,1 & 32,9 & 36,2 & 38,6 \\
\hline 20 & 7,43 & 8,26 & 9,59 & 10,9 & 12,4 & 15,5 & 19,3 & 23,8 & 28,4 & 31,4 & 34,2 & 37,6 & 40,0 \\
\hline 21 & 8,03 & 8,90 & 10,3 & 11,6 & 13,2 & 16,3 & 20,3 & 24,9 & 29,6 & 32,7 & 35,5 & 38,9 & 41,4 \\
\hline 22 & 8,64 & 9,54 & 11,0 & 12,3 & 14,0 & 17,2 & 21,3 & 26,0 & 30,8 & 33,9 & 36,8 & 40,5 & 42,8 \\
\hline 23 & 9,26 & 10,2 & 11,7 & 13,1 & 14,8 & 18,1 & 22,3 & 27,1 & 32,0 & 35,2 & 38,1 & 41,6 & 44,2 \\
\hline 24 & 9,89 & 10,9 & 12,4 & 13,8 & 15,7 & 19,0 & 23,3 & 28,2 & 33,1 & 36,4 & 39,4 & 43,0 & 45,6 \\
\hline 25 & 10,5 & 11,5 & 13,1 & 14,6 & 16,5 & 19,9 & 24,3 & 29,3 & 34,4 & 37,7 & 40,6 & 44,3 & 46,9 \\
\hline 26 & 11,2 & 12,2 & 13,8 & 15,4 & 17,3 & 20,8 & 25,3 & 30,4 & 35,6 & 38,9 & 41,9 & 45,6 & 48,3 \\
\hline 27 & 11,8 & 12,9 & 14,6 & 16,2 & 18,1 & 21,7 & 26,3 & 31,5 & 36,7 & 40,1 & 43,2 & 47,0 & 49,6 \\
\hline 28 & 12,5 & 13,6 & 15,3 & 16,9 & 18,9 & 22,7 & 27,3 & 32,6 & 37,9 & 41,3 & 44,5 & 48,3 & 51,0 \\
\hline 29 & 13,1 & 14,3 & 16,0 & 17,7 & 19,8 & 23,6 & 28,3 & 33,7 & 39,1 & 42,6 & 45,7 & 49,6 & 52,5 \\
\hline 30 & 13,8 & 15,0 & 16,8 & 18,5 & 20,6 & 24,5 & 29,3 & 34,8 & 40,3 & 43,8 & 47,0 & 50,9 & 53,7 \\
\hline
\end{tabular}




\title{
Apêndice B
}

\author{
Banco de Dados
}

Tabela B1 - Dados (simulados) para os quatro métodos de Manejo M1, M2, M3 e M4.

Tabela B2 - Dados (simulados) para as quatro espécies florestais E1, E2, E3 e E4. 


\begin{tabular}{|c|c|c|c|c|c|}
\hline MMF & $\mathrm{OC} 1=0$ & $\mathrm{OC} 2=5$ & $0 C 3=10$ & OC4=15 & $0 C 5=20$ \\
\hline M1 & 94,615 & 117,854 & 134,403 & 136,058 & 136,519 \\
\hline M1 & 96,053 & 117,628 & 133,247 & 134,909 & 135,398 \\
\hline M1 & 95,785 & 118,546 & 136,020 & 137,251 & 139,248 \\
\hline M1 & 94,358 & 117,248 & 133,796 & 134,482 & 135,317 \\
\hline M1 & 97,530 & 121,034 & 137,164 & 138,871 & 140,947 \\
\hline M1 & 99,210 & 120,537 & 137,811 & 139,005 & 139,881 \\
\hline M1 & 97,077 & 119,585 & 135,360 & 136,358 & 137,428 \\
\hline M1 & 95,955 & 118,884 & 135,719 & 136,661 & 138,842 \\
\hline M1 & 93,440 & 116,993 & 133,718 & 134,770 & 135,865 \\
\hline M1 & 95,738 & 115,370 & 132,002 & 132,618 & 133,880 \\
\hline M1 & 97,165 & 117,845 & 134,598 & 136,152 & 137,478 \\
\hline M1 & 96,523 & 118,165 & 134,463 & 135,796 & 137,458 \\
\hline M1 & 97,986 & 120,246 & 136,374 & 137,515 & 138,089 \\
\hline M1 & 97,433 & 119,589 & 135,920 & 137,615 & 137,963 \\
\hline M1 & 96,975 & 115,940 & 133,516 & 134,294 & 136,606 \\
\hline M1 & 94,282 & 114,849 & 129,462 & 129,582 & 131,759 \\
\hline M1 & 96,286 & 118,640 & 135,319 & 135,481 & 136,623 \\
\hline M1 & 96,998 & 122,128 & 140,127 & 141,131 & 141,937 \\
\hline M1 & 97,488 & 119,215 & 135,240 & 137,065 & 138,849 \\
\hline M1 & 96,578 & 118,723 & 136,597 & 137,852 & 139,700 \\
\hline M1 & 98,518 & 123,491 & 140,273 & 141,052 & 142,956 \\
\hline M1 & 96,216 & 119,143 & 135,979 & 136,864 & 138,866 \\
\hline M1 & 96,894 & 119,068 & 136,033 & 135,548 & 136,601 \\
\hline M1 & 99,217 & 121,152 & 137,255 & 138,262 & 140,089 \\
\hline M1 & 96,559 & 118,692 & 135,229 & 136,172 & 137,476 \\
\hline M1 & 96,431 & 117,869 & 134,368 & 135,415 & 136,818 \\
\hline M1 & 98,167 & 120,253 & 136,733 & 137,879 & 139,034 \\
\hline M1 & 95,750 & 118,697 & 134,855 & 135,960 & 137,998 \\
\hline M1 & 96,882 & 120,296 & 137,077 & 139,813 & 142,695 \\
\hline M1 & 97,571 & 118,331 & 134,219 & 135,167 & 136,721 \\
\hline M2 & 95,524 & 119,377 & 138,797 & 141,366 & 143,684 \\
\hline M2 & 95,183 & 115,833 & 132,058 & 132,745 & 134,317 \\
\hline M2 & 98,479 & 123,586 & 142,984 & 145,502 & 147,988 \\
\hline M2 & 95,777 & 120,546 & 138,930 & 140,872 & 142,232 \\
\hline M2 & 95,456 & 114,688 & 130,722 & 133,677 & 135,715 \\
\hline M2 & 98,218 & 121,870 & 139,487 & 142,192 & 143,360 \\
\hline M2 & 99,552 & 123,743 & 140,865 & 142,502 & 143,009 \\
\hline M2 & 97,429 & 121,215 & 140,035 & 142,699 & 144,428 \\
\hline M2 & 100,079 & 123,403 & 140,474 & 143,084 & 144,876 \\
\hline M2 & 99,240 & 123,466 & 141,219 & 142,647 & 144,880 \\
\hline M2 & 98,572 & 120,566 & 138,339 & 140,418 & 141,991 \\
\hline M2 & 98,252 & 120,106 & 136,414 & 137,878 & 138,522 \\
\hline M2 & 96,555 & 120,718 & 137,932 & 140,042 & 140,344 \\
\hline M2 & 94,814 & 115,831 & 131,590 & 133,398 & 133,746 \\
\hline M2 & 96,648 & 117,260 & 133,026 & 133,048 & 134,014 \\
\hline
\end{tabular}




\begin{tabular}{|c|c|c|c|c|c|}
\hline M2 & 97,457 & 121,472 & 138,184 & 140,837 & 142,141 \\
\hline M2 & 98,106 & 119,486 & 136,627 & 138,044 & 139,312 \\
\hline M2 & 97,650 & 122,648 & 139,788 & 141,364 & 141,901 \\
\hline M2 & 96,234 & 119,658 & 136,677 & 137,573 & 138,233 \\
\hline M2 & 97,942 & 120,732 & 138,082 & 138,231 & 138,949 \\
\hline M2 & 99,815 & 120,827 & 137,285 & 138,834 & 140,553 \\
\hline M2 & 97,010 & 119,731 & 136,787 & 138,605 & 141,199 \\
\hline M2 & 95,475 & 116,825 & 132,716 & 134,045 & 134,626 \\
\hline M2 & 95,713 & 120,039 & 138,905 & 141,486 & 142,676 \\
\hline M2 & 96,377 & 117,109 & 133,783 & 134,336 & 136,398 \\
\hline M2 & 96,410 & 119,202 & 137,199 & 139,641 & 142,359 \\
\hline M2 & 96,222 & 122,665 & 142,341 & 144,833 & 145,794 \\
\hline M2 & 95,516 & 119,257 & 136,077 & 138,273 & 138,943 \\
\hline M2 & 96,356 & 119,606 & 136,540 & 138,115 & 138,560 \\
\hline M2 & 96,177 & 118,587 & 136,929 & 138,243 & 140,310 \\
\hline M3 & 98,190 & 132,620 & 143,707 & 150,691 & 151,215 \\
\hline M3 & 96,132 & 131,374 & 142,860 & 150,846 & 152,044 \\
\hline M3 & 97,196 & 130,957 & 141,585 & 148,872 & 149,666 \\
\hline M3 & 99,092 & 132,450 & 143,637 & 150,820 & 150,724 \\
\hline M3 & 95,969 & 129,566 & 142,517 & 149,520 & 150,775 \\
\hline M3 & 99,141 & 131,534 & 142,251 & 147,740 & 147,884 \\
\hline M3 & 95,626 & 132,910 & 144,658 & 151,802 & 151,973 \\
\hline M3 & 97,487 & 132,187 & 143,261 & 148,352 & 148,689 \\
\hline M3 & 95,834 & 130,839 & 142,035 & 148,042 & 148,633 \\
\hline M3 & 94,928 & 129,418 & 142,391 & 149,925 & 151,542 \\
\hline M3 & 95,432 & 129,824 & 140,424 & 148,069 & 148,505 \\
\hline M3 & 98,470 & 131,710 & 143,227 & 149,088 & 149,241 \\
\hline M3 & 95,895 & 133,746 & 146,470 & 154,394 & 155,885 \\
\hline M3 & 97,122 & 130,906 & 142,270 & 149,865 & 151,462 \\
\hline M3 & 95,431 & 128,241 & 139,941 & 147,753 & 149,340 \\
\hline M3 & 97,822 & 132,356 & 143,929 & 150,325 & 150,988 \\
\hline M3 & 95,406 & 132,782 & 145,263 & 153,038 & 154,337 \\
\hline M3 & 95,795 & 128,897 & 139,542 & 145,387 & 146,524 \\
\hline M3 & 99,205 & 132,448 & 142,860 & 148,644 & 149,511 \\
\hline M3 & 97,058 & 132,026 & 144,084 & 152,295 & 153,700 \\
\hline M3 & 95,522 & 130,215 & 141,126 & 146,948 & 148,250 \\
\hline M3 & 94,468 & 125,865 & 137,559 & 145,002 & 146,324 \\
\hline M3 & 97,106 & 128,254 & 137,562 & 142,820 & 143,124 \\
\hline M3 & 96,724 & 132,536 & 143,382 & 150,759 & 150,886 \\
\hline M3 & 95,748 & 130,729 & 141,664 & 147,756 & 147,084 \\
\hline M3 & 96,909 & 133,096 & 147,964 & 157,443 & 159,481 \\
\hline M3 & 97,532 & 132,145 & 143,396 & 150,894 & 150,201 \\
\hline M3 & 98,125 & 131,411 & 142,013 & 148,978 & 149,930 \\
\hline M3 & 95,548 & 129,175 & 141,480 & 149,520 & 150,649 \\
\hline M3 & 97,121 & 127,784 & 138,000 & 145,102 & 145,242 \\
\hline M4 & 98,079 & 132,939 & 145,355 & 152,622 & 153,402 \\
\hline M4 & 96,861 & 128,322 & 138,876 & 145,122 & 146,096 \\
\hline M4 & 96,411 & 129,530 & 140,862 & 147,055 & 146,907 \\
\hline
\end{tabular}




\begin{tabular}{l|c|c|c|c|c}
\hline M4 & 95,807 & 130,430 & 141,989 & 148,971 & 147,874 \\
\hline M4 & 98,857 & 134,534 & 147,961 & 155,958 & 156,575 \\
\hline M4 & 95,472 & 130,218 & 142,139 & 149,195 & 150,062 \\
\hline M4 & 98,595 & 135,659 & 148,153 & 156,275 & 155,918 \\
\hline M4 & 97,645 & 131,035 & 142,191 & 148,573 & 149,839 \\
\hline M4 & 96,617 & 129,745 & 142,200 & 149,818 & 150,448 \\
\hline M4 & 97,962 & 132,890 & 145,852 & 154,337 & 155,329 \\
\hline M4 & 98,595 & 135,041 & 148,558 & 155,240 & 154,901 \\
\hline M4 & 96,963 & 130,770 & 142,606 & 148,884 & 150,063 \\
\hline M4 & 100,186 & 136,245 & 147,691 & 153,940 & 153,350 \\
\hline M4 & 94,085 & 127,957 & 139,836 & 145,867 & 146,367 \\
\hline M4 & 94,135 & 125,779 & 136,651 & 143,743 & 145,685 \\
\hline M4 & 96,041 & 129,792 & 142,479 & 150,761 & 152,554 \\
\hline M4 & 97,495 & 131,818 & 144,526 & 151,940 & 152,834 \\
\hline M4 & 95,771 & 129,718 & 143,371 & 151,745 & 153,444 \\
\hline M4 & 97,270 & 129,914 & 140,504 & 148,362 & 149,407 \\
\hline M4 & 95,349 & 128,752 & 140,114 & 147,923 & 149,302 \\
\hline M4 & 95,344 & 130,492 & 142,311 & 149,867 & 150,730 \\
\hline M4 & 97,071 & 130,555 & 143,306 & 152,105 & 153,363 \\
\hline M4 & 99,090 & 134,932 & 148,511 & 155,649 & 156,663 \\
\hline M4 & 97,367 & 131,371 & 140,545 & 147,749 & 147,793 \\
\hline M4 & 94,519 & 127,862 & 140,369 & 147,380 & 148,782 \\
\hline M4 & 97,446 & 130,066 & 141,061 & 147,140 & 146,949 \\
\hline M4 & 99,836 & 132,356 & 143,712 & 150,136 & 150,865 \\
\hline M4 & 98,800 & 131,947 & 143,215 & 149,599 & 149,927 \\
\hline M4 & 98,891 & 132,498 & 142,649 & 148,547 & 148,808 \\
\hline M4 & 98,736 & 134,371 & 146,193 & 154,098 & 154,887 \\
\hline & & & & & \\
\hline
\end{tabular}

\begin{tabular}{c|c|c|c|c|c}
\multicolumn{5}{c}{ Tabela B2 - Dados simulados das quatro espécies E1, E2, E3 e E4. } \\
ESPÉCIE & OC1=0 & OC2=5 & OC3=10 & OC4=15 & OC5=20 \\
\hline E1 & 9,9190 & 14,5882 & 17,2448 & 19,5898 & 21,1701 \\
\hline E1 & 9,6132 & 14,4511 & 17,1552 & 19,6230 & 21,2862 \\
\hline E1 & 9,7196 & 14,4053 & 16,9914 & 19,4964 & 20,9532 \\
\hline E1 & 9,9092 & 14,5695 & 17,2364 & 19,6196 & 21,1014 \\
\hline E1 & 9,5969 & 14,2523 & 17,1020 & 19,4376 & 21,1085 \\
\hline E1 & 9,9141 & 14,4687 & 17,0701 & 19,2062 & 20,7192 \\
\hline E1 & 9,5626 & 14,6201 & 17,3591 & 19,7473 & 21,2762 \\
\hline E1 & 9,7497 & 14,5417 & 17,1913 & 19,4158 & 20,9579 \\
\hline E1 & 9,5934 & 14,4033 & 17,0442 & 19,3755 & 20,9486 \\
\hline E1 & 9,4929 & 14,2361 & 17,0869 & 19,4903 & 21,2159 \\
\hline E1 & 9,5432 & 14,2916 & 16,8509 & 19,3790 & 20,9307 \\
\hline E1 & 9,9470 & 14,4881 & 17,1872 & 19,3829 & 20,8937 \\
\hline E1 & 9,5995 & 14,7121 & 17,5764 & 20,0712 & 21,8393 \\
\hline E1 & 9,7122 & 14,3997 & 17,0724 & 19,4955 & 21,2047 \\
\hline E1 & 9,5431 & 14,2165 & 16,7929 & 19,2079 & 20,9076 \\
\hline E1 & 9,7922 & 14,5592 & 17,2715 & 19,5423 & 21,1399 \\
\hline E1 & 9,5406 & 14,6071 & 17,4316 & 19,8951 & 21,6072 \\
\hline
\end{tabular}




\begin{tabular}{|c|c|c|c|c|c|}
\hline E1 & 9,5795 & 14,2997 & 16,7450 & 18,9016 & 20,5134 \\
\hline E1 & 9,9205 & 14,5694 & 17,1552 & 19,4537 & 20,9315 \\
\hline E1 & 9,7059 & 14,5229 & 17,2913 & 19,7984 & 21,5180 \\
\hline E1 & 9,5522 & 14,3237 & 16,9351 & 19,1034 & 20,8950 \\
\hline E1 & 9,4469 & 13,8562 & 16,5071 & 18,8503 & 20,4854 \\
\hline E1 & 9,7106 & 14,2179 & 16,5074 & 18,5796 & 20,0374 \\
\hline E1 & 9,6724 & 14,5790 & 17,2070 & 19,5987 & 21,1394 \\
\hline E1 & 9,5749 & 14,3802 & 16,9997 & 19,2083 & 20,5932 \\
\hline E1 & 9,6909 & 14,6406 & 17,7557 & 20,4676 & 22,3287 \\
\hline E1 & 9,7532 & 14,5360 & 17,2075 & 19,6292 & 21,0281 \\
\hline E1 & 9,9125 & 14,4552 & 17,0416 & 19,4973 & 20,9902 \\
\hline E1 & 9,5549 & 14,2093 & 16,9788 & 19,4376 & 21,0909 \\
\hline E1 & 9,7121 & 14,0573 & 16,6800 & 18,8633 & 20,3339 \\
\hline E2 & 9,9079 & 14,6233 & 17,4426 & 19,8409 & 21,4763 \\
\hline E2 & 9,6961 & 14,2254 & 16,7971 & 18,8659 & 20,4534 \\
\hline E2 & 9,6411 & 14,2483 & 16,9154 & 19,1172 & 20,5670 \\
\hline E2 & 9,5907 & 14,3473 & 17,0399 & 19,4962 & 20,7164 \\
\hline E2 & 9,9957 & 14,7987 & 17,7553 & 20,2747 & 21,9205 \\
\hline E2 & 9,5472 & 14,3241 & 17,0567 & 19,3954 & 21,0087 \\
\hline E2 & 9,9595 & 14,9225 & 17,8984 & 20,3158 & 21,8287 \\
\hline E2 & 9,7645 & 14,4139 & 17,0629 & 19,4445 & 20,9915 \\
\hline E2 & 9,6617 & 14,2720 & 17,0640 & 19,4895 & 21,0629 \\
\hline E2 & 9,7962 & 14,6289 & 17,5142 & 20,0638 & 21,7461 \\
\hline E2 & 9,9595 & 14,8545 & 17,9471 & 20,1812 & 21,6861 \\
\hline E2 & 9,6963 & 14,3847 & 17,1127 & 19,4992 & 21,0088 \\
\hline E2 & 10,0196 & 14,9870 & 17,7229 & 20,0122 & 21,4690 \\
\hline E2 & 9,4095 & 14,0753 & 16,7923 & 18,9757 & 20,4914 \\
\hline E2 & 9,4135 & 13,8357 & 16,3981 & 18,6866 & 20,3973 \\
\hline E2 & 9,6041 & 14,2771 & 17,0975 & 19,5989 & 21,3576 \\
\hline E2 & 9,7495 & 14,5111 & 17,3431 & 19,7522 & 21,4108 \\
\hline E2 & 9,5771 & 14,2691 & 17,2045 & 19,7269 & 21,4822 \\
\hline E2 & 9,7270 & 14,2905 & 16,8605 & 19,4171 & 20,9170 \\
\hline E2 & 9,5349 & 14,2727 & 16,8137 & 19,2300 & 20,9023 \\
\hline E2 & 9,5344 & 14,3541 & 17,0773 & 19,4957 & 21,1022 \\
\hline E2 & 9,7071 & 14,3611 & 17,1967 & 19,7737 & 21,4708 \\
\hline E2 & 9,9090 & 14,8425 & 17,9413 & 20,2344 & 21,9328 \\
\hline E2 & 9,7367 & 14,4508 & 16,8654 & 19,2074 & 20,6910 \\
\hline E2 & 9,4519 & 14,0758 & 16,8443 & 19,1607 & 20,9709 \\
\hline E2 & 9,7446 & 14,3073 & 16,9273 & 19,1282 & 20,5729 \\
\hline E2 & 9,9936 & 14,5592 & 17,2454 & 19,5177 & 21,1351 \\
\hline E2 & 9,9900 & 14,5142 & 17,1858 & 19,4479 & 20,9898 \\
\hline E2 & 9,9991 & 14,5749 & 17,1179 & 19,4411 & 20,9873 \\
\hline E2 & 9,9736 & 14,7808 & 17,5432 & 20,0329 & 21,6996 \\
\hline E3 & 9,4615 & 12,9749 & 16,1284 & 17,6877 & 19,1127 \\
\hline E3 & 9,6053 & 12,9392 & 15,9896 & 17,5382 & 18,9559 \\
\hline E3 & 9,5795 & 13,1501 & 16,3224 & 17,8426 & 19,4949 \\
\hline E3 & 9,4359 & 12,8974 & 16,0555 & 17,4840 & 18,9444 \\
\hline E3 & 9,7530 & 13,3137 & 16,4597 & 18,1962 & 19,7326 \\
\hline
\end{tabular}




\begin{tabular}{|c|c|c|c|c|c|}
\hline E3 & 9,9210 & 13,2591 & 16,5493 & 18,0707 & 19,5987 \\
\hline E3 & 9,7077 & 13,1555 & 16,2432 & 17,7267 & 19,2401 \\
\hline E3 & 9,5955 & 13,1993 & 16,2863 & 17,7659 & 19,5919 \\
\hline E3 & 9,3440 & 12,8692 & 16,0463 & 17,5201 & 19,0351 \\
\hline E3 & 9,5739 & 12,6907 & 15,8402 & 17,2405 & 18,7586 \\
\hline E3 & 9,7165 & 12,9740 & 16,1519 & 17,6998 & 19,2471 \\
\hline E3 & 9,6523 & 13,1082 & 16,1356 & 17,6535 & 19,2443 \\
\hline E3 & 9,7996 & 13,2271 & 16,3649 & 17,8770 & 19,4739 \\
\hline E3 & 9,7433 & 13,1559 & 16,3104 & 17,8900 & 19,3148 \\
\hline E3 & 9,6975 & 12,7534 & 16,0219 & 17,4582 & 19,1248 \\
\hline E3 & 9,4292 & 12,6444 & 15,5354 & 16,8470 & 18,4463 \\
\hline E3 & 9,6296 & 13,1604 & 16,2383 & 17,6138 & 19,1272 \\
\hline E3 & 9,6999 & 13,4342 & 16,8152 & 18,3470 & 19,8712 \\
\hline E3 & 9,7499 & 13,1137 & 16,2288 & 17,8185 & 19,5929 \\
\hline E3 & 9,6579 & 13,1695 & 16,3916 & 17,9338 & 19,5580 \\
\hline E3 & 9,9519 & 13,5840 & 16,8328 & 18,3368 & 20,0138 \\
\hline E3 & 9,6216 & 13,1057 & 16,3175 & 17,8053 & 19,5952 \\
\hline E3 & 9,6994 & 13,0976 & 16,3240 & 17,6214 & 19,1241 \\
\hline E3 & 9,9217 & 13,3267 & 16,4706 & 18,1041 & 19,6139 \\
\hline E3 & 9,6559 & 13,1661 & 16,2275 & 17,7024 & 19,2466 \\
\hline E3 & 9,6431 & 12,9766 & 16,1243 & 17,6040 & 19,1687 \\
\hline E3 & 9,9167 & 13,2278 & 16,4080 & 17,9373 & 19,4648 \\
\hline E3 & 9,5750 & 13,1667 & 16,1946 & 17,6748 & 19,3199 \\
\hline E3 & 9,6992 & 13,2326 & 16,4492 & 18,1887 & 19,9773 \\
\hline E3 & 9,7571 & 13,1264 & 16,1063 & 17,5717 & 19,1409 \\
\hline E4 & 9,5524 & 13,1315 & 16,7756 & 18,3776 & 20,1172 \\
\hline $\mathrm{E} 4$ & 9,5193 & 12,7526 & 15,8471 & 17,2569 & 18,8044 \\
\hline E4 & 9,9479 & 13,5956 & 17,1593 & 18,9153 & 20,7199 \\
\hline E4 & 9,5777 & 13,2601 & 16,7916 & 18,3264 & 19,9125 \\
\hline E4 & 9,5456 & 12,6169 & 15,6866 & 17,3780 & 19,0001 \\
\hline E4 & 9,9219 & 13,4167 & 16,7396 & 18,4850 & 20,0704 \\
\hline $\mathrm{E} 4$ & 9,9552 & 13,6117 & 16,9158 & 18,5253 & 20,0213 \\
\hline E4 & 9,7429 & 13,3337 & 16,8042 & 18,5509 & 20,2201 \\
\hline $\mathrm{E} 4$ & 10,0079 & 13,5743 & 16,8569 & 18,6022 & 20,2966 \\
\hline $\mathrm{E} 4$ & 9,9240 & 13,5813 & 16,9463 & 18,5441 & 20,2986 \\
\hline $\mathrm{E} 4$ & 9,9572 & 13,2623 & 16,7207 & 18,2545 & 19,8787 \\
\hline E4 & 9,9252 & 13,2117 & 16,3697 & 17,9373 & 19,5331 \\
\hline $\mathrm{E} 4$ & 9,6555 & 13,2791 & 16,5518 & 18,2055 & 19,6482 \\
\hline E4 & 9,4914 & 12,7524 & 15,7908 & 17,3419 & 18,7244 \\
\hline $\mathrm{E} 4$ & 9,6649 & 12,8986 & 15,9631 & 17,2964 & 18,7620 \\
\hline E4 & 9,7457 & 13,3619 & 16,7033 & 18,3218 & 19,8997 \\
\hline $\mathrm{E} 4$ & 9,9106 & 13,1446 & 16,3952 & 18,0757 & 19,5037 \\
\hline $\mathrm{E} 4$ & 9,7650 & 13,4914 & 16,7759 & 18,3773 & 19,8661 \\
\hline $\mathrm{E} 4$ & 9,6234 & 13,1625 & 16,4012 & 17,8845 & 19,4926 \\
\hline $\mathrm{E} 4$ & 9,7942 & 13,2805 & 16,6910 & 18,1000 & 19,5929 \\
\hline E4 & 9,9915 & 13,3020 & 16,4754 & 18,1914 & 19,6774 \\
\hline E4 & 9,7010 & 13,1704 & 16,4156 & 18,1487 & 19,7679 \\
\hline E4 & 9,5475 & 12,8618 & 15,9259 & 17,4259 & 18,8476 \\
\hline
\end{tabular}




\begin{tabular}{c|c|c|c|c|c}
\hline E4 & 9,5713 & 13,2043 & 16,7886 & 18,3945 & 19,9746 \\
\hline E4 & 9,6377 & 12,8820 & 16,0552 & 17,4637 & 19,0959 \\
\hline E4 & 9,6410 & 13,1122 & 16,4639 & 18,1533 & 19,9303 \\
\hline E4 & 9,6222 & 13,4932 & 17,0809 & 18,8413 & 20,4112 \\
\hline E4 & 9,5516 & 13,1183 & 16,3292 & 18,1055 & 19,5920 \\
\hline E4 & 9,6356 & 13,1567 & 16,3848 & 18,0850 & 19,5384 \\
\hline E4 & 9,6177 & 13,1557 & 16,4315 & 18,1016 & 19,6434 \\
\hline
\end{tabular}




\section{Apêndice C}

\section{Programas SAS}

Tabela C1 - Operações com vetores e matrizes.

Tabela C2 - Uniformidade da matriz de covariância comum de M1, M2, M3 e M4.

Tabela C3 - Teste de Mauchly e correções de Greenhouse-Geisser e de Huynh e Feldt da matriz de covariância comum de M1, M2, M3 e M4.

Tabela C4 - Análise de variância multivariada, capítulo 4, seção 4.2.1.

Tabela C5 - Análise de inventários florestais com parcelas permanentes considerando apenas um tratamento M1.

Tabela C6 - Análise de inventários florestais com parcelas permanentes considerando dois tratamentos M1 e M2.

Tabela C7 - Análise da homogeneidade de matrizes de covariâncias dos métodos de manejo M1, M2, M3 e M4.

Tabela C8 - Análise de perfil de M1, M2, M3 e M4.

Tabela C9 - Análise agrupamento pelo método de Ward para M1, M2, M3 e M4.

Tabela C10 - Coincidência de perfis, pelo teste "t" de Student, entre M3 e M4 (Proc IML).

Tabela C11 - Teste de coincidência de perfis entre M3 e M4 (Proc GML).

Tabela C12 - Análise de regressão: Mitscherlich para M1, M2, M3 e M4.

Tabela C13 - Análise de regressão: Mitscherlich para M3 e M4.

Tabela C14 - Análise de componentes principais e de fatores para as espécies E1, E2, E3

Tabela C15 - Análise discriminante para as espécies E1, E2, E3 e E4.

Tabela C16 - Análise de correlação canônica considerando os grupos métodos de manejo (M1, M2, M3, M4) versus o grupo das espécies (E1, E2, E3, E4). 


\section{Tabela C1 - Operações com vetores e matrizes.}

proc IML;

reset print;

$A=\left\{\begin{array}{lllllllll}4.66616 & 2.58743 & 0.46108, & 2.587432 & 3.80823 & -0.99984,0.46108 & -0.99984 & 2.82892\end{array}\right\}$;

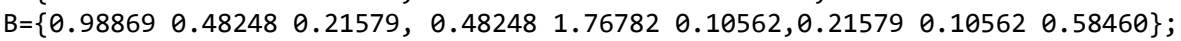

$C=\left\{\begin{array}{lllllll}1.16530 & 0.48109 & 1.12600,0.48109 & 3.53454 & 0.39000,1.12600 & 0.39000 & 3.10924\end{array}\right\}$;

$D=\left\{\begin{array}{lllllll}1 & 3 & 1,2 & -1 & 2,3 & 2 & 3\end{array}\right\}$;

TITLE'Transposta da matriz A';

$\mathrm{TA}=\operatorname{transp}(\mathrm{A})$;

TITLE' Traço da matriz A';

$\operatorname{trA}=\operatorname{trace}(A)$;

TITLE 'Matriz comum $S$ ';

$\mathrm{S}=(\mathrm{A}+\mathrm{B}+\mathrm{C}) / 3$;

TITLE 'Multiplicao das de matrizes $A$ e $B$ ';

$A B=A * B$;

TITLE 'Produto direto entre $A$ e $B$ ';

$P I=A @ B$;

TITLE 'Potencia de uma matriz';

$\mathrm{A} 2=\mathrm{A} * * 2$;

TITLE 'Potencia de uma matriz por elemento';

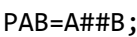

TITLE 'Determinante da matriz $A$ ';

$\operatorname{DETA}=\operatorname{det}(A)$;

title'Matriz inversa de $A^{\prime}$;

$\operatorname{INVA}=\operatorname{INV}(A)$;

title'Autovalores da matriz A';

AUVALA=EIGVAL (A);

title'autovetores da matriz $A^{\prime}$;

AUVETA=EIGVEC $(A)$;

title 'Inversa generalizada de $D$ ';

IGD=ginv (D);

QUIT;

\section{Tabela C2 - Uniformidade da matriz de covariância comum de M1, M2, M3 e M4}

PROC IML;

RESET PRINT;

$\mathrm{N}=120$;

$\mathrm{g}=4$;

$\mathrm{P}=5$;

$\mathrm{S}=\left\{\begin{array}{lllll}2.1061385 & 2.2793064 & 2.2381011 & 2.1368150 & 1.9697176,\end{array}\right.$

$2.2793064 \quad 4.77188045 .5400208 \quad 5.84515305 .5819684$,

$2.23810115 .5400208 \quad 7.2915454 \quad 7.9891048 \quad 7.9497102$,

$2.1368150 \quad 5.84515307 .9891048 \quad 9.3602169$ 9.5140274,

$1.9697176 \quad 5.58196847 .9497102 \quad 9.5140274 \quad 10.153285\}$;

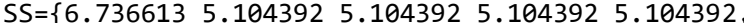

5.1043926 .7366135 .1043925 .1043925 .104392 ,

$5.1043925 .104392 \quad 6.736613 \quad 5.104392 \quad 5.104392$,

5.1043925 .1043925 .1043926 .7366135 .104392 ,

$5.1043925 .1043925 .1043925 .1043926 .736613\}$;

$\mathrm{DETS}=\mathrm{DET}(\mathrm{S})$;

DETSS $=\mathrm{DET}(\mathrm{SS})$;

DELTA $=(D E T S / D E T S S)$;

$M=-(\mathrm{N}-\mathrm{g}) * \operatorname{LOG}(\mathrm{DELTA})$;

$A=p *((p+1) * * 2) *(2 * p-3)$;

$B=6 *(N-g) *(p-1) *(p * * 2+p-4)$;

$A 1=A / B$;

$W=(1-A 1) * M$;

QUIT;

Tabela C3 - Teste de Mauchly e correções de Greenhouse-Geisser e de Huynh e Feldt da matriz de covariância comum de M1, M2, M3 e M4.

PROC IML;

RESET PRINT;

$\mathrm{p}=5$;

$\mathrm{N}=120$;

$\mathrm{g}=4$;

$\mathrm{V}=\mathrm{N}-\mathrm{g}$

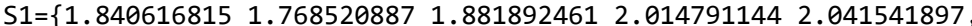

1.7685208873 .4672887923 .7861480614 .0587354514 .045605964 ,

$\begin{array}{llllll}1.881892461 & 3.786148061 & 4.573169082 & 4.850746154 & 4.855668095 \text {, }\end{array}$

$2.014791144 \quad 4.0587354514 .850746154 \quad 5.472367651 \quad 5.519759071$, 


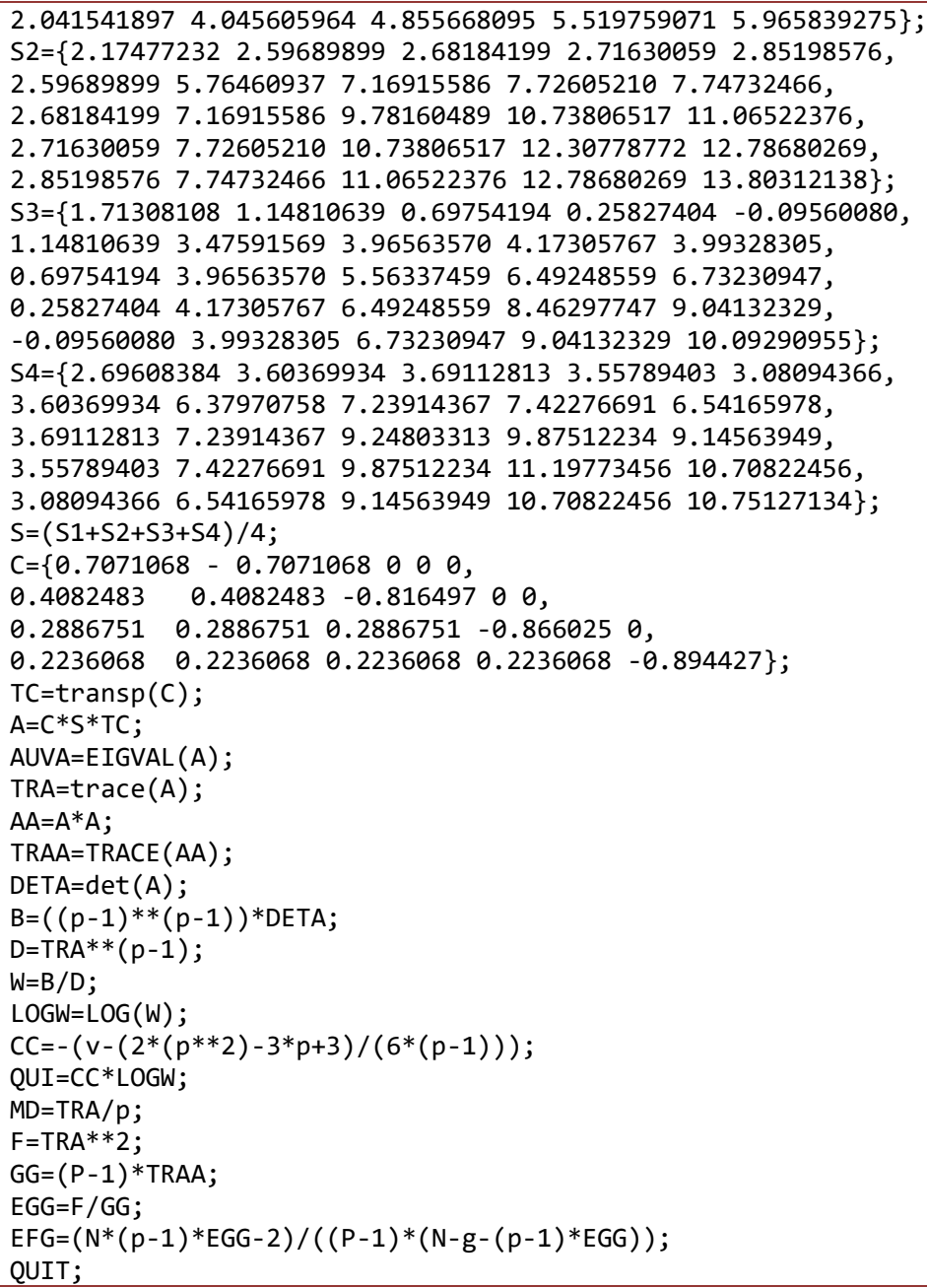

Tabela C4 - Análise de variância multivariada, capítulo 4, seção 4.2.1. title 'ANALISE DE VARIANCIA MULTIVARIADA'; data MANAVA;

input LABEL \$ TRAT \$ X1 X2;

CAN1 $=0.930763 * X 1-0.365623 * X 2 ; \quad C A N 2=0.998026 * X 1-0.062803 * X 2$;

list;

cards;

\begin{tabular}{llll} 
A & A & 4.59461 & 1.01091 \\
A & A & 4.83847 & 1.09984 \\
A & A & 4.63485 & 1.36393 \\
A & A & 4.13427 & 0.75682 \\
A & A & 4.41401 & 0.99222 \\
A & A & 4.49093 & 1.03807 \\
A & A & 4.34527 & 1.01440 \\
A & A & 4.76123 & 1.24293 \\
A & A & 4.85951 & 1.28071 \\
A & A & 4.54754 & 0.96189 \\
$B$ & B & 5.89330 & 1.30597 \\
B & B & 6.39272 & 1.02454 \\
B & B & 6.04890 & 0.95112 \\
B & B & 5.83597 & 1.18565 \\
B & B & 5.90154 & 0.82137 \\
B & B & 6.29682 & 1.28870 \\
B & B & 5.77850 & 1.09699 \\
$B$ & B & 5.51812 & 0.98971 \\
B & B & 5.69376 & 1.24628 \\
B & B & 6.17353 & 1.12303 \\
C & C & 4.62760 & 1.13162 \\
C & C & 4.57793 & 0.80946 \\
C & C & 4.63142 & 0.96004 \\
C & C & 4.37465 & 0.85200 \\
C & C & 4.55534 & 0.82440 \\
\hline & & &
\end{tabular}




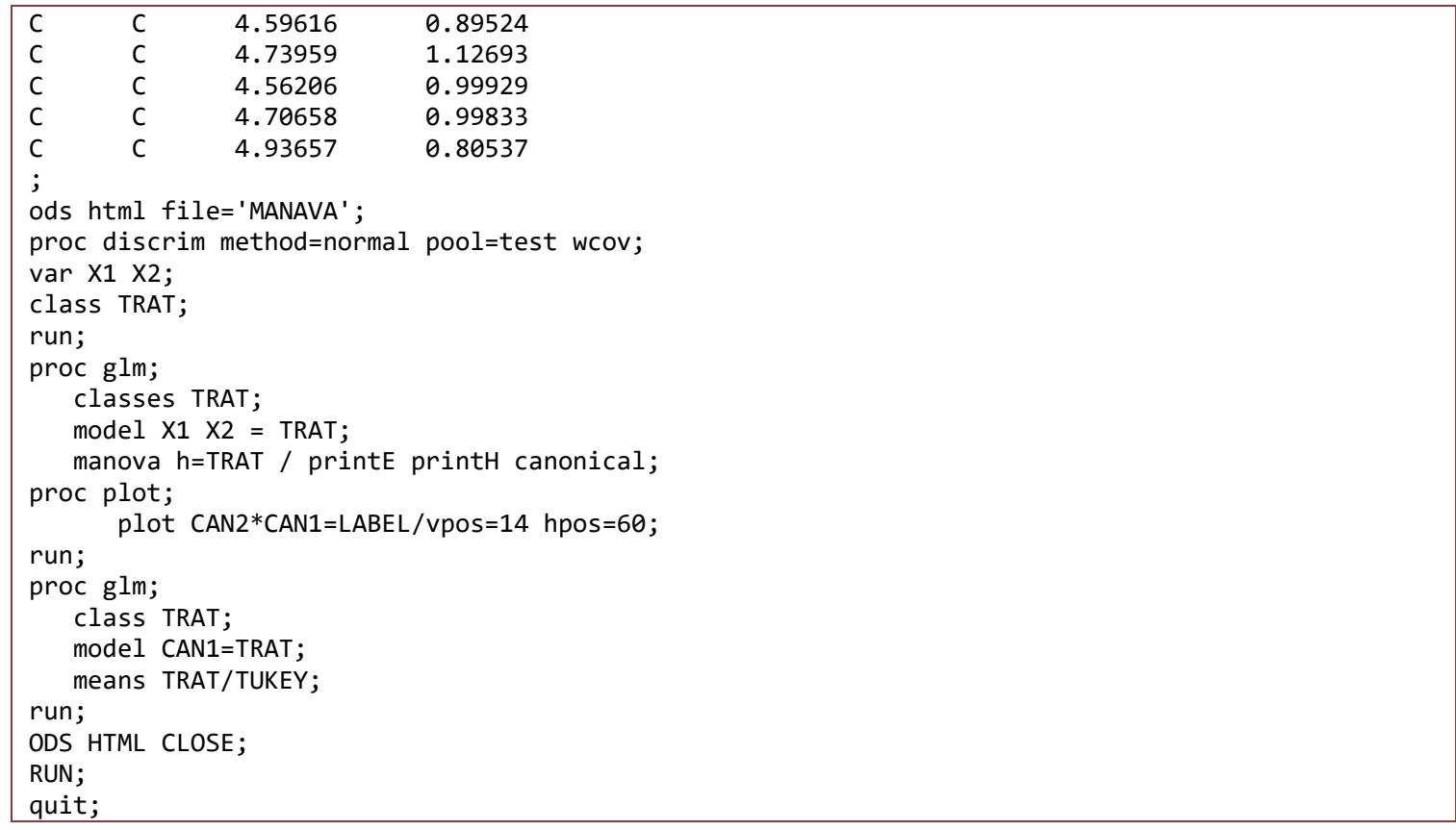

Tabela C5 - Análise de inventários florestais com parcelas permanentes considerando apenas um tratamento M1.

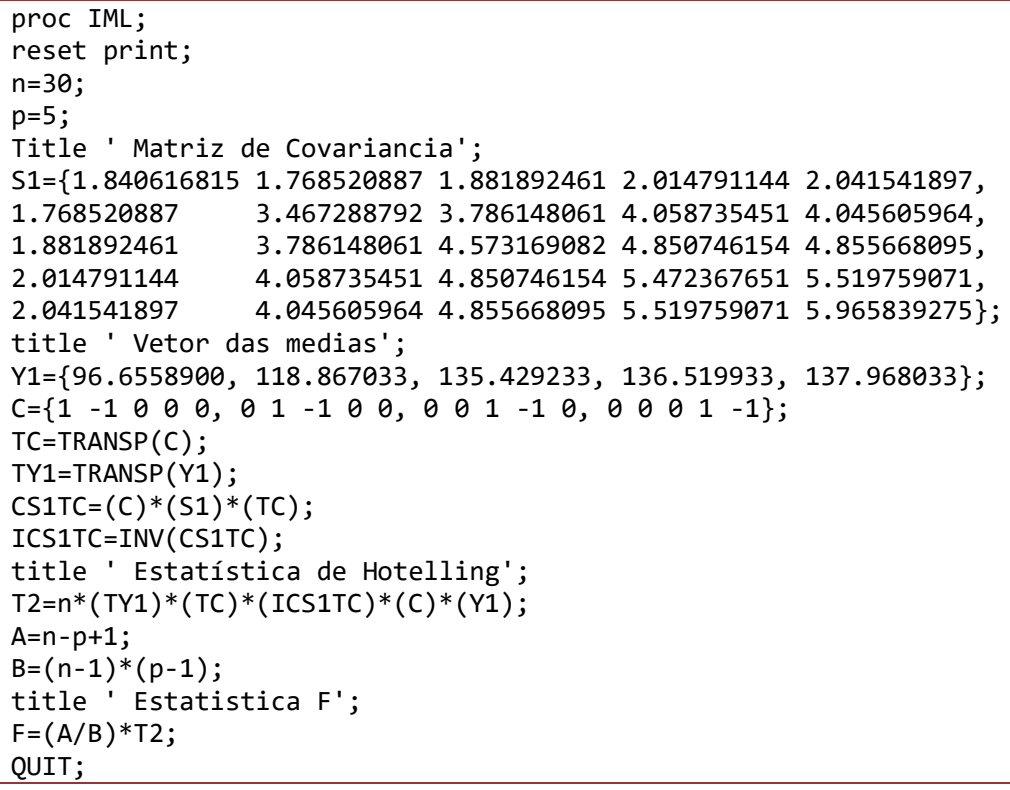

Tabela C6 - Análise de inventários florestais com parcelas permanentes considerando dois tratamentos M1 e M2.

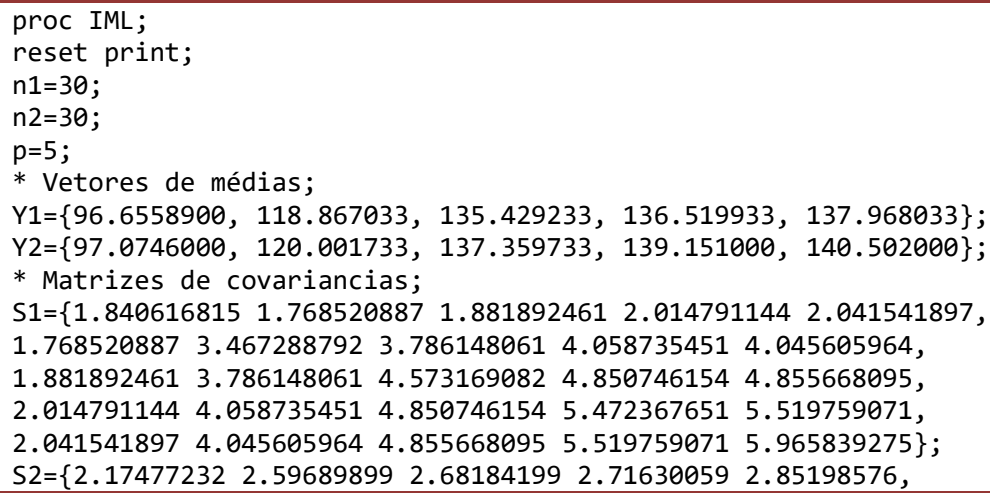




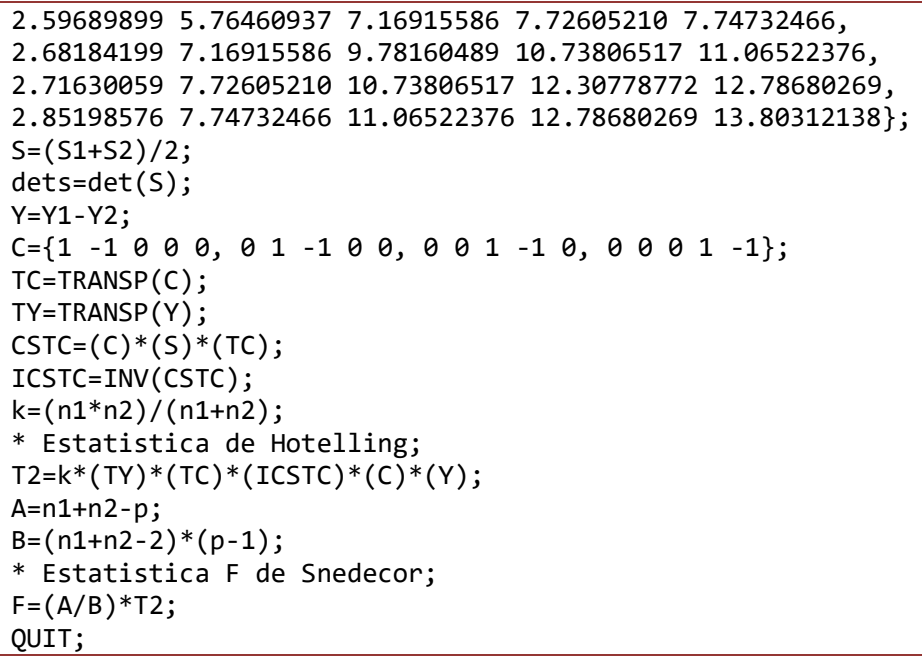

Tabela C7 - Análise da homogeneidade de matrizes de covariâncias dos métodos de manejo M1, M2, M3 e M4.

title 'TESTE DE HOMOGENEIDADE DE MATRIZES DE COVARIANCIA';

data MM;

input MMF \$ OC1 OC2 OC3 OC4 OC5;

cards;

$\begin{array}{llllll}\text { M1 } & 94.6152 & 117.854 & 134.403 & 136.058 & 136.519 \\ \text { M1 } & 96.0525 & 117.628 & 133.247 & 134.909 & 135.398 \\ \text { M1 } & 95.7847 & 118.546 & 136.020 & 137.251 & 139.248\end{array}$

DADOS TABELA B1 DO APÊNCICE

$\begin{array}{llllll}\text { M4 } & 98.800 & 131.947 & 143.215 & 149.599 & 149.927 \\ \text { M4 } & 98.891 & 132.498 & 142.649 & 148.547 & 148.808 \\ \text { M4 } & 98.736 & 134.371 & 146.193 & 154.098 & 154.887\end{array}$

ods html file="MM";

ods graphics on;

proc discrim method=normal pool=test wcov;

var OC1 OC2 OC3 OC4 OC5;

class MMF;

run;

ods html close;

run;

quit;

\section{Tabela C8 - Análise de perfil de M1, M2, M3 e M4.}

data PERFILMUL (keep = MM OC1 OC2 OC3 OC4 OC5)

ANAUNI (keep = MM OCASIAO VOL);

input MM \$ OC1 OC2 OC3 OC4 OC5;

output PERFILMUL;

VOL $=$ OC1; OCASIAO = 'OC1'; output ANAUNI;

VOL $=$ OC $2 ;$ OCASIAO $=$ 'OC2'; output ANAUNI;

$\mathrm{VOL}=$ OC 3 ; OCASIAO $=$ 'OC3'; output ANAUNI;

$\mathrm{VOL}=$ OC4; OCASIAO = 'OC4'; output ANAUNI;

VOL $=$ OC5; OCASIAO $=$ 'OC5'; output ANAUNI; cards;

$\begin{array}{llllll}\text { M1 } & 94.6152 & 117.854 & 134.403 & 136.058 & 136.519 \\ \text { M1 } & 96.0525 & 117.628 & 133.247 & 134.909 & 135.398 \\ \text { M1 } & 95.7847 & 118.546 & 136.020 & 137.251 & 139.248\end{array}$




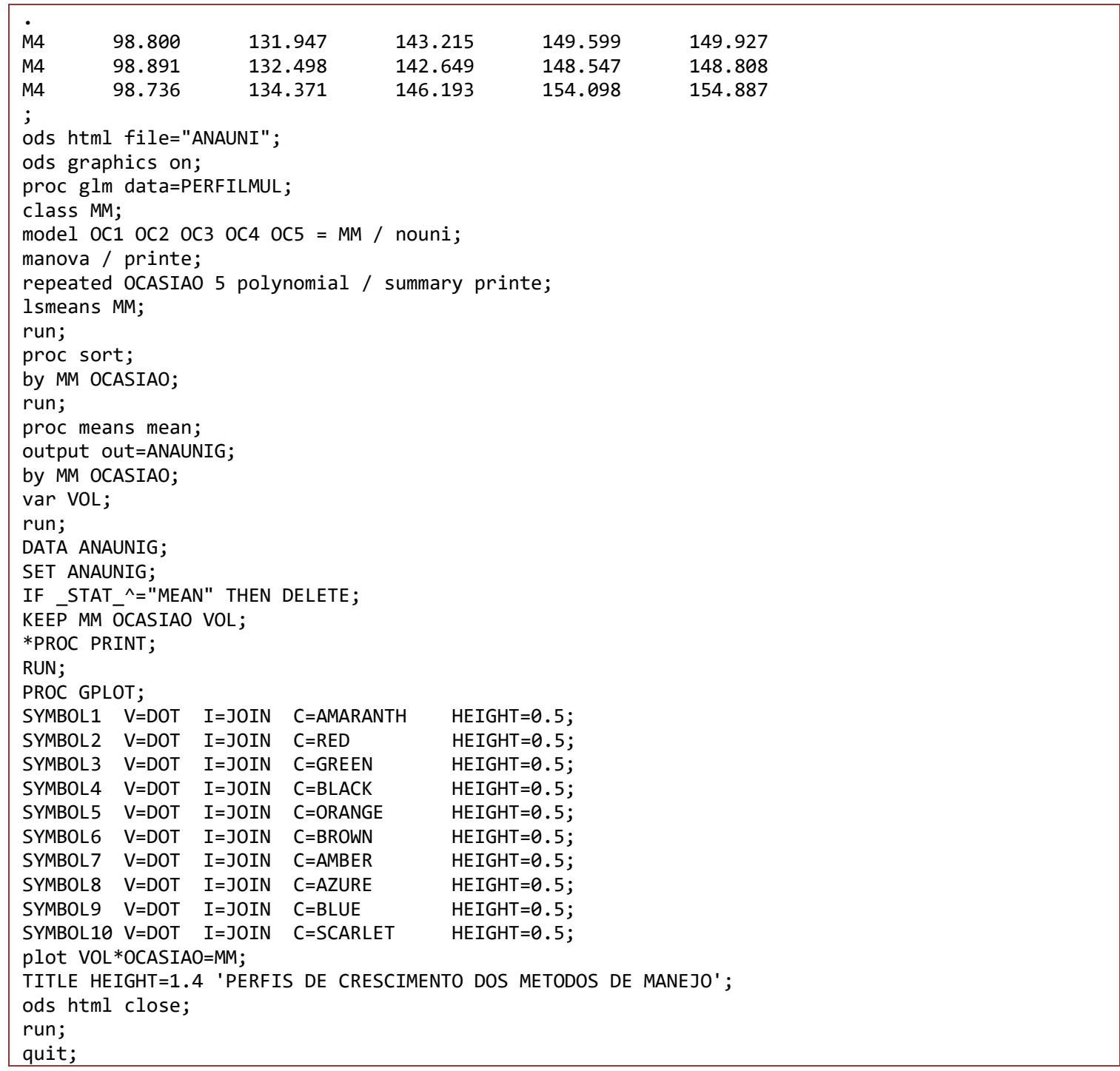

\section{Tabela C9 - Análise agrupamento pelo método de Ward para M1, M2, M3 e M4.}

\section{Programa 1: Usado o Proc IML.}

proc IML;

reset print;

Title ' $A=M 1, B=M 2, C=M 3, D=M 4$ ' ;

$A=\{96.6558900,118.867033,135.429233,136.519933,137.968033\}$;

$B=\{97.0746000,120.001733,137.359733,139.151000,140.502000\}$;

$C=\{96.7343267,130.933367,142.368600,149.356333,150.126967\}$;

$D=\{97.1765333,131.251267,143.126200,150.286700,150.970800\}$;

$C 1=(5 * 5) /(5+5)$;

$\mathrm{DAB}=(\mathrm{A}-\mathrm{B}) *(\mathrm{~A}-\mathrm{B})$;

$D A C=(A-C) *(A-C)$;

$\mathrm{DAD}=(\mathrm{A}-\mathrm{D}) * *(A-D)$;

$\mathrm{DBC}=(\mathrm{B}-\mathrm{C}) \cdot *(\mathrm{~B}-\mathrm{C})$;

$\mathrm{DBD}=(\mathrm{B}-\mathrm{D})^{*} *(\mathrm{~B}-\mathrm{D})$;

$\mathrm{DCD}=(\mathrm{C}-\mathrm{D}) \cdot *(\mathrm{C}-\mathrm{D})$;

$M A B=(A+B) / 2$;

$M C D=(C+D) / 2$;

$M=M A B-M C D$;

$\mathrm{C} 1=(5 * 5) /(5+5)$;

$\mathrm{C} 4=(10 * 10) /(10+10)$;

$\mathrm{DABCD}=(C 4 / C 1) *\left(M^{-} * M\right)$;

$\mathrm{SQCD}=(C-M C D)^{*} *(C-M C D)+(D-M C D)^{*} *(D-M C D)$;

$S Q A B=(A-M A B)^{*} *(A-M A B)+(B-M A B)^{*} *(B-M A B)$;

$M T=(A+B+C+D) / 4$;

$\operatorname{SQABCD}=(A-M T) * *(A-M T)+(B-M T) \cdot *(B-M T)+(C-M T)^{`} *(C-M T)+(D-M T)^{`} *(D-M T)$;

$\mathrm{SQABCDF}=(A-M T) *(A-M T)+(B-M T) * *(B-M T)+(C-M T) * *(C-M T)+(D-M T) * *(D-M T)-S Q C D-S Q A B$;

QUIT; 


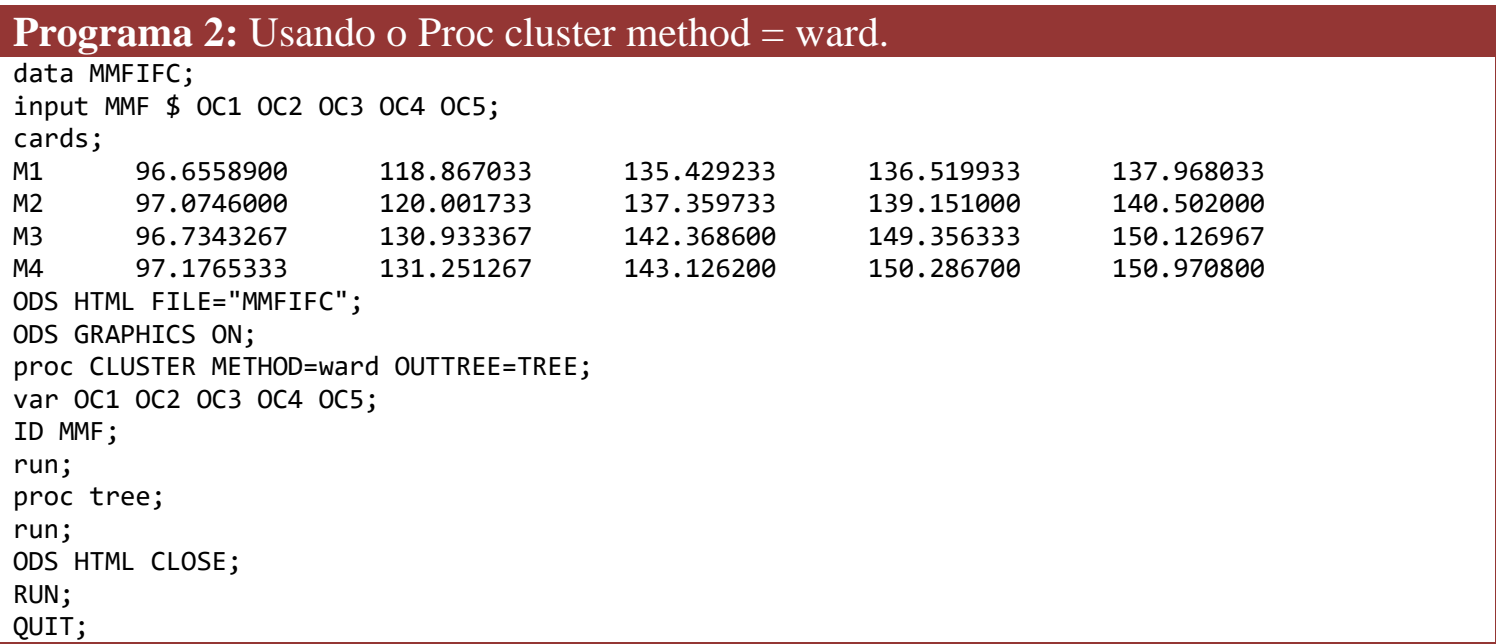

\section{Tabela C10 - Coincidência de perfis, pelo teste " $t$ " de Student, entre M3 e M4 (Proc} IML).

proc IML;

reset print;

$\mathrm{n} 2=30$;

$\mathrm{n} 3=30$;

YM3 $=\{96.7343267, \quad 130.933367,142.368600,1149.356333,150.126967\}$;

YM4 $=\{97.1765333, \quad 131.251267, \quad 143.126200, \quad 150.286700, \quad 150.970800\}$;

$\mathrm{S} 3=\left\{\begin{array}{llllll}1.71308108 & 1.14810639 & 0.69754194 & 0.25827404 & -0.09560080,\end{array}\right.$

1.148106393 .475915693 .965635704 .173057673 .99328305 ,

$0.69754194 \quad 3.96563570 \quad 5.563374596 .492485596 .73230947$,

$0.25827404 \quad 4.173057676 .492485598 .462977479 .04132329$,

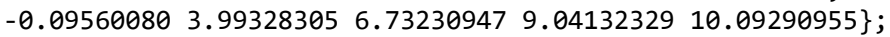

$\mathrm{S} 4=\left\{\begin{array}{lllll}2.69608384 & 3.60369934 & 3.69112813 & 3.55789403 & 3.08094366 \text {, }\end{array}\right.$

3.603699346 .379707587 .239143677 .422766916 .54165978 ,

$\begin{array}{lllll}3.69112813 & 7.23914367 & 9.248033139 .87512234 & 9.14563949 \text {, }\end{array}$

$\begin{array}{llllll}3.55789403 & 7.42276691 & 9.87512234 & 11.19773456 & 10.70822456 \text {, }\end{array}$

$\begin{array}{lllll}3.08094366 & 6.54165978 & 9.14563949 & 10.70822456 & 10.75127134\} \text {; }\end{array}$

$\mathrm{J}=\{1,1,1,1,1\}$;

$\mathrm{TJ}=\operatorname{TRANSP}(\mathrm{J})$;

$A=\mathrm{TJ} *(\mathrm{YM} 4-\mathrm{YM} 3)$;

$\mathrm{S}=(\mathrm{S} 3+\mathrm{S} 4) / 2$;

$\mathrm{B}=(\mathrm{TJ} * \mathrm{~S} * \mathrm{~J})$;

$C=(1 / \mathrm{n} 2+1 / \mathrm{n} 3)$;

$\mathrm{D}=\mathrm{B} * \mathrm{C}$;

$\mathrm{E}=\mathrm{SQRT}(\mathrm{D})$;

* Estatistica $t$ de student;

$\mathrm{TT}=\mathrm{A} / \mathrm{E}$;

QUIT;

\section{Tabela C11- Teste de coincidência de perfis entre M3 e M4 (Proc GML).}

\section{data MMF43;}

input MMF \$ oc1 oc2 oc3 oc4 oc5;

cards;

$\begin{array}{llllll}\text { M3 } & 98.1902 & 132.620 & 143.707 & 150.691 & 151.215 \\ \text { M3 } & 96.1315 & 131.374 & 142.860 & 150.846 & 152.044 \\ \text { M3 } & 97.1961 & 130.957 & 141.585 & 148.872 & 149.666 \\ \text { M3 } & 98.1245 & 131.411 & 142.013 & 148.978 & 149.930 \\ \text { - } & & & & & \\ \text {. } & & & & \\ \text { M3 } & 95.5484 & 129.175 & 141.480 & 149.520 & 150.649 \\ \text { M3 } & 97.1212 & 127.784 & 138.000 & 145.102 & 145.242 \\ \text { M4 } & 98.079 & 132.939 & 145.355 & 152.622 & 153.402 \\ \text { M4 } & 96.861 & 128.322 & 138.876 & 145.122 & 146.096 \\ \text { M4 } & 96.411 & 129.530 & 140.862 & 147.055 & 146.907\end{array}$

98.800

131.947

143.215

149.599

149.927 


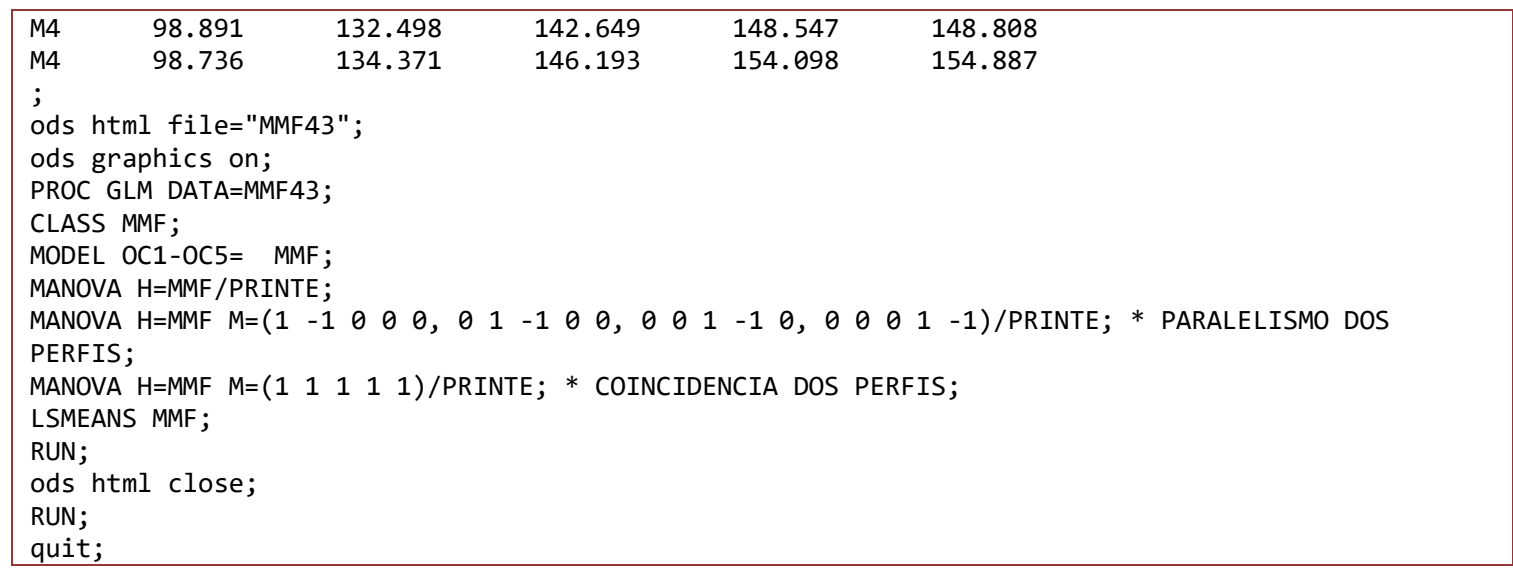

\section{Tabela C12 - Análise de regressão: Mitscherlich para M1, M2, M3 e M4.}

\section{DATA ANAPERFIL;}

INPUT MMF \$ OCASIAO VOL D1 D2 D3 D4;

CARDS;

$\begin{array}{lllllll}\text { M1 } & 0 & 94.615 & 1 & 0 & 0 & 0 \\ \text { M1 } & 0 & 96.053 & 1 & 0 & 0 & 0 \\ \text { M1 } & 0 & 95.785 & 1 & 0 & 0 & 0\end{array}$

DADOS ADAPTADOS DA TABELA B1 DO APÊNCICE B

$\begin{array}{lllllll}\text { M2 } & 0 & 95.524 & 0 & 1 & 0 & 0 \\ \text { M2 } & 0 & 95.183 & 0 & 1 & 0 & 0 \\ \text { M2 } & 0 & 98.479 & 0 & 1 & 0 & 0\end{array}$

$\begin{array}{lllllll}\text { M3 } & 0 & 98.190 & 0 & 0 & 1 & 0 \\ \text { M3 } & 0 & 96.132 & 0 & 0 & 1 & 0 \\ \text { M3 } & 0 & 97.196 & 0 & 0 & 1 & 0\end{array}$

$\begin{array}{lllllll}\text { M4 } & 0 & 98.079 & 0 & 0 & 0 & 1 \\ \text { M4 } & 0 & 96.861 & 0 & 0 & 0 & 1 \\ \text { M4 } & 0 & 96.411 & 0 & 0 & 0 & 1\end{array}$

$\begin{array}{lllllll}\text { M4 } & 20 & 149.927 & 0 & 0 & 0 & 1 \\ \text { M4 } & 20 & 148.808 & 0 & 0 & 0 & 1 \\ \text { M4 } & 20 & 154.887 & 0 & 0 & 0 & 1\end{array}$

ods html file="anaperfil";

ods graphics on;

PROC nlin Method=gauss;

Parms b0 $=144$ b1 $=0.05122$ b2 $=7.5$;

model $\mathrm{VOL}=\mathrm{b} 0 *(1-10 * *(-\mathrm{b} 1 *($ OCASIAO $+\mathrm{b} 2)))$;

output out $=p$ p=VOLE $r=$ resid;

run;

quit;

proc reg;

model VOLE=VOL;

test $\mathrm{VOL}=1$, intercept $=0$;

run;

proc corr;

Var VOLE VOL;

run;

QUIT;

PROC nlin Method=gauss;

Parms b0 $=144$ b1 $=0.05122$ b2 $=7.5$;

model $\mathrm{VOL}=\mathrm{b} 0 *(1-10 * *(-\mathrm{b} 1 *($ OCASIAO $+\mathrm{b} 2)))$; 


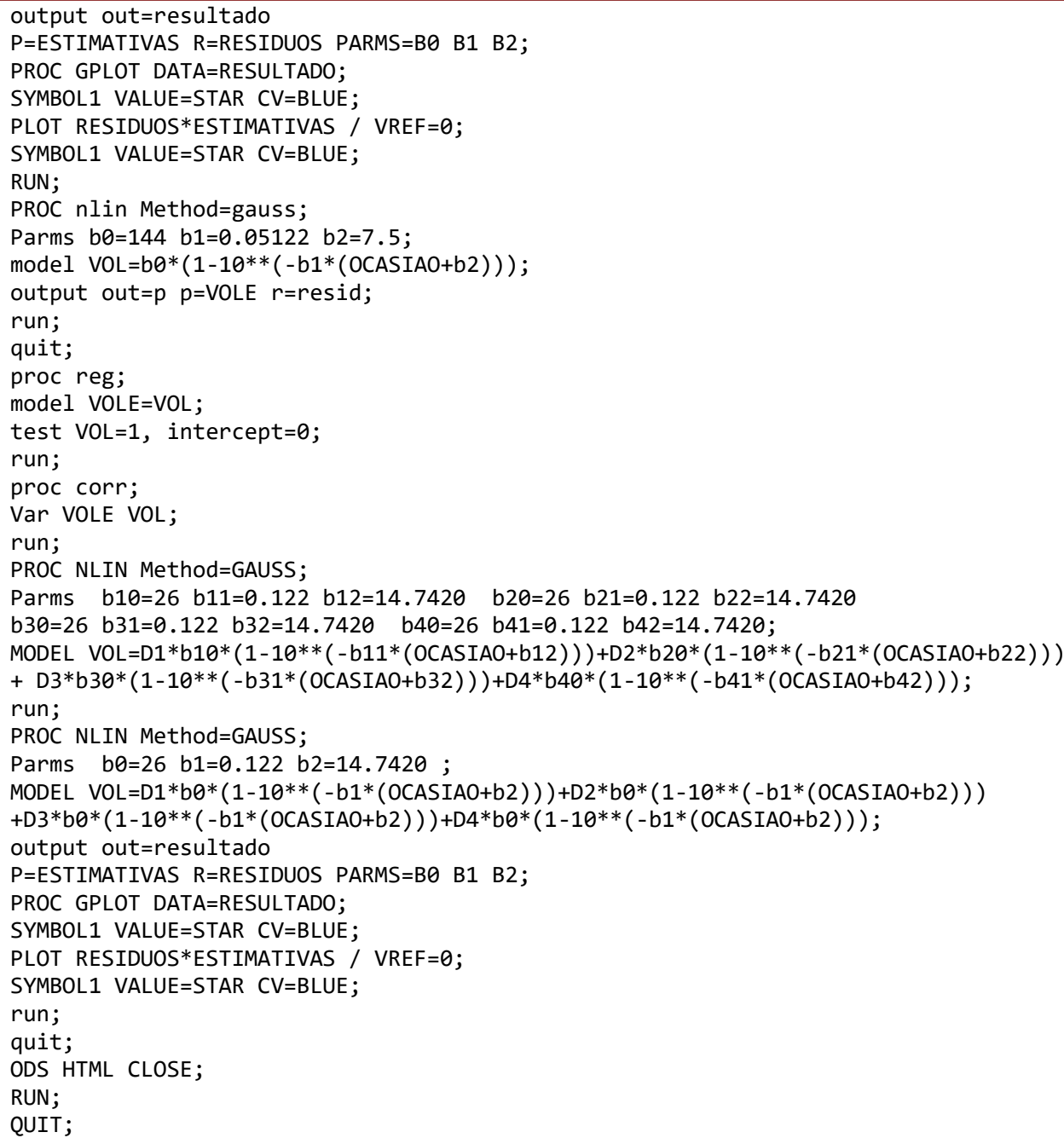

\section{Tabela C13 - Análise de regressão: Mitscherlich para M3 e M4.}

DATA ANAPERFIL;

INPUT MMF \$ OCASIAO VOL D3 D4; CARDS;

$\begin{array}{lllll}\text { M3 } & 0 & 98.190 & 1 & 0 \\ \text { M3 } & 0 & 96.132 & 1 & 0 \\ \text { M3 } & 0 & 97.196 & 1 & 0 \\ \text {. } & & & & \\ \text {. } & & & & \\ \text { M3 } & 20 & 149.930 & 1 & 0 \\ \text { M3 } & 20 & 150.649 & 1 & 0 \\ \text { M3 } & 20 & 145.242 & & 1 \\ \text { M4 } & 0 & 98.079 & 0 & 1 \\ \text { M4 } & 0 & 96.861 & 0 & 1 \\ \text { M4 } & 0 & 96.411 & 0 & 1\end{array}$

$\begin{array}{lllll}\text { M4 } & 20 & 149.927 & 0 & 1 \\ \text { M4 } & 20 & 148.808 & 0 & 1 \\ \text { M4 } & 20 & 154.887 & 0 & 1\end{array}$

ods html file="anaperfil";

ods graphics on;

*MODELO COMPLETO OMEGA*

title1 'MODELO COMPLETO OMEGA';

PROC NLIN Method=GAUSS;

Parms b30 $=26$ b31 $=0.122$ b32 $=14.7420$

b40 $=26$ b41 $=0.122$ b42 $=14.7420$;

MODEL VOL $=$ D $3 * b 30 *(1-10 * *(-b 31 *($ OCASIAO $+b 32)))$ 


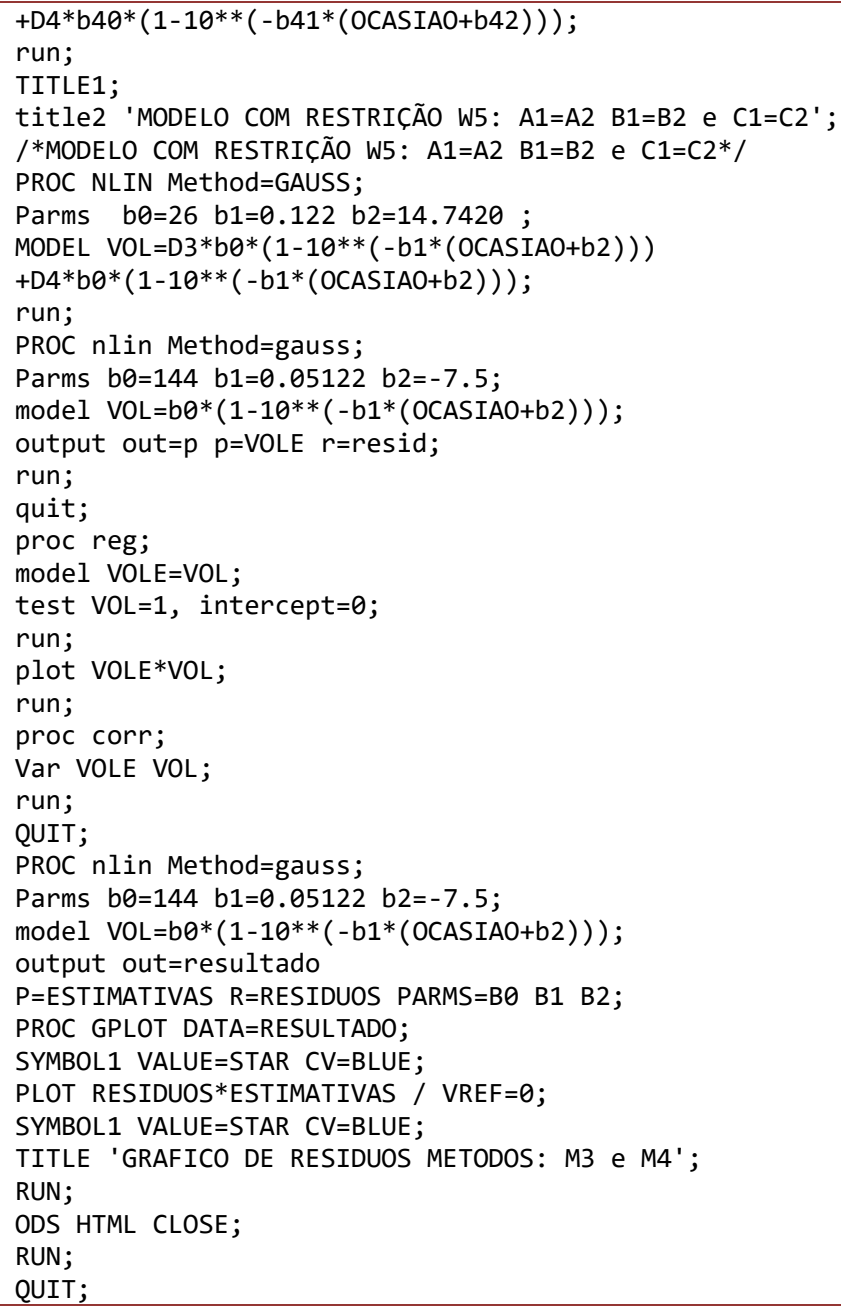

\section{Tabela C14 - Análise de componentes principais e de fatores para as espécies E1, E2,}

\section{E3 e E4.}

data especie;

input label \$ esp \$ oc1 oc2 oc3 oc4 oc5;

cards;

$\begin{array}{lllllll}A & \text { E1 } & 9.9190 & 14.5882 & 17.2448 & 19.5898 & 21.1701 \\ A & \text { E1 } & 9.6132 & 14.4511 & 17.1552 & 19.6230 & 21.2862 \\ A & \text { E1 } & 9.7196 & 14.4053 & 16.9914 & 19.4964 & 20.9532\end{array}$

DADOS DA TABELA B2 (APÊNDICE B)

-

$\begin{array}{lllllll}D & \text { E4 } & 9.5516 & 13.1183 & 16.3292 & 18.1055 & 19.5920 \\ \text { D } & \text { E4 } & 9.6356 & 13.1567 & 16.3848 & 18.0850 & 19.5384 \\ \text { D } & \text { E4 } & 9.6177 & 13.1557 & 16.4315 & 18.1016 & 19.6434\end{array}$

ods html file="ESPECIE";

ods graphics;

proc princomp COV out=prin;

var oc1 oc2 oc3 oc4 oc5;

run;

proc plot;

plot prin $2 *$ prin $1=L A B E L / v p o s=14$ hpos $=60$;

title1'A=E1 B=E2 C=E3 D=E4';

run;

proc factor nfactors $=2$ mineigen=0.01 rotate=varimax converge=0.01 out=prova3 all;

var OC1 OC2 OC3 OC4 OC5;

run; 
proc print data=prova3;

proc plot;

plot factor $2 *$ factor $1=\mathrm{LABEL} / \mathrm{vpos}=14 \mathrm{hpos}=60$;

run;

ODS HTML CLOSE;

RUN;

QUIT;

\section{Tabela C15 - Análise discriminante para as espécies E1, E2, E3 e E4.} data ESPECIE;

input LABEL \$ ESP \$ OC1 OC2 OC3 OC4 OC5; cards;

\begin{tabular}{|c|c|c|c|c|c|c|}
\hline A & $\mathrm{E} 1$ & 9.7922 & 14.5592 & 17.2715 & 19.5423 & 21.1399 \\
\hline$A$ & E1 & 9.5406 & 14.6071 & 17.4316 & 19.8951 & 21.6072 \\
\hline A & E1 & 9.5795 & 14.2997 & 16.7450 & 18.9016 & 20.5134 \\
\hline • & $S$ DA & ELA B2 & ICE B & & & \\
\hline A & E1 & 9.9125 & 14.4552 & 17.0416 & 19.4973 & 20.9902 \\
\hline$A$ & E1 & 9.5549 & 14.2093 & 16.9788 & 19.4376 & 21.0909 \\
\hline$A$ & E1 & 9.7121 & 14.0573 & 16.6800 & 18.8633 & 20.3339 \\
\hline B & E2 & 9.6041 & 14.2771 & 17.0975 & 19.5989 & 21.3576 \\
\hline B & E2 & 9.7495 & 14.5111 & 17.3431 & 19.7522 & 21.4108 \\
\hline B & E2 & 9.5771 & 14.2691 & 17.2045 & 19.7269 & 21.4822 \\
\hline . & & & & & & \\
\hline - & & & & & & \\
\hline$\dot{B}$ & E2 & 9.9900 & 14.5142 & 17.1858 & 19.4479 & 20.9898 \\
\hline B & $\mathrm{E} 2$ & 9.9991 & 14.5749 & 17.1179 & 19.4411 & 20.9873 \\
\hline B & E2 & 9.9736 & 14.7808 & 17.5432 & 20.0329 & 21.6996 \\
\hline C & E3 & 9.4292 & 12.6444 & 15.5354 & 16.8470 & 18.4463 \\
\hline C & E3 & 9.6296 & 13.1604 & 16.2383 & 17.6138 & 19.1272 \\
\hline C & E3 & 9.6999 & 13.4342 & 16.8152 & 18.3470 & 19.8712 \\
\hline . & & & & & & \\
\hline - & & & & & & \\
\hline$c$ & & & & & & \\
\hline C & E3 & 9.5750 & 13.1667 & 16.1946 & 17.6748 & 19.3199 \\
\hline C & E3 & 9.6992 & 13.2326 & 16.4492 & 18.1887 & 19.9773 \\
\hline C & E3 & 9.7571 & 13.1264 & 16.1063 & 17.5717 & 19.1409 \\
\hline D & E4 & 9.7457 & 13.3619 & 16.7033 & 18.3218 & 19.8997 \\
\hline D & E4 & 9.9106 & 13.1446 & 16.3952 & 18.0757 & 19.5037 \\
\hline D & E4 & 9.7650 & 13.4914 & 16.7759 & 18.3773 & 19.8661 \\
\hline . & & & & & & \\
\hline - & & & & & & \\
\hline$\cdot$ & & & & & & \\
\hline D & E4 & 9.5516 & 13.1183 & 16.3292 & 18.1055 & 19.5920 \\
\hline D & E4 & 9.6356 & 13.1567 & 16.3848 & 18.0850 & 19.5384 \\
\hline D & $\mathrm{E} 4$ & 9.6177 & 13.1557 & 16.4315 & 18.1016 & 19.6434 \\
\hline ; & & & & & & \\
\hline & rint & & & & & \\
\hline & & & & & & \\
\hline & LABE & ESP \$ & $C 20 C 30$ & & & \\
\hline$A$ & E1 & 9.9190 & 14.5882 & 17.2448 & 19.5898 & 21.1701 \\
\hline$A$ & E1 & 9.6132 & 14.4511 & 17.1552 & 19.6230 & 21.2862 \\
\hline A & $\mathrm{E} 1$ & 9.7196 & 14.4053 & 16.9914 & 19.4964 & 20.9532 \\
\hline - & & & & & & \\
\hline - & & & & & & \\
\hline$\dot{A}$ & & & & & & \\
\hline A & E1 & 9.9092 & 14.5695 & 17.2364 & 19.6196 & 21.1014 \\
\hline A & $\mathrm{E} 1$ & 9.5969 & 14.2523 & 17.1020 & 19.4376 & 21.1085 \\
\hline$A$ & E1 & 9.9141 & 14.4687 & 17.0701 & 19.2062 & 20.7192 \\
\hline B & $\mathrm{E} 2$ & 9.9079 & 14.6233 & 17.4426 & 19.8409 & 21.4763 \\
\hline B & E2 & 9.6961 & 14.2254 & 16.7971 & 18.8659 & 20.4534 \\
\hline B & E2 & 9.6411 & 14.2483 & 16.9154 & 19.1172 & 20.5670 \\
\hline - & & & & & & \\
\hline - & & & & & & \\
\hline - & & & & & & \\
\hline B & E2 & 9.5907 & 14.3473 & 17.0399 & 19.4962 & 20.7164 \\
\hline B & E2 & 9.9957 & 14.7987 & 17.7553 & 20.2747 & 21.9205 \\
\hline B & E2 & 9.5472 & 14.3241 & 17.0567 & 19.3954 & 21.0087 \\
\hline C & E3 & 9.4615 & 12.9749 & 16.1284 & 17.6877 & 19.1127 \\
\hline C & E3 & 9.6053 & 12.9392 & 15.9896 & 17.5382 & 18.9559 \\
\hline
\end{tabular}




\begin{tabular}{|c|c|c|c|c|c|c|}
\hline \multicolumn{7}{|l|}{$\cdot$} \\
\hline C & E3 & 9.7996 & 13.2271 & 16.3649 & 17.8770 & 19.4739 \\
\hline C & E3 & 9.7433 & 13.1559 & 16.3104 & 17.8900 & 19.3148 \\
\hline C & E3 & 9.6975 & 12.7534 & 16.0219 & 17.4582 & 19.1248 \\
\hline D & E4 & 9.5524 & 13.1315 & 16.7756 & 18.3776 & 20.1172 \\
\hline D & E4 & 9.5193 & 12.7526 & 15.8471 & 17.2569 & 18.8044 \\
\hline D & E4 & 9.9479 & 13.5956 & 17.1593 & 18.9153 & 20.7199 \\
\hline \multicolumn{7}{|l|}{. } \\
\hline D & E4 & 9.6555 & 13.2791 & 16.5518 & 18.2055 & 19.6482 \\
\hline D & E4 & 9.4914 & 12.7524 & 15.7908 & 17.3419 & 18.7244 \\
\hline D & E4 & 9.6649 & 12.8986 & 15.9631 & 17.2964 & 18.7620 \\
\hline \multicolumn{7}{|c|}{$\begin{array}{l}\text {; } \\
\text { ODS HTML FILE="ESPECIE"; } \\
\text { ODS GRAPHICS ON; } \\
\text { proc print; } \\
\text { proc discrim method=normal out=SAIESP data= ESPECIE testdata=ESPCLAS testlist; } \\
\text { var OC1 OC2 OC3 OC4 OC5; } \\
\text { class ESP; } \\
\text { proc plot data=saiesp; } \\
\text { plot oc } 1 * \text { oc } 5=1 \text { abel/vpos }=14 \text { hpos=60; } \\
\text { proc print data=SAIESP; } \\
\text { ODS HTML CLOSE; } \\
\text { run; }\end{array}$} \\
\hline
\end{tabular}

Tabela C16 - Análise de correlação canônica considerando os grupos métodos de manejo (M1, M2, M3, M4) versus o grupo das espécies (E1, E2, E3, E4).

data ACCAP;

input LABEL\$ ESP \$ OCE1 OCE2 OCE3 OCE4 OCE5 MMF \$ OCM1 OCM2 OCM3 OCM4 OCM5 @@; cards;

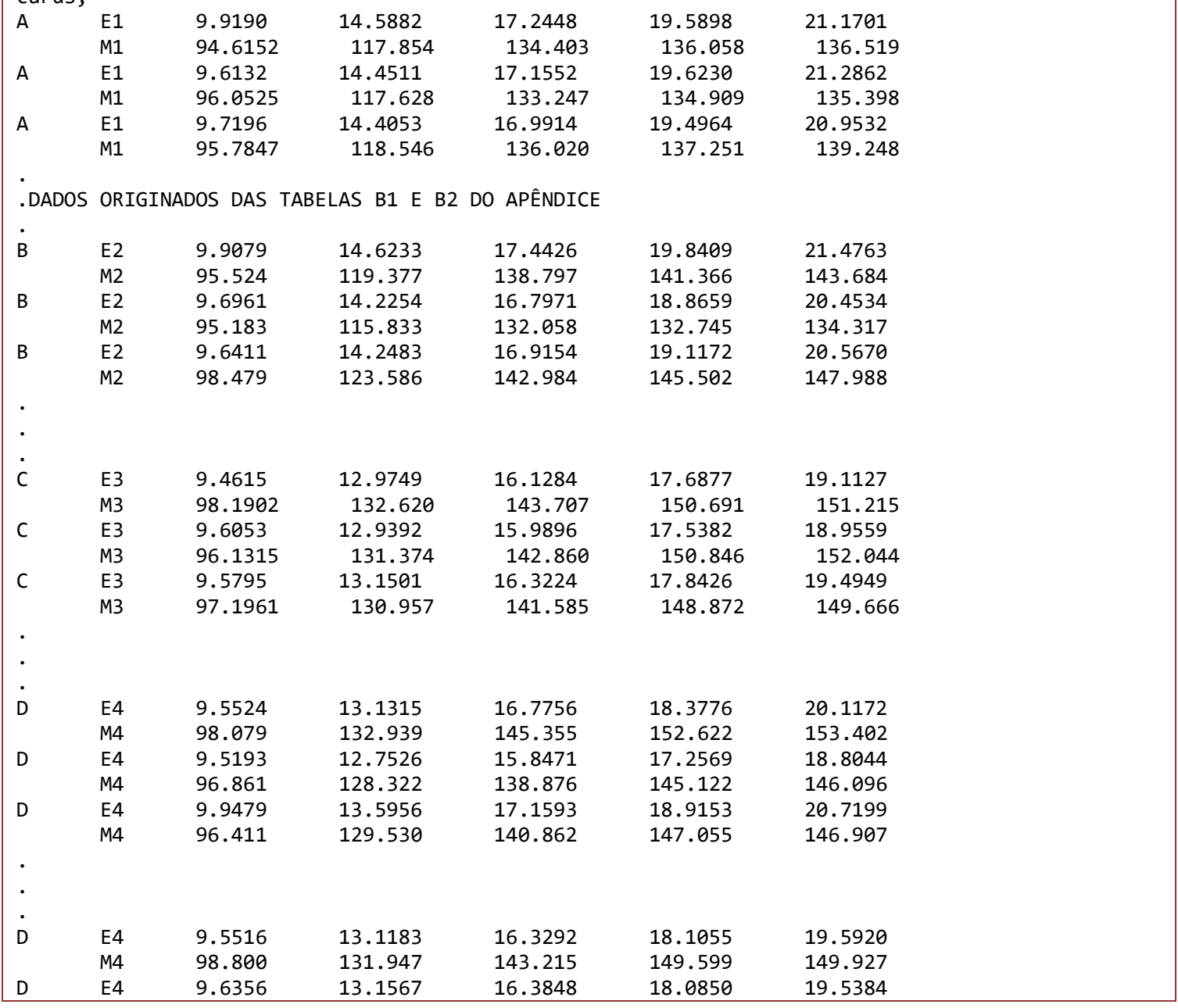


Análise Multivariada em Inventário Florestal Contínuo

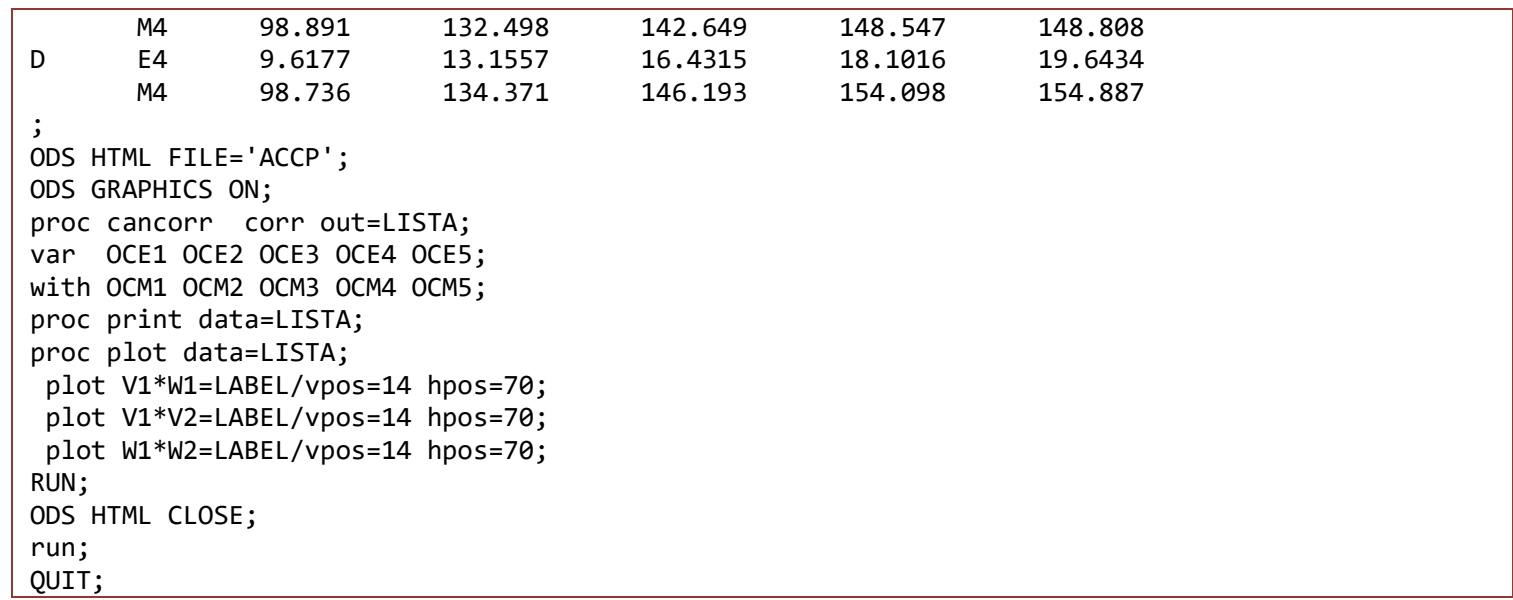




\section{www.poisson.com.br contato@poisson.com.br}

@editorapoisson 